\title{
THE HUMAN MECHANISM
}

PHYSIOLOGY, HYGIEN AND

$$
\text { SANITATION }
$$

\section{HOUGH AND SEDGWICK}




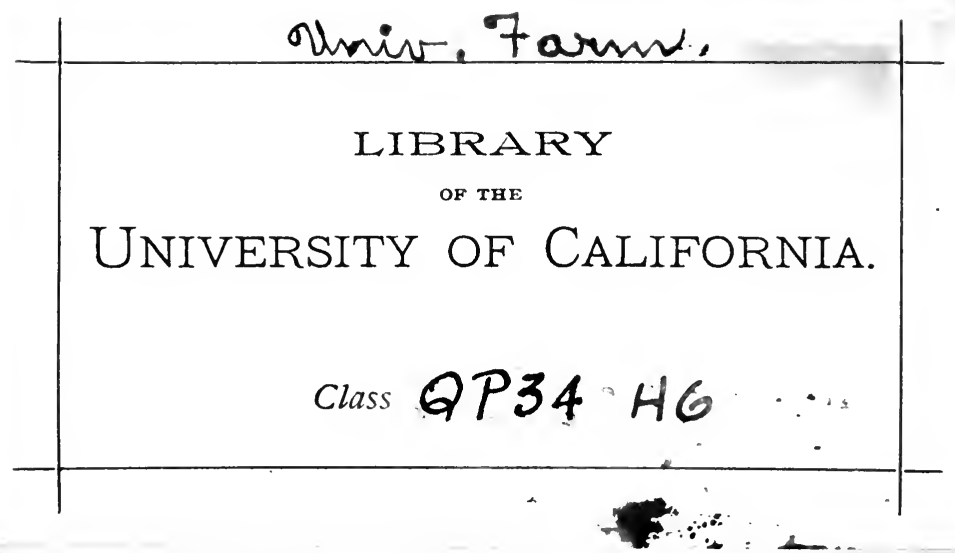

$\gamma$ m 



\section{THE HUMAN MECHANISM}

ITS PHYSIOLOGY AND HYGIENE AND THE SANITATION OF ITS SURROUNDINGS

BY

\section{THEODORE HOUGH ANd WILLIAM T. SEDGWICK}

Professor of Physiology in the University of Virginia; sometime Instructor in Fersonal Hygiene, Boston Normal School of Gymnastics
Professor of Biology in the Massachusetts Institute of Technology; Author of "Principles of Sanitary Science and Public Health," etc.

The fundamental conception of the living body as a physical mechanism . . . is the distinctive feature of modern as contrasted with ancient physiology

HuXLEY,

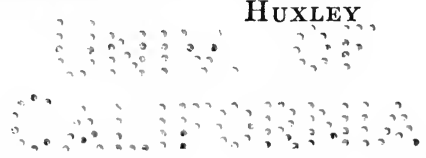

\section{GINN \& COMPANY}




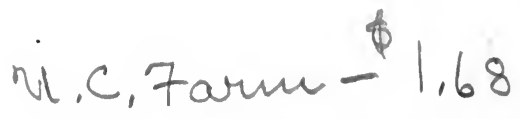

Entered at Stationers' Hall

COPYRIGHT, 1906, BY

Theodore Hough and William T. Sedgwick

ALL RIGHTS RESERVED

69.12

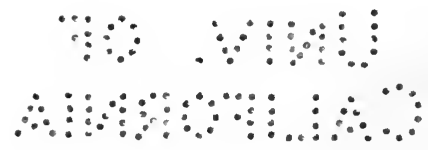

Cbe Atbenaum Press 


\section{PREFACE}

The authors of this work believe that extensive and fundamental changes must be made in the elementary teaching of physiology, hygiene, and sanitation, if these subjects are ever to occupy in the curriculum of education the place which their intrinsic importance requires. This text-book is a contribution toward effecting these changes, and has been prepared both as a demonstration of what the authors believe to be needed and as a practical aid toward securing the end in view.

The health and efficiency of the human body have rarely, if ever, been more highly esteemed than they are to-day, and yet no subject of similar importance is so generally neglected in the schools, or, when taught, taught less effectively. Several causes have contributed to this curious state of things, but undoubtedly one of the most important is that the teaching has been too largely anatomical and too remotely connected with the activities and problems of daily life.

In the present text-book, anatomy has been reduced to its lowest terms and microscopic anatomy or histology touched upon only so far as seemed absolutely necessary. Space has thus been gained for more physiology and, especially, for more hygiene than is usual, and also for the elements of sanitation, - a new and comparatively easy subject, but one of the very first importance in all wholesome modern living.

That point of view which regards the human body as a living mechanism is to-day not only the sure foundation iii

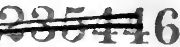


of physiology, hygiene, and sanitation, but is also surprisingly helpful in the solution of many questions concerned with intellectual and moral behavior. This view, therefore, we have not hesitated to expound and emphasize. Avoiding that form of physiology which looks chiefly at the organs and overlooks the organism, we have constantly kept in view the body as a whole, in order that physiology may become the interpreter of the common physical phenomena of the daily life and find in hygiene and sanitation its natural application to conduct.

We believe with Matthew Arnold that "conduct is three fourths of life," and that this is no less true of the physical than of the moral and the intellectual life. We therefore make no apology for fixing upon conduct as the keynote of this work, and the right conduct of the physical life as the principal aim and end of all elementary teaching of physiology, hygiene, and sanitation.

In those portions of the book devoted to public hygiene and sanitation the authors have kept in view the importance of this subject in all education for good citizenship. Sanitary science and the public health can be advanced only as they are supported by an intelligent public opinion, which appreciates the nature of the problems involved, the frequent duty of subordinating personal liberty to the public good, and the importance of rendering hearty support to public officials in the discharge of difficult and often delicate tasks.

It has not seemed wise to include in the text extensive directions for laboratory work. The opportunities and facilities for such work vary to such an extent in different schools that the largest discretion must here be left to the teacher. Many demonstrations and experiments described or referred to can, however, easily be performed, when advisable, with comparatively little trouble or expense. 
We are greatly indebted to Professor Werner Spalteholz for his kind permission to copy certain figures from his Hand Atlas of Anatomy, ${ }^{1}$ a book which we recommend as a most useful reference work for anatomical study. Our acknowledgments are also due to Professor Schottelius, from whom we have taken many of the figures of bacteria given in Part II. In the preparation of most of the original figures we have had the assistance of Dr. Percy G. Stiles, whose skill as a draftsman, combined with his appreciation, as a physiologist, of our point of view, has greatly facilitated this part of our work.

The index has been prepared with special reference to its use as a glossary of anatomical terms.

1 Werner Spalteholz, Hand Atlas of Human Anatomy; translated by L. F. Barker. G. E. Stechert, New York. 
Digitized by the Internet Archive in 2007 with funding from Microsoft Corporation 


\section{CONTENTS}

\section{PART I. PHYSIOLOGY}

Chapter

PAGE

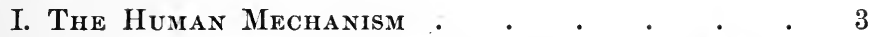

II. The Structure (Anatomy) of the Human MechANISM .

III. The Finer Structure of Two Typical Organs, Glands and Muscles. The Connective Tissues. The Lymphatic System • • .

IV. The Work of the Human Mechanism the ReSUltant of the Work of Its Different ORGans AND Cells . . . . . . . . 44

V. Work, Fatigue, and Restoration . • . . 55

VI. The Interdependence of Organs and of Cells 65

VII. The Adjustment or Coördination of the Work of Organs and Cells . . . . . . 70

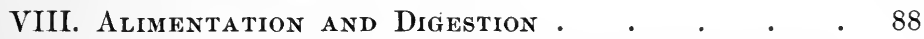

The Supply of Matter and Power to the Human Machine . . . . . . . . $\quad$. 88

Digestion in the Mouth. The Teeth. Enzymes . 98

Digestion in the Stomach . . . . . 106

Digestion and Absorption in the Small Intestine and

IX. The Circulation of the Blood . $\quad . \quad$. 132

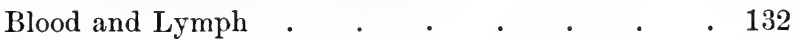

Mechanics of the Circulation of the Blood and of the

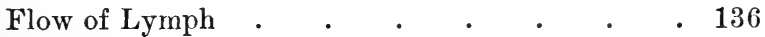

The Adjustment of the Circulation to the Needs of

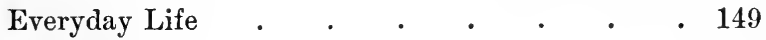

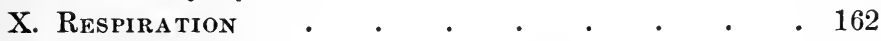

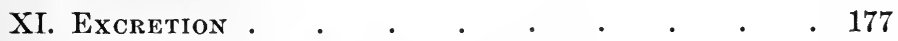

XII. Thermal Phenomena of the Body • • $\quad 187$

The Constant Temperature . . . . . 187

The Regulation of the Body Temperature $\quad$. 199 
The Sources of the Power and Heat of the Human

Mechanism . . . . . . . . . 211

Special Effects of the Different Nutrients . . 217

Flesh, Fat, and Glycogen · • • • • • 227

The Choice of Food and Nutrients . . . 233

XIV. Sense Organs And Sensations . . . . 244

XV. The Nervous System . . . . . . . . 266

Its Anatomical Basis . $\quad . \quad$. $\quad . \quad$. $\quad .266$

The Physiology of the Nervous System . . . 273

PART II. THE HYGIENE OF THE HUMAN MECHANISM AND THE SANITATION OF ITS SURROUNDINGS

XVI. INTRODUCTORY .

Hygiene the Right Use and Proper Care of the Human Mechanism . . . . . . . 291

Health and Disease . . . . . . . 293

The Three Great Factors of Disease . 296

\section{Personal Hygiene}

XVII. Muscular Activity 304

The Ministry of Muscular Activity to the Body as a

Whole

General Muscular Exercise

Muscular Exercises for Special Purposes. Corrective

Work. The Gymnasium . . . . . 321

XViII. The Hygiene of the Nervous System. Rest AND Sleep. • • • • • • • • 334

XiX. The Hygiene of Feeding . . . . . 347

XX. Food Accessories, Drugs, Alcohol, and Tobacco 357

XXI. The Prevention and Care of Colds and Some

Other INflammations . . . . . . 380

XXII. The Care of the Eyes and Ears . . . 395 
Chapter

XXiII. The Hygiene of the Feet . . • . 403

XXIV. Bathing $. \quad . \quad . \quad . \quad . \quad . \quad . \quad .413$

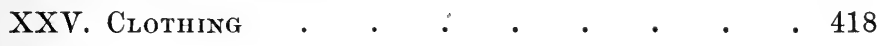

Domestic Hygiene and Sanitation

XXVI. The House: Its Site, Construction, FurnishINGS, AND CARE . . . . . . . 425

XXVII. The Warming and Lighting of the House . 434

XXVIII. The Air Supply of the House. Ventilation 442

XXiX. The Water Supply, Plumbing, and Drainage of the House. Garbage and Rubbish • 451

\section{Public Hygiene and Sanitation}

XXX. Public Health. Infectious and Contagious Diseases. Microbes . . . . . 463

XXXI. Some Microbic Diseases and their Prevention. Vaccination and Antitoxic Serums 477 XXXII. Public Supplies of Food, Water, and Gas.

Public Sewerage. $\quad$ • . $\quad$. $\quad 505$

XXXiII. The Hygiene and Sanitation of Traveling, Public Conveyances, Public Houses, etc. 520 XXXiv. Public Protection of the Public Health . 529 XXXV. The Health of Nations . . . . . 535 



\title{
THE HUMAN MECHANISM
}

\author{
Part I
}

PHYSIOLOGY 



\section{CHAPTER I}

\section{THE HUMAN MECHANISM}

1. The Human Body a Living Organism. - The human body, as compared with bodies of water such as lakes and seas, or with heavenly bodies such as the sun, moon, and stars, is a small mass of matter weighing on the average, when fully grown, about $150 \mathrm{lb}$. and measuring in length about $5 \mathrm{ft} .9 \mathrm{in}$. It is neither very hot, as is the sun, nor warm in summer and cold in winter, as are many bodies of water, but in life and health has always almost exactly the same moderate temperature, namely, $98.6^{\circ} \mathrm{F}$. or $37.0^{\circ} \mathrm{C}$. The human body is not homogeneous, as is the substance of a lake, that is to say, alike in all its parts, but very unlike, the various parts - eyes, ears, legs, heart, brain, muscles, etc. - being known as organs, and the whole body, therefore, as the human organism.

The most remarkable peculiarity of the human body, however, is that it is a living organism. A watch has unlike parts - spring, dial, hands, case, etc. - which are essentially its organs, and the watch might, therefore, be called an organism; yet it never is so called. We speak of a well-organized army, navy, government, society, church, or school, but never of a well-organized automobile, typewriter, printing press, or locomotive, - apparently for the reason that in army, navy, or school living things play a principal part, while in mere machinery life is wholly wanting. The highest compliment we can pay to a machine is to say that it seems "almost alive," but it is not a compliment to any human being to describe him as " a mere 
machine." What the vitai property is, what we mean by the terms "life" and "living," no one can exactly tell. About all we know of it is that some of the commonest elements of matter - carbon, hydrogen, oxygen, and nitrogen, with a little sulphur, phosphorus, and a few other elements - frequently occur combined as living matter; and that this living matter has marvelous powers of growth, repair, and reproduction, besides a certain spontaneity, originality, and independence, which lifeless matter never displays. "While there is life there is hope" for any plant or any animal, but this saying does not apply to any lifeless machine, however complex or wonderful.

2. The Human Body a Living Machine or Mechanism. - By a machine we mean an apparatus, either simple or complex, and usually composed of unlike parts, by means of which power received in one form is given out or applied in some other form. This power may be received, for example, in the form of heat, or electricity, or muscular effort, or as the potential energy of fuel; and it may be given out as heat, or electricity, or light, or sound, or as mechanical work, or in any one of many other ways. One of the simplest of all machines is a stove, an apparatus composed of a few simple parts by means of which the potential energy or power of fuel - wood, coal, gas, or oil - is liberated and applied as heat, for warming or cooking. A lamp is a still simpler machine in which the potential energy or power of gas or oil is liberated and converted into useful light. A candle is a lamp so simple that it almost ceases to be a machine, and yet the wick is really an apparatus for securing proper combustion of wax or tallow to provide good light.

Machines of greater complexity are watches or clocks, pieces of apparatus composed of many unlike parts which receive power in comparatively large amounts for a short time during the process of winding, store it as potential 
energy in coiled springs or lifted weights, and liberate it slowly in the mechanical work of moving the hands of the timepiece over a dial. Still more complex is a locomotive or an automobile, machines in which the power of coal, oil, gasoline, or other fuel, or the electricity of a storage battery, is applied to swift locomotion. But the most wonderful of all machines is the human body, a complicated piece of apparatus in which the power stored in foods, such as starch, sugar, butter, meat, milk, eggs, and fish, is transformed into that heat by which the body is warmed, and into that muscular, nervous, digestive, or other work which it performs.

For delicate and intricate machinery the term "mechanism" is often employed, and we may therefore describe the human body either as the "human organism," or the "human machine," or, perhaps best of all, as the HUMAN MECHANISM.

The study and the science of the construction (structure) of this mechanism is called its anatomy; of its ordinary behavior, operation, or working, its physiology; of its proper management, protection, and care, its hygiene. This text-book is devoted chiefly to an account of its operation and care, i.e. to its physiology and hygiene; but as any true comprehension of these subjects depends upon some preliminary knowledge of the parts of the mechanism itself, we shall begin by considering briefly the structure or anatomy of the human machine. 


\section{CHAPTER II}

\section{THE STRUCTURE (ANATOMY) OF THE HUMAN MECHANISM}

Anatomy is studied partly by dissection, which reveals chiefly those organs which are visible to the naked eye, and partly by microscopic examination, which gives a deeper insight into the detailed arrangement of the cells and tissues of which the organs of the mechanism are composed. The present chapter is devoted to structures or organs shown by dissection, - the gross anatomy of the body, - as distinguished from its microscopic anatomy (histology). ${ }^{1}$

1 Further explanation of the structure of the human machine will be given as it may be needed in subsequent chapters. At this point it is of the utmost importance that the student thoroughly master the general relations of the more important organs one to another; this, however, is not to be done by extensive reading, and still less by memorizing verbal descriptions; the aim should rather be to acquire from figures and diagrams, or better yet from actual dissection, where that is possible, a correct mental picture of the structures involved. Far more can be learned by constructing drawings or diagrams from memory than by the mere memorizing of text. The drawings may lack finish and may be at first difficult to execute; but so long as they represent the relations of the organs one to another they accomplish their purpose; beyond this point the more accurately they are drawn the better.

Moreover, drawing is a great aid to dissection. It not only fixes in the memory what is seen but it compels close observation; when one draws an object he is forced to note details and relations of structure which would otherwise escape observation. Nor is the freehand drawing which is required for our purpose as difficult as is often supposed by those who have never seriously used it. Let the student attempt to reproduce an object from his memory of its picture; begin with one which is not too complicated (such as the figure of the peritoneum and mesentery on page 14). Where be does not know how to represent a special structure, let him refer to the original from which he may get suggestions; then 
The human mechanism is composed of different parts, such as head, neck, trunk, arms, hands, legs, and feet, and each of these in its turn is composed of lesser parts. Arms and hands, for example, are covered by skin, which may be moved over underlying soft parts; at the ends of the fingers the place of the skin is taken by nails, while scattered over and emerging from its surface are hairs. Through the skin may be seen the veins, which may be emptied of the purplish blood they contain by pressing one finger on a part of the vein near the finger, and pushing another finger along the vein toward the wrist; so long as pressure is maintained by both fingers the vein remains collapsed, but on removing the first finger it fills again with blood. Finally, through the soft parts (flesh) may be felt the hard bones. In general these various parts of which the body is composed are known as its organs, and because it possesses organs it is called an organism (p. 3).

1. The Skin. - The body is everywhere covered by a complex protective and sensitive organ, the skin. Only the eyes and nails seem to be exceptions; but as a matter of fact the exposed surface of the eye is covered by a very thin, transparent portion of the skin, and the nails are really modified portions of skin.

2. Subcutaneous Connective Tissue.-On cutting through the skin we find that it is bound to the underlying flesh (chiefly meat or muscle) by what is known as connective tissue, the structure of which we shall study in the next chapter. Meanwhile we may notice that it contains blood vessels, that at some places it is more easily stretched than

close the book and draw from memory; any completed part of the work may be compared with the original and possible improvements discovered.

Such practice may well precede drawing from an actual dissection and will pave the way to the latter. At all events let the student understand thoroughly that in the present chapter the figures, supplemented if possible by actual dissections, form the main objects of study; the text is strictly subordinate to the figures. 
at others, and that when a flap of skin is pulled away from the muscles, this subcutaneous tissue fills with air. It often contains large quantities of fat.

3. Muscles and Deeper Connective Tissues. - The subcutaneous connective tissue sometimes connects or binds the skin directly to bone, as in parts of the head; usually, however, in the neck, trunk, and limbs the underlying tissue is the red flesh, or muscle, familiar to us as "lean of meat." If the skin be removed from the forearm, it at once becomes evident that this mass of meat or flesh is composed of a number of muscles which may be separated from one another more or less completely. In doing this it will be found that the muscles are held together by connective tissue in most respects quite similar to that immediately under the skin. Further dissection will show that one or another form of this tissue is the means of binding other organs together; thus the muscles are joined to the bones by a very dense, compact, and strong form known as tendon; the bones are united by a somewhat similar form known as ligament; and so on. The physical characters of the tissue differ widely, according to its situation and the use subserved; but one form shades more or less into another, and we have no difficulty in recognizing the general similarity which leads us to group them all together in one class.

4. Muscles attached to Bones. - When a muscle is carefully dissected away from neighboring muscles and other organs, it is almost always found that it is attached to one and usually to two bones; this union is frequently made by means of a tendon, as in the case of the large muscle of the calf of the leg, which is attached at one end to the bone of the thigh and at the other to that of the heel. A good example of the direct attachment of muscles to bones is furnished by those muscles which lie between the ribs (see Fig. 141). In either case the shortening of the muscle brings closer together the bones to which it is attached. 
5. Definition of Some Anatomical Terms. - Before proceeding further we must agree upon the exact meaning of certain anatomical terms. We often speak of one part of the body as being "above" or "below," "before" or "behind," another. Such terms, however, are confusing, because their meaning depends upon the position of the body at the time they are used. For example, when one is lying on his back the head is in front of, or before, the trunk; but when he is standing on his feet it is above the trunk.

Now the body is certainly divided into right and left halves, which are much alike externally, though this likeness is not so marked in the internal parts. Right and left then have their ordinary meanings, and that without regard to the various positions the body may take.

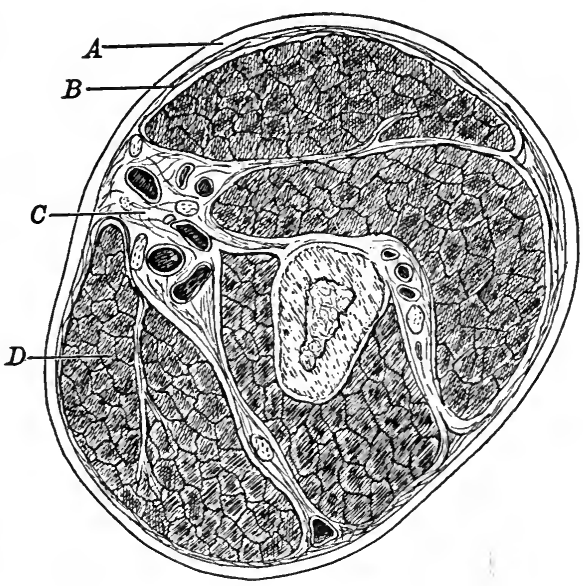

Frg. 1. Cross section of arm

$A$, skin ; $B$, subcutaneous connective tissue, binding the skin to the muscles $D$, and continuous with the connective tissue which binds together the muscles ; $C$, blood vessels and nerves

\section{To indicate that}

any part is nearer the head than another part, we say that the former is anterior to the latter; to indicate that the latter is further away from the head, we say it is posterior to the former.

Finally, the region popularly known as the back is called dorsal (Latin, dorsum, back), that opposite the back being called ventral (Latin, venter, belly). Thus the nose is on 
the ventral side of the head; the toes are at the posterior extremity of the foot.

6. The Body Cavities. - There is one striking and important structural difference between the trunk and the

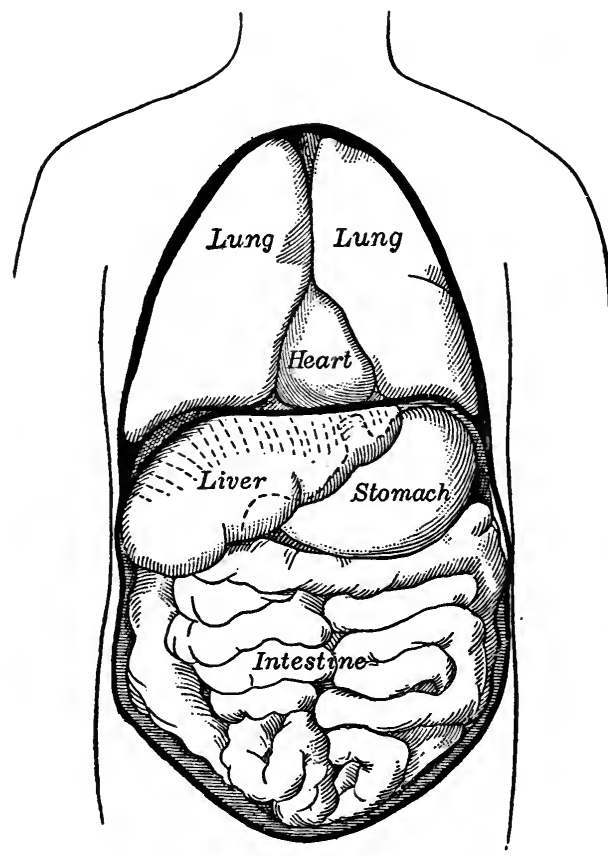

Fig. 2. The thoracic or pleural, and the abdominal or peritoneal, cavities filled with organs limbs; the former contains a central body cavity, completely filled, however, with various organs, while the arms and legs are each composed of a continuous mass of tissues, viz. muscle, blood vessels, nerves, bone, etc., all bound together by connective tissue.

The cavity of the trunk, or body cavity, is subdivided transversely by the dome-shaped muscle known as the diaphragm into two cavities, - an anterior, known as the thoracic or pleural cavity, and a posterior, known as the abdominal or peritoneal cavity. Both cavities are lined by a thin, smooth, shiny membrane, that of the thoracic being known as the pleura, and that of the abdominal as the peritoneum.

Filling the pleural cavity are found the heart, lungs, oesophagus, windpipe or trachea, and many great blood vessels; filling the abdominal cavity, the stomach, the small 
intestine, the large intestine, the liver, the pancreas, the kidneys, the spleen, and other organs together with numerous large and important arteries and veins. In both cavities the lining membrane (pleura or peritoneum) is folded back over the organs; that is to say, the organs do not really lie in the cavities, but only fill them as the hand would fill a bladder one wall of which it pushes in against the other. The surfaces of the organs, like the walls of the cavity, are consequently smoothly covered and glide over one another with very little friction. The preservation of these pleural and peritoneal linings in their

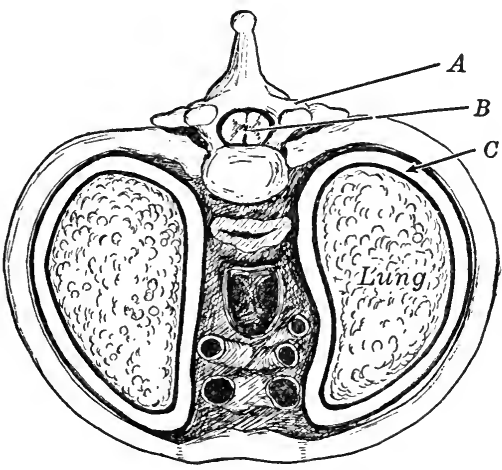
normal condition is a matter of great importance; when inflamed or otherwise injured their surfaces become roughened, and adhesions of connective tissue often develop between them which

Fig. 3. Cross section of the thorax anterior to the bifurcation of the trachea

$A$, a vertebra of the spinal column; $B$, spinal cord; $C$, the pleural cavity (which is exaggerated for the sake of clearness), the surface of the lung being actually in contact with the body wall. The œesophagus, trachea, together with several large arteries and veins are shown in the mediastinum ventral to the vertebra, and in the order named

fasten the organs together, or to the walls of the cavity, so that surgical interference is sometimes necessary. Pleurisy is such an inflammation of the pleura, peritonitis of the peritoneum; and both are very serious conditions.

7. Attachment of the Organs to the Walls of the Pleural and Peritoneal Cavities. - The pleural cavity is completely divided by a median partition of connective tissue (the mediastinum), within which are found the trachea, the 
aesophagus, the great blood vessels, and-lying within a special cavity of its own - the heart. Approximately halfway from the anterior to the posterior border of the mediastinum the trachea divides within that membrane into two tubes or bronchi, which pass through the mediastinum outward to the right and left lung respectively. The

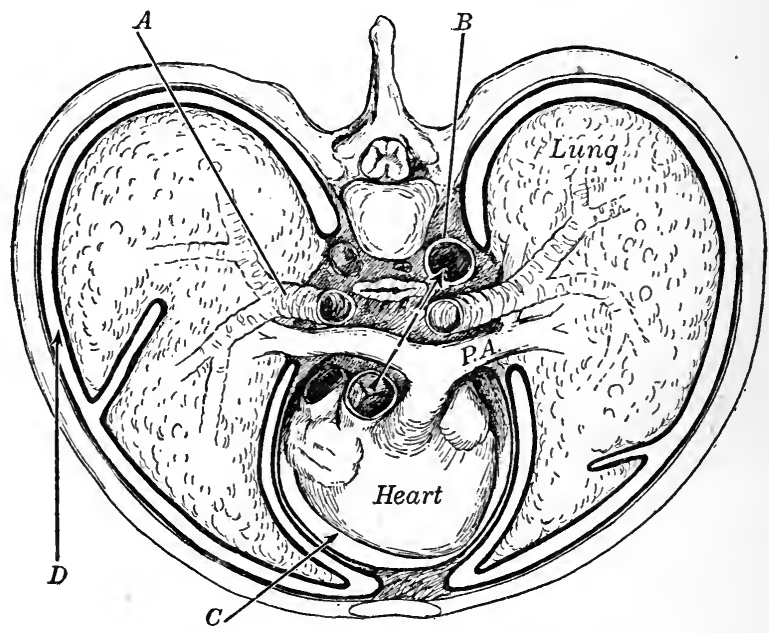

Fig. 4. Cross section of thorax posterior to bifurcation of trachea $A$, bronchus, entering the lung; $B$, the aorta cut at its origin and again at the descending part of its arch; $C$, the pericardial space; $D$, the pleural cavity; P.A., the pulmonary artery

pleural lining of the mediastinum is pushed outward by these tubes, and, as they end in the lungs, forms the pleural covering of the latter (Fig. 5). Consequently the organs of the pleural cavity either lie within the mediastinum (heart, œesophagus, trachea, etc.) or else are covered by extensions of the mediastinal pleura (bronchi and lungs).

The abdominal cavity is not similarly separated into right and left halves; but a membrane, the mesentery, passes ventrally from the dorsal wall to the stomach and intestine, which are slung in it somewhat as a man lies in a hammock. The line of attachment of this mesentery to the 
small intestine is much longer than that of its attachment to the body wall; hence it has the general shape of a ruffle, or flounce, - an arrangement which permits the suspension of the very long intestine ( 20 to $25 \mathrm{ft}$.) from the comparatively short median dorsal body wall (see Fig. 136). The great arteries and veins lie in the mesentery near the dorsal body wall and branches are distributed from them to the intestine within this expanding membrane (see Fig. 143).

The kidneys do not lie movably suspended in the abdominal cavity, as do the intestines, but are large organs, one on each side, situated near the spinal column and dorsal to the abdominal cavity from which they are separated by the peritoneum. Arteries and veins are sup-

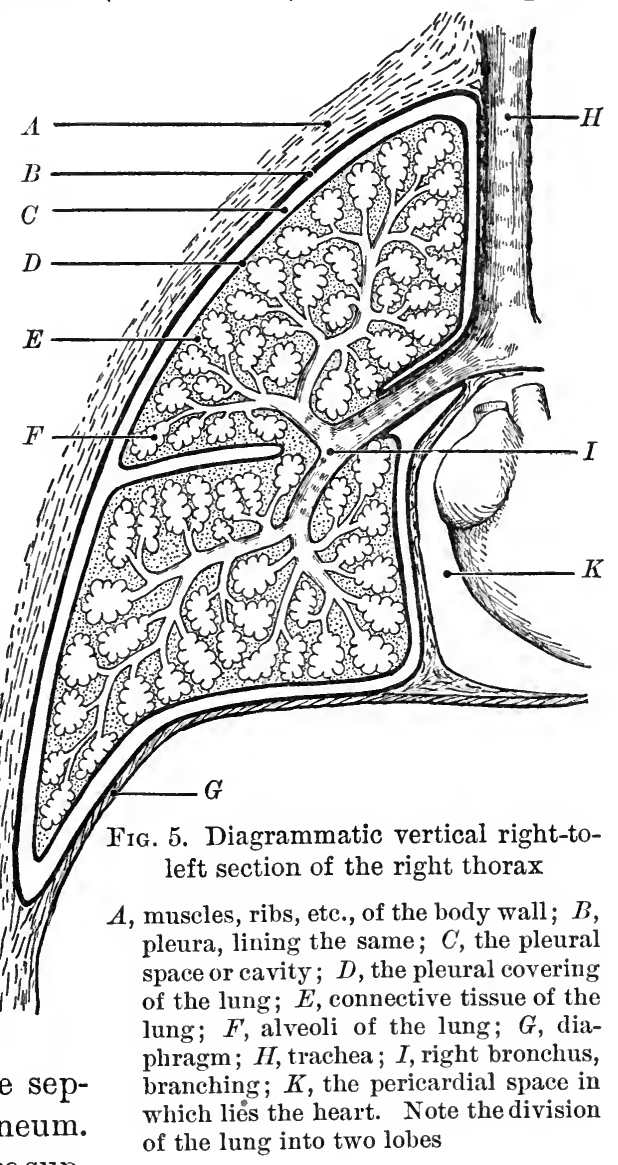
plied to them from the large median artery and the median vein already referred to (aorta and vena cava, Fig. 15), and these renal arteries and veins are likewise outside the abdominal cavity. 
The relation of the other organs to the peritoneum is more complicated, notably in the case of the liver; but in all cases

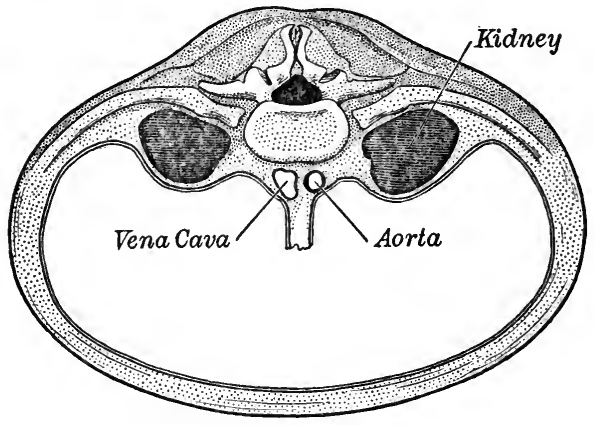

Fig. 6. Diagrammatic cross section of the abdominal cavity

Showing the relation of the kidneys and great blood vessels to the peritoneum. The intestine has been removed, the cut border of the mesentery being shown the organs are inclosed, or wrapped, either in a fold of the peritoneum, as is the kidney, or in a fold of the mesentery, as is the intestine; and their blood and nerve supplies run to them in similar folds.

8. The Axial Skeleton.-The bones and cartilages of which the skeleton is composed may be classified into an axial skeleton (of the head, neck, and trunk) and an appendicular skeleton (of the arms and legs). The axial skeleton comprises (1) the backbone or vertebral column, (2) the ribs and breastbone, and (3) the skull.

9. The Backbone or Vertebral (Spinal) Column. - This is composed of separate irregular ringlike bones or vertebrce placed one above another, and bound together by bands of strong connective tissue known as ligaments. It is customary to divide the backbone into the following regions.

a. Cervical. 7 vertebræ of the neck.

b. Thoracic. 12 vertebræ of the chest, to which ribs are attached.

c. Lumbar. 5 vertebræ of the "small of the back."

d. Sacral. 5 vertebræ (fused together) to which the large hip bones are attached.

e. Coccygeal. 4 or 5 very small simple vertebræ (constituting the skeleton of a rudimentary tail and corresponding to the tail of lower animals). 


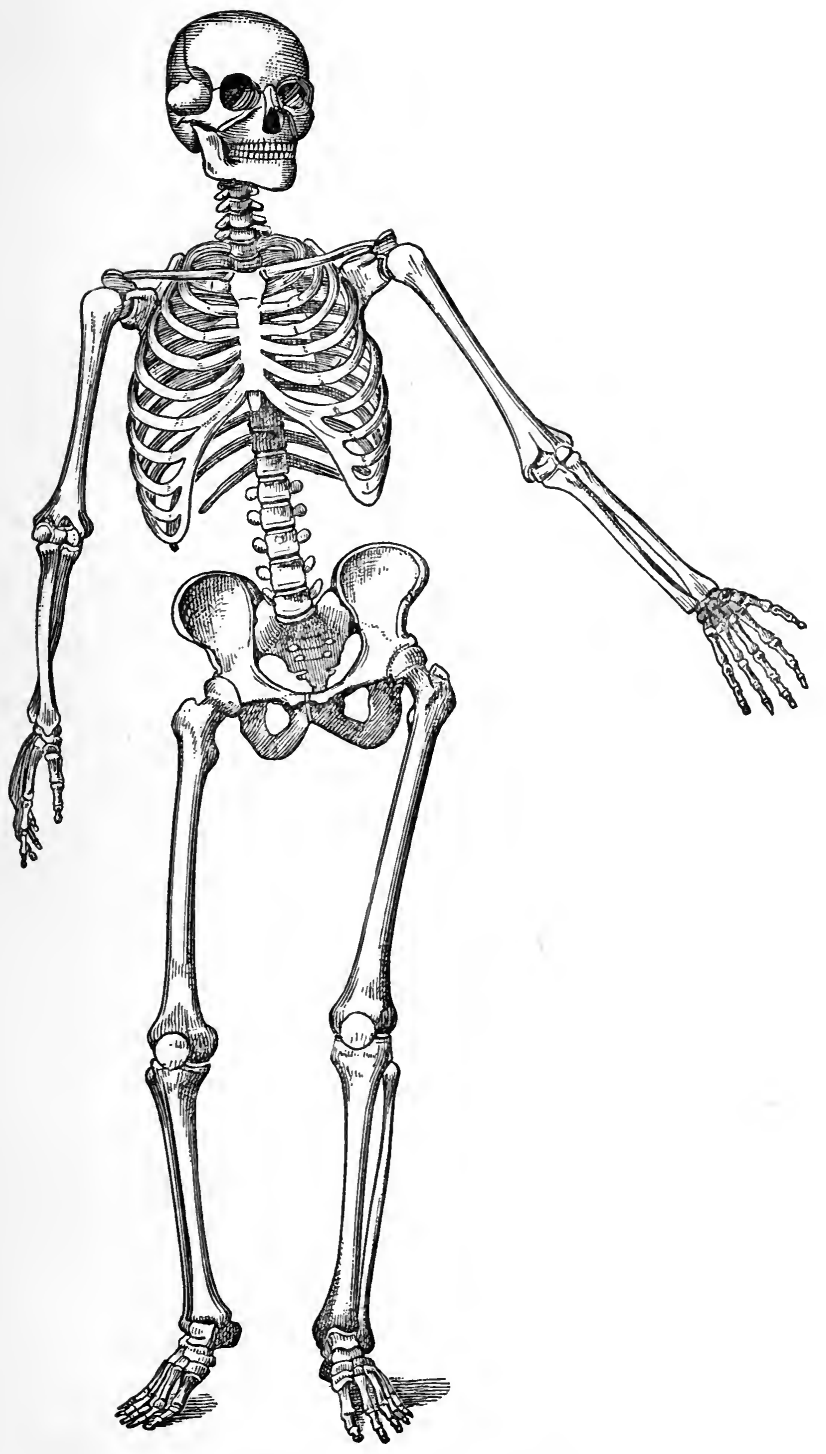

FIG. 7. The skeleton entire 
When one looks at the spinal column from behind, the vertebræ are seen to be placed one upon another, but all

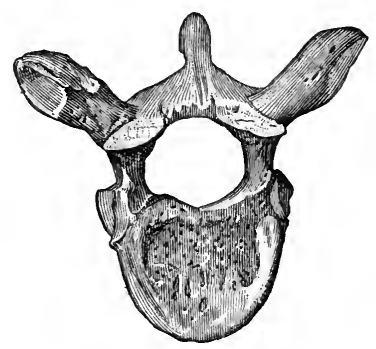

Fig. 8. Sixth thoracic vertebra

Seen from above in the median dorsoventral plane of the body (see Fig. 7). Seen from the side, however, several curves come into view, as shown in Fig. 10. On the ventral side, in the cervical and upper thoracic region, the curvature is slightly convex, in the thoracic region it is quite concave, in the lumbar region slightly convex, and in the sacral-coccygeal region again concave. It may well be asked how these separate vertebræ, piled; as it were, one above another, maintain their proper relative positions. This is partly due to the shape of the individual vertebræ, partly to the ligaments (p. 17) which pass from one vertebra to another and limit the movements of each, and partly to the action of muscles which are placed upon opposite sides of the vertebræ and by their antagonistic action hold them in place. The action of muscles and ligaments upon the bones may be illustrated by two blocks of wood held together by two rubber bands $\left(m, m^{\prime}\right.$, Fig. 11) slightly stretched; so long as each pair of opposite bands pulls with the same force,

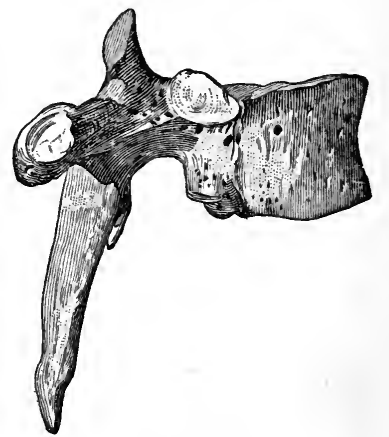

Fig. 9. Sixth thoracic vertebra

Seen from the side the blocks are kept in what we may call their resting position. Here the rubber bands represent two of the antagonistic muscles, which, by maintaining a steady and equal pull on the opposite sides of the vertebræ, keep 
them in place. Should one pull harder than its antagonist, as when a muscle contracts (see Chapter IV), the antagonist will be stretched and the bones become inclined somewhat toward one another, as shown in the figure.

This principle of muscular antagonism is quite general in the maintenance of the proper relative positions of bones in the body. Almost every joint is the theater of such plays of antagonistic muscles, which serve the double function of keeping the bones in proper position with regard to one another, and of producing movement at the joint, the amount of this movement being limited by the slack but inextensible connective-tissue ligaments which bind the bones together. In Figure 11 both the shortening of the muscle and the slackness of the ligaments is purposely exaggerated, in order to represent more clearly the functions of these tissues. Ligaments may also guide the movement of bones by preventing motion in one direction or another.

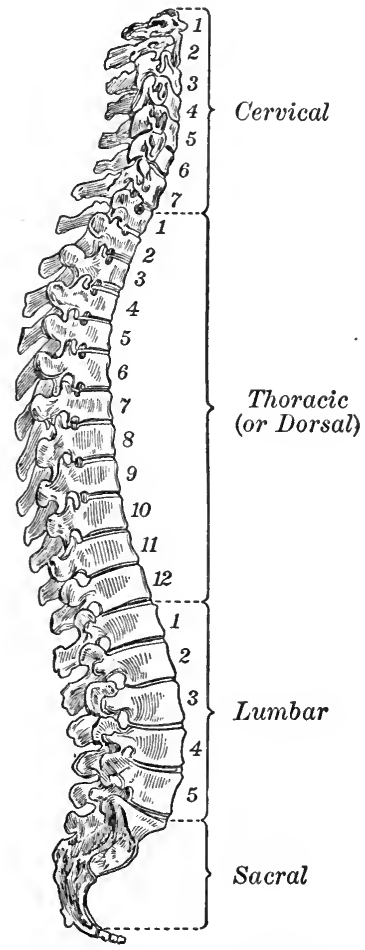

FIg. 10. The vertebral column

Seen from the side

10. The Ribs. - Each rib consists of a bony and a cartilaginous portion. The former articulates (i.e. forms a joint with) the vertebral column, while the latter continues this bony portion to the ventral median breastbone, to which it is directly joined. The ribs form the framework for the thorax and may be lifted or 

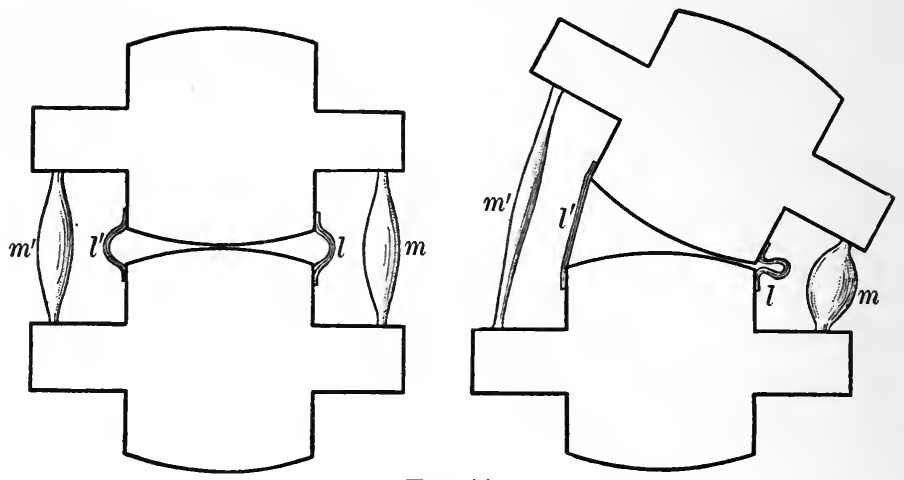

Fig. 11

Model showing the action of muscles on two vertebræ, and of the ligaments $\left(l, l^{\prime}\right)$ in limiting the amount of movement. The contraction of the muscle $m$ stretches its antagonist $m^{\prime}$. The amount of movement is greatly exaggerated

lowered by muscles which connect them with the vertebral

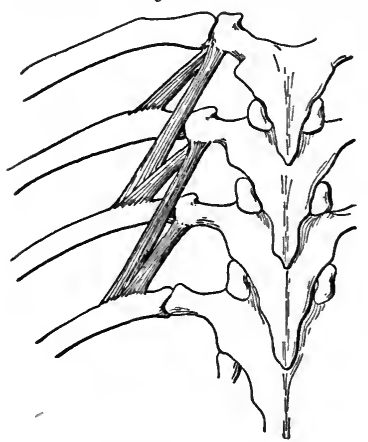

Fig. 12. Dorsal view of vertebræ and ribs

Showing some of the muscles which lift or raise the ribs column and other parts of the skeleton (see Fig. 12).

11. The Skeleton and the Central Nervous System. - The skull consists of the bones of the face and those of the cranium, the latter holding the brain. It is supported on the spinal or vertebral column whose ringlike vertebræ inclose a bony canal continuous with the cranial cavity. This is known as the spinal or vertebral canal in which lies the spinal cord, ${ }^{1}$ - the continuation of the central nervous system posterior to the brain.

1 The terms "spinal cord," "spinal column," and "spinal canal" are sometimes confused by beginners. The spinal column is the entire bony framework formed by the vertebræ, - the whole backbone; it surrounds the spinal canal which, in turn, contains that part of the nervous system known as the spinal cord. 
Nerves, which pass through small openings in the cranium and between the vertebræ, leave the brain and cord and end in the muscles, skin, glands, and other organs of the body (see Chapter VII).

12. The Appendicular Skeleton. - The bones of the arm, leg, hand, and foot may readily be felt, and are sufficiently familiar. We may, however, call attention to the similarity in the number and form of the bones of the arms and legs, a similarity which is not only helpful in mastering their names and arrangement, but is also suggestive of the similarity of function in quadrupeds, both limbs in these animals being organs of locomotion.

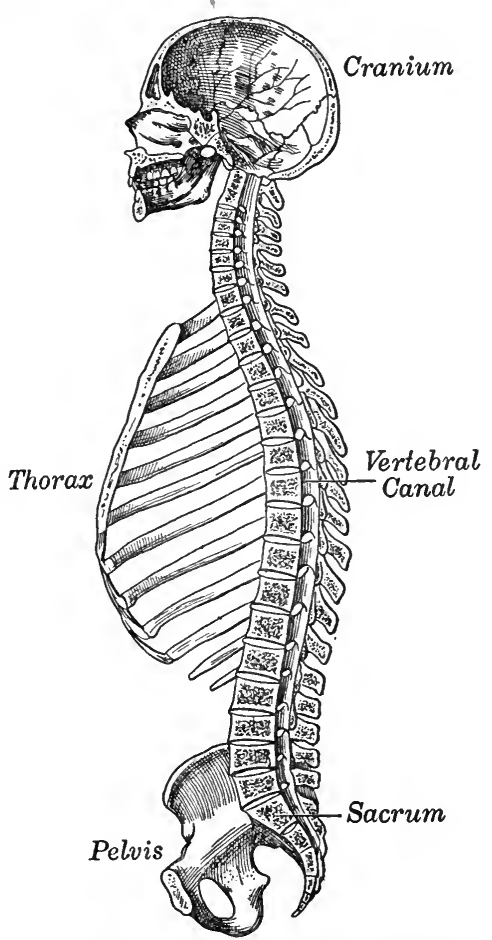

FIG. 13. Median dorsoventral section of the skeleton

\section{LEG}

Femur, single long bone of the thigh.

Tibia and fibula, two nearly parallel bones of the lower leg. Seven small irregular bones of the ankle and heel.

Five parallel bones of the instep. Bones of $\{$ Great toe, two bones. toes Other toes, three bones. 
The legs are attached to the vertebral column by the large hip bones, which articulate directly and immovably with the sacrum $^{1}$; but the humeris, or bone of the upper arm, articulates on each side with one of a pair of bones which form the shoulder girdle, or skeleton of the shoulder

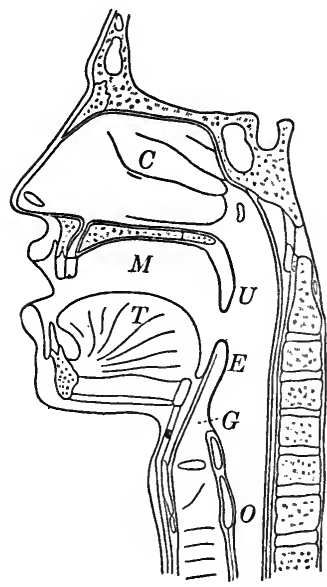

Frg. 14. Diagrammatic median dorsoventral section of the nasal and throat passages

$C$, nasal cavities; $M$, mouth cavity; $T$, tongue; $E$, epiglottis; $G$, glottis, or opening from the pharynx into the trachea; $U$, the end of the soft palate; $O$, œsophagus region; this pair consists of the collar bone (clavicle) ventrally and the shoulder blade (scapula) dorsally. The clavicle articulates with the head of the breastbone; otherwise the shoulder girdle, with the arm attached to it, is connected with the axial skeleton by muscles only. A wide range of movement is thus secured at the shoulder joint.

13. Organs of Digestion. - The digestive system consists essentially of a long tube, the alimentary canal, passing through the body. ${ }^{2}$ Into this tube at various points ducts from a number of glands pour $d i$ gestive juices. The alimentary canal begins with the mouth cavity and its familiar organs, the teeth, the tongue, etc.; this cavity opens posteriorly into that of the pharynx, into which also opens the nasal cavity, separated from the mouth only by the palate (see Fig. 14).

On the ventral side of the pharynx just beyond the root of the tongue is the slitlike opening of the windpipe (see Section 14); posteriorly the pharynx is continued in the long gullet, or osophagus, a tube which passes downward

1 The sacrum and the two hip bones together form the pelvis.

${ }^{2}$ See Fig. 135 for the general arrangement of the organs of digestion. 
through the neck and thorax (within the mediastinum) to join the stomach, which it enters immediately after passing through the diaphragm.

The stomach is a large pouch, with contractile walls permitting adaptation of its size to the bulk of food it may contain. Its situation is shown in Fig. 135, which also shows how it opens on the right side of the body into the very long, coiled, small intestine. The coils of this part of the tube may be followed for from twenty to twenty-four feet, to the large intestine, into one side of which it opens. The large intestine, or colon, consists of three portions: the first ascending on the right side to the general level of the stomach, the second passing transversely at this level from right to left, and the third descending on the left side to the rectum, the posterior terminal portion of the digestive tube.

Numerous glands pour secretions through ducts into the digestive tube, the following being the more important with their places of discharge: salivary glands (see Chapter III) - mouth; liver - beginning of small intestine; pancreas - beginning of small intestine (see Fig. 50). Smaller glands empty into the stomach and intestines at numerous places.

14. The Organs of Respiration. - The organs of respiration consist of the right and left lungs (see Fig. 5) from each of which a single bronchus (pl., bronchi) leads to the trachea, or windpipe. The walls of the trachea and bronchi are kept from collapsing by successive rings of cartilage. Anteriorly the trachea opens into the pharynx through the larynx, or voice box, the cartilages of which may be felt in the throat at the root of the tongue. The familiar hoarseness which accompanies inflammatory roughening of the lining of the larynx shows how important is this organ in the production of the voice. The respiratory and digestive paths cross in the pharynx, the former reaching the exterior through the nose, the latter through the mouth. 
15. The Organs of Circulation. - The position of the heart and the great blood vessels in the thorax has been described on page 11. The heart is essentially a large mass of muscle, containing a cavity which is divided into right and left halves, wholly separate from each other. The cavity on each side is divided into that of the large ventricle with very thick walls, and that of the much smaller auricle. The heart is thus composed of right and left auricles and right and left ventricles. Valves are so placed in the heart as to allow blood to flow in one direction only (see Fig. 64).

The arteries are tubes which carry the blood to the tissues, and from each side of the heart a single artery takes its origin, - the pulmonary artery from the right ventricle and the aorta from the left ventricle. The pulmonary artery supplies the lungs with blood, while all other organs are supplied by the aorta.

The veins are tubes which conduct the blood from the various organs to the heart. Beginning in the tissues as microscopic tubes, they unite to form larger and larger tubes as they approach the heart; those visible through the skin of the hand may be regarded as of medium size; as the union goes on, the size of the vessels increases until finally at the heart there are only two great veins on the right side (superior vena cava and inferior vena cava) and four on the left (pulmonary veins). The venæ cavæ bring blood back from those portions of the body which are supplied by the aorta, that is to say, from all parts of the body except the lungs; the pulmonary veins bring blood back only from the lungs, that is to say, from the organs supplied by the pulmonary arteries. The venæ cavæ empty into the right auricle, the pulmonary veins into the left auricle. The general arrangement of heart, arteries, and veins is shown in Fig. 15, and the figures in Chapter IX (especially 65 and 66 ) should also be consulted. 
The blood flows in the following circuit.
Pulmonary circulation $\left\{\begin{array}{l}\text { Right ventricle to } \\ \text { Pulmonary artery to } \\ \text { Lungs to } \\ \text { Pulmonary veins to }\end{array}\right.$
Left auricle to
Left ventricle to
Systemic circulation
Aorta and its branches to
All organs of the body (except the lungs) to
Veins which unite to form the venæ cavæ to Right auricle to Right ventricle.

Thus the blood which leaves the left ventricle flows to the different organs of the body (except the lungs), and returns by way of the veins to the right side of the heart; thence it passes through the lungs and again to the left auricle and ventricle, thus completing the "circulation." The term "circulation," strictly speaking, is applied to the entire circuit which the blood must traverse until it returns again to the point from which it started; it is often convenient, however, to use it to denote the course from the right ventricle to the left auricle, or from the left ventricle to the right auricle; in this case we speak of the former as the pulmonary and of the latter as the systemic or aortic circulation. In this sense there may be said to be a "double" circulation.

The veins have thinner walls than the corresponding arteries, and those of the systemic circulation contain purplish or even bluish blood, while the arteries of the same circulation contain bright scarlet blood. The bright color of the arterial blood is due to the fact that it contains more oxygen. The change from purple to scarlet occurs in the lungs, and the reverse change in the organs supplied by branches of the aorta. Consequently the blood of the pulmonary arteries is blue or venous in color, and that of the pulmonary vein scarlet or arterial. 


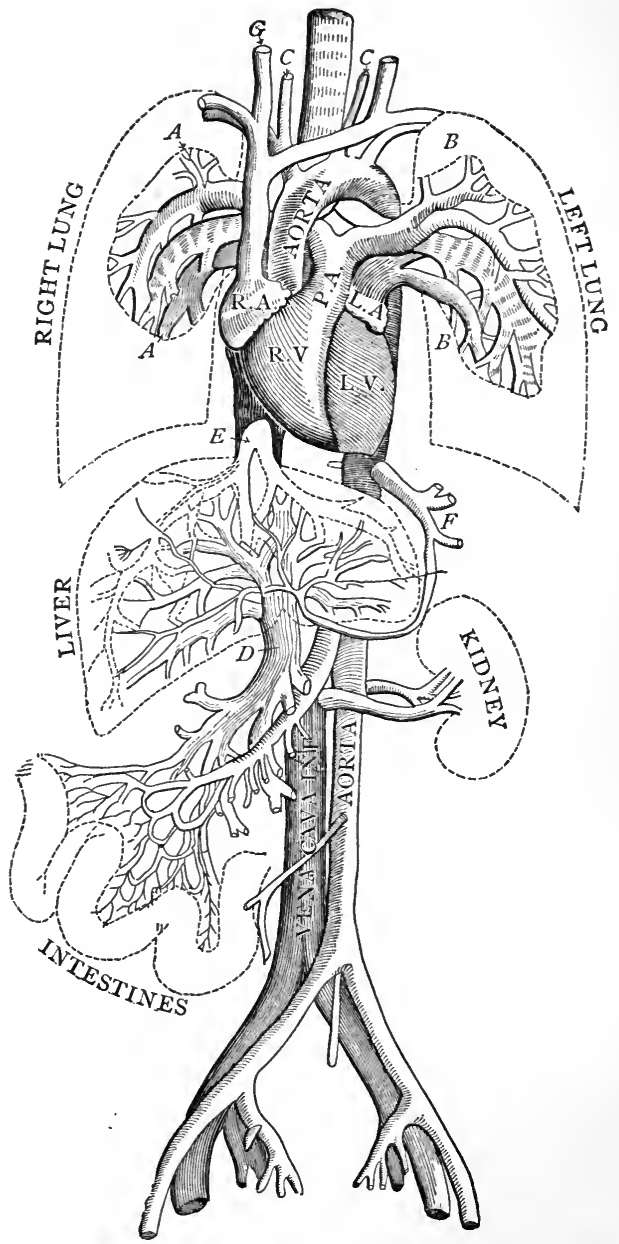

Fig. 15. Diagram of the circulation of blood

$R . A$., right auricle; L.A., left auricle; R.V., right ventricle; $L . V$. , left ventricle; $P . A$., pulmonary artery; $A$, pulmonary artery and vein of right lung; $B$, pulmonary artery and vein of left lung; $C$, carotid artery to head, showing branch of left subclavian artery; $D$, portal vein; $E$, hepatic vein; $F$, hepatic artery; $G$, jugular vein, bringing blood from head and neck 
16. The Course and Branches of the Pulmonary Artery and Vein. - Soon after leaving the right ventricle the pulmonary artery divides into two branches, one going to each lung. Each of these further divides as it plunges into the substance of the lung alongside the bronchus. The course of the four pulmonary veins may be similarly traced into the lungs, from which they bring the blood back to the heart (Fig. 15).

17. The Course and Branches of the Aorta. - The aorta passes anteriorly from the left ventriçle, but very soon arches dorsally and posteriorly, forming the arch of the aorta (Fig. 15); the general course of the artery can be best understood from the figures, or from actual dissection. The arch of the aorta is continued in the large dorsal aorta which passes posteriorly on the left side of the mediastinum near the spine,

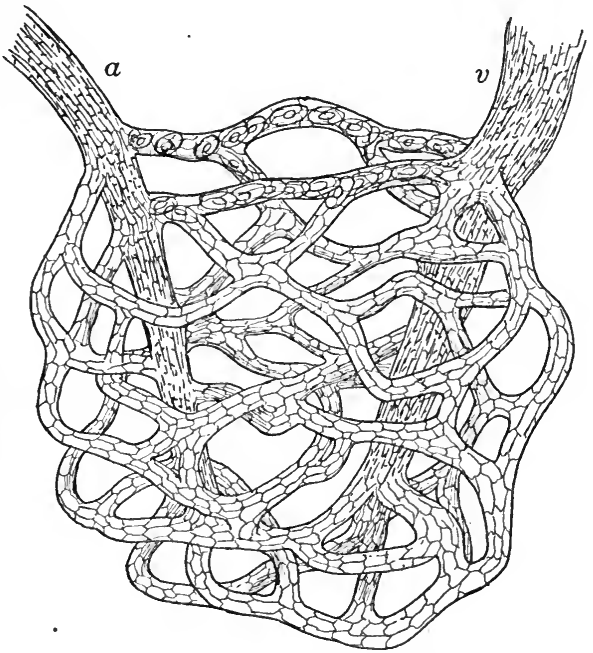

Fig.16. A network of capillaries, with the artery $a$ and vein $v$ (highly magnified) phragm, to the lower portion of the abdominal cavity, where it divides into two large arteries which supply blood to the hips and legs. From the arch of the aorta three large arteries pass to the head, neck, shoulders, and arms; from the thoracic dorsal aorta arise a number of small arteries which supply the muscles and other organs of the thoracic wall; immediately after passing through the diaphragm two large branches 
go to the stomach, spleen, liver, pancreas, and a large part of the small intestine; posterior to these the renal arteries pass right and left to the kidneys, and still further down a large artery supplies the lower small intestine and the large intestine. The supply to the legs has already been mentioned. Other small arteries arise from the abdominal

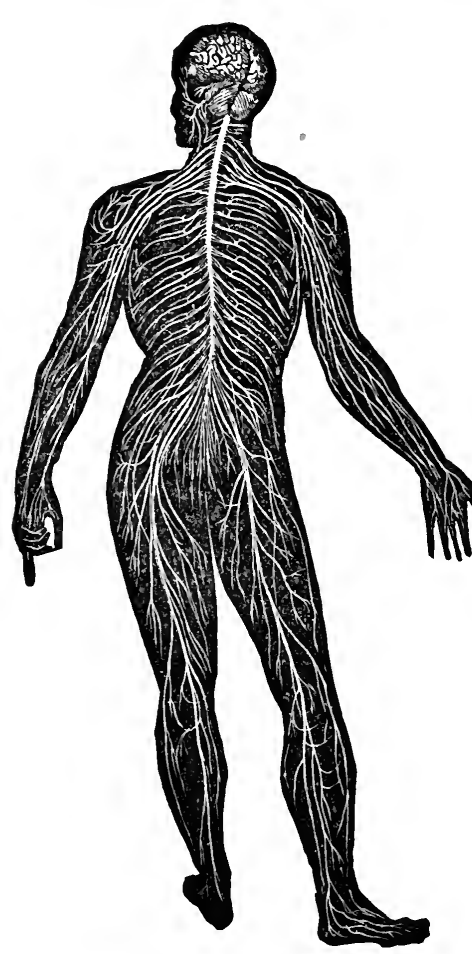

FIG. 17. The general arrangement of the nervous system (dorsal view) aorta and are distributed to the muscles and skin of the back. The arteries to the stomach and intestine lie in the mesentery (Fig. 143) and their course may be readily traced in a dissection.

18. The Course and Branches of the Venæ Cavæ. - The blood which has thus been distributed from the aorta returns to the opposite side of the heart through the veins which ultimately form the two venæ cavæ. In general, it may be stated that the veins of those organs which are anterior to the diaphragm form the superior vena cava, while those posterior to the diaphragm form the inferior vena cava. The larger veins usually run near and approximately parallel to the larger arteries. This is the case with those from the arms and legs, the kidneys, and the muscles of the trunk. One notable and very important exception, 
however, is found in the venous supply of the stomach, spleen, and intestines, the veins of which unite to form a single large vein (portal vein) which passes to the liver, where it breaks up into smaller vessels; the blood which has thus passed through the liver is finally collected in the hepatic vein and poured by this into the inferior vena cava just before the latter passes through the diaphragm on its way to the right ventricle (Fig. 15).

19. The Capillaries. - The blood which enters an organ through the arteries passes to its veins through a system of microscopic tubes (Fig. 16), the capillaries (Latin, capilla, a hair); these may be readily seen under the microscope in the web of a frog's foot. From the foregoing description of the course of the circulation it will be observed that generally the blood must pass through one set of capillaries in going from the aorta to the venæ cavæ, or from the pulmonary artery to the pulmonary vein; but the blood which flows through the capillaries of most of the abdominal organs (stomach, intestines, spleen) must pass also through a second set of capillaries, viz. those of the liver, before it can return to the heart.

20. Organs of the Nervous System. The skull and the spinal column (p. 18) are chiefly occupied by the brain and the spinal cord respectively, and from Fig. 18. Nerve trunks each of these principal organs of the of the right arm

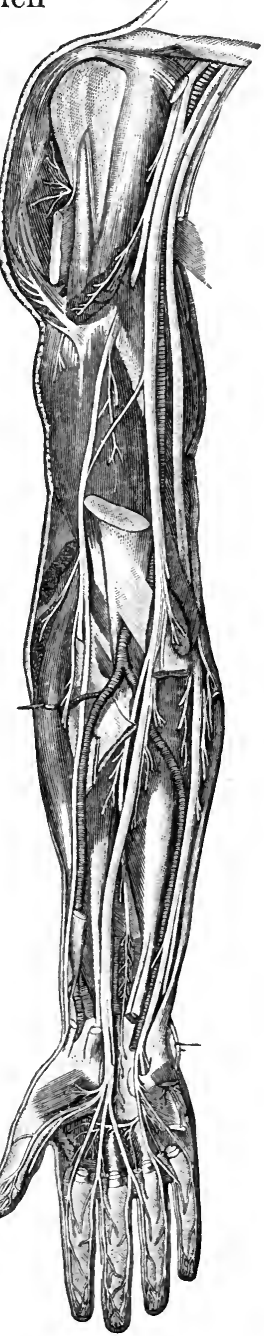


nervous system branches consisting of cords of nervous substance, the nerves, pass out through small holes in the skull or spinal column and are distributed to all the other organs, where they terminate in peculiar structures called end organs. The optic nerve, for example, ends in the retina, the auditory nerve in the inner ear, and motor nerves in muscles, - the nerve endings in these different organs differing materially in structure and arrangement.

Figure 17 gives some idea of the general arrangement of the nervous system. The nerves to the shoulder, arm, and hand will be seen to arise from the cervical region of the spinal cord; those for the trunk, from the dorsal and lumbar regions; those for the legs, from the sacral region. The head and face receive nerves from the posterior portions of the brain. The dissection of the arm in Fig. 18 shows more accurately the main nerve trunks to that region. Further information with regard to the structure of the nervous system will be given in Chapters VII, $\mathrm{XIV}$, and $\mathrm{XV}$. 


\section{CHAPTER III}

\section{THE FINER STRUCTURE OF TWO TYPICAL ORGANS,}

GLANDS AND MUSCLES. THE CONNECTIVE TISSUES. THE LYMPHATIC SYSTEM

In the previous chapter we have examined the general construction of the human machine as regards its more conspicuous parts or organs, and especially their location,whether internal or external, dorsal or ventral, anterior or posterior, on the right or on the left, - their relations to certain important cavities, and their combination to constitute the mechanism which we call the human body. We must now push our examination further and investigate the finer structure of some of the more important parts of the machine. For this purpose we may select two typical organs, a gland and a muscle, the one unfamiliar, by name at least, to most people, the other well known in the form of steaks, chops, roast beef, and other meats.

1. What is a Gland? - A gland is a mass of tissue, generally softer than muscle, and of no special size or shape, though often rounded or egg-shaped. The gland most easily seen is the milk gland or udder of the cow. This is a large mass of soft tissues devoted to manufacturing or secreting milk. In general, glands are manufacturing organs for the preparation of saliva, gastric juice, bile, tears, sweat, or other secretions. Some have tubes or ducts through which their secretions are carried away; others have no such outlets and hence are known as ductless glands. Glands vary in size from some which are microscopic to the huge liver, which is the largest single organ in the human body (see Fig. 2). The pancreas or 
"sweetbread" is an excellent gland for the beginner to dissect or study.

2. A Typical Gland. - If we have before us the whole or a part of any typical gland, we find that we are dealing with a comparatively soft and sometimes even pulpy mass held together by a loose mesh or network of harder, tougher, and more or less fibrous materials. A pancreas or a liver, if entire, shows conspicuous lobes, and in the pancreas these lobes are plainly subdivided into smaller lobes or lobules. In favorable specimens tubes may be seen connected with the gland, some of which are blood vessels supplying blood to the gland, and one of which is a duct draining away from it the liquid which the gland has man-

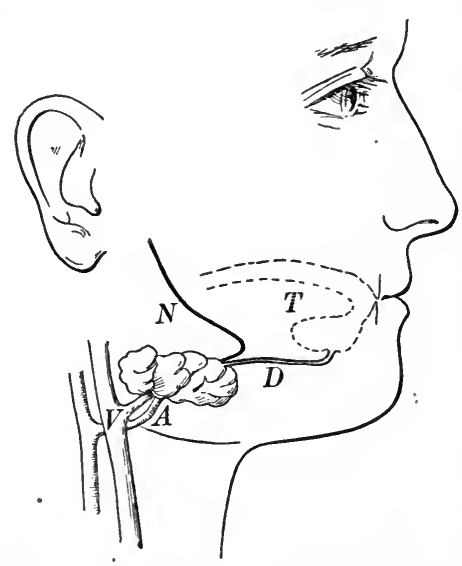

Fig. 19. Diagram of submaxillary gland

$D$, its duct; $N$, its nerve; $A$, its artery; $V$, its vein; $T$, tongue ufactured or secreted. After a preliminary examination of this sort of some edible gland, we may consider in greater detail one of our own salivary glands, of which we have three on each side of the head, namely, one parotid, one sublingual, and one submaxillary gland.

3. The Structure of the Submaxillary Gland. - The two submaxillary glands lie, one on each side of the face, embedded in the tissues between the lower jaw and the upper portion of the neck. From each gland a duct passes forward in the tissues forming the floor of the mouth, into which it opens by one of the small eminences, or papilloe, under the tongue. Through this duct the gland pours into the mouth its secretion, saliva. 
If the gland were to be cut in two in any direction with a sharp knife, we should see at once that it is composed of separate parts, or lobes, and that these lobes are still further divided into smaller portions, or lobules. The lobules and lobes are bound together with a rather loose connective tissue which is continuous with a somewhat denser layer surrounding the gland and forming its capsule; the connective tissue between the lobes forms the primary septa (sing., septum), and that between the lobules the secondary septa. The relation of these structures is shown in

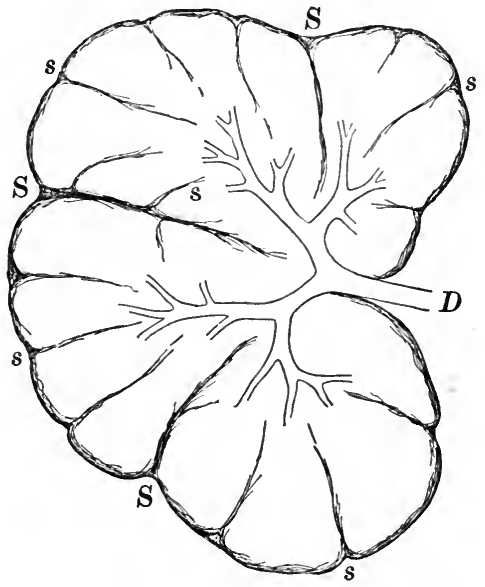

Fig. 20. Diagram of a cross section of a gland

Fig. 20. With the aid of Showing its division by primary septa $(S)$ the microscope we find that each lobule is still further divided by connective tissue into flaskinto lobes, and by secondary septa $(s)$ into lobules; also the origin of the larger branches of the duct $(D)$ in the lobes and lobules. The beginnings of the duct are shown in Figs. 21 and 22

shaped structures or alveoli (sing., alveolus); in these the secretion, saliva, is manufactured, and from them it is discharged into the duct of which the alveoli are the blind ends (Fig. 21).

The whole gland may be compared to a large bunch of grapes ; the main tubular duct of the gland branches (in the septa of connective tissue) very much as the stem of the bunch of grapes branches; and just as the branches and subbranches of the stem lead, when followed up, to the grapes themselves, so the branches of the duct lead to the alveoli of the gland. If now we pack the bunch of 
grapes in a small basket of sawdust or cork waste, as Malaga grapes are packed, so that the sawdust fills up loosely the spaces between the individual grapes and the branches of the stem, we shall have something with which

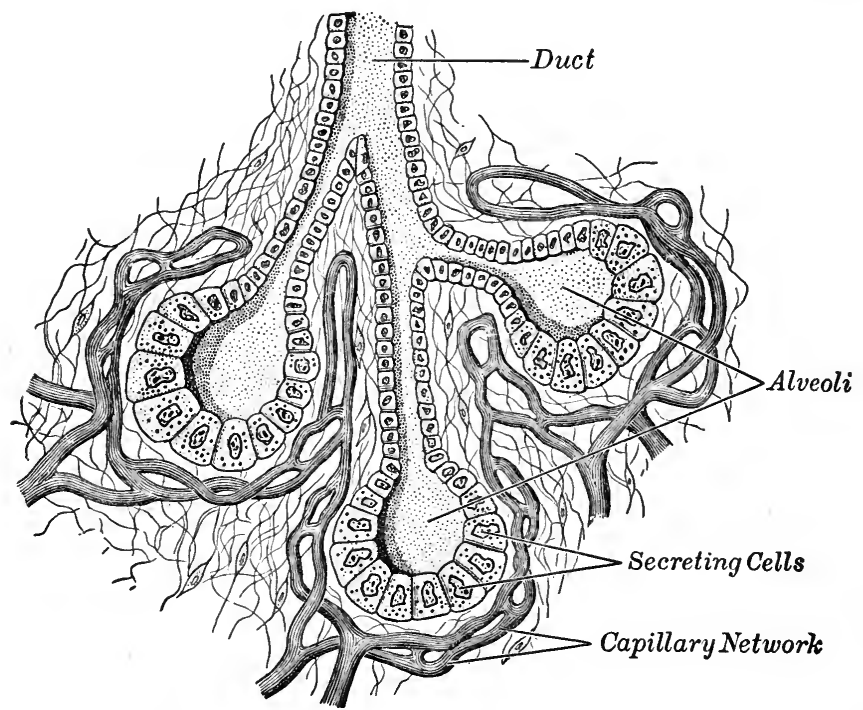

Fig. 21. The origin of the duct of a gland in alveoli, together with the connective tissue and blood vessels

to compare the arrangement of the connective tissue in relation to the rest of the gland, - the sawdust standing for the connective tissue in which the ducts and alveoli are embedded, and the basket for the capsule.

4. Minute Structure of Ducts and Alveoli. - The alveoli are, however, neither empty shells like glass flasks, nor solid masses like grapes, but rather hollow bags lined with a layer of relatively thickish, closely set cells, all very much alike. Each of these cells consists of two portions, a small central body, the nucleus, and a larger surrounding mass, the cytoplasm. And we may state at once that all 
organs of the body are composed of cells, differing, it is true, in different organs, or in different parts of the same

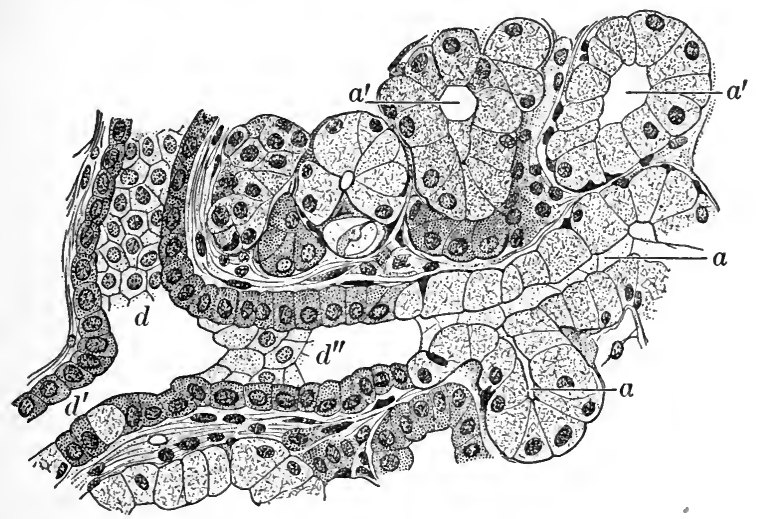

Fig. 22. Section of a portion of a salivary gland (magnified 500 diameters). After Kœlliker

The duct $d$ divides into the two branches $d^{\prime}$ and $d^{\prime \prime}$, one of which ends in the alveoli, $a, a$. Neighboring alveoli, $a^{\prime}, a^{\prime}$, whose ducts are not in the plane of the section, are also shown. In some cells the section does not include the nucleus, which would be in the preceding or the succeeding section

organ (as in the duct and the alveolus of the gland); but all consisting of two never-failing parts, — nucleus and cytoplasm.

The muscle and the gland consist of cells, just as all the branches of the military service - the infantry, the cavalry, the artillery, the engineers, etc. - consist of men. The cell is the anatomical or fundamental unit of these organs, as the soldier is the fundamental or anatomical unit of the army; in both cases the anatomical units, differing in equipment and training, perform different kinds of work, yet have the same essential structure; and the cells are combined into brigades, divisions, or corps, as tissues and organs; they make of the body an army organized to fight its way through the vicissitudes and against the obstacles of life. 
5. The Structure of the Biceps Muscle. - The biceps muscle is familiar as the mass of flesh lying on the front of the upper arm and bulging somewhat when the arm is bent at the elbow, especially when one "feels his muscle," or when a weight is being lifted by the hand. Figure 23

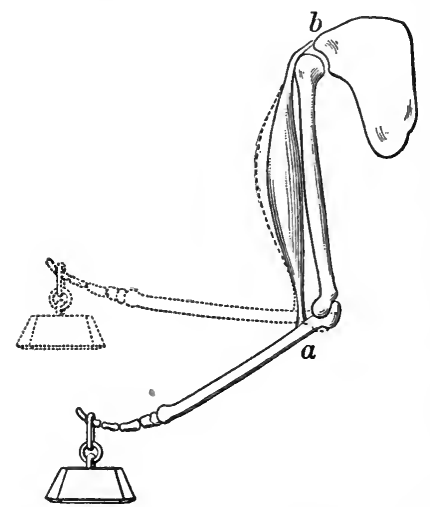

FIG. 23. The biceps muscle of the arm

The resting condition is shown by the solid lines, the contracting condition by the dotted lines shows this muscle with the bones to which it is attached. It consists of two portions : a central, thick, red part, known as the belly, soft when the muscle is at rest, hard when it is contracted; and cordlike strings, or tendons, two at the upper end and one at the lower, by means of which the muscle is attached to two bones of the shoulder girdle and to one of the forearm. When the belly of the muscle shortens the points $a$ and $b$ are brought closer together and the arm is bent, or flexed, at the elbow. This drawing together, or contraction, is the special work, or function, of muscles in general.

Every one has seen the cross section of a muscle in a raw beefsteak. This shows that the muscle as a whole is surrounded by a sheath of connective tissue which contains more or less fat; septa pass inwards, dividing the muscle into lesser red masses known as fasciculi, or bundles, and these are further subdivided into secondary fasciculi by secondary septa, very much as the gland is subdivided into lobules.

A longitudinal section shows that the fasciculi run from tendon to tendon, and microscopic examination proves that the general connective tissues of the belly of the muscle 
are continuous with that of the tendon. The tendon itself is a peculiarly strong and inextensible variety of connective tissue, consisting chiefly of parallel fibers which are specially fitted to transmit to the bone the pull of the belly of the muscle.

6. The Muscle Fibers. - Examination of the structure of one of the finer fasciculi in the belly of the muscle shows that it is composed of threads, or fibers, which at first sight differ greatly from the secreting cells of the gland. These are the muscle fibers. They are 1 to $1 \frac{1}{2}$ inches in length and from $\frac{1}{250} \overline{0}$ to $\frac{1}{2} 50$ of an inch in thickness, thus being from 250 to 2500 times as long as wide, and comparable in

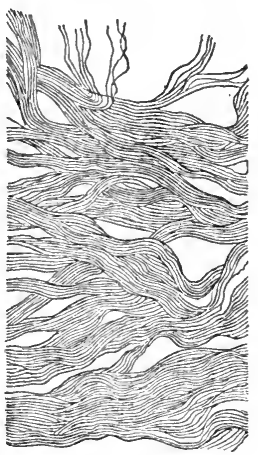

FIg. 24. Tendon (highly magnified) Showing the fiber bundles separated shape to a long leather shoestring rather than to a sausage.

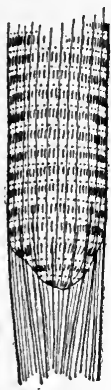

Fig. 25. One end of a muscle fiber

Showing the attachment of the tendon fibers to the sarcolemma

Each fasciculus contains hundreds or even thousands of fibers. The fibers always run lengthwise of the fasciculus, but, as a usual thing, do not extend its entire length, as obviously follows from the fact that a single fasciculus of the biceps is several inches in length. The fibers are inclosed in a very thin transparent membrane, the sarcolemma, and are bound into bundles, or fasciculi, by the same fine connective tissues seen between the alveoli of a gland. To the end of the sarcolemma are attached fine fibers of connective tissue which pass into the tendon (Fig. 25). Indeed, the fibers of the tendon are the collected fibers from the sarcolemmas of all the muscle fibers. For this reason the part of the muscle near the tendon is "tough meat," while that in the belly of the muscle is tender, owing to the smaller number of connective tissue fibers. 
7. The Muscle Fiber is a Cell. - The muscle fiber at first sight does not seem like the typical cell already described, with nucleus and cytoplasm; for when examined in the fresh condition the only obvious points of structure seen in it are striking cross striations consisting of alternate dark and light bands. It has been shown, however, by ingen-

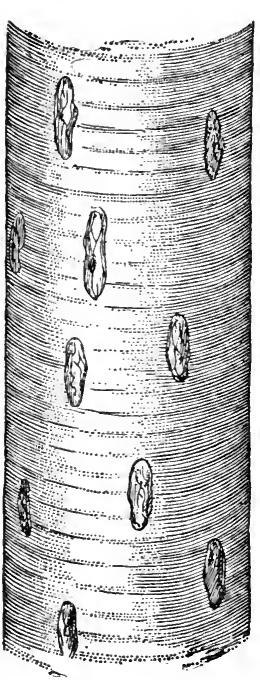

Fig. 26. Part of a muscle fiber

Specially prepared to bring out the numerous nuclei ious and careful study, that the cross striations are optical appearances produced by the peculiar shape of extremely minute longitudinal rods in the cytoplasm of the muscle fiber, and that, immediately under the sarcolemma, there are numerous characteristic nuclei which are easily brought into view by suitable treatment. Briefly, then, the muscle fiber is a cell with many nuclei, in whose cytoplasm are found peculiar structures, the myofibrils; upon superficial examination these myofibrils not only obscure the nuclei but give to the whole fiber the appearance of cross striation.

8. How far is the Structure of Glands and Muscles Typical of All Organs?Both the gland and the muscle are thus composed of cells. Although differing considerably in the two organs, these cells possess certain general and fundamental features in common, for each one contains a nucleus (or nuclei) and surrounding cytoplasm. Is the same thing true of all other organs? The muscle and the gland are examples of organs which do active work, but some other organs perform purely passive functions. Such are the bones, which do no work themselves, but upon which the work of mechanical motion is done by the muscles; the tendons, which transmit the pull of muscles; the 
ligaments, which limit and sometimes guide the motion of bones; and the connective tissues which bind together other parts of the body. None of these is a working organ in the sense that a muscle or a gland is a working organ, and we are not surprised to find that their structure departs from that of the muscle and gland in that, while nucleated cells are present in all of them, the great mass of the organ is composed of lifelessmatter between the cells. In the tendon this consists of very strong parallel fibers (Fig. 24); the ligament shows much the same structure; a bone consists
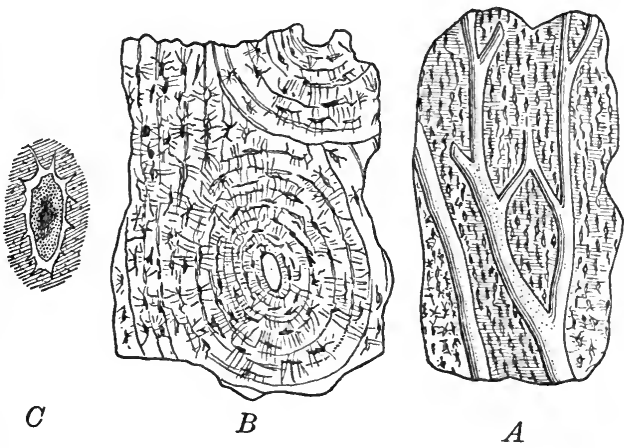
chiefly of lifeless Fig. 27. Longitudinal $(A)$ and transverse $(B)$ secmaterial containtions of bone

ing large amounts Showing the branching and communicating canalsof mineral matter, with cells lying here and there in spaces which in which are blood vessels and nerves - surrounded by the lifeless bone substance. In this are spaces connected with one another by very minute channels. Each of these spaces contains a living cell shown in $C$

communicate with one another by means of minute channels. The connective tissues, like that which binds the skin to the underlying muscles, or that which forms the sheath and septa of glands and muscles, consists essentially of lifeless fibers running in all directions and thus ready to limit the extent of any pull tending to separate unduly the adjacent organs. To organs and tissues of this kind we may give the name of supporting organs and tissues, and they form almost the sole exception to the general rule that the essential part of a tissue consists of its cells. 
The latter statement is true of all organs and tissues which do work, - the active organs of the body. In the case of the supporting tissues the cells which they contain are the fundamental units of the organ, since they make the intercellular lifeless substance. But the part which the organ plays in the work of the body as a whole is performed by

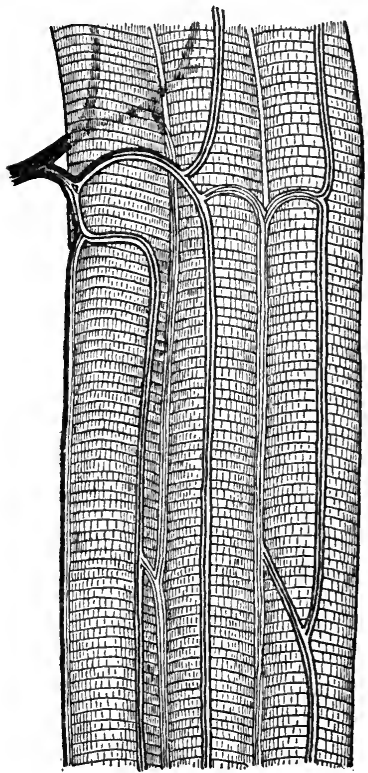

Fig. 28. Three muscle fibers and an artery breaking up into capillaries between them the lifeless substance (fibers, etc.) which the cells have manufactured and keep in repair.

9. The Blood Vessels are Closed Tubes in Connective Tissue. - The arrangement of connective tissues is fundamentally the same in the gland and the muscle. As their name implies, these tissues serve the obvious purpose of binding the anatomical units into organs, but they also perform other functions equally important.

We have seen (Chapter II, Section 19) that each organ receives blood through one or more arteries, and that this blood flows away from the organ through one or more veins. If a colored fluid mass which would afterwards set, e.g. a warm solution of gelatin colored with carmine, had been forced into the arteries before we began our examination, we should find that this mass would everywhere be confined in a system of closed tubes which merely lie in the connective tissue. The artery entering the muscle branches into smaller and smaller arteries in the general sheath of the organ, or in its branches, the septa; from these finer arteries an exceedingly rich network of small thin-walled 
tubes is given off to the finest connective tissue which surrounds the cells themselves; these tubes are the capillaries. They ultimately unite to form the larger veins, which can be traced in the septa to those veins which gross dissection reveals as leaving the organ (see Fig. 19). Through these tubes - arteries, capillaries, and veins the blood flows; and it is important for us to understand that it is everywhere confined to them in its passage through the organs; nowhere does it come into direct contact with the living cells (save those lining the vessels). Whatever exchange of matter or energy takes place between the blood and the living cells must be through the walls of the blood vessels. ${ }^{1}$ These walls are relatively thick in the arteries, usually somewhat thinner in the veins; in the capillaries, however, they are very thin, and it is through these thin capillary walls that all interchanges of matter take place. That the connective tissue surrounding the capillaries bears an important relation to the Fig. 29. Superficial and some deeper circulation we shall now see.

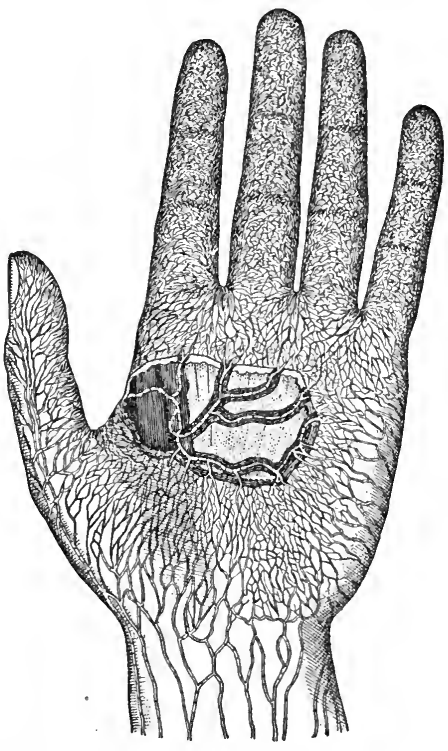

\section{The Lymph Spaces of the Connective Tissue. The} Lymph. - Careful examination shows that the fine connective tissue within which the capillaries are embedded

1 The term " blood vessel " is sometimes confusing to the beginner, since it suggests a utensil for holding liquids, like a cup or kettle or barrel. In anatomy the term "vessel" is applied to tubes, ducts, or canals through which blood or lymph flows. 
is not a solid or continuous mass, but rather a mass or

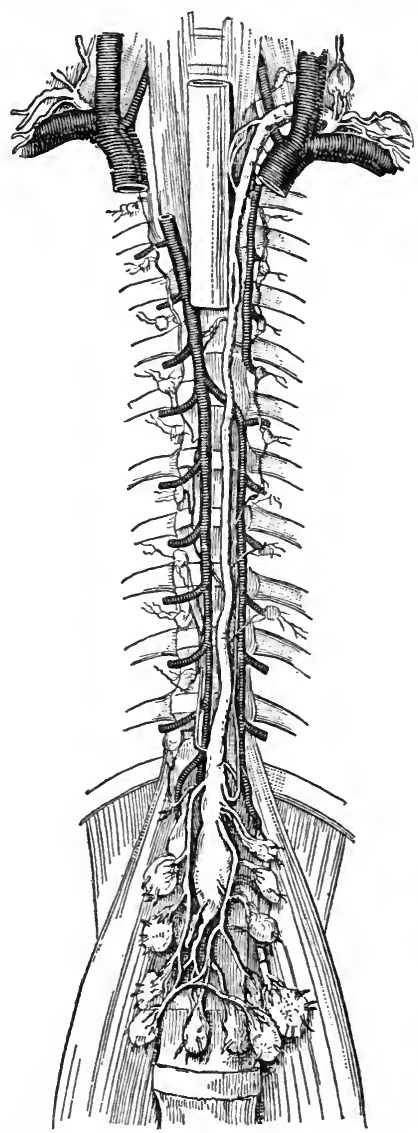

FIG. 30. The two main lymphatic trunks (in white), with their openings into the great veins near the heart

The larger of these trunks - that on the left side and known as the thoracic duct-returns all the lymph except that from the right side of the head, neck, and the right arm and shoulder region mesh of extremely fine fibers or bundles of fibers, with here and there "connective-tissue cells" which keep the fibers in repair. The connective tissue, therefore, is everywhere channeled by irregular spaces running between the fibers and other structures; these spaces communicate freely with each other and contain a colorless liquid known as lymph; the spaces of the connective tissue may thus be conveniently described as lymph spaces. They serve as communicating channels between the cells and the walls of the capillaries.

\section{Origin of the Lymph. -} The lymph which the spaces contain is derived partly from water and soluble food materials which have passed through the capillary walls from the blood, and partly from material produced by the neighboring cells (see the next chapter); on the other hand, the cells absorb from the lymph substances which the latter has received from the blood, while the blood, in turn, takes from the lymph substances discharged from the cells. The lymph thus 
becomes the means of communication, the middleman, between the living cells of the organs and the nourishing blood, and forms the immediate environment of the cells themselves. In other words, the cells of muscles, glands,

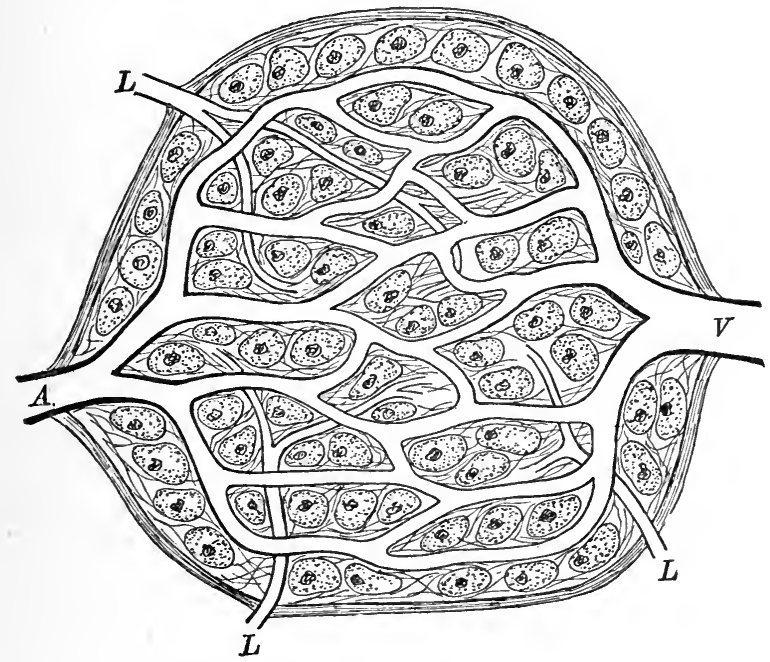

Fig. 31. Diagram of the relation of the cells of an organ to its blood vessels, lymphatics, and connective tissue $A$, artery; $V$, vein ; $L$, lymphatic

and other organs live in lymph, just as the human body as a whole lives in air, or a fish in water.

12. The Lymphatics. - Besides the veins, which convey blood away from an organ, a second system of tubes or vessels passes out through the capsule. These tubes arise in the lymph spaces of the connective tissue and unite with similar tubes from other regions to form larger and larger trunks, known as lymphatics, which ultimately form one or two great trunks and open into the great veins near the heart (see Fig. 30). Through these direct outlets the surplus lymph of the organ flows in a varying but for 
the most part continuous stream. This flow of lymph away from an organ is of the very greatest importance in maintaining the normal environment of the cells.

13. Function of the Lymph Flow from an Organ. - It is clear from inspection of Fig. 31 that there is a steady flow of liquid from the capillaries, through the lymph spaces of the connective tissue, over the surfaces of the living cells or of any intervening capillaries, to the lymphatics. The cell is thus bathed not by a stagnant medium but by one which is in gentle movement; one which brings to all parts of its surface the food which it needs and immediately carries away from all parts of its surface to the adjacent capillaries the products of its activity. By providing this outlet from the lymph spaces the lymphatics render possible the movement of lymph within the organ itself, whereby material is readily transferred from the cell to the capillaries, and from the capillaries to the cell.

14. Distribution of Nerves to Muscles and Glands. - The distribution of nerves resembles that of the arteries, the larger nerve trunks being found in the septa and their fine ultimate branches being distributed by way of the connective tissue which surrounds the cells, in whose neighborhood or even within whose substance they finally end. As we shall see in subsequent chapters, it is the function of the nerves to arouse the gland cells or muscle fibers or other cells to activity.

15. Summary. - Disregarding for the moment those peculiarities of arrangement, shape, and structure of the cells which are connected with the special work of each organ, (e.g. the arrangement of gland cells to form a blind tube, or of the connective tissue and fibers of muscle so as to exert a pull on a bone), we may say that the typical structure of an organ would be represented in Fig. 31 . The whole is surrounded by a capsule, receives a blood supply through a system of closed tubes, and contains the special 
cells upon whose activity its characteristic work depends. These cells are held together by a fine connective tissue whose numerous and freely communicating spaces contain a fluid, the lymph, which is free to flow out through a second system of tubes, the lymphatics. Nerves from the brain or spinal cord are also distributed in the connective tissue to the cells of the organ.

Before concluding this description of the finer structure of organs, a word may be added with regard to the physical nature of the cell substance. In its literal meaning the word "cell" is a misnomer, since it suggests a hollow space inclosed by solid partitions or walls. Plant cells do, in fact, usually have such walls around their cytoplasm (Chapter VIII), and this cytoplasm frequently contains spaces (vacuoles) filled with a solution of salts, sugar, and other dissolved material. But neither the cell wall nor vacuoles are of universal occurrence, each being rarely found in the animal cell, and often absent even in the plant cell. Fifty years of thorough investigation has reduced the number of essential cell constituents to the cytoplasm and the nucleus. The ultimate structure of these is still unsettled; but it would seem that the cytoplasm is a mixture of highly viscous, liquid materials, often containing minute granules in suspension, and possibly the nucleus is of a similar physical character. In these fluid substances occur those "vital" or "life" processes to the study of which we now proceed. 


\section{CHAPTER IV}

\section{THE WORK OF THE HUMAN MECHANISM THE RESULT- ANT OF THE WORK OF ITS DIFFERENT ORGANS AND CELLS}

From common experience we know that the work of the body as a whole is the resultant of the work of its various organs. We know that an act as simple as the taking of food involves the coöperation of the arm, fingers, eyes, teeth, tongue, and other organs. But we do not often realize that the work of each organ is equally the resultant of the work of its component cells. Eyes, hands, and tongue we can see and even watch at their work, but the cells of muscles and glands and nerves are invisible. If we could see them and delve into their recesses, we should there discover activities no less remarkable. As we cannot do this directly and with the naked eye we must do it indirectly and make use of experiments, chemical analyses, microscopic studies, and all other available methods. And we may again choose for detailed consideration two typical organs, muscle and gland, the structure of which we have studied in the last chapter.

1. Physiology of the Salivary Glands. Working Glands and Resting Glands. - The function of the salivary glands is the secretion or manufacture of saliva for use in the mouth, and one of the first things we notice about this act of secretion is that it is not constant but intermittent. Most organs have periods of activity, or work, followed by periods of inactivity, or rest, and these glands are no exception. Physiologists frequently speak of "working glands" 
and "resting glands." We all know that our own salivary glands work more effectively at some times than at others. The mouth "waters" at the sight of food; when we are in the dentist's chair the flow of saliva often seems excessive; and at other times our mouths are "parched" or "dry."

2. The Chemical Composition of Saliva. - The saliva is sometimes thin and flows readily, while at other times it is thick and viscous, or glairy. This difference is caused by the fact that the amount of water in it varies under different conditions. At all times, however, it is a fluid which consists of water containing certain solids in solution. The amount of these solids varies from five to ten parts in a thousand of saliva, and they consist chiefly of three groups of compounds. The first is mucin, familiar to us as the chief constituent of the phlegm or mucus discharged from the nose and throat, and giving to the fluid its viscous character; the second group consists of substances known as enzymes, which have the power of changing starch to sugar; these we shall study in detail in the chapters on digestion; the third group consists of mineral or inorganic salts, of which ordinar, table salt, or sodium chloride, is the most important. As we shall see, the salts and water are derived directly from the blood, while the mucin and enzymes are manufactured by the gland.

3. Blood Supply of the Working Gland. - Whenever a gland is actively working there is an increased flow of blood through it. For this reason the resting gland is slightly pink, while the working gland becomes distinctly red. Since the secretion of saliva requires water, and this can be obtained only from the blood, it is easy to see why an abundant blood supply is essential to activity. Other constituents of the saliva, such as the inorganic salts, likewise come directly from the blood. 


\section{The Relation of Nerves to Gland Work. Irritability.}

Nerves pass, as we have seen (p. 30), from the central nervous system to the salivary glands. These nerves are essentially bundles of nerve fibers which are distributed to the neighborhood of the gland cells, their final endings not yet having been conclusively demonstrated. But it is known that they are the means of conveying to the gland an influence, called a nervous impulse, and that nervous impulses cause the gland to secrete saliva. It is also a fact that when these nerves are cut or injured in any way, so that the gland is no longer in connection with the brain and spinal cord, saliva is not secreted, even when food is placed in the mouth. Evidently the activity of the gland is normally aroused by nervous impulses from the brain and spinal cord, just as the activity of a receiving instrument in a telegraph office is aroused by the electric current which comes to it over a wire, or as a mine is exploded by the same means. The gland then stands ready for the act of secretion, and is thrown into activity by a nervous impulse from the central nervous system. We speak of this action of a nerve upon the organ in which its fibers end as stimulaiion, and that property of an organ in virtue of which it may be aroused by a stimulus as irritability.

All the working organs of the body (in contradistinction to the supporting organs, p. 37) are in this sense irritable, and most of them receive nerves which set them to work. Irritable tissues may, however, be artificially stimulated by other means than by nervous impulses. Of these means an electric shock is the most familiar; others are the sudden application of heat, the presence of certain foreign substances in the blood, and even a sharp blow.

We have now to inquire what it is that happens in the gland when it is stimulated by a nervous impulse.

5. The Response of the Gland to Stimulation by its Nerve. - The visible result of stimulation of the gland is 
the discharge of saliva into the mouth. Something must have happened in the gland which has led to the passage of water and other substances from the blood (and lymph) through the gland cells into the duct. But something more has happened, for the saliva contains several substances which are not found in the blood. The gland has evidently contributed something to the saliva. How were these contributions to the secretion made?

When a gland has been resting for some time microscopic study shows that the cytoplasm of its cells becomes loaded with small granules, at times so numerous as to obscure the nucleus itself. As secretion goes on these granules disappear from the cell, presumably contributing something to the secretion. If the secretion continue for several hours, it is found that the granules have disappeared and that the size of the cell is often distinctly smaller than before secretion began.

The "resting" gland is therefore by no means an idle gland, but gradually stores within its cytoplasm something in the form of granules, which under the influence of nervous impulses or other forms of stimulation more or less rapidly disappear in the "secretion."

6. Activity of the Gland involves Chemical Change within its Cells. - It might be supposed that the granules manufactured during rest are merely dissolved or washed out of the cells in the copious stream of water and salts which during secretion passes through from the blood and lymph to the duct. If this were so, it would be possible to dissolve from the gland a substance exhibiting in general the same properties as the secretion itself. But this is not generally the case. Extracts of fresh glands commonly fail to exhibit the characteristic properties of normal secretions, and we are compelled to believe that the activity of a gland means something more than the mere discharge of previously stored substances; that is to say, the material of the 
granules in the resting cells is not simply set free when the gland secretes, but is at the same time chemically changed. In the digestive juices, for example, we have active substances called enzymes, which, it has been shown, are derived from other substances called zymogens, in the gland cells. The chemical change from the one into the other is as essential to the process of secretion as is the visible flow from the duct.

These facts then present to us the picture of the cell as the working or physiological unit, as we have already seen that it is the anatomical unit of the gland (p. 32). The work of the gland is the sum of the work of its constituent cells. During the period of rest these cells manufacture from the blood zymogens or other substances which they store away in the form of granules within their cytoplasm. When they are stimulated by the nervous impulse a chemical change takes place in them, the zymogens are changed to enzymes and other substances, and these, together with the water, salts, etc., derived from the blood, form the secretion.

7. Physiology of Muscular Contraction. - At first sight muscles and glands seem to differ in action or function no less than in form and structure. No two acts are apparently more unlike than lifting a weight by the muscles of the arm and the secretion of saliva by the salivary glands. But beneath obvious and important differences there are profound and fundamental similarities in the processes which occur in the two organs during activity. Like the gland, the muscle is set to work or stimulated by a nervous impulse; its contraction is accompanied by an increased blood supply; and, most important of all, the work or contraction is accompanied - indeed precededby chemical changes much more profound than that of the transformation of zymogen into enzyme. These chemical changes supply the power for the work. 
Muscles retain the power of contraction for a time after removal from the body. If such isolated muscles are inclosed in an air-tight space and made to contract by electric stimulation, carbon dioxide $\left(\mathrm{CO}_{2}\right)$ gas will be given off and sarcolactic acid $\left(\mathrm{C}_{3} \mathrm{H}_{6} \mathrm{O}_{3}\right)$ will be found in the muscle. Thus the activity of the muscle, like that of the gland, involves chemical change ; and just as glandular activity produces an output called a secretion, so muscular activity produces an output consisting of substances usually described as waste products.

8. Combustion or Oxidation in Working Muscle. - The source of the carbon dioxide, sarcolactic acid, and other waste products, as of the mucin, enzymes, and other substances produced by the active gland, must in the long run have been the matter taken into the body of the animal in the food. When, however, we compare the intake of food with the output of waste products of muscular contraction, we find that whereas the food is poor in oxygen the waste products are rich in that element. Somewhere, between the entrance of the food and the oxygen into the body and their final outgo as waste products, the two have chemically combined, very much as the coal and the oxygen which enter the furnace of an engine combine to form the gases which are discharged from the smokestack. This combination of substances with oxygen always occurs when anything is burned, a process which is called combustion or oxidation. The work of a muscle, like that of the engine, is the result of such combustion or oxidation.

9. Storage of Fuel Substances in the Muscle. - In one respect, however, the oxidation which gives rise to muscular contraction differs from that which gives power to a steam engine. At the time of the work the engine requires a draft, i.e. a constant supply of free oxygen to its furnace; shut this off and the fire goes out, - the engine comes to rest. 
With the muscle this is not so. An isolated muscle, from which every trace of free ${ }^{1}$ oxygen is removed, may be made to work in a vacuum, and in doing so it produces carbon dioxide and other waste products just as it does when it is surrounded by air. The presence of free oxygen at the time is not necessary to muscular work or to the production of waste products by the muscle.

To account for this remarkable fact we must suppose that the muscle has the power of storing oxygen within itself, not as free oxygen but in combination with other substances which upon the application of a stimulus readily yield it up to combine with the elements of the food, thus forming the waste products.

We cannot attempt to explain in an elementary work the details of this process; but enough has been said to show that during the period of rest the muscle, like the gland, has been engaged in storing something within itself. As the gland stores its zymogens ready to undergo chemical change into the enzymes, so the muscle stores from the food and oxygen what we may term fuel substances. It is these which readily change into waste products when the muscle is stimulated, thus completing the analogy so far as chemical activity of the two organs is concerned.

10. Nature of the Stored or Fuel Substances of Muscle. - The stored material of a muscle is not in the form of granules, as is that of the gland, nor has it been extracted from muscles, as zymogens have been extracted from glands. But it is clear that the material made by the muscle fiber from the food and oxygen must consist of unstable bodies, like gunpowder or dynamite, ready to go off, as it were, at the slightest touch; and, as in the case of these explosives, the change to the stable substances produced by their discharge gives the power for work.

1 i.e. oxygen uncombined with other elements; as the oxygen in the atmosphere. 
The comparison with gunpowder is so instructive that we may enter into it in greater detail. Gunpowder is a mixture of charcoal, sulphur, and saltpeter and contains no free oxygen. The oxygen which the mixture contains is chemically combined with potassium and nitrogen in the saltpeter $\left(\mathrm{KNO}_{3}\right)$, and upon the application of an appropriate stimulus, in the form of a spark for example, this oxygen is given up to unite with the carbon of the charcoal according to the following equation:

$\underset{\text { carbon }}{3 \mathrm{C}}+\underset{\text { sulphur }}{\mathrm{S}}+\underset{\text { saltpeter }}{2 \mathrm{KNO}_{3}}=\underset{\begin{array}{l}\text { carbon } \\ \text { dioxide }\end{array}}{3 \mathrm{CO}_{2}}+\underset{\text { nitrogen }}{\mathrm{N}_{2}}+\underset{\begin{array}{l}\text { potassium } \\ \text { sulphide }\end{array}}{\mathrm{K}_{2} \mathrm{~S}}$

Here again we may speak of the storage of oxygen during the chemical processes by which the saltpeter was made, just as the muscle has stored oxygen received from the blood in the form of some substance which readily gives it up, upon the application of a stimulus, - whether a nervous impulse, an electric shock, or a sharp blow, - to form with the carbon and hydrogen derived from the food the highly oxidized and stable waste products.

11. Similarity between the Work of a Gland and that of a Muscle. - These two tissues (glandular and muscular) comprise a very large part of the organs of the body. Among glands are the salivary glands, those in the lining of the stomach and intestine, the pancreas, the liver, the thyroid, the tear glands, and the kidney. The skeletal muscles comprise nearly half the total weight of the body; while among the muscular or contractile tissues must be classed the heart, half or more of the walls of the stomach, the intestine, the arteries, and the veins.

In the present chapter we have described in broad outline the work of these two organs in order that the student may see for himself that they present striking points of similarity; and these points of similarity are the 
very points which are typical of the action of most living cells. They may be summed up as follows:

1. Both gland cells and muscle fibers manufacture from raw materials of the blood something which they store within themselves (zymogen, fuel substance).

2. When the gland cell or muscle fiber which contains these substances is set to work or stimulated by a nervous impulse, or in other ways, a chemical change takes place, and the stored substance disappears. At the same time products of the chemical change made their appearance, e.g. the enzymes or ferments of certain glands and the "waste products" of muscular activity.

3 . In all the cells of the animal body the general character of these working chemical changes is that of a combustion or oxidation, certainly at times indirect, possibly at other times direct.

12. Cells as Factories. - These and similar chemical activities lie at the basis of the work of every cell of the human mechanism. Not only in the cells of glands and muscles, not only in all the other cells of higher animals, but in those of lower animals and of plants as well, oxidation and other chemical changes are invariable accompaniments of life. The exact nature of the change differs in different cells, and is adapted to the work which the cell must perform for the body as a whole. Hence the universal need of carbon and hydrogen and nitrogen in the food, and of oxygen obtained in one way or another. The cell is fundamentally a chemical laboratory, or, better still, a chemical mechanism fitted to employ chemical processes which occur within it for definite and useful ends.

13. The Relation of Chemical Change to the Work of Muscular Contraction. - The student will readily recall many examples which show that the act of combustion supplies the power necessary for doing work upon surrounding objects. The explosion of gunpowder will shiver solid 
rock; a hogshead of water may be evaporated by the heat of a fire and its vapor conveyed by tubes to other places where it may be condensed again into water; here the act of combustion has just as truly done work by moving the hogshead of water as if the water had been moved from the one place to the other by human effort. In both these cases it is comparatively easy to trace every step of the process and thus see clearly the relation between the combustion and the work done. In the case of the muscle fiber, on the other hand, the relation is not so obvious, but only because we do not so readily see the plan and mechanism of its constituent parts.

A man who has lived all his life as a hunter in the woods and sees for the first time a shoe factory may not understand how the combustion of coal in the furnace of the engine does so many and varied things, because he is not familiar with the construction and operation of machinery. We ourselves are in a somewhat similar position with regard to the muscle fiber; but, like the factory, it is a mechanism so arranged that the power supplied by oxidation of food is applied to accomplish a certain end, - the shortening or contraction of the fiber in which it occurs, and so the lifting of a weight.

It is now generally believed that the rodlike myofibrils (p. 36) form an essential part of this mechanism. But we cannot discuss this question more extensively at the present time. Our only purpose in introducing it is to give to the student that most fundamental conception of physiology, - cells as living mechanisms capable of using the power obtained from the oxidation of the food to do definite kinds of work.

14. Recapitulation. - The present chapter is entitled The Work of the Human Mechanism the Resultant of the Work of its Different Cells. We have traced the character of the work done in the case of the gland and the muscle, 
and have found that it is fundamentally the work of the cells of which the organs are composed. The cells of other organs are similarly constructed to do other kinds of work, and the character of their chemical changes and of the mechanisms for utilizing power varies accordingly; all, however, showing the same fundamental plan of working engines. We can now readily appreciate the meaning of the heading of the chapter; the body is a community of groups of cells of different kinds, each kind doing some work more or less peculiar to itself ; in addition to the two groups (gland and muscle cells) which we have studied, there are nerve cells in the brain, spinal cord, and elsewhere; cells which make blood corpuscles; cells which keep in repair the connective tissues, - bone, gristle, tendon, and ligament; and many more, such as cells which manufacture or themselves form the lining of free surfaces, like the skin, the alimentary tract, the air passages, etc. The sum total or net result of the activities of these and other cells makes up the work of the body as a whole; the work of the body - the human organism, the human mechanism - is thus the outcome or resultant of the work of its different component cells. 


\section{CHAPTER V}

\section{WORK, FATIGUE, AND RESTORATION}

While it is true, as shown in the last chapter, that capacity for work is one of the principal characteristics of the human body, no experience of daily life is more familiar than that work is followed by fatigue. This is true both of individual organs and of the organism as a whole; for fatigue may be either local, as when some one muscle is tired from hard work, or general, as when weariness affects all organs, - those which have been resting as well as those which have been working.

We use the word "fatigue" in two different senses, and it is important that a distinction be clearly made between them. In the one sense the word means the diminution of working capacity due to work. In testing one's strength of grip or of back a second test if made immediately shows less work done than at the first test; and this is true whether or not we are conscious of fatigue or of diminished working power. If, however, a certain time be allowed for rest, the second test will give as good results as the first.

In the other sense the word refers to the feeling of fatigue which frequently, though not always, accompanies the diminution of working power. We may even "feel tired" when we have been doing nothing, and conversely, under the influence of excitement or other causes, we may experience no feeling of fatigue even when we are near the limit of our working power. Often in an exciting game the players do not know at the time that they are tired or even 
that their working power is lessened; and stories are told of soldiers in hasty retreat who feel that they must " drop in their tracks" until the discharge of musketry close behind stimulates them to move faster than ever. The report of the rifles did not change the working power of the muscles, but only removed for the time being the feeling of weariness in the nervous system, which had interfered with getting from these muscles their best work.

The consciousness of fatigue has its seat in the nervous system, and its study must be postponed until we have learned something of the physiology of the brain and spinal cord. In the present chapter we are not immediately concerned with this side of the question, but rather with the diminution of working power produced by work. Such fatigue must be measured not by our sensations but by the work accomplished, whether that work be physical

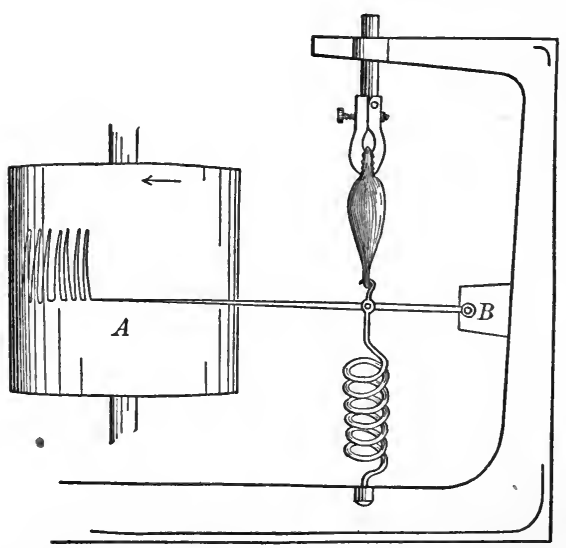

FIG. 32. Diagram of apparatus for recording successive muscular contractions or mental. And as we studied the physiology of work in its simplest form in a single working organ, such as a muscle or a gland, so we can best begin our study of diminished working power or fatigue in one of these same organs, namely, the skeletal muscle.

1. Fatigue of an Isolated Muscle and of a Muscle with Intact Circulation. - The course of fatigue in a muscle is best studied by causing the muscle to contract to its utmost, at regular intervals of time, against the resistance 
of a suitable spring. If now we record the height of each contraction, we obtain a series which shows at once the effect of the work on the working power, i.e. the course of fatigue. Fig. 32 gives a diagram of the arrangement of such an experiment with an isolated muscle, i.e. a living muscle detached from the rest of the body. One tendon is made fast in a rigid clamp, while the other is attached to the spring, which is stretched by the contraction when the muscle is stimulated. The length of the line written by the lever $A B$ records what the muscle is capable of doing at the time; and if the records of successive contractions are made on the smoked surface of a slowly revolving drum, as in the figure, we have at once a record of the course of fatigue.

Such fatigue tracings may also be taken from a muscle within the body, and hence with its circulation intact. Thus the work of the biceps muscle

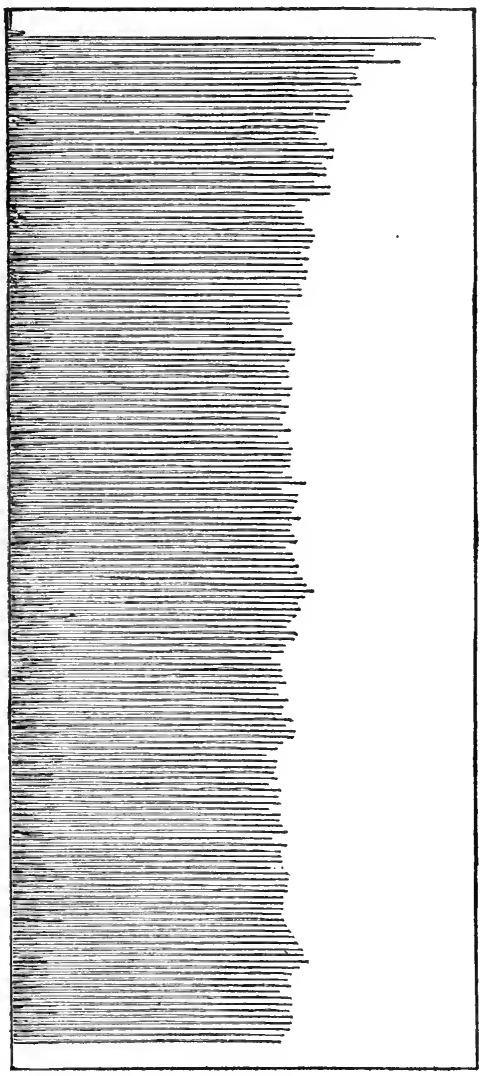

Fig. 33. Record of the successive contractions of the flexor muscles of the elbow joint

Showing the gradual decrease in working power to a fatigue level. The muscle contracted once every three seconds against the resistance of a strong spring, which was stretched each time as far as the strength of the muscle permitted 
in flexing the arm at the elbow (Fig. 23) may be recorded by instruments essentially similar to that used with the excised muscle. And in Fig. 33 we have reproduced a tracing of this kind.

It is evident that a continuous line joining the highest points reached by the several contractions will represent graphically the course of fatigue, and in Fig. 34 the line $a$ represents this so-called "curve of fatigue" in the experiment whose results are given in Fig. 33. It falls off at first rather rapidly, then more and more slowly, until at last it becomes parallel with the base line. In other words, the muscle in this case finally finds a constant level of working power. This may be called the fatigue level.

The broken line $b$ in Fig. 34 gives the result of a fatigue tracing with the isolated muscle. It will be seen

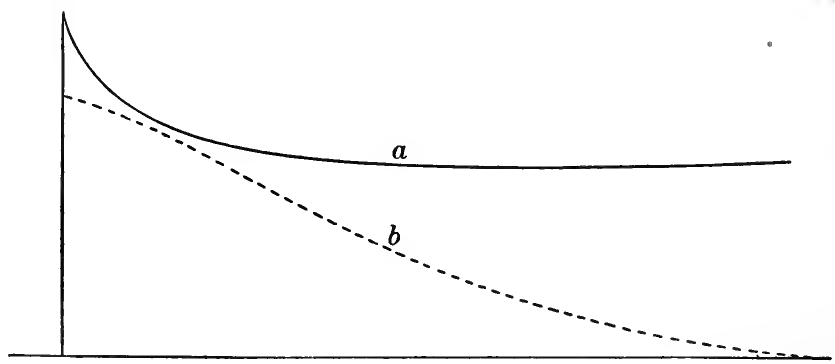

Fig. 34. Curves of fatigue

$a$, from a muscle with intact circulation; $b$, from an isolated muscle

that the fall in the height of contraction continues until at last the muscle no longer responds to stimulation. The contrast thus brought out between the effect of work upon muscles with and those without the circulation shows that the circulation of the blood through the working organ in some way maintains the working power.

The height of the fatigue level in the same muscle at different times is very closely dependent on the rate at 
which the muscle works. Thus with a contraction every four seconds instead of every three seconds the fatigue level would be higher than in Fig. 33 ; with a contraction every second it would be much lower. When the contractions come every nine or ten seconds there is usually no falling off in the work done, the time between contractions being sufficient for the complete recovery of working power.

This picture of fatigue hardly agrees with our feeling of fatigue, for the decline of working power begins at once, or at most after a very small number of contractions, whereas we usually notice fatigue only after work has gone on for a considerably longer time. One does not feel tired from walking, for example, during the first ten or twenty minutes of the walk. We need not discuss here just what makes us unconscious of the beginnings of fatigue; but it is important to understand that whether we are or are not aware of its presence, fatigue is the invariable and immediate result of all muscular work.

Weariness is simply the conscious sensation of fatigue, but fatigue is a physical condition of living cells and organs. Moreover, its phenomena are by no means confined to muscular work. When a gland is stimulated to vigorous secretion a diminution is sooner or later noted in the amount of the secretion, and there is good reason to believe that nerve cells may also become tired from continued activity. Fatigue, then, in one word, is a natural condition of an organ accompanying work, and we may proceed to inquire into its exact cause.

2. Waste Products as a Cause of Fatigue. - When blood which has been circulating through a fatigued muscle is sent through a resting muscle, the resting muscle shows signs of fatigue, even though it has itself done no work. Apparently the blood has extracted from the working muscle something which has the power of lessening the working capacity of a fresh muscle. 
The same thing is illustrated by another experiment. A muscle which is deprived of its circulation, e.g. by clamping its arteries and veins, is fatigued by vigorous work; it is then found that although when left to itself a slight recovery takes place, this recovery is much more marked if we first pass through its blood vessels a weak solution of salt. Here no food is supplied; the salt solution has only removed something from the fatigued muscle, which in consequence of this treatment recovers some of its working power.

Again, the mere exposure of a resting muscle to blood containing sarcolactic acid, or to blood heavily charged with carbon dioxide $\left(\mathrm{CO}_{2}\right)$, produces the condition of fatigue. Now in the last chapter it has been shown that both sarcolactic acid and carbon dioxide are waste products of muscular activity; and these and other facts have led to the view, now generally received, that the waste products of the active organ interfere with the work of the organ and so constitute one of the main causes of fatigue. It is apparently for this reason that the injection of an extract of worked muscle fatigues fresh muscle, for the extract contains waste products. It is for the same reason that washing out a fatigued muscle with salt solution produces partial recovery, for the waste products of activity are in this way partially removed. We can also understand why fatigue always accompanies vigorous work. Waste products then necessarily accumulate and clog the living mechanism, because they cannot be removed by the blood as fast as they are formed by the muscle cells. No fatigue occurs with only a single contraction every ten seconds or more, because between contractions sufficient time is given to insure the complete removal of wastes.

3. Loss of Fuel in the Working Muscle as a Cause of Fatigue. - The blood, however, not only removes the wastes but also brings new food and oxygen with which 
the muscle makes good the loss of explosive fuel; and it may well be - although it is not absolutely proved - that recovery from fatigue depends upon both of these good offices of the blood. We have certainly one well-established cause of fatigue, namely, the presence of the waste products of activity; and we recognize the probability that the depletion of explosive fuel may also contribute to the result. But whether the first of these causes alone is sufficient to explain it, or whether both work together, we can understand that the maintenance of a good blood supply is of the first necessity, and that undue fatigue can be avoided only by working at a moderate rate. It is an old and physiologically true saying that "it is the pace that kills."

4. Explanation of the Fatigue Level. - In the experiment with the isolated muscle no waste products were removed, nor were new food and oxygen supplied; hence the wastes in the muscle increased with each contraction, until at last their accumulation prevented all contraction. In the normal muscle the wastes likewise accumulate for a time; and this is why the curve of work at first falls (Fig. 33). It does not continue to fall, because as the wastes within the muscle increase in amount the blood carries more and more of them away in a given time. The quantity of waste removed thus continues to increase until the same quantity is carried away from the muscle between two contractions as the muscle produces with each contraction. When this happens no further accumulation of waste is possible and the fatigue level is established.

5. General Fatigue resulting from Muscular Activity. - Every one knows that after a day's tramp it is not simply the worked muscles which are unfit for good work, but that the brain, too, is tired, for hard mental work is then difficult or well-nigh impossible; and it is generally the fact that long-continued muscular work fatigues the brain 
more than brain (mental) work itself. The obvious explanation of this fact is that the waste products of muscular activity have accumulated in the blood more rapidly than the body can get rid of them, and so have fatigued the other tissues, including the nerve cells of the brain, just as the injection of the extract of a tired muscle lessens the working power of a fresh muscle. No doubt these same waste products may similarly fatigue gland cells; for experience seems to show that the secretion of digestive juices is not so active when one is suffering from muscular fatigue, and that it is not wise to eat heavy meals when one is tired out. We can also understand why long-continued, vigorous muscular action produces marked fatigue in nerve cells and gland cells, while the activity of the latter produces only inappreciable fatigue in the muscles; for the amount of chemical change and the production of wastes is far greater in the case of muscular work than in that of nervous or glandular activity.

6. The Analogy of the Engine. - In previous chapters we have compared the living body with a machine or locomotive engine; both do work, and both obtain the power for work from the direct or indirect oxidation of food or fuel. What we have now learned about fatigue suggests an extension of the same comparison. Every locomotive suffers impairment of its working power with use, and special measures are taken to limit this impairment as much as possible; the gases and smoke are carried away at once by the chimney or smokestack; the furnace is provided with a grate so that the ashes shall not accumulate and shut off the draft; the bearings are oiled and foreign matters removed; above all, as the consumption of fuel goes on, the loss is made good by stoking.

The continuance of the work of the engine requires two things, - fresh supplies of fuel and the removal of wastes. Obviously the blood performs these same offices for the 
cell. It supplies to the cell fuel (food) from the alimentary canal and oxygen from the lungs, and it carries away the waste. Provision is thus made to maintain the human machine in working order and good condition during its activity. If the blood flows too slowly through the muscle, the same thing happens as in the locomotive when the fireman neglects to rake the fire or to put on new fuel; the efficiency both of the human engine and of the locomotive may be impaired either by the undue accumulation of the waste products of its own activity or by the neglect to supply proper food or fuel.

7. The Cell a Living Machine. - The similarity of the cell to the lifeless engine is more than a mere analogy. The cell is not only like - it actually is - an engine, machine, or mechanism, but it is also a living mechanism. This means that it possesses properties and powers not shown by the locomotive. Both may be injured by overwork or by accident, but only the living mechanism is itself capable of repairing damage; the locomotive must be sent to the shops and be repaired by work done upon it by other machines; if the boiler rusts, it must be taken out and a new one put in ; if the wheels wear unevenly, they must be made true again by turning in a lathe, or new ones must be substituted; when the grate burns out a new one must be put in its place. The living cell, on the other hand, itself makes these repairs from the same material that supplies its fuel. Indeed, the material for the repair of the living cell is to some extent the same thing as the fuel; and when food is not supplied with sufficient frequency this living machine actually consumes its own substance for fuel, - as happens, for example, during periods of starvation. Nothing of this kind, of course, is seen in any locomotive.

These are merely suggestions of the striking differences between the living engine and the locomotive. But we 
cannot pursue this subject further in an elementary work like the present; enough has been said to indicate the difference, while the facts given in this and the preceding chapter show that so far as the resemblances go, they are complete. Both machines do work, both require power for their work, both derive their power from the oxidation of fuel, both are provided with special mechanisms which transform energy into definite kinds of work, both are clogged by the accumulation of waste products, and hence both require care for their best efficiency. Yet the full recognition of the fact that the cell is an engine, a machine, a mechanism, should not make us forget that other fact that it is more than this: that it is a living engine, a living machine, a living mechanism, capable not only of fatigue but also of self-repair ; and, perhaps most wonderful of all, the organism which these cells compose is capable of sensations of weariness in fatigue, and of refreshment and new power after feeding and rest, - powers and sensations utterly lacking in any lifeless machine however perfect. 


\section{CHAPTER VI}

\section{THE INTERDEPENDENCE OF ORGANS AND OF CELLS}

\section{Are Cellular "Waste Products" necessarily Harmful?}

We have now learned that the active living cells of the body are the seat of chemical changes which produce new substances; that the accumulation of these products of activity tends to limit the working power of the cells in which they are produced, and may even depress the activity of other cells to which they are carried by the blood. In the case of the skeletal muscles we have spoken of the carbon dioxide, the sarcolactic acid, etc., as "waste products," meaning thereby that they are incapable of serving as sources of power for the work of the muscle; and this term, together with the fact that they constitute one cause of fatigue, is apt to mislead us into supposing that they can be of no further use to the body, or, even more, that they are necessarily harmful and that their presence in the blood is objectionable.

These conclusions, however, do not necessarily follow from the facts. It does not even follow that a substance which produces fatigue is for that reason undesirable. Most adults can recall times when because of long-continued application to mental work, or because of worry or other nervous strain, they have become overexcitable and restless, and have been unable to obtain the sleep of which the body as a whole stands in need. At such times sleep is often best secured by producing general fatigue through muscular work. The waste products, by their very act of fatiguing the overexcited nerve cells, may be of service to 
the body as a whole. And it is probably true that not only in such abnormal conditions but also in the daily conduct of life the fatigue of moderate muscular activity contributes its share toward inducing healthful and refreshing slumber.

Thus far we have considered the chemical activities of each organ as contributing to the work of the organ in which they occur, and, because of the accumulation of waste products, as the occasional cause of undue interference with efficient activity, both in the working organ and elsewhere. And yet the familiar case which we have just cited suggests another view of the matter. The products of the chemical activity of one organ may be of service to other organs, and so to the body as a whole; and while their too rapid accumulation in the blood is undesirable, their presence in moderate amounts may be beneficial, and may contribute to the normal environment of the cells of. the body.

It must never be forgotten that the secretions of the salivary glands, the stomach, and the pancreas as truly contain products of cellular activity as does the blood which leaves a working muscle. It is also probable, although it has not yet been directly proved by experiment, that if such a secretion were to accumulate within and around the gland cells, it would limit their activity and produce a true fatigue. The constituents of the saliva, for example, are waste products of the gland, in the sense that they do not serve for the manufacture of new saliva, but we do not call them waste products chiefly because we know they are still useful to the body.

2. The Thyroid Gland. - This view of the case is strikingly emphasized in the physiology of the thyroid gland, a small organ in the neck, the two chief lobes of which lie alongside the trachea. For a long time its use was not understood, and at times it was even supposed that 
it played an unimportant part in the life of the body as a whole. It has been found, however, by experiment that removal of the thyroid is followed by a disease in all respects similar to one which had long been observed in human beings, and especially in children; and this fact suggested that the disease is due to the failure of the thyroid to perform its normal functions.

The subject was further cleared up by the discovery that after the removal of the thyroid in a lower animal the disease in question could be prevented by feeding the animal thyroids or even by giving to it a certain substance extracted from them. Evidently the thyroid manufactures and

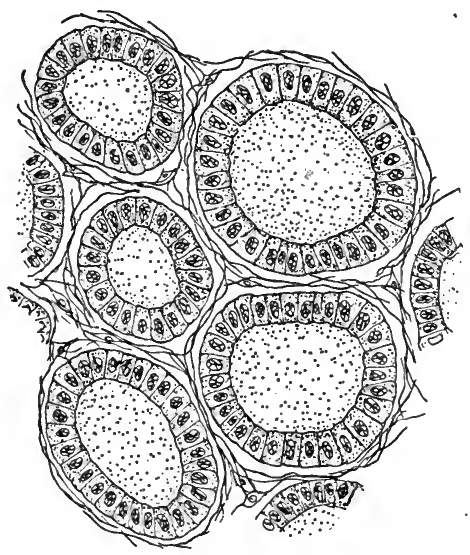

Fig. 35. Cross section of the thyroia gland

The cells secrete into the closed sacs, which they surround, the internal secretion, which then passes out between the cells into the lymph spaces of the connective tissue discharges into the blood a peculiar substance necessary to the healthy life of the cells of the body; and when the gland fails to manufacture this substance it can still be supplied artificially by introducing it into the blood by absorption from the alimentary canal.

3. Internal Secretions. - In our study of secretion in Chapter IV (p. 44) we dealt only with glands which discharge their principal products through a duct into some part of the alimentary canal; such glands are the salivary glands, the pancreas, and the liver. Other glands send ducts to the surface of the body, - for example, the sweat glands which discharge perspiration upon the skin, and the lachrymal glands which discharge the tears on the eyeball. In the 
case of the thyroid, on the other hand, we have an example of an organ which, like those just mentioned, manufactures a special substance from the blood, but, having no duct, contributes the products of its manufacture to the blood, for the use of other cells. This process is spoken of as internal secretion, to distinguish it from ordinary secretion, in which case something is discharged on a free surface like the skin, or into the alimentary canal, the nasal cavity, or the air passages.

4. Other Examples of Internal Secretion. - Certain other organs similarly manufacture and contribute to the blood material for use elsewhere. Lying immediately above the kidney are two small bodies, the adrenal or suprarenal bodies, which, like the thyroids, were until recently supposed to be of minor importance. It is now known that these also contribute to the blood an internal secretion which is absolutely indispensable for the healthy life of other organs.

A still more remarkable discovery has shown that the pancreas not only manufactures an important digestive juice (pancreatic juice) which it discharges into the intestine through its duct (pancreatic duct, see Fig. 50), but also produces another substance which is necessary in order that other organs may use the sugar which is in their food. Here we have an example of an organ which produces both an ordinary and an internal secretion, and the same thing seems to be true of the kidney, as it certainly is of the liver.

Thus, through the medium of the blood the chemical activity of one organ may affect the life of other organs favorably or unfavorably. All the cells of the body help to make the blood what it is, many of them contributing to it something useful or even necessary to other cells. The work of the body is not merely the sum total of the work of its separate cells, each working for itself alone and performing a single function. Between the cells an 
exchange of products often takes place, so that cells become both serviceable to and dependent upon one another for the material needed to carry out their own special chemical activities. And what is true of cells is no less true of organs; these also are interdependent, ministering to one another.

5. The Coöperation of Cells and Organs, and the Net Result. - In the foregoing paragraphs we have repeatedly spoken of the work of the cells (and organs) as if this work were the sole end and object of their existence. If, however, we turn back to Chapter IV and recall its principal lesson, namely, that the net result (resultant) of the work of the individual cells and organs of the body is the work of the body itself, then we shall perhaps begin to realize that no matter how important to the cell or to its neighbors individual cell work may be, this is even more important as an indispensable constituent in the work of the body as a whole. Any one can observe the human mechanism actively working at home or in field or factory; or running, leaping, or sleeping; or engaged in professions or business or trade; but only the physiologist realizes how these most various acts are merely the net result of the lives and activities of myriads of individual cells, - - lives in which the cells not only minister and are ministered unto, but also work together both for mutual benefit and for the benefit of the body as a whole.

Least of all do we commonly realize that it is not merely the operation, but the coöperation, - not merely the work, but the combined, and especially the harmonious or orderly, work of the myriad cells of the body, united as they are into one grand army, - that underlies the effective work of the human mechanism as a whole. To the study of the mechanism of this coöperation we may now proceed. 


\section{CHAPTER VII}

\section{THE ADJUSTMENT OR COÖRDINATION OF THE WORK OF}

ORGANS AND CELLS

A great physiologist once said, "Science is not a body of facts; it is the explanation of facts." Some of the most important chapters of science are those which seek to explain facts so well known and obvious that we are apt to forget that they need explanation. When anything irritates the lining of the nasal cavity we sneeze; when it irritates the larynx we cough; when it irritates the exposed surface of the eyeball we wink. These three facts are well enough known; but it is safe to say that any one considering the matter for the first time would find it difficult to explain how it comes about that anything going "down the wrong way" does not make us sneeze or wink, but sets us to coughing. The answer to the general question thus raised is the subject of this chapter, which considers the adjustment of the work of the individual cells and organs of the body, each to do its work at the proper time, and so to play its due part in the work of the organism as a whole.

The more we think of it, the more wonderful does this fact of adjustment appear. The millions of living cells are in a way individual units, and communities of individuals do not invariably work together. Let us compare the human body in this respect with bodies or groups of men or boys. In a game of football each team is a body of eleven individuals, and each individual is assigned to a definite position to do definite things as occasion arises. Theoretically, 
under given conditions of the game it is the work, or function, of the "left tackle" to prevent a certain player of the opposing side from making a certain play. But there is always a doubt whether he will do this thing, or "lose his head" and do something else, leaving his man free to do what he pleases. In the latter case that organism which we call a football eleven would act very much as the human organism would act if it were to wink and not cough when a foreign body lodges on the lining membrane of the larynx.

Evidently we have something here to explain. Why are the actions of the body purposeful, that is, adapted to accomplish the right thing at the proper time? and in the more complicated actions, how is the work of the different units - the organs and the cells - adjusted, or coördinated; that is to say, how is each one made to do its proper share of the work? Let us begin with the study of a very simple action, - that of winking.

1. Winking is caused by the contraction of muscle fibers which run transversely across the eyelid in a curved course. As they are attached most firmly at the regions $A$ and $B$ (Fig. 36), their shortening straightens their arched course and so brings the two edges of the eyelid into contact. The work of this muscle is obviously purposeful, for the wink takes place when the eyeball needs protection; it is also co-

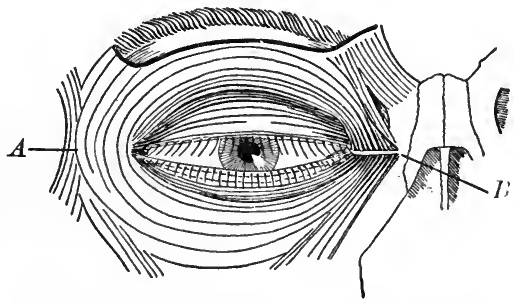

Fig. 36. The muscular mechanism of winking ordinated, since the act is executed by a number of fibers working together, for if only those of the lower eyelid were to contract the lids could not be closed.

The muscle fibers which work together to produce the wink do not originate their own activity. They merely do 
what they are stimulated to do by the nervous impulse, which acts upon the muscular fuel substance somewhat as a fuse acts upon a charge of gunpowder. Even the amount of contraction is determined by the strength of the nervous impulse, a strong impulse producing greater contraction than a weak impulse. In health the muscle fibers are the
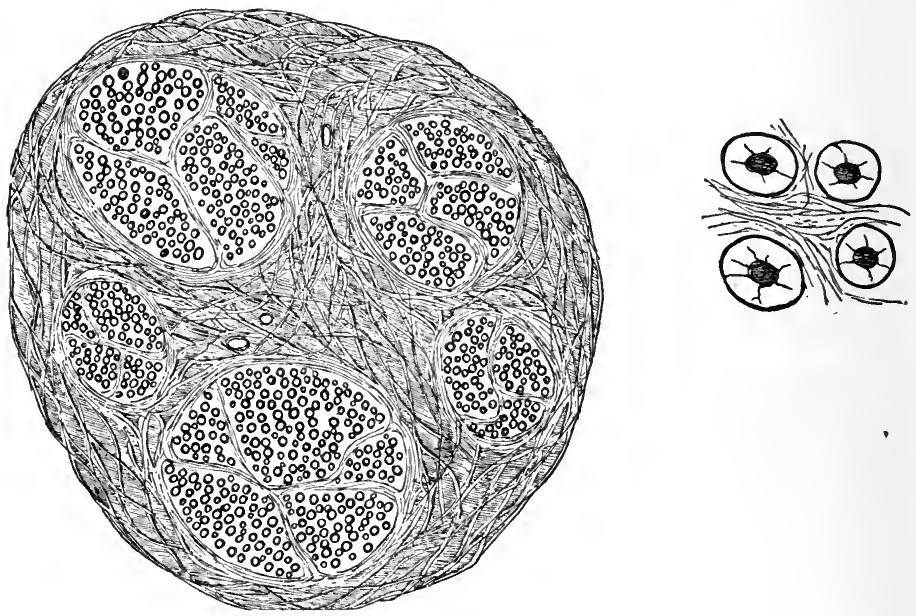

Fig. 37. Cross section of a nerve

Showing five bundles of nerve fibers bound together by connective tissue containing a few blood vessels. On the right are shown four fibers more highly magnified, the dark center being the axon, around which is the white or fatty sheath, both axon and fatty sheath being inclosed within the fine membrane, the neurilemma. Compare Fig. 38

obedient servants of the nerves, and if they act in a purposeful and coördinated manner, it is because the nerves stimulate them to act in this way. The explanation of purposeful and coördinated action must therefore be sought not in the muscles but, behind these, in the nervous system, to the study of which we now turn.

2. Structure of a Nerve. - A nerve, like a muscle, may be separated into long fibers (Fig. 38) which are bound together by connective tissue containing blood vessels, lymph 
spaces, and lymphatics. The nerve fiber, which is the essential part of the nerve, just as the muscle fiber is of the muscle, differs somewhat in structure in different nerves ; it generally consists of a central threadlike core surrounded by a fatty sheath, the latter being, therefore, shaped like a hollow cylinder, - which, however, is interrupted at intervals of about one millimeter, - and both of these are enveloped in a delicate membrane comparable to the sarcolemma of the muscle fiber. Such fibers are from about $\frac{1}{50 \overline{0}}$ to $\frac{1}{10 \overline{0}}$ of an inch in diameter (compare the diameter of a muscle fiber, p. 35).

There are, however, nerve fibers $R$, node of Ranvier at which which have no fatty sheath, and others which are destitute of mem-
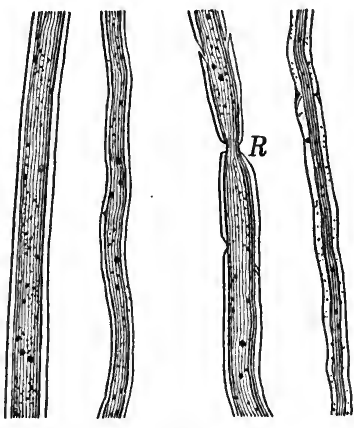

Fig. 38. Four nerve fibers (highly magnified) the fatty sheath is discontinuous

brane. The essential part of the fiber is the threadlike portion in the center; this is never absent from nerves and is known as the axon, or axis cylinder.

3. The Axon of a Nerve Fiber is a Branch of a Nerve Cell. - By suitable methods these axons may be traced along the nerve of which they form part, and even into the brain and spinal cord; it is then found that they pursue an uninterrupted course and ultimately become continuous with the cytoplasm of a nerve cell. Nerve cells are found in the brain, in the spinal cord, in enlargements (ganglia) on certain nerves, and even alone in the connective tissue of many organs of the body, as the heart, the intestine, etc. By far the greater number are in the brain and spinal cord, and in some cases the axons to which they give rise are of very considerable length; those of the muscles of the foot, for example, reach from cells in the sacral region of the spinal cord to the extremity of the foot. Such fibers would 
be over a yard long and less than $\frac{1}{1000}$ of an inch wide, and we may regard the cell whose main portion is in the sacral cord as sending out a branch, or process, from this region to the foot.

Furthermore, recent investigations have led to the generally accepted conclusion that each axon is a part of only

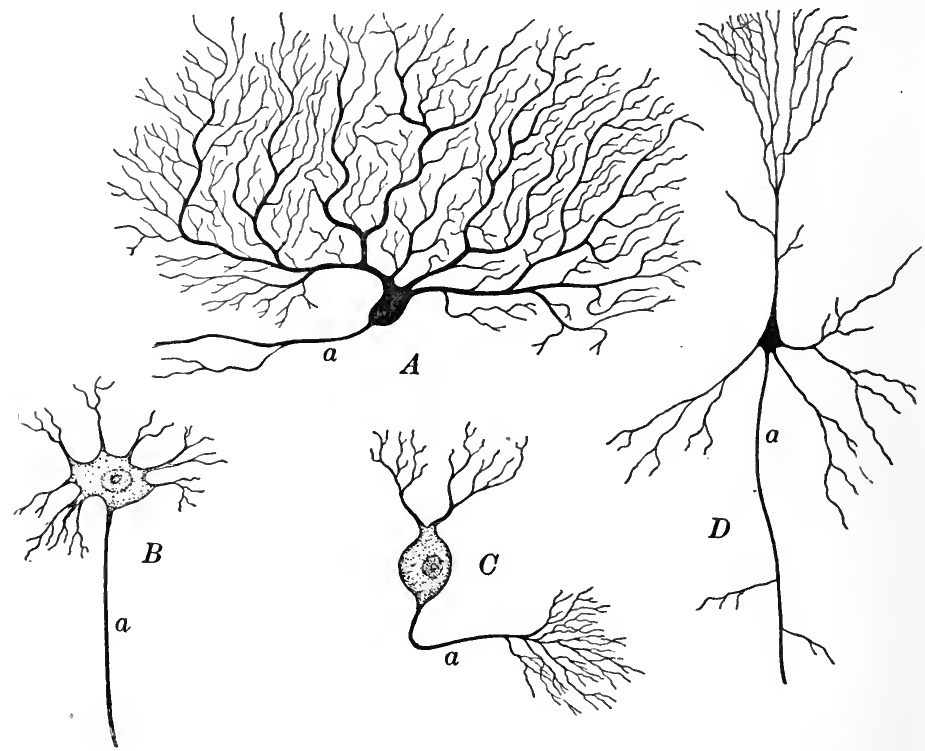

FIg. 39. Four nerve cells

$A$ and $C$, from the cerebellum; $B$, from the gray matter of the spinal cord; $D$, from the cerebrum; $a$, the axon. The cells $A$ and $D$ are stained so that the main body and the dendrites (p. 76) are a uniform black; $B$ and $C$, so as to show the nucleus and cytoplasm

one nerve cell; a single cell may give off more than one axon, but the axon is never connected with more than one nerve cell. Of these cells and of their connections with nerve fibers we can get a more definite picture by an examination of the structure of the spinal cord.

4. Structure of the Spinal Cord. - When the vertebral canal is opened a whitish cord is found within it, - the 
spinal cord, - from each side of which arise thirty-one pairs of nerves, or, in general, one pair for each vertebra. One nerve of each pair arises on the ventral side of the cord, the other on the dorsal side. These nerves are known as the ventral and dorsal nerve roots ${ }^{1}$ respectively. On the dorsal nerve root some distance from the cord there is a slight enlargement, or ganglion. Just outside this ganglion the two roots unite, and from their union nerves pass to the skin, the muscles, the blood vessels, the viscera, etc. Dorsal

The spinal cord itself in cross section shows a darker central core, known as the gray matter, surrounded by an outer lighter portion, the white matter. The
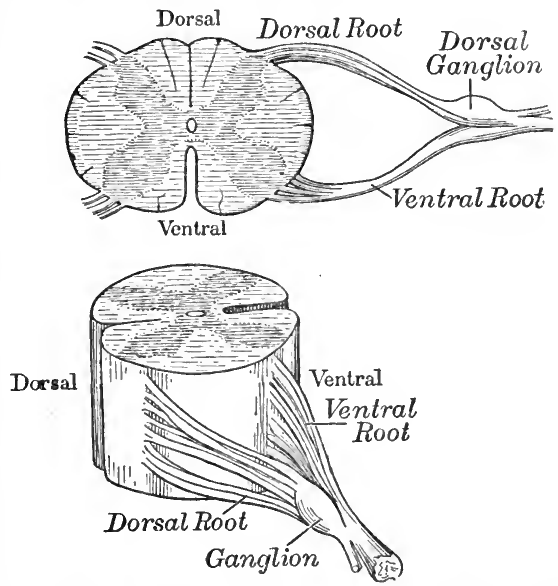

Fig. 40. The origin of the dorsal and ventral nerve roots of a segment of the spinal cord

white matter consists essentially of nerve fibers which run lengthwise of the cord and here and there send branches into the gray matter; it may be regarded as a large nerve. The gray matter, on the other hand, contains a mesh of fibers, and in addition numerous nerve cells. There is the same difference everywhere between the white and gray matter of the nervous system; the arrangement in the brain is not so simple as in the cord, but here also the white matter consists of fibers running from one part of the nervous system to another, while the masses of gray matter always include collections of nerve cells.

1 The older anatomical terms, and those even to-day more generally used, are anterior and posterior instead of ventral and dorsal. 
5. Fibers of the Ventral, or Anterior, Nerve Root. These fibers may be traced into the spinal cord. It is then found that the nerve cells from which they arise lie in the gray matter in the immediate neighborhood of the root to which they belong; i.e. the fibers of the roots do not come from higher or lower parts of the cord, or from the brain. It has also been found that when they are stimulated they throw muscles into contraction and produce effects on the blood vessels and glands, but they do not give rise to sensations, nor produce other effects in the cord itself. In other words, the fibers of the ventral root conduct impulses from the cells of the spinal cord outward; they do not conduct impulses from outside into the spinal cord. Hence they are known as efferent fibers (Latin, ex, out of ; ferre, to carry).

The nerve cells from which these fibers arise consist of a mass of cytoplasm around the nucleus and of one or more outgrowths of this cytoplasm, usually more or less branched. These outgrowths, or processes, divide and subdivide, ultimately forming in the gray matter exceedingly fine terminal branches like those of a tree in the air. Such processes are known as dendrites (Greek, dendron, a tree). The nerve cells in question have numerous dendritic processes; in other nerve cells there may be but one, and still others possess no dendritic processes at all. In all cases the general appearance of the cell depends largely upon the number and manner of branching of these processes. Thus it happens that nerve cells differ from one another in appearance just as a Lombardy poplar, an oak, an elm, and a maple differ, although all show the fundamental characteristics of a tree (Fig. 39).

In subsequent portions of this work it is unnecessary for us to go into the details of the form of the nerve cells to any extent; the student need only understand henceforward that nerve cells consist of a central mass of 
nucleated cytoplasm from which proceed outgrowths, or processes, which are of two kinds, - (1) those which become axons of nerve fibers, and which form an essential part of all nerve cells; and (2) the dendrites, which are usually but not always present. The whole structure, including the central cell body with its dendrites and axons, is an anatomical unit, - a cell. To this entire cell the term neurone is given. The neurone is the cellular unit of the nervous

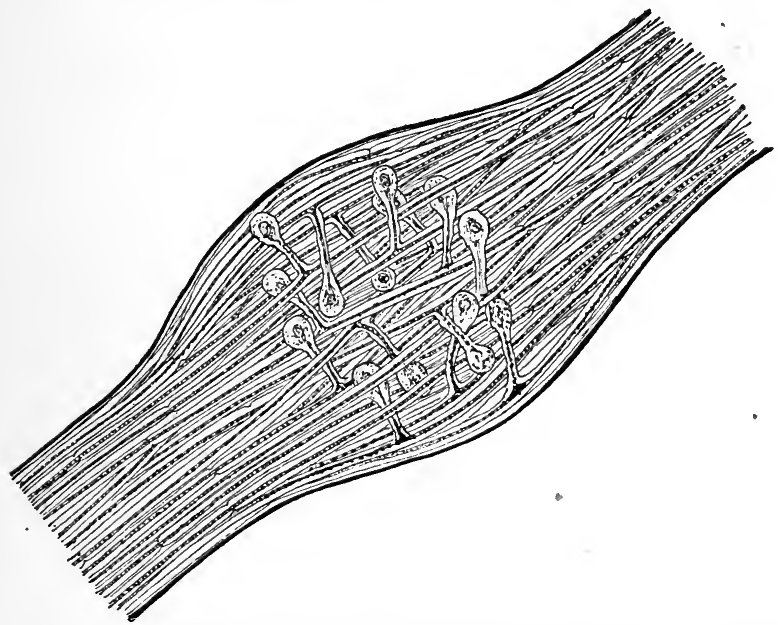

FIg. 41. Semidiagrammatic longitudinal section of a ganglion of the dorsal (posterior) root

system, just as the muscle fiber is the cellular unit of the muscle, and the gland cell of the gland.

6. Fibers of the Dorsal, or Posterior, Roots. - The ventral roots, as we have seen, are entirely efferent in function; that is, they conduct impulses only away from the spinal cord. The dorsal, or posterior, roots, on the other hand, are found to be essentially afferent (Latin, ad, to; ferre, to carry), i.e. they carry impulses from outside toward and into the spinal cord. This is shown by the fact that when 
these roots are destroyed by disease muscles can still be thrown into contraction, glands will still secrete, etc., -i.e. there is no interference with efferent impulses, - but no sensations are received from the part of the body to which these nerves are distributed; pinching the skin is not felt; the flesh may be burned and its owner be entirely unconscious of it. Since these results never follow destruction of the ventral roots, we must conclude that impulses enter the cord solely by the dorsal roots precisely as they leave the cord solely by the ventral roots.

It has been stated above (p. 75) that there is a ganglion on the dorsal root. Microscopic study of this ganglion shows that the fibers of the dorsal root pass through it, and that each fiber gives off at right angles to itself a branch which becomes continuous with a pear-shaped nerve cell of the ganglion. These cells have no other processes. We may express the relation between the pear-shaped cells of the ganglion and the fibers of the dorsal root by saying that the single axon from the main cell body divides into two in the ganglion, one branch passing outward to the periphery, and the other centrally into the spinal cord (Fig. 41).

7. Endings of the Peripheral Branches of the Neurones of the Dorsal Root in Sense Organs. - The peripheral branch ultimately ends in some "sense organ," one of the most important of which, so far as the spinal nerves are concerned, is the skin. The eye, the ear, the nose, the mouth, are examples of other sense organs, and they all contain the peripheral endings of afferent neurones. Each is sensitive to some special influence from without, as the eye to light, the ear to sound, etc.; and when stimulated they start nerve impulses moving inwards along the nerves toward the brain or cord.

8. Ending in the Spinal Cord of the Central Branch of the Neurones of the Dorsal Root. - The other or central 
branch passes into the spinal cord. It does not, however, like the neurones of the ventral root, there become continuous with the nerve cells of the gray matter, ${ }^{1}$ but divides, on entering the cord, into an ascending and a descending branch (Fig. 42), each of which runs for a longer or shorter distance in the white matter of the cord. Indeed, many of the ascending branches extend as far anteriorly as the lower parts of the brain. As shown in the figure, these branches give off at right angles to themselves subbranches, the collaterals, each of which enters the gray matter and ends there by breaking up into a tuft of extremely fine fibrils, the synapse. The synapse is in close proximity to and possibly in a kind of anatomical continuity with the dendrites, or the main body of nerve cell of the gray matter. Each afferent neurone, then, is a cell whose main body is in the ganglion of the dorsal root, and whose branches, or arms, reach out, one of them to a peripheral sense organ and the other to the gray matter of the spinal cord and brain, where they end in synapses. By means of the synapses the afferent neurone excites or stimulates other neurones.

\section{Anatomical Relation of Afferent to Efferent Neurones.}

- We may now put together what we have learned about the neurones of the ventral and those of the dorsal root; we then obtain a plan like that shown in Fig. 42, and such, in principle at least, represents the manner in which the afferent neurone is brought into relation with efferent neurones.

Afferent and efferent fibers enter and leave portions of the brain in much the same way, although the separation into ventral and dorsal roots is not obvious. We may therefore take the above scheme as typical of the relation between these two kinds of neurones, - those of the brain as well as those of the cord.

${ }^{1}$ It is, as has already been pointed out (p. 78), part of a nerve cell in the ganglion of the dorsal root. 
10. Application of These Facts of Structure in the Explanation of Purposeful and Coordinated Action. - The diagram in Fig. 43 readily explains why the sudden appearance of an object in front of the eye causes us to wink and not cough; that is to say, it explains the purposeful character of this so-called reflex action. The formation of the image of the object on the retina, a sense

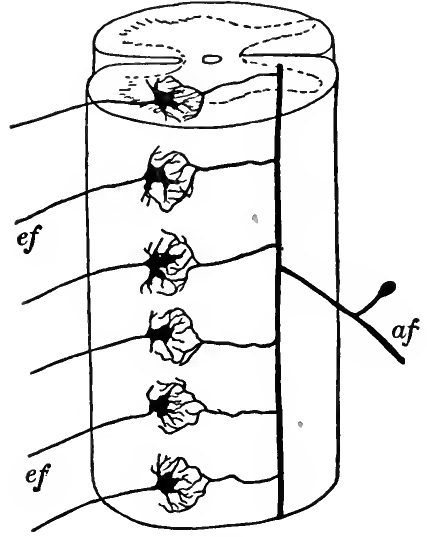

$A$

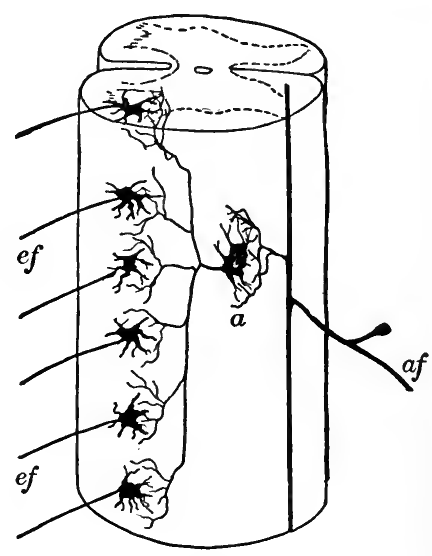

$B$

Fig. 42. Relation of afferent $(a f)$ to efferent $(e f)$ neurones of the spinal cord

In $A$ the single afferent neurone branches into six collaterals, each of which ends in a synapse around an efferent cell. In $B$ the connection is made through the agency of the cell $a$, as explained in Section 13

organ, starts impulses along the fibers of the afferent optic nerve; these fibers extend into the brain, and their synapses end around and stimulate those efferent nerve cells which stimulate the muscles of the eyelid. The action is purposeful because the fibers of the optic nerve end around these cells, and not around those which, for example, innervate ${ }^{1}$ the muscles which open the mouth or flex the finger (Fig. 43).

1 i.e. supply with nerve fibers. 
Our diagram also gives the basis of coördination, the combination of the work of different muscle fibers in orderly harmonious action. The system of collaterals on the central branch of the afferent neurone is obviously a mechanism to combine the action of the efferent neurones in this way. The diagram also gives a clew, at least, to the explanation of another element of coördination: when two or more muscles work together to accomplish a given act, one of

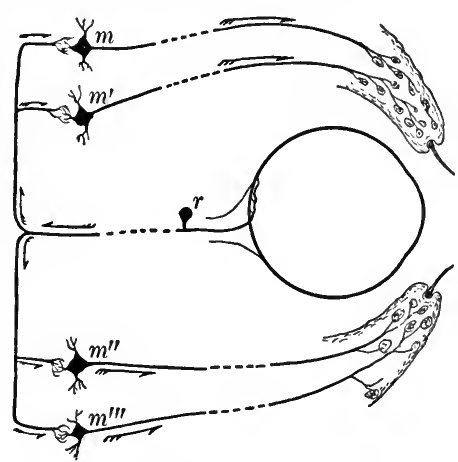
the muscles usually works harder than another; not only must they work together, but the amount of force exerted by each must be adjusted to the needs of Fig. 43. Diagram of the nervous mechanism by which a wink is produced by the sudden appearance of an object in front of the eye

$r$, afferent neurone of the optic nerve; $m, m^{\prime}, m^{\prime \prime}, m^{\prime \prime \prime}$, efferent neurones to the muscles of the eyelid

the movement as a whole. This adjustment is most probably effected by differences in the connection of the synapses with their cells; thus those muscles which contract most forcibly are innervated by neurones whose dendrites and main cell body come into more intimate contact with the synapses of the afferent neurone; or the number of fibrils of the synapse may be greater in their case than in the others. These, however, are only possibilities; the whole subject requires further elucidation.

11. Definition of Reflex Action. - An action such as we have just been studying is known as a reflex ${ }^{1}$ action. By

1 The word literally suggests the idea of reflection from the afferent to the efferent neurones, as light is reflected from a surface; but the student has already learned enough to understand that efferent impulses are something more than mere mechanical reflections, or rebounds, of afferent impulses. 
this we mean an action called forth by the more or less direct action of afferent upon efferent neurones, and without the intervention of the will. The afferent neurone may be stimulated by some external agent, such as light, heat,

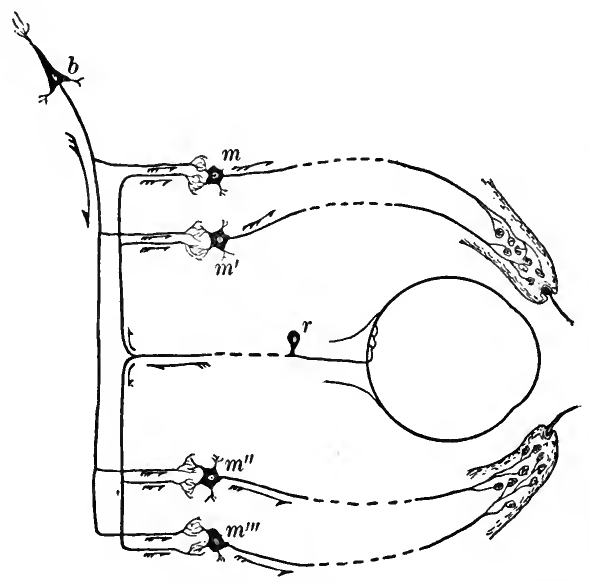

Fig. 44. Diagram of the nervous mechanism represented in Fig. 43, with the addition of the neurone $b$ (see Section 12) sound, pressure, etc., or by some condition within the body itself, as when diseased or abnormal conditions of the stomach or some other organ induce vomiting.

It is a common error to suppose that all actions which are not called forth by the will are reflex. The essential feature of a true reflex is the more or less direct action of the afferent impulses on efferent neurones, and not merely its nonvolitional character. There are, in fact, involuntary actions in which the efferent neurones are directly stimulated not by afferent neurones but by the condition of the blood, or in other ways. Such actions are not reflex, though they may be either involuntary or unconscious, or both. They are known, in general, as automatic actions, and we shall meet examples of them as we proceed with the study of the various functions of the body.

12. Actions resulting from Stimulation by the Will. A wink is not always a reflex action. We can wink "on purpose," or, otherwise expressed, a wink may be called forth by the will, and entirely apart from the sudden 
appearance of some object in front of the eye. Here the muscles of the eyelid act in exactly the same manner as in a reflex wink, which means that they are stimulated in the same way by the same efferent neurones. Thus far the mechanism is the same in the two cases, but the source of stimulation of the efferent neurones must be different.

In later chapters of this book we shall bring forward evidence to show that the exercise of the will (volition) requires the coöperation of the highest portion of the brain or cerebrum. Nerve cells in the gray

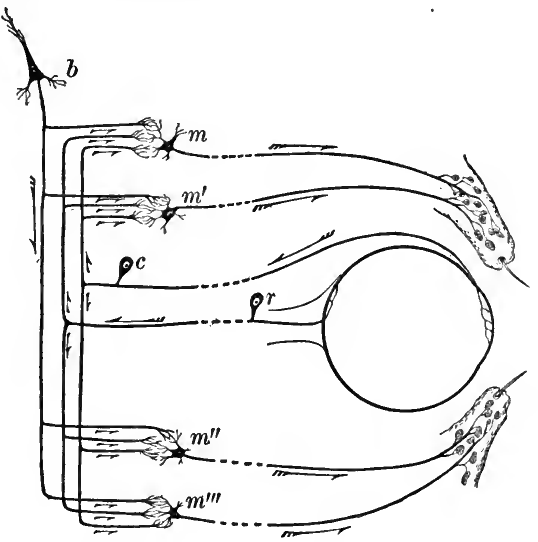

Fig. 45. The nervous mechanism shown in Fig. 44, with the addition of the afferent neurone $c$, from the cornea (see Section 12)

matter of the cerebrum send off axons which pass downward to those portions of the brain and spinal cord from which the motor or efferent neurones arise; with the neurones of these nerves they make exactly the same kind of connections (collaterals and synapses) as are made by the afferent fibers from the retina which excite the reflex (see Fig. 44 , in which $b$ is the cerebral neurone).

The collaterals and synapses of the cerebral neurone (which, it will be observed, is entirely confined to the central nervous system) simply duplicate those of the afferent neurone; hence the two neurones produce the same result.

There is, however, still a third way in which winking may be stimulated. When the cornea of the eye begins to dry, a reflex wink spreads tears over the eyeball. In this case we have to deal with a second reflex, the afferent 
neurones being not those in the optic nerve, but those in what is known as the trigeminal, the sensory nerve of the cornea. Our scheme thus becomes that shown in Fig. 45.

13. The "Master" Neurone. - The multiplication of collaterals and arborizations which this scheme involves would seem to be largely avoided by the presence of a

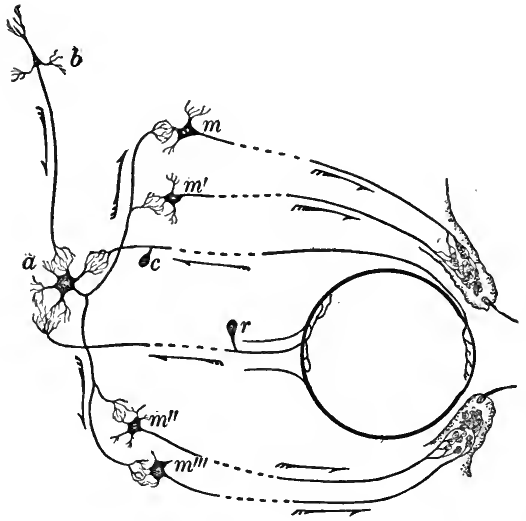

Fig. 46. The "master" neurone third neurone between those which stimulate the action and the efferent neurones which directly act on the muscles (Fig. 46).

In this way, when a wink is produced, whether from the cerebrum or from the retina or from the cornea, the single cell $a$ is stimulated; and this in turn stimulates the groups of efferent neurones which immediately innervate the muscles of the eyelids. Many of the nerve fibers of the cord and brain belong to neurones which perform the same function as that attributed to the cell $a$ in our diagram. They are entirely confined to the brain or cord, and group together those efferent cells which by working together produce a coördinated action.

The organization of the nervous system is, in fact, much like that of a large manufacturing establishment. The nerve cells which send axons to the muscles, glands, blood vessels, etc., may be compared with the operatives, each with his special task to perform; over these are foremen, or "bosses," from whom they take their orders, or, in physiological language, who stimulate them to do their 
work, and who would correspond to cells like $a$ in Fig. 46. The foremen in turn receive orders, now from one department of the establishment, now from another, as the work of their operatives is needed in making one or the other of the products offered for sale. So the "master" neurones receive stimuli from the brain, or from afferent nerves, as the needs or the desires of the organism as a whole require their activity. The comparison is instructive and may easily be carried out in greater detail by the student himself.

By this time the student has, no doubt, made the discovery that a mere wink is after all an extremely complex event; and yet winking is very simple compared with many of our actions, such, for example, as throwing a stone. Here not only muscles which produce motion at the shoulder, elbow, wrist, and finger joints are called into play, but also muscles which maintain the erect position and balance of the body as a whole. The entire mechanism involved baffles the imagination to conceive; and yet any boy can perform the act. He can do it, however, because his motor neurones are grouped together into a perfectly well-organized army which executes at once the bidding of its commander-in-chief, - the will.

We have given in this chapter only the merest glimpse into the complexity of one part of the wonderful human mechanism. No watch, no machine which man has ever invented or constructed can for a moment compare with this living machine in complexity or in perfection. Yet, like all machines, this one can be abused, it can get out of order, it can even break down. And we have already learned enough to understand why this is so. Some neurones may be injured by overwork, or may degenerate from disuse; indulgence in stimulants or narcotics may poison the governing nerve cells; above all, constant failure to lead a normal life may deprive these precious cells 
of their sole means of repair. The human body is a machine designed for use, even for hard use, and it thrives upon right use; but it is a machine too delicate and too complex to be abused with impunity.

When one thinks of the hundreds, perhaps thousands, of movements which the body makes, and of the combination of these movements into definite actions or work, and then reflects that the muscle fibers which execute any movement are thrown into orderly contraction by nerve cells which are themselves commanded by higher nerve cells; that these in turn are marshaled, as it were, by still higher cells when the separate movements they evoke are to be combined into a still more complicated action, - one begins to appreciate the complexity of the organization of the nervous system. The number of the nerve cells is measured by hundreds of thousands, and their efficiency in directing the working organs of the body, so as to meet the demands of life, depends not only upon the integrity of the neurones but also upon the perfection of their organizations, i.e. their grouping into squads, companies, regiments, brigades, divisions, and corps, ready to yield instant and obedient response to the command of the higher officers of the will, or to the signals of those pickets, - the sense organs and their afferent neurones, - which everywhere guard the outposts and give information of the need for action.

Moreover, this army of neurones, like any other army, becomes efficient by work, by drilling, by practice, even by battle. Like the soldiers of a regular army, the neurones may be overworked, and the efficiency of the military body may suffer thereby; but they may also work too little; the perfection of their development, of their organization, depends on the practice they get with reasonable activity. To this point we shall return; but meantime the student can safely make the application for himself. Such comparison and such application are not only instructive but 
intensely practical in their bearing upon the affairs of everyday life, - upon that right conduct of life which is the first duty of every man, every woman, every child.

14. The Activity of the Individual Neurones. - We have thus far emphasized the grouping of the neurones and their organization into an army, and have said but little of what takes place in the individual neurone during activity. We have once or twice, to be sure, spoken of the discharge of a nervous impulse from the cell, and the passage of a nervous impulse along the fiber, but have avoided any discussion as to what these nervous actions really are. To go into these matters in detail would take us too far afield. We may simply say that there is every reason for believing that the activity of the nerve cell, like that of the muscle fiber, involves chemical changes in the cell, - a consumption of stored material and the production of carbon dioxide, water, acids, etc. It has been shown, in fact, that the nucleus and cytoplasm actually change their form and appearance after work, somewhat as gland cells do after prolonged activity. The general physiology of the individual nerve cells would thus be similar to that of the individual muscle fibers and gland cells described in Chapter IV.

The nerve impulse which passes along the axon of the nerve fiber, on the other hand, appears to be a physical change rather than an explosive chemical decomposition, - comparable, for example, rather to the conduction of a current of electricity through a salt solution than to the progress of chemical change along a fuse of gunpowder.

The nervous system participates in every act of human life, and as we study further the functions of the body we shall constantly extend our knowledge of its working. The present chapter is intended to give only some indispensable preliminary information. To other of the more important functions and relations of the nervous system we shall return in Chapters XIV and XV. 


\section{CHAPTER VIII}

\section{ALIMENTATION AND DIGESTION}

\section{A. The Supply of Matter and Power to the Human Machine}

1. Power and the Materials for Repair supplied separately to Lifeless Machines. - Living and lifeless machines are alike in that worn-out parts must be renewed and that power must be supplied to do work. In the lifeless machine these two requirements are supplied separately. A factory and its equipment of machinery are kept in repair and enlarged (grow) by means of bricks, lumber, steel, belting, new pieces of machinery, etc., which are brought into the building; while the power which runs the machinery comes in quite separately as fuel, or water power, or electric power.

2. Power and the Materials for Growth and Repair supplied to the Human Machine in the One Form of Foods. With the human mechanism this is not so. Materials for growth and repair, and power for running, are introduced from without not separately, but together, both being supplied in the one form of food. As it does its life work the human mechanism, like a lifeless machine, not only consumes power but its parts deteriorate, and it is the double function of the food we eat to make good this double loss. Some foods possibly serve only as means of power ; others merely make good the loss of essential parts of the mechanism; while still others may serve both purposes.

3. Food as a Source of Power. - Experiment and experience alike prove that foods are the source of power for work. Bread, butter, starch, sugar, beef, and the like may 
be dried and then burned as fuel, giving power to an engine. The occasional use of Indian corn or wheat for fuel, in the West, the employment of hams and bacon as fuel by steamers short of coal, the explosion of flour dust in mills, and similar phenomena further illustrate by the teachings of experience the fact that these foods are rich in energy, or power.

When we say that the food must supply power to the body we mean that the power which it contains must be available to the body. A lump of coal may be a source of power, as is shown by its use in a locomotive; but a lump of coal would be of no use as food, because the body has no such means of burning it as has the engine. Again, nitroglycerin contains chemical elements needed in the food; but although when exploded in a dynamite cartridge it may furnish power enough to shatter heavy armor plate, its energy is not available to the body.

Thus, to recapitulate, food $(a)$ makes good the loss of living substance in the body, $(b)$ it supplies material for growth, and $(c)$ it supplies power for the work which the body is to do. It also performs one more important function which will be more clearly understood hereafter; for (d) by its oxidation food provides the heat usually required to keep up the body temperature. The detailed consideration of this subject, however, must be postponed to Chapter XII.

4. Chemical Composition of Foods ; Nutrients. - The human race has learned by long experience that certain things do, and that other things do not, meet the demands of the body for food. Perhaps no animal uses so many different materials as man in satisfying sensations of hunger and thirst. Some foods are taken from the animal and some from the vegetable kingdom, and their variety is greatly increased by special modes of preparation. But however numerous the foods from which we prepare the dishes 
served at different meals, chemical analysis shows that the essential constituents of all foods belong to a comparatively small number of chemical groups. These classes, or groups, may be called nutrients; and as all the members of the same group undergo practically the same processes of digestion, and perform similar functions in nourishing the body, it will be equally accurate and more convenient, in treating of this part of physiology, to speak of the different nutrients, and not of beef, mutton, fish, eggs, bread, milk, butter, etc.

From the point of view of digestion the most important nutrients are the proteids, the albuminoids, the carbohydrates, the fats, the inorganic salts, and water; and the student must at this point become thoroughly familiar with what is meant by these fundamental terms.

5. The Group of Proteids. - We may obtain a working idea of what a proteid is by recalling some of the foods in which proteid preponderates or is easily seen. Such foods are the white of egg, the lean of tender meat (muscle fibers), the curd of milk, the tenacious gluten of wheat. Proteids also exist in relatively large quantities, though not so readily seen, in yolk of egg, beans, peas, oats, and other grains.

Proteids contain carbon, hydrogen, nitrogen, oxygen, and sulphur. Some of them contain phosphorus, and some iron. Chemically they are exceedingly complex substances, the proteid molecule consisting of a very large number of atoms. We cannot write the chemical formula for proteid as we write $\mathrm{H}_{2} \mathrm{O}$ for water ; we know the proportions by weight in which the elements are combined, but we have not yet discovered how many atoms of each element there are in the molecule. It should be noted that the proteids are the most important nutrients which contain nitrogen and sulphur.

Many proteids readily become insoluble. Examples of this are the hardening of the white of egg or the lean of meat by cooking, and of the casein or curd of milk by 
rennet or " junket tablets." This change is known as coagulation, and most of our proteid food is eaten after having been coagulated in the process of cooking.

Proteids occur only within the living cells of plants and animals, or as the products of these living cells. They form, as we shall more clearly see later, the basis of the living cell, and are constantly disintegrating within the cell into simpler substances. Hence there is a constant cellular loss of proteid, which in the animal body can be made good only from proteid in the food. Plants, on the other hand, have the power of manufacturing proteids from sugars and certain mineral salts, which latter supply the needed nitrogen and sulphur. The plant kingdom is, therefore, in the long run the sole source of proteid food for animals, for while some animals (carnivores) get their proteid entirely by eating the flesh of other animals, the latter (herbivorous animals) in turn have obtained their proteid from plants.

6. The Group of Albuminoids. - Closely related to the proteids in chemical composition, and indeed derived from them, is a group of bodies known as the albuminoids. Physiologically they cannot entirely replace proteids in our diet, but they can replace them partially. The most important of these substances in use as food is the fibrous connective tissue, whose fibers in the uncooked state contain the albuminoid substance collagen, which by heating in the presence of water is converted into the closely related gelatin. Other albuminoids are of minor practical importance.

7. The Group of Carbohydrates. - Within the cells which make up a potato or a grain of wheat one often sees a large number of granules of starch. This starch was derived originally, with the aid of the sunlight and the green parts of the plant, from carbon dioxide obtained from the air, and water absorbed by the roots. It is a typical carbohydrate (a term shortly to be explained) and constitutes the chief 
carbohydrate food of the world. It is readily changed into the other members of the same group, - the gums, or dextrines, and the sugars. 'The latter are often formed in the plant itself, as in sugar maples, sugar cane, sugar beets, and in such fruits as apples, pears, and peaches.

Carbohydrates are compounds of the elements carbon, hydrogen, and oxygen, the last two usually, but not always, occurring in the proportions of two atoms of hydrogen to one of oxygen. The carbohydrates actually used as foods belong for the most part to three closely related groups. The members of the simplest, the monosaccharides, have the formula $\mathrm{C}_{6} \mathrm{H}_{12} \mathrm{O}_{6}$, and the most common are grape sugar and fruit sugar; the second group includes cane sugar and milk sugar, each of which has the formula $\mathrm{C}_{12} \mathrm{H}_{22} \mathrm{O}_{11}$; the third group includes the gums and starches which have the formula $\left(\mathrm{C}_{6} \mathrm{H}_{10} \mathrm{O}_{5}\right)_{n}$ where $n$ is some factor which multiplies the numbers 6,10 , and 5 . Thus while the exact formula for starch is not known it is safe to say that $n$ is as large as 25. This would make the above formula $\mathrm{C}_{150} \mathrm{H}_{250} \mathrm{O}_{125}$. The molecule of starch therefore contains a very large number of atoms of carbon, hydrogen, and oxygen, but no nitrogen.

8. The Group of Fats. - Fats are familiar to us in such forms as butter, lard, olive oil, and the fat of meat. Like the carbohydrates they are compounds of carbon, hydrogen, and oxygen, although the oxygen is always present in small quantities. The formula for one of the fats is $\mathrm{C}_{51} \mathrm{H}_{98} \mathrm{O}_{6}$, and this composition is typical of all of them.

Fats may be split up into certain acids (fatty acids) and glycerin $\left(\mathrm{C}_{3} \mathrm{H}_{8} \mathrm{O}_{5}\right)$, and when treated with alkalis, like caustic soda or caustic potash, they form soaps. They are insoluble in water. Like the carbohydrates they contain no nitrogen.

9. Oxidizable and Nonoxidizable Nutrients. - All the above nutrients may and do combine with oxygen within 
the cells of the body, although the way in which this chemical union is brought about is one of the unsolved problems of physiology. While all of the nutrients may be burned after being dried, such combustion requires a high temperature. Within the body they are not only burned (i.e. combined with oxygen) at a temperature rarely exceeding $39^{\circ} \mathrm{C}$. $\left(100^{\circ} \mathrm{F}\right.$.), but they undergo oxidation while in a moist state, or even in solution. However this oxidation may be effected within the cell, there can be no doubt that it yields the heat for keeping the body warm and the power for its work.

The remaining groups of nutrients, the inorganic salts and water, are, for the most part, not oxidized in the body.

10. The Groups of Inorganic Salts and Water. - These nonoxidizable nutrients are absolutely necessary for the proper nourishment of the body. Their presence in the blood and lymph, and in the living cells, is indispensable to the processes of life. The salts are taken in small quantities, partly as salt itself, partly as portions of the various foods we eat. During growth they furnish much of the mineral matter of bones, and since the body is daily losing salt, it is necessary that salt be supplied in the food. Salts, however, are not acted on to any large extent in the alimentary canal by the processes of digestion; they are largely absorbed in the same form as eaten. Hence they do not concern us at present to the same extent as do the oxidizable nutrients which generally have to be chemically changed, or digested, before they can be absorbed for use in the body. And the same thing is true of water.

11. Indigestible Material in Food. - When we say that a food is digestible we mean that when taken into the alimentary canal, if not already in solution, it is chemically acted upon by the digestive juices so as to be dissolved and made capable of being absorbed into the blood. The greater part of the food we eat consists of digestible 
substances, but many foods contain a certain amount of indigestible material, and some a very considerable amount.

The most conspicuous example of such material is cellulose, a member of the same group of carbohydrates to which starch belongs. It occurs in almost all vegetable foods, and since in the human alimentary canal cellulose

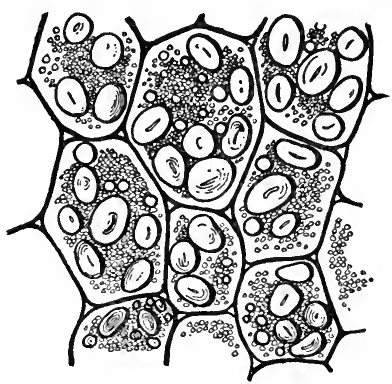

Fig. 47. Part of the seed of the bean

Showing the larger starch and the finer proteid granules inclosed within the cellulose cell walls

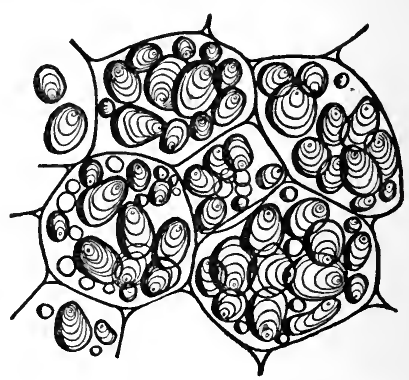

Fig. 48. Section of potato

Showing starch granules inclosed within the cellulose cell walls

is for the most part unaffected, it cannot be absorbed and necessarily forms an important part of the feces. Other indigestible substances are the outer skin of animals, e.g. the skin of fowls, and certain portions of the connective tissue of meat.

12. Animal and Vegetable Foods. - The classification of foods into animal and vegetable not only describes the origins of foods from the two great kingdoms of living things but also defines important differences between them with reference to digestion. These differences may be summed up as follows.

Animal foods are generally rich in proteids and poor in carbohydrates, while vegetable foods are generally poor in proteids and very rich in carbohydrates, especially starch. In the second place, animal foods contain relatively little indigestible material, while vegetable foods, as they occur 
in nature, contain large amounts of indigestible cellulose. In the third place, the digestible materials of vegetable foods (the proteids, carbohydrates, and fats) are often contained within a plant cell which is surrounded by a cellulose membrane impermeable to the digestive juices; before they can be digested this membrane must be ruptured in one way or another. In the case of many animal foods, on the other hand, especially meat and fat, the cells (muscle fibers and fat cells) which contain the essential nutrients are held together by connective tissue made up largely of fibers of an albuminoid nature. These fibers are soluble in the juices of the stomach, in which the cellulose which holds together the vegetable foods is insoluble. The full importance of these differences will be evident before we have finished the study of digestion.

13. Composition of Some Common Foods. - The following table gives the percentage composition of some of the more common foods.

\begin{tabular}{|c|c|c|c|c|c|c|c|}
\hline & & WATER & ProteID & Starch & SUGAR & $F_{A T}$ & SALts \\
\hline Bread & . & 37 & 8 & 47 & 3 & 1 & 2 \\
\hline Wheat flour. & . & 15 & 11 & 66 & 4.2 & 2 & 1.7 \\
\hline Oat meal. & . & 15 & 12.6 & 58 & 5.4 & 5.6 & 3 \\
\hline Rice . . & . & 13 & 6 & 79 & 0.4 & 0.7 & 0.5 \\
\hline Peas & . & 15 & 23 & 55 & 2 & 2 & 2 \\
\hline Potatoes & . & 75 & 2 & 18 & 3 & 0.2 & 0.7 \\
\hline Milk . & . & 86 & 4 & - & 5 & 4 & 0.8 \\
\hline Cheese & . & 37 & 33 & - & - & 24 & 5 \\
\hline Lean beef & . & 72 & 19 & - & - & 3 & 1 \\
\hline Fat beef & . & 51 & 14 & - & - & 29 & 1 \\
\hline Mutton & . & 72 & 18 & - & - & 5 & 1 \\
\hline Veal & & 63 & 16 & - & - & 16 & 1 \\
\hline White fish & 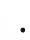 & 78 & 18 & - & - & 3 & 1 \\
\hline Salmon . & . & 77 & 16 & - & - & 5.5 & 1.5 \\
\hline Egg . & & 74 & 14 & - & - & 10.5 & 1.5 \\
\hline Butter. . & • & 15 & - & - & 一 & 83 & 3 \\
\hline
\end{tabular}


14. The Process of Alimentation. - Before corn, wheat, meat, vegetables, and other food materials can be taken into the body and made to yield up to it the material and power which they contain, they must, in most cases, undergo various preparatory or preliminary processes or treatments which shall make them easier or better to eat, or more attractive. The most familiar of these processes is cooking, but it is by no means the only one. In the case of animal food the animal must be captured, if wild, or raised, if domesticated. It must be killed, skinned, dressed, cut up, and the meat in many cases "ripened" by keeping, or "cured" by smoking, salting, drying, or corning. So, also, with plant food, such as cereals, vegetables, fruits, nuts, and the like; these must first be found, if wild, or grown, if domesticated. They must then be separated from the rest of the plant, - threshed, if wheat, rye, oats, or barley; husked and shelled, if corn; dug up or removed from the earth, if vegetables like potatoes, celery, radishes, or lettuce. Fruits and nuts must be separated or picked from vine or tree; milk, drawn from animals; and even salt, water, and condiments like mustard and pepper, separated from the earth or the sea or from plants. After collection and further preparation by winnowing, grinding, or cleaning, elaborate cooking is applied to many forms of food before it is put upon the table; and even then, at the last moment before it is eaten, a further separation, as of meat from bone, must be made either by the carver or by the eater himself.

To this entire process of the supply and preparation of food for eating the term "alimentation" may be conveniently applied. Reflection will show that it is largely a process of food refining, the principal result being a concentration of the nutrients at every step. It is also a separation of the comparatively useful from the comparatively worthless (as food); and just here, and in these two 
points, - refining and the separation of good from poor materials, - we may recognize a true process of digestion, but one external rather than internal: a refining in the field, the mill, and the kitchen rather than in the stomach; in the environment rather than within the organism.

15. Digestion, External and Internal. - The word "digestion" comes from the Latin dis and gerere, to tear apart or separate. A "digest" of the laws of any state or country is a compact, concentrated statement of those laws from which the unimportant parts have been separated and omitted. Now one of the principal objects and functions of digestion is to separate the nutritious and therefore important part of the food from the innutritious and therefore unimportant. Another and no less important function is to prepare this food that it may be more readily absorbed and utilized by the body proper. A survey of the processes of alimentation and digestion shows that all are tributary to these ends, and that we may with advantage consider alimentation (including cooking) as a kind of external digestion, which applies to the food a treatment not only preliminary to but also preparatory for ordinary or internal digestion. Digestion as commonly understood thus becomes only the last part of a much longer process.

\section{The Alimentary Canal a Digestive Laboratory. -} Many animals low in the scale of life, and all plants, are destitute of those tubes running through the body, which are called alimentary canals, but all animals (except some parasites) above the lowest are provided with such tubes or canals. These are really no more than places, or spaces, in which food is prepared for absorption, and they always closely resemble chemical laboratories or refineries in which raw materials are altered or refined by the action of chemical reagents. The laboratories are of several kinds, - mouth, stomach, intestine, — and the reagents correspondingly different, — salivary juice, gastric juice, 
pancreatic juice, bile, intestinal juice, etc. To the detailed consideration of internal or ordinary digestion we may now proceed.

\section{B. Digestion in the Mouth. The Teeth. Enzymes}

17. Digestion. - The food upon which the body depends for its supplies of matter and power enters the body proper through the lining membranes of the alimentary canal into which it is received through the open mouth. Inasmuch, however, as these lining membranes contain no visible openings, the food must somehow soak, or "diffuse," through them, and in order to do this, solid foods must be greatly changed and prepared for absorption by being brought into solution.

But this is not all. Some foods are soluble but still not suitable for the use of cells and tissues. Common cane sugar (saccharose), for example, is very soluble; but before it can be used by the cells it must be converted into the closely related but somewhat different sugars known as dextrose and levulose. Again, proteid food is absolutely indispensable for all animals, but ordinary proteids pass through membranes like those of the alimentary canal with great difficulty; they are, therefore, before absorption, turned into other and more diffusible bodies known as peptones. Starches and fats also require special treatment to make them available or acceptable for absorption. We shall begin our study of the processes of digestion with those which take place in the mouth, and these are the crushing or grinding of the food by the teeth and its mixture with saliva.

18. Structure of a Tooth. - A tooth has three parts, the crown, or exposed portion; the neck, a narrow constriction at the edge of the gum ; and the root, or roots, by which the tooth is fixed in the jawbone (see Fig. 138). 
The tooth consists of a hard body surrounding a central pulp cavity, filled with a loose connective tissue containing blood vessels and nerves, which enter the pulp cavity by a minute opening on the tip of each root. Elsewhere the pulp is surrounded by the hard parts of the organ, which consist of three different tissues. Immediately surrounding the pulp, both in the root and in the crown, is the dentine, which makes up the main bulk of the tooth; this is a hard structure, containing some 65 per cent of mineral matter, chiefly carbonates and phosphates of calcium. It is channeled, as shown in the figure, by minute canals, the dentinal tubules, which run into the pulp cavity. In the root the dentine is covered with cement, which is virtually bone in structure and composition. In the crown, or that part of the tooth not covered by the gum, the dentine is covered with enamel, the hardest substance in the body. This contains in the adult from 95

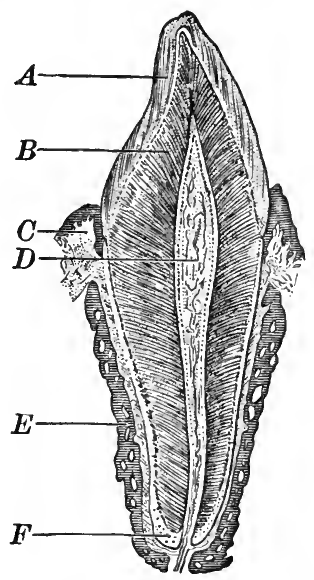

FIG. 49. Section of an incisor tooth. After Spalteholz

$A$, enamel ; $B$, dentine; $C$, gum; $D$, pulp; $E$, jawbone; $F$, cement to 97 per cent of very insoluble mineral matter, and is the protective covering of the tooth. In structure, enamel consists of columns, hexagonal in section, set together so as to form a practically impenetrable mosaic covering. It is thus admirably fitted to protect the dentine, and indeed the whole tooth, from mechanical injury, from chemical erosion, and from bacterial action.

19. Care of the Teeth. - Too much stress can hardly be laid on the preservation of the teeth. Apart from considerations touching personal appearance, the teeth are of great importance in masticating the food. Mastication, or 
chewing, is one of the many acts of digestion, and when the power of chewing is impaired, the efficiency of the whole digestive process is to that extent lessened; other portions of the alimentary tract, especially the stomach, must then do, as far as possible, what should have been done by the teeth; digestion is hindered; some kinds of food are never properly digested, and the opportunity for bacterial decomposition of the food is greatly increased, because of the prolonged exposure of the food to bacterial action. Besides, there is always the possibility that a decaying tooth will cause the formation of an abscess (an ulcerated tooth), often a most painful and sometimes a dangerous thing.

20. Decay of the Teeth is usually, if not always, due to the action of bacteria, which produce acids by fermenting food particles in the mouth. These acids dissolve away the lime salts of the enamel and the dentine; the enamel, however, is acted upon very slowly and with great difficulty; so long as it is intact, the underlying dentine, which is dissolved much more readily, is protected; but if for any reason the enamel becomes worn away, its absence should be made good by filling the tooth, thereby preventing access of foreign substances, and especially of destructive bacteria, to the dentine.

The action of bacteria upon the enamel is favored by the formation of a hard deposit known as tartar, a mixture of lime salts precipitated from the saliva; and especially apt to be deposited between the lower teeth and in the neighborhood of the gums. Sometimes tartar is even deposited under the gums, in which case it is inaccessible to the action of a brush. Because of this tartar crust, bacteria and the acids which they produce are not properly rubbed away by the movements of tongue and cheeks, and hence the importance of its artificial removal. This is greatly facilitated by using a tooth powder which contains 
some substance, like precipitated chalk, not hard enough to injure the enamel but exerting friction enough to break up the deposit. At least once or twice a week a good tooth powder should be used in cleaning the teeth. At other times the brush and water are sufficient. After using powder, indeed always after brushing the teeth, the mouth cavity should be very thoroughly rinsed out.

It is, however, very difficult, and at times impossible, to remove the tartar entirely by the use of powder and brush. For this reason the teeth should be examined by a dentist at least once a year, the accumulated tartar thoroughly removed, and the teeth polished. In this way the beginnings of decay are detected and measures can be taken to prevent its further progress. Further advice as to the care of the teeth can and should be obtained from a good dentist.

Decay of the teeth caused by bacteria is also prevented by removing as far as possible the food supply of these organisms, to whose growth and activity nothing is more favorable than particles of food between, or otherwise in contact with, the teeth. The ideal plan is to brush the teeth with water and rinse out the mouth after each meal; in most cases this is perhaps more than is required; it may be suggested, however, that the teeth should be brushed at night as well as in the morning, and that brushing them at night accomplishes more toward restraining bacterial action than does brushing them in the morning.

By thus exercising care the teeth may usually be preserved in efficiency until old age. The unpleasant necessity of using false teeth is almost always the result of neglect of the teeth in earlier life.

21. Chemical Action of Saliva. - We have already stated that the main function of the saliva is to lubricate the food and so aid mastication and swallowing. But if saliva 
is brought in contact with starch, a very characteristic chemical action occurs, and this we must study in some detail, chiefly because it is typical of many similar chemical effects produced by other digestive juices. To demonstrate the effect in question some starch paste should be prepared. This is not a clear solution like salt or sugar, but an opalescent liquid, which does not become clear upon passing through an ordinary filter. A characteristic test for starch - the blue color produced when a few drops of a solution of iodine are added to it - may be used to detect its presence in the course of the following experiments.

\section{Experiment I}

Three tubes or small beakers containing starch paste are prepared; one of them should be cooled nearly to the freezing point by packing ice around it, and some filtered saliva should be cooled in the same way. A second portion of the filtered saliva is to be heated to the boiling point, while a third is to be used without further treatment. To the cooled starch paste add the cooled saliva, keeping the cooled mixture surrounded by ice; to the second portion of the starch paste add the boiled saliva, and to the third the remaining portion of saliva. Mere observation of the three test tubes will soon show that while the first two remain opalescent, the last soon becomes clear. After this change has occurred, a little of the third mixture may be removed, diluted with water, and tested with iodine; the color now produced is no longer a pure blue, but purplish, i.e. a mixture of blue and red; some minutes later the iodine test gives a portwine color, and still later no color at all. This shows that under the action of the saliva the starch has disappeared from the third test tube. The intermediate port-wine color is due to the formation of dextrines, or animal gums, into which the starch is first transformed; but subsequently this, also, disappears. Meanwhile, the starch in the first two test tubes shows no change either in its opalescent appearance or in.its original reaction with iodine. Keep these test tubes, the cooled one still surrounded by ice, for the subsequent examination described in Experiment III. 


\section{Experiment II}

Let us now inquire what has become of the starch in the third test tube. The solution looks clear and has a sweetish taste. Moreover, if boiled with a mixture of sodium hydroxide and copper sulphate, it gives a red precipitate indicating the presence of sugar. These simple tests, then, prove that saliva first changes starch into dextrines and subsequently changes these dextrines into sugar.

22. The Salivary Ferment, or Enzyme. - This change of starch first into dextrine and then into sugar is caused by ptyalin, a constituent of the saliva which belongs to the general group of enzymes or unorganized ferments. A striking feature about the action of ptyalin is the fact that it is not destroyed or consumed in producing the reaction. This is unlike what usually occurs to substances taking part in chemical reactions. When wood combines with oxygen in burning, both the wood and the oxygen disappear and in their place the products of combustion smoke, gas, etc. - make their appearance. In the salivary digestion of 'starch three substances enter into the reaction, - starch, water, and the enzyme; two of these, starch and water, disappear; the third, the enzyme, remains over after the reaction is completed and continues capable of transforming new starch paste into sugar.

We shall repeatedly meet with other enzymes (pepsin, trypsin, etc.) in our further studies of digestion.

\section{EXPERIMENT III}

Returning now to our experiments with the starch paste, we find that neither test tube 1 nor 2, whether tested for starch or for sugar, shows any change. The student should now remove a portion of the cooled mixture and allow it to rise to the room temperature; it will soon be found that the starch gradually changes into sugar, which shows that the cold has prevented or inhibited the action of the enzyme but has not destroyed it. If mixtures of saliva and starch be kept at different temperatures, it will be found that as the 
temperature of the mixture approaches that of the body $\left(98.6^{\circ} \mathrm{F}\right.$.), the more quickly is the transformation into sugar effected. The activity of the enzyme is dependent upon the temperature. If, however, we use still higher temperatures, one will eventually be found which destroys the enzyme. This temperature differs with different enzymes, but all are destroyed before the boiling point of water is reached; and no matter how long we may keep a mixture of starch and boiled saliva, no change of starch into sugar will occur.

\section{Experiment IV}

Still another instructive experiment may be made upon the action of saliva on starch. Prepare five or more small beakers of starch paste and add to the first one a drop of filtered saliva, to the second two drops, to the third three drops, and so on ; then observe the time required in each case for the disappearance of the iodine reaction. This experiment will show that while a very small amount of saliva will transform an indefinite amount of starch to sugar, the more saliva present the more rapidly will the transformation occur; and the same thing is true of all enzymes.

\section{Experiment V}

Finally, dilute some starch paste with an equal volume of 0.4 per cent hydrochloric acid (this will, of course, make a 0.2 per cent solution of the acid). Now add a few drops of saliva. It will be found that no reaction takes place. Saliva will not act in an acid medium of this strength; and it can be easily shown that it acts most vigor- ously in a neutral or faintly alkaline solution. This result is of much practical importance, because the gastric juice contains approximately 0.2 per cent of hydrochloric acid, and may therefore be expected to interfere with salivary digestion.

While we are eating, the food obviously stays too short a time in the mouth to allow the conversion of any large amount of its starch into sugar before it is swallowed. Whatever actual work the saliva may do in bringing about this chemical change must evidently be done chiefly in the stomach, and this will be studied in the next section.

We have dwelt at length upon the enzyme action of saliva not merely for its own sake but rather because 
the behavior of the salivary juice is typical of the action of other of the digestive juices and of enzyme action in general. All the other juices of the alimentary canal, with the single exception of the bile, contain enzymes, and it will greatly help our understanding of the digestive action of these enzymes if that of the salivary enzyme be first mastered.

Digestion in the mouth, then, consists first, of a mechanical process of chewing, by which food is crushed or comminuted ; second, of a physical process of moistening, by which dry foods are prepared for the act of swallowing; and third, of a chemical process, the chief part of which is the conversion of starch into sugar by enzyme action. ${ }^{1}$

1 Those who have studied the elements of chemistry will find the following facts helpful to a fuller understanding of the action of saliva on starch. Saliva first causes the large molecule of starch to unite with water, and in so doing to split up into one or more molecules of sugar and a certain gum (dextrine). The saliva next causes the gum or dextrine thus formed to combine with water and break up into one or more molecules of sugar and a different gum, or dextrine. The repetition of this process ultimately transforms all the dextrine to sugar. The process may be conveniently represented as follows.

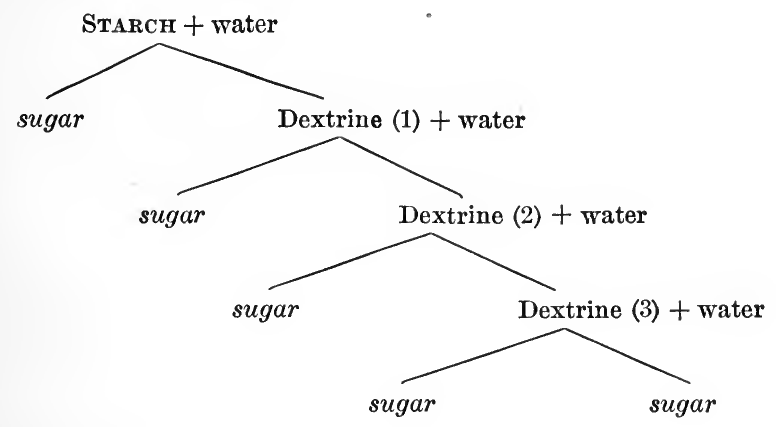

(The original substance is given in capitals and the end products in italics.) Thus in the above diagram one molecule of starch by these successive processes of cleavage would yield five molecules of sugar. In point of fact, one molecule of starch undoubtedly yields much more sugar than 


\section{Digestion in the Stomach}

According to popular ideas the stomach is the chief organ of digestion; in fact, however, it is an organ in which the food which has been swallowed is temporarily stored while undergoing a preliminary preparation for the more important digestive changes which are to take place in the intestine. In this preparatory process, to be sure, some of the

this. Assuming as we did on page 92 that the formula for starch is $\mathrm{C}_{150} \mathrm{H}_{250} \mathrm{O}_{125}$, the reaction would be

$$
\begin{aligned}
& \mathrm{C}_{150} \mathrm{H}_{250} \mathrm{O}_{125}+25 \mathrm{H}_{2} \mathrm{O}=25 \mathrm{C}_{6} \mathrm{H}_{12} \mathrm{O}_{6} \text {. } \\
& \text { starch water sugar }
\end{aligned}
$$

In reactions like the above, the sugar is known as the end product, while the various dextrines are known as intermediate products. It is also obvious that this change from starch to sugar involves a reduction in size of the molecule; one large molecule is split up into many smaller molecules. This reduction in size of the starch molecule appears to be of fundamental physiological importance, in that the carbohydrate food thus acquires physical properties which render it capable of passing through the lining membrane of the intestine in the process of absorption.

As stated in the text, the chemical action of the enzyme at first sight appears very unlike the ordinary chemical reaction where all substances concerned disappear in contributing to the final result. For example,

$$
\mathrm{HCl}+\mathrm{KOH}=\mathrm{KCl}+\mathrm{H}_{2} \mathrm{O} \text {. }
$$

Here for every molecule of hydrochloric acid which disappears, one molecule of potassium hydrate likewise disappears. The distinction, however, is only apparent. The reaction between liydrochloric acid and potassium hydrate should be compared with the reaction between starch and water, not between starch and saliva; and there are numerous examples in chemistry of this action of a third body in producing or accelerating a chemical reaction between two other bodies. Thus hydrochloric acid acts very slowly, if at all, at ordinary temperatures on pure zinc; if, however, a drop or two of platinum chloride be added, vigorous union takes place:

$$
\mathrm{Zn}_{2}+2 \mathrm{HCl}=2 \mathrm{ZnCl}_{2}+\mathrm{H}_{2} \text {. }
$$

Here again the platinum chloride by its mere presence produces the reaction without itself contributing anything to the compounds formed. Such reactions are in general known as catalytic, and are by no means confined to living things. Their frequent occurrence, however, in the chemical processes of animal and plant life is a striking fact. 
food is incidentally changed into those forms in which it is taken into the blood; but this action is incidental and subordinate to the main function.

23. Form and Structure of the Stomach. - The stomach is a large pouch into which open two tubes, - the œesophagus (gullet) toward the left side and the intestine on the right (see Fig. 50). The two regions into which these

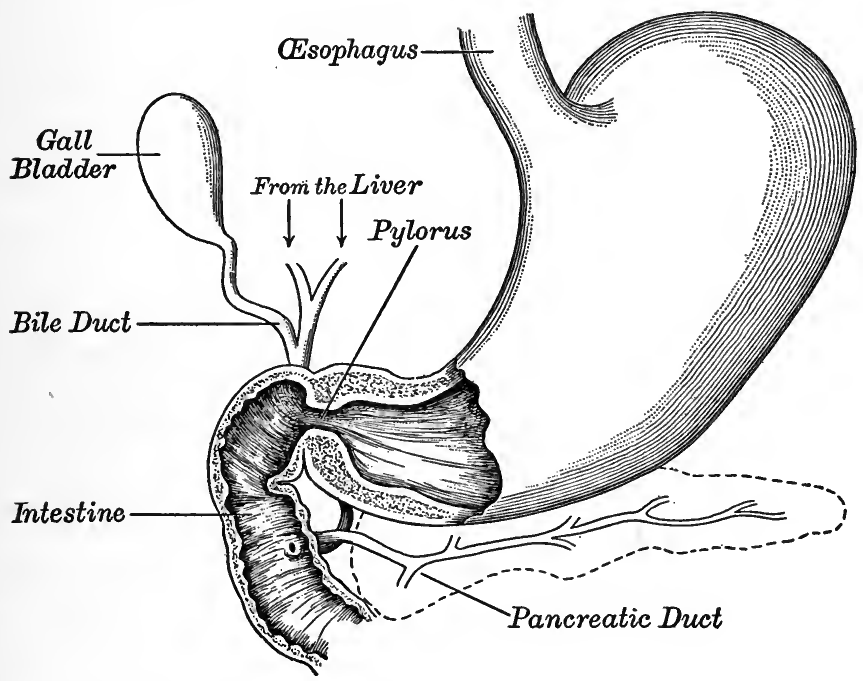

Fig. 50. Stomach, beginning of small intestine, and entrance of bile and pancreatic ducts

During digestion the bile flows directly from the liver into the intestine; at other times the opening of the bile duct is closed, and the bile passes into the gall bladder, where it is stored

tubes open are different in structure, and are known as the cardiac (left) and pyloric (right) portions of the stomach; the cardiac portion differs from the pyloric portion in having greater diameter and thinner walls. The entire inner surface is lined by a membrane some three or more millimeters in thickness, crowded with comparatively simple glands which pour their secretion, the gastric juice, 
into the stomach, very much as sweat glands discharge perspiration on the skin (see Fig. 51).

The glandular membrane is one of the two principal components of the stomach wall; the other is the mus-

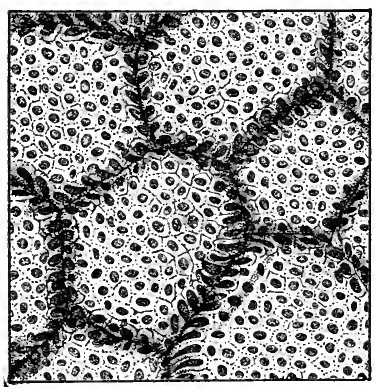

Fig. 51. The inner surface of the stomach (magnified about 20 diameters)

Showing the openings of the glands. The lining glandular membrane is thrown into folds

cular or contractile tissue which forms a second coat outside the other and closely united to it by connective tissue containing the larger blood vessels, lymphatics, nerves, etc. The muscular coat is comparatively thin in the cardiac, and comparatively thick in the pyloric region, the thickening in the latter region being caused chiefly by muscle fibers circularly arranged.

24. The Gastric Juice. - The gastric juice is a clear, thin liquid, which contains, among other things, about 0.2 per cent of hydrochloric acid and two enzymes, pepsin and rennin. The latter changes casein, one of the proteids of milk, into an insoluble body, - the curd, - which is subsequently acted upon by other enzymes of the gastric and pancreatic juices in the same manner as are other proteids. ${ }^{1}$

The other enzyme of the gastric juice, pepsin, is of much greater importance. It acts only in the presence of free acids, and on entering the intestine is destroyed by the alkaline pancreatic juice; hence its action is confined to the stomach. It produces no effect upon pure fats (although it plays an important part in the digestion of adipose tissue), nor upon carbohydrates, such as starch and sugar. Its part in digestion consists in its action upon two constituents

1 Rennin is the essential constituent of "junket tablets," which are made from the stomachs of calves. 
of the food, - the proteids and the connective tissue of animal foods. The fibers of connective tissue are closely related to the proteids in chemical composition, and the action of the enzyme is essentially the same on both; their complex molecules are split up into smaller molecules, and the new substances formed pass into solution in the gastric juice. By thus dissolving the connective tissue, which holds together the muscle fibers, fat cells, etc., animal food is considerably subdivided and made to present a greatly increased surface to the further action of digestive juices. It is also well to remember that the gastric juice dissolves connective tissue much more rapidly than does any other of the digestive juices; and that this action upon connective tissue is really more important than that upon proteids, although the latter is usually more emphasized. Proteids not acted on in the stomach are rapidly digested by the pancreatic juice in the intestine; connective tissue, on the contrary, escaping solution in the stomach, is dissolved but slowly in the intestine.

The action of the gastric juice, as regards both proteids and connective tissues, consists essentially in splitting up larger molecules into smaller ones. This recalls the action of saliva in converting starch into dextrines and sugars (p. 105). In the case of proteids the products formed are known as proteoses and peptones, substances readily soluble in the gastric juice.

The student is, however, warned against supposing that because gastric juice is able to transform the proteids of the food to peptones, it actually does exert this action upon all the proteid eaten. In point of fact, as proteid foods are divided into smaller and smaller particles in the stomach, they are discharged into the intestine where their digestion is completed by the pancreatic juice. In man the pancreatic, and not the gastric juice, is the main agent of proteid digestion. 
25. The Stomach at Work. - Having now gained a general idea of the chemical changes which occur in the stomach, we may next proceed to consider what actually happens when food enters that organ. And here our knowledge has been gained partly by examining the gastric contents at different periods of digestion, partly by observing the movements of the stomach by the aid of the Röntgen rays, ${ }^{1}$ and partly by other means.

As soon as food enters the stomach, and even while it is still in the mouth, the gastric glands begin to discharge the gastric juice, and continue to do so during the four or more hours of gastric digestion. When the meal is fluid or is small in amount this gastric juice is thoroughly mixed with it; when, however, the food is more or less solid and bulky only the outer layers, which are in immediate contact with the walls of the stomach, are mixed with the juice. At least this is true at the cardiac end; the cavity of the pyloric portion is so small, and the amount of movement there so great, that all portions of the pyloric contents are thoroughly mixed with gastric juice; in the much larger cardiac portion the central mass of the food may receive no gastric juice, and thus remain, for an hour or more after the meal, neutral or alkaline in reaction. Under these circumstances very considerable amounts of starch may continue to undergo the salivary digestion begun in the mouth.

Any chemical action is aided by agitation, since the reacting compounds are thus brought into more intimate union; and observation of the working stomach shows that while the cardiac portion makes no movements, but

1 For this purpose food is swallowed which contains enough of the harmless salt, bismuth subnitrate, to make the stomach contents impervious to the Röntgen rays. The changes produced in the shape of the food mass by the corresponding movements of the walls of the stomach are then observed by means of shadows cast upon the fluorescent screen. 
merely keeps up a steady contraction and thereby exerts a moderate pressure upon its contents, the pyloric portion executes, from a very early stage of digestion and throughout the whole process, a series of movements which gradually bring about a thorough mixture of the contents and rub down the softened food into smaller and smaller masses. These movements consist of waves of constriction which arise at the beginning of the pyloric portion and pass onward to the pylorus itself, a new wave beginning about once every ten seconds, and consuming from thirty to forty seconds in passing to the pylorus. Consequently there are always two or more slowly moving waves in the pyloric end of the stomach at one time. ${ }^{1}$

The pyloric end of the stomach is thus the seat of a combined chemical and mechanical action on the food. The vegetable foods are softened, while the connective tissue of the animal foods is dissolved away; in addition, the food is mixed with a considerable amount of liquid supplied
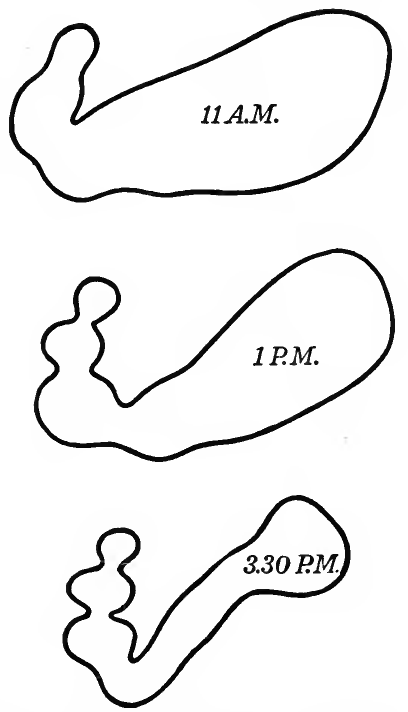

Fig. 52. Outline of the contents of the stomach of a cat at three stages of the digestion of a meal taken about 11 A.M.

Showing the peristaltic constrictions which pass over the pyloric portion, and the diminution of the quantity of food in the cardiac end. (Full description given in Section 25)

by the secretion of gastric juice. The contents of the pyloric end of the stomach thus ultimately come to consist of minute solid masses suspended in a liquid, the

1 These movements of the stomach and intestine are best shown in zoetrope figures, which may be obtained from the Harvard Apparatus Company, Boston, Mass. 
consistency of the whole being that of moderately thick pea soup. This product of the work of the stomach is known as chyme.

26. The Expulsion of Chyme into the Intestine. - The openings of the œsophagus and intestine into the stomach are usually closed; the former is opened, normally, only during the act of swallowing, while the latter opens at irregular intervals during the process of gastric digestion. The opening of the pylorus allows the waves of constriction moving over that region of the stomach to discharge the semifluid chyme into the intestine. If, however, a large mass of solid food arrives and is driven against the walls, the pylorus reflexly closes, thus guarding the entrance of the intestine from the passage of food not yet ready for intestinal digestion. The pressure exerted by the sustained contraction of the walls of the cardiac end of the stomach adds to the food in the pyloric region new portions from time to time, and the same combined chemical and mechanical process already described is continued until the whole mass is reduced to chyme and driven into the intestine.

This brief sketch of the working of the stomach shows that this organ serves the two main functions of storing the food and of making it more accessible to the digestive fluids of the intestine. When the chyme is delivered to the intestine the mechanical difficulties in the way of absorption are practically gone; the surface of the food exposed to digestive action is now immensely increased by its subdivision, and the work remaining for the intestine is almost wholly the chemical duty of changing the constituents of the chyme into substances which are soluble and ready for absorption.

Serious troubles arise when, for one reason or another, gastric digestion goes wrong, because the subsequent processes of digestion are largely dependent upon the 
preparation which the food receives in the stomach. Gastric digestion may be impaired in one of three ways : first, the gastric juice may not be secreted in proper amount or proper strength; or, second, the stomach may not execute its movements efficiently; or, third, the gastric juice secreted may not be able to get at the food readily, owing to improper cooking or insufficient mastication. The study of the conditions which produce these troubles, which taken together constitute indigestion, or dyspepsia, will be postponed to the chapter on the Hygiene of Feeding (Part II).

27. The Stimulus to the Secretion of the Gastric Juice. - The first requirement for the work of the stomach is the secretion of sufficient gastric juice. Of late years the brilliant researches of physiologists have shown that the secretion of gastric juice, like that of saliva, is excited by impulses from the nervous system, and that these impulses are called forth in two ways.

(a) The "Psychic" Secretion. - When agreeable or appetizing food is offered to an animal, and especially when such food is taken into the mouth, a secretion of gastric juice follows, which may continue for fifteen minutes or more. This secretion occurs

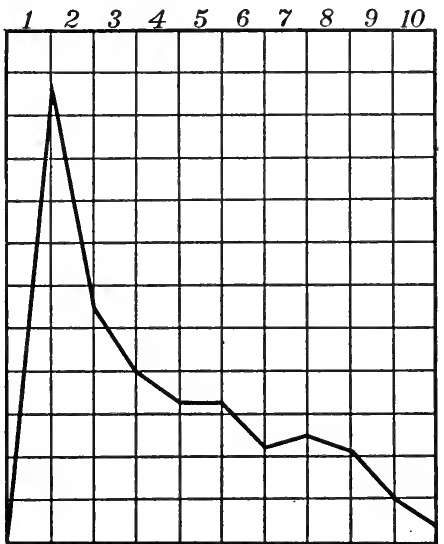

FIG. 53. The curve of the "psychic" secretion of gastric juice

Vertical lines represent half-hour periods after taking the meal; horizontal lines, relative amounts of gastric juice secreted when the food has been in the mouth only ten or fifteen seconds, and even when it is merely offered to a hungry animal and not taken into the mouth at all. Again, it 
occurs only when the animal is conscious; for if food be introduced into the stomach of a sleeping dog, it evokes only the most scanty secretion of gastric juice after the animal has awakened. Moreover, both the amount and the efficiency of the juice secreted vary directly with the enjoyment of the meal. When meat is given to a dog which is not hungry, no such abundant secretion of gastric juice occurs as during hunger.

It is clear that we have here to deal with a nervous process more complicated than the simple reflex, and that the efferent discharge to the stomach occurs as the result of nervous processes taking place in the brain in connection with the enjoyment of food. In other words, the more the food is desired or enjoyed, the more efficient will be this secretion of the gastric juice.

It is known that this "psychic secretion" will continue for several hours after an ordinary meal, increasing in amount during the first hour or more, and gradually diminishing from that time onward (Fig. 53).

(b) Reflex Secretion from Stimulation of the Stomach itself by the Products of Digestion. - We have just seen that the mere contact of most foods with the walls of the stomach does not call forth a secretion of the gastric juice. This is shown by the experiment of placing food in the stomach of a sleeping animal; such food, even when appetizing and nutritious, has been known to remain in the stomach for twelve or more hours after the animal had awakened without evoking a secretion of gastric juice. Yet it is also known that when digestion of food has been begun by the action of the "psychic" secretion, the peptone formed from proteids arouses a second secretion which increases in amount as the first, or "psychic," secretion diminishes. That the peptone formed from proteids is the true cause of this secretion is proved by the fact that the introduction of peptones into the stomach of a sleeping 
animal evokes this secretion, although, as already stated, ordinary food, which contains no peptones, does not. This second secretion continues throughout the period of gastric digestion.

The secretion of gastric juice must be kept up throughout the entire period of gastric digestion, to make good its loss by the discharge of chyme into the intestine; and it would seem that this great need is met only by the combined action of the "psychic" stimulus and the stimulus of peptones produced during the process of digestion. Without the "psychic" secretion peptones are not formed fast enough to induce sufficient subsequent secretion; without the "peptone" stimulus the psychic "secretion does not suffice to complete the digestion of a hearty meal, a labor which may require four or five hours. It is also plain that proteids should form part of large meals, since it is only from proteid that peptones can be made.

\section{Digestion and Absorption in the Small Intes- tine aND IN the LaRge Intestine}

Every few minutes during the process of gastric digestion the pylorus opens and the stomach forces a few cubic centimeters of chyme into the intestine. Chyme consists of water holding in solution certain products of digestion, and in suspension relatively large quantities of undissolved matter, and has the consistency of moderately thick pea soup. The suspended matter consists, among other things, of small bundles of muscle fibers (from meat), fat ${ }^{1}$

1 The fat of meat consists of connective tissue whose cells are greatly swollen with drops of fat. In typical adipose tissue the connective-tissue cell becomes one large fat droplet surrounded by the thin layer of the cell cytoplasm with its nucleus. These "fat cells," like the muscle fibers of meat, are thus held together by the fibers of connective tissue, and are set free when the latter are digested and dissolved away by the gastric juice (see Figs. 83-85). 
melted by the heat of the body and set free from adipose tissue by the digestion of its connective tissue, bits of coagulated proteid, such as casein from milk or the white

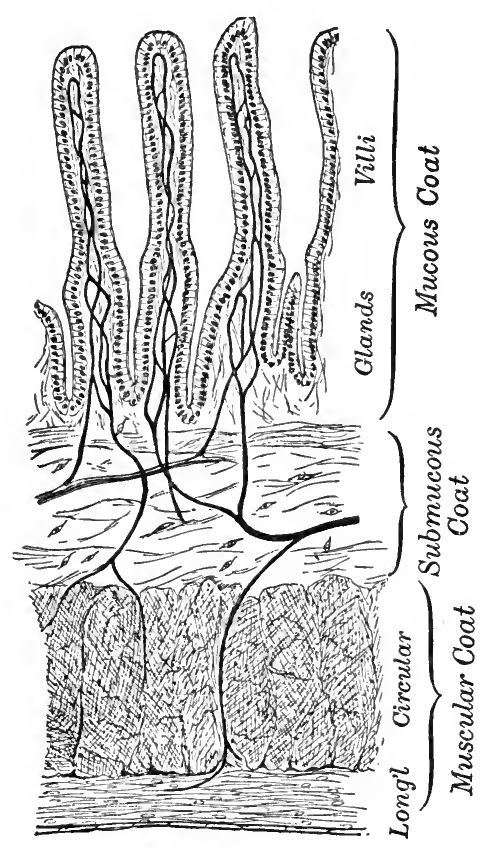

Fig. 54. Longitudinal section of the small intestine

The submucous coat consists of connective tissue and contains the larger blood vessels from which the mucous and muscular coats are supplied with blood

of egg, together with other proteids, starches, and fats of animal or vegetable foods.

The work of intestinal digestion is essentially chemical in nature, and consists largely in the transformation of the suspended constituents of chyme into soluble substances which can be absorbed into the blood and furnish nourishment for the body; proteids are thus changed into peptone-like bodies, starches into sugars, and fats probably into fatty acids and soaps. These changes are accomplished by digestive reagents, or juices, secreted into the intestine somewhat as the gastric juice is secreted into the stomach, while the mixture of food and digestive juice is being driven along the intestine by the action of its muscular walls ; meanwhile the products formed are being absorbed into the blood through the lining membrane of the intestine.

28. The General Structure of the Intestine. - The main functions of the intestine, like those of the stomach, are indicated in the structure of two of its coats, the muscular 
coat and the glandular mucous membrane. The fibers of the former are arranged in two layers, an inner layer in which they are circularly disposed around the mucous membrane of the gut (see Fig. 54), and a much thinner outer layer in which they run lengthwise. The contraction, or shortening, of the circular fibers constricts the bore, or lumen, of the tube; and it is essentially this result that the muscular work of the intestine produces.

In the structure of the inner or mucous membrane two points are of importance to us. In the first place numerous simple tubular glands discharge into the intestinal tube an important digestive juice, the intestinal juice; in the second place, finger-like processes, or villi $(0.5-0.7 \mathrm{~mm}$. long by $0.1 \mathrm{~mm}$. thick), arise from its surface and project into the intestinal cavity. These are important organs of absorption. The entire surface of the villi, the glands, and the plane surface of the intestine between these structures is lined with a continuous membrane composed of columnar cells, which separates blood vessels and lymphatics in the intestinal wall from the cavity of the intestine (see Fig. 55).

29. The Pancreas and the Liver. - Besides the intestinal juice thus added from the glands of the mucous membrane to the contents of the intestine as they are passed along its length of twenty or twenty-five feet, two other juices, the pancreatic juice and the bile, are mingled with the chyme from two large and important glandular organs whose ducts open near the stomach. These glands are the pancreas and the liver. It is not necessary for our present purpose to describe the minute structure of these organs; it is enough for the student to understand that they are glands (p. 29), which pour their secretions through ducts into the intestine, very much as the salivary glands pour their secretions into the mouth.

The food in the intestine is thus treated with three principal secretions, or chemical reagents, - the pancreatic 
juice, the bile, and the intestinal juice; and we may now pass on to a study of these reagents and the chemical reactions they cause in the chyme.

30. The Pancreatic Juice is a strongly alkaline liquid, and consequently, when mixed with the acid chyme, it

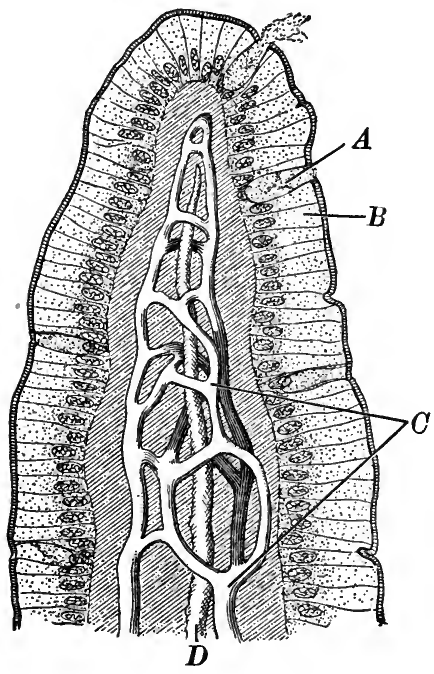

Fig. 55. Longitudinal section of the tip of a villus

Showing the columnar lining cells $B$, through which the products of digestion must pass on their way to the blood vessels and lymphatics. The connective tissue between the columnar cells and the vessels is indicated diagrammatically and without showing its structure. $A$, cell which manufactures mucus ; $C$, capillaries ; $D$, lacteal or lymphatic

neutralizes most if not all the hydrochloric acid of the latter. Thus it happens that while the food in the stomach is strongly acid, in the intestine it becomes at once either neutral or alkaline. Under these circumstances the pepsin of the gastric juice becomes inactive (see p. 108), and is soon destroyed by the pancreatic juice, so that it plays no further rôle in proteid digestion. This is henceforward carried on by an enzyme of the pancreatic juice, trypsin, which acts most vigorously in a neutral or slightly alkaline medium. It forms from the proteids of the food the same general class of peptone-like substances produced by the action of the gastric juice. In addition to trypsin the pancreatic juice contains at least three other enzymes; one of which seems to be closely related, if not identical with, the rennin of the stomach, although the exact part it plays in digestion is not yet clear. The other two enzymes are much more 
important. One of them, amylopsin, is practically identical with the ptyalin of the saliva, and changes starch into sugar, much as happens in salivary digestion. The other enzyme, lipase or steapsin, acts upon fats, changing them into fatty acids and glycerin. We cannot go into the details of the somewhat complicated digestion of fats. The change, like that of proteids into peptones and of starches into sugar, involves the formation of a smaller molecule, either of fatty acids, or soaps, or both, and it is probably in these forms that fats are received from the intestine by the villi.

The pancreatic juice thus contains a special enzyme for each of the three great classes of nutrients, — proteids, fats, and carbohydrates, - and thoroughly completes their digestion, after they have undergone the preparatory processes effected by cooking, mastication, and gastric digestion. Pancreatic juice is by far the most important of the digestive juices in producing the chemical changes of digestion. In this respect, also, we may say it is of primary importance in the work of intestinal digestion, the other two juices, the bile and the intestinal juice, acting as aids in its work.

31. The Bile contains no enzymes of importance in digestion. It is in fact partly an excretion, some of its constituents being waste products which are poured into the intestine only to be ultimately discharged from the rectum. Other constituents of the bile play an important rôle in the digestion and absorption of fats, as is shown by the fact that, if bile be prevented from entering the intestine, from forty to sixty per cent of the fat eaten fails of absorption and is discharged with the feces. It is probable that this is because certain soaps formed in pancreatic digestion are not soluble unless bile is present. When these soaps are not dissolved they are not only themselves not absorbed, but, by being precipitated and adhering to 
other still undigested food, prevent the ready access of the enzymes, and so greatly delay digestion.

32. The Intestinal Juice, like the bile, contains no enzymes of primary importance, although it contains some which play a subordinate rôle. It is, however, characterized by its large content of alkaline salts, especially sodium carbonate. This is of importance, since two processes not yet emphasized but constantly occurring in the intestine produce acid; these are $(a)$ the splitting of the fats into fatty acids and glycerin, and $(b)$ the bacterial decompositions of carbohydrates and (to some extent) of proteids. The sodium carbonate of the intestinal juice which, it will be remembered, is being secreted along the entire length of the intestine, neutralizes these acids and so maintains the reaction of the contents at an approximately neutral point. This reaction is most favorable for the action of the enzymes present.

33. Action of the Muscular Coat of the Intestine. - Few points in the structure and functions of the intestine are more striking than the provisions made for promoting mechanically by muscular movements the effective mixture of the food received from the stomach with the digestive juices and abundant contact of the digested foods with absorbing surfaces. Consider, to begin with, the structure of the intestine. The average length of the small intestine is about twenty feet; its average circumference is some three inches; this of itself would give an absorbing surface of some five square feet. But this surface is increased fivefold or more by the finger-like villi and by folds of the mucous membrane; so that we may estimate the total area of the absorbing surface as probably not less than twenty-five square feet, an area almost twice as great as that of the skin. But this is not the whole story. If the digesting mixture in the intestine were kept in constant motion, it is clear that contact of all parts of it with the 
villi would be greatly facilitated; and such a motion, or agitation, is in fact maintained by contraction of the muscles of the intestinal walls and the consequent movement of the contents.

34. Divisive or Segmenting Movements. - The movements of the intestine, like those of the stomach, are best

$A$
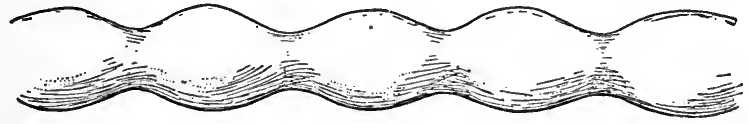

$\boldsymbol{B}$
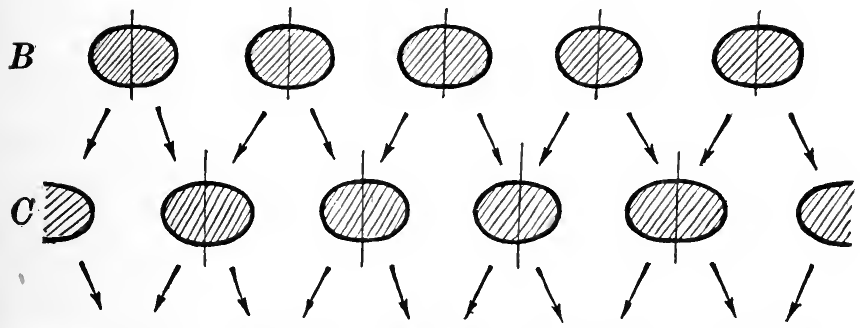

$\downarrow$

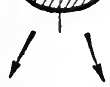

\section{$D$}
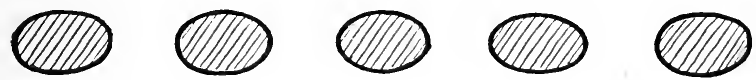

Fig. 56. The divisive or segmenting movements of the small intestine

$A$, surface view of a portion of the intestine, showing six constrictions which divide the contents into five segments, as shown in $B$; as these constrictions pass away new ones come in between them and divide each segment of the contents into two, the adjoining halves of neighboring segments fusing to make the new segments shown in $C$. Repetition of this process results in the condition shown in $D$

studied by means of the Röntgen rays. These studies have shown that the food is not distributed continuously along the entire length of the intestine, but is divided into a number of separate portions which lie in different loops of the intestine. This is partly due to the intermittent character of the discharge of the chyme from the stomach. A certain number, sometimes all, of these masses of food will be seen to undergo division into small segments, 
obviously produced by a series of constrictions of the walls, as shown in Fig. 56. The next moment these are replaced by a second series of constrictions between the first. Each segment is thus divided into two, and the neighboring halves of these segments fuse. The next moment the second series of constrictions is replaced by the first, and this process continues at times for many minutes with no change in the general position of the food mass. These divisive, or segmenting, movements occur from twenty to thirty times a minute, and it has been estimated "that a slender string of food may commonly undergo division into small particles more than a thousand times while scarcely changing its position in the intestine."

35. Peristalsis. - Every now and then a ring of constriction, instead of being confined to one place, moves onward, pushing before it the contents of the tube. A contraction of this kind is called peristaltic. The effect produced is much the same as when the contents of a rubber tube are emptied by squeezing it along between the thumb and finger; and it is by this means that the contents of the small intestine are from time to time moved onwards from the stomach toward the large intestine.

Thus each consignment of chyme delivered from the stomach immediately receives its share of pancreatic juice and of bile, and the final transformation of the digestible foods takes place as the whole is driven from time to time along the intestine by peristaltic contractions, the efficiency of the contact of the food with the digestive juices, as well as its exposure to the absorbing surfaces, being greatly enhanced by the agitation produced by the movements of constrictive division carried out by the circular muscles between periods of peristaltic activity. The efficiency of digestion and absorption probably depends as much on the movements carried out by the muscular coat as 
upon the chemicul processes effected by the enzymes and the ether constituents of the digestive juices.

So far as is known, the stimulus to these movements is afforded by the presence of material within the intestine, and especially by the contact of solid particles with the walls; but we cannot enter into detail on this point further than to say that nervous factors are known to play an important part.

36. Absorption is the name given to the passage of digested food materials from the cavity of the intestine into the blood. The word itself perhaps suggests that the products of digestion are received into the blood without change, as a sponge might absorb a solution of peptone, sugar, fatty acids, soaps, and inorganic salts. Such, however, is by no means the case, and the actual physical and chemical processes of absorption are extremely complicated, - far too complicated to be discussed here. Suffice it to say that the intestine is not lined by a dead membrane but by living cells, and through these guardian cells the products of digestion must pass to enter the blood (see figure of a villus, p.118).

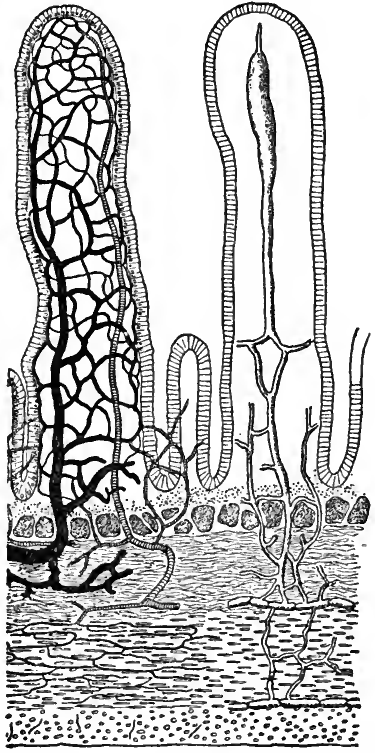

Fig. 57. The intestinal structures concerned in absorption

In one villus is shown the close network of blood vessels immediately under the lining membrane; in the other villus, the central lymphatic or lacteal. Observe that the products of digestion must first be exposed to absorption by the blood vessels before they can enter the lacteal

It is known that the peptone-like bodies are not allowed to enter the blood (except in mere traces), and it is generally believed that they are again built up into certain 
definite proteids which ean nourish the tissues. Again, the fats, though they enter the lining membrane as soaps or fatty acids, appear in the blood largely as fats. The grape sugar, on the other hand, formed in the digestion of starch, probably passes into the blood unchanged.

The present state of our knowledge does not justify further discussion of the full significance of these changes. But what has been said is enough to show that the chemical changes of digestion do not end with those produced by enzymes or other agents in the cavity of the intestine, but that these are succeeded by other important changes effected during absorption. The object of the whole process of alimentation, digestion, and absorption would seem to be that of supplying food to the muscle fiber, the gland cell, the nerve cell, etc., through the blood as an internal medium, or middleman, in that form which is best fitted for the use of the tissues. No matter how varied the forms of proteid (meats of various kinds, beans, peas, white of egg, curd of milk, etc.), all would seem to enter the blood, after being worked over by the processes of digestion and absorption, in the form of a few definite compounds, possibly of proteid nature, which are utilized by the tissues with comparative ease; and something similar is true of fats and carbohydrates. It is very instructive to find that whereas grape sugar and fruit sugar, when introduced directly into the blood, nourish the tissues of the body as well as when they are received from the alimentary canal, the closely related cane sugar when so injected is useless; taken as a food into the alimentary canal, the latter is there changed into grape sugar and fruit sugar, and absorbed in these forms, it nourishes the body. Similarly, the raw or unboiled white of egg is useless as a food unless it first be changed into other proteids by the processes of digestion. Indeed, it is in general correct to look upon the chemical processes of internal digestion as 
merely initiating a long series of successive chemical changes, which, beginning in the intestine, are continued in the process of absorption and only completed in the

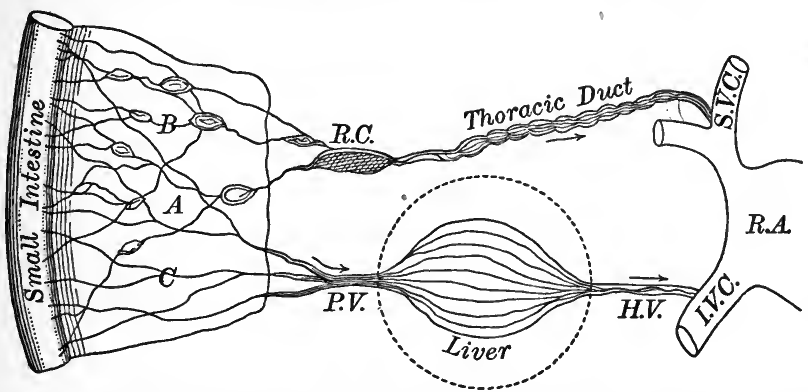

FIG. 58. The paths by which the products of digestion enter the general circulation

Those which are absorbed by the blood vessels $C$ of the intestine pass by the portal vein $P . V$. to the liver before they can enter the right auricle $R . A$. through the hepatic vein $H . V$. and the inferior vena cava I.V.C. Those products which are absorbed by the lacteals pass directly to the superior vena cava S.V.C. through the thoracic duct

tissues themselves. The last stage of these changes we have yet to study in the chapters on nutrition.

37. Microbic Life in the Intestine. - Occurring simultaneously with the chemical changes produced by the digestive juices are others produced by microbes (Part II), which are always found in the intestine in large quantities. The acidity of the gastric juice keeps down the numbers of these germs in the stomach, and under healthy conditions greatly limits their activity in that organ. We have seen, however, that some portions of the contents of the stomach are not acid in reaction during certain periods of digestion; and it not infrequently happens for this reason that unhealthy living, and especially improper feeding, may result in serious gastric indigestion with excessive bacterial decomposition of the food. The production of gas, leading to flatulence or belching, is one of the most familiar results of such bacterial action. 
In the intestine the approximately neutral or even alkaline reaction is much more favorable to bacterial life and growth, and we accordingly find that the number of microbes gradually increases from the stomach to the large intestine. It is not the microbe itself, however, which is of importance to the organism as a whole, but the substances which it produces from the foods. Most of these are either harmless themselves or else are readily changed into harmless substances, either before or soon after entering the blood; it has even been suggested that some are useful and that microbic life is a valuable aid to digestion, - an idea that has no substantial basis; others are poisons, but are normally present in such minute quantities as to be entirely negligible; more rarely they are produced in large quantities and may cause various ill effects either locally or upon the body as a whole.

The production of undue quantities of such harnful substances, most of which are derived from proteids, is chiefly dependent upon the food supply of the bacteria. This is normally kept low by the speedy and efficient removal of the peptones. Native ${ }^{1}$ proteids are acted on comparatively slowly by bacteria, and in any case must first be changed into peptones before they can be further broken down into harmful bodies. If, however, the processes of absorption quickly and efficiently remove the peptones formed both by the bacteria and by the enzymes, subsequent harmful decomposition of the food is prevented, for there are normally no bacteria in the blood. It is, therefore, of great importance to maintain the efficiency of absorption. This can be done in general only by leading a normal life, - by taking sufficient muscular exercise, by

1 A " native" proteid is a proteid in its natural form, or as it occurs in nature before being changed by digestion or other chemical action. The proteids in food are largely native proteids, or else, what amounts to the same thing, as far as the action of bacteria is concerned, native proteids coagulated by heat. 
proper habits of sleep and rest, by proper feeding, and so on. The hygienic conduct of life tends to maintain all functions of the body in proper working condition, those of the digestive organs included; and nothing else can be depended on, in the long run, to do this. To this subject we shall return in the chapters on hygiene, when dealing directly with the personal conduct of life.

We have thus far been dealing only with those microbes commonly found in the intestine. At times foreign microbes find entrance, and these may be the cause of such diseases as typhoid fever, dysentery, cholera, etc. The action of these occasional intruders will be more fully dealt with in Part II.

38. Digestion in the Large Intestine. - The large intestine contains no villi, and its glands secrete a somewhat different intestinal juice from that of the small intestine. It is a juice characterized by a larger percentage of the viscous substance, mucin, familiar in the nasal discharge during a cold, and in the thick, glairy saliva sometimes secreted. This juice serves chiefly to lubricate the walls of the intestine and to prevent harmful friction with the more solid contents.

In the small intestine the amount of water added by se-

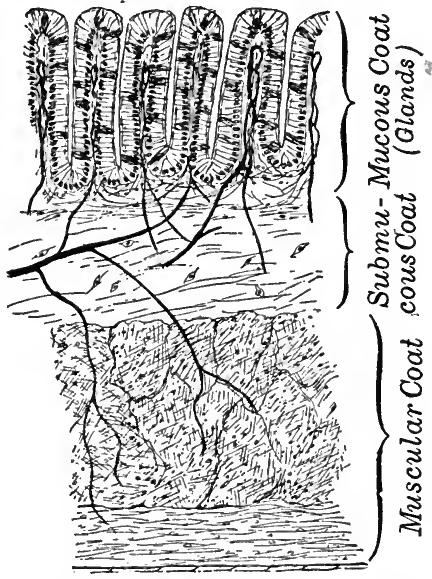
cretion balances that absorbed, so that the consistency of the contents undergoes but little FIG. 59. Longitudinal section of the large intestine

Note the absence of villi change from the stomach to the beginning of the large intestine. This consistency, it will be remembered, was (approximately) that of moderately thick pea soup. During 
the passage through the small intestine the digested portions of the food are being removed by absorption, while the indigestible elements are left behind. Among the indigestible elements of food are certain connective tissues of the animal foods, but especially the cellulose (p. 94), which forms the cell wall of plant tissues. The large intestine receives from the small this indigestible material, together with a certain variable but usually comparatively small proportion of the proteids, fats, and carbohydrates which have thus far escaped digestion; in addition there are certain constituents of the digestive juices which are not absorbed, and some (e.g. certain constituents of the bile) which are distinctly excretory products.

The digestion of proteids, fats, and carbohydrates may to some extent continue in the large intestine, and the products of digestion be absorbed. This process, however, tends to be checked, owing to the fact that here the processes of secretion fall behind those of absorption, so that the contents of the intestine gradually become more and more solid, being finally collected as the feces in the lower colon and rectum. As the intestinal contents become more solid, the peptones and other digestive products are less efficiently removed while bacterial action greatly increases, giving to the feces their characteristic odors. And while the substances which are responsible for the offensive odors of the feces may themselves be harmless to the body, it must be remembered that such odors are an indication of bacterial action capable of producing other substances, which may be, and very often are, the cause of headaches, general malaise, etc., or of even more serious troubles.

39. The Elimination of Intestinal Waste. - Those who are "blessed with a good digestion" sometimes find it hard to realize that the preparation of food for absorption through the delicate membranes lining the alimentary 
canal is a difficult and complex process, requiring much delicate physical and physiological apparatus and involving various and important chemical reactions. Even when they realize this, they rarely appreciate the indispensable coöperation and fine adjustment of the several parts and processes concerned. It is just here, however, that a clear understanding is important, for without this it is not easy to see how disorders of digestion arise.

Let us then remember that the efficient handling of the food in the stomach is aided by the preparatory crushing it receives in the process of mastication ; that in the stomach an adequate and efficient secretion of gastric juice must take place, and that this begins as the result of nervous events connected with our enjoyment of the food when eaten; that the continued secretion of gastric juice is secured, in turn, by stimulation of the mucous membrane of the stomach by the peptones which the "psychic" secretion has formed from the proteids of the food ; and finally that the chemical action of the gastric juice is aided by the peculiar contractions of the muscular coat of the stomach. All these agencies working together deliver the food to the intestine in a finely divided state, well adapted and indeed absolutely necessary to secure the proper contact of the food with the pancreatic juice, the bile, and the intestinal juice.

The flow of pancreatic juice, in turn, is partly the result of the action of the hydrochloric acid of the chyme on the walls of the intestine, while the efficiency of the action of the pancreatic enzymes depends upon the simultaneous action of the bile and the intestinal juice; lastly, the chemical action of these juices, as well as the final act of absorption, requires the coöperation of the muscular coat. Healthy conditions with respect to bacterial action similarly depend upon all else occurring as it should. Digestion, in short, is a chain of events, each depending upon 
those which have gone before, and to a large extent upon those which are taking place at the same time.

Keeping these facts in mind, it is easy to appreciate the possibility of diarrhea or constipation, the latter consisting in the retention of wastes, the poisonous constituents of which may be absorbed into the body and cause discomfort, headaches, and malaise. When all the digestive processes work together properly there should be a perfectly natural and regular evacuation of the bowels. The frequency of such evacuation varies somewhat and is largely a matter of habit; with some people it is twice a day, with others once every other day, but with the vast majority it is normally once every day and at about the same time. Where this is not the case there is reason to believe that some part of the work of digestion is not being properly performed. The trouble is not ordinarily in the mechanism governing the actual discharge of the feces from the rectum, but in a derangement somewhere else; it may be entirely the fault of the mechanism of peristalsis, or it may be due to imperfect secretion. In all cases it means that something is wrong, and the remedy should be sought not in drugs or pills but in search for and removal of the cause. A moment's consideration will show the reasonableness of this position. If a watch loses time because it needs cleaning, we do not seek a remedy in drugs, but in its cleaning, better adjustment, and good care; and the remedy for diarrhea or constipation should in all cases be sought for in the better conduct of life. Is enough muscular exercise being taken? Is the diet properly chosen? Are we drinking enough water? Especially, is the food of sufficient bulk, and does it contain enough laxative material (such as fruit)? And, above all, are we getting enough sleep? Are we overworking, or do we work too long at a time without resting? Is our clothing warm enough, or are we overclad? Such are the questions which should be 
seriously asked. The student of personal hygiene cannot lay to heart too seriously the truth that the man who goes from day to day, from week to week, from year to year, neglecting the warnings of diarrhea or constipation, only reaps the harvest of his folly when in later years he suffers loss of health and at times bodily discomfort; and it is nothing short of impiety to marvel under such circumstances at the "mysterious" ways of a Providence which so "afflicts" his creatures. It is no exaggeration to say that the regular discharge of the wastes is quite as important as the regular feeding of the body, and that no less pains should be taken to form good habits in the one case than in the other. Many of the headaches, many of the bad feelings, and many of the bad tempers of the world are due to neglect of this simple fact. No city, however well fed or beautiful, the drains of which are choked with filth, can long remain either wholesome or attractive, - and the human body is essentially a city populous with living cells. 


\section{CHAPTER IX}

\section{THE CIRCULATION OF THE BLOOD}

\section{A. Blood AND LyMPH}

1. The Blood as a Common Carrier. - In previous chapters some of the more general features of the circulation have already been touched upon. In studying the parts of the body the student has become somewhat acquainted with the heart, the arteries, and the veins ; in considering the typical structure of the organs (Chapter III) he has seen how the arteries are connected with the veins by a system of communicating tubes, the capillaries, through the thin walls of which interchange takes place between the lymph and the blood ; and in studying the interdependence and coöperation of the cells and organs (Chapter VI) he has learned how the blood leaving each organ returns to the heart, there to be mixed with that coming from all other organs and thence pumped first to the lungs and then to the rest of the body. The need of a circulation is obvious; for the food received from the alimentary canal and the oxygen received from the lungs must somehow be carried to the muscle fibers, the nerve cells, the gland cells; the cellular wastes must be taken away to the organs of excretion, and the internal secretions of the body must be transported from the organs in which they are made to those in which they are to be used. In other words, it is a necessary corollary to the fact that no cell or organ "liveth unto itself" that there should be some common carrier of matter and of energy from one organ to another. Such a common carrier is the blood. The 
analogy of the blood system of the body with the railway system of a country is instructive. As different persons and different communities in any country make different products and have different needs, it becomes more and more necessary that the means of communication between them be extensive and efficient. Hence the remarkable growth of the railroads, or "common carriers," of any country in which industrial development produces increasing division of labor.

The blood, which is thus the common carrier first between the various organs, and second between each organ and the outer environment, is the net product of the united work of all the organs ; from the alimentary canal it receives water and the products of digestion; from the lungs it receives oxygen;
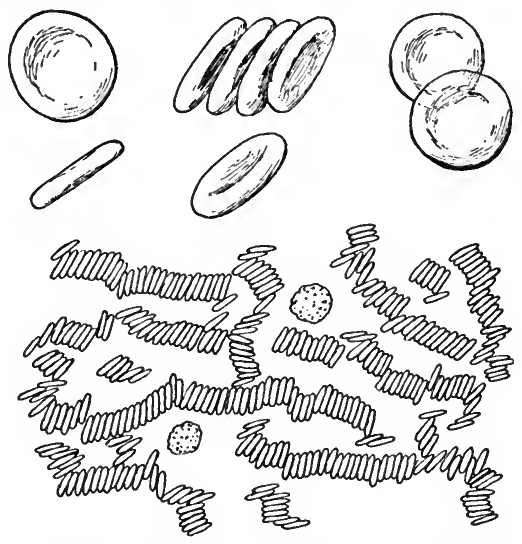

Fig. 60. Structure of a drop of blood as seen under the microscope

Above are shown nine red corpuscles, highly magnified; below, less highly magnified, the appearance of the blood soon after being drawn. Two white corpuscles are shown, and the red corpuscles stick together, forming " rouleaux." Size of red corpuscle, $7.7 \mu$ wide, $2-4 \mu$ thick. Diameter of white corpuscle, $5-10 \mu$. Number of red corpuscles, $4,500,000-5,000,000$ per cubic millimeter. Number of white corpuscles, 4500-13,000 per cubic millimeter, according to the state of digestion, etc. Surface area of all the red corpuscles of the blood, 3000 square meters $(30,000$ square feet or approximately four times the size of a baseball diamond. $(1 \mu$ or micron $=0.001$ millimeter)

each organ contributes its share of waste products or of internal secretion, while some influence the composition of the blood by removing from it certain things that it contains.

2. The Microscopic Structure of the Blood. - Examined under the microscope the blood is seen to consist of a 
liquid portion, the plasma, crowded with small solid bodies, the corpuscles. These are of two kinds: the red corpuscles, - biconcave disks containing a pigment, hemoglobin, which gives the red color to the blood; and the white corpuscles, which are colorless, nucleated cells.

Important data on the number, size, and surface area of the corpuscles will be found in connection with Fig. 60 .

3. The White Blood Corpuscles. - The white blood corpuscles really comprise several different kinds of cells, hav-
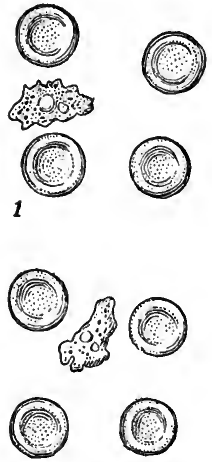

3
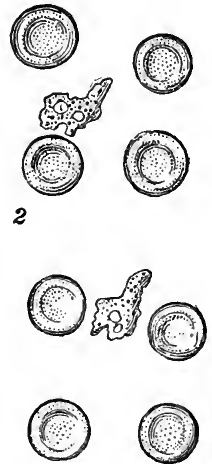

4

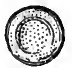
ing different functions, the study and explanation of which belong to advanced rather than elementary physiology. It is enough for our purpose to state that these cells are not confined to the blood, but work their way out of the blood vessels between the cells of the capillary walls and are often found in the lymph spaces of the tissues as wandering cells. The latter term refers to their movement from place to place. The cytoplasm of the white corpuscle is a thick, viscous fluid without constant or definite form. In locomotion the cytoplasm flows slowly from some part of the surface in the direction of motion, forming what is known as a pseudopodium (from the Greek; meaning a false foot), as shown in Fig. 61 ; the rest of the body of the corpuscle then flows into the pseudopodium. By the continuation of this process the white corpuscles make their way through the spaces of the connective tissue. Locomotion by means of pseudopodia is frequently spoken 
of as amoboid, from the amoba, a unicellular animal which moves in the same manner.

4. The Red Blood Corpuscles. - The red corpuscles are pigmented, biconcave disks, with no nucleus ; they are normally confined to the blood vessels and are carried around passively in the blood current without active movements of their own. The main function of these corpuscles is to carry oxygen from the lungs to the tissues, a function which will be further studied in connection with respiration. They contain a pigment, hemoglobin, which gives to the blood its red color and carries the oxygen.

5. The Blood Plasma is an exceedingly complex fluid whose general composition is represented as follows: water, 90 parts; solids, 10 parts, - proteids, 8 parts ; inorganic salts, 1 part; extractives, 1 part.

Under the extractives are included a very large number of substances which, though present in small quantities, are interesting to the physiologist because they are largely products of the chemical activities of the body, and as such give information about the nature of the chemical changes occurring in the organs.

Finally, it should be remembered that the cells of the body generally are bathed with lymph, not with blood; in other words, that the lymph and not the blood is the immediate environment of the cells. Lymph is sometimes described as blood minus its red corpuscles, but this statement, though convenient, is not strictly correct, since the amount of waste products in lymph must be greater than in blood, while the amount of food material must be less (see Chapter IV). Much as the blood is a product of the united chemical activity of all the organs of the body, so the lymph of each organ is derived from the cells of that organ and from the blood flowing through it. Lymph thus has a double origin, and of course shows very considerable differences of composition in different organs. 


\section{B. Mechanics of the Circulation of the Blood AND OF THE FLOW OF LYMPH}

The greatest discovery ever made in physiology was that of the circulation of the blood. As late as the settlement of the earliest English colonies in America it was thought that the blood moved back and forth in the blood vessels, as the waters in the sea ebb and flow; but of any circulation, in the sense of a steady stream returning to its source, there was no idea; and it was not until 1621

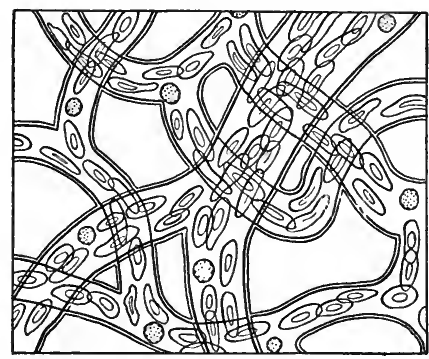

Frg. 62. The circulation of the blood as seen in the small arteries and capillaries of the web of a frog's foot that William Harvey, an English physician, proved beyond the shadow of a doubt that the blood in the body of all the higher animals flows like a stream always in one direction, ultimately returning to its source.

Tests made upon various animals have shown that this circulation is accomplished in the surprisingly short time of from twenty to thirty seconds; which means that the whole mass of the blood (in man about twelve pints) passes between three and four thousand times a day through the various organs of the body, bringing to them their food, carrying away their wastes, and in general helping to maintain normal conditions. By what hydraulic machinery is this marvelous work done?

6. The Motive Power of the Circulation as a Whole. The Beat of the Heart. - Whenever a mass of liquid is kept in motion we naturally look first for the motive power. In answering the question, "What makes the blood circulate?" we shall find that while there are several causes, 
one of these, namely the beat of the heart, is vastly more important than all the others combined. This fact is now so familiar that it is hard to realize that we owe to Harvey not only the discovery of the circulation, but also the discovery of the meaning of the heart beat. Before his time, to be sure, the living heart had been seen at work, alternately shrinking in size and then swelling, the shrinking being called systole and the swelling diastole. But these changes in size were regarded as the results of the contraction and expansion of certain "vital spirits" which the arterial blood was then supposed to contain, and not as muscular contractions and relaxations. Harvey showed that the heart is a powerful muscle, and that its systole is a muscular contraction; that during systole it becomes hard, just as the biceps muscle does when it shortens, and during diastole soft and flabby; he also proved that with each systole the heart drives or spouts blood into the large arteries (the aorta and the pulmonary artery), and that this blood is prevented from flowing back into the heart during diastole by membranous valves at the very beginning of the large arteries in question.

7. The Heart a Muscular Force-Pump. - The beat of the heart, even to its most minute detail, is one of the most important as well as one of the most interesting subjects in physiology; everything in the body hangs on its proper efficiency and regulation, and it cannot be too thoroughly studied. For our present purposes it will suffice to describe the heart as composed essentially of a pair of muscular force-pumps. Dissection shows that it is divided into right and left halves (see Fig. 65), completely separated from each other, and that each half consists of two chambers, - an auricle and a ventricle. The auricles, into which the great veins open, have thin muscular walls and are comparatively small in size; the ventricles, on the other hand, from which the great arteries arise, have thick 
muscular walls, especially the left ventricle. The ventricles indeed constitute the principal part of the force-pump; the auricles merely facilitate the work of the ventricles, and for purposes of elementary study may be mostly neglected. The student should, if possible, examine for himself and actually handle the auricles, ventricles, and great blood vessels of a sheep's heart, which in size and structure sufficiently resembles the human heart. Figures 15 and 142 should also be consulted.

8. The Mechanics of the Heart Beat. - All force-pumps consist of two indispensable parts, - some device for pressing upon a liquid within a chamber, and valves at the
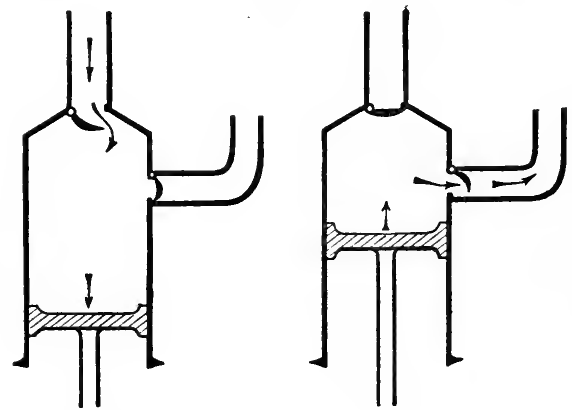

FIG. 63. Diagram of the action of a force-pump openings of the chamber so arranged as to allow the passage of the liquid in one direction only. Each ventricle of the heart is really such a pump, and is provided with two sets of valves, one set at the inlet, between the auricles and the ventricles, and the other at the arterial outlet. These valves permit blood to pass only from the great veins through the auricles and on through the ventricles to the great arteries. The contraction of the muscular wall of the ventricles produces pressure on the blood within their cavities; this pressure quickly and easily closes the auriculo-ventricular valves, and finally forces open the shut valves at the openings of the great arteries. In this way the right ventricle drives venous blood into the pulmonary artery, and the left ventricle arterial blood into the aorta. With the relaxation of the ventricles 
(diastole) pressure falls within their cavities, and were it not for the valves at the mouths of the aorta and the pulmonary artery, blood would regurgitate, or flow back, into the heart; but this "slip" (as it is called in hydraulics) the valves prevent, and the ventricles again fill through the onlyopen channel, i.e. the one leading from the great veins and the auricles. Thus by contractions rhythmically repeated the heart continues to spout or deliver blood from the two sets of
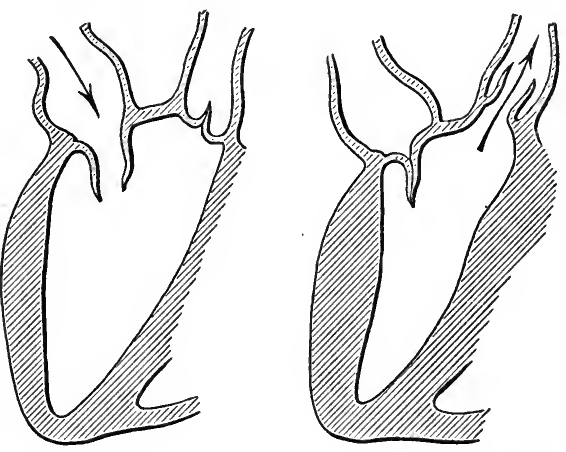

great veins into the two sets of great arteries. It is plainly a double force-pump, or, better, a pair of force-pumps lying and working side by side.

9. The Arterial and the Venous Reservoirs. - To understand the exact nature and result of the work of the heart we must now consider the relation of this living pump to the pipe system (arteries, capillaries, and veins) with which it is connected. The student should first trace the general course of the circulation in the simple diagrammatic representation given in Fig. 65. This shows that the blood which enters the aorta from the left ventricle must return to the right side of the heart and pass through the lungs before it can again reach the aorta. As the physical principles of the circulation are the same for the systemic and the pulmonary vessels, we shall confine our attention to the former.

In the first place we may observe that the heart pumps the blood into what is practically a large reservoir (the 


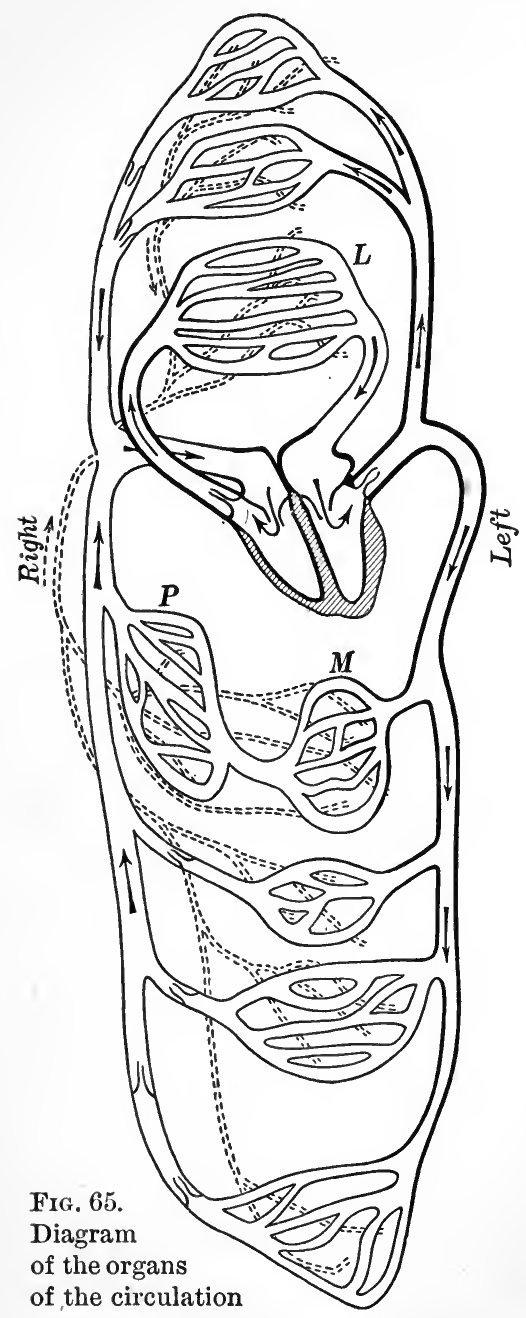

$L$, pulmonary circulation; $M$, circulation through the organs suspended by the mesentery, the blood being carried to the liver $P$ before it returns to the heart. The circulation through other organs, such as brain, muscles, skin, and kidneys, is indicated. Lymphatics are represented by dotted lines larger arteries), and that the blood flows from this reservoir to a second reservoir (the larger veins) by various routes; for the vessels of the different organs represent many alternative courses which the blood may take in flowing from the arterial to the venous reservoir. The blood stream indeed may be compared with a stream supplying water power to a series of mills in a manufacturing town. The larger arteries from the main source of pressure (the heart) correspond to the headrace from above the dam, while the larger veins correspond to the tailrace. The water flows from the one into the other only through the smaller sluices, or penstocks, which supply the mills. So, in the vascular system, a part of the blood 


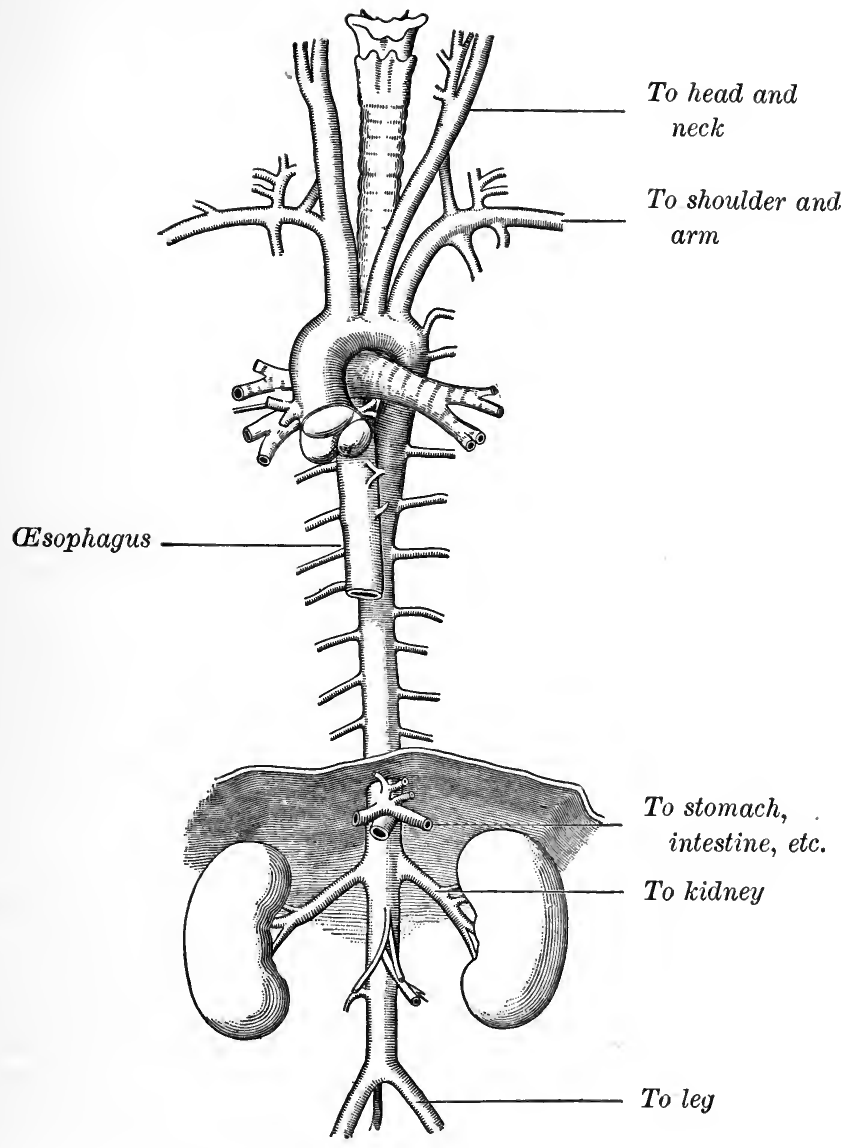

Fig. 66. The aorta and its main branches

At the beginning are shown the three pocket valves, which prevent regurgitation of blood during diastole 
pumped into the arterial reservoir, or aorta, finds its way into the venous reservoir by way of the skin, another part by way of the digestive organs, another by way of the brain, still another by way of the kidneys, and so on; but the flow in every case is essentially the same, namely from a reservoir of high pressure to one of lower pressure.

\section{The Driving Force for the Flow of Blood from the} Aorta. Pressure in Arteries and Veins. - The hydraulic conditions in the aorta may be illustrated by means of the following simple piece of apparatus. To an ordinary rubber syringe attach a piece of elastic rubber tubing, the other end of which is closed by a detachable nozzle. If now the nozzle be removed and water pumped into the tube, it will be found that the flow from the open end consists of squirts or spouts and continues only during the stroke of the pump; if, however, we attach the nozzle, and again pump water into the tube, the resistance caused by the small orifice of the nozzle prevents the water from flowing out of the tube as fast as the syringe pumps it in. The tubing becomes distended with water. Since, however, the tube is elastic, ${ }^{1}$ and so tends to return to its original size, it forces the liquid out through the nozzle even between the strokes of the pump. The immediate cause of the steady flow from the nozzle is therefore the elasticity of the rubber tube. The intermittent stroke of the pump produces distention of the tube, and the elasticity of the distended tube constantly forces the water out of the nozzle.

Closely similar conditions obtain in the arterial reservoir. Here the outlet is also through very small tubes,

1 An elastic body is one which returns to its original shape when it has been stretched, compressed, or otherwise deformed. Elasticity must not be confounded with "extensibility," or the property of allowing stretching. Thus when we "pull" taffy we deal with a body which is very extensible but which is practically inelastic. A body indeed may be extensible only with difficulty, but possess a very high degree of elasticity; ivory is a good example of this kind. 
the small arteries, whose bore is not greater than $\frac{1}{50}$ or $\frac{1}{100}$ of an inch; which fact introduces the same condition as does the nozzle of our apparatus, i.e. a resistance to the outward flow of the blood. Consequently the blood cannot flow out of the aorta as rapidly as it is driven in, and the extensible and elastic walls are necessarily stretched. The immediate effect of the heart beat is to keep the arterial reservoir overfilled or distended, so that the elastic reaction of its walls is brought into play; and it is this elastic reaction of the arterial walls which is the immediate cause of the steady outflow through the small arteries and capillaries.

The force of compression, or pressure, exerted by the elastic arterial walls is primarily exerted upon the blood within them; and the more the arteries are distended the greater will be the pressure exerted on the blood. A liquid thus under pressure tends to find an outlet; should any part of the arterial wall be weak, as sometimes happens in diseased conditions, it is bulged outward; and, for the same reason, a flow of blood will take place through such outlets as are presented by the smaller arteries and capillaries. Moreover, the greater the pressure of the blood in the arteries, the more rapid will be the flow into the capillaries. Hence it is customary to use the arterial blood pressure as a measure of the force of elasticity exerted by the distended arterial wall.

The veins, on the other hand, are less elastic than the arteries; they are indeed more like mere conducting tubes through which the blood can flow back to the heart. They are not overfilled (since, for one reason, there is no resistance to the flow of blood out of them into the heart), and hence venous blood pressure is low.

Thus we have the conditions favorable for the flow from the aorta to the great veins, - a high pressure in the arterial reservoir and a low pressure in the venous reservoir. 
It is the function of the heart, by continually pumping the blood from the veins into the arteries, to keep the arterial reservoir distended, thus maintaining a difference of pressure in the two reservoirs. It is this difference of pressure which drives the blood through the organs.

11. The Distribution of the Blood among the Organs. Some organs require more blood than others, and the same
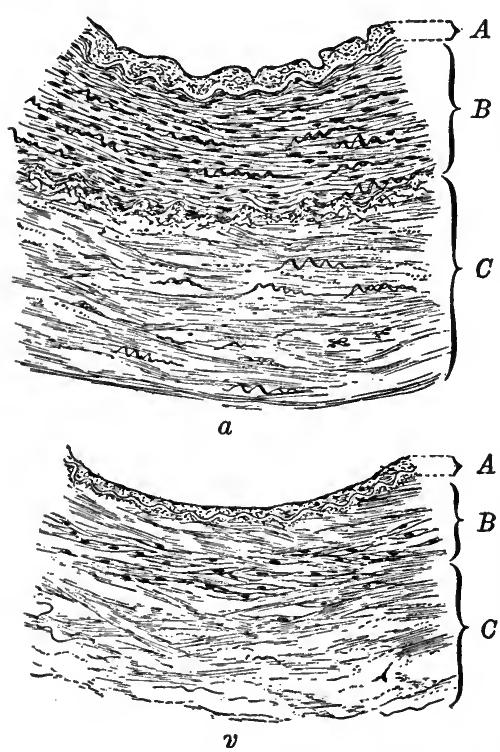

Fig. 67. Cross sections of portions of the wall of a smaller artery $(a)$ and a smaller vein $(v)$

$A$, internal coat; $B$, middle coat, with muscle fibers ; $C$, outer coat of connective tissue. The contraction of the circularly disposed muscle fibers narrows the bore of the tube organ often requires more blood at one time than at another. Thus muscles and glands, the seat of very active chemical changes, require more blood than a tendon; and a gland requires more blood during the process of secretion than during rest. How is the supply of blood to the organs regulated to meet their varying needs? In the first place, some organs are more vascular than others; those requiring a larger supply of blood receive a greater number of arteries from the arterial reservoir and have a closer network of capillaries. But in addition to this, these smaller arteries contain circular muscle fibers whose contraction diminishes the bore of the tube. When an organ needs more blood, the muscle fibers of its small arteries relax, thus permitting the arterial tubes to widen or dilate; 
just as when we want the water to flow faster from a faucet, by turning the spigot a little further we widen the outlet from the pipe. When less blood is needed the small arteries are caused to constrict, just as a spigot may be partially turned off (see Sections 25-27). In this way the flow of blood to any organ is regulated to meet the varying needs of the organ in question. ${ }^{1}$

12. Secondary Aids to the Circulation. - In the preceding discussion we have seen that the cause of the flow of blood through the organs is the difference of pressure in the two reservoirs. We have further seen that this difference of pressure is maintained by the heart beat in pumping blood from the venous into the arterial reservoir. A moment's consideration will show that anything which hastens the flow of blood from the veins into the heart, and so lowers pressure within the veins, would similarly aid the circulation, since, with the same arterial pressure, more blood will flow into an empty vein than into one which is partially filled.

(a) The Breathing Movements. - There are two factors which thus tend to empty the veins. The first is the suction exerted on the blood within the veins by breathing movements. The exact mechanism by which this is accomplished must be left for consideration in the chapter on respiration. Suffice it to say here that just as the enlargement of the thorax, when we take in a breath, sucks air into the lungs, so it also sucks blood from the large veins outside

1 In order that the student may become more familiar with these fundamental hydraulic principles of the circulation, such questions as the following should be answered: (1) What are the two principal factors whose variations change the amount of arterial pressure? Illustrate by an example or model. (2) How would the dilation of all the arteries of the intestine affect the general arterial pressure? (3) What would be the effect upon the amount of blood flowing through the skin under this condition? (4) How would dilation of the arteries of the skin affect the blood flow through the brain? 
the thorax into those which lie within it; because of the thickness of the walls of the arteries, the same effect
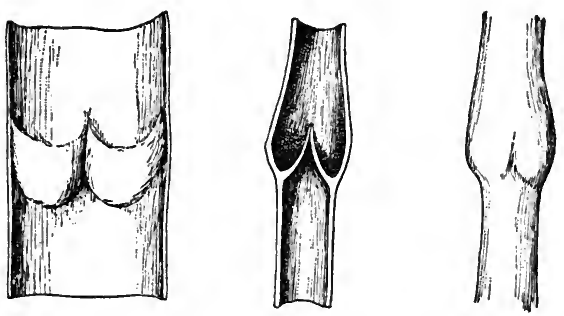

FIG. 68. The pocket valves in the veins

On the right is shown the external appearance of the vein at the valves when the latter are closed; on the left a vein slit lengthwise and opened; in the middle a longitudinal section of a vein

occurs to only a very slight extent in the arterial reservoir. During expiration, on the other hand, the reduction in size of the thorax forces air out of the lungs, and we might expect that it would similarly force blood from the veins within the thorax into those

without. And this it certainly would do if the veins were not provided with valves which allow the blood to flow only toward the heart. In general, therefore, both inspiration and expiration aid the circulation, the former by sucking blood into the thoracic veins and so emptying those outside, the latter by making this blood in the intrathoracic veins flow on more rapidly to the heart, whence it is pumped into the arteries. In a word, deep breathing greatly promotes a good circulation.

(b) Intermittent Compression of the Veins. - The other secondary factor of the circulation is intermittent compression of the veins, and in ordinary life this is brought about in two ways: (1) Whenever a muscle contracts it thickens and hardens; the veins and capillaries which are between the fibers and fiber bundles, or in the connective tissue between two contracting muscles, will thus have the blood squeezed out of them into the large veins; when the muscle relaxes the empty veins and capillaries will readily fill from the arteries, since the valves of the veins will prevent any backward flow of the blood from the larger 
veins. Alternate contractions and relaxations of muscles, therefore, aid the flow of blood through this so-called "pumping" action on the veins. (2) A similar pumping action on the veins is exerted by alternate flexions (bendings) and extensions at any joint. In general, flexions force the blood out of the veins, while extensions allow them to fill. When we remember how largely most of our usual muscular actions consist of alternate flexions and extensions of joints, and alternate contractions and relaxations of muscles, as, for example, in walking and running, we can at once appreciate how greatly muscular activities must aid the circulation. When to the effect of these we add the suction action of the deepened breathing movements the effect upon the circulation becomes very great.

13. Massage. - The action of massage is only another illustration of the same principle. By rubbing the legs and arms in the direction of the heart, the blood contained in their veins is forced onward and the circulation aided, precisely as when a muscle contracts or one member of a limb is flexed upon another.

14. The Lymphatics. - Important as are the suction action of the breathing movements and the pumping action of contracting muscles as aids to the circulation of the blood, they are even more important as causes of the flow of lymph along the greater lymphatic trunks toward the heart. Reference to the general method of origin of lymphatics, as described in Chapter III, will show that the lymph in the lymph spaces, unlike the blood in the capillaries, has not behind it a high-pressure reservoir; there is no such force from behind to send it onwards, since the lymphatics arise blindly in the tissues. What, then, makes the lymph flow along the lymphatics toward the heart?

The lymphatics resemble the veins in structure, having thin walls and pocket valves; like the veins, most of them 
originate in extra-thoracic organs, and join or combine to form larger trunks as they proceed toward the thorax. All of them finally unite in two large lymphatics within the thoracic cavity, and these open into the great veins near the heart. (Figures 30 and 65 should be consulted in this connection.) It is at once clear that the breathing movements must exert on the lymph within these thinwalled vessels exactly the same suction action as they exert on the blood in the veins; and anything which increases this suction action, such as the deepened breathing movements during muscular activity, must necessarily increase the flow of lymph from every organ of the body. On the other hand, a pumping action on the lymph in the organs results from all rhythmic movements of parts of the body with reference to one another, since each change of position carries with it some change of external pressure on lymphatics. Familiar examples are the movements of arms and legs in locomotion, of the diaphragm in breathing, and of the lungs in respiration.

It has also been supposed that a third cause of the lymph flow is the passage of waves of constriction (peristalsis, ef. p. 122) over the larger lymphatics. This, however, probably plays only a minor part.

Finally, in the formation of lymph from the blood, more water generally passes from the capillaries to the lymph spaces than from the lymph spaces into the capillaries. Under these circumstances, at least at certain times, the lymph spaces become distended and a certain low pressure obtains in them. This we may speak of as the "active force" of lymph formation, and it constitutes a fourth factor in causing the lymph flow.

We have already pointed out the importance of the lymph flow in maintaining the lymph currents about the living cells; we are now able to appreciate the importance of those agents which secure this flow. As enumerated 
above, they are four in number: (1) suction action of the breathing movements ; (2) pumping action of muscular or passive movements ; (3) active force of lymph formation ; (4) peristaltic contractions of the large lymphatics.

Of these the fourth is at least doubtful and in no case of great importance; the other three may therefore be regarded as the chief causes of the lymph flow, and of these the first and second are brought into most effective action by muscular activity; this deepens the breathing movements and so increases their suction action on the lymph, while the movements of the body exert on the lymphatics a pumping action, which is largely lacking during complete inactivity. The great practical importance of this aspect of the subject will be discussed beyond in those chapters which deal with the hygiene of muscular activity (Part II).

\section{The Adjustment of the Circulation to the Needs of Everyday Life}

The total quantity of blood in the body (ten to fourteen pints) is not enough to furnish a working supply to all organs at the same time; and since, in general, whenever an organ works it receives more blood, and when it is at rest it receives less, our daily life with its changes of activity among the organs makes necessary frequent adjustments of the circulation to the needs of the organs at various times.

Some of these adjustments are matters of familiar experience. The increased flow of blood to the skin on a warm day makes the veins stand out and the face red, and we are conscious of the more rapid heart beat during muscular activity, even in an act so simple as running upstairs. Other adjustments are not so evident, but betray themselves by their results, as happens after a hearty meal when the 
demand of the digestive organs for blood lessens the supply to the brain and we feel disinclined to hard mental work. We may begin our study of these adjustments by learning what occurs in the circulation during some of the more common activities and events of daily life.

\section{The Circulation during Exposure to Heat and Cold.} - When the skin is exposed to cold its blood supply is greatly diminished; the veins no longer stand out prominently on the hand, and if a small area of skin be made pale by pressing upon it (thus driving the blood out of its capillaries), the pallor passes off very slowly. This simple experiment shows that blood is flowing but slowly from the arterial reservoir into the skin. Conversely, on a warm day the veins stand out prominently and the red color instantly returns upon the removal of pressure. These variations in the supply to the skin are due, as we have already seen (p. 144), to changes in the diameter of the arteries of the skin, which changes serve like the spigot of an ordinary water faucet to regulate the flow of liquid.

The changes in the blood-flow through the skin are accompanied by corresponding but inverse changes in the internal organs. On a cold day the stomach and intestines, the pancreas, the liver, the kidneys, etc., are richly supplied with blood, while on a warm day their blood supply is diminished. In the former case the blood withheld from the skin finds its way into the internal organs; in the latter case the skin draws upon these organs for its needed supply. The circulation in the internal organs compensates for that in the skin.

16. The Reason for Compensatory Changes. - We have seen that it is the function of the heart to keep the arterial reservoir adequately distended with blood, thus supplying a steady driving force for the flow of blood through the organs. When the small arteries of the skin widen on a warm day blood escapes more rapidly into the skin 
from the arterial reservoir. This alone would diminish the amount of blood in the reservoir unless the heart pumped more blood, or unless the dilation or widening of the cutaneous arterioles were compensated by a constriction elsewhere, so that the total drain on the reservoir remained the same. In the case in question it is the latter of these alternatives which is adopted, and the reservoir is kept filled without calling on the heart to pump more blood.

Conversely, on a cold day the diminution of the outflow into the skin would lead to a backing up or accumulation of blood in the great arteries, and so to their increased and perhaps undesirable distention, if the dilation of the arterioles of internal organs did not provide an outlet for the surplus blood.

Nowhere, perhaps, is this principle of compensatory dilation or constriction of arteries in one region, to allow for the effect of the opposite change in some other region, so highly developed or so fully applied as in the reactions of the body to changes in external temperature.

17. The Circulation during Muscular Activity.-During muscular activity the arterioles of the muscles and of the skin are dilated, the former in order to supply more blood to the working organ, the latter to aid in the discharge of the excess of heat produced by the contracting muscles. The heavy drain upon the arterial reservoir by these two large areas (among the largest in the body) is compensated to some extent by the constriction of the arteries of the digestive and other internål organs. This alone, however, would not suffice to keep the arterial reservoir filled; and we accordingly find that the heart beats more rapidly and more powerfully, pumping more blood into the aorta in a given time.

It is very important to remember that muscular activity is the one condition of life which materially increases the work of the heart; at other times the greater demand 
of blood for the working organ is met more or less successfully by withdrawing blood from a resting organ, while the supply to the whole arterial system, and hence the work of the heart, remains approximately unchanged. During muscular exercise, and then only, is the heart called upon for decidedly increased work ; and, like the skeletal muscle, its strength, its ability to meet strain and emergencies, and to withstand fatigue depend to a great extent upon the training given it in this way.

Muscular activity also influences the circulation indirectly by increasing the action of its secondary driving forces, - the suction action of the respiratory movements and the pumping action of the contracting muscles on the

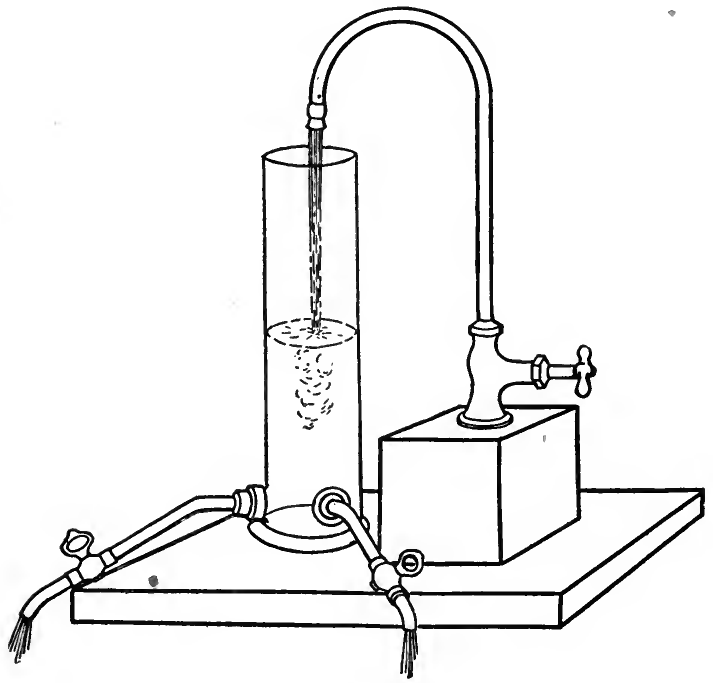

Frg. 69. Simple apparatus to illustrate the relation between the output of the heart, the peripheral resistance, and the general arterial pressure

The amount delivered by the faucet represents the output of the heart, and is one factor in keeping up arterial pressure; two alternative routes of outflow, each capable of regulation, represent the arterioles to different organs. Compensatory constrictions and dilations and other hydraulic conditions described in the text may readily be imitated 
veins. These are among the most important effects of this agent upon the flow of blood, but they are too complicated for detailed discussion here.

It is sometimes stated that muscular exercise "quickens" the circulation. This is true in the sense that the heart pumps more blood into the pulmonary artery and the aorta than during rest. From this it follows that during exercise more blood flows through the lungs, and that blood flows more rapidly out of the arterial reservoir ; but it does not mean that blood flows more rapidly through all organs, for the digestive and other internal organs at such times actually receive less blood. Indeed, we may say that the quickening of the circulation during exercise is chiefly confined to three important organs, - the muscles, the skin, and the lungs; in other organs the change is relatively slight, as, for example, in the brain; while in still others, notably those of the digestive system and the kidneys, the speed is diminished.

18. The Circulation during Sleep. - An adequate blood supply is necessary to the full activity of the brain; when the circulation in this organ is seriously interfered with, imperfect mental action or even unconsciousness is a result. Thus when all the arterioles of the body dilate, or the heart beat is slowed down, in consequence of some sudden "shock," so that pressure in the arterial reservoir falls too far, the driving force for the flow of blood through the brain, in common with other organs, is diminished, and the person loses consciousness, or faints. Most cases of fainting are traceable to one or the other of these causes.

The most familiar and most common example of unconsciousness, however, is that of sleep, which in so many respects resembles fainting as to suggest that the unconsciousness in both cases is due to the same cause, namely, a lessened blood supply to the brain. Unquestionably, the amount of blood flowing through the brain is greatly 
lessened during sleep. The evidence for this statement cannot be given here in full, but it is known that where accident has destroyed a part of the rigid bone of the
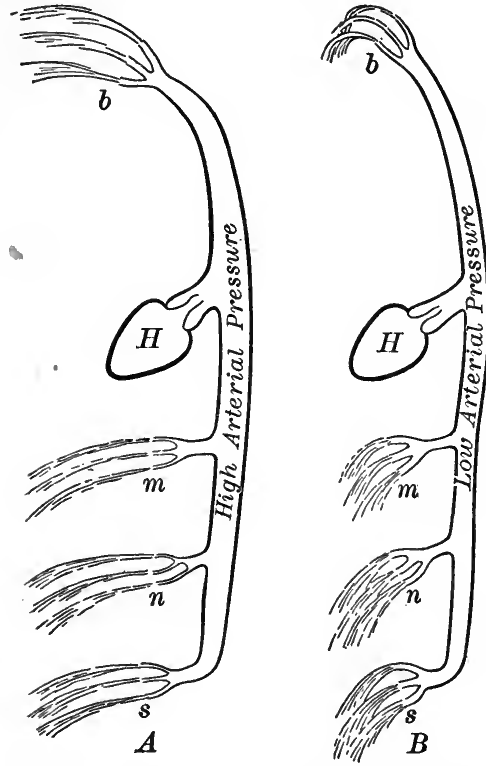

FIG. 70. Showing the relation between general arterial tone and the supply of blood to the brain

In $A$ the arterioles of the organs $m, n, s$ are constricted, raising general arterial pressure which forces a large amount of blood through the brain $b$. In $B$ the arterioles of $m, n$, and $s$ are dilated, general arterial pressure is low, and less blood is forced through the brain. $H$, heart

volving the personal interest or mental concentration of the subject of the experiment.

It is thought by some that other vascular areas, that of the abdominal cavity, for example, behave in this respect in the same way as the skin; but on this point

skull, and the wound has been covered over by connective tissue and skin, the scar sinks in during sleep, _ indicating less blood in the brain, - and returns to the level of the general surface of the head when the subject awakens.

Upon this point of diminished blood supply to the brain during sleep almost all physiologists are agreed; there is also general agreement that the arm and the leg increase in volume when we go to sleep, and this is thought to be due to a dilation of the arteries of the skin. It is very significant, on the other hand, that the arm shrinks in volume when the brain is active in mental work, and especially in mental work in- 
the evidence is not conclusive. It is, indeed, not improbable that these other vascular areas play some part in the regulation of the flow to the brain, but it is not likely that they stand in the same intimate relation to it as does the skin.

The fact is clear, however, that a close relation exists between cutaneous circulation and the maintenance of proper vascular conditions in the brain. Mental work, for example, is more difficult for most people in very warm weather because at that time the cutaneous arterioles are widely dilated; and, on the other hand, it is easy to understand why the constriction of the vessels of the skin by cold makes it difficult to go to sleep without sufficient bedclothing.

19. The Circulation during the Digestion of a Meal. After eating a meal, more blood is needed in the secreting digestive glands (especially the stomach and pancreas), and also in the intestinal organs of absorption, the villi. And this need is greatest during the first hour or two, when there is the largest amount of food to be worked upon. We find, accordingly, that the arteries of these organs then dilate to such an extent that the mucous membrane of the stomach and intestine, which is pale pink while those organs are at rest, now becomes very red on account of the large amount of blood flowing through them.

There is probably some compensation for this in other organs, but it is an imperfect compensation. The drowsiness which is apt to come on after a hearty meal is probably an indication that these compensations are not complete, and that owing to the fall of arterial pressure the brain is not receiving its normal blood supply.

20. Some Practical Applications. - We may pause here to consider some important practical applications of these facts. While the most active secretion is in progress 
nothing should be done which will take blood away in large quantities from the stomach. Muscular exercise, for example, then, as always, dilates the arterioles of the muscles and skin and constricts those of the digestive organs; this is obviously an unfavorable vascular condition for the act of secretion; if the meal be a light one, so that comparatively little of the digestive juices are required, no harm may be done by taking exercise after a meal; but where the meal is heavier it is almost always unsafe, especially in warm weather. Similar considerations, which are likewise in full accord with experience, indicate that it is unwise to eat as large meals in very warm weather as in cooler weather; the larger the meal the greater the amount of gastric juice required to start its digestion; but in warm weather the arteries of the stomach and intestine tend to be constricted (see p. 150), so that it is difficult to secure an adequate blood-flow through these organs, and their efficiency is to this extent impaired.

It is sometimes stated that mental work immediately after meals causes indigestion by taking blood away from the digestive organs and sending it to the brain. It is very doubtful, however, whether the increased blood-flow to the brain is secured largely at the expense of that to the digestive organs. While instances, might be cited of indigestion among people who do mental work upon a "full stomach," it must be remembered that these are usually people who fail to take proper exercise or sufficient sleep and rest; the indigestion from which they too frequently suffer is more often attributable to these causes than to the fact that the digestive organs are deprived of their proper blood supply.

21. The Mechanism of the Regulation of the Flow of Blood. - Having thus considered exactly what takes place in the circulation during some of the more important events of daily life, we may next inquire briefly into the 
physiological mechanism by which these adjustments are secured. Its most important features are the regulation of the inflow from the heart into the arterial reservoir and the regulation of the outflow through the arterioles and capillaries of the organs. These two must be adjusted to each other in order that the reservoir may remain full, and thus the driving force for the flow through the organs be maintained. We shall go into the details of this very beautiful but complicated mechanism only far enough to enable the student to appreciate certain principles of fundamental importance in the practical conduct of life.

22. The Regulation of the Pumping Action of the Heart. - The amount of blood which the heart pumps varies considerably from time to time. At times it may be as low as three quarts a minute, and at other times as high as twelve quarts, the quantity being largely determined by the drain made at the time upon the arterial reservoir. It will be seen at once that this involves a wide range of adjustment.

The beat of the heart is primarily due to events which take place within the heart itself. We have seen that this beat is a muscular contraction. But the cardiac muscle differs from the skeletal muscle in that it does not require an impulse from the central nervous system to throw it into activity. When the heart is cut off from connection with the rest of the body it continues to beat for a time, and if supplied with warm blood it may be kept beating for hours. We express this by saying that the heart beat is automatic, by which we mean that the heart contains within itself a complete mechanism for doing its own work.

23. The Augmentor and the Inhibitory Nerves of the Heart. - Nevertheless, the heart receives from the central nervous system two pairs of nerves, which are able to influence the rate and the force of the automatic beats. One 
pair of these nerves carries from the spinal cord to the heart impulses which stimulate that organ to beat more rapidly or more forcibly, or both. Hence these are known as the augmentor or accelerator nerves.

The fibers of the other pair of nerves produce exactly the opposite effect. Running from the lower part of the brain, they carry to the heart impulses which slow the beat or lessen its force, or they may produce both effects at the same time. They act, as it were, like a brake on a wheel, checking the activity of the automatic beat. These fibers are known as inhibitory fibers, and their action is a case of inhibition.

24. Inhibition. - In the examples of nervous action which we have thus far studied, the nervous impulse has uniformly thrown some cell into activity. The stimulation of muscle fibers to contract, of gland cells to secrete, and of nerve cells in the execution of reflexes will be readily recalled. To this same class of nervous actions must now be added that of the augmentor nerves of the heart, for they excite the heart to greater activity.

In the inhibitory nerves, on the other hand, the nervous impulse produces exactly the opposite result. Instead of setting organs to work or stimulating them to more vigorous action, they diminish activity, and in extreme cases check or stop it altogether. In our subsequent studies we shall meet with many examples of this effect; but we may say at once that inhibition is as characteristic and as important a feature of the nervous system as is excitation (see p. 285).

25. The Regulation of the Outflow from the Arterial Reservoir. Arterial Tone. - Wound around the walls of the arterial tubes, especially the smaller arteries (arterioles) which deliver blood from the arterial reservoir to the organs, are peculiar muscle fibers. Their contraction diminishes the size and bore of the tube, and when they 
relax, the tube and its lumen become wider. As a usual thing these smaller arteries are kept somewhere midway between extreme constriction and extreme dilation by the sustained moderate contraction of their muscle fibers. On a day of moderate temperature, for example, the arterioles of the skin are moderately narrowed by this action of their muscle fibers. During colder weather these fibers contract more than usual, and so lessen the size of the tube, while during warm weather they relax somewhat and widen it; but ordinarily they are never contracted to their utmost, nor are they often completely relaxed.

This condition of sustained activity of the arterial muscles is known as arterial tone, and in general any sustained activity of a living cell is spoken of as tonic activity, or tone. Since, as we have seen, the total quantity of blood in the body is not enough to fill completely and distend all the blood vessels when they are widened to their utmost, it follows that the maintenance of arterial tone is essential to that overfilling of the great arteries which supplies the driving force for the flow of blood through the organs. If every arteriole were to lose its tone, blood would flow out of the reservoir more rapidly than the heart could possibly pump it in; we should have somewhat the same condition of affairs as if, in our artificial model (p.142), the small nozzle which affords resistance to the outflow were removed. Arterial pressure would fall and, the driving force being thus removed, the blood would remain at rest in the capillaries and veins of the organs; the circulation would cease because blood would not return to the heart to be pumped. The maintenance of arterial tone is consequently no less essential to the circulation than is the beat of the heart itself.

26. Vasoconstrictor Nerves. - The muscle fibers of the arteries receive nerves which stimulate them to contract, for if these nerves are cut, the arteries lose their tone 
(dilate). We conclude, therefore, that the ordinary maintenance of arterial tone is, in part at least, a function of the nervous system. The muscle fibers of the arteries, in other words, remain in tonic activity because the neurones which supply them with nerve fibers are in tonic activity; and we can understand how general arterial tone may be increased or decreased by the condition of the central nervous system, by reflexes, by the nervous "shock" of surgical operations, etc.

Neurones which maintain the proper amount of arterial tone are known as vasoconstrictor neurones. They obviously do for the muscles of the arteries what the motor nerves do for the skeletal muscles, and the augmentors do for the heart.

27. Vasodilator Nerves. - Arteries, however, receive a second set of nerves, which have exactly the opposite function, i.e. to make their muscle fibers relax, and so lead to a widening or dilation of the artery. These nerves do for the tonic contraction of the arteries what the inhibitory nerves of the heart do for the heart beat; they diminish or abolish an existing activity and thus give us our second example of inhibitory nerves. They are known as the vasodilators.

The vasodilators are not regularly in tonic activity like the vasoconstrictors. They are called into action, reflexly or otherwise, when it is necessary that an organ receive more blood than usual; at other times the vasoconstrictors are free to exert their tonic stimulation and so regulate the flow of blood to the organ.

28. Importance of the Nervous System to the Vascular Adjustments of Daily Life. - It is impossible within the limits of the present work to enter further into the mode of action of these arterial nerves, or to discuss other minor influences which regulate the circulation of blood. Our main purpose is to show the student that the proper 
coördinated working of the nervous system is as important in adapting the work of the heart and blood vessels to the hourly needs of daily life as it is in producing purposeful movements of the skeletal muscles. Every change of occupation and activity, every change of surrounding conditions of temperature, moisture, wind, etc., necessitates some special adjustment of the vascular system; and this adjustment is dependent upon the same sort of coördinating nervous action which we have already compared with the operations of a large army. In spite of the fact that we are for the most part unconscious of it, it is none the less a part of our daily life; and the fatigue induced within the mechanism of vasoconstrictor and vasodilator neurones by their continued activity probably contributes a large share to that general bodily fatigue which leads us to seek recuperation in rest and sleep.

The apparatus, the operation, and the regulation of the flow of blood and lymph afford an excellent illustration of the fact that the human body, at least in this particular, is a complex machine. But while we of to-day look upon it with somewhat less of awe than did our ancestors, and while there is for us less of mystery and more of mechanism in it, we gain, on the other hand, a wholly new revelation of its intricacy, and a fresh sense of its marvelous delicacy, beauty, and perfection of adjustment. The mere fact that every one of us carries in his bosom a powerful double force-pump of remarkable design, original construction, and extraordinary power, capable in many instances of successful and unremitting service for more than three quarters of a century, should be, in itself alone, enough to excite admiration and respect for the entire mechanism of which it is only one part, and to awaken within us a desire to use that mechanism "as not abusing it." 


\section{CHAPTER $\mathrm{X}$}

\section{RESPIRATION}

1. Breathing is not the Fundamental Act of Respiration. - We have found in studying the chemical changes which underlie cellular activity (Chapter IV) that muscle fibers and gland cells and, we may now add, nerve cells, take in oxygen and give out carbon dioxide. This cell breathing is the essential act of respiration, for respiration is only another name for the oxidative processes of the living body. Respiration of this kind (and of this kind only) is universal among living things. The one-celled animal, for example, takes its oxygen directly from the free oxygen of the water in which it lives, and discharges its carbon dioxide into the same surrounding medium. Every one of the thousands of cells of which the human body is composed repeats this same process, taking its oxygen from, and discharging its carbon dioxide into its surrounding medium, - in this case the lymph. The breathing movements, which renew the air in the lungs, and the circulation of blood, which affords the channel of communication between the lungs and the tissues, are merely accessory mechanisms rendered necessary by the distance of the cells and the lymph from the surface of the body. Their principal function is to keep the lymph supplied with oxygen and to remove from it the carbon dioxide. In other words, breathing, though ministering to respiration, is not respiration itself.

2. The Quantity of Oxygen and of Carbon Dioxide in the Lymph Surrounding the Cells of the Body. - The cell is the true seat of oxidation. Within its imperfectly understood 
mechanism are found the conditions which lead to the union, direct or indirect, of oxygen with the proteids, the carbohydrates, and the fats of the food.

The cell draws oxygen from the surrounding lymph very much as a burning match draws oxygen from the surrounding air. Consequently the amount of oxygen dissolved in the lymph is generally comparatively small, and would be removed altogether were it not constantly renewed from the blood.

For similar reasons the lymph must be relatively rich in carbon dioxide, since it is this fluid which directly receives the gas (in solution) from its source of manufacture, the working cell. ${ }^{1}$

3. The Quantity of Oxygen and of Carbon Dioxide in the Plasma of Arterial Blood. - It is through the lungs that the body as a whole receives its oxygen and discharges its excess of carbon dioxide. Consequently arterial blood contains more oxygen and less carbon dioxide than venous blood. The actual figures are as follows:

100 cc. of arterial blood contain $100 \mathrm{cc}$. of venous blood contain
Oxygen

$20 \mathrm{cc}$.

Carbon Dioxide

Nitrogen
38 cc. 1-2 cc. $45-50 \mathrm{cc}$. 1-2 cc.

These figures apply to the whole blood, i.e. to plasma and corpuscles; but what is true of the whole blood is true in a general way also of the circulating plasma, which consequently enters the capillaries ${ }^{2}$ relatively rich in oxygen and poor in carbon dioxide, thus presenting exactly the

1 The gases oxygen and carbon dioxide are, of course, dissolved in the liquid lymph and blood plasma. A liquid exposed to a gas absorbs or dissolves the gas. Thus, $100 \mathrm{cc}$. of water when exposed to atmospheric air at $0^{\circ} \mathrm{C}$. dissolves $4 \mathrm{cc}$. of oxygen and $2 \mathrm{cc}$. of nitrogen.

2 The total time consumed by the blood in passing from the capillaries of the lungs through the heart to those of the rest of the body seldom exceeds five or six seconds. Hence the amount of the gases in the blood entering the capillaries, for example, of a muscle is practically the same as in the blood leaving the lungs. 
reverse composition, in respect to these gases, of that found in the lymph surrounding the living cells.

4. The Passage of 0xygen and of Carbon Dioxide between the Lymph and the Blood Plasma. - In the capillary regions of all parts of the body except the lungs we have two fluids, the lymph and the blood plasma, containing

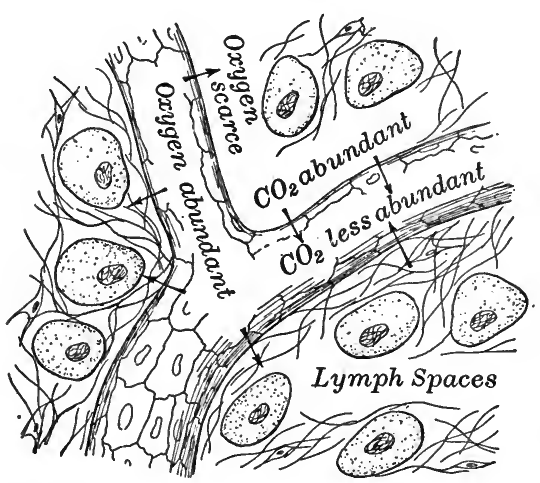

Fig. 71. The passage of oxygen and carbon dioxide between the blood and the lymph in the tissues

very different amounts of oxygen and carbon dioxide, and separated from each other by the exceedingly thin membrane of the capillary wall. Under such conditions both gases will tend to equalize, and each gas will pass through the membrane from that liquid in which it is more abundant to that in which it is less abundant; that is to say, the oxygen will pass from the blood plasma in which it abounds to the lymph in which it is scarce; and the carbon dioxide in the other direction, from the lymph to the blood plasma (see Fig. 71). Hence the blood enters the veins richer in carbon dioxide and poorer in oxygen than it left the arteries.

5. The Red Corpuscle as a Carrier of 0xygen. - The blood plasma under the conditions of temperature and pressure to which it is exposed can hold in mere solution only a small amount of oxygen, far too little to meet satisfactorily the demands of the resting tissues, and utterly inadequate for the much greater needs of the working tissues. This difficulty is met and the oxygen-carrying capacity of the blood vastly increased by the peculiar properties of the 
coloring matter, or pigment, of the red corpuscles. This substance, known as hemoglobin, readily forms with oxygen a compound (oxyhemoglobin) whenever the amount of oxygen is high in the medium surrounding it; if, however, much oxygen is removed from its surrounding medium, the oxyhemoglobin breaks up or dissociates into hemoglobin and free oxygen. Applying this to the conditions in the capillaries, we find that $100 \mathrm{cc}$. of arterial blood contain less than $1 \mathrm{cc}$. of free oxygen in the plasma, but about $19 \mathrm{cc}$. of oxygen combined in the oxyhemoglobin of the red corpuscles. When the blood enters the capillaries of living tissues, oxygen passes, as we have seen, from the plasma into the lymph, so that the oxygen content of the plasma is reduced. When this reduction goes below a point which is quickly reached, dissociation of the oxyhemoglobin occurs, and the oxygen thus set free in the plasma is drawn away by the lymph, from which it is in turn drawn by the cell, the real seat of oxidation.

It is important that the student should clearly understand that the cell is the master of the respiratory processes, not their servant, - "We may lead a horse to water or fetch water to a horse, but we cannot make him drink," - and that the cause of this movement of oxygen step by step from the corpuscle to the plasma, thence to the lymph, and thence to the cell, is the utilization of oxygen by the cell for its life work, not the presence of oxygen in the blood; and the chemical activities within the cell, not the amount of oxygen brought by the blood, primarily determine how much oxygen shall pass to it from the blood. This fundamental truth will be emphasized as we proceed.

6. Gaseous Exchanges of the Blood in the Tissues. - How much oxygen is taken from the blood by the tissues and how much carbon dioxide is received? This question might be answered for the body as a whole by comparing the 
amounts of these gases in the blood of the large arteries and in that of the large veins.

Oxygen Carbon Dioxide Nitrogen $\begin{array}{lrrr}100 \mathrm{cc} . \text { of arterial blood contain } & 20 \mathrm{cc} . & 38 \mathrm{cc} . & 1-2 \mathrm{cc} . \\ 100 \mathrm{cc} \text {. of venous blood contain } & 8-12 \mathrm{cc} . & 45-50 \mathrm{cc} . & 1-2 \mathrm{cc} .\end{array}$

It appears from these figures that there is always a very considerable amount of carbon dioxide in arterial blood, and a very considerable amount of oxygen in venous blood; in other words, that the tissues do not remove all the oxygen nor do the lungs remove all the carbon dioxide from the circulating blood. What the processes of respiration accomplish is to keep the gaseous content of the arterial blood nearly constant at the figures given above; and except in the case of suffocation (asphyxia), the gaseous content of arterial blood does not vary widely from that given. It was formerly assumed that in muscular exercise the increased consumption of oxygen and production of carbon dioxide by the working muscles made the arterial blood more venous, i. e. poorer in oxygen, or richer in carbon dioxide, or both; but it is now known that the arterial blood during exercise contains about the same amounts of oxygen and carbon dioxide as during rest.

Not only is this true, but it has also been proved that the amount of oxygen given above is almost all that can be taken up by the blood, when it flows through the lungs under the atmospheric pressures to which we are ordinarily subjected. An elementary text-book is not the place for the details of this subject; but even the beginner should understand that neither by deeper nor by more rapid breathing, nor by any change in the character of breathing, is it possible to increase appreciably the amount of oxygen absorbed by the same volume of blood flowing through the lungs. The assertion, too frequently heard, that some special form of breathing movements leads to more efficient 
oxidation of the body wastes betrays lamentable ignorance of this fundamental fact of physiology. This, however, is not denying that one type of breathing movement may still, for other reasons, be preferable to another, nor affirming that deepened breathing may not sometimes be desirable. Breathing movements accomplish other things than oxygenation of the blood, and we may now proceed to study their physiology.

7. Structure of the Lungs. - In Chapter II the anatomical relations of the air passages (trachea, bronchi, etc.) and lungs have been described. The student at this point should consult especially Fig. 5 (p. 13) in order to obtain a clear idea of the structure of the lungs. The bronchi which enter the lungs branch, much as the ducts of a gland, and their ultimate branches end in the alveoli, which, like those of a gland, consist of a single layer of cells, but in this case of very thin, flattened cells. Fig. 72 shows two of these alveoli dissected, and Fig. 73 a section taken lengthwise through the

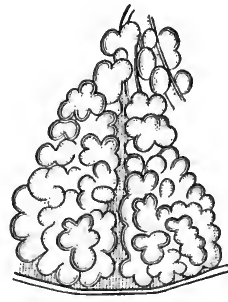

Fig. 72. Two adjacent alveoli of the lung

Showing the air cells same. Connective tissue binds together the alveoli and bronchial tubes, thus forming the lobes of the lungs. In this connective tissue - and hence between the alveoli are the larger blood vessels, branches of the pulmonary artery and pulmonary veins. The arterioles supply an exceedingly close network of capillaries (Fig. 139), which are in direct contact with the lining cells of the alveolus, so that the blood in these capillaries is separated from the air in the alveolus only by the thin capillary wall and the equally thin layer of flattened alveolar cells. Under these circumstances the exchange of oxygen and carbon dioxide takes place readily between the air in the lungs and the blood in the capillaries. Finally the absorbing surface of 
the alveolar wall is greatly. increased by being arranged in the form of pits or air cells, ${ }^{1}$ as shown in Figs. 5, 72, 73, and 139.

8. Purpose of Breathing Movements. - As the blood is constantly giving up carbon dioxide to, and taking oxygen

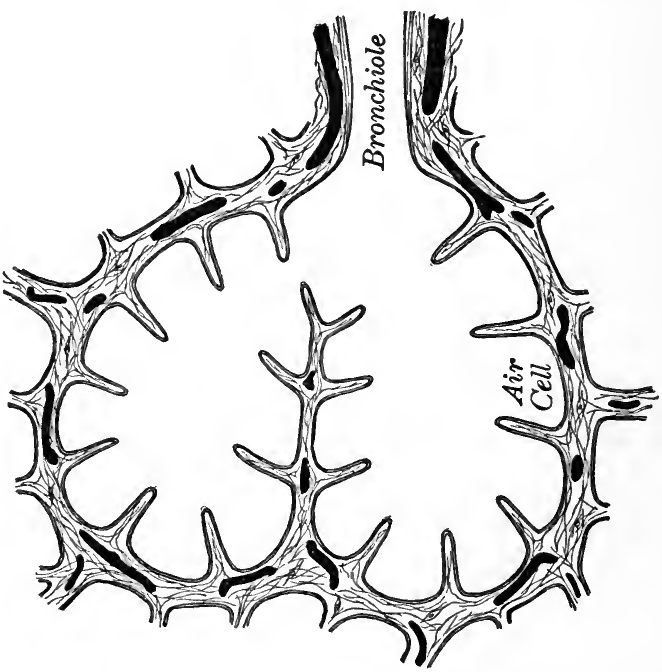

Fig. 73. Diagram of a longitudinal section of two alveoli with their common bronchiole and showing, in black, the larger blood vessels in the connective tissue

The capillary network belonging to these vessels is shown in Fig. 139

from the air of the lungs, this air would soon cease to be of use in purifying the blood, were it not for the breathing movements, whose function is to replace the vitiated air within the lung with pure air from without. Breathing is, accordingly, an act of ventilation of the lungs, and it is the stoppage of this ventilation which produces suffocation or asphyxia.

1 The word "cell" is here used to represent a hollow space, and not with its usual histological meaning. 
9. Mechanics of the Breathing Movements. - A knowledge of the mechanism of the breathing movements is of much practical importance, especially in hygiene, and may be understood without great difficulty by the study of the model shown in Fig. 74. The trachea and the bronchi are represented by the glass tube, and the lungs by an elastic bag, $L$, at the end of the tube. The lungs lie in the large air-tight thorax which incloses the pleural or thoracic cavity (p. 10). This thoracic wall is represented in the model by a glass bell jar closed beneath by a sheet of thick rubber, $D$. The cavity of the bell jar represents the pleural cavity, and the rubber represents the diaphragm (see Fig. 134). The condition of the lung in the pleural cavity may be still further imitated in the model by partially exhausting the air in the bell jar. The distensible and elastic
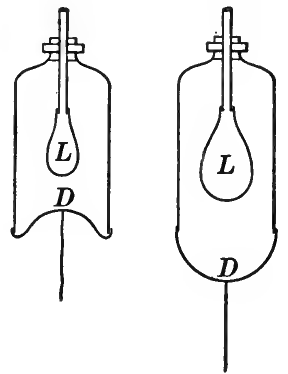

Fig. 74. Model of the action of the thoracic walls and lungs in respiration. See Section 9

rubber bags now inflate, because the atmospheric pressure within them is greater than the pleural pressure without. We shall not stop to explain why pleural pressure is less than that of the atmosphere; but knowing the fact, it is easy to understand why the lungs should be distended, so as virtually to fill those portions of the thoracic cavity not occupied by the heart, great blood vessels, and other organs. ${ }^{1}$

If now the size of the pleural cavity of our model be enlarged by pulling downwards the sheet of rubber at the bottom, the pressure will be still further lowered in the cavity and the lungs will expand. At the same time air is sucked in through the glass trachea and mixes with

1 The student is again warned against supposing that the pleural cavity is a large space filled with air; in this respect the model is misleading, since the lungs and other organs completely fill the thoracic cavity. 
that in the model lungs. When the pull is released, the pleural pressure rises, the lungs grow smaller, and air is forced out. In this way the mechanism of the ventilation of the lungs may be imitated in essential particulars.

In life the pleural cavity is enlarged during inspiration by the contraction of the diaphragm and the elevation of the ribs. Both of these are movements effected by the action of skeletal muscles. The understanding of the elevation of the ribs need give no difficulty; muscles, some of which are shown in Fig. 12, pull upwards on the ribs; and the attachment of the ribs to the vertebral column and the breastbone (sternum) is such that when they are raised the diameter of the thorax is increased dorso-ventrally and from side to side. The diaphragm, on the other hand, is a kind of circular muscle with a central fibrous or tendinous portion from which the bundles of muscle fibers radiate outwards to its edges. Any shortening of these fibers evidently diminishes the diameter of the diaphragm; and because of its form (that of a dome directed upwards into the thoracic cavity), contraction of this muscle must increase the size of the lower thorax. ${ }^{1}$

There are three typical modes of breathing: (1) The predominantly costal or "rib" breathing. Here the diaphragm is but little used. It is the type characteristic of those who impede movements of the lower ribs and abdomen with constricting clothing, such as tight corsets. (2) The predominantly abdominal. Here the ribs are little used while

1 The action of the diaphragm is often described as increasing the antero-posterior (head to foot) dimension of the thorax; but this can happen only when the diaphragm is free to descend, and it can descend only when, by displacing downwards the contents of the abdominal cavity, it causes the well-known respiratory movements of the abdominal walls. These "abdominal movements" may, however, be prevented by the simultaneous contraction of the abdominal muscles. In this case the diaphragm cannot descend, and its contraction can only raise the lower ribs to which it is attached. The mechanism in these two methods of using the diaphragm is clear from Fig. 75. 
the diaphragm does most of the work, the abdominal muscles being relaxed so that the belly-wall has its maximum of movement. This type of breathing involves great relaxation of tone of the abdominal muscles, which is a serious disadvantage. (3) The lateral costal. Here the abdominal muscles act at the-same time as the ribs and diaphragm.
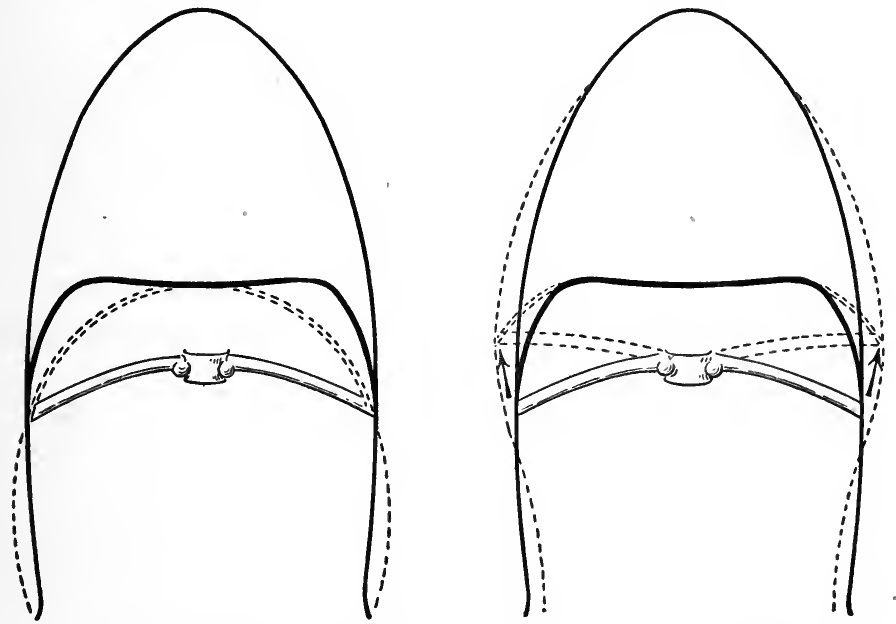

Fig. 75. Action of the diaphragm in "abdominal" and in "lateral costal" breathing

Solid lines represent position of body wall, diaphragm, and ribs during expiration; dotted lines, the same during inspiration. The left-hand figure represents abdominal breathing, the diaphragm becoming more convex, displacing downward the abdominal viscera, and forcing outward the abdominal body wall. In the lateral costal type the diaphragm raises the lower ribs and the abdominal walls may actually move inward, owing to the contraction of their muscles

This form of breathing produces the highest pressures on the contents of the abdominal cavity, and maintains the tone of the abdominal walls, without diminishing the efficiency of the oxygenation of the blood. It also forces'the use of the upper ribs to a much greater extent than does the predominantly abdominal type of breathing (Fig. 75). 
It is seldom that one or another of these types is used in its entirety, and the advantages of one form over another are often greatly exaggerated. The following statements may, however, be taken as summing up the essential practical points.

1. The breathing movements should be such as to use all portions of the lungs. In the abdominal type there is little or no movement of the upper thorax. The result is that the apical or upper lobes of the lungs do not share in the enlargement and contraction of the lungs; they are poorly ventilated, their lymph current - which largely depends upon these movements - becomes sluggish, and because of these unfavorable physiological conditions there is greater liability to disease. More than sixty per cent (some observers claim as many as eighty per cent) of the beginnings of the lung ravages of pulmonary consumption are found in this portion of the lung; and this is believed to be due to the lack of movement which results from the failure to use the upper thorax.

2. Actual study of the breathing movements in people who have not worn constricting clothing indicates that the enlargement of the thorax in inspiration is effected by the approximately equal action of the diaphragm and of the muscles which elevate the ribs.

3. The abdominal muscles should to some extent contract with the diaphragm. This is especially important in those whose occupation is more or less sedentary, as it is the most convenient means of giving to these muscles the use which is essential to the maintenance of their strength, and the consequent prevention of that loss of tone which takes away from the organs of the abdominal cavity one of their chief supports. (Consult Part II, Chapter XVIII.)

4. There are good reasons for thinking that it is important to develop properly the muscles of the upper thorax and especially those which lie in the triangle between the 
root of the neck, the collar bone, and the shoulder blade. When these muscles are not developed, especially in thin people, the wall of the thorax in this region sinks inward during inspiration; under these circumstances this portion of the thorax is not enlarged during inspiration, the apical lobes no longer share in the expansions and contractions of the lungs, and imperfect ventilation of this part of the lung results.

10. Secondary Effects of the Breathing Movements. The student will now be better able to understand the part taken by the breathing movements in facilitating the return of blood and lymph to the heart. The enlargement of the thorax during inspiration sucks blood and lymph in toward the great veins by the same process that it sucks air into the lungs. ${ }^{1}$ Especially in the case of the lymph flow is this a most important factor. Moreover, in the lymphatics of the lungs, situated as they are entirely within the thorax, the movements of the lungs during respiration pump the lymph onwards and are of special importance in this respect. Much of the invigorating effect of muscular exercise, popularly ascribed to better oxygenation of the blood and tissues, is really attributable to the greatly improved lymph flow from all organs which results from the deepened respiration in muscular activity.

11. The Nervous System as a Factor in Respiration.The muscles of the diaphragm and those of the ribs, like the biceps and other muscles which act upon the skeleton, are stimulated to contraction by nervous impulses from the brain and spinal cord. Every movement of respiration is called forth and regulated, in accordance with the needs of the body at the time, by the coördinated action of a number of nerve cells. Those which are most intimately

1 The model of the thorax (p. 169) may be made to show this action in the veins and lymphatics by the addition of a second bag to represent the blood vessels. 
concerned with respiration are found in different parts of the central nervous system, from the lower portion of the brain to the end of the first half of the spinal cord, inclusive; and there is good reason for thinking that nerve cells in the lower portion of the brain send out the stimuli to those of the cord, and through them excite the muscles to contract. Their precise mode of stimulation, however, is too complex for description in an elementary work on physiology.

12. The Circulation as an Essential Part of the Mechanism of Respiration. - The consumption of oxygen and the production of carbon dioxide thus involve an interchange of these gases between the blood and the tissues (internal respiration) on the one hand, and between the blood and the air in the lungs (external respiration) on the other. But to carry out these gaseous exchanges a third factor is obviously necessary, namely, a means of communication between the two, so that the oxygen absorbed in the lungs may be carried to the tissues, and the carbon dioxide produced in the tissues be carried back to the lungs. This communication is provided, as has been shown in earlier chapters, by the circulation, which thus becomes an essential part of the respiratory mechanism.

We have already seen that under the most varying conditions $100 \mathrm{cc}$. of arterial blood always contain approximately $20 \mathrm{cc}$. of oxygen and $38 \mathrm{cc}$. of carbon dioxide; and that this is practically all the oxygen this amount of blood can hold. From this it follows that so long as the amount of blood pumped by the heart in a given time remains constant, no more oxygen will be carried to the tissues, even if we breathe more deeply. In other words, increased ventilation of the lungs without any accompanying increase in the rate and force of the heart beat will not supply more oxygen to the tissues. The beat of the heart is as important to proper tissue respiration as are the deepened breathing movements; and we find accordingly that these two 
events are closely coördinated. Greatly increased tissue respiration invariably carries along with it increased work on the part of the heart.

A large number of measurements of the respiratory exchanges ${ }^{1}$ under different conditions and activities of our life has shown that these are increased by the taking of food, by exposure to cold, by awaking from sleep, and, above all, by muscular activity. The last three really belong under the one head of muscular activity; for when we are exposed to cold we move about more actively, or if we do not, we "shiver"; and the same thing is true of awakening from sleep. The taking of food does not lead to any remarkable increase in gaseous exchange, except when one passes from a period of prolonged fasting to one of feeding; but this event is so abnormal that it may be neglected. Consequently we have left the one condition of muscular activity as the important means of increasing tissue respiration.

And this increase is at times very great. Even the muscular activity necessary to maintain the erect position in sitting and standing, as compared with the complete relaxation of sleep, doubles the gaseous exchange ; gentle exercise (a walk of three miles an hour) more than doubles that of rest; and vigorous, yet by no means excessive, exercise will increase it tenfold. These increases mean corresponding, though not absolutely proportionate, demands on the heart, and emphasize the importance of keeping that organ in an efficient working condition. Breathlessness, for example, usually indicates, in part at least, that the heart fails to respond properly to the demands made uponit, these demands being greater than it can meet without undue fatigue; it is a warning that we are pushing the heart too hard, a warning which we will do well to heed. Generally it is also a warning that we

1 i.e. oxygen absorbed and carbon dioxide discharged in a given time. 
are not getting sufficient muscular activity; the heart fails to meet the emergency of some unusual exertion because all along it has not been kept in proper training; so that while we should, as stated, heed the warning not to push the heart so hard for the time being, we should also act upon the equally important warning that it needs practice or training, - a training which can be given only by reasonable, regular, muscular activity.

The training of muscular activity is therefore not only a training of the muscles but also of the heart. But this is not all. The work of the circulatory and respiratory mechanisms must be adjusted or coördinated, the one to the other. When, for example, the deepened breathing movements accompanying muscular activity rush the blood back more rapidly to the heart (p.145), it becomes necessary for the heart to adjust the character of its beat to the new conditions; and this adjustment is the work of the nervous system. Time is, however, required to make the adjustment, so that it is wise to "warm up" gradually to more vigorous work. We can also understand how by physical training this process of adjustment comes to be shortened; for we have not only trained the heart by giving it more work to do, but we have also trained those portions of the nervous system which regulate its beat. 


\section{CHAPTER XI}

\section{EXCRETION}

We have seen that through the chemical activity of the cells of the body waste products are produced from the food, and this largely by a process of oxidation. We have also learned that the presence of undue quantities of these wastes interferes with many normal activities of the cells. In the organs of the body, as in the houses of a city, wastes tend to accumulate, and both for health and for decency, they must be removed. It is this removal which is known by the general name of excretion.

1. The Waste Products of the Human Mechanism.-We may first consider the nature of the more important wastes, and then the means by which they are eliminated from the body.

The oxidation of fats takes place according to the following equation:

$$
\mathrm{C}_{51} \mathrm{H}_{98} \mathrm{O}_{6}+71 \mathrm{O}_{2}=51 \mathrm{CO}_{2}+46 \mathrm{H}_{2} \mathrm{O} \text {. }
$$

In other words, they are changed to carbon dioxide and water. The same thing is true of the carbohydrates:

$$
\mathrm{C}_{6} \mathrm{H}_{12} \mathrm{O}_{6}+6 \mathrm{O}_{2}=6 \mathrm{H}_{2} \mathrm{O}+6 \mathrm{CO}_{2} \text {. }
$$

In the case of the proteids and such allied compounds as gelatin the chemical changes are more complex and the products of the change more numerous. These substances, unlike the fats and carbohydrates, contain nitrogen in addition to carbon and hydrogen, and their waste products comprise both nitrogenous and non-nitrogenous compounds. Of the former, urea $\left(\mathrm{CON}_{2} \mathrm{H}_{4}\right)$ occurs in largest 
quantity; indeed, it constitutes most of the nitrogenous excretion of the body; other nitrogenous substances are found in small quantities, among which may be mentioned uric acid $\left(\mathrm{C}_{5} \mathrm{H}_{4} \mathrm{~N}_{4} \mathrm{O}_{3}\right)$, the nearly related xanthin bases, hippuric acid, and creatinin. The non-nitrogenous wastes of proteids are chiefly carbon dioxide and water; others that we need not mention occur in small quantities.

The main excretions of the body are, therefore, carbon dioxide, water, and urea, together with small amounts of other nitrogenous and non-nitrogenous compounds. In addition to these the body is constantly excreting inorganic salts, sodium chloride being the largest in amount.

2. The Organs of Excretion. - Excretion, like absorption, must obviously take place either through the outer surface of the body, - the skin, - or else in organs like the lungs, the intestine, or the kidneys, which directly communicate with this outer surface; and we actually find that waste products leave the blood through all of these channels. An organ may, however, be essential to the proper removal of a given waste product, or it may remove the waste product only incidentally in performing its essential functions. Thus the skin removes a small amount of carbon dioxide from the body merely because a certain amount of this gas diffuses from the blood as it flows through the skin. It is not necessary to the health of the body that the skin should excrete this carbon dioxide, for the lungs are quite capable of doing the work, and would do so if for any reason such excretion through the skin were prevented. Without the lungs, on the other hand, the carbon dioxide would rapidly accumulate in the blood and cause death. The lungs are essential to the removal of this waste; the skin is not. Similarly, the perspiration contains small amounts of urea and other wastes which are removed in large quantities by the kidneys. It is not necessary that the skin should, remove any of these, for 
the healthy kidney can and does, when necessary, remove them. They appear in the perspiration because they are in the blood from which the perspiration is formed, and because the cells of the sweat glands allow them to pass through, just as the skin allows the passage of carbon dioxide.

These considerations are of practical importance in the hygiene of the skin. It is not necessary to induce perspiration merely to remove waste products from the body. If the human skin, like that of the cat, and the dog, contained no sweat glands, the waste products would be as thoroughly removed as they are now ; and in cold weather, when no perspiration is being secreted, the excretion of waste is as complete as when in warm weather perspiration is abundantly secreted.

The most important organs of excretion are the lungs and the kidneys; next to these should be placed the intestine. Each of these is essential to the elimination of one or more of the waste products from the blood. The skin, on the other hand, as just shown, is only incidentally an organ of excretion. The chief wastes leaving the body by each of these channels are given in the following table, the incidental excretions being in italics.

Lungs : carbon dioxide, water.

Kidneys: urea, uric acid, and other nitrogenous compounds, salts, water.

Intestine : bile pigments, nitrogenous compounds, etc.

Skin : urea, etc., salts, water.

The structure and action of the lungs and intestine have already been described; so that we have left for study the kidneys and the skin.

3. Structure of the Kidneys. - Each kidney is a beanshaped gland whose duct, the ureter, runs to the urinary bladder. As the ureter enters the kidney at the center of the depression in that organ it expands to form a large 
basin, known as the pelvis of the ureter. Into this basin open the hundreds of glandular tubules of which the bulk of the kidney is composed. Each tubule, like the alveolus

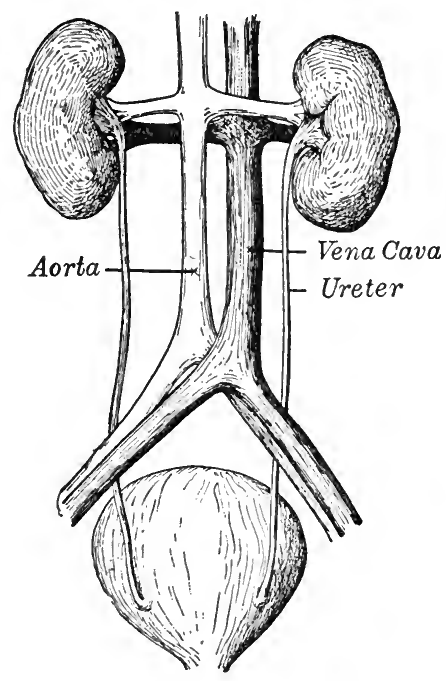

Fig. 76. Dorsal aspect of the kidneys, ureter, urinary bladder, and abdominal aorta and vena cava

and ducts of the gland described in Chapter III, consists of a single layer of cells, which separate the blood and lymph from the lumen of the tubule; and the formation of urine by the kidney is essentially an act of secretion.

4. The Secretion of Urine. - The urine is being constantly secreted from the blood, at one time more rapidly than at another, but under normal conditions never ceasing altogether. Passing down the tubules, it collects at the upper portion of the ureter, and successive peristaltic waves carry it from this point to the urinary bladder, an organ with muscular walls, in which the urine accumulates and from which it is from time to time discharged. Secretion by the kidney, however, presents some peculiarities which make it unlike the secretion of the gastric juice or the saliva. We have seen that the secretion of these latter juices is called forth by nervous impulses; but in the kidney the main factor which determines the amount of urine secreted is the amount of blood flowing through that organ.

It has been shown that any increase of blood-flow through the kidney is accompanied by an increased secretion of urine, and vice versa. The same thing is seen in 
everyday experience. The three factors which increase the activity of the kidneys in healthy people are (1) exposure to cooler temperature, (2) drinking large quantities of water, and (3) the taking of proteid food. Exposure to cold, or, more accurately, the cooling of the skin, causes constriction of the cutaneous arterioles and a compensating dilation of those of internal organs. This means an increased blood-flow through the kidneys and an increase in the amount of urine secreted. The absorption of water or of proteid food also increases the activity of the kidneys by increasing the blood-flow through them.

These changes in the quantity of the urine secreted are, generally speaking, only changes in the

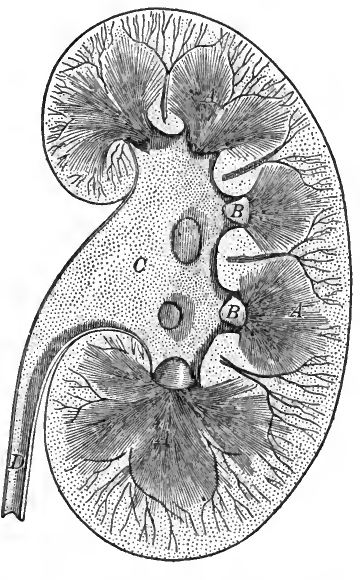
amount of water rather than in the amount of urea, uric acid, salts, and other dissolved constituents. Certain constituents of the urine, however, are not very soluFig. 77. Vertical section of the kidney. Diagrammatic

The tubules $(A)$ of the gland open, on the papillæ $(B, B)$, into the pelvis $(C)$ of the ureter $(D)$ ble, so that it is not well to have water, the only solvent of these substances in the urine, unduly diminished. A very scanty secretion of urine during the day is, in general, a distinct indication, especially in warm weather, that insufficient water is being taken. Many persons drink too little water rather than too much.

5. The Structure of the Skin. - The skin is an organ which performs several functions, the most important being (1) that of protecting the underlying structures from drying and mechanical injury; (2) that of assisting in maintaining the constant internal temperature of the body; 
and (3) that of receiving the external stimuli of pressure, heat, and cold. Incidentally, as we have seen, the skin is

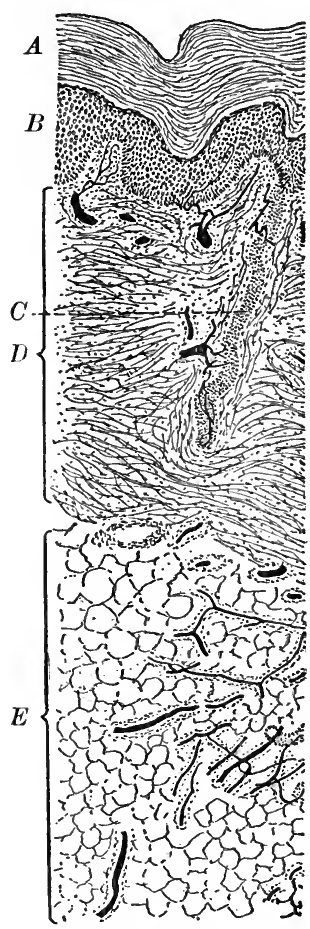

Fig. 78. Cross section of skin

$A$, horny layer of epidermis ; $B$, deeper layer of epidermis; $C$, duct of sweat gland ; $D$, the dermis; $E$, subcutaneous connective tissue (p. 7). The blood vessels are injected to show black. Compare Fig. 82 an organ of excretion. We may therefore describe its structure and excretory function in this connection, reserving the study of its other functions for Chapters XII and XIV.

The skin consists of an outer layer, the epidermis, and an inner layer, the dermis, cutis, or corium. The dermis consists of connective tissue richly supplied with blood vessels, lymphatics, and nerve fibers, together with sense organs of touch. The fibers of the dermal connective tissue are most dense near the epidermis ; in the deeper portions the network is loose and the lymph spaces larger, the connective tissue of the dermis passing insensibly. into that of the subcutaneous connective tissue.

The cells of the more open portions of the dermal network, and especially those of the subcutaneous tissue, store up more or less fat within their cytoplasm. The subcutaneous tissue, indeed, is one of the most important organs in the body for the storage of fat. Connective tissue in which large amounts of fat are stored is known as adipose tissue (see p. 230).

The outer surface of the dermis is not flat, but contains mound-like projections known as papillo, which project into the overlying epidermis. Some of these papillæ contain nerve endings 
of the sense of touch, while others contain capillaries, which are found also in other portions of the dermis. The dermis is the vascular organ of the skin, blood vessels being entirely absent from the epidermis (see Figs. 81, 82).

The epidermis consists of many layers of cells, the number of layers being very great, a hundred or more on the palms of the hands and the soles of the feet; in other places less exposed to pressure they may not exceed twenty. The cells which rest immediately upon the dermis are cylindrical or columnar in shape; those immediately above them are more or less spherical or polygonal, with minute spaces between them for the flow of lymph. Both the cylindrical and the spherical cells are alive, and it has been shown that they grow and divide. As we pass farther from the dermis with its blood supply and nearer the surface with its exposure to drying, the cells show signs of degeneration and are gradually transformed into dead, flattened, horny scales, which, packed together and known as the horny layer, form the outer portion of the epidermis. These scales are being constantly rubbed off and their loss made good by the growth and multiplication of the spherical cells beneath. Such a covering or lining is therefore well fitted for surfaces which are exposed to friction or drying. The mouth, the part of the pharynx used in swallowing, the œsophagus, and the rectum are lined with the same tissue. Afferent nerves are found in the lower layers of the epidermis.

The hairs, the sweat glands, and the nails are modified portions of the epidermis. Of these the hairs and the sweat glands are of sufficient importance for our purposes to merit some description.

6. Structure of a Hair and a Hair Follicle. - A hair grows from the bottom of a pit, the hair follicle, which extends downward into the dermis or even into the subcutaneous tissue. Microscopic examination shows that this follicle is 
lined with a continuation of the epidermis, just as a gland of the stomach or intestine is lined by an ingrowth of the cells of its surface. At the bottom of the follicle is a

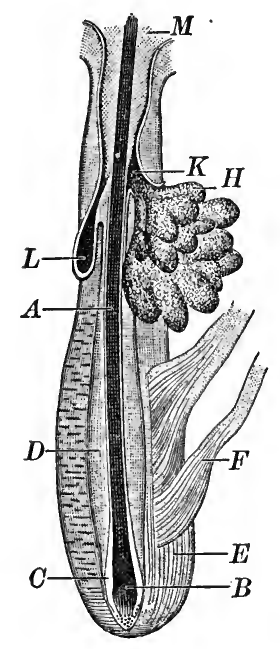

Fig. 79. Hair and hair follicle

$A$, root of hair ; $B$, bulb of the hair; $C$, internal root sheath; $D$, external root sheath; $E$, external membrane of follicle; $F$, muscular fibers attached to the follicle; II, compound sebaceous gland with its duct $K ; L$, simple sebaceous gland; $M$, opening of the hair follicle papilla, and the hair which grows out from this papilla to the free surface bears to the cylindrical and spherical cells of the papilla the same relation that the horny layer of the epidermis bears to the similar underlying cells. We accordingly find that the hair is composed of horny scales closely pressed together into the well-known threadlike structure. The scales of the outer layer, which form the covering for the hair, are, however, flattened and overlap somewhat like the shingles on the roof of a house.

Opening into the hair follicle, one or more sebaceous glands discharge an oily secretion which lubricates the hair and the horny layer of the epidermis, and so prevents drying and chapping.

7. The Sweat Glands are tubular prolongations of the epidermis through the dermis into the subcutaneous tissue. Here the tube becomes much coiled, forming the secreting recess, which is richly supplied with blood vessels and also receives nerves. It is a simple tubular gland formed as an ingrowth from the epidermis (see Figs. 81 and 82).

8. The Secretion of the Perspiration, like the secretion of the gastric juice, is under the control of the nervous system. When the nerves going to the sweat glands of a given area of skin are cut or otherwise injured, the secretion of 
perspiration ceases over that area; and the appearance of cold beads of perspiration as the result of fright shows how events taking place in the nervous system may excite these glands to activity apart from the presence of their normal stimuli, - the application of heat to the skin and the liberation of heat within the body by muscular and other activities. The distinction should be made between the so-called "sensible" and "insensible" perspiration, the latter name being given to the perspiration the water of which evaporates as fast as secreted; the former to that which does not evaporate so rapidly and hence remains for a time on the surface of the skin. When the water evaporates, the dissolved solids (salts, urea, and other compounds) remain behind on the skin.

9. Value of Profuse Perspiration in the Care of the Skin. - While the skin is not primarily an organ of excretion, the perspiration contains a certain amount of waste sub-

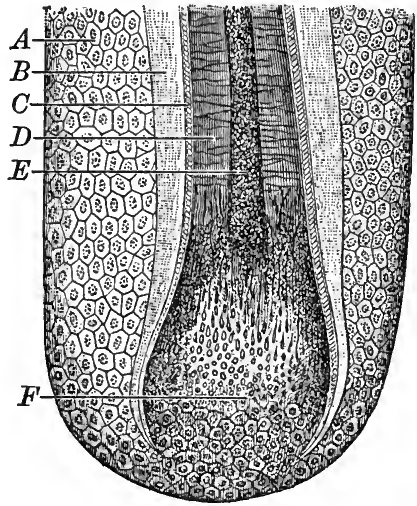

Fig. 80. Magnified section of the lower portion of a hair and hair follicle

$A$, membrane of the hair follicle, cells with nuclei and pigmentary granules; $B$, external lining of the root sheath; $C$, internal lining of the root sheath; $D$, cortical or fibrous portion of the hair shaft; $E$, medullary portion (pith) of shaft; $F$, hair bulb, showing its development from cells from $A$

stances and salts which are left by the evaporation of the water upon the surface, and, to some extent, in the mouths of the ducts of the sweat glands; this is especially the case when evaporation takes place about as rapidly as the perspiration is discharged. When the secretion of perspiration is more abundant, as during muscular work, or at very high temperatures, or, in general, where it does not evaporate 
as rapidly as discharged, the accumulation of solids in the ducts of the glands is washed out. For this reason a

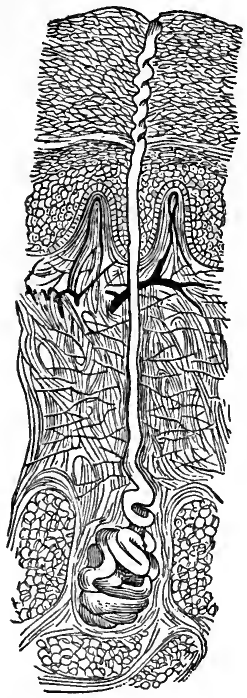

Fig. 81. Sweat gland slightly magnified

Note the coiled form of the tube in the subcutaneous tissue. Compare Fig. 82 vigorous perspiration followed by a bath is a useful hygienic measure in the care of the skin, although it is not necessary, as is sometimes supposed, in order to secure the efficient elimination of wastes from the blood.

10. The Skin as an Organ of Absorption. - While it is true that water as perspiration may readily find its way out through the skin, such escape is effected chiefly by the sweat glands which are under the strict control of the nervous system. Apart from this the skin is virtually water-tight; and, oiled as it is by the secretion of the sebaceous glands, it serves both to keep in the water, which forms so important a part of the tissues, and also to keep out water which might otherwise soak into the body, as, for example, during bathing. This waterproof characteristic also makes it next to impossible for us to absorb food materials by way of the skin. A "milk bath" may be at times useful in the care of the skin, because the fat or oil of the milk may supply any deficiency in the sebaceous secretion and so insure lubrication of the epidermis; but it cannot be regarded as a means of supplying food to the body. 


\section{CHAPTER XII}

\section{THERMAL PHENOMENA OF THE BODY}

\section{A. The Constant Temperature}

1. The Constant Temperature. - No characteristic of the human mechanism is more remarkable than its constant temperature. Whether we are awake or asleep, by night or by day, at work or at rest, at home or abroad, in summer or winter, in the tropics or the polar regions, in subterranean caves or on lofty mountain peaks, the temperature of healthy human beings is always nearly the same. So steady is this temperature that an increase or decrease of two or three degrees gives just cause for anxiety, and a change of seven or eight degrees is looked upon with alarm.

In many modern laboratories constant temperatures are obtained by the use of a thermostat, the apparatus of which is visible and easily understood; but no such special apparatus regulates the constant temperature of the human body, and we have rather to seek an explanation in the coördinated activities of organs already familiar, such as muscles, skin, blood vessels, and especially the all-controlling nervous system.

2. Temperature and Chemical Changes. - Every chemical reaction takes place more readily under some external physical conditions than under others, and among these conditions none is more important than temperature. We have already seen this fact illustrated in the case of the enzymes. At the freezing point, 'saliva exerts no action upon starch paste; as the temperature rises, the activity of the enzyme increases up to a certain point, and then 
diminishes more or less rapidly until a point is finally reached at which its peculiar chemical properties are destroyed. (See Experiment III, p. 103.)

3. Temperature and Vital Activities. - When we come to the activities of living cells, - activities which, it will be recalled, depend on chemical changes, - precisely the same thing holds true, and in so striking a manner as to create a widespread but erroneous impression that this dependence upon temperature is peculiarly characteristic of living things. The one-celled animal, Amœba, moves about more actively and digests more food at $20^{\circ} \mathrm{C}$. than at $10^{\circ} \mathrm{C}$.; bacteria grow more rapidly at the room temperature than near the freezing point; the pitch of the note made by a cricket rises with the temperature, indicating that the movements of the wing covers which produce the sound are being made more rapidly; and in the winter sleep of hibernating animals we have a beautiful example of the decline of vital activities with the fall of external temperature.

Nor are the living cells of the human body exceptions to this rule. The rate of the heart beat varies directly with the temperature of the blood, and the character of the breathing movements is influenced by the same cause; a cooled muscle contracts more slowly, a cooled gland secretes less abundantly. If the temperature of the body itself falls, every vital activity is depressed, and death itself may result from undue cooling.

4. The Constant Temperature of the Body. - This depression of nervous, muscular, and glandular activity results, however, only from a fall of the temperature of the body, not of that of the surrounding air or other medium. These two things are by no means the same, as may be readily seen from the fact that a clinical thermometer placed in the mouth indicates almost the same temperature of the body on warm and on cold days; even while we are shivering with 
cold, the thermometer gives about the same reading as when we are enjoying the warmest summer weather. The temperature of the body remains nearly constant, regardless of changes in the temperature of the air around it.

We have only to appeal to experience to see that this is not the way in which lifeless matter generally behaves; a stone, the earth, a piece of iron is warmer on a warm day and colder on a cold day; in general, lifeless things take the temperature of the medium in which they are placed, and this is one of the fundamental principles of physics. Nor do most living things act differently; the temperature of a plant or a tree, of an earthworm, a frog, a turtle, a snake, does not differ greatly from that of its surroundings. It is only birds and mammals which show this remarkable power of maintaining an approximately constant body temperature notwithstanding wide limits of change in that of the surrounding air. Such animals are known as warmblooded because they are usually warmer than surrounding objects; those animals which do not thus maintain a constant temperature, on the other hand, are known as coldblooded. ${ }^{1}$

It is clear that the power to maintain a constant body temperature is of the utmost importance in enabling an animal to counteract the varying conditions of climate. Were it not for this power, man would be a hibernating animal; with the coming of winter all his activities would gradually be slowed down, and long before our rivers and ponds had begun to freeze all business, industrial life, and intellectual life would come to a standstill; it would not be possible for the human race to people every zone

1 A cold-blooded animal exposed to a temperature of $99^{\circ} \mathrm{F}$. is as warm as a warm-blooded animal. Such animals are so called because they usually feel colder when handled than do warm-blooded animals ; but this is merely because the temperature of the air (which is also their temperature) is usually lower than the temperature of warm-blooded animals. 
of the earth, - the shores of Alaska or Iceland as well as the banks of the Ganges or the Amazon.

And yet here again we are dealing with a reaction which is not limited to man, or even to mammals and birds. There are some inanimate objects of human invention which show similar power. The automatic valves which are used in some of our house furnaces to regulate the draft are so arranged that as the external temperature goes down the draft is opened more widely and the temperature of the furnace remains the same or even rises. The best incubators for hatching eggs are provided with simple devices which maintain a temperature as constant as does the hen sitting on her nest; and we have already referred to the laboratory thermostat.

5. The Temperature of the Body not absolutely Constant. - The term "constant" as applied to the temperature of warm-blooded animals is not, however, to be taken too literally. No animal has an absolutely constant temperature. In the first place there are slight variations from time to time under the changing conditions of life. The temperature is higher by from one to four degrees during muscular exercise than during rest; it varies during the day, being highest in the afternoon and lowest in the small hours of the morning; it is often raised half a degree or more by taking food, and marked changes of surrounding temperature may cause a change of one degree or even more in that of the body. These changes between $97.5^{\circ}$ and $99.5^{\circ} \mathrm{F}$. are of everyday occurrence and are entirely normal; so that when we speak of the temperature of the body being constant we mean that it varies only within narrow limits, or that it is constant in comparison with that observed in cold-blooded animals.

6. The Temperature of Different Organs. - Nor is this all; some parts of the body have a higher temperature than others. Thus the temperature of the liver is often as high 
as $107^{\circ} \mathrm{F}$; ; that of the muscles varies between $99^{\circ}$ and $105^{\circ} \mathrm{F}$.; that of the blood in the right side of the heart is usually a degree or so higher than that of the blood in the left side. But it is in the skin that we meet with the widest variations from the general average. Every one knows that on a very cold day the temperature of the skin may be far below $98.6^{\circ} \mathrm{F}$.; indeed, the experience of "frosted" ears or feet shows that at times cutaneous temperature may descend to, or even below the freezing point itself; and it is very exceptional indeed when the skin temperature is above $92^{\circ}$ or $93^{\circ} \mathrm{F}$, even on very hot summer days. These variations are due to the fact that the skin is the organ which is immediately exposed to the changing environment, and hence peculiarly subject to cooling influences. It is therefore customary to distinguish between an outer body zone of variable temperature and the more constant temperature of internal organs.

7. Measurement of the Body Temperature. - The great equalizer of the body temperature is the blood. That which has flowed through the skin comes away cooled; that which comes from an organ like the liver or a working muscle, in which active oxidations or other chemical changes have taken place, is heated. In the great veins and in the heart the warmer blood is mixed with the cooler; so that theoretically what we mean by the body temperature would be that of the blood which the left side of the heart pumps to the organs. It is, of course, impracticable to measure this; and we must take instead the temperature of organs which are well supplied with this blood, themselves producing little or no heat, and at the same time not exposed to cooling influences. The most accurate measurements of temperature are made in the rectum, and there are times when reliable readings can be obtained only in this way. It is customary, however, to take the temperature in the mouth, the bulb of the thermometer being placed under the 
tongue and the lips kept closed. For many purposes this is sufficiently accurate, although at times it may introduce a comparatively large error. Subject to the variations mentioned above, the rectal temperature is about $99.6^{\circ} \mathrm{F}$, that of the mouth being $98.6^{\circ}$.

8. The Feeling of Cold or Warmth is not a True Test of the Body Temperature. - It is well at this point to warn the student against confusing the body temperature with sensations of cold or warmth. Since sensations of heat and cold originate only in the skin, the mouth, and perhaps the upper part of the osophagus, - parts which belong to the zone of variable temperature, - it is clear that our feelings give us no information as to the temperature of the internal parts of the body. This fact is strikingly illustrated in the case of a "chill," when the internal temperature is almost always really above, and not below, the normal; and the feeling of warmth produced by muscular activity or by warming one's self at a fire merely indicates a higher temperature of the skin, not a higher temperature. of internal organs.

Having now learned the more obvious facts about the constant temperature of the body, we have next to inquire by what means this constant temperature is maintained. Such an inquiry is of the utmost importance in the study of human physiology and hygiene, since the effort to keep a constant temperature involves at times the introduction of physiological conditions intimately related to the maintenance of the highest standard of health.

9. The Production and the Transfer of Heat. - The temperature of any body of matter can remain constant only as long as the amount of heat it produces or receives (from objects warmer than itself) exactly equals the amount it gives out; the temperature rises when the heat produced plus that received is greater than that lost; and it falls when the balance of the account is on the other side. 
In applying this principle to the human body we must first remember that the body produces or liberates heat. The chemical changes, largely oxidative in character, which are at the basis of the work of its muscles, glands, nerve cells, etc., liberate heat just as truly as the burning of coal in the furnace of an engine liberates heat. Heat production is therefore an indispensable result of cellular and organic activity, and it is greatest in those organs, like the muscles and liver, which carry out the most active chemical processes. The body is warm for the same reason that a stove is warm; that is, because combustion (oxidation) is going on within it.

In the second place, the heat liberated within the body, like that liberated in a stove, may be transferred to surrounding objects. Every one knows that when a warm body is brought near a colder one, the former becomes colder and the latter warmer. Heat has been transferred from one to the other, and this transfer continues until the two bodies reach the same temperature. The clothing is warmed by contact with the body; so is the air in immediate contact with the skin; and conversely the body may be warmed by contact with anything warmer than itself, a hot-water bottle, for example.

It is not, however, necessary that two salid bodies be in actual contact in order that heat may pass from one to the other. A stove warms all the objects in a room, although few of them are touching it; and the human body may lose heat to, or receive heat from, objects at a greater or less distance. There are, indeed, three methods of transferring heat from one body to another, and before we go farther it is important that these methods of heat transfer be thoroughly understood. They are known as conduction, convection, and radiation. In the case of conduction and convection the means of transferring heat, or the "vehicle" of transfer, is some form of matter, either a solid, or a 
liquid, or a gas ; radiation, on the other hand, occurs independently of the presence of ordinary matter between the two bodies, though on our earth the air, at least, is almost always present.

10. Conduction. - Whenever heat is transferred directly from one mass of matter to another with which it is in contact, such transfer is known as conduction. A good example is the heating of a poker in a fire; the heat of burning coal is communicated directly to the outer particles of iron, and then from one particle of the iron to another. The particles of iron do not move up and down the length of the poker; each one simply passes on to the next the heat it has received, and finally those of the handle communicate their heat to the hand. All transfer of heat along solid objects, or from one mass of matter to another with which it is in immediate contact, is by means of conduction.

Solids and liquids are much better conductors of heat than gases, and air when perfectly still is one of the poorest conductors of heat with which we have to deal. It is a familiar fact that the skin is chilled much more rapidly by water than by air of the same temperature (why?); and we shall learn in hygiene that warm fabrics owe their warmth mainly to the amount of poor-conducting air stagnant within their meshes.

11. Convection. - When a warm body is surrounded by a fluid such as water or air, heat is similarly conducted to the adjacent layer of water or air, which thus becomes warmer; but, unlike the case of the solid, this heated layer now moves off, carrying its heat with it to other parts of the gas or liquid, and so communicating it to other matter with which it subsequently comes in contact. This method of heat transfer is known as convection, which, it will be seen, depends at bottom upon conduction, but which is at the same time conduction modified by the 
movement of a heated gas or liquid. So long as the air around us is at rest it does not remove heat readily from the skin, since air is a poor conductor. Air in motion, on the other hand (as in fanning), cools the skin more rapidly, because as each part of the air is heated it is moved away and replaced by colder air. In this case the air cools the skin by convection (Latin con-, with; vehere, to carry).

The transfer of heat from the internal heat-producing organs to the skin affords an excellent example of the difference between conduction and convection; for some of this heat passes by direct conduction through the subcutaneous tissue to the overlying skin, while some of it is carried to the surface by convection in the blood stream. When the arterioles of the skin are dilated, convection is an important means of heat transfer to the surface; when, in the reverse case, the cutaneous arterioles are constricted to their utmost, convection becomes relatively unimportant and direct conduction alone remains as the chief means of heat transfer to the skin. Moreover, when the subcutaneous tissue contains large amounts of fat, it is a poor conductor of heat, and for this reason fat people when sitting still on cold days often feel colder than lean people do.

12. Radiation. - Heat is thus removed from the skin by conduction, and at times to an even greater extent by convection. But there is still a third method of heat loss, known as radiation, by which heat can be transferred across a space in which there is neither solid, liquid, nor gas, and in which conduction and convection are consequently impossible. The most familiar and striking example of radiation is the transfer of heat from the sun to the earth, since there is no atmosphere in the greater part of the more than ninety millions of miles of space which separate us from that intensely heated body.

Any detailed consideration of radiation belongs to the domain of physics rather than physiology, and would be 
out of place here. It is enough for our present purposes to understand that, whether a solid body be in an atmosphere of air, or in a transparent liquid, or even in a vacuum, it transfers or loses heat by direct radiation to colder objects about it. From an open fire heat may be transferred by conduction to andirons or walls in direct contact with it; or by convection through heated air currents to the chimney top; or, finally, by radiation to persons standing in front of it. In the latter case the heating is chiefly by radiation, since there is no contact with the fire, and such air currents as exist are mostly composed of cool air sucked towards and into the chimney by its draft. It is for these reasons that open fires are said to "roast people in front and freeze them behind." Conversely, the human body, if warmer than its surroundings, may lose heat by conduction, convection, and radiation to cooler objects in the vicinity.

The practical importance of these facts is seldom realized. It often happens that the air in contact with the skin is of the proper room temperature; and yet, if one is sitting too near a cold wall or window, enough heat may be lost by radiation from the skin to the cold wall, through the warm air, to chill the skin materially, causing a loss of heat and a "cold."

13. Laws of Conduction and Radiation. - For our purposes the two most important factors which determine the loss of heat by conduction and radiation are (1) the difference in the temperature of the two objects; and (2) the distance between them. In general, the greater the difference of temperature the more heat will be lost from the warmer to the colder object; thus the skin loses heat rapidly by these means when surrounding objects are at $0^{\circ} \mathrm{F}$., but only slowly when they are at $90^{\circ}$. It is also clear that as soon as the temperature of surrounding objects and of the atmosphere is as high as that of the 
body $\left(98.6^{\circ} \mathrm{F}\right.$.) no further heat can be lost by conduction and radiation; and that above $98.6^{\circ} \mathrm{F}$. heat is conducted and radiated to the body, not from it.

Furthermore, the greater the distance of the colder object from the body the less heat will the body lose to it. Here heat loss takes place inversely as the square of the distance; i.e. when we are twice as far away from a cold (closed) window we lose only one fourth as much heat through it by radiation; if we are three times as far away, we lose only one ninth as much, and so on. Consequently we rapidly diminish radiation from our bodies by sitting farther away from the walls of a room ; and it is important to have our living rooms large enough to make it unnecessary to sit near the windows or near a cold outer wall in very cold weather.

14. Heat Output by the Evaporation of Perspiration. In one other way heat may be lost from the body. The change from the liquid to the gaseous state (evaporation) always involves absorption of heat from surrounding objects. This is readily illustrated by the simple experiment of blowing a steady current of dry air over the dry hand, and comparing the cooling thus produced by convection with that produced by blowing the same current against the moistened finger. ${ }^{1}$ The cooling in the latter case will be much greater than in the former, and is caused by the evaporation of the water. Liquids like ether, which evaporate more rapidly than water, will produce even greater feeling of cold on the skin.

In the secretion of perspiration we have this principle applied to the uses of the body. The student should understand clearly at the outset, however, that it is the evaporation of the perspiration, not the secretion of it, which abstracts heat from the body. Perspiration may be

1 In this experiment the temperature of the room and of the current of air must be so low that they will not induce active perspiration. 
secreted in large quantities, but if it does not evaporate, as happens on a very moist, humid, muggy day, when the atmosphere already contains about as much aqueous vapor as it can hold, - it takes little or no heat from the skin. Nor is the efficiency of the perspiration as a cooling agent measured by the amount of visible or " sensible " perspiration, for this is only the perspiration which has not evaporated; the true measure of the cooling effect would be the perspiration which has evaporated and of which we are not conscious.

It is important to note that the evaporation of perspiration (or of water from the lungs and air passages) is the only means of cooling the body when objects around it are warmer than the body itself. In this case conduction, convection, and radiation only add heat to the body; but even their combined action may often be overcome by an abundant evaporation of perspiration. Men have remained for some time in rooms whose temperature was as high as $260^{\circ} \mathrm{F}$., or $48^{\circ}$ above the boiling point of water, without any marked rise of the body temperature and without severe discomfort, the temperature of the body being kept down solely by the evaporation of perspiration from the skin. In order to make this means of cooling possible, it is absolutely essential that the air be dry and capable of taking up moisture. No one can survive long at such temperatures in moist air.

15. Summary. - In conclusion we may recapitulate the ways in which the body may gain or lose heat.

It may GAIN heat by conduction, convection, or radiation from warmer surroundings. The amount of heat it receives from without is, however, very small compared with that derived from the oxidations taking place within itself. These oxidations constitute the chief sources of its heat.

It may LOSE heat (1) by conduction, convection, or radiation to things external to itself. . Here are included not 
only the heat lost from the skin but also that lost in heating the expired air and the excretions ; (2) by evaporation of water from the skin, the lungs, and the air passages.

It is quite impossible to give definite figures showing just what percentage of the total heat-loss occurs by each of these channels, because this differs at different times; on a very cold day, for example, none is lost in perspiration because no perspiration is secreted, but much is lost by radiation and convection; on a hot day the conditions are reversed. On a warm day when the wind is blowing convection plays a more important rôle than it does when the air is quiet. During vigorous exercise on a cool day we lose heat by evaporation of perspiration; when we are at rest at the same temperature little or no heat is lost by this means. A little reflection will show that most of the output of heat from the body is from the skin. The heat which the body loses in the urine and feces is almost negligible, and that which is lost by heating the expired air and evaporating the water which this air carries off as aqueous vapor probably never exceeds ten or twelve per cent of the whole. From eighty-five to ninety per cent of the total output of heat is by way of the skin; so that it need not surprise us to find that it is the skin which plays by far the most important rôle in checking or facilitating, as the case may be, the output of heat.

\section{B. The Regulation of the Body Temperature}

In the preceding section we have learned the essential facts concerning the production and output of heat by the human body, and have also seen that a constant temperature is maintained because heat production and output are kept equal. We have now to study the means employed to adjust heat production to heat loss, and heat loss to heat production. For this purpose we may begin by considering 
what happens during the two conditions of daily life which most conspicuously threaten the heat balance, i.e. during changes in external climatic conditions and during internal conditions produced by muscular activity. The former disturbs the balance by changing the rate of conduction, convection, radiation, and evaporation from the surface; the latter, by producing more heat within the body.

16. Reactions of the Body at Rest to Changes of External Temperature. - The more important climatic conditions which affect the transfer of heat are temperature, atmospheric moisture (or humidity), and wind. We may study first the influence of changes of temperature, assuming for the present that the air remains of moderate humidity and that there is little or no wind.

On a warm day, when the thermometer stands at $90^{\circ} \mathrm{F}$. or more, and one is lightly clad and sitting still, perspiration is secreted abundantly and the arterioles of the skin are widely dilated. Let us now suppose that the day gradually grows cooler. The perspiration becomes less abundant, and, as the falling temperature nears $70^{\circ} \mathrm{F}$., ceases to be of consequence; but the blood-flow through the skin, though lessened, is still considerable. Below $70^{\circ}$ the perspiration ceases, so that heat is now lost almost entirely by conduction, convection, and radiation.

As the temperature falls below $70^{\circ} \mathrm{F}$, the veins are no longer conspicuous on the hand and arm, which shows that the loss of heat is being checked by limiting the blood supply to the skin. At the same time the arterioles of internal organs are dilating (see p. 150), so that the liver, the kidneys, the mucous membranes of the alimentary canal, and those of the air passages contain an increased amount of blood.

By the time the temperature has fallen to $60^{\circ} \mathrm{F}$, or thereabouts, the cutaneous arterioles have constricted to their utmost, the blood-flow through the skin has nearly 
ceased, and the organism has no means at command by which to restrict the further output of heat. If in this emergency heat production were to remain constant while external temperature continued to fall, the temperature of the body would be lowered, for conduction and radiation not only continue but increase. That it is not usually lowered is due solely to the fact that more heat is then produced within the body; the oxidations (and hence heat production) which have remained fairly constant in amount between $90^{\circ} \mathrm{F}$. and $65^{\circ} \mathrm{F}$. external temperature now increase to compensate the inevitable loss, and continue to increase as the atmospheric temperature continues to fall.

17. The "Dangerous Region" of Atmospheric Temperature. - It is important to understand that the methods of regulating the temperature of the body differ with different external temperatures. Below $60^{\circ}$ the only available method is to vary heat production; above $98.6^{\circ}$ the sole method is to evaporate perspiration; between $70^{\circ}$ and $98.6^{\circ}$ the evaporation of perspiration is combined with variations in the blood-flow through the skin; and finally we have left the region between $60^{\circ}$ and $70^{\circ}$, when the vascular changes in the skin play the most important rôle.

In this region between $60^{\circ}$ and $70^{\circ}$ three or four degrees make all the difference between the ideal room temperature and one which is decidedly dangerous. At $66^{\circ}$ or $68^{\circ}$ the blood is properly distributed between the skin and the internal organs, and there is no excess in either. At $60^{\circ}$ or $61^{\circ}$, on the other hand, the blood is forced back upon the internal organs, thus threatening serious congestions and other unhealthful conditions which we shall consider at length in our study of hygiene (see Part II, Chapter XXI). It is because the temperature of a room may fall from $66^{\circ}$ to $60^{\circ}$ so gradually that we do not notice it until the internal damage is done, whereas it could not fall to $50^{\circ}$ or $40^{\circ}$ without our noticing it and correcting the 
trouble, that more colds are taken in the former case than in the latter. In other words, as the temperature goes below $65^{\circ}$ the body seems at first to rely wholly on the vascular mechanism of temperature regulation, and does not begin to produce more heat until this resource has been utilized, not only to its utmost, but even to an extent inconsistent with health. The "dangerous region of room temperature," then, may be placed between $60^{\circ} \mathrm{F}$. and $65^{\circ} \mathrm{F}$.

18. The Influence of Atmospheric Moisture or "Humidity." - The amount of water vapor which the atmosphere contains influences the output of heat in two ways. In the first place moist air conducts heat from the skin more readily than dry air. We do not notice this action of moist air in very warm weather because the difference of temperature between the body and the air is then so slight that neither moist nor dry air conducts any large amount of heat from the surface. But when this difference of temperature is greater, the effect of humidity in facilitating conduction becomes quite evident. Moist air at $65^{\circ} \mathrm{F}$. is distinctly chilly when one is sitting still, while dry air at this temperature is comfortable.

As soon, however, as the evaporation of perspiration becomes necessary to maintain the constant temperature, the presence of moisture, so far from aiding the output of heat, actually interferes with it; for evaporation will not take place readily into moist air. This is the reason why a high degree of humidity makes us feel warmer on a warm day, whereas, as stated above, it also makes us feel colder on a cold day. In the latter case it acts by conducting heat more rapidly from the skin; in the former by interfering with evaporation, which is then the chief means of heat output.

1 The student is reminded that in the above discussion the figures given apply only when one is lightly clad and sitting still, when the humidity is moderate, and when the air is comparatively quiet. 
19. The Effect of Stagnant vs. Moving Air; the Aërial Blanket. - On a perfectly still day the layer of air about the body becomes warmed by the skin, and, so long as it is not removed, forms an air-blanket which goes far to keep the skin warm; for air is a poor conductor of heat. As soon, however, as a breeze springs up convection comes into play and the skin is cooled more rapidly. In stagnant air, moreover, the evaporation of the perspiration tends to saturate this air-blanket with water vapor, so that further evaporation is rendered difficult. Accordingly, when perspiration is not being secreted, moving air cools the body by increasing convection; and when the skin is moist it cools the body both by increasing convection and by facilitating the evaporation of perspiration.

20. The Influence of Muscular Activity. - External temperature, moisture, and wind tend to disturb the balance of the heat account chiefly by changing the rate at which heat is lost from the body. But the balance may also be disturbed by changing the amount of heat liberated within the body. This occurs most conspicuously during muscular activity, for the chemical changes (or oxidations) which then take place necessarily involve the liberation of large amounts of heat within the muscles; and this heat must evidently be got rid of, if a constant temperature is to be maintained.

The reactions which then occur are familiar to every one. The arterioles of the skin are dilated (while those of internal organs are constricted) and perspiration is secreted. These are the same reactions which are noticed when the body is exposed to external warmth, and their purpose is the same in both cases, - to facilitate the escape of heat. But in the one case they are made necessary by the fact that climatic conditions interfere with the output of heat, in the other by the fact that more heat is being liberated and hence more must be got rid of. 
Seldom indeed is so severe a strain imposed upon the mechanism of heat dissipation as during vigorous muscular exertion, and especially when the external conditions are not favorable for radiation, convection, and the evaporation of perspiration. Caution is then urgently indicated lest we make the strain too great. It is a practical point to remember in this connection that some forms of muscular exertion introduce conditions for getting rid of the surplus heat much more readily than others; this is especially true of those which involve movement of the body as a whole. Bicycle or horseback riding, by creating a breeze, renders the cooling of the body a much easier matter than does sawing wood, or swinging Indian clubs, or gymnastic work in general ; again, a particular form of exercise on a dry day, when the perspiration can evaporate readily, may be safe, while it would be decidedly inadvisable on a muggy day, even though the temperature were somewhat lower. Indeed, by this time the student must have learned that the thermometer alone is no safe indicator of the difficulty of heat elimination in warm weather.

21. Relations of Climatic Conditions to Mental Work and Sleep. - During mental work the brain requires an increased supply of blood, and this is obtained partly by diminishing the supply to the skin (constriction of cutaneous arteries); during sleep, on the other hand, the supply to the brain is diminished, and this is ordinarily effected by dilating the arteries of the skin (see p. 153). Mental work is difficult on very warm days, partly because it is difficult to bring about cutaneous constriction; and it is especially difficult on warm, muggy days, since the maintenance of the constant temperature then requires an excessive cutaneous dilation, and the brain is quite unable to command its needed blood supply.

During sleep, on the other hand, the arterioles of the skin should dilate, and this they cannot readily do when 
the skin is exposed to cold. To "sleep warm" is good advice, and is based on sound physiological principles.

22. Digestion and the Regulation of the Constant Temperature. - During digestion, and especially during its earlier stages, when secretion is at its maximum, a large supply of blood is needed in the stomach, the pancreas, and the intestine. This cannot readily be secured when the blood is being sent in large quantities to the skin in order to cool the body. We have seen all along that the two great vascular areas of the skin and digestive organs are more or less antagonistic or compensating in their vasomotor reactions. When the blood is present in large quantities in the skin it is present in smaller quantities in the stomach, the intestine, the pancreas, the liver; and, vice versa, these organs can best obtain an adequate blood supply when the demands of the skin are not excessive. Consequently digestion is more difficult in warm than in cold weather, and we should then eat less at a time, even if we have to eat somewhat more frequently.

During the digestion of a meal the chemical activities of secretion, the peristaltic muscular movements, etc., somewhat increase heat production in the body; and this increase, though not great, is at times great enough to make us feel distinctly warmer. When one is slightly chilly, for example, he often feels warmer after eating something, even though the meal be cold; and on a very warm, muggy day, when the blood-flow through the skin is already excessive and its temperature unduly high, the digestion of a meal often adds to the discomfort, because the larger production of heat leads to further dilation of the skin vessels.

23. Subcutaneous Adipose Tissue a Hindrance to the Output of Heat. - In the last chapter we saw that heat may be brought from the internal heat-producing organs to the skin in two ways, - by the circulation (convection), or else 
by direct conduction through the tissues. How much will take one course and how much the other depends partly upon the conducting power of the tissues in question. Fat is a poor conductor of heat; and when it is stored, as is usual in fat people, in large quantities in the connective tissue immediately under the skin, it so interferes with direct heat conduction that a larger proportion of the transfer of heat to the surface must be by the circulation; the cutaneous arterioles must be more widely dilated and the perspiration, if necessary, must be increased. Hence muscular exertion and exposure to warm weather produce more discomfort in fat than in lean people because of the larger quantity of perspiration and the heating of the skin by the greater cutaneous blood-flow; such people readily become "overheated." On the other hand, contrary to popular belief, when fat people are exposed to cold weather they are often more chilly when sitting still than lean people, probably because, since the blood-flow through the skin is lessened, their skin has not the same source of warmth in the direct conduction of heat from subjacent tissues. Fat people thus retain the heat of the body better, and should maintain more easily the constant internal temperature; but this very retention of heat within the internal organs keeps heat away from the skin, which consequently becomes cold and produces the feeling of chilliness.

24. The Mechanism of Temperature Regulation. - The preceding pages have shown us that temperature regulation depends chiefly on three physiological mechanisms : (1) the vasomotor system, which controls the distribution of blood between the skin and the internal organs; (2) the sweat glands ; (3) the mechanism of heat production. The first of these has already been described in the study of the circulation. The heating of the skin stimulates afferent nerves which reflexly dilate the arteries of the skin and 
also simultaneously constrict those of internal organs. This reflex, then, is dependent on the temperature of the skin; anything which heats the skin causes a reflex dilation of its arterioles and lessens the supply of blood to internal organs.

The secretion of perspiration is also under the control of the nervous system. The sweat glands, like the salivary

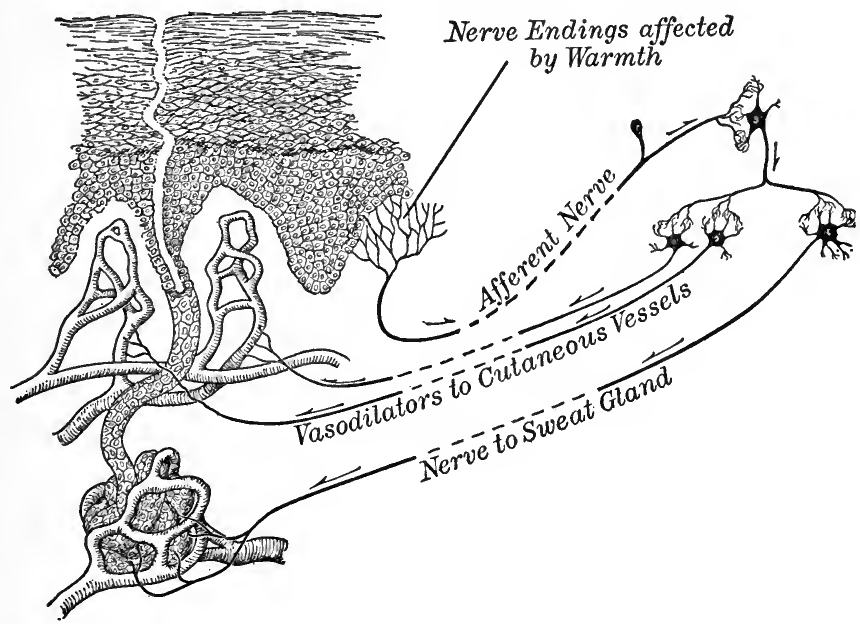

Fig. 82. Diagram of the cutaneous reflexes of temperature regulation Showing the epidermis, blood vessels of the dermis, a sweat gland, and the nervous mechanism governing blood-vessels and sweat glands

glands, receive nerves, and secrete only in response to their stimulation. When the nerves going to the sweat glands of any region are injured, exposure of these glands to external warmth produces no perspiration; stimulation of their nerves, however, produces a copious secretion.

25. The Skeletal Muscles the Main Organs in the Regulation of Heat Production. - The third mechanism of heat regulation is that whereby the amount of heat produced is increased as it is needed, and experiment has shown that 
the main organs here concerned are the skeletal muscles. When these are in any way prevented from contracting, the increase of heat production on exposure to cold does not occur.

Certain drugs, for example, interrupt the connection between muscles and their motor nerves, and when an animal under their influence is exposed to a falling temperature the increase of heat production below $60^{\circ} \mathrm{F}$. is not observed; the temperature of the body rapidly falls. Similarly, experiment has shown that if a human being, exposed to a falling temperature, is careful to lie in as relaxed a condition as possible, thus suppressing as far as possible the activity of the skeletal muscles, the usual heat production fails to appear.

From these facts we conclude that below $60^{\circ} \mathrm{F}$. cold stimulates cutaneous afferent nerves, which reflexly excite the skeletal muscles of the body, and that the heat produced by their contraction tends to compensate the increased loss of heat from the skin.

26. Shivering. - The contraction of the muscles thus produced does not necessarily result in movement. Almost every joint at which motion ordinarily takes place is acted upon by antagonistic muscles; for example, the elbow, finger, knee, and ankle joints are moved by extensor and flexor muscles, but only when one group is contracting to a greater extent than the other. If the two groups contract to the same extent, the action of the one neutralizes that of the other, so far as movement is concerned; but both contract, ${ }^{1}$ and in contracting produce heat. This is what happens when external cold stimulates all the muscles of the body to increased contraction. Movement does not usually result because the extensors and flexors are more or less equally balanced. There are times, however,

${ }^{1}$ A muscle may contract without shortening or producing movement, as when we pull on a weight we cannot lift. 
when this contraction of the antagonists is not well balanced, and we "shiver." Shivering is the extreme expression of this heat-producing reflex.

We are often conscious of this increased muscular action during cold, even when we are not shivering. Every one knows the difference between the "bracing" effects of a cool or cold day and the "relaxed," "slack-twisted" feeling on a warm day; and this is largely traceable to the sensations which come from the contracting muscles in the former case and to the absence of such sensations from the inactive muscles in the latter. To put it in another way, cold increases the tone of the skeletal muscles (see p. 159). A skeletal muscle on a cold day is never completely relaxed; like the unstriped muscles of the arteries, it is in a condition somewhere between extreme contraction and extreme relaxation.

27. The Regulation of the Body Temperature a Function of the Nervous System. - We may close this brief account of thermal phenomena of the body by recalling to the attention of the student what must now be obvious at a glance, namely, that a constant temperature is maintained by the coördinating action of very many nervous reflexes. The action of the vasomotors of the skin and of the internal organs, of the nerves of the sweat glands and of the motor nerves of the skeletal muscles, must all be so adjusted with regard to one another that exactly the right balance is preserved amid all the variations of heat production and of climatic conditions which affect heat-loss. Success in this adjustment depends upon the skill with which the coördinating nervous system does its part. With the single exception of muscular exertion, no condition of life makes such far-reaching, or such imperious demands upon the system as a whole as does the maintenance of the proper internal temperature. Mental work and the efficiency of digestion are examples we have 
already studied - and more could easily be cited - of functions which, important as they are, are subordinated, even sacrificed, to prevent a marked rise or fall in the temperature of the blood.

To such an extent is the nervous system as a whole adapted to maintain the constant temperature that the failure to do this, as shown by the presence of fever, or by the even more serious subnormal temperature, becomes one of the most important indications that something has gone wrong. We know already how the nervous system intervenes in every function of our lives, and how the wellbeing of the body as a whole depends upon the adjustments which it brings about. It is for these reasons that, when it is no longer able to exercise that supreme control of the constant temperature which is one of its most characteristic features in health, the physician's orders usually are to "go to bed and be perfectly quiet." The body is then in no condition to make demands on the nervous system for action; and a person who refuses to heed the plain warning which his temperature holds out has nothing but his own foolishness to blame if he suffers serious consequences. 


\section{CHAPTER XIII}

\section{NUTRITION}

\section{A. The Sources of Power and Heat for the Human Mechanism}

1. Food and Nutrition. - The one source of supply of matter required for the growth and repair of the human machinery, and of power required for its work, is food; and the study of the various ways in which different kinds of food serve the needs of the body, and the value of each kind of food, under different circumstances or for different purposes (such as athletic training, mental work, life in cold climates, in hot climates, etc.), is the study of nutrition. We shall limit our consideration of this great subject to a few elementary and practical topics, beginning with the supply of heat and power to the body.

2. The Fuel Value of Food. - In any locomotive engine the same amount of a given fuel will enable the engine to pull a train of the same weight for the same distance over the same track, provided, of course, the engine itself, the bearings of the wheels, etc., are in the same condition. When a ton of coal is put into the tender, it is with the expectation that it will move the train a certain distance. Thus there is a definite relation between the fuel burned and the work done. Every engineer knows also that the same weight of different fuels will carry the train different distances; a thousand pounds of wood, of bituminous coal, and of anthracite coal have different fuel values.

The same weight of a given fuel when burned will always yield exactly the same amount of heat, as is proved 
by burning the fuel under conditions which enable us to measure the heat given off. The simplest means of doing this is perhaps with the ice calorimeter, - a metal box within which the fuel is burned, the box being everywhere surrounded by a thick layer of ice. The heat produced in burning the fuel is measured by the amount of ice melted.

In this way we may find the relative amounts of work which can be done with two different fuels; for it has been discovered by actual experiment that if one kind of fuel produces twice as much heat as another, it will also do twice as much work.

Now food is the fuel for the muscular work of the body and also for the liberation of heat. Consequently, if we determine how much heat is liberated when a certain amount of proteid, or fat, or carbohydrate is burned in a calorimeter, we know how much work it may do in the body; or at least we know that it can do no more than the amount indicated by the calorimetric experiment.

3. Units of Heat and Work. - In order to measure we must have units of measurement. Common units of length are the inch or centimeter; units of area are the square yard, the square meter, or the acre; units of volume, the quart or peck; units of weight, the pound or kilogram. And we express the results of these measurements by saying that a thing is so many inches long or of so many pounds weight. What are the units of heat and work?

Like all units, these are arbitrarily chosen: The unit of heat, known as the calorie, is the amount of heat necessary to raise one gram (one cubic centimeter) of water one degree Centigrade. The unit of work is the amount of work done in lifting a kilogram (2.2 lbs.) to the height of one meter (39.37 in.) from the surface of the earth against the attraction of gravitation. This is known as the kilogrammeter. Thus when a man weighing sixty kilograms goes up a 
flight of stairs ten meters high his muscles do 600 kilogrammeters of work. ${ }^{1}$

Finally, it has been found that the same fuel which when burned will liberate one calorie of heat will supply the power to do 0.423985 kilogrammeters of work. By this we mean that not more than 0.423985 kilogrammeters of work can be obtained from it. Not every engine is so perfectly constructed as to get from a certain fuel its full working capacity; indeed, most engines transform only a small fraction of the power of their fuel into work, the rest escaping as heat, - in the smoke, or by radiation, conduction, and convection from the furnace, boiler, etc. But by the method above outlined it is possible to find the maximum amount of work which can be obtained from a given weight of fuel.

Applying the same methods to food, we find that:

1 gram of dried proteid yields

1 gram of dried carbohydrate yields

1 gram of fat yields
4100 calories.

4100 calories.

9300 calories.

These figures are known as the fuel values of proteids, carbohydrates, and fats.

But the total possible power which may be obtained by actually burning a certain substance under the most favorable conditions is one thing, and the amount of power which the muscles may obtain from it is quite another. When coal is burned in an engine it does work; but the human body would get no energy for its muscular work from eating coal. So that we have now to inquire from what nutrients (p. 89) the muscles get their energy for work, and from what nutrients the body derives its heat.

4. The Power for Muscular Work. - Few questions in physiology have been more thoroughly investigated than

1 Work may also be expressed in foot pounds. (How many foot pounds equal one kilogrammeter?) 
this. In the first half of the nineteenth century many investigators, impressed with the fact that the muscle fiber yields, on chemical analysis, large quantities of proteid and only traces of carbohydrates and fats, believed that the energy for muscular contraction comes entirely from the consumption of the proteid of the muscle substance. If this were true, it would necessarily follow that proteid is the proper food to yield the energy for muscular contraction, while fats and carbohydrates would simply be oxidized to give heat.

This view was disproved by the following epoch-making experiment of physiology. Two observers determined for three successive days the nitrogen excreted by themselves; since almost all this nitrogen comes from proteid, this gave the amount of proteid consumed by the body. On the first and third days no vigorous muscular work was done; on the second day they climbed a mountain 1956 meters ( $6415 \mathrm{ft}$.) high. As one man weighed 66 kilograms and the other 76 kilograms, the work done in lifting the body to the top of the mountain in the two cases was 129,096 and 148,656 kilogrammeters respectively. The proteid which was oxidized in this time could in the two cases have yielded power for only 68,690 and 68,376 kilogrammeters of work. In other words, the proteid did not begin to yield sufficient power for the muscular work done in lifting the body to the top of the mountain; something else than proteid must have been oxidized for that purpose, and that something must evidently have been carbohydrate or fat, or both.

Again, it was noticed that there was no increase of proteid disintegration on the day of work; this remained practically unaffected by muscular contraction. Numerous other experiments made since that time have shown the same thing. Muscular exercise does not necessarily increase proteid disintegration, and the power for it can be obtained, in large part at least, from fats and carbohydrates. 
In the experiment above referred to no determinations were made of the excretion of carbon dioxide. Since then numerous experiments have been made in which, on an abundant mixed diet, both the nitrogen and the carbon of the excretions were accurately determined. These have shown that while muscular exercise does not necessarily increase proteid disintegration, it invariably increases the production of carbon dioxide. If the carbon of the carbon dioxide came from proteid, it would be accompanied by increased excretion of nitrogen derived from the brokendown proteid. The fact that it is not so accompanied can only mean that it came from the oxidation of something which did not contain nitrogen, i.e. from fat or carbohydrate, or both.

But while muscular work does not necessarily or even usually increase proteid decomposition, and the power for the same may be derived largely, if not entirely, from carbohydrates and fats, it has been shown conclusively that under certain conditions this power may come entirely from proteid. In one experiment a large and very lean dog was fed for several weeks on an abundant diet of lean meat, containing practically no carbohydrate or fat; during this time the dog did large amounts of work in a treadmill and in other ways; and since it was found that this work could be done for weeks at a time on the meat diet, we conclude that the proteid must have been the sole source of power for the work; it must also have served as the source of heat production, for the normal temperature of the animal was maintained.

5. Summary of Considerations on the Supply of Power for Work. - These and other experiments show (1) that the animal body can get its energy for mechanical work and for the production of heat from proteid, or from carbohydrate, or from fat; (2) that when the animal is on an abundant mixed diet, even vigorous muscular work does 
not increase the oxidation of proteid, ${ }^{1}$ but that it does enormously increase that of carbohydrates and fats. The probable meaning of this is to be sought in the fact that proteid decomposition depends primarily not on muscular work but, as we shall see later (p. 222), on the amount of proteid eaten; while the oxidation of fats and carbohydrates depends entirely on the demands of the body for energy, and is largely independent of the amount of these foods eaten.

6. The Supply of Energy for Heat Production. "Heating" Foods. - In studying the phenomena of heat production in the body we found that when the body needs more heat in order to maintain its normal temperature, this heat is supplied chiefly by greater chemical activity in the muscles (p. 207). The contraction or tone of the muscles increases in response to stimuli from the same motor nerves which stimulate them to activity when they do external mechanical work. Heat production in the body, from the standpoint of nutrition, is therefore, as far as we know, largely a case of increased muscular activity; and here, as in the case of mechanical work, the energy can be obtained from one kind of food as well as from another. Contrary to popular ideas, we have no conclusive evidence that one kind of food supplies heat more readily than another. What is required in cold weather is more food, whether proteid or carbohydrate or fat. We shall see that there are good reasons for not unduly increasing the proteid of the diet under any conditions (p. 225), and hence in this special case it is probably better to increase the non-nitrogenous foods to a greater extent than the proteids, though not because they are better "heating" foods.

1 Under the abnormal conditions of excessive muscular work (e.g. sixday walking matches or bicycle races) the proteid oxidation is often increased. 
There is another reason why the fats and carbohydrates may well be increased in cold climates, and that is that they are par excellence the foods which lead to the storage of fat. The storage of fat in the subcutaneous tissue, we have already seen, greatly facilitates the maintenance of the body temperature by interposing a poor conductor of heat between the skin and the more internal heat-producing organs; and this gives to these foods a special value in a cold climate.

It is often pointed out that inhabitants of cold climates eat more fat, while the non-proteid food of those who live in warm climates consists chiefly of carbohydrates, and the conclusion is drawn that fat is a better food for cold weather. This reasoning, however, neglects the fact that vegetables, from which alone carbohydrates are obtained, are not common in cold climates. The people in such climates get their non-proteid foods from the most convenient source, - the adipose tissue of animals. The question is further complicated by considerations of digestibility of the two kinds of food, and cannot be answered offhand.

\section{B. Special Effects of the Different Nutrients}

Our study in the preceding section of the sources of power and heat has led to the conclusion that while one nutrient may be preferable to another, either proteid or carbohydrate or fat may meet these two needs of the body. In other words, no marked difference in use has thus far developed for the oxidizable nutrients. In the present section we desire to dwell upon the special uses of the nutrients, i.e. the uses which each subserves and which cannot be replaced by others.

The more important nutrients are, as we have seen in Chapter VIII, proteids, carbohydrates, fats, inorganic salts, 
and water. These may now be conveniently classified as follows :

\section{$A$. Compounds of carbon which undergo marked chemical change, by oxidation or otherwise, into waste prod- ucts within the body}

$B$. Substances excreted from the body largely in the same form as absorbed
1. Proteids
2. Carbohydrates
3. FAts
Non- proteids

The proteids contain carbon, hydrogen, nitrogen, oxygen, and sulphur; some of them also contain phosphorus and some contain small quantities of iron. The carbohydrates and fats contain only carbon, hydrogen, and oxygen. All three are oxidized in the body to the waste products with which we have already become acquainted (see Chapter XI). The usual inorganic salts of the food, on the other hand, do not undergo oxidation; and, while they may change into other chemical forms during their stay in the body, it is still true, on the whole, that they are excreted in the same form as that in which they were absorbed. And the same thing is true of the water we drink.

7. The Albuminoids and Extractives. - In addition to the above, the connective tissues of animal foods contain a sixth nutrient which is composed of the same elements as proteids, i.e. carbon, hydrogen, nitrogen, oxygen, and sometimes sulphur. By far the most important member of the group is the collagen of the fibers of connective tissues, bone, etc., and the gelatin into which this collagen is converted when it is boiled with water. These so-called albuminoids may do the work of proteids in part, but they cannot replace the proteid entirely.

Finally, most foods contain small quantities of other substances, known as extractives, which are of use to the body

1 See Chapter VIII for definition of the terms in this table. 
chiefly because they contribute to the taste or flavor of the food. Some of them are thought to exert a stimulating effect, while still others are of importance in disease. For our present purposes, however, they are of minor importance, and we shall confine our attention to the nutritive value of the proteids, carbohydrates, fats, salts, and water.

8. The Inorganic Salts and Water. - We may begin with these because, so far as they concern us in an elementary work like this, their uses are most easily understood. Inorganic salts (chlorides, sulphates, carbonates, and phosphates of sodium, potassium, calcium, etc.) are found in all living cells, in the blood and the lymph, and are constantly being removed from the blood in the urine and the perspiration. This loss must be made good by the food. While inorganic salts are not oxidized by the body, their presence in the blood and tissues is for various reasons absolutely necessary. The craving of herbivorous animals for salt, in which their food is deficient, is well known, and in parts of India salt famines have occurred during which the price of salt was higher than that of gold.

The loss of water from the lungs, skin, and kidneys is made good from two sources. In the first place, proteids, carbohydrates, and fats all contain hydrogen; and when they are burned within the body their hydrogen combines with oxygen to form water, thus :

$$
\begin{gathered}
\mathrm{C}_{6} \mathrm{H}_{12} \mathrm{O}_{6}+6 \mathrm{O}_{2} \\
\text { sugar oxygen }
\end{gathered}
$$

In this way, on an average diet, almost one pint of water may be produced within the body in a day; and we are able to understand how at times, despite the constant loss of water and the comparatively small amount drunk or taken in the food, the tissues do not dry up. Usually, however, this supply' is insufficient, and it is necessary to use water as an article of food. 
9. The Proteid and the Non-Proteid Foods: Three Points of Difference. - We may pass now to the study of those food stuffs which are compounds of carbon, and which undergo marked chemical change into waste products within the body. We have already separated those food stuffs - proteids, carbohydrates, and fats - into two divisions, the proteids and the non-proteids, because in at least three respects the members of the two divisions play different rôles in the nutrition of the body. They differ (1) in their availability to serve in repairing losses of living tissue; (2) in their effects upon the chemical changes in the body; and (3) in their relation to the storage of fat. In all these respects the fats and carbohydrates resemble each other closely, while they differ from the proteids.

10. The Waste and Repair of the Living Cells. - Certain organs of the body, notably the muscles and glands, consist chiefly of living cells, thus presenting a striking contrast to other organs, like bones, tendons, ligaments, and cartilage, in which the living cells are few in number, the main mass of the organ consisting of lifeless matter between the cells. If, therefore, we make a chemical analysis of organs of the first kind, we can obtain some insight into the composition of the living cell. The results of the analysis of muscle (lean meat) will serve as a type of what is found in such cases.

Composition of lean meat:

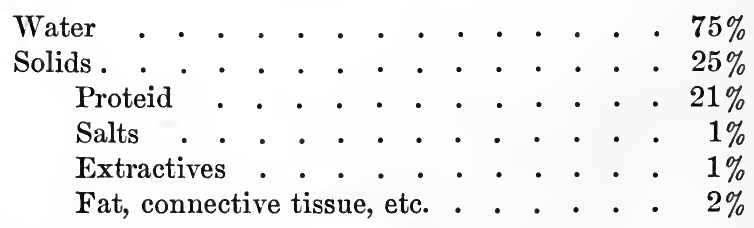

We do not know in what form the proteid which we find in dead muscle is present in living muscle; but even if it is not in the precise form which we know as lifeless 
proteid (such as the white of an egg), it is still true that this proteid constitutes the chemical basis of the living substance. Hence we may say that the living cell is largely composed of a proteid-like substance containing among other elements carbon, hydrogen, nitrogen, oxygen, and sulphur.

11. The Proteid-like Substances of the Living Cell are constantly disintegrating. - Actual study of the excretions of the human body shows that under all conditions of its life nitrogenous substances and sulphates are given off. Since the nitrogen and sulphur which these contain are found only in the proteids of the food, it follows that there is a constant disintegration of proteid going on in the body. This is observed during fasting and even when an abundance of fats, carbohydrates, salts, and water, but no proteid, is taken in the food. The disintegration of proteid seems to be as truly a part of the life of the body and of its constituent cells as is the consumption of oxygen; and all the facts show that whether we are doing muscular work or mental work, whether we are asleep or awake, whether the weather be warm or cold, in short, no matter what may be the conditions of life, this disintegration of proteid is going on in every living cell. We shall subsequently learn that it is greater at one time than at another, but that it never ceases. Hence it follows that proteid food, which alone contains the nitrogen and sulphur needed to make good this loss, is an indispensable part of the diet; and it has been shown that when their diet contains no proteid, animals starve as surely as they do when they eat no food at all. Fats and carbohydrates, on the other hand, may be excluded from the diet without fatal, and sometimes without harmful results.

12. The Effect of Proteids and Non-Proteids on the Consumption of Material in the Body. - The amount of coal or other fuel burned in an open fire depends to a large extent 
on the amount put on the fire, and is independent of the need for heat in the room. Is this true of the food taken into the body?

Actual study of the subject has shown that the proteid and the non-proteid foods differ greatly in this respect. When more proteid is eaten, digested, and absorbed, more proteid is consumed in the body and more waste products produced. Here there is some resemblance to an open fire. Feeding more of proteid does actually lead to greater oxidation of proteid in the body.

With the non-proteid nutrients this is not true, or at least not to the same extent. The body uses only as much of them as its needs require, or but little more than this, and stores the excess for future use. It behaves toward them somewhat as the fire would behave toward the fuel if, independently of the amount of fuel fed, it burned only so much as was necessary to heat the room to the proper temperature. The behavior of the body toward them suggests the action of the automatic regulator which closes the draft of a furnace as the temperature of the room rises, and so limits the consumption of fuel according to the need for heat. The body, then, behaves toward proteid somewhat as an open fire does toward the fuel put upon it, while in its behavior toward fats and carbohydrates it is more.like a self-regulating furnace.

13. The Relation of Proteid Feeding to the Quantity of Proteid-like Substance in the Living Cells of the Body. - We have shown in the last paragraph that when more proteid is eaten more is consumed in the body. We may now point out another important relation of proteid to nutrition. It has been found that if a man, whose food meets all requirements of his body so that he is in good health, does full work, and is neither gaining nor losing in weight, increases the amount of proteid in his food, not all the extra proteid is disintegrated into waste products, but that some of it is 
retained in the body, apparently increasing the total amount of living cell substance. If now the increased proteid diet be continued from day to day, it will be found that the amount of proteid thus reserved or the amount of living cell substance thus gained becomes less from day to day, until finally the amount of proteid disintegrated exactly equals that absorbed. The condition thus finally attained is known as nitrogenous equilibrium, which means that the nitrogen of the intake (proteid food) exactly equals the nitrogen of the output (urea, etc.); and so long as the same amount of proteid is fed this state of nitrogenous equilibrium continues. Such experiments, therefore, prove that increasing the proteid of the diet not only leads to increased proteid consumption but also to an increase of the stock of proteid-like material within the cells of the body.

Furthermore, if after the establishment of nitrogenous equilibrium the amount of proteid in the diet be decreased, the body for a time excretes more nitrogen than it takes in; that is to say, it consumes all the proteid of the food and, in addition, some of the proteid-like substance of its own living cells. Obviously the amount of proteid within the living cells of the body depends on the amount of proteid absorbed from the alimentary canal. We may, therefore, conclude that (1) the amount of proteid digested and absorbed is a determining factor in the amount of proteid consumed or disintegrated in the body ; and (2) it is a determining factor in the proteid contained within the body. In other words, to maintain a given amount of proteid in the living cells, a certain amount of proteid is necessary in the food; less than this will lead to loss of proteid, more will increase it.

With the non-proteid foods the case is different. Eating more fat or carbohydrate does not necessarily change the amount of these substances consumed or oxidized. The body seems, on the whole, to use as much of them as it 
needs (see pp. 213-217), and to store up the rest chiefly as fat. Here then is the second point of difference between proteid and non-proteid foods ; the amount of the former which the body disintegrates varies conspicuously with the amount fed, while the disintegration of the latter is chiefly determined by other causes.

14. The Effects of Proteids and Non-Proteids respectively on the Storage of Fat within the Body. - The third point of difference between proteid and non-proteid food is that the latter readily lead to the storage of fat, - in other words, that they are "fattening foods"; while the former are converted into fat only to a much smaller extent, and normally are possibly not turned into fat at all. This subject will be more fully discussed in the next chapter.

15. Summary. - In three respects, then, the proteid and non-proteid foods exert different nutritional effects upon the body: first, only the proteids can repair waste of living tissue; second, the mere act of eating more proteid leads to increased proteid disintegration and to increased chemical change in the body; while the excess of nonproteids over and above the needs of the body for power and heat is stored away for future use; and third, fat is formed chiefly, if not entirely, from the non-proteid foods.

On the other hand, many requirements of the body may be met by either class of food stuffs, though one may do better than the other. In the previous chapter we found, for example, that both proteids and non-proteids may supply power and heat. In some respects it is a matter of indifference whether we eat proteid or carbohydrate or fat; in other respects it is a matter of considerable importance.

16. Other Considerations with Regard to Special Effects of Proteids and Non-Proteids. - We have repeatedly spoken above of the amount of a nutrient "eaten, digested, and absorbed," instead of merely the amount "eaten." This was done to emphasize the fact that the nutritional effects 
we have described are produced by the material which passes into the blood by absorption, and not merely by that which is swallowed. For, in the first place, a part of the food eaten is not digested at all, but leaves the body with the bowel discharges; the amount of food thus lost to the organism varies with different individuals. Observations are on record which show that at times as much as one third of the proteid eaten thus escapes the action of the digestive juices. Nor is this all. We have seen that more or less of the food eaten is consumed within the intestine, and sometimes even within the stomach, by bacteria. Proteids, carbohydrates, and fats thus destroyed by bacterial action produce none of the effects described above. These effects are produced only by those foods which are digested and absorbed into the blood.

These considerations partly explain the striking fact that some people remain lean although they are "great eaters," while others grow fat on a comparatively meager diet. In other words, it is one thing to take food into the alimentary canal and quite another thing to digest, absorb, and in acceptable form distribute it to the living cells of the body.

Another point is of very considerable practical importance and should be reckoned with in the choice of food. The carbon dioxide and water into which the fats and carbohydrates are oxidized in the cells are at once taken up by the blood and carried directly to the lungs and other excretory organs where they are got rid of. With the waste products of proteid disintegration the case is different. Proteid does not break down in the cells at once into urea, etc., but largely into intermediate products; these are thereupon taken to other organs, chiefly the liver, where they are chemically changed into urea; and it is only after this series of chemical changes that the proteid waste is carried in its final form to the kidneys for removal 
from the body. Evidently it is a more serious task to eliminate the waste products of proteids than those of fats and carbohydrates. While the body is no doubt quite capable of caring for large amounts of these proteid wastes, there are reasons for thinking that there is such a thing as overburdening the liver and even the kidneys with them. Consequently, although proteids yield heat and the power for muscular work as readily as do carbohydrates, it may be better to use carbohydrates for these purposes rather than proteids.

Finally, questions of digestibility often complicate the problem of meeting the nutritional demands of the body. It may well be that of two diets we may choose one rather than the other because it is more digestible, and not because, when digested, its constituents more perfectly meet the demands of the body. Thus 100 grams of proteid +100 grams of fat +300 grams of carbohydrate have together the same approximate nutritional value as 100 grams of proteid +50 grams of fat +415 grams of carbohydrate (since 50 grams of fat are equivalent to 115 grams of carbohydrate); but a man whose digestive system cares for fat with difficulty might well choose the latter diet rather than the former.

17. Are there Special Foods for Special Organs or for Special Work? - This question is raised by the flaming advertisements of "nerve" foods, "brain" foods, foods "good for muscle," or for other parts of the body, which are often seen in public journals or public places. The student who has mastered the foregoing chapters of this book hardly needs to be told that in the swiftly circulating blood all cells of all tissues have a common ration and practically sit at one table, very much as officers and soldiers do while engaged in actual warfare. No one then thinks of giving to officers brain food and to common soldiers muscle food, but rather to both a good, general, "all-around" food supply. 
Moreover, any one who will recall the complex chemical processes of digestion cannot have forgotten that all foods are greatly changed during that process, so that if a special food is eaten, there is no evidence that it ever reaches any special cells, such as those of brain or muscle, in or even near its original form; or, if it should reach the desired organ or tissue, that it would be either wanted or used there. Very likely some persons sometimes take special foods or drinks with apparent or temporary advantage, due to change of diet or to a real increase of food supply; but that their improved feeling or condition is due to any special feeding of brain or muscle or other tissue is unlikely; and the same or even better effect could probably have been obtained by the same amount of attention paid to a change of diet, increased muscular activity, rest, sleep, or any one of a number of the factors of hygienic living.

\section{Flesh, Fat, and Glycogen}

18. Flesh and Fat. - People of the same height differ greatly in weight. We recognize this fact in the use of the adjectives "stout" and "thin," or "lean." We also recognize the fact that a man of heavy build may owe his weight to fat or to muscle. Sometimes we hear a man say, "I weigh the same as formerly, but I have got less muscle and more fat," or vice versa. This expresses a physiological distinction which is not always made in our common use of words; for when we say some one is "stout" or "fleshy" or "corpulent," we do not state whether his greater weight is due to muscle or to fat, although we more commonly mean that it is due to fat.

It will be convenient for our purposes if we agree upon a definite use of terms to express this difference. In a piece of beefsteak there are three well-known parts, - the lean meat, the fat, and the connective tissue; and this 
composition of one of the organs of the body corresponds to the composition of the body as a whole, which consists of three kinds of material: (1) the living cells, represented by the muscle fibers, gland cells, nerve cells, etc.; (2) the connective and supporting tissues, such as tendon, ligament, bone, and cartilage ; and (3), stored away within certain cells, lifeless matter or food material, which may be called upon to supply in part the needs of the body when food is not being absorbed from the alimentary canal. Fat is one of the two most important members of the third class.

With the second of the above classes (connective and supporting tissues) we are not concerned in the present chapter, since they are not rapidly consumed in the work of the body and hence do not suffer large loss of substance, which must be made good from the food. With the first and third groups, however, we are immediately concerned, since they stand in intimate relation to the cellular activities, and so are subject to chemical change.

To the essential living substance of the cells, which we have seen is built chiefly from proteid, we give the name flesh, and it is only with this meaning that we shall subsequently use the term. Flesh, then, would include the living substance of the muscle fibers, of the cells of the pancreas, kidney, liver, nervous system, etc.; in short, all the material of the cell which is essential to its life. It does not include the lifeless matter which may be stored away within these cells. Thus a cell of the liver may contain drops of lifeless fat in addition to its living flesh.

19. The Increase of Flesh. - The fat and flesh of the body may vary in amount at different times; one of them may change while the other remains constant, or both may change simultaneously. The amount of each is, to a large extent, independent of that of the other; and we have now to inquire what is known about the conditions which 
determine how much flesh and how much fat the body contains at any given time.

We have already described in the preceding chapter one of the conditions of the storage of flesh in the body, namely abundant proteid feeding. Simply feeding more proteid certainly leads at times to the formation of more flesh. The student will review what has been said on page 223 . But it is by no means certain that this is the only condition that leads to increase of flesh. The growth of muscles by use suggests that under conditions of active muscular work more of the proteid eaten is retained in the body as flesh, and less broken down to waste products, than when little muscular work is done. The whole subject needs further elucidation.

20. The Hoarding of Inactive or Lifeless Food Material. The Storage of Fat. - The body not only adds from time to time to its stock of living flesh, but also stores lifeless material for future use, and that, for the greater part, in two forms, - fat and glycogen. Fat may be stored as drops of oil in the cytoplasm of any cell of the body. Muscle fibers, for example, contain at times large quantities of this substance, and are then said to have undergone fatty degeneration. Under normal conditions, however, the presence of considerable quantities of fat in muscle fibers or nerve cells or most gland cells is unusual. In the cells of connective tissue, on the other hand, fat is readily stored under normal conditions, and the adipose tissue or fat of the body is simply connective tissue whose cells are loaded with droplets of fat. Figs. 83-85, with their explanation, will show how this takes place. But while fat may be normally stored in any of the more open connective tissues, it is especially in the subcutaneous tissue, the great omentum, the mesentery, and some other situations that its chief storage takes place. From these storehouses it is drawn upon as a reserve food material when the immediate supply 


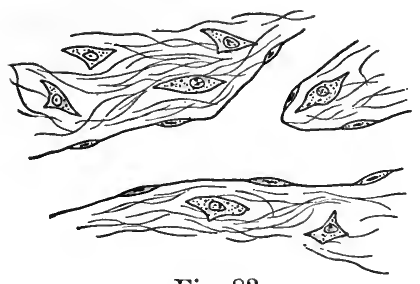

Fig. 83
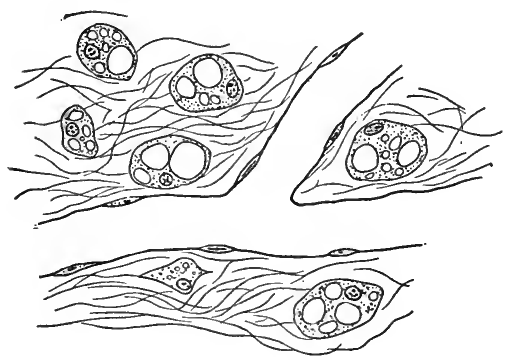

FIG. 84

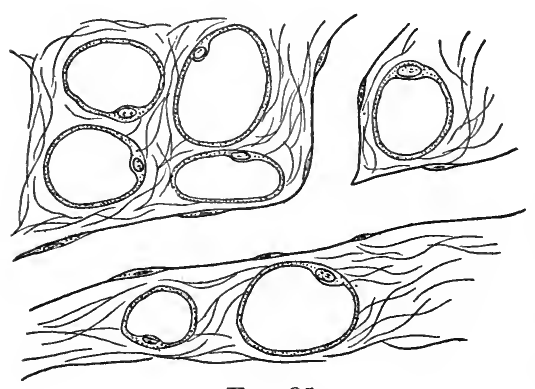

Fig. 85

Figs. 83-85. Three successive stages in the transformation of ordinary connective tissue into adipose tissue

A portion of the capillary network is shown, surrounded by the fibers, among which are several cells. The accumulation of fat droplets in the cell cytoplasm is shown in Fig. 84, and the fusion of these to form one large droplet, surrounded by the cytoplasm, is seen in Fig. 85 of food from the alimentary canal becomes inadequate for the work of the body. The exact mechanism by which it is stored in a cell at one time and discharged at another is not fully understood. Some of the conditions under which it is accumulated, and some of those under which it disappears, are known; but we do not know the whole story. Some people lay up fat in larger quantities than others on the same diet, and apparently while doing the same amount of work; and some keep lean under conditions apparently the most favorable for growing fat.

It was formerly believed, and is still sometimes supposed, that the animal body forms fat only from the fat of the food; that to get fat we must eat fat. This was disproved by a number of experiments, especially one by Liebig, who kept 
account over a long period of the fat in the food supplied to a cow, and found that the fat given off in the cow's milk far exceeded that in her food. In another experiment four pigs out of a litter of eight were killed and the total amount of fat in their bodies determined. The other four pigs were fattened for a time, then killed, and the fat in their bodies eventually determined. Assuming that the second set of four pigs originally had the same quantity of fat as the first four, the difference between the two quantities of fat found would give the quantity of fat the last four had stored up. Meantime, strict account had been kept of the fat supplied in the food of the last four, and it was shown that for every 100 parts of fat fed to them these pigs had laid up 472 parts of fat. They had evidently manufactured fat from some substance other than the fat in their food.

21. Fats can be stored from Fats and Carbohydrates in Food. - There is no doubt that fat may be both stored away and manufactured from the fats in the food. There is also no doubt that large quantities of fat may be and often are manufactured and stored from the carbohydrates (sugars, starches, etc.) of the food; so that, while there is some truth in the idea that one may get fat by eating fat, it is equally true that we can get fat by eating other foods.

22. Are Proteids a Source of Fat? - Whether fats are normally made in the body from proteids is a more difficult question. There is no undisputed case on record of such manufacture and storage; and while the facts do not yet justify us in denying the possibility, there can be little doubt that such transformation does not take place to any great extent, and it is possible that in the mammalian body it does not normally occur at all.

Fats, then, are manufactured readily from fats and carbohydrates, and sparingly, if at all, from proteids. Their disappearance during starvation, when they are drawn 
upon to supply power and heat for the body, shows that they serve as a true reserve food material. They are a kind of food capital or hoard, saved and laid up by the body against a rainy day.

23. The Hoarding of Inactive or Lifeless Food Material. The Storage of Glycogen. - In many cells of the body, but especially in those of the liver and to a less extent in those of the skeletal muscles, there is found a carbohydrate substance known as glycogen. 'This substance belongs to the same group of carbohydrates as starch and dextrines (see Chapter VIII), and is sometimes called animal starch. Like them it is changed into sugar by the action of saliva and pancreatic juice, whence its name ( $\gamma \lambda v \kappa u ́ s ~ s w e e t ;-\gamma \epsilon \nu \eta^{\prime} s$, former). The same change occurs on the death of the cells in which it is contained, the sugar thus formed giving to such tissues a sweetish taste. This is often noticed, for example, in liver and in scallops (the shell muscle of Pecten). The total amount of glycogen in the human body may reach 100-200 grams (3-7 ounces), one half of which is concentrated in the liver and the other half scattered about in the other tissues of the body.

Experiments have shown that glycogen is not formed from the fat in food; that it is formed in small quantities from proteid; while its chief source is the carbohydrates of the food.

The blood may be said to be always sweet, its constant percentage of sugar (2-3 grams per 1000 cubic centimeters of blood plasma) being a striking fact, and one that we should hardly have anticipated. One might suppose that the sugar in the blood would increase, as does the amount of fat, during active digestion and absorption; and that, after digestion had ended, it would diminish. As a matter of fact the amount of sugar in the blood remains practically constant for many hours after the completion of digestion, and this despite the fact that the tissues are constantly abstracting 
sugar from the blood. Evidently the blood must be supplied with sugar from some other source than the alimentary canal, and there must be somewhere in the body a compensating mechanism controlling the sugar supply of the blood.

Experiments have shown that sugar is absorbed from the alimentary canal entirely by the intestinal blood vessels. It must pass, therefore, through the liver by the portal vein (see Fig. 58, p. 125) before going to the rest of the body. The liver, thus standing at this great gateway to the circulation, would seem to act as the carbohydrate storehouse, or savings bank, of the body. Any excess of sugar in the portal blood is there transformed into glycogen and deposited, saved until it is needed, and then "paid out," as sugar, when the ready supply is exhausted. Other tissues doubtless aid in preventing an undue richness of sugar in the blood, acting likewise as temporary storehouses for this form of food. If it were not for some such provision as these together make, the eating of a quantity of candy or of maple sugar might easily add to the blood enough sugar to damage the tissues.

The body is thus not immediately dependent upon the food which is being absorbed from the alimentary canal at the time. In the glycogen and fat it has within itself a stock of hoarded non-proteid material upon which it may call to meet its requirements for power and heat, and by which it is protected against temporary shortage of these nutrients in the diet. When, on the other hand, the proteid of the diet is lacking, the body can make good the deficit only by yielding up its own flesh.

\section{The Choice of Food and Nutrients}

When we buy food for the table we do not, of course, ask for proteids, carbohydrates, fats, salts, etc. Foods sold in markets are mixtures of these nutrients in varying 
proportions. Moreover, most meals consist of mixtures of several foods, and the foods used at one meal differ from those used at another, so that the diet becomes what is known as a "mixed diet." In this way two results are secured: first, the food is rendered more appetizing, - an important physiological and hygienic consideration; and, second, we avoid the undue preponderance of one nutrient over another, which would almost surely follow the use of a single kind of food. Lean meat, for example, contains a large amount of proteid and little fat or carbohydrate; potatoes, on the other hand, contain little proteid, but a large amount of carbohydrate; by using the two at the same meal we obtain better proportions of proteid and carbohydrate; and if butter be used on the potatoes, we add fat to the dietary.

These three nutrients play different rôles in nutrition, and it is not a matter of indifference whether any one of them is absent, deficient, or present in excess. In other words, while the proportions of the different nutrients is not the chief consideration in the selection of food, - such things as digestibility, flavor, bulk, amount of indigestible material, etc., also entering into the decision of the question, - it is nevertheless an important consideration, and there can be no doubt that some persons are poorly nourished because they err in this respect. We have, therefore, to consider when we may need more proteid, or more fat, or more carbohydrate in the food in order to meet properly the different conditions of daily life, such as prolonged or hard muscular work, exposure to cold or warm weather, mental work, and so on.

24. The Effect of Occupations and of External Conditions upon the Consumption of Food. - Since it is the function of food to make good the material losses of the body, we naturally ask, first of all, how the various occupations and conditions of life influence the excretions, for this would 
give us some information as to the material whose loss must be made good. Many experiments have been made to determine this ; but their results may be briefly summarized by saying that only three things seem to affect directly the consumption of food in the body, viz. muscular activity, changes of temperature, and the act of feeding 'itself. The changes thus produced in the quantity and quality of the chemical activities of the body have already been fully described in previous chapters. Muscular work increases the oxidation of fats and carbohydrates, and when these are not present in sufficient quantities to yield energy for the work, it also increases the disintegration of proteid. Exposure to cold seems to have the same effect. As to the influence of the third factor mentioned above, - the act of feeding itself, - it will be recalled that the quantity of proteid disintegrated depends on the quantity fed, and that the quantity of fat stored depends chiefly on the quantity of fat and carbohydrate eaten.

25. Feeding for Muscular Activity. - The hard-working man must be well fed if he is to do his best work. Not only is his appetite greater, but he cannot continue hard. work successfully for any length of time on a meager diet. No further argument is needed to show that the diet in muscular work should be relatively abundant. But should its character also be changed? From the fact that when the diet remains unchanged muscular work increases the oxidation of fats and carbohydrates, while it affects that of proteid only as the other nutrients fail, some physiologists have concluded that the increased food taken should be non-proteid rather than proteid. It is, however, actually found that when those who are engaged in hard work are free to choose their diet according to the dictates of appetite, they use more proteids as well as more carbohydrates and fats. Similarly it has been found that when a race horse is being fed for the track he does better work 
when fed on those grains which (like oats) contain rather large proportions of proteid than on those which (like corn) contain small proportions of proteid. This probably means that any animal, man or horse, doing hard work is better off for having more living flesh with which to do that work easily, and this flesh can be gained (see p. 222) only by eating more proteid. Moreover, when once the flesh weight has been increased to the desired amount it can be maintained only by continuing with the same quantity of proteid food (p. 223). While, therefore, it is probable that the greater part of the increase of the diet for steady and continued muscular work should fall on the fats and carbohydrates, it is reasonable to eat more proteid and thus maintain a greater flesh weight. When, on the other hand, the "greater work" consists of a day or so of increased effort by one who is otherwise engaged only in moderate muscular exertion, there is not the time for an increase of flesh, and it is not clear that any nutritional gain would accrue from eating more proteid after the day of work; at the same time there is no reason to think that harm would result from a moderate increase of this nutrient.

In this connection it is easy to see why muscular work should antagonize the accumulation of fat in the body, since it involves the consumption of those foods (carbohydrates and fats) which, when present in excess of requirements, furnish the material for the manufacture and storage of fat. Hence the most reasonable treatment of obesity is to limit the use of non-proteid foods and to indulge in hard muscular work.

26. Feeding for Cold and for Warm Weather. - The appetite is normally better in cool weather than in warm, and this corresponds to the greater need for food, one function of which is to supply by its oxidation the heat necessary to maintain the temperature of the body. Moreover, we tend to do less muscular work in warm weather 
than in cold, and this is an important factor in the problem. The needed increase of heat on cold days may be obtained from the increased use of fats and carbohydrates, although there would seem to be no good reason why proteids may not also be increased. We have seen that eating more proteid readily leads to the oxidation of more proteid, and so to the production of more heat. Hence, in warm weather, when the body finds difficulty in securing the needful output of heat, it is unwise to indulge too freely in proteid foods, since their very presence within the system necessarily increases the amount of heat to be got rid of. There is a widespread popular impression that it is not wise to eat as much meat in summer as in winter, and the impression is probably correct, although it applies also to vegetable foods, like beans and peas, which contain relatively large quantities of proteid. For a discussion of the relative value of fats and carbohydrates in cold and warm climates, see p. 216.

27. Feeding for Mental Work and Sedentary Occupations. - Mental work requires special attention to diet chiefly for two reasons: in the first place it involves for the greater part a sedentary life, and in the second place the processes of digestion are liable to interfere more or less with the most effective working of the brain. Thus far no effect of mental work upon the character of the chemical changes within the body has been conclusively proved beyond those effects which are obviously the result of the sedentary life which accompanies it. Hence feeding for mental work is largely a question of feeding for the sedentary life. We do not then need so much food, and especially do not need so much non-proteid food. To eat as much carbohydrate and fat as when we are leading an active life would be to have these nutrients present in excess, and thus to lead either to the storage of fat or else to overtaxing the digestive organs which, in the absence 
of reasonable muscular activity, may not be able to do their best work.

This being the case, it is evidently advisable to use rather easily digestible food, especial attention being paid to its proper preparation in cooking. Pork and beans, for example, may do well enough for men working on a farm in cold weather, but they are not the best foods for a clerk who is confined to his office during the greater part of the day, or for the college student. Moreover, special attention should be paid to the eating of food. "Quick lunches," inadvisable at all times, should then be avoided altogether, and the food should be well chewed. These matters will be fully discussed in the chapter on Alimentation, or Right Feeding (Part II).

There is also every reason to believe that mental activity, especially when it involves certain feelings or moods, may exert no inconsiderable effect on the nervous processes of digestion. We have seen that the secretion of gastric juice depends to a large extent upon the enjoyment we obtain during the act of eating; and the man or woman whose absorption in mental work, or in the cares and annoyances of life, is so complete that the food is eaten with little more attention to its taste and flavor than if it were so much paper, is certainly laying the foundation of indigestion, and so of bad nutrition.

The above considerations point clearly to the need of special attention to diet during mental occupations, using that term in its broader sense to include all occupations which involve mental rather than muscular work. It must not be supposed, however, that we can correct the effects of a sedentary life by mere attention to diet. As we shall show in Part II, the human mechanism is adapted to a life of muscular activity, and on the whole cannot remain healthy for a long time without reasonable use of this factor of health; it is also adapted to the use of 
a reasonable amount of rest, and cannot thrive when subjected to undue nervous strain. There are times in every life when we cannot get all the muscular activity and rest we need, and when we must necessarily be subjected to severe nervous strain. When such is the case the special measures we have outlined should be taken with regard to diet; but we should not be deceived into thinking that attention to diet can in the long run replace other essential factors of healthy living. In other words, normal, healthy nutrition depends not alone on the amount of proteids, fats, and carbohydrates we eat, not alone on their preparation, not alone on the manner in which we take them, nor alone on all these things taken together; it depends no less on the hygienic conduct of life in all respects. The best nutrition is in the end the same thing as the best health, and this is assured only to those who "keep the whole law."

28. General Conclusions as to the Choice of Nutrients. We may sum up as follows. The amount of fats and carbohydrates needed depends primarily on the amount of muscular work done, on the amount of heat needed to maintain the constant temperature of the body, and (in cold climates) on the quantity of subcutaneous fat desirable to assist in preventing undue loss of heat from the internal organs. The amount of proteid needed, on the other hand, is not so easily determined. Increased proteid feeding increases proteid destruction within the body and leads to a greater flesh weight. The human machine will continue to live and do its work on very different quantities of proteid in the food; that is to say, when it disintegrates very different quantities of proteid and when it possesses very different flesh weights. Just what quantity is best is a question to which the facts at present at our disposal do not justify a final answer. But we are within safe limits when we say that it is well to avoid both extremes; too much proteid 
probably throws undue work on the organs of excretion; too little leads to an undesirable reduction in the living flesh or working equipment of the body.

In the foregoing pages we have refrained from giving definite figures as to the amount of each nutrient needed under different conditions of life, partly because such figures mean but little to those who can go no more extensively into the subject than the majority of those who read this book, but chiefly because any figures we may give are apt to be misleading. So much depends on individual peculiarities of height and general build of the body, and so much on the occupations and other conditions of life, that to deal with the subject effectively would take us far beyond the limits of the present work. In actual practice we cannot depend upon the chemical balance and the use of dietary tables to secure the correct proportion of nutrients in our food; but knowing the general composition of our foods, we can by judicious selection vary the amount of nutrients, and thus, with the help of appetite, adapt our diet more or less successfully to the varying conditions of life. To change the proteid we have only to change the quantity of those foods like meat, eggs, and beans, which contain relatively large quantities of proteid; and we similarly vary the quantity of non-proteid by changes in the consumption of foods like bread, potatoes, rice, butter, etc., which contain relatively large amounts of carbohydrates and fats.

29. The Question of Vegetarianism. - Closely connected with the matters discussed in the present chapter is the question whether the human machine thrives best on a mixed diet or on vegetable food alone. No one would seriously suggest that man should live on animal food alone; it is true the Eskimos do this, but with them it is a case of necessity, and no one would recommend to people at large the adoption of their diet. In animal foods 
the non-proteid nutrients are represented chiefly by fat, and we have every reason to believe that an undue amount of fat in the diet severely taxes the digestive powers; animal foods also contain too little indigestible residue to give the proper stimulus to the movements of the alimentary canal.

Vegetable foods, on the other hand, generally contain enough indigestible cellulose to meet the latter requirement, and most of them also contain all three nutrients. With few exceptions, however, the carbohydrates are present in marked excess. In order to obtain from such foods the proper quantity of proteid, it is generally necessary to eat too much of carbohydrate. Some vegetable foods, notably peas and beans, are not open to this objection since, although they have a very high percentage of carbohydrate, they have also a relatively higher percentage of proteid; and it is possible by judicious selection and mixture of foods to obtain a nutritious diet from vegetable foods alone.

This is much easier to do if milk and eggs are added to the diet, and many people who call themselves vegetarians do, as a matter of fact, make extensive use of these animal foods. With them vegetarianism means simply the use of a diet from which meat is excluded. Such persons are not really inconsistent, as is often charged, for the question of vegetarianism is practically the question whether man should or should not eat meat.

Against the use of meat two different lines of argument are used. The first is that man has no right to take animal life when he can obtain his food without doing so. With this argument we have here no concern, since we are dealing solely with the physiological and hygienic aspects of the subject. People who have conscientious scruples against the use of meat may fulfill every requirement of nutrition without going contrary to the dictates of conscience. 
The second argument is that animal food is not a healthy or wholesome food, and to this we must give some attention. It is claimed that while the animal proteids and fats may be unobjectionable, meat contains other substances which exert an unfavorable action on the body. Although it is true that meat contains small quantities (about one per cent) of organic substances other than proteids, carbohydrates, and fats, it has not been proved that any of them are harmful to a healthy man or woman. Nor is it an answer to this argument to urge that in gout and rheumatism meat is often limited or even prohibited by the physician. No one - not even a vegetarian — would suggest that starchy foods should be given up because persons suffering from diabetes must use them sparingly. In other words, it has not been shown that the eating of meat is the cause of rheumatism, nor even that it is one of the causes of the disease; on the contrary, it is more in accord with our knowledge of the subject to regard the disease as originating in other causes; and, only when it has once obtained a foothold in the body, is it rendered worse, or its treatment more difficult, by the use of meat. It does not seem, therefore, that any valid hygienic reason has yet been given for excluding all meat from the human dietary.

Nevertheless, as a standing protest against some careless habits of feeding, vegetarianism has a certain value. Too frequently the quantity of meat eaten during a meal is out of all proper proportion to the bread and vegetables. This means that the proteid of the diet is in excess, that the food is too concentrated, and that there is an insufficient quantity of indigestible waste to exert a proper laxative effect on the movements of the intestine. Take, for example, some of our "course" dinners, - oysters, soup, fish, roast, game, dessert (perhaps mince pie), crackers and cheese, coffee. Here nearly every course consists of some form of proteid, while only a few contain, in addition, a 
small quantity of vegetables. A very common method of ordering meals at a restaurant is to order a heavy por-. tion of meat, - a sirloin steak or mutton chops, - and to make the meal largely from this proteid food, while the vegetables play only a minor rôle. It is against such things as these that vegetarianism properly protests; but there seems to be no good reason why all should abstain from meat because some people eat it to excess. In general, poorly cooked vegetables are less digestible than poorly cooked meat. Hence to attempt to live on a purely vegetable diet, under the actual conditions of modern life, is to run serious risk of impairing the digestion. We should welcome all attempts to improve the preparation of vegetables and all other foods by better cookery, and vegetarians have done much to help forward this reform. But so long as the actually available food is cooked as it is, it is wiser to continue the mixed diet of meat and vegetable foods which man has freely chosen in the past, and against whose reasonable use no valid objections have yet been urged. 


\section{CHAPTER XIV}

\section{SENSE ORGANS AND SENSATIONS}

\section{The Human Mechanism a Conscious Mechanism.}

Thus far we have repeatedly compared the human mechanism with lifeless mechanisms, and the points of similarity are most interesting and instructive. In the supply of power, the elimination of wastes, the interdependence and coöperation of parts, the adjustment to the changing conditions of work, and in many other respects the resemblance holds good. But in one respect there is no likeness whatever. When a human mechanism is not in good working order or is tired, it may be aware of the fact; when an engine is damaged in any way, the engine does not know it. Events taking place in the living animal body arouse in it, and in it only, conscious sensations.

Sensations are always called forth by the condition of some organ or by the condition of the body as a whole. When several hours have passed since the taking of food we feel hungry; or of drink, we feel thirsty; when anything touches the skin a sensation of touch is aroused; if it presses very hard, that part of the skin feels painful; if the tongue is acted upon by sugar or salt, we get a sensation of taste; if light enters the eye, it produces conditions in that organ which arouse in us sensations of color. In all these cases the conscious sensation is due to the condition of some part of the body.

2. The Reference of Sensations. - Sometimes we refer the sensation to the part of the body which is first affected, or to the body as a whole, and sometimes we refer it to 
external objects. Thus, if in driving a nail the hammer misses the nail and hits a finger, we refer the pain to the finger and not to the hammer; and we similarly refer sensations of hunger and thirst to the body and not to external objects. If, on the other hand, the skin is cooled by a piece of ice, we do not say that the skin is cold, but that the ice is cold; we refer the sensation to the external object which causes it, not to the skin in which it actually originates. In the case of the sense of sight, this reference of the sensation to the external object which sends light into the eye is so complete that unless we stop and reflect upon it, we do not realize that it is the condition of the eye of which we are conscious, rather than the condition of the external object at which we are looking.

3. Sense Organs. - A few sensations, like pain, are aroused by the condition of most, if not all, parts of the body; there is no one organ set apart to produce them. Some, like hunger, although at times more or less general in origin, are commonly aroused by the condition of some one organ ${ }^{1}$ which ordinarily performs other functions. Other sensations arise in organs set apart for the purpose and constructed to react to only one kind of stimulus (special sense organs, or organs of special sensation). To this latter class belong the eye, the ear, the olfactory mucous membrane of the nose, the touch organs in the skin, etc. We therefore speak of general sensations and special senses, although no sharp line of division can be drawn between the two.

4. The Brain the Seat of Sensation. - In all cases however the sensation, although originating elsewhere, is developed in the brain and not in the sense organ. If the optic nerve be cut, blindness ensues, although light falling on the retina produces the same effect in the eye itself as when the nerve is intact; it even starts nervous impulses

1 In the case of hunger, the stomach. 
toward the brain; but, since these impulses go no farther than the cut, they excite no sensation of light. And the same thing is true of other sensations. Conversely, after the amputation of a limb it often happens that sensations are felt, as if they came from the lost member. In this case the stump of the cut nerve is stimulated in some way, and the impulses thus sent to the brain excite the same sensations as if they came from the usual endings of the nerve. When one hits his "funny" or "crazy bone" (i.e. directly stimulates the ulnar nerve) the sensations developed in the brain may be referred to the fingers in which the nerve originates.

In the development of every sensation, therefore, we have to distinguish between $(a)$ what takes place in the sense organ or end organ, $(b)$ the passage of a nervous impulse from this organ to the central nervous system, and $(c)$ the events which the arrival of the nervous impulse excites in the brain. It is only the last $(c)$ that, strictly speaking, we can call sensation. The sense organs and their afferent fibers are merely tributary mechanisms which serve to excite the sensations in the brain. We are not aware that it is the brain which is thus active, for we refer the sensation either to the organ or to some external object.

5. The Sense of Sight. The Eye. - Sight is one of the most highly specialized of the senses. The eye is the only organ in which originate sensations of light or color, and it is a wonderfully constructed apparatus, the function of which is to stimulate the optic nerve by rays of light. It is essentially a living camera in which, by means of a lens, an image of things around us is formed upon the retina; just as in the photographer's camera the lens forms an image on the ground glass or on the sensitive plate or film.

6. Structure of the Eye. - The eyeball consists of three concentric coats surrounding and inclosing transparent 
substances through which rays of light pass to the retina. The outer or sclerotic coat (the white of the eye) is composed of very tough, dense connective tissue, and forms the protecting covering of the eye. Over a small area in front this coat is transparent, and this part of it is known as the cornea. Inside the sclerotic is the middle coat or choroid, richly supplied with blood vessels and containing in its connective tissue large quantities of black pigment, which prevents the passage of light into the eyeball except through the cornea. The choroid lines the sclerotic everywhere except in front, where in the region of the cornea it leaves the sclerotic and projects toward the long axis of the eye as a kind of curtain, the iris, - that part of the eye which is black or gray or blue. The pupil is the dark round opening, or hole, in the iris. Immediately inside the choroid is the third and innermost coat, the retina. This is a thin membrane, not more than one eightieth of an inch in thickness, and lining the chamber of the vitreous humor as far forward as the ciliary region (Fig. 86). The retina is the part of the eye sensitive to the stimulation of light. Here also begin the fibers of the optic nerve, which passes through and perforates the choroid and sclerotic coats behind on its way from the retina to the brain. These and other parts of the eye may be easily seen by dissecting the eye of an ox or sheep.

7. The Lens and the Muscle of Accommodation. - Immediately behind the pupil is the lens, a biconvex, transparent, compressible, and elastic body fastened by a circular ligamentous sheet to the choroid coat immediately above and behind the iris. The lens and its suspensory ligamentous sheet thus divide the eye into two distinct chambers: the one, in front of the lens and behind the cornea, filled with a watery fluid, the aqueous humor; the other, behind the lens and surrounded by the retina, filled with a jellylike, transparent substance, the vitreous humor (Figs. 86, 89). 
The elastic choroid coat is not long enough to reach around and inclose the vitreous humor without stretching, and hence it constantly exerts a steady, elastic pull or tension on the ligament of the lens. This tension flattens the

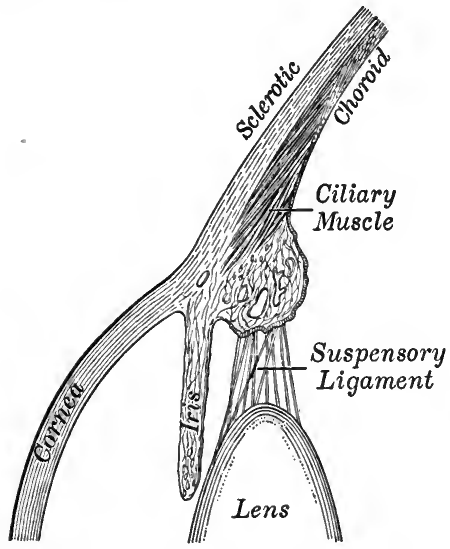

Fig. 86. Vertical section through the ciliary region of the eye

Showing the structures concerned in accommodation (see Section 7). This should be compared with the perspective view into the hemisphere of the eyeball, shown in Fig. 147 compressible lens (i.e. makes it less convex), and the lens is always in this flattened condition in the resting eye ; for example, when one is asleep. The same condition should obtain, as we shall learn, whenever we are looking at distant objects.

The pull of the tense choroid on the lens is, however, overcome at times by the action of the sheetlike ciliary muscle. The fibers of this peculiar muscle originate in the sclerotic coat around and just outside the cornea, and diverge radially outward and backward to end in the choroid beyond the attachment of the suspensory ligament of the lens. Figure 87 shows how the contraction of this muscle, fixed as it is near the cornea, must draw the choroid forward and so ease the pull of the latter on the ligament of the lens. When this happens, the lens, owing to its own elasticity, assumes its independent (more convex) shape.

The curvature of the lens is thus variable, and is determined by the action of this muscle of accommodation. When the ciliary muscle is at rest, the lens is kept flattened by the pull of the choroid on the ligament; when the muscle contracts, this pull is eased off (or slacked) and 
the lens becomes more convex. The entire operation is known as accommodation, and we may now inquire what part accommodation plays in vision.

8. The Formation of an Image by a Lens. - The eye is a camera, in that it forms on the retina an image of objects in front of the cornea; and it is the first essential of clear vision, just as it is the first essential of photography, that this image be sharp, or at least distinct. A simple experiment will show that clear vision of near and of distant objects cannot be had by the eye at the same time. Hold up a pencil or a pen about ten inches from the eye and look first at it and then at some object far away. Both can be seen, but only one at a time clearly ; and often an effort is required to shift from the far to the near object.

The change which occurs in the eye in the act of accommodation is illustrated in the following experiment. A wooden or pasteboard box

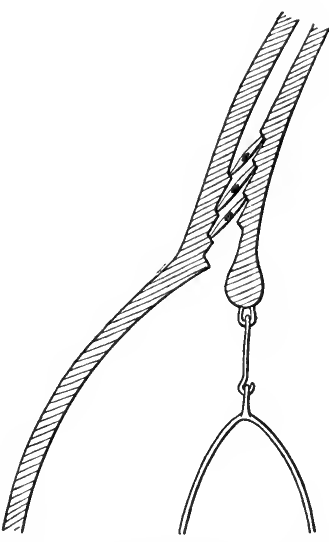

Fig. 87. Diagram of the mechanism of accommodation

The ciliary muscle is represented as three fibers passing obliquely from the sclerotic to the choroid (approximately 8 by 5 by 4 inches) is fitted with a piece of ground glass on one side and provided with a convex lens on the opposite side. This is a rude camera, and some object is now placed at such a distance that the lens forms an image of it on the ground glass, which is now in focus for the object. If, later, the object be moved nearer to the lens, the focus is changed; the image on the glass becomes blurred, and in order to make it distinct it will be found necessary to use a more convex lens.

Essentially the same change occurs in the eye in accommodating for near objects: the lens must be made more convex; 
and this, it will be remembered, involves work on the part of the muscle of accommodation (see p. 248). We can thus understand why, in general, it is too much of "near work," and especially near work necessitating very distinct vision, that tires the eye. The ideal condition of the eye, regarded merely as a camera, is that in which distant objects are focused on the retina when the muscle of accommodation is completely relaxed and the lens is thus flattened to its utmost by the elastic pull of the choroid coat

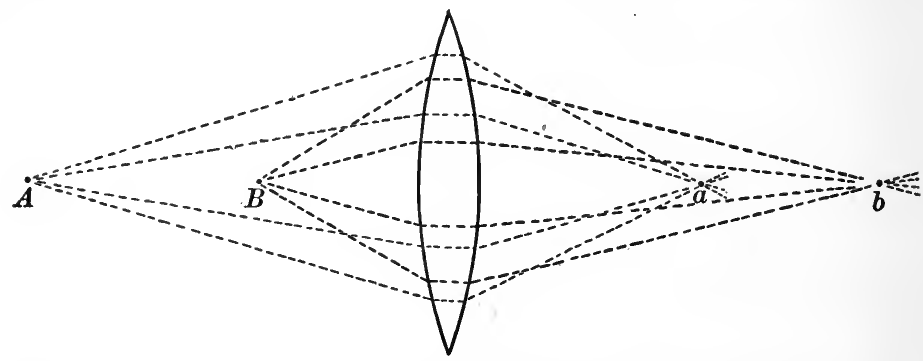

FIG. 88. Action of a convex lens in bringing to a focus the rays of light diverging from a single point

The rays from $A$ are focused at $a$; those from $B$, at $b$

(p. 248); for in this case the eye is rested by looking at distant objects, and works only when looking at near objects. Such an eye is known as an emmetropic eye (Fig. 90, $E$ ):

Unfortunately, not all eyes meet this requirement. The eyeball may be either too short or too long; so that, with the muscle of accommodation relaxed, the position of the perfect focus for distant objects is either before or behind the retina; the eye no longer sees distant objects distinctly when it is at rest, because then the retinal image is blurred. To understand more fully the undesirable consequences of this condition, we must learn how convex lenses produce images of objects.

9. The Action of a Convex Lens on Rays of Light. - The rays of light diverging from a single point and entering a 
convex lens are bent so that all come together again in a point behind the lens, or, as it is said, are brought to a focus. This is shown in Fig. 88, as is also the fact that rays of light diverging from more distant points come to a focus behind the lens sooner than those diverging from nearer points.

Now a lens forms an image of an object because all the rays of light from each point of the object are focused in

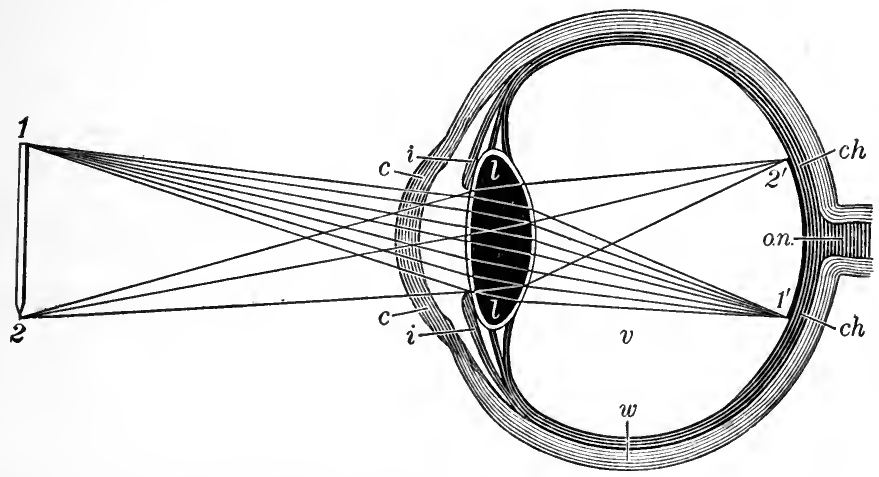

FIG. 89. Diagram showing the formation of an image on the retina 1,2 , the object; $1^{\prime}, 2^{\prime}$, the image of the same; $c$, cornea; $i$, iris; $l$, lens; $v$, vitreous humor; $w$, sclerotic; ch, choroid; on, optic nerve

corresponding points behind the lens. This is shown in Fig. 89, where all the rays diverging from 1 are focused at $1^{\prime}$, all those from 2 at 2 , and those from intermediate points of the object at intermediate points of the image.

If the rays from each point meet in front of the retina and then diverge before reaching the retina, the retinal image is blurred; and the image is also blurred if the retina is so near the lens that the rays from each point have not yet come to a focus. The more convex the lens the more will the rays of light be bent; consequently we use the muscle of accommodation (which makes the lens more convex) to get clear images of near objects (see Fig. 88). 
10. Myopia, Hypermetropia, and Presbyopia. - In the emmetropic eye (Fig. 90, $E$ ) the distance between the retina and the lens is such that light from distant points
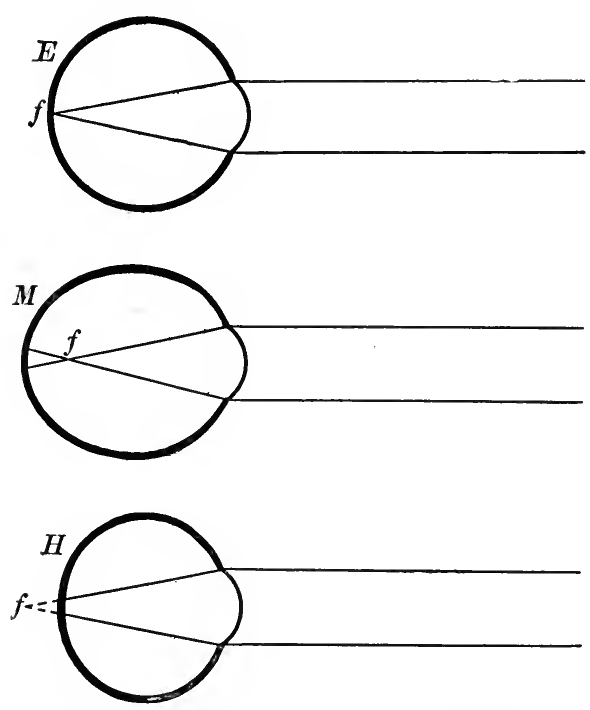

Fig. 90. Course of the rays of light from a distant point

Through the emmetropic $(E)$, the myopic $(M)$, and the hypermetropic $(H)$ eye, the musele of accommodation being relaxed. (The rays diverging from a distant point would enter the eye practically parallel)

clearly. To correct such vision it would be necessary to make the lens still less convex, and this the eye is unable to do. (Why?) Such an eye is known as myopic, or nearsighted, and its defect must be corrected by the use of concave glasses, which act as if the lens were made flatter, and so throw the focus farther back upon the retina. A myopic eye generally has clear sight for very near objects because, as stated above, the nearer the object the farther back is the image formed. 
On the other hand, the eyeball may be too short, fore and aft (Fig. 90,H), so that, when the ciliary muscle is relaxed, light from distant points has not yet been brought to a focus when it reaches the retina (hypermetropia). Such an eye must accommodate not only for near but also for distant objects, and its muscle of accommodation can never rest so long as the eye is being used. Moreover, to see near objects the ciliary muscle must work much harder than in the normal eye, and it often happens that, even with its utmost effort, the rays are not sufficiently bent to focus them on the retina; so that a book, for example, must be held at arm's length to be read. Persons having such eyes form one class of those said to be "farsighted," and their trouble can be corrected by the use of convex glasses.

As old age approaches, changes occur in the lens, in consequence of which it no longer becomes as convex as formerly in response to the action of the muscle of accommodation (presbyopia, from $\pi \rho \epsilon \in \beta v$, old). Some, though not all, results of this condition resemble those of hypermetropia; but the two differ in cause. Hypermetropia is due to shortness of eyeball; presbyopia, to failure of accommodation.

11. Astigmatism. - We have thus far been dealing with those optical imperfections due to improper distance between the lens and the retina. Another and frequently more serious trouble, known as astigmatism, results when the curvature of the cornea (and sometimes of the lens) is not perfectly regular; i.e. when these surfaces are not segments of perfect spheres, but resemble in curvature the side of a lemon. In this case the rays of light from a point are not brought to a focus again in a point behind the lens; and remembering the importance of sharp focusing in securing distinct retinal images, the student will see that this defect must seriously interfere with clear vision. The 
optics of astigmatism are too complicated to be explained in an elementary work, but the defect reveals itself generally in an inability to see with equal clearness lines run-

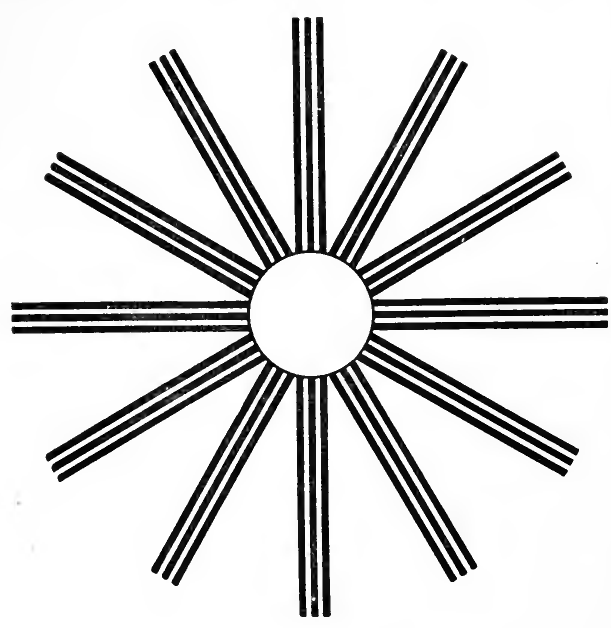

Fig. 91. A test for astigmatism ning in different directions. Thus some of the lines in Fig. 91 will be sharply defined and black while one is looking with one eye at the white center, and others will be blurred and lighter in color.

Astigmatism is of special importance in reading, because the lines of printed letters run in different directions. The effort to see clearly the printed page is often severe, and results in headaches and other general disturbances of health, the true cause of which is often unsuspected. The trouble may usually be, corrected by the use of so-called "cylindrical" glasses, i.e. glasses which compensate the defects of curvature in lens and cornea.

12. Accommodation and "Near Work." - The abovedescribed defects of the eye as an optical instrument may usually be successfully corrected by the use of proper glasses, which should, generally speaking, be prescribed by a good oculist and not by an optician. Glasses may be used for various reasons, - as a matter of convenience, as where a person with slight myopia wears them merely to see distant objects clearly; or of necessity, as when the myopia is more pronounced; or they may serve the much 
more important purpose of relieving the muscle of accommodation of undue work in reading or sewing, and thus of avoiding "eye strain." A hypermetropic eye should always be provided with glasses, since otherwise its muscle of accommodation cannot be rested by looking at distant objects. But since it is near work which requires the greatest effort of accommodation, it is in reading, writing, drawing, sewing, etc., that the eye strain is apt to be greatest. As this kind of work is constantly increasing in modern life, the need for the complete correction of such defects becomes more and more necessary. Those whose occupations require long-continued use of the eyes should see to it that these precious organs are used only under the most favorable conditions, and that all strain is as far as possible relieved.

13. The Importance of Sufficient Illumination, etc.When we try to read in a poorly lighted room we naturally hold the printed page closer to the eye in order to get from its bright parts the greatest possible amount of light, and also because the images thus formed are larger. But this clearly involves more severe work on the part of the muscle of accommodation in order to give the lens its unusual convexity. Such work frequently results in injury to the parts concerned, and should be avoided as far as possible. Many an eye has been injured, especially in early life, by reading too late in the waning light of evening before lighting a lamp.

It is also of the highest importance that printed matter be held steady, as the effort of accommodation is otherwise rendered more difficult. Consequently it is in general a bad thing to read, and especially to read fine or poorly printed matter, on any but the steadiest railroad train. For the same reason, for all kinds of near work an unsteady or flickering light is very objectionable.

14. Visual Sensations. - We have shown (p. 245) that the sensation of sight does not develop in the eye, but in 
the brain, as the result of nervous impulses sent thither over the fibers of the optic nerve from the retina. Just how the light falling upon the retina originates these impulses cannot be discussed here; suffice it to say that the character of the impulse differs according to the color of the light ${ }^{1}$ stimulating the retina; the lens focuses upon the retina a flat, colored picture of the objects at which it is looking, just as a photographic camera does, or as the painter represents a scene on canvas. One part of the retina is thus stimulated by light of one color, and another part by light of another color, or by another shade of the same color; and the different kinds of impulses started in the fibers of the optic nerve ultimately, upon their arrival in the brain, excite in consciousness what we know as visual sensations. The sensations which we get from the retina are therefore primarily sensations of color.

15. Visual Judgments. - But when we look at an object we get more than mere sensations of color. The world does not appear to us as a flat surface, of different colors, like the painter's canvas. When we look at the wall of a room we know that it is a flat surface, and when we look at a box we know that it has not only length and breadth but also thickness. If we were dependent entirely upon the retinal image for our idea of the box, it would look as flat as the wall; that it does not appear so is because we receive other information about the box than that which comes from the retina. We have to accommodate the lens differently for the near and the far edges, and we have learned by experience that this necessity indicates depth, or different distances of different parts of the object. Again, we see the box with both eyes, and the images formed on the two retinas are not exactly the same. One eye sees more of one side, the other eye more of another

${ }^{1}$ In this and the following paragraphs white, black, and gray are regarded as colors. 
side; and while we are not conscious of this fact, we have really learned by experience and by the actual handling of objects that these slightly different sensations from the two eyes are produced only by solid objects. Again, when we look at any point on the near edge of a box the two eyes are converged by their muscles to a greater extent than when we look at a point on the far edge, and we have learned that these different pulls of muscles and positions of eyeballs indicate that the object is not flat, but has depth. The importance of binocular vision in the estimation of depth or distance from the eye is most strikingly illustrated by attempting, with one eye closed, to bring together the points of two pencils held in the hands and moved from side to side at arm's length.

Consequently when we look at anything we get a number of sensations; from the retina, those of color and the position of the color spots with reference to one another; from the muscular efforts of accommodation and of convergence of the eyeballs, those which reveal the property of depth in what we see. And from all of these, fused together and interpreted in the light of experience, we construct a visual judgment of the nature of the object.

16. Optical Illusions. - That our vision is essentially the result of unconscious judgments is strikingly shown by the fact that these sometimes deceive us. Thus the parallel vertical lines in Fig. 92, when crossed by the oblique lines, seem to be inclined toward each other. The retinal images of the lines are parallel, and we falsely judge them inclined, this error of judgment arising from the presence of the oblique lines. In other words, our final idea of the lines does not correspond to their image on the retina.

Many other examples might be given showing that our visual idea of the world around us is not a simple sensation or impression, but an unconscious inference, judgment, or conclusion built up from a number of simple 
sensations taken separately or blended together, and compounded with results of lifelong experience. In looking at a piece of fine silk or cloth we seldom stop to think that its tissue may be resolved into many simple component threads; and in quite the same way we fail to realize that

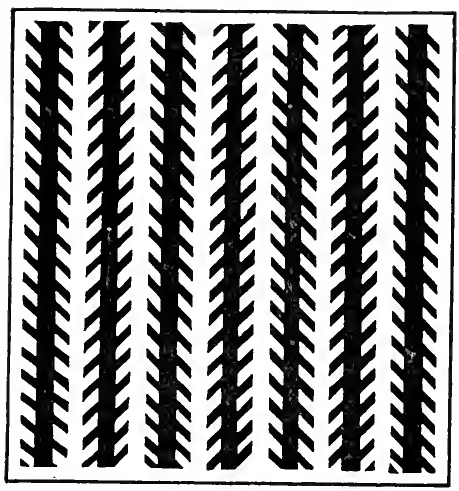

Fig. 92 even our quickly formed judgments of the size, distance, form, or color of objects are likewise tissues woven out of many threads, most of which we have been slowly and laboriously spinning since childhood's days in the hidden factory of individual experience.

17. Sound and Hearing. When the string of a violin, piano, or harp "sounds," one can observe that it is in rapid vibration; and the same thing is true of all sounding bodies. These vibrations are imparted to the air, water, or other surrounding medium, and through this medium they are transmitted as waves of sound. It is these waves or vibrations which, on entering the ear, excite the sensation of sound. The more rapid the vibrations the higher is the pitch of the note, and the greater their amplitude the louder the sound.

The ear is an organ specially adapted to receive these vibrations of air and to transform them into nervous impulses. It is subdivided by anatomists into the outer ear, the middle ear, and the inner ear.

18. The Outer Ear. - The outer ear consists of the expanded pinna (or that part which we commonly call "the ear") and a tube along which the vibrations of sound pass inward to the tympanic membrane, or drum. 
Glands along this canal secrete wax which guards the approach to the drum. It is a bad habit to pick at this wax, and especially to dig into the ear with any pointed

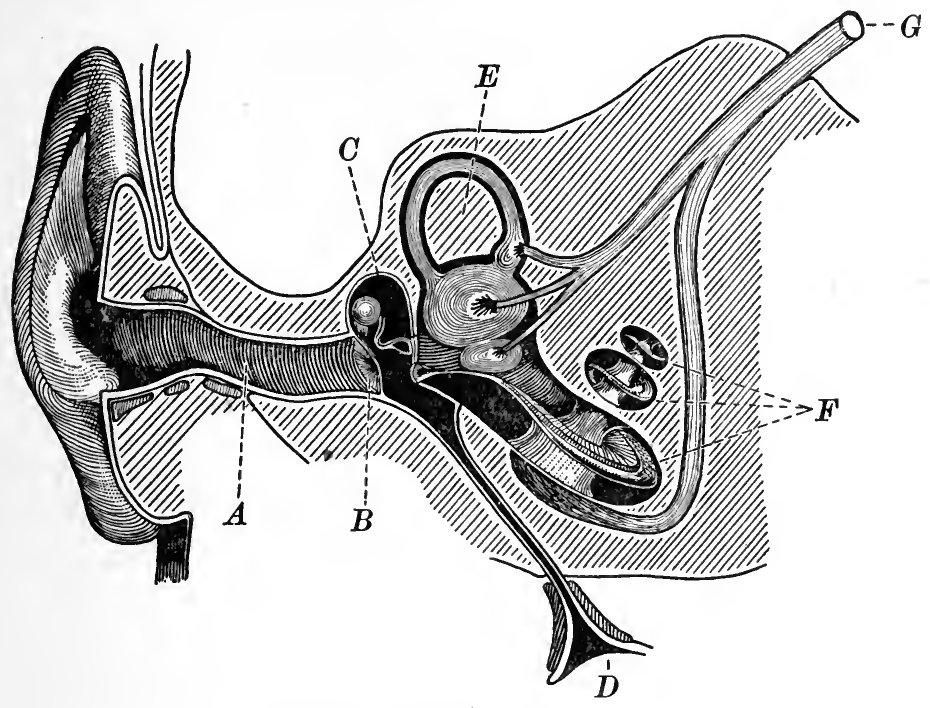

Fig. 93. Diagram of the ear

$A$, the auditory canal, leading to the tympanic membrane $B ; C$, cavity of the tympanum, communicating by the Eustachian tube with the pharynx $D$; $E$, semicircular canals ; $F$, cochlea ; $G$, auditory nerve

instrument, for there is always danger of perforating the drum. If trouble is suspected, a physician should be consulted.

19. The Middle Ear. The Eustachian Tube. - The tympanic membrane separates the outer from the middle ear, or tympanum, a small cavity lying in the temporal bone of the skull and communicating with the throat or pharynx by means of the Eustachian tube. The air which it contains is consequently under the same pressure as that of the atmosphere without, and the tympanic membrane is not normally bulged inward or outward by inequality of pressure 
on its two sides. The opening of the Eustachian tube into the pharynx is, however, closed except when one swallows, and hence swallowing often relieves the drum from undue pressure of air in the middle ear.

The cavity of the tympanum also communicates, with a network of spaces, or sinuses, in the temporal bone. Because

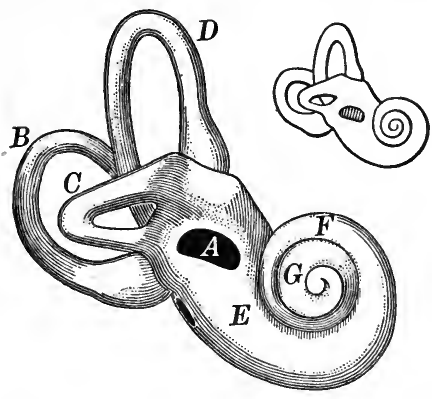

Fig. 94. The bony labyrinth, its actual size being shown in the smaller figure

$B, C, D$, the semicircular canals; $A$, the oval window, by means of which the vibrations of the stirrup bone are transmitted to the cochlea; $E, F, G$, the whorls of the cochlea. Compare Fig. 95 of these connections of the middle ear with the throat, on the one hand, and with the temporal sinuses on the other, inflammatory processes in the nose and throat during a cold sometimes extend into the Eustachian tube, the tympanum, and even into the temporal sinuses, causing serious trouble, and occasionally deafness.

Passing directly across the tympanum, from the drum on its outer side to the cochlea on its inner side, is a chain of three very small bones, the ear ossicles (hammer, anvil, and stirrup). These bones are bound together and attached to the walls of the tympanum by ligaments, and are so arranged that when sound waves set the tympanic membrane in vibration this motion is transmitted by the ossicles to a portion of the inner ear known as the cochlea.

20. The Inner Ear. - The structures of the inner ear lie in the temporal bone, on the side of the tympanum opposite the drum. They consist of a system of small bony spaces and tubes, the bony labyrinth, within which lies a corresponding membranous labyrinth. Forming part of the lining of the membranous labyrinth are very sensitive 
cells, and between these cells are the endings of the nerve fibers which connect the ear with the brain. The cells of the inner ear are sensitive to the vibrations which have been transmitted across the tympanum by the ossicles, just as the retina is sensitive to light; and as the retina is the origin of the fibers of the optic nerve, so the inner ear

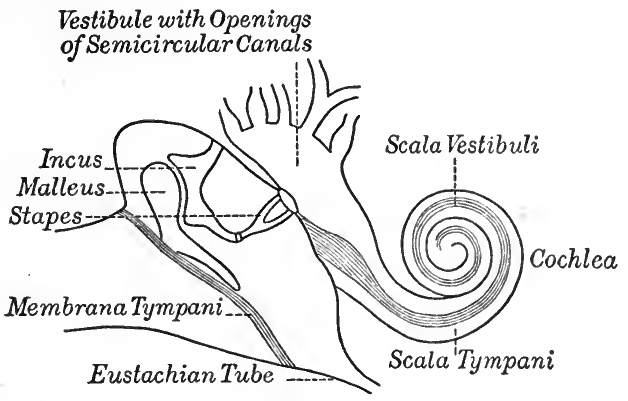
is the origin of FIG. 95. Diagrammatic representation of the memthose of the auditory nerve.

21. Taste and Smell. - The end

The scala vestibuli and scala tympani are the two porthe structures shown in Figs. 93 and 94

organs of taste are small rounded eminences, or papilloe, on the dorsal surface of the tongue, and from these the fibers of the nerves of taste pass to the brain. The end organs of the nerve of smell are situated in the upper portion of the nasal cavity and consist of delicate cells very sensitive to the presence of odors. Sensations of taste are frequently confounded with those of smell. An onion, for example, has little or no taste, as can be shown by placing a bit on the tongue when one is holding the breath; none of the flavor of the onion is perceived. On the other hand, sour, sweet, bitter, and salt are true taste sensations. The unconscious blending of tastes with odors in forming our ideas of the nature of objects recalls the formation of visual judgments by the combination of retinal with other sensations (p. 256).

22. Cutaneous Sensations. - The skin is the place of origin of at least three sensations, - touch, cold, and 
warmth. These sensations are distinct, as is shown by the observation that on certain points of the skin some of them may be felt, but not others. This fact is usually interpreted to mean that each sensation has its own set of end organs and nerve fibers. Especially striking is the fact that warmth and cold are never felt by the same spot

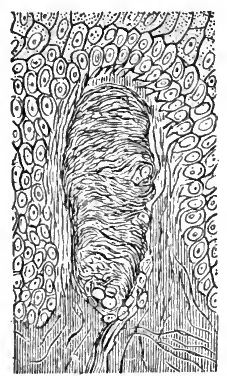

Fig. 96. A tactile corpuscle in one of the papillæ of the dermis ; an end organ of the sense of touch of skin, which seems to prove conclusively that they are separate sensations.

The afferent nerves of cold and warmth not only carry into the brain those impulses which give rise to the corresponding sensations but also serve as one important means of stimulating the reflexes which help to regulate heat production and heat output (see Chapter XII).

23. The Sense of Position. - The expression "the five senses" has become proverbial, and comes from the time when sight, hearing, taste, smell, and touch were the recognized special senses. To-day, however, we must add to these not only warmth and cold but still others, most conspicuous among which is the sense of position. When the eyes are closed we are aware of the position of the various parts of the body. We know whether the arm is bent at the elbow or straight; whether the head is looking forward or is turned to one side or the other. And while we are aware of these things, partly from tactile sensations, there is conclusive evidence that afferent impulses from the muscles, tendons, and joints also play an important part in the result.

When one is blindfolded and lies flat on a revolving table which can be turned noiselessly in one direction or the other, the subject of experiment can form fairly correct judgments as to the angle and direction through which 
the table is turned. Here there is no change of character either in the tactile impulses or in those from the muscles, tendons, and joints, for the subject of experiment lies still and is only passively moved. It is believed that in this case the sensations in question come from the movements of the lymph in portions of the inner ear. One part of this, the cochlea, is undoubtedly concerned with the perception of sound; but another part, the three semicircular. canals, are now believed to be end organs of this sense of position.

The impulses which make us aware of the position of parts of our bodies also play a very important rôle in reflexly guiding our movements. Upon this we shall dwell at greater length in subsequent chapters (see especially Chapter XV).

24. Sensations of Pain. - Most organs of the body may also give rise to impulses which, on their arrival in the brain, cause sensations of pain. It is still, perhaps, an open question whether this sensation, like sight, smell, and hearing, is aroused by its own mechanism of end organs and afferent nerves, or whether it is called forth by the excessive stimulation of the nerves of the other senses; but for the discussion of this question the reader must consult more advanced works on physiology.

Pain is a useful danger signal, since it effectively calls attention to abnormal conditions and incites us to the adoption of active remedial measures. Remedies, however, should not be confined to the abolition of unpleasant sensations, but should be directed to the removal of their cause. A toothache from a decaying tooth may often be stopped, for a time at least, by the use of chloroform or other anesthetic drugs, but the drug only stops the pain; it does not check the progress of decay or repair the damage. Again, a bronchial cough may be unpleasant, and even painful, but we should not rest content with the 
use of some drug or cough medicine which merely lessens the irritability of the inflamed surface of the air passages, and so, perhaps, stops the cough without curing the disease.

Pain is a warning that some abnormal condition needs attention. Sometimes that attention may be supplied by the sufferer himself, or by his friends, but often skilled medical advice is needed. Too frequently, for the sake of economy or from feelings of modesty, or even because of an unwillingness to acknowledge illness either to the world or to oneself, the mistake is made of postponing the visit to the physician, the patient meanwhile bearing discomfort and perhaps actual suffering in the hope that he will soon be better and that the trouble will "cure itself." Sometimes, of course, it does cure itself; but sometimes it does not; and remediable disease has too frequently been allowed to run on in this way until some vital spot is attacked or the trouble has become too grave for medical skill to overcome. Many diseases, like a fire, may be extinguished at the start, but if not attended to, grow rapidly into a conflagration beyond control. Pain is one of the most trustworthy warnings that attention to the mechanism itself or to our operation of it is necessary; and we have no right, either for our own sake or that of our friends, to neglect its warnings. While there are times when it is an act of heroism to endure suffering and to keep the knowledge of it to oneself, there are other times when to do this is not only foolish but wrong.

25. Hunger and Thirst. - No account of the physiology of sensations would be complete without some reference to those very common experiences of life,-hunger and thirst. We have already spoken of them as sensations which are referred to the body and never to external objects, thirst usually being referred to the mouth and throat, and hunger frequently to the stomach; but hunger and even thirst may sometimes affect us as sensations coming from the 
body as a whole, in which case they are usually indistinguishable from certain forms of general fatigue.

It is not yet fully understood how hunger and thirst are excited, though it is probable that they arise partly from the conditions of the stomach and throat respectively, and partly from conditions of the body as a whole. But they are as definite sensations and as truly adapted to guide us in the choice of food as sight is adapted to picture to us the world in which we live. So long as the body is normally occupied and healthy they may usually be trusted; but there are abnormal conditions of sedentary life, in the midst of a superabundance of tempting food, when they become less trustworthy; and in some forms of dyspepsia the sensation of hunger is never absent, no matter how often one eats. In such cases the very effort to satisfy hunger only aggravates disease. Conditions of this sort should not prevail if proper attention be paid to the general hygienic conduct of life. Broadly speaking, appetites, like fire and dynamite, are good servants but bad masters. 


\section{CHAPTER XV}

\section{THE NERVOUS SYSTEM}

\section{A. Its Anatomical Basis}

In the preceding chapter we have repeatedly emphasized the fact that sensations of all kinds are developed in the brain from nervous impulses coming from the sense organs;

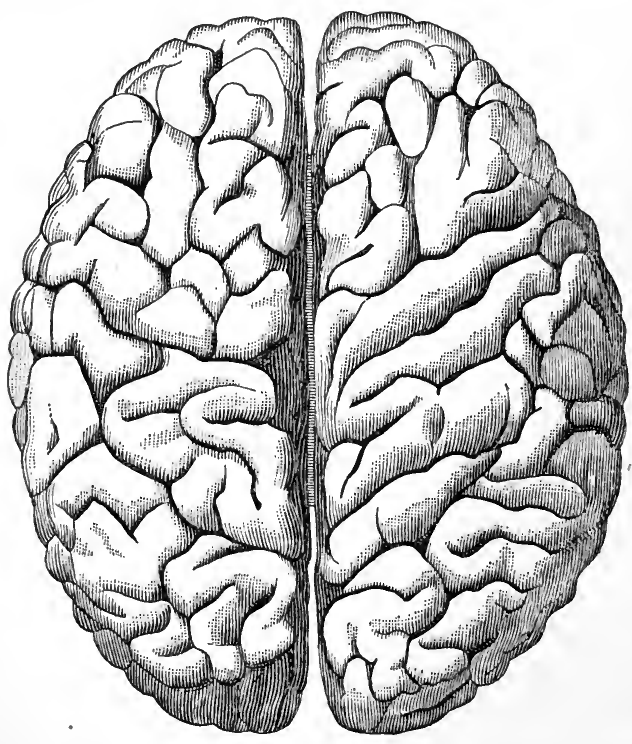

Fic. 97. The human brain viewed from above. The cerebral hemispheres completely cover the rest of the brain

vous system, and we have now to inquire what is known of the mechanism by which these results are brought about. chapter (VII) we have seen that without reaching the brain, or at least without affecting consciousness, these afferent impulses may give rise to reflex action. A reflex action or a conscious sensation, or both a reflex action and a conscious sensation, may therefore result from the entrance of a nervous impulse into the central nerand in a previous 
For this purpose we must first learn something more of the anatomy of the spinal cord and brain.

1. Fundamental Structure of the Nervous System. The Brain of a Frog. - The human spinal cord and brain are so
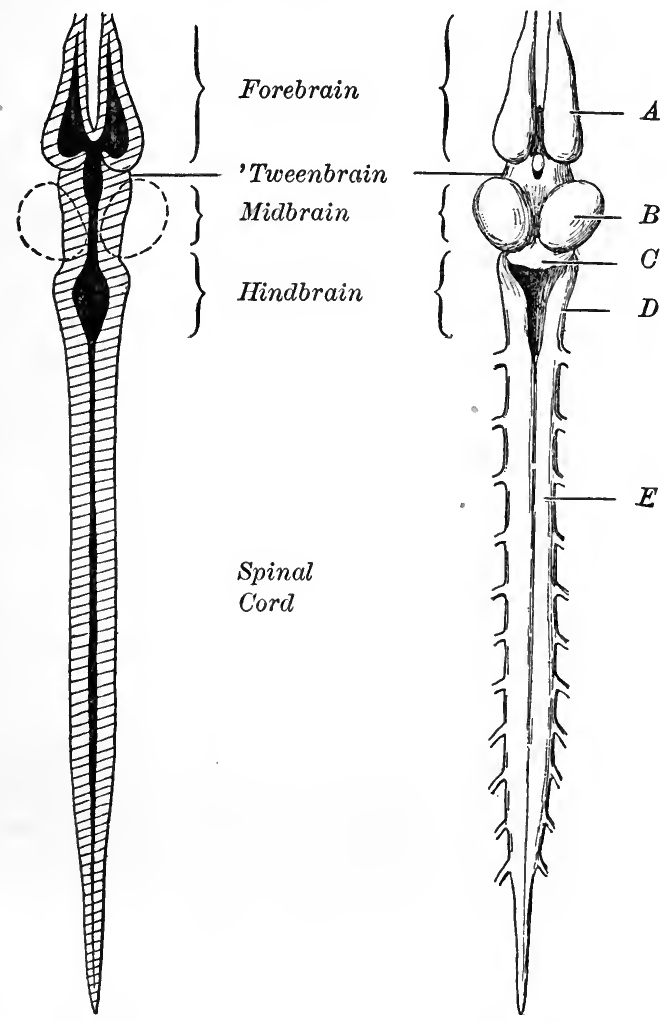

FIg. 98. The brain and spinal cord of the frog

On the left is a longitudinal, right to left section, showing the central canal and the ventricles of the brain ; on the right the dorsal view of the brain and cord. $A$, the cerebral hemispheres; $B$, the optic lobes; $C$, the cerebellum; $D$, the bulb; $E$, the spinal cord

complicated that it is best to study first the nervous system of a simple vertebrate like the frog; for the fundamental 
plan of structure is the same in both. The spinal cord is a relatively thick-walled tube, the walls of which are composed of white and gray matter, the minute bore, or lumen, of the tube being known as the central canal. The arrangement in the brain is similar, but here the central space is no longer a small tube of even bore, but consists for the

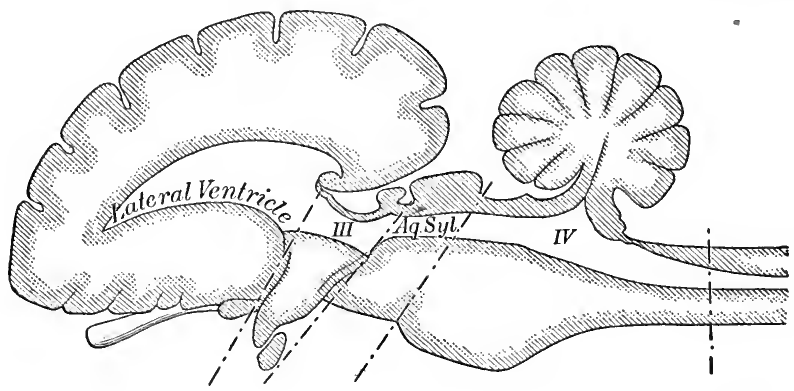

FIG. 99. Diagrammatic median longitudinal section of a mammalian brain. After Edinger

For convenience the cerebrum with its lateral ventricle is represented as a single organ in the median plane, instead of two hemispheres on either side of this plane and each with its own lateral ventricle. The division into forebrain, 'tweenbrain, midbrain, and hindbrain is marked by the broken lines

greater part of irregular cavities known as the ventricles of the brain, while the walls consist of masses of gray and white matter varying in size and shape.

Figure 98 will assist the student in understanding this plan of structure. Anteriorly the spinal cord is continued in the $b u l b^{1}$ whose central cavity is the fourth ventricle. Part of the dorsal wall of this ventricle forms the cerebellum, which in the frog is only slightly developed, but which in higher vertebrates (birds and mammals) becomes a large and conspicuous organ. Anteriorly the fourth ventricle is connected with the third by a tube, the aqueduct of Sylvius. The thick walls of this aqueduct contain

1 The older term for the bulb is the medulla oblongata, to distinguish it from the medulla spinalis, or spinal cord. 
various masses of gray matter whose names need not detain us; the walls of the third ventricle are similarly composed of large masses of gray matter scattered among

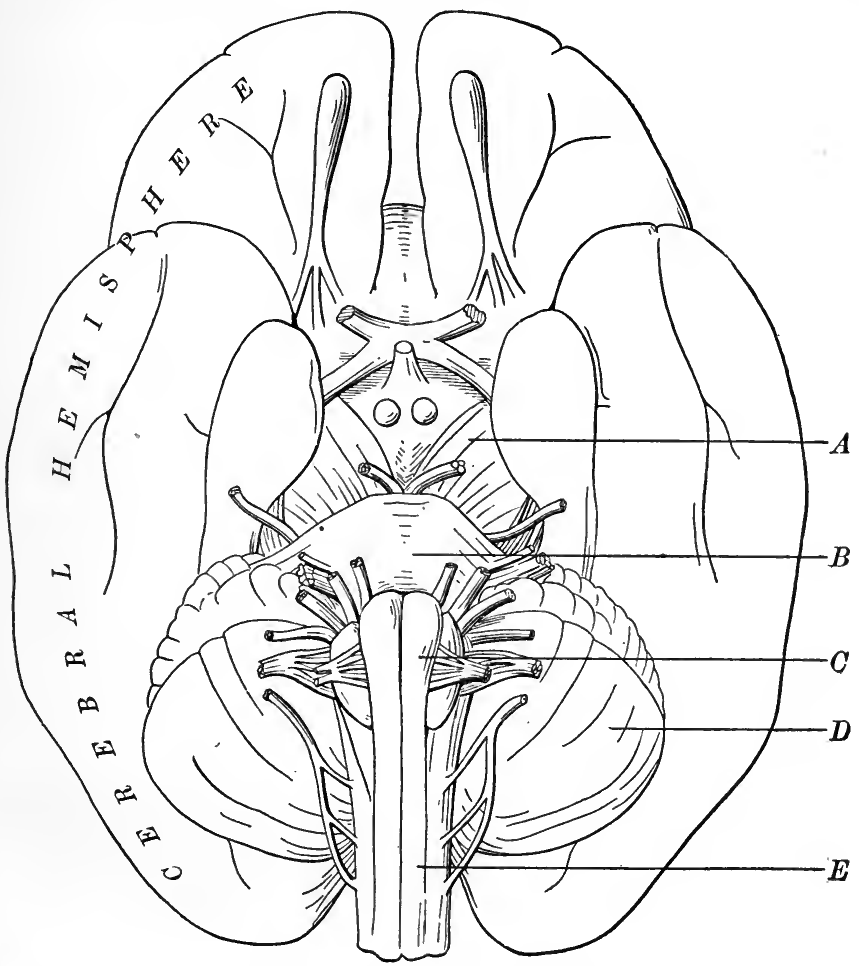

FIG. 100. The base of the human brain, showing the cranial nerves

$A$, crus cerebri, composed largely of nerve fibers which connect the hindbrain with the 'tweenbrain and forebrain; $B$, pons Varolii, the anterior floor of the fourth ventricle, connected laterally with the cerebellum; $C$, bulb; $D$, cerebellum; $E$, spinal cord

the fibers of the white matter. Still farther forward two openings from the third ventricle, one on the right and one on the left side, lead into the large lateral ventricles, the nervous tissue of whose walls is the cerebrum, or the 
cerebral hemispheres. It is convenient to divide the brain into the forebrain, surrounding the lateral ventricles; the 'tweenbrain, surrounding the third ventricle; the midbrain, surrounding the aqueduct of Sylvius; and the hindbrain, surrounding the fourth ventricle.

2. The Brain of the Mammal is built on the same fundamental plan as that of the frog, and differs from it mainly

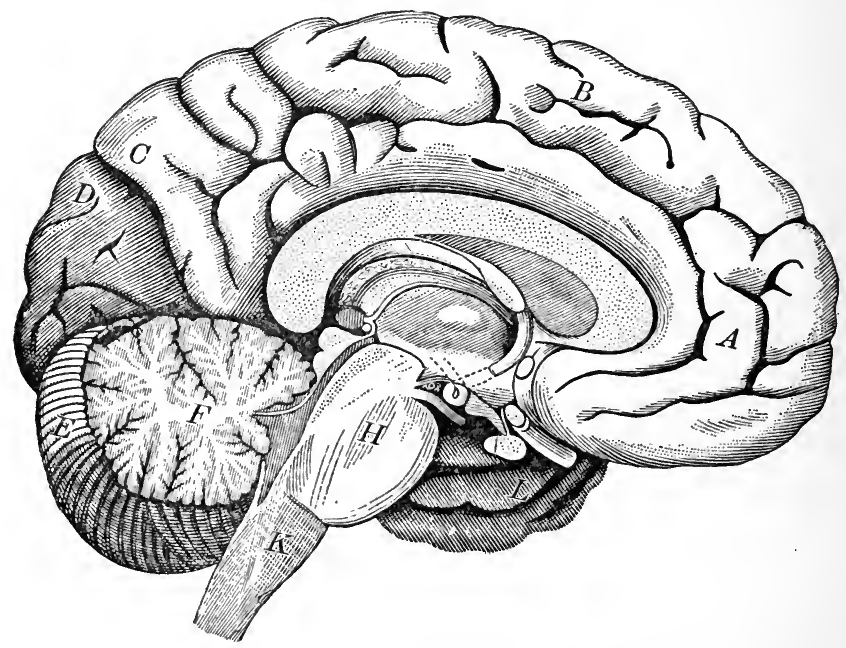

FIG. 101. Median longitudinal section of the human brain

$A, B, C, D, L$, convolutions of the median surface of the cerebrum; $E, F$, the cerebellum, showing in the plane of section the inner white matter and the outer gray matter; $H$, the pons Varolii; $K$, the bulb

in the greater number of neurones and in the complexity of their connections with one another. This results in great thickening of the ventricular walls and the formation of a very complicated anatomical structure. Mammals are especially characterized by an enormous development of the cerebral hemispheres, which in man grow to such proportions upwards and backwards as to overhang and completely cover the other structures on the dorsal side. 
But even these large masses of nervous tissue, no less than the smaller cerebrum of the frog, are composed entirely of the gray and white matter forming the walls of the lateral ventricles.

By comparing the brain of a frog (Fig. 98) with those of the rabbit, cat, and monkey (Fig. 146), and finally with the human brain (Figs. 97, 100, 101), a fairly good idea may be had of the increasing complexity of the brain as we pass from the lower to the higher animals. Especially noteworthy is the greater relative prominence of the cerebrum. In the frog this organ is small and inconspicuous; in the rabbit it is much larger, but its surface is smooth; in the cat there is a further increase in size, and the surface is thrown into folds, or convolutions; and this increase in size and surface folding - carried yet farther in the monkey - reaches its highest development in the human brain.

3. The Cranial Nerves. - Nerves enter the 'tweenbrain, midbrain, and hindbrain somewhat as they enter the spinal cord; and, although their separation into dorsal and ventral roots is not obvious, the neurones to which their nerve fibers belong are in all respects analogous to the neurones of the spinal nerves. They may serve as the paths of reflexes (e.g. a wink is a reflex from the optic or the trigeminal nerve to the facial nerve), and their relation to the cells of the cerebrum and other higher portions of the brain is essentially the same as that of the spinal nerves. Figure 100 will give the points of entrance or exit of these nerves from the human brain.

4. Histological Structure of the Brain.-Microscopic study of the brain shows an aggregation of neurones similar to that seen in the spinal cord. These neurones differ greatly in shape (see Chapter VII, p. 74), in the number of their dendrites, and in the abundance of their connections with other neurones. The regular arrangement in 
the cord of central gray matter surrounded by white matter is wanting; instead, masses of gray matter occur here

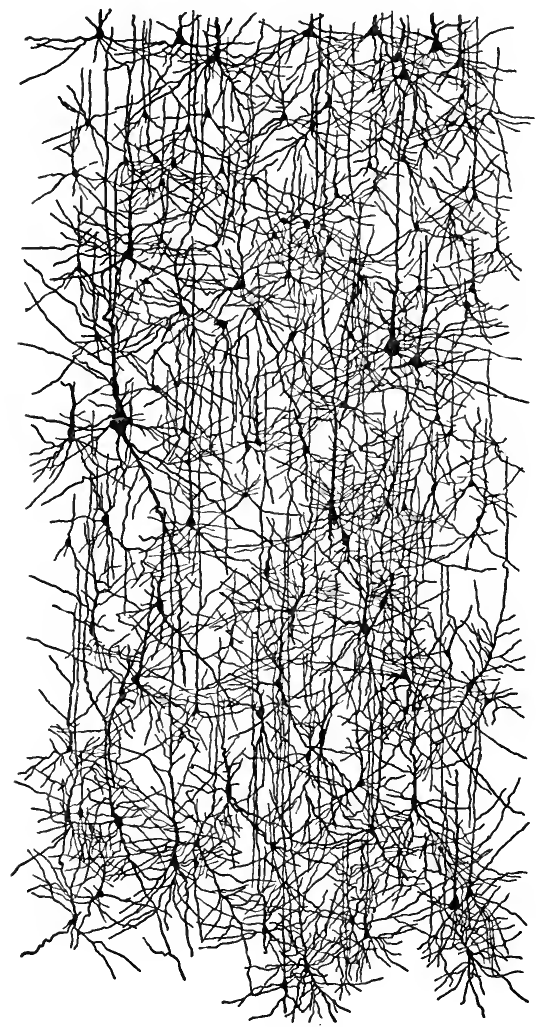

FIG. 102. A portion of the gray matter (cortex) of the cerebrum (highly magnified). After Kölliker

Note the large number of dendrites. The axons are the fibers of uniform diameter rumning lengthwise of the drawing. One of these cells is shown in Fig. $39, D$ in both; the brain as well as the cord does its work because the connections of its neurones with one another

and there among the bundles of nerve fibers of which the white matter is composed. In the cerebrum and cerebellum the external surface consists of gray matter and is known as the cortex of the cerebrum and cerebellum respectively. These cortical structures form the most complicated system of nervous tissue in the body, and the cerebral cortex is intimately concerned with the highest functions of the brain. (See Figs. 102, 103, and 104.)

The figures give some idea of the variety and complexity of the neurones of the brain. But however different, at first sight, the brain may be from the spinal cord, the anatomical plan of organization is the same 
bring about coördinated action. The secret of the structure of the brain, as of the cord, lies in the nature of the connections of its units, the neurones, one with another.

\section{B. The Physiology of the Nervous System}

Whenever through accident, disease, or otherwise, some portion of the nervous system is destroyed, functions dependent upon it are no longer performed, or at least are not performed normally. A very large number of observations have been made upon both animals and men in this condition, and these have made it possible for us to obtain some idea of the part played in normal life by each part of the brain and cord. We shall attempt

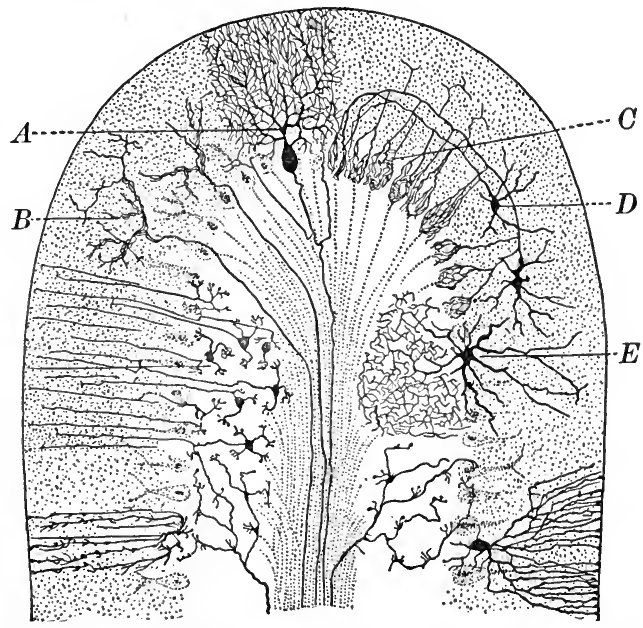

Fig. 103. Transverse section of a convolution of the cerebellum. After Ramon y Cajal

The figure represents only a few of each kind of nerve cells and nerve endings. $A, D, E$, cells; $B, C$, nerve endings (synapses)

here to sketch only a few of the more important outlines of the picture, which the reader may complete by more extensive study of physiology and psychology.

We shall choose for study the case of a single animal, the frog, the anatomical structure of whose brain has been given in the last chapter. The phenomena shown by the 
frog are, however, as far as we shall describe them, in general true of higher vertebrate animals.

We shall therefore study (1) the behavior of a frog

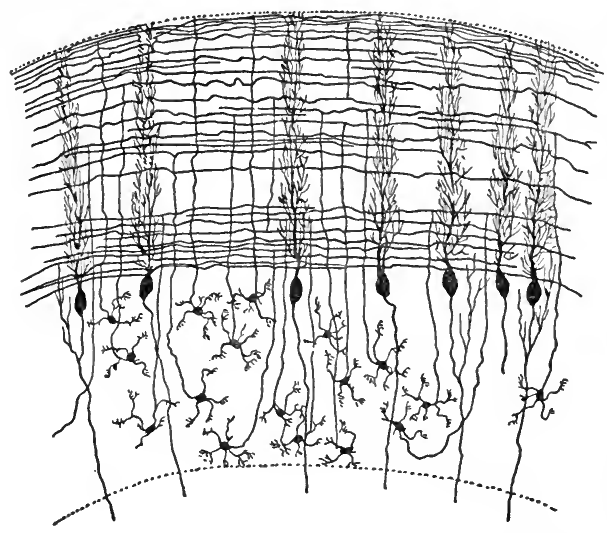

whose brain has been destroyed, i.e. a frog which possesses no part of its central nervous system except the spinal cord ; (2) the behavior of a frog with spinal cord and bulb intact, but destitute of midbrain, 'tween-

Fig. 104. Section of the cortex of the cerebellum brain, and cere(at right angles to that shown in Fig. 103). brum; (3) the After Ramon y Cajal

behavior of a frog with spinal cord, bulb, midbrain, and 'tweenbrain, but destitute of the cerebrum.

The behavior of these incomplete animals will each be compared with that of a normal frog, which, of course, possesses a complete nervous system.

5. The Behavior of a Brainless Frog, i.e. a frog which possesses of its nervous system only the spinal cord. Such a frog can carry out only reflex actions of a comparatively simple character. It lies flat upon its belly and, like the normal frog, bends its hind legs under its flank, but does not sit erect by supporting the head and upper trunk on the fore legs. There are no respiratory movements ; the vasoconstrictor tone of the blood vessels is impaired or absent, as are also many other of the most important reflexes.

But if one leg be pulled gently backward, the animal will bend it again to its normal position under the body. If 
the toe be pinched, the leg will suddenly be drawn away; and if the skin of the flank be irritated by a bit of filter paper moistened with acid, the paper will be kicked off by the leg of the same side.

These are all purposeful ${ }^{1}$ and coördinated actions, and make upon the inexperienced observer the impression that the frog is aware of the stimulus and acts intelligently. But the mere fact that an act is purposeful and coördinated does not show that it is a conscious act; our movements of respiration, winking, coughing, and sneezing are purposeful and coördinated, but we know well enough that they, as well as more complicated actions, may and often do occur in the complete absence of consciousness. One of the first lessons that the student of animal behavior must learn is not to make the mistake of regarding an action as conscious merely because "it looks so," or is purposeful and more or less highly coördinated.

The spinal cord alone, then, and without the help of the brain, is capable of maintaining a small part of the normal posture of the resting frog, and also of executing some of the simple reflexes, especially those involving movements of the hind legs ; but it does not seem to be capable of originating actions or of doing any except reflex actions.

6. The Behavior of a Frog with Spinal Cord and Bulb only. - In this case there is no new feature in the maintenance of posture ; the frog lies on its belly and executes the same reflexes as before. The respiratory movements, however, go on in a normal manner; the vasomotor tone of the arteries is maintained, most vasomotor reflexes may be produced with ease, and the heart may be reflexly inhibited. As compared with the brainless frog, the number of actions which the animal can execute is increased, and the reflex movements become somewhat more complicated;

1 The word purposeful is used here in the same sense as in Chapter VII (p. 71) and does not include conscious purpose in its meaning. 
but the differences are slight, as compared with those seen in the animal which has the 'tweenbrain and midbrain in addition to the hindbrain and cord.

7. The Behavior of a Frog with Spinal Cord, Bulb, Midbrain, and 'Tweenbrain; that is to say, a frog with the entire nervous system exclusive of the forebrain, or cerebrum. The following points are especially noteworthy: $(a)$ the sitting posture maintained at rest; $(b)$ balancing movements; and $(c)$ more complicated movements of locomotion.

(a) Such a frog, unlike those already described, sits erect exactly like a normal frog; and this fact shows that complete maintenance of the normal posture requires the coöperation of higher portions of the nervous system than the bulb and spinal cord, but does not involve the coöperation of the cerebrum.

(b) If the frog be placed on a rectangular block of wood, and the block slowly turned so that the frog tends to slip off backwards, it will crawl up and over the descending edge, keeping itself perfectly balanced. By continuing to turn the block the frog can be made to creep around it almost indefinitely. Thus it not only maintains the erect position but also corrects loss of equilibrium by appropriate balancing movements.

(c) If the frog be stroked upon its belly, it will croak; if its lips be touched with a blunt pin, it will brush the pin away with its forefoot. Most important of all, if it be thrown into the water, it will swim; and when it reaches a solid object it will crawl out upon it and come to rest. In short, the animal will carry out almost any movement of which a normal frog is capable, provided the proper stimulus is applied; but without this it will do nothing, though capable of doing so much.

The facts thus far brought forward show that the neurones of the 'tween-, mid-, and hindbrains, and of the spinal 
cord, constitute nervous mechanisms which can maintain the normal posture, correct loss of balance, and even carry out the usual acts of locomotion. The more of the nervous system which the animal retains the more complicated are the movements, as we should expect when we remember the increase in the number of neurones, and the greater complexity of coördination thereby rendered possible.

8. Comparison with the Normal Frog. - The behavior of a frog lacking only the forebrain (or cerebrum) differs from that of a normal frog in two most significant respects. In the first place, the animal rarely makes any movement without obvious external stimulation; if protected from drying, it will often sit motionless for days, or even weeks. Such is not the conduct of an animal which is aware of what is going on around it, or of its own sensations or feelings, i.e. of a conscious animal. In the second place, the frog shows the most remarkable regularity and persistency in making repeatedly the same response to the same stimulus; if its lips be touched thirty times with a blunt needle, it will brush at the offending object every time in the same way with the same forefoot. We should certainly not expect a conscious animal to do this; for, after trying one plan of action a few times, it would realize that its efforts were unavailing, and would try something else, such as jumping away. This same peculiarity is met with in all animals deprived of the cerebrum. They act like mere complicated and faithful machines; they do not act as if they were thoughtful, original, or wise.

Especially striking is the avoidance of objects during locomotion. This fact looks at first sight as if the animal were aware of the presence of the obstacle in its path; but a dog without a cerebrum, even when it has been without food for a day or more, will go to one side of a piece of meat and pass it by. He acts as if unaware of the nature of the object, of its use as food, etc. The image of 
the piece of meat formed on his retina seems to generate nervous impulses which pass to the brain by way of the optic nerve and reflexly guide the movements of the dog; but these impulses do not inform the animal of the nature of the object, and we have no reason to believe that the dog is aware of the existence of the meat.

When we consider our own experience we find that we too, as we walk along a crowded street, avoid objects, not only without noticing them but without even being aware of their presence. Here again the afferent impulses from the retina pass to the nervous system and reflexly guide our walking without affecting consciousness at all. And the wonderful feats of somnambulism, where the "eyes are open" but "their sense is shut," where the sleeper maintains his balance and avoids stumbling in situations where he would almost inevitably fall if he were aware of his surroundings, show how perfect is this very complicated mechanism of locomotion, which seems to be complete even in the absence of the cerebrum.

We are, indeed, so accustomed to regard our actions as volitional and conscious that we rarely consider the large part which reflexes from the eye, the ear, the skin, the muscles, and the joints play in guiding them. We will to do a certain thing, to walk to a certain point, for example; perhaps the first step is a volitional act, but subsequent steps, the suiting of these steps to slight unevennesses of the path, the avoidance of many obstacles, the maintenance of the balance of the body as a whole, - for we walk not only with the legs but with the entire body, - all these things take place apart from any exercise of the will, and, for the greater part, in the entire absence of consciousness, although consciousness may, of course, at any time intervene. Reflex actions thus play a most important part even in the execution of those movements which we think of as distinctly conscious acts. 
9. Connections of the Cerebrum with Lower Portions of the Nervous System. "The Way Out." — Granting that the nervous events at the basis of consciousness occur within the cerebrum, how do these events influence the muscles, the glands, and other organs which do the bidding of the will? What is the way out from this seat of consciousness? This path has already been referred to in Chapter VII (p. 82). Cells in the gray matter of the cerebrum give off axons which pass downward through the structures of the 'tween-, mid-, and hindbrain into the white matter of the spinal cord. These axons give off along their course collaterals which end in arborizations around nerve cells of the lower portions of the nervous system, and by bringing groups of these cells into coördinated activity produce definite volitional movements. The student should review carefully in this connection what has already been said with reference to these neurones (see Fig. 145, v).

10. Connections of the Cerebrum with Lower Portions of the Nervous System. "The Way In." - The fact that afferent impulses from our sense organs of sight, hearing, etc., may affect consciousness indicates that there must be some connection between afferent neurones and the cerebral hemispheres, since only when the latter are present does a nervous impulse produce a conscious sensation. The connection is not, however, so direct as in the case of efferent impulses. The neurone of the dorsal root may be traced as far as the bulb, but no farther; from this point the impulse can find its way to the cerebrum only by new neurones, and of these it would seem that there are several. These relations are indicated in Fig. 145, where the efferent neurones are represented in black and the afferent in red.

This diagram brings out the fact of increasing complexity of reflexes as we proceed to the more anterior portions of the nervous system. In the spinal cord the collaterals 
of the afferent neurone act upon the efferent neurones; in the structures of the midbrain and the 'tweenbrain the afferent tract makes connection with more and more complicated and extensive systems of these efferent neurones or motor mechanisms. The range of possible movement is increased to include most of the usual actions of the animal, and some of these actions represent a very high degree of coördination. Finally, in the cerebrum the highest of all these connections is made; here take place those events of whose nature we have thus far been quite unable to form any conception, but which play some part in the genesis of conscious sensations and in the closely related dispatch of volitional impulses. We can now understand why it is that removing this highest portion of the nervous system leaves untouched not only the simpler reflexes but even the more complicated reflexes of locomotion, of swimming, of flight, etc.

11. Walking an Endless Chain of Reflexes. - The reflex mechanism of walking may not be clear at first sight, although the facts we have already given in this chapter (p. 278) leave no doubt that the movements of locomotion are guided to some extent by afferent impulses.

In a previous chapter (p. 262) it was shown that afferent impulses from the skeletal muscles, the joints, etc., enable us to form judgments of the position of parts of our body with reference to each other; we have also seen that afferent impulses from the semicircular canals contribute to this same sense of position. ,The afferent neurones, however, which serve as the path of these impulses to the cerebrum, send off collaterals to the lower portions of the brain, as shown in Fig. 145, and in this way make the reflex connections which execute the act of locomotion.

With each position which the body assumes, a certain combination of these afferent impulses passes into the central nervous system, and each position gives rise to its 
own combination of impulses. It is clear (Fig. 145) that these impulses, having entered the central nervous system, have before them one or the other or both of two destinations: they may proceed onward to the cerebrum and there give rise to the knowledge of the position of parts of the body; or they may go by way of collaterals to the cerebellum and other lower nerve centers, and there influence the efferent or motor mechanisms of the more complicated reflex actions.

It is further necessary to understand that the result of the arrival of the afferent impulses at the cerebrum or the lower centers depends on the condition of those portions of the nervous system at the time. Thus the impulses affect consciousness only when we heed them; when one is sitting still and thinking of other things they help to maintain equilibrium by their influence on certain lower nerve centers which control the muscles, but they do not stimulate new movements ; finally, during locomotion they not only maintain the balance of the body but also call forth the next movement, because at that time the efferent motor mechanism of the lower centers is free to act.

In walking, for example, as we take the forward step with one foot, the combination of these afferent impulses is constantly changing; this change of combination obviously changes the stimuli acting upon the efferent or motor cells, and the movement progresses, changing in character each instant until the foot taking the step is planted firmly on the ground and the step with the other foot begun. Here again the same kind of changing reflex movement goes on until the second step is completed and the parts of the body are in the same position with reference to one another as when the first step was begun; we return in this way to the original combination of afferent impulses and so begin again the same cycle of movements; the third step is like the first. Locomotion is thus an 
endless chain of reflexes. The character of the act may be modified by new afferent impulses from the eye (as when we unconsciously avoid objects), or from touch when we tread upon some obstacle; or volition may modify it, as when we give attention to what we are doing. But so long as the character of the path or of surrounding objects remains the same and volition does not intervene, the act progresses as an endless chain of reflexes.

Essentially the same thing is true of swimming, of running, and of many other actions. They are fundamentally reflexes from the sense organs of position, though their character may be modified either reflexly, by new afferent impulses, or volitionally, by the intervention of new impulses from the cerebrum. Such an act as dancing differs from. the others only in the greater extent to which the volitional impulses play upon the chain of reflexes of which the action is fundamentally composed.

12. Actions resulting from Nervous Processes originating within the Cerebrum. - A very large part of the activities of the body are thus fundamentally reflex actions; they do not require the aid of consciousness for their execution. And it is fortunate for us that this is the case; one has only to imagine a human being who has to give his attention, or "his mind," as we often say, to every adjustment of the digestive, respiratory, and vascular systems required to meet the changing necessities of life; who has to keep his thoughts on every movement of walking or running; who has to be constantly on guard against loss of balance even when sitting still. Such' a being is almost inconceivable; he would "go crazy" in a single ' day; but we can in this way realize to what extent the reflex mechanisms of the body perform the menial offices of life, leaving the mind free for higher things:

Speech is the result of movements in which the muscles of respiration - those of the larynx, those of the tongue, and 
those of the lips - coöperate to produce articulate and intelligible sound. The act of writing also consists of a series of movements in which the muscles of the arm and hand coöperate to make thought visible; performing on a musical instrument, modeling a figure in clay or marble or bronze, painting a picture, - all these things occur to us as examples of movements which are not fundamentally refiex, and from which the character of an endless chain is absent. Such are the highest actions of the body, and the movements of which these actions are made up are chosen and directed by the will.

These higher actions, like consciousness, depend upon the presence of the forebrain. When a certain area of the cerebrum is destroyed by disease, the power of speech is lost; when another part is destroyed, the skilled use of the hand is lost; destruction of other portions affects in the same way others of these skilled movements. In such cases locomotion, the maintenance of balance, the movements of respiration, etc., may be and usually are unaffected; the patient merely loses the power of doing one or more of those things which, in no sense reflexes, involve the selection of disconnected and to some extent independent movements giving expression to some original thought, sentiment, or idea.

The neurones of the cerebrum and their connections thus constitute nervous mechanisms whose activity is essential to consciousness, - to our seeing, our hearing, our smelling, and, more than this, to our understanding of what we see, or hear, or smell, - nervous mechanisms whose activity is also necessary to the expression of our thought in action. It is because of this fact that, when the cerebrum is removed, the animal becomes merely a complicated reflex machine, acting only as it is immediately stimulated from without, or by events taking place within its own body. 
13. Effects of Anesthetics on the Nervous System.-When a person passes under the influence of an anesthetic the first function to disappear is consciousness ; the ether or the chloroform first paralyzes this highest and most complex connection between the afferent and the efferent sides of the nervous system. In this condition the patient may groan and struggle, for he is in somewhat the same state as the animal without cerebral hemispheres. The use of the surgeon's knife will still produce movements ; respiration may be affected so as to result in groans and other movements which the inexpert observer, perhaps in alarm, attributes to severe suffering; and yet when the patient awakes he tells us he knew nothing of what passed and felt no pain. It is important to realize that the signs of pain are never reliable evidence of its existence.

If the anesthesia be pushed further, even these more complicated reflexes disappear. In the ordinary major operations of surgery, the ether or the chloroform is given until it interrupts not only the cerebral connections between the afferent and efferent paths, but also those of the lower portions of the brain; it is even administered until only a few reflexes are left, such as the wink when the cornea is touched, the contraction of the pupil when the eye is exposed to light, etc., - these serving as useful tests of the condition of the patient. If, for example, the pupil no longer contracts to light, it is an indication that the anesthesia is going too far, - too near the point where the nervous mechanism of respiration, etc., will be paralyzed. The giving of ether is then suspended until these reflexes are again well established.

After the operation, as the ether or chloroform is eliminated from the system, the reflexes return in the reverse order; and the unconscious movements, groans, incoherent, or even more or less coherent, talking (comparable with talking in one's sleep) are sometimes most harrowing to 
the feelings of those who do not understand that they are all unconscious acts. The physician and nurse who remain unmoved may even be wrongly charged with lack of feeling because they do not waste sympathy where they know there is neither suffering nor consciousness.

\section{Inhibitory Phenomena in the Nervous System. - We} have learned that some nerves excite organs to activity, while others diminish activity or abolish it altogether (p. 158). The beat of the heart is quickened by one set of nerves and slowed by another; the circular muscular fibers of the arterioles are excited to contract by vasomotor nerves, their tonic constriction is paralyzed or inhibited by vasodilators, and many other examples might be drawn from the action of neurones on peripheral organs of the body.

Precisely the same thing is true in the brain and spinal cord. Afferent impulses may not only reflexly excite neurones to activity but may also inhibit the existing or threatened activity of other neurones, as when a sneeze is stopped by biting the upper lip or by pinching the nose; or an action may be inhibited by a volitional impulse from the cerebrum, as when the breathing movements are voluntarily stopped for a while, or when we similarly stop a wink or a sneeze. These are all examples of inhibition, not of the skeletal muscles concerned but of the neurones which innervate them, - in other words, of the inhibition of one neurone by another.

It must be understood that inhibition is as essential a part of the activity of the nervous system as is excitation. Just as the driver of a team must urge on one horse while he restrains another, so in all more complicated actions, probably in all actions, reflex or volitional, the orderly movement is as much the result of holding one neurone in check as of stimulating another one to work, or to work harder. Consciousness proves its presence most conclusively by suppressing reflexes which would otherwise 
inevitably occur, and by bringing about new movements to meet the desired end. Even in the highest processes of the most highly organized of nervous systems, viz. those in which human action originates, the man reveals his character and influences the world around him by what he does not do, - by what he refrains from doing, sometimes at the cost of severe struggle against impulse, instinct, or passion, - quite as much as by what he does. Education, even, has been defined as the "training of inhibitions and the control of reflexes."

15. Use and Disuse as Factors in Individual Development, Training, and Efficiency. - When we consider the marvelously complicated character of the nervous mechanisms which control our actions, we naturally wonder how this intricate machinery can be built, and why it does not more frequently get out of order. We cannot say that a simple and comprehensive answer will not some day be given to these questions; but to-day we have no adequate answer whatever. The neurones with which we must work in life are born with us, but in most cases efficient connections must subsequently be made between them, thus perfecting the mechanisms they compose; and this perfecting of the nervous machine comes with use. The use of a nervous mechanism is generally essential to its proper development, just as the use of a muscle is essential to its strength. If the child never tried to walk, the neurones which carry out the movements of walking would not develop; not only do the muscles of an arm strapped down to the side of the body waste away and become practically bands of connective tissue, but the neurones concerned in the actions which the arms should execute degenerate and are ultimately irreparably injured.

Provision is made from earliest life for the proper development of these neurones, and the establishment of irritable connections between them by use; out of the first 
aimless movements of the head and eyes and hands and legs of the baby the simpler coördinating nervous mechanisms are one by one brought to perfection; then comes the training of those reflexes which maintain the erect position, and of those which govern locomotion; then play comes in, with its ceaseless activity, increasing still further the number of movements which the nervous system can make, and correspondingly enlarging the possibility of human achievement. As the child grows older the family calls upon him to contribute some share to its life or support; new activities, in the shape of chores about the house or the farm, now share with play the work of the nervous system; activity becomes less general, more special. Finally, the youth settles down to some definite occupation or pursuit, and the more strictly this is adhered to the narrower becomes the range of activity; the more constantly a few systems of neurones are used, the more rarely are others called into play.

16. The Physical Basis of Habits. - All this indelibly writes its history in the nervous system. No fact is more significant, or of greater physical and moral import, than that the doing of any act so affects the connections of neurones with one another as to make it easier to do the same act again under the same conditions; that refraining from doing something toward which we are inclined similarly renders more easy the inhibitory processes concerned when the same conditions impel us toward it again. We are largely what we make ourselves by the training which our actions give to the nervous system.

And what activity thus does for the development of power it does also for the maintenance of power. An efficient nervous mechanism of any kind once acquired does not remain efficient without use. The man who has developed a rugged constitution in colder climates and then lives for years in the tropics, constantly exposed to 
warm climate, finds on return to the home of his youth that the mechanism of heat regulation does not readily adjust itself to cold damp winds and blizzards; the athlete who has learned to execute the greatest variety of "tricks" in the gymnasium and then settles down to a sedentary life finds after some years that he is almost as helpless as the man who gave no attention to such training. It is unnecessary to multiply examples. Efficiency in any direction is the result of continued use of organs, and especially of continued training of the nervous system. As we fit ourselves to do some few things, and to do them well, we have not time to conserve by use the efficiency of all the nervous mechanisms we have acquired; we must to some extent sacrifice the more general actions for those which are more special and useful. But it must not be forgotten that this can be carried too far; that a certain amount of general activity is a condition of healthy living, and that one of the problems of life to solve, and to solve aright, is how to distribute our activity between the two. To the consideration of these questions we shall return in our study of personal hygiene. 


\title{
THE HUMAN MECHANISM
}

\author{
PARt II
}

THE HYGIENE OF THE HUMAN MECHANISM AND THE SANITATION OF ITS SURROUNDINGS 



\section{CHAPTER XVI}

\section{INTRODUCTORY}

A. Hygiene and Sanitation: the Right Use and Proper Care of the Human Mechanism

In Part I of this work we have examined in some detail the normal structure and workings of the human mechanism. We have now to consider that care and use of the mechanism which constitute a wise and proper conduct of life, to the end that we may possess and enjoy the greatest possible measure of bodily health, strength, and usefulness.

The owner of any valuable lifeless mechanism, such as a watch, a yacht, or a piano, or of any valuable living mechanism, such as a prize-winning dog or horse, gives close attention to the proper care, management, and use of his property; and if, as often happens, great sums are invested in such property, this care is naturally all the greater. Comparatively few persons own such things, but every one of us - every man and woman, every boy and girl - begins life possessing a far more valuable mechanism which we call the human body. Some of these mechanisms are of marvelously strong and perfect construction; they seem to be equal to any amount of work and to suffer but little from careless operation or unfavorable surroundings. Many are not so strong, but yet with reasonable care give excellent service. Others require constant, intelligent attention, without which they readily get out of order, but with which they do fair and even good work. Much of the best work of the world has been done by persons in poor health; Darwin never had robust 
health; Heine was an invalid in his later years; Milton was blind; Sir Walter Scott was always lame; Pasteur was partially paralyzed during much of his life. On the other hand many, originally robust, have not only broken down and failed to do good work for themselves and their fellows, but have actually become a burden to the world, simply because they have refused to give to their bodies the care which they would freely bestow on a watch or a bicycle.

The proper management and operation of the human mechanism requires not only care, but intelligent care. A locomotive is intrusted only to an engineer who knows its construction, who can detect the evidences that something is wrong, who knows how much steam to apply at different times, what to do on various grades, how to start his engine safely, and how to bring it to rest. By lessons on anatomy and physiology, in Part I, we have endeavored to impart to the student the same preliminary knowledge of the construction and workings of the human mechanism which any one intending to be an engineer must have of machinery before he can master the practical operation of his engine. The chapters immediately following are concerned with the proper care and management of the mechanism under the various conditions, of daily life.

The principles governing the proper care and right use of the human mechanism and its surroundings form the subject-matter of hygiene and sanitation; and practical hygiene and sanitation consist in the application of the principles of physiology and sanitary science to the conduct of physical life. Their object is the preservation and promotion of health, the prevention of premature death, and the establishment and maintenance of the highest possible working efficiency of the human mechanism. 


\section{B. Health and Disease}

All things vary from time to time in respect to their condition. They may be cold or hot, wet or dry. Machines may be in good condition, - in good working order, - or the reverse; and the human body, the human machine, is no exception. It may be cold or hot, wet or dry, in good condition or in bad condition.

Health is a condition of the human mechanism in which all the organs are sound and in good working order. "Perfect health" is much the same thing, since imperfect health suggests lack of soundness, i.e. disease. "Robust health," "strong health," "sound health," "good health," and all similar terms are easily understood as signifying various states or conditions of the human mechanism. The common use of such a term as "broken health" testifies to the popular recognition of the fact that health is the normal or sound condition of the machine. The terms "poor health," "weak health," "feeble health," etc., are obviously contradictory in themselves, and really refer to states or conditions in which health is either imperfect or altogether wanting.

1. Degrees of Health. - There are, nevertheless, degrees or variations in health as in other conditions. A stone may be very wet or very dry, or only slightly wet or half dry. A watch may be in tolerably good condition or in excellent condition. Likewise the human body may be in fair health only, or in excellent health, or in "splendid" health.

2. It is the Aim of Hygiene and Sanitation to secure the Best Health Possible under Any Given Conditions. - In youth fair health is not enough to expect, but in extreme old age anything beyond this may be impossible. Some trades or industries are, from their very nature, unhygienic and correspondingly dangerous. Moreover, the degree of health, as of other things, is largely determined in each 
individual by personal ambition, resolution, and effort. Many persons go through life on too low a plane of health simply because they are too unambitious, too careless, or too indolent to make the effort needed to rise to any higher plane. Without strong desire for improvement, improvement rarely comes.

One characteristic of the human body in health is that it does its work with ease; but when it passes into an unsound or abnormal condition this characteristic tends to disappear, and dis-ease, disturbance, or difficulty in operation takes its place. A "jumping" toothache, the "sore" throat of diphtheria, the "uneasiness" of dyspepsia, the "pains" of rheumatism, the " racking" cough of advanced consumption, the "splitting" headache of incipient typhoid fever are good examples.

Disease is a condition of the human mechanism in which one or more of the organs is unsound or abnormal, or in such poor working order as to interfere seriously with the welfare of the entire mechanism.

3. Degrees of Disease. - We speak of "mild disease," "severe disease," and even of "malignant disease," using phrases which testify to the fact that there are degrees of disease or disturbance of the human mechanism, no less than of its health, and the attention which we give to disease is largely determined by its severity. When we have a cold in the head we regard the disease as a trifling matter and expect it to pass away of itself in a day or two. We are apt to summon a physician only when we find that we have a fever, or when some trouble, which at first seemed of small consequence, "hangs on" or seems to be getting worse instead of better. We are especially apt to call in the doctor when we are actually suffering pain and are unable to find relief, - all of which facts bear witness to our practical recognition of the truth that there are various degrees of disease as well as of health. 
4. Different Attitudes assumed towards Disease. - Various attitudes are assumed by different persons towards health and disease. One attitude, represented perhaps by the practice of the majority of people, is to go about one's work, whatever that may be, giving no thought whatever either to the maintenance of health or to the avoidance of disease ; in other words, to pay no attention to the mechanism and to do nothing to keep it in order; to wait until something happens, some breakdown occurs, some disease has clearly developed, and then hastily to take a dose of medicine, or, finally, to call a physician. This we may call the attitude of heedlessness.

$A$ second attitude is that of neglecting any active cultivation of health, but carefully attempting to avoid those things which are liable to produce disease. In this case persons often give great attention to the choice of diet, to protection against cold, to the purity of their drinking water, their food supplies, etc., fixing their attention wholly on the agents of disease and assuming that, if these be kept at a distance, the body will take care of itself. This may be called a half-hygienic attitude.

A third attitude - the reverse of the second - consists in actively cultivating abounding health by attention to those things which are believed to build up a strong constitution, in the belief that no disease can attack a strong and vigorous body. Such persons concentrate attention on health and underestimate the possibilities of succumbing to attacks of disease. This also is a half-hygienic attitude, although in practice perhaps somewhat safer than the second; very many, perhaps all, diseases are less likely to appear in a strong and vigorous body than in one which is not in sound health. But if the experience of the race teaches anything, it is that strong men, seemingly in perfect health, often succumb to attacks of disease. It is not safe, even for a healthy man, to swallow the germs of 
Asiatic cholera; it is not safe, even for a healthy man, to prick his finger with a knife which has been used in lancing a boil. Without in the least undervaluing the importance of maintaining health and physical vigor as preventives of disease, we cannot too strongly affirm that these are not absolute preventives, that they are not reliable preventives, and that in some cases they are not preventives ${ }^{1}$ at all.

$A$ fourth (and the only right) attitude toward health and disease is that which actively seeks to maintain in the mechanism the highest possible degree of health under all conditions, and at the same time constantly takes all reasonable precautions to ward off attacks of the external agents of disease. This is the true hygienic attitude, as indicated by reason and modern science; and this attitude of mind we shall endeavor in the following pages to encourage, justify, and strengthen in students or readers of this work.

\section{The Three Great Factors of Disease}

5. The Three Great Factors of Disease. - Keeping always in mind the truth that the human body is a machine or mechanism, and agreeing to regard any condition as one of dis-ease in which the body does not do its work smoothly or with ease, we perceive that there are three great causes of disease of the body, just as there are three

1 The truth of this fact is illustrated when there appears among a people some disease to which neither they nor their ancestors have regularly been exposed; the ravages of epidemics of Asiatic cholera in Europe and America, and the history of the great plague in Europe in the seventeenth century, or of yellow fever in our own southern states, being cases in point (see Daniel Defoe, Journal of the Plague Year; James Ford Rhodes, History of the United States, I, p. 400). The North American Indians, who wère presumably strong and healthy, were decimated by measles, - a comparatively mild disease, - when this was brought among them by the early settlers of this country. 
chief causes of trouble in the running of a locomotive. These are (1) imperfections in the mechanism itself; (2) unskillful operation and care; and (3) unfavorable external conditions. Let us consider carefully the part played by each of these in the maintenance of health and the prevention of disease.

(a) Imperfections in the Mechanism. - The wheels of an engine may not be perfectly true, some of its valves may leak, some bearing may be unduly exposed to dust. So is it with the human body. Wonderful as is the human mechanism, it is never perfect. A valve in the heart may leak and permit "regurgitation" of the blood; a defect in the structure of the spine may make it hard to hold the trunk in its normal posture; the glands of the stomach or pancreas may be made of poor material and so secrete an ineffective digestive juice; in short, any organ may be of poor construction and so have imperfect capacity for work. Such constitutional defects may be born with us, or they may be acquired by some accident or other circumstance which leads to irreparable and permanent injury. Where they exist they must be recognized and reckoned with in what we attempt to do, although their cure or compensation is by no means hopeless. The deaf mute adapts himself to a lack of hearing, and in spite of it communicates with his fellows; and men and women with serious organic troubles may often lead useful and, on the whole, healthy lives.

Again, every human body possesses as the outcome of its construction or constitution more or less capacity to endure hardship and to struggle for continued existence. In the strong this capacity, loosely called vital resistance, may be very great, and in the weak or feeble very small; but in order that life shall continue at all, every human body must have more or less of it. It is required to withstand heat and cold, underfeeding and overfeeding, the attacks 
of parasites, the work and the play of life, the infirmities of age. If it be very great, almost all hardships can be endured, almost all diseases avoided or overcome; if it be very small, as it often is in old age, even the grasshopper may become a burden.

As we pass middle life and old age creeps over us we find this power of vital resistance lessened. Of all people who enter their seventieth year, a much larger percentage die before reaching their next birthday than is the case with those entering their twentieth year. This can only mean that the ability to cope with unfavorable conditions is lessened as age advances. The body shows by growing feebleness that it is wearing out, and ultimately succumbs to disease which in earlier life would have been a matter of small consequence. Hence it follows that old people must reckon with a poorer constitution, and must give greater care than the young to the bodily machine.

(b) Unskillful Operation and Care. - The most perfect engine will behave badly in the hands of an ignorant, unskillful engineer or fireman. There is a proper method of firing, a proper method of starting; and when a grade is to be ascended it must be taken in the proper way. When these things are not done rightly the engine is very apt to suffer damage, even to acquire structural or constitutional defects; and in no such case can it be expected to do its best work, or to do any work, with perfect ease. Human life involves the operation of a much more delicate engine or mechanism. The human body is a machine calculated to do work, and when we say that it is alive, we mean that it makes use of the potential energy of foods to accomplish ends which no lifeless machine can accomplish; but it does not do this life-work without management or operation. It is the faithful servant of an intelligent will, and it may be worked or used wisely or unwisely, skillfully or unskillfully. This engineering, management, direction, or operation of 
the human mechanism constitutes the physical conduct of life, and is one of the most fundamental and important elements in the maintenance of health.

(c) Unfavorable External Conditions. - Again, the best work of an engine requires more than good construction and skillful operation; it also requires favorable conditions and surroundings. If the road-bed be poorly ballasted or the rails rusty and uneven; if the weather be so cold as to make it impossible to keep up full steam in the boiler; if the water tanks be not kept supplied with water, or the coaling stations with fuel, poor work and often actual injury to the mechanism itself - constitutional injury - is the result. Finally, if by chance a stone has rolled upon the track, or a signal has been wrongly set and a collision results, a good locomotive may be disabled or even ruined.

So with the human mechanism. Like all other living things it cannot continue its work under certain external conditions. It cannot live without food in a desert; it cannot endure exposure to extreme cold without protection; it cannot keep sound in a room with leaky gas fixtures, or in a cell which admits no sunshine. It must have pure drink and pure food, and it must avoid exposure to the contagion of diseases against which it has no sure defense.

6. Definition of "Environment." - We have already used and shall hereafter often use the terms "environment" and "environmental," and a word in explanation of them may be serviceable at this point, although they have been defined briefly in Part I and will be further considered beyond. They are used in this work, as in biology in general, for that portion of the universe not ourselves, and generally for those portions of it comparatively near to us. A man's home, for example, is a part of his normal environment, and so are his relatives, friends, and neighbors, his horses, dogs, and pets, his clothing, and his surroundings generally. In the same category come, however, more remote things, 
such as the village, town, city, or state in which he lives. Even the sun, the intermittent light from which causes day and night, with their corresponding alternate sleeping and waking, working and resting, though so far away, is a part of man's environment. We have referred in Part I to the blood and lymph as the environment of the cells of the body, and such they are; but this internal environment (of the cells) need not be confounded with the external environment (of the body). This latter may be concisely defined as everything outside ourselves.

A close relation always exists between the body and its environment, by which the former is, so to speak, in harmony with or adapted to the latter. Just as the ship is adapted to the sea; the punt, the shell, and the canoe to quiet waters; the bicycle to good roads, — so also man's body reaches its best development and does its best work, not in the tropics or at the poles, but in the more temperate zones to which it appears to be better adapted. A perfectly healthy organism might, perhaps, be defined as one perfect in construction or constitution, and perfectly adapted to its environment.

7. Man and His Environment. - Man is absolutely dependent on a favorable environment. He cannot, like a fish, live long in the sea; or, like a bird, in the air. $\mathrm{He}$ cannot even make his way to the poles of the earth; and at an elevation of six or seven miles in a balloon or on a mountain he perishes. Fire quickly destroys, and molten iron or boiling water quickly kills him. A constant temperature of even $100^{\circ} \mathrm{F}$. causes him great discomfort, if not danger. Intense light is painful to him, intense noise disagreeable. The same thing is more or less true of other animals. They flee from fire, shudder or cry at great noises, are often dazzled by intense light.

But while the environment thus narrowly limits and controls human life and activities, man may, to a great 
extent, choose or control his immediate environment, a power which he shares with many other animals. The beaver and the muskrat build rough shelters or houses, many birds build nests, foxes dig burrows, and some birds and other animals migrate from one environment to another. Man also seeks or makes for himself the shelter of caves, huts, wigwams, houses, or hotels. These he heats if cold, or cools if hot. He lights them by windows by day, and by artificial light by night. He provides for himself, instead of the ground, couches to lie upon, chairs to sit upon, and tables upon which to place his food. All this is a kind of artificial adaptation of the environment to man, and, if wisely done, is an important aid to health, since it tends to secure for the body that favorable environment which is one of the greatest factors of health. Man can, moreover, modify his surroundings at pleasure and make them what they should be, or at least more nearly what they should be. Fires are used in winter, shades and fans in summer; ventilation replaces bad air with good air; by proper drainage, filth and dampness are avoided; by artificial lighting, darkness is done away with; by cultivating. the soil and by raising cereals and cattle, the food supply is maintained. Thus in many ways the environment is controlled and healthful conditions are promoted or maintained. Failing all else, man may travel for a while and find benefit in temporary change ; or, like thousands arriving in America, may abandon one environment and emigrate to another.

8. Scope and Subdivisions of Hygiene and Sanitation.The considerations dwelt upon in the foregoing pages indicate the scope and possibilities of the science of hygiene. Given the constitution of any individual as it is at any one time, we must seek to maintain or place that constitution in a condition of health, or efficient working order, in two ways : first, by the proper care and operation of the mechanism 
itself, including the proper direction of its activities ; and second, by providing for it favorable surroundings or environment. The former we call hygiene, the latter sanitation. Each of these efforts reacts on the constitution; improper operation of the muscles in muscular work, or improper use of the nervous system in mental work, may "undermine" a strong constitution and lower its vital resistance; similarly, a bad climate, a neglect of the ventilation of living and sleeping rooms, the use of polluted water or milk or other food, exposure to the contagion of disease or to excessive cold without proper clothing, - all such failures to provide a proper environment may injuriously affect the constitution or structure of the body.

It is impossible to draw any sharp line between the care, management, and operation of the body mechanism, and the care and control of the environment, and it is neither necessary nor desirable to do so; but we shall begin our detailed study with those things which concern chiefly the care, use, and operation of the mechanism itself, — such, for example, as the proper direction of muscular and nervous activity; alimentation or right feeding; the use and abuse of stimulants, narcotics, and other drugs; bathing, clothing, the care of the eyes and ears, etc. These matters which concern chiefly the individual or the person constitute that part of our subject known as personal hygiene.

We shall then proceed to consider those matters of health which concern not only individuals but communities of individuals, such as families, cities, states, and nations, for example, the site, ventilation, heating, and plumbing of the dwelling house; the control of food supplies, as to their purity; public supplies of water and milk; sewage disposal; the infectious and contagious diseases. All these things require the coöperation of many individuals, either as families, or as citizens of an entire town, city, or nation. Hence they are classed under domestic hygiene and 
sanitation and public hygiene and sanitation. The exact meaning of these terms will be explained in Chapters XXVI and XXX.

It should be understood that the following statements on the hygienic conduct of individual and social life are not equally applicable to all who read them. For one, those on muscular exercise are more important than those on nervous strain ; for another, those on domestic hygiene are more important than those on the hygiene of the person. No attempt has been made to indicate the relative importance of any part of the subject, either by the order in which it is treated or by the amount of space devoted to it. The application must be made by each reader for himself, with strict reference to himself and to the conditions of his own life and environment. The principal desire and aim of the authors is to persuade every one who reads this book, not merely to study and to know himself as a physical mechanism with the same earnestness and thoroughness with which he would study a valuable watch, a bicycle, a yacht, or an automobile, but also to use that mechanism scientifically -i.e., intelligently, carefully, and skillfully, - to the end that life may be longer, more useful, and more enjoyable. 


\title{
PERSONAL HYGIENE
}

\author{
CHAPTER XVII
}

MUSCULAR ACTIVITY

\section{A. The Ministry of Muscular Activity to THE Body as a WhOLE}

It is sufficiently obvious that it is through muscular activity that we do many necessary, useful, or otherwise desirable things ; and it is also a matter of common experience that muscular activity is required in order to build up strong muscles. A very considerable amount of it is required in order that the laborer may do his work, and a similar amount is necessary in order that one may become an athlete. But the effects of muscular activity on the body as a whole are not so obvious; while a large number of people think that it is " a good thing" and a smaller number are convinced that it is absolutely necessary to the best of health, yet we not infrequently hear men and women seriously question the latter proposition and even venture to doubt the truth of the former.

Now there is nothing in hygiene more clearly established than that muscular activity is absolutely essential to healthy living. The effects of a sedentary life may not show themselves at once, but almost without exception they will assert themselves in the end. Muscular work, in other words, not only enables us to influence our surroundings, not only builds up strong muscles, but in other and equally important though unseen ways ministers to the health of the 
body as a whole. It is the purpose of this section to present this, the most important hygienic side of our subject, by describing some of the physiological effects which muscular activity produces in the body, and the hygienic value of each of these effects.

\section{The Present Use of the Term "Muscular Activity."}

- In the present chapter the term "muscular activity" is used in a somewhat general sense, and without attempting to set sharp limitations upon it. Strictly speaking, of course, muscular activity would include all work done by the muscles of the body, and this is of various kinds. Even those persons who do no manual labor unconsciously perform muscular work; the heart works on, the breath comes and goes through orderly muscular contractions; sitting and standing, speech, gestures, mastication, - all these things involve muscular activity, and do, as a matter of fact, contribute something to the maintenance of the healthful conditions of the body. It is not improbable that they are the physical salvation of thousands of people leading sedentary lives. At the other extreme are those who perform severe manual labor, or who engage in vigorous exercises or purposely cultivate exceptional physical strength.

We are not, however, directly concerned at present with either of these extremes, nor with those forms of muscular activity so common to-day in workshops where, hour after hour, the workman performs the same task over and over again. We are rather concerned with those forms of muscular work which are seen in a lumber camp or on the farm; which present the characteristic of variety and involve the use of the musculature of the body as a whole; in short, those forms of activity by which until very recently the human race has supported itself in its daily life. Such things as brisk walking, running, rowing, woodchopping, swimming, tennis playing, would thus be placed 
in the same class, since they involve a use of the muscles similar to those which we have mentioned.

2. The Physiological Effects of Muscular Activity and their Hygienic Value. - We may now turn to the hygienic value of the more important physiological effects of these general muscular activities, leaving for subsequent consideration exercises designed for special purposes, such as much of our gymnasium work.

(a) The physical and chemical changes in the working organ are greater than those accompanying any other bodily activity. The output of carbon dioxide by the body per minute is increased at once from three- to tenfold with what would be termed moderate or vigorous exertion, while digestion seldom increases it more than one fifth, and mental work shows practically no effect upon it. Large quantities of heat are likewise liberated and the temperature of the muscle rises several degrees. These physical and chemical changes are mentioned first because the hygienic effects upon the body as a whole are to be traced to them as the primary cause.

(b) As the result of these changes in the muscles new physical and chemical conditions are introduced into the blood and lymph. The excess of carbon dioxide is entirely excreted by the lungs, so that the blood carried to the other organs by the arteries shows no increase in this substance; but other waste products (such as salts of sarcolactic acid), whose elimination requires the coöperation of other organs than the lungs, are found in the arterial blood in larger quantities than during rest. The chemical and physical characteristics of the immediate environment of every cell of the body is thus changed, and profoundly changed. Let us now consider the reaction of other organs to these changes in the muscles and in the blood and lymph.

(c) Some of the most striking effects of muscular work are those which are connected with the heat-regulating mechanism. 
The large liberation of heat by the working muscle necessitates active measures to get rid of that heat and maintain the constant temperature. The small arteries of the skin dilate, while those of internal organs constrict, perspiration is secreted, and all these processes are carried out in a coördinated manner. The nervous mechanism of heat regulation is given a new form of activity, and thus receives valuable training in adjusting itself to the changing conditions with which it has to cope in daily life.

(d) Closely connected with the foregoing is the (temporary) relief afforded to any congestion of blood in the internal organs. Sedentary occupations usually involve more or less overfilling of the blood vessels of the stomach and intestine, the pancreas, the liver, the spleen, and the kidneys; they also involve the absence of those movements of the trunk whose pumping action affords a marked assistance to the flow of blood through the abdominal organs (p. 147). The congestion thus caused is not a good thing ; it almost certainly renders the organs concerned more liable to inflammatory processes (see Chapter XXI), and, if there has been established any tendency to catarrhal conditions (see p. 381), it aggravates that tendency. Popular experience has long associated with health a good color of the skin; and, while it is not safe to make such an inference in all cases, pallor very frequently means internal congestion, unhealthy digestive functions, and greater liability to cold in the head or the chest.

(e) Muscular activity is the only thing which can be depended upon to increase the work of the heart. While this fact makes caution and moderation necessary for persons having certain forms of heart disease, yet for the vast majority of people it is of the greatest hygienic importance to accustom the heart to reasonably hard work. Only in this way does it receive the training necessary for its proper development and for the maintenance of its strength. 
Emergencies will arise when the heart is called upon for severe effort, brief or prolonged. The familiar example of the sudden "sprint" for a car is a case in point; and there are times, as in pneumonia, when the issue in sickness is largely determined by the endurance of the heart. In too many such cases, if the patient escapes the fatal issue, it is only with a permanently weakened heart.

It is important not only that the heart should be kept ready for emergencies but also that it be kept in condition for vigorous work as a regular duty of daily life. One of the worst of "vicious circles," as physicians call them, is the acquirement of a weakened heart by abstention from proper muscular exertion, and, as a consequence of this weakened heart, increasing disinclination to exertion of any kind whatever. The failure to take proper exercise leads to deterioration in strength and endurance on the part of the heart; and this cardiac deterioration, with the resulting discomfort of breathlessness, leads in turn to abstention from muscular activity.

(f) Muscular exercise is the one agent which increases the depth and frequency of the respiratory movements. The hygienic importance of this does not lie in the better oxidation of wastes, since, so far as we have any accurate knowledge on the subject, it would seem that the processes of respiration during sedentary life more than supply the existing demands of the tissues for oxygen. The increased respiration is rather of importance because of the secondary effects of the respiratory movements in promoting the flow of blood, and especially the flow of lymph (see p. 147).

It is probable that the "freshening effects" of muscular exercise are to a very large extent attributable to the improved lymph circulation in the tissues, and this effect, it will be remembered, is felt in the immediate environment of almost every cell in the body. The suction action of inspiration quickens the lymph flow from all organs 
outside the thorax (p. 173), and the increased pumping action of the respiratory movements themselves aids the lymph flow from the lungs and other organs within the thorax. Waste products are more completely removed from the lymph spaces surrounding all cells, and thus one of the most important of fatigue conditions is relieved (see p. 59). Where lymphatics are subject to the pumping action of contracting muscles and of the alternate flexion and extension of joints, the suction action of the respiratory movements is reënforced. This pumping action especially affects the lymphatics of the arms and legs, and those of the abdominal cavity (through the action of the diaphragm and the trunk movements).

The increased respiratory movements also contribute to greater mobility of the ribs and to the better ventilation of the lungs. During vigorous exercise all lobes of the lungs are used, and the dangers attendant upon disuse of the apical lobes (p. 172) are largely obviated.

(g) Moderate exercise exerts a favorable effect upon the digestive organs, although the precise action involved is very complicated. Here also it improves the lymph flow, thus promoting absorption and producing better conditions in all digestive glands and in the muscular apparatus of the digestive tract; it prevents continued congestion and the unfavorable attendant conditions. It is probably also a direct stimulus to peristalsis, for unquestionably the exercises which involve movements of the trunk often prove a peculiarly efficient remedy for constipation.

The above summary is very far from a complete enumeration of the effects of muscular exercise upon the organism, but it will suffice to show how essential an element such exercise is in the life of the body. The training of the heat-regulating mechanism, the training of the heart, the improved lymphatic environment of every cell resulting 
from increased breathing movements and from the pumping action of mechanical motion, the relief of internal congestions and the favorable influence upon digestive functions, - all these things are fundamental to healthful cell life.

\section{Muscular Activity a Necessity for All. - We often} hear of men and women who live to old age and do large amounts of mental work with seemingly little or no muscular activity; and it is sometimes suggested that the experience of these people proves that exercise is unnecessary. There are also on record a few cases of men who can drink large quantities of whisky without getting drunk; but it will not be contended that most men can do likewise. As to any line of right hygienic conduct there are some among the hundreds of millions inhabiting the earth who can do the reverse with impunity; but they are not to be taken as safe guides. The cases are very few indeed where abstinence from muscular activity persisted in as the rule of life is without disastrous results; the bad effects do not always come in a day or a week or a year, but sooner or later they almost invariably show themselves. We must never fail to distinguish carefully between the immediate and remote effects of any line of conduct; and nowhere is this caution more needed than in observing the effects of a sedentary life, the evil results of which, though sometimes long postponed, usually appear sooner or later.

Some muscular exercise is a hygienic necessity for every period of life ; it belongs to no one age. Youth is the time when athletic sports, games, and all kinds of activity are most agreeable, most necessary, and most enthusiastically pursued. In old age the changes which take place in the arterial walls necessitate caution as to severe exertion. But these are only the extremes. Rarely indeed do we meet with people who would not be benefited by a walk of several miles a day, at a rate of three or four miles an hour; and it cannot be too strongly insisted that the 
inability to do this with enjoyment and profit is in almost every case because the habit of taking exercise is not kept up. The heart is not as strong as it once was; the connective-tissue elements of the muscles, the ligaments, etc., become sore upon taking exercise, not because of any inevitable "old-age change," but because the ability to do the work easily has not been maintained by constant practice.

It would be amusing, if it were not sad, to see how the average adult American will try almost everything which holds out the slightest promise of maintaining some sort of health rather than take muscular exercise, - alcoholic drinks (to dilate cutaneous vessels), Turkish baths, massage, patent medicines, - anything rather than a horseback or bicycle ride, or a brisk walk, or some other simple and perfect remedy which stands within easy reach. It is not to be expected that when these exercises are first tried after years of sedentary life, they will be enjoyed; and too often the man or woman, instead of persisting patiently, draws the conclusion that the time for such things has gone and only resignation to old age is in order. When young men and women begin life, it should be with a clear conception of the danger of falling into habits of muscular inactivity, and with a conscious and strong determination to avoid this danger.

4. The Conservation of the Enjoyment of Muscular Activity. - Muscular activity is so necessary for health, for the enjoyment of life, and for usefulness, that the ability to take it should be conserved at all costs. We should not only keep " in practice" by making it as much a daily habit as eating or sleeping, but we should also avoid those unfavorable conditions which interfere with our enjoyment of it. Some will not walk a step more than necessary because, by the use of improper shoes, they have acquired deformed feet, unable to support the weight of the body; sometimes a sunstroke, following incautious exposure to 
the hot sun, leaves the heat-regulating mechanism so injured that muscular exertion except in cool weather becomes unsafe or even dangerous; exposure to dampness often brings rheumatism, an almost insuperable barrier to pleasurable movement of any kind; some infectious diseases leave their trace in the form of an incurable organic weakness which makes muscular activity inadvisable. These things should, of course, be avoided for their own sake; they should be avoided also because of their serious indirect effects on health.

5. General Character of the Most Useful Exercises. To specify the exact forms or amounts of muscular exercise advisable would take us beyond the scope of the present work. Here as elsewhere the student must work out his own salvation. In the following chapters we shall discuss, as far as possible, the characteristics of some special exercises; for the present a few general suggestions may prove useful. The muscular activity which formed part of the life of our ancestors may be described as generally moderate, though at times vigorous or hard; only exceptionally did it involve extreme endurance or great muscular strain. Our ancestors were not, as a rule, given to "tugs of war," or to putting up heavy dumbbells, or to making inordinately long runs, or to "giant swings" in the gymnasium; nothing like a hundred-yard dash or a four-mile boat race was a common occurrence among them. Where work of this kind had to be done it was left to those who, by reason of exceptional strength, were especially fitted for it; mankind as a whole did no such work, and it is not necessary (or even advisable) for most of us.

Nor can it be claimed that the cultivation of great muscular strength was a common practice. There was a much higher average of strength than among us, and we should probably be better off were our average higher than it is; 
but if we can judge at all from the history of mankind, such training as that required to break some college strength test is not demanded for hygienic purposes. Nor does our own experience tell a different story. Very strong men are no healthier nor longer-lived than those of only average strength, and, in general, the athletic ideal is not the hygienic ideal. It is not necessarily unhygienic, but it is not required for purposes of health.

It is not desirable that exercise taken for general hygienic purposes shall be unduly fatiguing. A moderate amount of fatigue is not unwholesome, since fatigue brings with it the desire for rest; nor is fatiguing exercise necessarily harmful. But exercise need not necessarily be of this character; and, in view of the other work of life, it is certainly better to avoid undue fatigue, especially when we cannot rest well afterwards. A walk of six or eight miles will do more good than one of forty or fifty.

6. Exercise for Women. - Muscular exercise is no less essential to the health of women than of men. Fortunately the day is past when false standards misinterpreted the truth that woman's most natural sphere in life is the home to mean that, tied down to the confining duties of household life, she should never know the joy of movement, except in dancing (and sometimes not even in that); and then proceeded to make sure of the result by clothing her in narrow, pointed, high-heeled shoes, heavy skirts, and tight-lacing corsets. The reaction from this state of affairs, at times going to the opposite and undesirable extreme, has unhappily at times produced in women exhibitions of mannishness which once led a lady to speak of "that terrible thing called muscular exercise." But disgust with these grotesque but avoidable consequences should not be allowed to blind us to the fact that a reasonable enjoyment of daily muscular activity is as much a necessity for women as for men. 


\section{B. General Muscular Exercise}

The present section deals with the use of muscular activity for its hygienic effect upon the body as a whole; the next section with its employment for special purposes. Exercises undertaken for their general good effect are frequently spoken of as "hygienic"; the term is, however, objectionable, and we shall speak of them as general muscular exercises.

General muscular exercise is of hygienic value because it produces the physiological results which have been enumerated in the preceding section, - results which have been shown to constitute essential elements of the normal internal environment of the cells of the body. To review the separate offices of this ministry to the normal conditions of the body :

1. General exercises should produce to a considerable extent those physical and chemical changes which accompany muscular contraction, with the resulting effects upon the physiological condition of the muscle itself and upon the general internal environment, the blood and lymph.

2. They should exercise, and so train, the heat-regulating mechanism.

3. They should tend to relieve vascular congestion in internal organs, bringing the blood in larger quantities to the skin.

4. They should afford training to the heart.

5. They should increase the ventilation of the lungs.

6. They should increase the flow of lymph in the lymphatics, and thereby improve the environmental conditions of all the cells in the body (see Chapter IV).

7. They should exert a favorable influence upon the digestive processes, promoting proper secretion and absorption, and tending to prevent unhealthful conditions. leading to constipation. 
Such being the physiological ends sought for, we may conclude, as to the character of such exercises:

1. They should consist of rhythmic rather than of sustained contractions. These involve less fatigue, are more enjoyable, and especially facilitate the flow of blood and lymph.

2. They should be vigorous, somewhat prolonged, and should usually be continuous. A brisk walk or a run meets most demands; so do bicycling and many games. The strolls or saunters which are too frequently mistaken for exercise do not meet the reasonable hygienic demands of the body; they involve only an insignificant increase of chemical activity in the muscles, they hardly affect respiration, they do not train the heart; in short, they do not produce adequate physiological effects to accomplish hygienic ends.

3. They should involve considerable movement on the part of the trunk as well as the limbs. Many excellent forms of exercise, such as bicycling, are somewhat deficient in this respect. It is not meant that sudden and violent trunk movements are called for, but that hygienic exercise should bring full change and relief from the constrained positions of the trunk imposed by the sedentary occupations of modern life. A vigorous walk, with its accompanying increase of breathing and trunk movements, fencing, and games which involve the throwing and catching of a ball are especially good in this respect.

4. They should be accompanied by full and free respiration. The importance of this requirement needs no comment. Constricting clothing should not be allowed to interfere, and, as far as possible, the trunk should be held erect, the neck and shoulders back, so as to permit the freest movement of the upper ribs.

5. It is advisable not to confine oneself wholly to one form of exercise. Similar considerations to those which 
hold in the choice of food apply to some extent to exercise. At the same time it must be admitted that perfect health can frequently be maintained to old age by using only one kind of general exercise, such, for example, as walking.

7. Considerations concerning Fatigue. - The relation of general exercise to fatigue is a matter of considerable interest and also of importance in correlating muscular work with the other work of life. Fatigue of the whole organism is a very complicated matter and involves much more than the total amount of chemical change in the muscles and of the resulting waste products in the system as a whole. We may, for example, be made very tired by unpleasant sensations from the joints and tendons, or by walking in shoes which do not permit free play to the bones, ligaments, tendons, and muscles of the foot; and this even when the amount of muscular exertion involved may have been slight. It is well known that merely standing still for a time will frequently cause more fatigue than will a longer time spent in walking.

Again, some forms of exercise throw a relatively large share of the total work on some muscle or small group of muscles, while others distribute the total work more evenly over larger groups. Walking and running are very unlike in this respect; in the former the weight of the body must be lifted from the ground with each step, - especially when we walk very erect, - by the extensor muscles of the leg, and chiefly by the extensors of the ankle joint; running, on the other hand, consists in a continual falling forward and the restoration of equilibrium by a more general action of the muscles of the body as a whole. A walk of four and a half miles an hour is much more fatiguing to a person in good training than a run of four and a half miles an hour, because in the former case a few muscles are thrown into very vigorous contraction and so give rise 
to severe local sensations of fatigue, sometimes accompanied by cramps in the muscles.

8. Some Examples of General Exercises: Cycling. Bicycle riding is remarkable for distributing the total work over large numbers of strong muscles, so that the amount done by each is relatively small; consequently, where there is but little hill-climbing or no strong head winds, local fatigue is but slight, although the total work done by the body is considerable. Actual measurements of the carbon dioxide excreted have shown that this is much greater per minute in a ride of eight miles an hour on a smooth level track, than in walking three and a half miles an hour; in other words, the total work is greater. The well-known increase of perspiration brought about by such moderate riding points in the same direction; the chemical changes in the body are greater and so is the associated heat production; and yet any cyclist knows that the conscious fatigue of the ride is as nothing compared with that of the walk. Moreover, in wheeling the weight of the body is not supported on the feet, and we are thus to a large extent relieved from the unpleasant sensations produced by pressure and jar in the ankle and knee joints. It is a characteristic of moderate or even fairly vigorous bicycle riding that it produces a maximum of chemical change with a minimum of fatigue. 'This is of great practical importance. The larger production of carbon dioxide involves deeper breathing, and, as the student now well knows, increased work on the part of the heart.

Within proper limits this is, of course, good for the heart; there is some danger, however, that in the absence of conscious fatigue we may throw upon that organ more work than is good for it, and medical experience leaves no doubt that many cases of injury to the heart have resulted from injudicious cycling; that is to say, from "scorching" 
against strong head winds and in "showing grit" by refusing to get off and walk up very steep hills. There are occasions when it is not wise to be too ambitious, and when " discretion is the better part of valor."

9. Some Examples of General Exercises: Games. - Somewhat similar considerations apply to most of our more active games, such as basket-ball, football, tether-ball, hockey, polo, etc. They are perfectly safe for healthy people when not played more vigorously than the training of the heart justifies; the fact that there is an element of danger in them is no reason why they should not be used, but it is a very good reason why they should not be worked to extremes, and especially why we should be sure, from competent medical advice, that there is in those who play them no organic trouble to begin with, and that players are in good training when they play most intensely.

The choice of the kind of muscular work and exercise involves so many considerations other than those which are strictly physiological and hygienic that it is impossible to give in an elementary treatise like this any detailed discussion of the special merits and defects of each. We often have other aims in view besides the purely hygienic; thus the group games, such as football, baseball, basketball, hockey, etc., train the spirit of coöperation and may be made useful means of moral training. In camping in the woods canoeing is not simply a means of exercise, but also a means of transportation; and under other conditions the same thing is true of horseback riding, rowing, etc. Woodchopping, digging, porterage, and plowing are valuable means of livelihood. It is believed, however, that the principles here given will help the individual to form a correct judgment as to whether his work in life supplies him incidentally or inevitably with the needed general muscular activity for hygienic purposes, and, if it does not, to plan to meet the want. intelligently. 
The combination of muscular exercise with some other pursuit is highly desirable, and when practicable often simplifies the hygienic conduct of life. But it is nothing short of a hygienic misfortune to lose the youthful love of activity for its own sake. It is well as we grow older to have golf, or a horse to be exercised (!), or a fishing preserve in the woods, to "take us out in the open air" and make us use our muscles. But a human being who is dependent upon something of this kind to drag him into activity cuts a sorry figure from a moral standpoint. Man's highest distinction is the fact that his actions may arise so largely from processes of psychic life within rather than from some immediate stimulus from without. The proper hygienic conduct of life involves moral fiber as well as physical fiber, and this is especially true of that absolutely essential part of hygienic conduct which depends upon the use of organs like the skeletal muscles, which are so largely subordinate to the commands of the will.

\section{Importance of Walking as a Means of Exercise. -} In their enthusiasm for athletic games and outdoor sports in youth, and for other outdoor activities in middle life, the American people are always in danger of losing their love for the various forms of walking, such as tramping and mountain climbing. Walking is the one form of general exercise for sound people which can always be had for the taking. For this reason, if for no other, it should ever be a part of all sound physical training to conserve the love of tramping and the ability to walk. Apart from the obvious fact that it is in this way that we can get closest to nature and the real beauty of the world in which we live, the possession of the love of the activity involved is one of the most precious possessions of our hygienic life. The man or woman who does not keep and improve this power by use must look forward to the same fate as the servant in 
the parable who hid his talent in a napkin, only to have it taken from him in the end.

11. Fresh Air not a Substitute for Muscular Activity. A word of warning is needed against the folly of supposing that fresh air is a substitute for muscular activity. Fresh air is one of our greatest hygienic blessings, and it is very desirable to live an outdoor life as far as possible. But too many think that lounging in the shade, or riding in the open air in an automobile, a carriage, or an electric car, does for them what muscular exercise alone can do. Especially as age creeps over us and the love of activity wanes from its disuse, more and more does the idea grow upon us that "fresh air" is everything. To many the possession of a comfortable carriage and a pair of thoroughbreds is a misfortune. At one of our most beautiful summer resorts some one said to a local physician, "Medical practice at such a place as this must be very unremunerative." "By no means," replied the man of experience; "people come here where they are tempted to overeat; in the place of exercise they lie back on the cushions of their carriages while they are driven about; their adipose tissue increases rapidly, and very soon it is true that to no class of people is the doctor so absolutely essential as to them." The student can easily make the application for himself. Indigestion, fatty degeneration, insomnia, loss of appetite, nervous prostration, and kindred ills rarely come to those who labor with their hands; and these ills can be largely prevented, even in those who must engage in sedentary occupations, by a wise and intelligent conduct of the physical life, and especially by the daily employment of an hour or so of vigorous general muscular activity properly correlated with the other work of life. 
C. Muscular Exercises for Special Purposes. Corrective Work. The Gymnasium

The muscles may be used not only to produce those general influences which are necessary to the maintenance of health but also to produce desirable special effects, among which the prevention and correction of faulty carriage and action are of great importance. In considering the use of muscular work for this purpose our subject naturally groups itself under two main divisions: first, faults of form or carriage of the body at rest, - in other words, a bad figure; and second, faults of handling the body while it is in motion, - in other words, awkwardness or clumsiness.

12. The Shape or "Figure" of the Body. - The human body may be chiseled in marble or molded in bronze, and the statue thus formed may recall to the mind the shape or figure of the person it represents. But the shape of the living body is not rigidly fixed, as is that of the statue. The bony skeleton is sometimes called a framework, which supports the muscles, viscera, skin, etc. While this is to some extent true, the organs are not rigidly supported by the skeleton, as the canvas is supported by the poles and ropes which constitute the framework of a tent. In other words, the bones of the skeleton are not rigidly joined together; they do not of themselves make a selfsupporting framework; the strong ligaments which pass from one bone to another simply limit or guide the movement of the bones (p. 16); they do not, strictly speaking, bind them together. If all organs save the bones and ligaments were removed, the skeleton would collapse. It is itself held upright by the muscles, which determine what position the bones shall have with regard to one another; and it is more correct to say that the muscles support the skeleton than that the skeleton supports the muscles. 


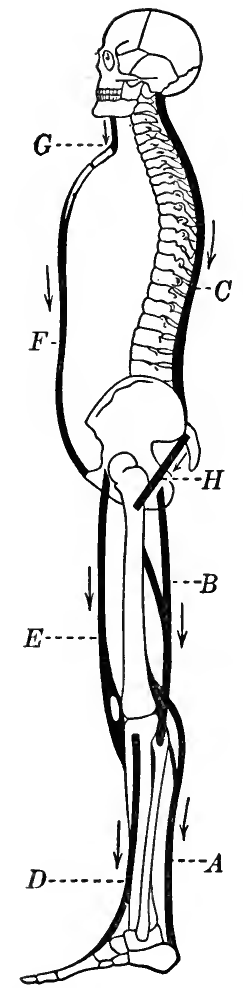

Fig. 105. Diagram showing the action of antagonistic muscles which keep the body erect. After Huxley

Arrows indicate the direction of the pull, the feet serving as a fixed basis of support. The muscles $A, B, H$, and $C$ keep the body from falling forward; $D, E$, $F$, and $G$ keep it from falling backward
13. Round Shoulders as a Type of Faulty Carriage. Their Cause. - The carriage of the shoulders well illustrates the closing statement of the last paragraph. Some people have square, while others have sloping, shoulders; in some the shoulders are held back so that the upper portion of the back is approximately flat, while in others they droop forward, thus causing the upper chest to be more or less contracted and the back "round." To some extent these differences may be due to hereditary structure; but they result, for the most part, from causes which are largely if not entirely under individual control. There is little or no excuse for round shoulders in healthy people, and the marked effect of training is evident in the fine bearing of well-trained soldiers. The truth of this statement is seen when we consider how the deformity is usually acquired, the chief causes being the following.

(a) Faulty Posture. - Round shoulders are uncommon among people whose work requires an erect carriage of the body; for example, among those. who carry things upon the head. With most, however, the occupations of daily life lead to bending forward over work; writing, drawing, sewing, lifting, gardening, paving, machine and tool work at once occur as examples. The trunk is held in such a position that the shoulders tend to fall forward of their 
own weight. This tendency is aided by the wrongly curved backs of most chairs, - which seem as if planned especially

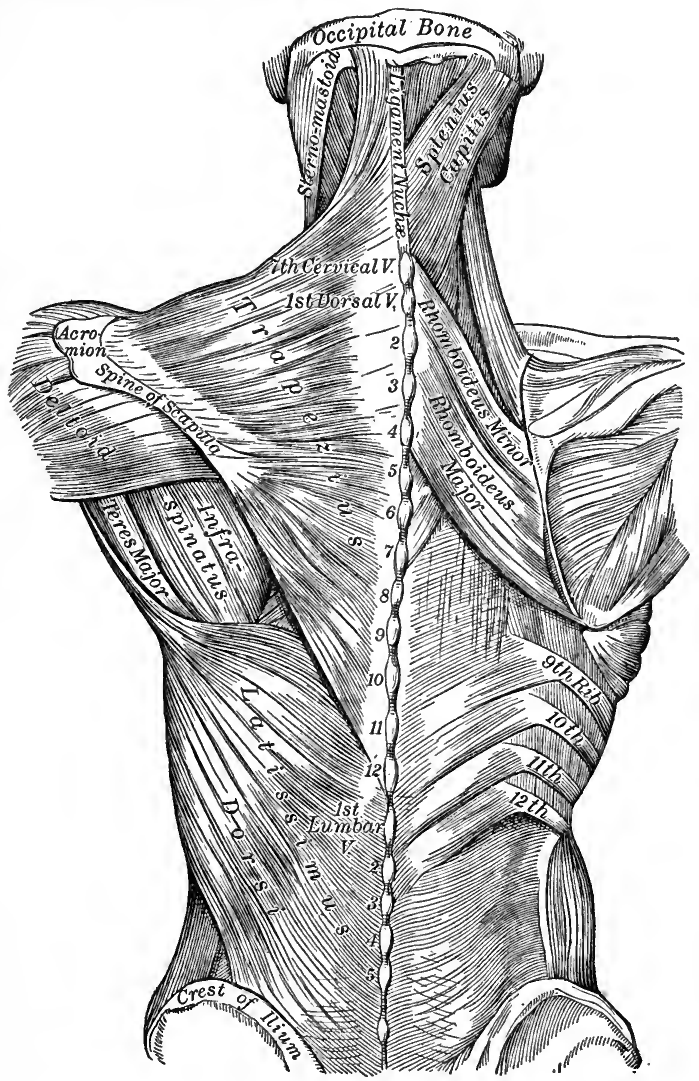

Fig. 106. Some of the muscles of the back

On the left side are shown those immediately under the skin ; by dissecting away this first layer, there are exposed the muscles shown on the right side

to force the shoulders forward, - and in boys by the use of many forms of suspenders.

(b) Improper Balance in the Play of Antagonistic Muscles. - The position of the shoulders with reference to the ribs, 
vertebral column, and breastbone is largely dependent upon the action of several groups of antagonistic muscles, the most important of which are those of the breast and those of the back. Figures 106 and 107 show the general antagonistic action of these muscles. The contraction of the great breast (or pectoral) muscle pulls the shoulder forward and nearer the breastbone; the contraction of the back muscles (rhomboideus, trapezius, and others) pulls them backwards and nearer the backbone. Both groups of muscles are kept in a state of sustained moderate contraction (or tone) by the nervous system; but if the back muscles relax, while those of the pectoral group remain in tonic contraction, the shoulder will be pulled forward and the back will be round. Obviously the maintenance by the nervous system of the proper balance in the action of these and other antagonistic groups of muscles is essential to correct carriage of the shoulder.

(c) Deficient Use of the Back Muscles, with or without the Excessive Use of the Breast Muscles. - Most occupations and

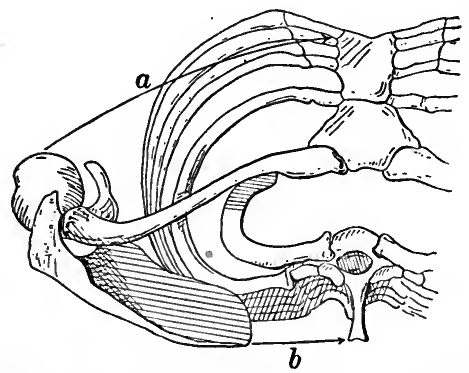

FIG. 107. The skeleton of the trunk seen from above. After Demeny

Showing the antagonistic play upon the shoulder of the muscles of the breast $(a)$ and back $(b)$ activities involve greater use of the breast muscles than of the back muscles. Striking a blow with a bat or an ax, throwing a ball, and similar actions are more usual than acts, like pulling taffy, which extend the arms and draw the shoulder blades closer together. Movements of the first kind obviously strengthen the breast and stretch the back muscles; those of the second kind have the opposite effect. Consequently any marked preponderance of pectoral action tends 
to elongate the back muscles; and unless this is counteracted by movements of the opposite character, which stretch the breast muscle, the pectoral and back groups become "set," as we may express it, in improper relative lengths.

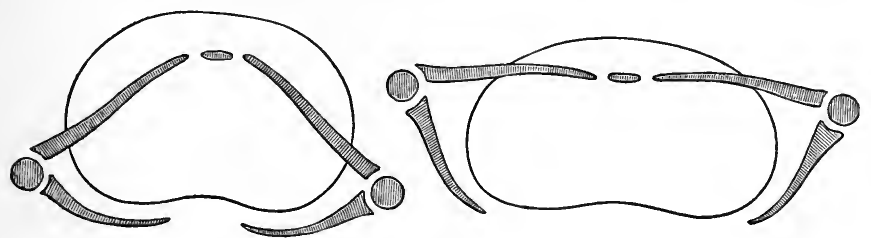

Fig. 108. Correct and incorrect positions of the shoulder girdle. After Demeny

The result is round shoulders. Consequently one of the most important things to have in view in gymnastic work is the use of movements which train the back muscles and stretch the pectorals, thus counteracting the effect of the one-sided use of these two groups of muscles in ordinary occupations.

14. The Period of Growth Especially Favorable for the Acquisition of Round Shoulders and Other Deformities. The length of a growing muscle is determined largely by the distance between its origin and insertion ${ }^{1}$ during the period of growth. The breast muscle will grow to be a longer muscle when the shoulders are held back by the back muscles than when they are habitually allowed to droop forward. In the former case the pectorals grow to sufficient length and do not tend to pull the shoulders forward and downward; and we avoid the excessive length of the back muscles, which makes it necessary for them to take up their own slack before they can keep the shoulders in position.

1 Where a muscle is attached by its two tendons, the point of attachment against which it usually pulls or is fixed is known as its origin, while the one it usually moves is known as its insertion. Thus the origin of the pectoral muscle is the breastbone and ribs, its insertion the shoulder and the upper arm. 
The student can now appreciate the fact that it is in youth, during the period of growth, that deformities are most readily acquired and most easily corrected; for the muscles, the ligaments, the bones, are then in their formative stage. In the case in point, if the boy or girl holds the shoulders properly, the pectoral and back muscles of each side adjust themselves to their proper length; and the shoulders grow into the correct form, just as the sapling which is not bent nor deprived of proper sunlight grows into the symmetrical, beautiful tree. During the period of growth, then, - say up to at least the twentieth year, we can hope to accomplish most in correcting and especially in preventing deformities. The correction and prevention of round shoulders evidently depends upon the proper training and use of the muscles which play upon the shoulder; it is therefore a legitimate part of gymnastic training, for gymnastic training is largely the art of learning to use the muscles properly.

Where there is a special defect to remedy or prevent, special exercises are required. These are of the general character of the "setting-up" drill of the soldier, and in the case in point we accomplish our purpose by using movements which in the first place stretch the pectorals and even overextend them; in the second place, give to the back muscles the exercise which they fail to get in our ordinary occupations, and so bring up their strength, their ability to withstand fatigue and to maintain the tonic contraction demanded of them; and which, in the third place, give us the knowledge of the correct position of the shoulders.

\section{Education of the Consciousness of Correct Posture.}

In explanation of the last point we may say that when one habitually carries the shoulders properly he feels that he is taking an awkward position when he allows the shoulders to droop; on the other hand, the man who 
habitually allows the shoulders to droop forward feels that he is in an unnatural position when he holds his shoulders back. This is largely because in the first case the back muscles and in the second the pectorals must be put on a stretch; it is also due to the fact that the sensations derived from the habitual posture, whether it be correct or incorrect, have impressed themselves on consciousness as signs of the normal conditions; to take any other position is to experience the feeling of something unusual or abnormal.

We learn of the position of parts of our body with reference to one another by sensations derived from the muscles, tendons, joints, etc. (see Chapter XIV, p. 262); and the sensations of position which result when we assume the habitual posture fix themselves in our thought as signs of the normal posture. Our practical, working idea of normal posture, indeed, is nothing more nor less than our experience of the sensations of position resulting from habitual posture. The man who never carries his shoulders back really knows nothing of their correct position, because the sensations from correct posture are lacking; he knows no more about them than a man blind from his birth knows of the color of a landscape. One of the first steps in correcting this and similar faults must be to experience the muscular sensations which come from correct carriage; and the more frequently these sensations are experienced, the better does the subject become acquainted with them, the more likely are they to replace his erroneous judgment.

It is only through the sense of position that we can hope to acquire the practical working knowledge of correct carriage. What we learn by reading about the matter or by looking at pictures or statues of the correct figure is of little use ; for such ideas come to us only through the eye, and we obviously cannot depend on our sense of vision 
to inform us whether we are carrying ourselves properly or not. We do not "see ourselves as others see us"; generally we do not "see ourselves" at all. It is only the sense of position that is capable of reminding us the instant we go wrong; and this sense can be trained properly only by actually assuming the correct posture.

16. The More Important Faults of Form and Carriage. - We may now pass to the consideration of the more important deformities, which it is the aim of special muscular exercises to prevent or correct.

(a) The failure to hold the neck erect (allowing it to bend forward). - This results naturally from the fact that the

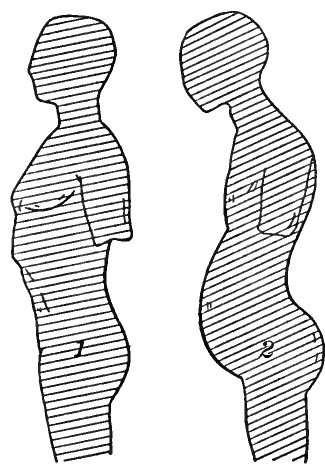

Fig. 109. The results of proper (1) and improper (2) carriage of the vertebral column. After Demeny weight of the head will do this, provided the tendency is not corrected by the proper training of the muscles of the back of the neck and trunk. The position of the head usually taken in reading, sewing, etc., is another cause of this bad habit.

(b) Round or stoop shoulders. These defects have already been sufficiently dwelt upon (p. 322).

(c) Too great backward (dorsal) convexity of the spine in the thoracic region, and too great forward (ventral) convexity of the spine in the abdominal region. - A certain amount of such curvature is normal in these regions (see Chapter II) ; but there is usually a tendency to excessive curvature because of the weight of the parts of the body which the spine must support. Every one knows that it is an effort to sit erect; and this feeling of effort comes from the fact that the spine is straightened, or rather its curvature kept normal, by the action of a rather complicated group of muscles, - the erectors of the spine. To 
sit, or stand, or walk erect involves the activity of these muscles; when they cease to act the faulty curvature becomes more pronounced. Hence the value of all exercises which tend to straighten the spine, - exercises, for example, in which, while standing on the feet, we try by our own muscular effort to make ourselves as tall as possible. They train and strengthen the muscles in question; they stretch their antagonists, just as throwing the shoulders back stretches the pectorals; and they impart to us by actual experience the sensation of being erect.

(d) Lateral curvature of the spine. - When the spinal column and its attached ligaments and muscles are properly developed there is little or no lateral curvature of the spine; the two halves of the body are symmetrical with regard to the median plane of the body, although a considerable amount of bending of the spine as a whole to one side or the other is possible. It is, however, quite possible, by maintaining incorrect positions, to acquire a more or less pronounced lateral curvature in which the muscles and ligaments of the concave side become shortened and those of the convex side lengthened. Perhaps nothing is so responsible for all these faults of curvature of the spinal column as improper positions at the school desk, and much can be done to prevent them by properly constructed school furniture and careful attention to correct position. But it is not wise to depend on these alone. No desk has been constructed in which correct posture can be indefinitely maintained with ease, and we have still in any case to contend with the force of gravity. Active exercises which straighten the spine should supplement the other measures. Experience has well established the fact that the true preventive and remedy lies in movements which elongate the spine.

(e) We have elsewhere (p. 172) pointed out the important action of the muscles of the abdominal wall in 
supporting the abdominal viscera, especially those, like the stomach, the spleen, and the intestine, which are suspended from the dorsal wall of the abdominal cavity. Fig. 137 will at once make clear how the relaxation or elongation of the abdominal muscles, by removing support from these viscera, permits their weight to pull unduly upon the mesentery, and so to stretch this support. It is also not improbable that the tense mesentery at times, by pressing upon thin-walled veins and lymphatics, interferes with the circulation of blood and the flow of lymph in some organs, and so leads to trouble. A pot-belly is not a thing of beauty, and there is every reason for thinking it to be undesirable from the hygienic point of view. It is prevented, in the first place, by every movement which prevents undue lumbar curvature of the spine, and, in the second place, by exercises of the abdominal muscles, which result in their improved tone. These, however, like all corrective exercises, must be followed up by maintenance of the correct position of the trunk.

17. Special Exercise for the Training of Nervous Coördination. - A man or woman may possess none of the deformities noticed above, - the anatomical form of the body may conform to the best ideals, - and yet the positions and movements of the body may be awkward, inexpert, ungraceful. In other words, the muscles may be well developed but the individual may be deficient in the power of easily coördinating their action in the accomplishment of desired ends. After what has been learned of the part which the nervous system plays in directing our actions, the brevity of any reference to this purpose of physical training will not mislead the student into thinking that it is of little importance. We have learned that the maintenance of equilibrium, when the body is at rest and when it is in motion, and the execution of complicated movements, both require training of the nervous system by use. 
The range of activities for which we can train is very extensive; playing upon musical instruments, the execution of gymnastic feats on the parallel and horizontal bars, the traveling rings, or the trapeze, are only a few examples of what can be done by the training of the nervous system by practice.

A large part of gymnasium work consists in this sort of training, and there is almost no limit to the forms of exercise to which we may train, - vaulting, jumping, balancing the body on one foot while various movements are made, the tricks of the parallel and horizontal bars, trapeze, etc. Is there any principle to guide us in the choice of what we shall do? In reply to this question we may say that the leading principle should undoubtedly be that of training for what will be useful, and while we need not discard all training which cannot be justified on this ground, that which is useful should not be sacrificed to that which is not useful. A large amount of skill is required to walk upon the hands with the feet in the air, and the thing can be done very gracefully by training; but it is certainly better to cultivate the habit of walking gracefully upon the feet. And yet one may see professional gymnasts who are extremely graceful while performing their tricks, but whose gait is clumsy and awkward.

18. Balance Exercises. - It is evident that by far the greater number of our customary coördinated movements are made on the feet. Hence the value of so-called balance exercises in the widest sense, whether they consist in the execution of difficult movements while standing on one foot or on the "walking beam," or in making a proper landing from a jump or a vault; all of them afford training of those reflexes by. which we retain control of the body in motion, thus securing grace of posture and carriage.

The general purpose of training these reflexes is the same as the purpose of those exercises which correct 
deformities; they do for the nervous mechanism of the movement what the others do for the skeletal parts and the muscles which play upon them ; they give the training of use and prevent atrophy from disuse.

Both these ends, the corrective and the so-called coördinative, are best secured by the use of gymnastic movements; and the increasingly sedentary character of much of our modern life correspondingly increases the value of gymnastic work, especially in the period of youth. It is well to learn and understand the most useful exercises, and even in adult life to have resort to them two or three times a week in order to hold fast what has been gained.

19. The Gymnasium as a Means of General Muscular Exercise. - Under the conditions of city life, especially in winter time, the gymnasium is also useful in supplying general exercise in the form of running, gymnastic games, etc. It is better to seek outdoor work as far as possible for this exercise, but there are times when those living in the heart of crowded cities cannot get to the country, and outdoor exercise in town is not all that is to be desired. While there is sometimes a tendency to extol unduly the value of gymnastic work, there is equally marked ignorance in other quarters as to what the gymnasium may accomplish. Our cities are vastly better off for their Y. M. C. A. and other gymnasia, and we cannot afford to discourage any means of properly directed physical training.

20. Hygienic Value of Corrective Work. - Before leaving the subject of corrective and coördinative work we may answer a question which is frequently asked: Has it, after all, any hygienic value? All will readily grant that this part of physical training has an æsthetic value, and that the cultivation of the taste for correct form and carriage in one's own person is to be commended. But is a man less healthy for being round-shouldered? The answer is that he may or may not be less healthy. The deformity 
of round shoulders carries with it the lessened use of the upper ribs in breathing; and while one man or woman may escape dangerous consequences, another may not, indeed we know does not, and it is the part of wisdom to avoid the danger as far as possible. In one a pot-belly may be consistent with perfect health, while in others it is not. One may go through life with some faulty curvature of the spine and not suffer from it; but thousands of persons have to consult physicians every year because of such faults. Many a man wears improper shoes without bad results; hundreds pay for it with flat foot and suffering which at times amounts to torture. There is not a single deformity enumerated above which may not prove a serious matter ; and when it is so easy to avoid most of them, it would seem from a hygienic point of view well worth while to do so.

The hygienic value of corrective and coördinative work is justified, however, still more effectively on another ground. The tendency to take general exercise is directly proportional to the excellence of the neuromuscular mechanism of the body. The man who is awkward and clumsy, who can make but few movements, does not enjoy general exercise as does the man who has good control of his muscles and can make many movements. It is probably not too much to say that a very large proportion of the people who settle down to a sedentary life with the coming of their thirty-fifth or fortieth year do this because they can do so little with the body, and because exercise is consequently monotonous and distasteful. We can undoubtedly preserve more readily the love of movement for its own sake when we have a body which can move freely and easily, skillfully and joyously, than when we have one which is never so much at home as in an easy-chair or upon a soft bed; and we have shown above (p. 311) how valuable is this joy of movement to the body as a whole. 


\section{CHAPTER XVIII}

\section{THE HYGIENE OF THE NERVOUS SYSTEM. REST AND SLEEP}

In no respect do the conditions of modern life stand in more striking contrast to the life of former times than in the increasing importance of mental work in contrast with muscular work as a means of support. Not only are there more professional men, - such as lawyers, editors, physicians, teachers, and the like, - but the character of modern business life involves no less the use of the nervous system both on the part of those who direct large enterprises and of those who occupy subordinate positions. The clerk in a bank, as well as the president or cashier, is "living by his wits" and is using his brain to an extent rarely seen until within the last century. Never was competition so keen; never has it been so necessary to inform oneself minutely as to market conditions of demand and supply; never before has the margin of profits been so small; never before has it been so necessary to avoid. waste; and never before has it been so difficult to protect oneself against novel and unforeseen conditions. Truly the modern business man must be ever awake, ever alert.

Nor is this all. With the introduction of the telegraph and telephone, communication between man and man is facilitated; the widespread employment of stenographers results in an increase of letters received and sent; and in other ways the number of matters demanding attention is multiplied many-fold. Moreover, the increase of wealth has enlarged the possibilities of life ; concerts, art 
exhibitions, books, crowd upon us; social engagements are multiplied; so that as a result we are kept ever on the alert, and the man or woman who does not firmly decline invitations, engagements, and efforts which would overcrowd life to no good purpose, experiences elements of distraction, or fatigue, or worry, which tell upon health and too often lead to what we call nervous prostration.

For no student of the practical problems of hygiene can shut his eyes to the marked prevalence of nervous prostration and even insanity, or fail to recognize the evident connection between these things and the intensity, the hurry, the unrestful character of the lives we lead. Probably there is no more pressing problem of practical hygiene than that which is here presented.

And even where there is no question of nervous prostration or insanity, a large number of people suffer from nervous troubles of one kind or another which interfere seriously with their work and with the legitimate enjoyment of life. We have seen how close is the connection between all parts of the nervous system, and also how conditions of the nervous system may and must influence nutritive and other functions of the body. The two are most intimately bound together, and many a man or woman fails to secure the blessing of good health because intense, unremitting work is demanded of the nervous system, such as would never be imposed on the muscles, or the stomach, or the skin. Consequently the avoidance of actual nervous prostration is but a small part of what must be accomplished by the hygienic conduct of life; a far more pressing practical problem is the lessening of daily strain, worry, and fatigue which are the precursors of the more serious troubles, and the avoidance of which affords the only sure means of defense against the all too common and distressing breakdowns of useful lives. 
Christian nations have always had in Sunday one day in seven when most of the work of life may be suspended and the strain relieved; and the preservation as far as possible of Sunday as a day of rest is a matter of sound hygienic policy. Recent years have also shown encouraging signs of relief from steady strain in shorter hours of labor, in the early closing of shops on Saturday, and in the more general use of vacations in summer. These are all to be welcomed and encouraged, but a large amount of strain still remains; the value of the Sunday rest is largely nullified when we go back on Monday morning to an intense application which continues without break until Saturday night.

1. What causes Nervous Strain? - The nervous system, in common with all other organs of the body, is unfavorably affected by unwholesome conditions or acts of life. Improper feeding, sedentary occupations, bad ventilation, or overheating of houses tell upon its working capacity; and the effects of such conditions upon the nervous system are often wrongly attributed to mental work or overwork. Especially is this true in the matter of muscular activity. Many break down with nervous prostration, not because the brain and spinal cord have been overworked, but because, in an excessive devotion to business, or science, or literature, or art, or pleasure, or even because of sheer idleness, the muscular system has been neglected and has accordingly failed to minister to the rest of the body. At the same time it often happens that despite proper muscular activity, proper feeding, etc., the element of strain is still present. Muscular activity itself entails extensive nervous work, and the nervous activity which participates in muscular work may be only a part of the sum total which produces overstrain, and even leads at times to nervous prostration itself.

2. Misdirected Nervous Activity. - It is very important to understand clearly that it is misdirected nervous activity, 
and not mental work in itself, or the concentration of attention which mental work requires, that leads to bad results. It is a part of our normal life to do mental work and to cultivate the power of close application to that work; it is a part of education to develop the power of concentration and attention against resistance and inclination, and experience shows that this may be entirely consistent with the maintenance of health. But when a student "crams" for an examination for two or three days, with the minimum of sleep during the period, and breaks down after it is over, it is not merely mental work which should be blamed for the result; for he would probably have broken down if he had attempted to work a typewriter during the same time, with no more relaxation or rest. The real cause of the trouble is the too long continued use of the nervous system.

3. Mental Work and Overwork. - Much nonsense is said and written about "working the brain too hard." If by this is meant working it too long at a time without rest, or without stopping for muscular exercise; if it means the attempt to do more sums in arithmetic, to read more Latin or German, to write a longer composition, or to master more science than the hours of study justify, and so prolong these hours of study to the neglect of other hygienic demands, no objection can be made to the phrase; but if it refers to the hard mental work and close application required for a reasonable time by a sum in mathematics or a passage in Latin, we may well hesitate to regard such work as in any degree dangerous. The world is overflowing with people who have never acquired habits of mental concentration and hard thinking; and yet their general health is no better than that of persons who have acquired such habits, while their mental powers often suffer severely by comparison.

In the physiological portion of this work the anatomical and physiological aspects of the nervous system were 
carefully described. One of the chief reasons for doing this was to impress upon the student the extreme complexity of the mechanism, the great number of parts (neurones) which are concerned in our actions, and the natural difficulty, as well as the necessity, of proper coördination. In the work of a muscle it is not so important if some of the fibers fail to do their work, provided the remaining fibers work harder, and so exert the same pull on the tendon, for the work will still be done. But it is not so with the nervous system; if ten or twenty neurones of a given nervous mechanism fail to work, the work will not be done at all, or will at best be done imperfectly. In any mechanism of interdependent parts, weakness of one part means weakness of the whole. The secret of efficiency in the nervous system as a whole is the maintenance of the efficiency of each and every unit.

\section{The Care of the Nervous Machinery. Rest and Sleep.} - If a locomotive is to be kept in the state of high efficiency, it must not be worked without ceasing until something goes wrong. When a train is to be pulled three hundred miles it is customary to ehange engines two or three times on the run; and these changes are made, not because the first engine cannot pull the train to its destination on schedule time, but because heating occurs, or dust finds its way into the bearings, or the strains and jars impair adjustment; and it prolongs the life of the machine and its good working to remove the dust, cool the parts, and otherwise frequently put the engine in perfect order. When an engine breaks down, it is usually because some one part has given way. With proper care a good machine should wear out but not break down.

The central nervous system, although infinitely more complicated than the steam locomotive, is far less durable as a mechanism. Its bearings are not made of hard steel, but of living, irritable protoplasm keenly susceptible to 
fatigue. In the numerous connections between neurone and neurone there is the same chance as in the steam engine that some one part will fail to do its work; and the main principle of its hygienic care is to oil the bearings and clean and repair the machinery, by repose and sleep, before the danger of a breakdown is imminent. Rest, and especially the rest of sleep, is the one preventive for these unfavorable conditions; by these alone is the fatigued neurone withdrawn from work and given the chance to repair itself and to return to its normal condition.

5. How Much Sleep is Advisable? - Different people undoubtedly require different amounts of sleep; but it is safe to say that the vast majority of adults require from seven to eight hours a day; children and young people require more. It is, however, an interesting question whether all of this should be taken at one time or not. Since the nervous life of to-day is more intense than was that of our ancestors, it is all the more needful that we keep the nervous system in a continuous state of high efficiency. To go about the duties and pleasures of life from early morning until late at night without a moment's rest is a great mistake; we are then doing what the engineer would do who should run his engine all day, feeding it with coal but without giving it a drop of oil, without tightening a nut, without cleaning a bearing. As the play of nervous activity goes on, now calling upon one combination of nerve cells, now upon another combination, those nerve cells which belong to more than one mechanism are called on for more than their share of work, and every mechanism to which they belong may be to that extent impaired. The stimulus of the will must be more vigorously applied, and as this becomes ineffective, the individual is tempted to use stimulants, as the whip is applied to tired and straining horses, or as blows were showered upon galley slaves in time of battle. 
Contrast with this the benefit of brief sleep during the day in facilitating night work. Some persons, it is true, do not seem to be thus benefited, but the vast majority are. And the benefit is out of all proportion to the time spent asleep. We are tired and work is difficult, not so much because the whole nervous system is exhausted, but because unfavorable conditions of fatigue, etc., have come in at important points; during even a short nap, with its marked muscular and nervous relaxation, normal conditions are restored and the whole mechanism then works on with less effort, less general fatigue, less local injury.

6. Nervous Rest in Change of Work. - Sleep is the very best means of insuring local nervous repair, because it is the only condition which involves complete relaxation. There is, however, some rest, or at least some refreshment, in mere change of employment; as when, for instance, we pass from mental work to physical exercise. Calling into play a new group of nerve cells gives a chance for rest to many cells which have previously been active. And at times we feel tired after mental work because we need muscular activity rather than sleep. The tired feeling may come not from tired nerve cells but from the want of what the muscles might furnish (see Chapter XVII). At such times muscular exercise to some extent, perhaps to a great extent, refreshes us; and in general we maintain a higher degree of working power by judicious variety of activity. But it must be remembered that, in the long run, neither muscular exercise nor any other change of occupation can take the place of the complete relaxation and refreshment found in sleep. It is, indeed, doubtful whether there is any change of employment which brings with it an entire change of nervous activity. A certain number of the same cells, already weary, are still kept at work, as has already been explained above; and it is by sleep alone that every cell has its natural opportunity 
for repair. Those who would define rest as "selected excitement" should bear this fact carefully in mind.

The cardinal principle in the care of the nervous system is thus the same as that in the care of the steam engine. Do not often call upon it for activity of any kind when conditions of undue fatigue are likely to be present. Go to the performance of every physiological activity, to digestion, to study, to muscular work, to social life, - for all these mean nervous activity, _ as far as may be with a rested nervous system. Of course to do this is not always possible; there are times when we must drive the body to mental work despite the fact that it is physically tired; but this ought to be the exception, never the regular order of life.

7. Examples. - Let us suppose that some one, man or woman, after application at sedentary work for six or eight hours, has some time free before the evening meal, and that, tired and perhaps nervous, relaxation is sought in a brisk walk, which is almost immediately followed by dinner. The effort which the digestion of this, perhaps the heaviest meal of the day, costs the nervous system shows itself in a stupid, almost somnolent condition which often follows. The body is trying to do hard work with a tired nervous system, some of whose bearings need oiling; its owner is making the mistake of continued activity without opportunity for the rest and repair which a nap of fifteen or twenty minutes, or even absolute idleness and complete muscular relaxation without sleep, for half an hour or so before the meal, might have given him.

Again, there are times in every one's life when some unusual strain must be borne; when, for example, after the day's work watch must be kept at a sick bed during the greater part of the night. Too often people will undertake this strain, expecting to " make up" the loss of rest when it is over, even when it is possible to prepare 
for it by an hour or so of sleep beforehand. We seldom work steam engines in this way. Should we treat the nervous system less carefully than a steam engine?

These examples must suffice. The application must be made by each individual according to his work in life. If work is undertaken which requires constant activity from early morn until late at night, the case is hopeless, and the only remedy is a change of occupation. Only gross ignorance of the plainest facts of human experience; as well as of physiological science, can excuse such conduct.

8. Muscular Relaxation in Sleep.-_Sleep and rest involve muscular relaxation. All have noticed, when falling off to sleep, the feeling of relief from strain; the framework of the skeleton seems to be held together less rigidly, and finally, as we lose consciousness, relaxation seems complete. And at times when sleep will not come, many have felt the inability to relax; when, as it has been well expressed, we seem to be afraid that the bed will slip away from under us and we must hold on to it. We have seen that during waking life the nervous system is continually sending out impulses which keep the muscles in a state of moderate contraction, and thus among other things liberate heat for the maintenance of the body temperature. Usually this tonic activity of the motor neurones must be more or less relaxed before sleep will come, and the inability to release it is one of the danger signals of the nervous system. There can be no doubt that when nervous work is pushed too hard against unfavorable conditions, the nerve cells develop a condition of excessive irritability, so that they are discharged by afferent impulses or other stimuli which would ordinarily not affect them ; and they maintain this irritable condition even in the presence of general bodily fatigue. Normal rest is, of course, extremely difficult or quite impossible under these conditions, which for this reason alone should be attended to at 
once. The trouble may be in some general or special unhygienic condition of life, - impaired digestion, insufficient muscular exercise, the presence of undue anxiety, etc.; these should be inquired into and remedied if present; but the trouble is usually the result of pushing activity of different kinds for too long periods without cessation. In other words, we have lost the ability to relax because we have not practiced relaxation.

9. Conservation of the Ability to relax. - The ability to relax is something which, like all phenomena of nervous life, depends on practice. Indeed, it is not improbable that it is something more than a mere process of desisting from activity, and that direct active processes of inhibition (see Chapter XV) are concerned in it. All have known people who can go to sleep the instant they lie down; and they can do this - it would almost seem by an act of the will - because they have long done it. It is a power which can indeed be cultivated too well ; by too frequent repetition of the process of taking a nap, and by sleeping too long at night, there may be acquired a diminished irritability of the nerve cells, which makes attention to work a very difficult matter, and long-sustained attention almost impossible. Those in this condition may escape the danger of nervous prostration, but they impair their usefulness in life.

The true path, as in other matters of personal hygiene, is that between these extremes. When one rises at seven or eight in the morning, a short period of rest in the afternoon is sufficient; the persistent practice of the act of relaxation every hour or less is apt to lead to loss of muscular tone and of nervous efficiency in general. At the same time, the habit of momentary relaxation in the midst of the day's work is a valuable aid, partly in bettering conditions at the time, but chiefly in retaining the power to relax when it is wanted for longer periods of rest. 
10. Drugs are Delusive and Dangerous. - The physiologist cannot condemn too strongly the substitution of stimulants for the proper regulation of work and rest. The reader will see at once what this course of action may be expected to accomplish ; the stimulant is an antagonist of relaxation; the nerve cell becomes more and more irritable as it is pushed harder and harder; finally it reaches either the condition of excessive irritability or else that of being unable to work without the stimulant. It has adapted itself to the presence of the stimulant in its environment, it is trained to work under those conditions, and it cannot work without them. It may be safely asserted that, in general, the time above all others when stimulants should not be used is when we are tired out ; to use stimulants regularly, day after day, in place of rest is shown by experience to be one of the most dangerous of mistakes.

Nor, on the other hand, can we condemn too strongly the use of narcotics to produce sleep. Probably none of these drugs are capable of producing normal sleep; and while in times of emergency the physician must have recourse to them, they should never be relied upon in place of the hygienic conduct of the whole life. Many of them, and some of those in common use, are very dangerous, and none of them is, known to be above reproach.

11. The Influence of Mental and Moral States. - Finally, it must also be remembered that psychical processes exert a profound influence upon the well-being of the brain and spinal cord. It is a matter of common experience that emotions, feelings, moods, etc., profoundly influence human conduct, and so indirectly affect health, especially the health of the nervous system. It is also certain that they exert a more direct physiological influence on the bodily functions; the changes which emotions produce in the heart beat are good examples of other changes which are none the less important because they do not lend themselves 
so readily to observation. The bestowal of a healthy attention upon the moral aspects of conduct is a legitimate and essential part of personal hygiene; and it is not too much to say that much of the ill health from which men and women suffer is to be traced primarily to the absence of sound moral sense or to its abnormal or perverted development. Care and worry often cause weariness and loss of sleep which even diversion and muscular exercise cannot overcome. They seldom trouble the young, but as age advances they are sometimes inevitable. Efforts should be made to avoid them, as far as possible, by a wise ordering of life, by forethought, thrift, economy, sobriety, honesty, and the like, which tend to "a light heart" and "a clear conscience." A heavy heart and a clouded conscience tend to unhappiness, anxiety, wakefulness, and other physical ills.

12. "Mental" Cures of Disease. - It has been shown that mental conditions are far from being without influence upon the activities of the body, even leaving out of account the voluntary muscles. The effect of emotions upon the heart has been referred to, and so has the psychic secretion of gastric juice. It is known that the movements of the alimentary canal are readily modified by events in conscious life. In the hypnotic state the effect of suggestion upon functions which we habitually regard as involuntary is even more striking. Facts like these have led many to the rash assumption that there is no limit to the domination of the mind over physiological processes. In numerous cases the ascendency which some have gained over certain forms of disease has been as surprising to others as it has been gratifying to themselves. Beyond question the righting of disordered functions and the suppression of pain have been frequently attained, and this fact makes it easy to see why so great a following has been drawn to a belief in the universality of mental power. 
But certain dangers are always involved in the attempt to overcome disease by resolutely forgetting it and denying its existence. The feeling of pain may at times be banished by believing that it does not exist, but this may be quite as undesirable as self-inflicted blindness or deafness. While relief from pain may frequently favor recovery by promoting rest and nutrition, it may at other times simply mean the loss of warnings which deserve to be heard. Where there is grave organic disease, this may move on to a fatal issue even while the deluded subject consistently ignores its course. It is not wise to try to annul the effects of a disease in consciousness when both cause and effect can be removed by rational medical treatment. Hypnotism may relieve a toothache, but it is not claimed that it will mend a decaying tooth. The dentist's filling, which does both, is the type of medical as contrasted with psychical methods in dealing with acute disease. Especially foolish is it to ignore or deny the actual presence of infectious or contagious disease, for here delay menaces not only the patient but those about him. The consequences of this folly, when confined to its deluded victim, may end in virtual suicide; when they extend to others, they may fall little short of manslaughter. 


\section{CHAPTER XIX}

\section{THE HYGIENE OF FEEDING}

The present chapter deals with certain hygienic considerations connected with the taking of food into the body, - its preparation, its cooking, its quantity, the frequency of our meals, and the adjustment of our habits of feeding to the other work of life. ${ }^{1}$

Mankind as a whole was probably never better fed than it is at present. The opening up of the New World with its vast fields of corn and wheat and its enormous pastures ; the introduction of improved methods of agriculture, agricultural machinery, and education in agriculture; and especially the improvements in transportation facilities and in arts of food preserving (such as refrigeration and canning), - all these have immensely increased the avaitable food supply of the world and made famine and starvation much more rare than formerly. It is now only in inaccessible places, such as the central parts of India, that great famines still occur.

And yet in the midst of abundance it is still true that many men and women are poorly nourished; for it is the absorption of food by the blood and not merely the eating of meals which supplies the needs of the tissues. Hence the problem of alimentation in its widest sense involves not only the growing of food on farms or in gardens, and

1 Many practical points connected with alimentation have already been considered in Part I (see chapters on digestion and nutrition). Special reference may be made to Chapter XIII, p. 233, on the Choice of Foods and Nutrients. 
the preservation of this food so that it may be delivered in proper form to the consumer, but also the eating of it in such form and quantities and at such times as will insure its proper utilization, by the processes of digestion, for the needs of the body.

1. Appetite as a Guide in Feeding. - Nature herself has provided us with guides in the choice of food, and these guides are the sensations of hunger and thirst, and what we sum up in general under the term "appetite." So long as these remain normal and unperverted, they are to be largely trusted; and, like all physiological functions, they are kept normal and unperverted, in the first place, by attention to the general health of the entire body. Appetite is apt to fail or become untrustworthy in the case of men or women who are suffering from lack of muscular activity or from mental worry. The care of the appetite is never a matter of direct attention to the appetite itself, but of maintaining the bodily conditions in which it normally acts. Consequently the basic principle in securing proper nutrition is attention to the general health. A patient suffering from indigestion once consulted a wise old doctor and began recounting the foods that agreed or disagreed with him, together with his innumerable symptoms, until the doctor interrupted him by saying, "The first thing you must do is to forget that you have a stomach." The present chapter is not written for people like this patient, or for invalids, or for others suffering from indigestion in any one of its thousand forms. It is written for those who can, and will, first of all, take the needful muscular exercise and the needful rest; who will pay proper attention to clothing and bathing, to the heating and ventilation of the home, to the avoidance of dampness and other unfavorable conditions; who will not abuse themselves by stimulants and narcotics. Those who prefer not to belong to this class, or who because of some 
constitutional disease cannot, must seek and depend upon medical advice as regards their habits of feeding.

At the same time, to insure proper digestion and nutrition, more is required than attention to general hygiene. What additional precautions are required in the taking of food by persons leading an otherwise healthy life? It is in answer to this question that we shall attempt to give some suggestions.

2. Good Cooking as an Aid in Nutrition. - It has already been pointed out that digestion begins with the preparation of the food by cooking, which serves three purposes.

1. It destroys parasites and disease germs. The importance of this will be shown and emphasized elsewhere (Chapter XXXII).

2. It renders the food more appetizing (see p.113).

3. It makes some foods more digestible by making them accessible to the action of the digestive juices; thus the connective tissue of animal foods, when heated in the presence of water, swells, and is more easily acted on by the gastric juice, so that tough meat in this way is often made tender by boiling. The cellulose walls of the vegetable foods, on the other hand, are softened by cooking, the starch granules are swollen, and their envelopes burst (see p. 94).

At the same time it is possible to render food less digestible by improper cooking. A piece of meat may "have the life cooked out of it"; and egg albumen, which in the raw state mixes rather easily with the gastric juice, may sometimes be boiled to a leathery consistency which renders the action of the digestive juices a slow process.

3. Chewing of Food an Aid to Digestion. - It is unnecessary to dwell at length upon the importance of chewing, or mastication. We have already seen that the word "digestion" is derived from the Latin words dis and gero, to tear apart or separate; and our studies of physiology have 
shown how the division of food into smaller and smaller masses is prerequisite to reasonable rapidity of solution and absorption. The student is also reminded of what has been said (p. 100) concerning the importance of caring for the teeth.

Vegetable foods especially should be well chewed, partly because the cellulose which holds them together is not readily acted on by the gastric juice, and partly because the thorough mixture with the saliva facilitates the gastric digestion of starch (p. 110). Meats also should be well masticated. The fact that a dog bolts his food with impunity is no guide for civilized man, since, for one reason, human gastric juice contains much less acid and so acts less readily upon connective-tissue elements. It is true that the "quick lunch" thrives in busy places, but no one considers it hygienic.

4. Feeding in Relation to Gastric Digestion. - In order that gastric digestion may be efficient it is, of course, necessary that gastric juice shall be secreted in proper amount, and we have learned that the first step toward this secretion consists in the pleasurable sensations connected with the satisfaction of appetite. Consequently it is one of the first hygienic requisites of gastric digestion that the food shall be appetizing, and that the condition of the body and especially of the digestive system shall be such that the food shall be eaten with relish. This is not the same thing as saying that food which is appetizing will be digested; it merely means that food is more digestible for being appetizing, and that, when it is not enjoyed, its stay in the stomach is apt to be unduly prolonged. For this and other reasons the appetite should not be impaired by eating candy, or by visiting the pantry between meals for something to eat; on the other hand, a good appetite should be encouraged by healthy living, by proper preparation of the food, and even, as far as possible, by agreeable 
table appointments. There was wisdom as well as pleasure in the old custom of having a jester at the dinner table, and there is reason in the saying, "Laugh and grow fat."

5. Excessive Quantity of Food. Overfeeding. - It is furthermore important that the amount of food eaten at one time be not excessive, and that the stomach under no circumstances be unduly distended. A large proportion of those cases of dyspepsia which have their origin partly or entirely in the conditions of feeding are due to overeating, which may take various forms. Too large a proportion of the total food may be taken at one meal, usually dinner; or too many meals may be taken, - three should suffice; or each of the three may be full-sized meals, - a very undesirable custom among those engaged in sedentary pursuits. We have seen that the one condition of life which calls for heavy feeding is that of muscular activity, whether in the performance of external work or for the production of heat in cold weather; a person who is engaged in some occupation which involves large amounts of muscular work can and should have three full meals daily; with others the habit is attended with considerable risk.

Gluttony has always been a vice of the idle and luxurious. As the world has grown wiser it has become less common, because a larger intelligence makes it plain that gluttony defeats its own ends, and that the secret of the greatest pleasure in eating, as in everything, lies in temperance, not in excess.

Many persons, however, without any desire or even any thought of gluttony, regularly overeat. These are usually healthy persons leading sedentary lives, "blessed," as they say, "with a good appetite," and because of quiet or even indolent disposition giving but small heed to muscular activity. As the years go by, such persons are apt to grow fat, and by and by to find themselves suffering from a weak heart, or shortness of breath, or something worse; seldom 
realizing, until it is too late, that overeating is the principal cause of their undoing. If sufficient manual labor or other exercise of the skeletal muscles is practiced, trouble from overeating rarely comes. It is the sedentary, inactive, and indolent who suffer most from this source; for them a good appetite often proves to be a curse rather than a blessing, and a poor appetite, by preventing overeating, has often been a blessing, though a blessing in disguise.

6. Fried Foods. - Caution is required in the use of fried foods. When a layer of fat varnishes over a particle of food the digestive juices do not readily penetrate the mass, and digestion is to that extent impaired. This is not of so much importance in intestinal digestion, since in that portion of the alimentary canal the layer of fat is itself digested and removed; the stomach, on the other hand, does not digest fat, and we can easily see how, because of its interference with the first processes of digestion in this organ, the use of too much fried food is unwise.

Moreover, in frying, care should be taken to have the temperature of the fat high enough to coagulate promptly the surface layers of the food, thus preventing the penetration of the fat into the food, which, moreover, should not be served swimming in fat, but as dry as possible. The frying pan is still used far too extensively in some parts of America. Most of our foods should be roasted, broiled, boiled, or baked, rather than fried.

7. Perspiration in Relation to the Hygiene of Feeding. The secretion of gastric juice is seriously impaired by excessive perspiration, especially when the loss to the system is not made good by drinking sufficient amounts of water. This is probably true of the secretion of all of the digestive juices, but it is especially important in the case of the gastric digestion, upon the proper performance of which the subsequent work of intestinal digestion so largely depends. Therefore, in general, smaller meals 
should be eaten in hot weather, - we have seen that we need less food at that time, - and heavy meals should not be taken immediately after vigorous exercise involving profuse perspiration. Indeed, it is a general rule that excessive loss of water by perspiration should be made good, as far as possible, by drinking water more freely.

8. Digestion and Bodily Fatigue. - Digestion, like all other functions of the body, involves to a very considerable extent the intervention of the nervous system; and we may repeat here the advice already given (p. 341) not to go tired to the digestion of a heavy meal. It is one of the objections, probably the chief objection, to evening dinners that they so frequently follow immediately upon a hard day's work, when the nervous system is in a poor condition for its share in digestive work. A rest of half an hour before dinner is, however, generally all that is needed, and usually prevents the mental heaviness which so often follows a full meal.

9. Mental Work after Meals. - An exaggerated importance has probably been given at times to the danger of mental work after meals. There is no proof whatever that the demand of the brain for greater blood supply will seriously interfere with that to the digestive organs. While it is true that indigestion often affects people who go straight from their meals to hard mental work, it is also true that these are usually people who take insuffcient muscular exercise, rest, and sleep. The relation of the circulation in the brain to that in the digestive organs is too imperfectly understood to justify some of the glib but shallow utterances frequently met with on this subject, especially when the statements in question are not clearly supported by experience. (See page 155.)

10. Muscular Activity after Meals. - Vigorous muscular activity immediately after meals is quite another matter. Here we know that blood is taken away from the digestive 
organs and sent through the muscles and skin; this fact suggests caution, and experience amply confirms the need of the caution thus suggested. Even here it is vigorous exercise, and especially after heavy meals, that is to be condemned.

11. The Use of Water as a Drink. - Many people, and especially many women, drink too little water. Water is constantly being lost through the lungs, skin, or kidneys, and this loss is only partially made good by the oxidation of the hydrogen of the proteids and fats. ${ }^{1}$ No rules as to the amount can be given, since it varies so much with temperature and the amount of muscular activity; but the habit of drinking no water between meals and but little at the table, in spite of popular opinion on the subject, is to be strongly deprecated. We have already shown that the abstraction of undue amounts of water by perspiration may seriously interfere with the secretion of the gastric juice, and there is every reason to believe that a deficiency in the supply of water to the blood similarly interferes with the secretion of the other digestive juices, and so, by impairing intestinal digestion, favors constipation.

Undue emphasis has been laid upon the danger of drinking water with meals. The reasons given - that such water unduly dilutes the gastric juice or takes the place of a normal secretion of saliva - are questionable. As a matter of fact, the water thus taken is soon discharged into the intestine and absorbed. It is true, however, that the use of too much fluid with the meal is apt to lead to insufficient mastication because it makes it easier to swallow the food; and from this point of view caution is advisable. It is probably also true that much drinking with meals tends to overeating, by facilitating rapid eating;

1 The water excreted from the body comes partly from the water drunk, but also partly from that formed by the union of the hydrogen of the food with oxygen. 
and it may be that this is one reason why fat people are usually great drinkers.

A further point in the hygiene of gastric and intestinal digestion is the avoidance of those inflammatory conditions of the bowels which follow exposure to cold. This subject will be dealt with in Chapter XXI. The student will also recall what has been said in Chapter XVII with regard to the importance of general muscular exercise, and especially of exercises involving the use of abdominal muscles.

12. The Importance of Coarse Foods. - In treating of the physiology of digestion it was pointed out that the presence of a certain amount of indigestible material in the food is helpful as a stimulus to the muscular action of the intestine. Food may be, and nowadays often is, especially in "delicate dining," too largely composed of very digestible substances, which leave an insufficient residue of solid material in the lower intestine to stimulate proper peristalsis. Our main reliance for the needed coarse or indigestible part of our diet is the cellulose of many of our vegetable foods; oatmeal and fruit are two of the more important foods which serve the purpose. Indian corn is also an important laxative, although its action is probably dependent on other things than cellulose. The laxative action of prunes and figs is well known. Graham flour, bran, "whole wheat," etc., which retain more or less of the hull of the grain, have the same action. In selecting the diet care should be taken, especially in winter, to include in it a sufficient quantity of such foods, and a judicious addition of coarse foods to any diet is probably wise.

13. The Individual must study his own Needs. - In thus sketching the broad outline of hygienic feeding little or no attention has been given to what we should or should not eat; and this has been done in order to discourage looking at the subject from this popular but entirely 
misleading point of view. It may be true that "what is one man's meat is sometimes another man's poison," but only in a very limited sense. Each individual in the course of his experience will learn that there are some things he cannot eat with impunity, and, if he be wise, will govern himself accordingly. But it must be remembered that man enjoys a wide latitude in the choice of his food. The vast majority of people, if they will but lead otherwise hygienic lives, can eat almost anything; and the inability to digest something which we have always eaten, or which others eat with impunity, should lead not so much to its exclusion from the diet as to an inquiry whether the trouble does not have its origin in the general unhygienic conduct of life. Those who treat such conditions by constructing a table of the things they can eat and another of those they cannot eat, and confine their diet to the former, usually find that as life advances the size of the latter table increases at the expense of the former. It is the same fallacy of dealing with the symptom instead of the disease, which leads others to treat constipation with cathartics, and still others to treat a bronchial cough with so-called "cough medicines." 


\section{CHAPTER XX}

\section{FOOD ACCESSORIES, DRUGS, ALCOHOL, AND TOBACCO}

1. Food Accessories and Drugs. - Through the alimentary and respiratory tracts there are received into the blood not only substances such as proteids, gelatin, fats, carbohydrates, salts, and water, which we have described as supplying the material for power and for growth and repair, but also other substances capable of modifying in one way or another the course of events within the body. The flavors which contribute to the enjoyment of foods play an important rôle in the secretion of the gastric juice; and yet the substances which cause these flavors are negligible as sources of power. Salt belongs under the same head; for we use in cooking more salt than is needed to make good the daily loss from the body, and we do this to develop an agreeable flavor in our food. Substances of this kind are spoken of as food accessories, and among them must be included coffee and tea, for their effect is not chiefly a matter of nutrition; certain constituents of tea and coffee absorbed into the blood affect the nervous system, and it is largely for this reason that we use them.

We may pass in this way from the necessary food accessories through those, like coffee and tea, which, while not essential, may still be regarded as part of the food of a large portion of mankind, to the great number of chemical compounds known as drugs, which also act by changing the course of events within the body; and it is difficult to draw any sharp line of distinction between those which 
occasionally serve as medicine or "stimulants" and those of which daily use is made as food accessories.

Animals as a rule take substances into their bodies only to satisfy hunger or thirst or appetite ; man alone takes, in addition to his nutriment, food accessories and drugs for the sake of their special effect upon the nervous system or other organs. Many of the numerous food accessories which human ingenuity has discovered or devised are harmless enough in the form used; but others contain substances which are capable of poisoning the body. It is an important part of the study of personal hygiene to learn of what these substances consist, what is their action on the human organism, and wherein lie their special dangers.

2. The Drug Habit. - It is a lamentable fact that large amounts of drugs are swallowed by men and women apart from any medical need which compels their use. In a subsequent chapter we shall show reasons for avoiding an undue dependence upon drugs as a remedy for various minor ills. Bad as this practice is, with its tendency to rely upon the uncertain action of a drug instead of taking proper hygienic care of the body, it is far worse to make habitual use of drugs for their special effects upon the healthy body, for the habit is one which is only too easily cultivated. There is no reason why a healthy human being, living a normal life amid healthful surroundings, should need to use drugs habitually, and a little consideration will show that the practice is dangerous.

3. Dangers of the Drug Habit. - When we eat meat, or vegetables, or when we breathe air, we take into the body materials needed for normal living. These things have always formed part of the food of the race, and, unless wrongly taken, do good and not harm. When, on the other hand, we take a drug, such as chloroform, or cocaine, or opium, or alcohol, or coffee, or tea, we take something 
which is foreign to the body, in so far as it has not been a regular constituent of animal food in the past. It is not needed, as proteid and salt and water are needed; there is no special preparation for its reception; and, while it may do good, there is danger that it may do harm.

In the second place, the exact action of many drugs is only imperfectly understood. In an emergency the physician uses them temporarily, for some effect which he desires to produce, thus tiding over a difficulty. He uses the drug only a few times at most, and is consequently not greatly concerned about unfavorable attendant effects ; it accomplishes some needed purpose, and if it does any harm, the organism may be trusted to recover from it. It is very different, however, with the habitual use of any drug. The very fact that it gives some new direction to the events taking place within the body means that abnormal conditions of life are being maintained; and we have already learned that abnormal conditions of life are apt to be unhygienic.

Again, the use of drugs is only too apt to be substituted for the hygienic conduct of life. We may, for example, take drugs to accomplish something which the healthy body should accomplish for itself without outside help. When any one drinks a cup of black coffee to facilitate mental work which his fatigued condition would not otherwise allow him to do, he is trying to get from a drug the power which he could and probably should secure by normal sleep. The coffee acts like a whip to a tired horse; the same work is done as might have been done had the horse been allowed a little rest; but the horse is not as well off when he does the work under the lash as when he does it in a properly rested condition. Similarly, persons suffering from sleeplessness often take drugs used to produce sleep (hypnotics), and, superficially at least, the sleep thus secured resembles normal sleep; but experience 
shows that few if any hypnotics can be used for any length of time without bad effects. Here again a drug is being depended upon to do what the normal body should do for itself. Pepsin tablets may be taken to aid digestion, and thereby an attack of indigestion may sometimes be prevented or relieved; but a healthy stomach should furnish its own pepsin; and the fact that it does not do so is a sure warning that something is wrong in the conduct of life. It is irrational to neglect the duty of attending to the cause of the ailment, and it is foolish to substitute temporary relief for permanent cure. Perhaps if the drug did all that the proper care of the body does, and did no more, no serious objection could be made to its use ; but there is probably no drug of which this is true, and for this reason it is foolish and rash to try to substitute the use of drugs for the hygienic conduct of life.

Lastly, if the drugs do not accomplish in the long run what should be done by the hygienic conduct of life, their extensive use becomes all the more dangerous in view of the unquestioned fact that we are apt thereby to become their slaves. Every man is the slave, broadly speaking, of the habits he forms, and it is only a question as to whether he will be the willing slave of good habits or the abject slave of bad habits. The man who leads a hygienic life is the slave of muscular activity, of correct feeding, of proper clothing, of rest, etc.; that is to say, these things become necessary to his life; he cannot get along without them. If for these proper agents of health he persistently substitutes some drug, whether it be alcohol, or tobacco, or coffee, or tea, or chocolate, or opium, the habit of using the drug is substituted for that of maintaining normal conditions. But since drugs cannot entirely take the place of such conditions, the constitution goes from bad to worse, and increasing dependence must be placed upon the drug. It is a safe rule that whenever we are uncomfortable or 
unhappy without the use of a certain drug we should cease using it until, with the help of hygienic living, we can get along without it.

There are people who are slaves of coffee, of tea, of chocolate, of patent medicines, of candy, and of soda water, just as truly as there are slaves of tobacco, or of alcohol, or of opium. It is worse to be the slave of alcohol than of coffee, because the evil consequences of alcohol are greater than those produced by the corresponding use of coffee; but it is by the same process in both cases that the man or woman becomes a slave to the drug, and that process is the formation of bad habits.

With these practical considerations about the use of drugs, - by which term it will be seen that we mean, not simply the medicines purchased from the apothecary, but all those substances which are taken into the body in order to give some new or abnormal direction to the course of events in the organism, - we may pass on to the discussion of those in common use.

4. Tea and Coffee.-Different as are these drinks in taste and appearance, their most important physiological effects are due essentially to the same substances, viz. caffeine (or theine) and tannic acid (or tannin). Caffeine is a very powerful stimulant, especially of the nervous system, and also of the heart, although probably to a lesser degree; tannin, on the other hand, is a bitter, astringent substance, which may considerably hinder digestion and directly injure the mucous membrane of the stomach. Tea contains about twice as much tannin as an equal weight of coffee, but as coffee is frequently made much stronger than tea the actual amount per cup may often be more nearly equal in the two drinks than these figures indicate. The amount of tannin dissolved in tea varies greatly with the method of preparation, and largely for this reason tea should not be boiled, nor allowed to steep too long. The 
proper method of making tea is to pour over the dry leaves water which has been brought just to the boiling point, and then to allow the infusion to stand, without further heating, for not more than a few minutes.

Both tea and coffee seem to have a slightly retarding influence upon gastric digestion. In healthy people this is of little consequence, but when the digestive powers are in any way impaired the use of these beverages may be inadvisable. The more important effect, however, of both tea and coffee is in their stimulating action on the nervous system. No satisfactory explanation has yet been given of the fact that some people can use tea and not coffee, while with others the reverse is true. It is probably safe to say that when used in moderation, tea and coffee are usually harmless to those leading an otherwise hygienic life. They should be used sparingly by nervous people and by those in whom digestion is feeble and slow (Hutchinson). Even by the perfectly healthy they should not be used to excess, nor should the habit be acquired of using them as the whip to the tired horse. Drinking strong coffee in order to keep awake for evening study is objectionable, and the substitution of afternoon tea for a little rest or sleep is also unwise.

5. Cocoa is made from the seeds of trees of the genus Theobroma, and chocolate is prepared from cocoa. In the solid form both are highly nutritious, as shown by the following average results of analyses.

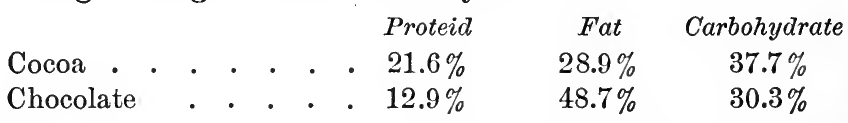

When used as a beverage, however, the nutriment derived from them is small. In addition, cocoa and chocolate both contain theobromine, a substance closely related chemically to caffeine and possessing much the same stimulating properties. In general, the same hygienic considerations which 
apply to the use of tea and coffee should guide us also in the use of chocolate and cocoa.

6. Soda Water and Similar Beverages. - Of these little need be said. In general they are harmless enough, especially to those enjoying perfect digestion. The large amount of sugar which they contain is apt to make matters worse in many cases of dyspepsia; by taking them frequently between meals the appetite for wholesome food is impaired, and excessive indulgence in them under any circumstances is needless and foolish.

7. Alcoholic Beverages. - In the case of an alcoholic drink we have to deal with something which, like tea and coffee and cocoa and "temperance drinks," is used as a beverage and to that extent must be classed in. the same group. Alcoholic drinks are, however, taken as stimulants and so resemble tea and coffee and cocoa, but they differ from all of these in their action upon the body. Moreover, their abuse gives rise not only to degraded moral and social conditions but is also attended with bad hygienic effects. Every one should be informed of their nature and of the dangers attending their use.

The common alcoholic beverages consist of (1) malt liquors, including beer and ale; (2) wines, such as hock, claret, Burgundy, sherry, and champagne; (3) distilled liquors, including brandy, whisky, rum, and gin; and (4) liqueurs and cordials. These groups are distinguished from one another largely by the method of preparation and by the amount of alcohol they contain. Malt liquors are fermented liquors which contain from three to eight per cent of alcohol; wines are also fermented liquors, but contain from seven to twenty per cent of alcohol; distilled liquors, on the other hand, are first fermented and then concentrated by distillation, and contain from thirty to sixty-five per cent of alcohol. In all these the most important constituent, so far as their physiological action 
upon the body is concerned, is the chemical compound known as ethyl alcohol $\left(\mathrm{C}_{2} \mathrm{H}_{6} \mathrm{O}\right.$ or $\left.\mathrm{C}_{2} \mathrm{H}_{5} \cdot \mathrm{OH}\right)$.

8. Fermentation. - The ethyl alcohol in each of these beverages is produced by the action of yeast on sugar, and this action is known as alcoholic fermentation. Yeast is a

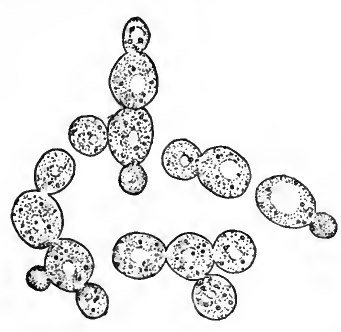

Fig. 110. Yeast cells unicellular plant, and when a small amount of it is added to a solution of grape sugar or fruit sugar it breaks up these substances, chiefly into alcohol and carbon dioxide gas. The latter passes off, while the alcohol remains behind in the solution. In addition to these chief products of fermentation there are always formed other products in small quantities, and to these, in part, the flavor of the fermented mixture is due. Different varieties of yeast produce different kinds of fermentation. Thus one variety (domesticated yeast) is used in making beer, and another (wild yeast) in making wine. The amount of alcohol produced differs with the yeast used, as do also the character and quantity of the secondary products. The growth of yeast, like that of all living ferments, is checked by the accumulation of the products of its own activity. Consequently when the alcohol produced reaches a certain percentage (usually less than ten per cent) the fermentation ceases. Alcoholic drinks which contain higher percentages of alcohol are prepared by special processes which will be described later.

9. Malt Liquors. - Malt consists of sprouted grains (chiefly barley). The grains contain a large amount of starch which during the process of germination is converted into sugar by diastase, an enzyme produced by the living cells of the plant, - the action of diastase being essentially similar to that of the ptyalin of the saliva. The germinating plant thus comes to contain considerable quantities of 
sugar, together with salts, proteids, and other substances. The watery extract of malt is known as wort, and it is this which, after being boiled with hops, is acted upon by the yeast. The liquid thus produced from wort by fermentation is known as ale, beer, stout, porter, etc., according to the conditions under which the fermentation takes place and the character of the malt and the yeast employed. German beers contain from three to four per cent of alcohol; ale contains from four to six per cent.

10. Wines. - Wine is produced by the fermentation of the juice obtained by crushing grapes, and the yeast comes from the "bloom" on the skin of the grapes. The juice, or " must," thus extracted is allowed to undergo fermentation, and the fermented liquid is wine. Most wines, however, are subjected to subsequent treatment. Some are allowed to ripen in wooden casks, during which process there take place chemical changes which give to each wine its peculiar flavor. In other cases the wine is "fortified" by the direct addition of alcohol. Wines differ from one another according to the variety of the grape used in making the must, according to the variety of yeast used for fermentation, and according to other circumstances.

11. Distilled Liquors and Spirits. - This group of alcoholic beverages contains the highest percentage of alcohol, and includes whisky, brandy, rum, and gin. In the making of all of these the essential procedure is the same; namely, first to produce fermentation in some sugary liquid, and afterwards to distill from the products of this fermentation its alcohol and some other volatile constituents. Whisky is made by distilling fermented corn or rye; brandy may be spoken of as distilled wine; rum is distilled from fermented molasses, and gin from a fermented mixture of rye and malt, - juniper berries and other substances being added to the distilled product. In 
general, distilled liquors contain from thirty to sixty per cent of alcohol.

With these differences of preparation, alcoholic beverages differ greatly among themselves, independently of the quantity of alcohol they contain, and some of their special effects are due to other constituents. The chief danger of most of them, however, lies in the action of the ethyl alcohol upon the system, and we shall confine our discussion to the effects of this substance. The problem is by no means a simple one, because these beverages are used in so many different ways by different people. Moreover, the results of their use differ according to the constitution of the person using them, and according to his other habits of life. Sweeping assertions are too frequently made, in good faith, only to be found false by experience in special cases, and in this way harm is done where good was intended. For example, it is often asserted that alcohol used in any amount whatever is a poison to the healthy organism. If this be so, it is the only known drug of which this is true. Dr. John J. Abel, from whom we shall extensively quote, says on this subject: "All poisons are capable of being taken without demonstrable injury in a certain quantity, which is for each of them a.special though sometimes very minute fraction of their toxic or lethal dose. There is no substance which is always and everywhere a poison." Alcohol is a drug, and, like many drugs, may be and frequently is used in poisonous doses; but it must not be supposed that its real danger lies in the fact that it always exerts a poisonous effect on the body.

12. The Physiological Action of Alcohol. - As to the immediate action of alcohol on the body we may say that it belongs in the same general class of drugs as the ether and chloroform used for anesthesia; in other words, its general action is that of an hypnotic or anesthetic. To 
quote again from Dr. Abel: "An exhilarating action is an inherent property of these substances in certain doses. Occasionally the physician meets with persons who have formed the habit of inhaling chloroform from the palm of the hand or from a lightly saturated handkerchief. The inhalation is usually carried on for a short time only, and its object is to induce a pleasant form of mental stimulation. Only occasionally is the inhalation of chloroform carried on until helpless intoxication occurs." And again : "That alcohol can produce as profound anesthesia as any of the substances named is also well known. In the days before anesthesia it was the custom of bone setters to ply their patients with alcohol in order to facilitate the reduction of difficult dislocations. . . . The anesthesia produced by alcohol is, however, not commendable, since it cannot safely be induced in a short time and is too prolonged. The quantity needed for surgical anesthesia would in many cases lead to a fatal result."

13. Is Alcohol a Stimulant? - The view of the action of alcohol just stated is, of course, borne out by the condition of a thoroughly intoxieated person; but it is opposed to the very general idea that alcohol, except in large doses, is to be regarded as a stimulant. Whether we shall call it a "stimulant" or not depends upon how we use that term. Some of the exhilarating effects of alcoholic drinks might lead us to speak of it in this way. People who have drunk wine often become more talkative, so that the first effects of intoxication often resemble those of stimulation. There is, however, strong reason for thinking that this action is only superficially, and not fundamentally, a case of stimulation, as we shall now see.

In studying the physiology of the nervous system we found that processes of inhibition are as important in its operation as are those of excitation; and in mental operations the course of our thinking is constantly checked or 
inhibited by the knowledge of facts opposed to the conclusions towards which we are tending. Probably it is this essential feature of all accurate and valuable mental work which is the first to be paralyzed by alcohol. The man who takes alcohol becomes fluent, not because he is stimulated, but because of the removal of checks whose presence may make him talk less fluently, but which at the same time make him speak more accurately. He may become witty, and may say some brilliant things; but he will almost always do and say some very erratic things.

The following (by Dr. Abel) appears to be a sound statement of our present knowledge of this important subject: "Alcohol is not found by psychologists to increase the quantity or vigor of mental operations; in fact, it clearly tends to lessen the power of clear and consecutive reasoning. In many respects its action on the higher functions of the mind resembles that of fatigue of the brain, though with this action is associated a tendency to greater motor energy and ease.

"In speaking of a certain type of individual James says: ' It is the absence of scruples, of consequences, of considerations, the extraordinary simplification of each moment's outlook, that gives to the explosive individual such motor energy and ease.' This description aptly applies to the individual who is under the influence of a 'moderate' quantity of alcohol. It tends to turn the inhibitive type of mind into the 'hair-trigger' type. We have said that the speech and the bearing of men, the play of their features, all bear witness to the action of alcohol on the brain; that it removes restraints, blunts too acute sensibilities, dispels sensations of fatigue, causes a certain type of ideas and mental images to follow each other with greater rapidity, and gives a ' cerebral sense of richness.'

"Larger quantities, such as are for most individuals represented by one or two bottles of wine (ten per cent 
of alcohol), may, according to the resistance and type of individual in question, cause a lack of control of the emotions; noticeably affect the power of attention, of clear judgment and reason ; and decidedly lower the acuteness of the several senses. In many individuals such quantities will develop so marked an anesthetic action that all phenomena of intoxication may be seen to follow each other in due sequence, finally to end in the sleep of drunkenness.

"There has been much discussion as to whether álcohol is in any sense a stimulant for the brain. We have seen that pharmacologists of high repute deny that it has this action, holding that alcohol is a sedative or narcotic substance which belongs to the same class as paraldehyde and chloroform; that its stimulating action is but fictitious; and that even the earlier phenomena of its action are to be referred to a paralyzing action on cerebral (inhibitory) functions. This theory assumes an unequal action on cerebral functions in the order of time. Kraepelin, however, holds that this is a purely subjective analysis, and that in the early stages of its action alcohol truly stimulates the motor functions of the brain; that a state of mental exhilaration, of ' motor excitability,' may coexist with undiminished power of perception and judgment. His psychological experiments on the action of alcohol, taken all in all, do not, however, entirely prove his position."

Some cases of apparent stimulation are really due to the fact that alcohol, when taken in the form of wines and distilled liquors, sets up an irritation in the mucous membrane of the mouth, œsophagus, and stomach, which reflexly excites the heart to greater activity or for the time being reflexly stimulates the nervous system. Such stimulation is, however, transient and, as the alcohol is absorbed into the blood, gives way to depression and even stupor.

It is neither possible nor necessary to state here in full the reasons which have led to what seems to the authors 
the erroneous view that alcohol in small doses is a stimulant and only in larger doses a depressant and hypnotic. Enough has been said to show that there are at least two opinions about the matter: that even if alcohol is at times a stimulant, it is an uncertain stimulant; and that its excitation is liable to give way at any time to depressing effects. A critical examination of the literature on the subject has failed to demonstrate to us a direct stimulating action of alcohol on any of the functions, such as the beat of the heart, respiration, digestion, etc. At times, especially in sickness, alcohol may be useful; but the evidence tends to the conclusion that where it exerts any physiological action on the healthy body at all, that action is usually depressing. This is notably true as to the beat of the heart, as to respiration, and as to the ability to do muscular work.

We have dwelt at length upon this question in order to disabuse the student's mind of the idea that alcoholic drinks can be safely depended upon as an aid in the performance of work. Few causes are more effective in leading to the abuse of alcohol than the idea that when one finds difficulty in doing a thing it may be accomplished more easily by having recourse to beer or wine or whisky for their "stimulating" effect. In general, so' far is this from being the truth that the person seeking such aid is really using a hypnotic and depressant. Obviously he would be acting more wisely to adopt other methods of accomplishing his end. Nor is this conclusion merely theoretical. Brain workers who wish to "keep a clear head" almost universally avoid alcoholic drinks, at least until work is over. And even among those who do drink it is customary to avoid drinking until the day's work is done.

14. Alcohol in Muscular Work. - That the general effect of alcoholic drinks is to depress rather than stimulate the powers of the body is furthermore indicated by the results 
of experiments on men doing heavy work, as, for example, soldiers on forced marches. In the Ashanti campaign the effect of alcohol as compared with beef tea was tested. "It was found that when a ration of rum was served out, the soldier at first marched more briskly, but after about three miles had been traversed the effect of it seemed to be worn off, and then he lagged more than before. If a second ration were given, its effect was less marked, and wore off sooner than that of the first.' A ration of beef tea, however, seemed to have as great a stimulating power as one of rum, and not to be followed by any secondary depression" (Lauder Brunton). The results of these and other experiments lead us to the conclusion that alcohol cannot be depended upon to increase the capacity for hard muscular work, and that in the great majority of cases it actually diminishes it.

\section{The Dilation of Cutaneous Arteries by Alcohol. -} One of the most important effects of alcoholic drinks is the dilation of the arteries of the skin, thus sending more warm blood to the surface. It is a common experience among persons not accustomed to alcoholic drinks that even a small amount "makes the face hot" and flushed, and the red face of the toper is proverbial. The result of this dilating effect is that the temperature of the skin rises and the individual feels warmer. Congested states of internal organs may thus be relieved, and this is probably one reason why men leading an exclusively sedentary life often use alcoholic drinks apparently to some advantage. But even these would do infinitely better to secure the same result by proper muscular activity.

Even if a temporary advantage appears to be gained in some cases or at some times, this has often to be paid for by bad secondary effects, such as impaired capacity for good work some hours later; and in mental work of the highest kind, such as original writing or composition, the 
after effects of alcoholic drinks are sometimes prolonged and easily detected by the subject of the experiment.

16. Alcohol as a Defense against Exposure to Cold.-Because of this effect upon the cutaneous circulation alcoholic drinks are frequently used by men exposed to cold, with the mistaken idea that the conditions within the body are thereby improved. The student has, however, learned (p. 192) that a feeling or sensation of warmth does not necessarily indicate greater heat production within the body; and he also knows that bringing the blood to the skin when the body is exposed to cold serves to increase the loss of heat. As a matter of fact, the internal temperature often falls when alcohol is taken under these conditions. The story is told of some woodsmen who were overtaken by a severe snowstorm and had to spend the night away from camp; they had with them a bottle of whisky, and, chilled to the bone, some imbibed freely while others refused to drink. Those who drank soon felt comfortable and went to sleep in their improvised shelter; those who did not drink felt very uncomfortable throughout the night and could get no sleep, but in the morning they were alive and able to struggle back to camp, while their companions who had used alcoholic drinks were found frozen to death. They had purchased relief from their unpleasant sensations of cold at the cost of lowering their body temperature below the safety point. This, if true, was, of course, an extreme case ; but it accords with the universal experience of arctic travelers and of lumbermen and hunters in northern woods, that the use of alcohol during exposure to cold, although contributing greatly to one's comfort for the time being, is generally followed by undesirable or dangerous after effects.

17. Alcohol as a Food. - There has been much discussion as to whether alcohol is or is not a food, i.e. whether its oxidation within the body may supply energy. This 
question must now be answered in the affirmative, although whether it can do more than supply heat to maintain the body temperature, i.e. whether it can also supply the power for muscular work, as do fats and carbohydrates, we cannot in the present state of our knowledge positively say. In many cases of sickness the oxidation of alcohol is probably a useful source of heat production, since it is absorbed quickly and without digestion; but the healthy man does not and should not use it in this way. The amounts which would be required to be of any considerable service as food are far beyond those in which it may be used with safety. In other words, in using alcohol for food, one would be obtaining heat at the cost of direct injury to many organs, and also at the cost of impaired working power. Moreover, men do not use alcohol as a food; they use it as a drug. So that while the action of alcohol as a food is of practical importance to the physician, who must deal with the abnormal conditions of disease, its action as a food is not a matter of practical importance to healthy people.

\section{Pathological Conditions Due to the Use of Alcohol.}

When alcoholic beverages are taken in excessive amounts we have the sad and degrading spectacle of a "drunken spree." Whether or not the drinker at first appears bright or witty, sooner or later there is presented the pitiable picture of complete loss of nervous coördination and control. The man becomes silly, or maudlin, or pugnacious, as the case may be, but always irrational; he staggers, stumbles, or falls ; and finally passes into a drunken stupor. In this event the victim of his own indulgence is said to be "dead" drunk, or "intoxicated," being as it were thoroughly poisoned. If such intoxication is frequently repeated, there is a complete breakdown of the nervous system; the victim of alcoholic indulgence becomes a raving maniac, and with disordered vision thinks he sees all about him snakes or foul vermin (delirium tremens). The silly or foolish stage 
of this poisoning sometimes provokes smiles or laughter in thoughtless observers, but none can witness the more serious consequences of repeated intoxication by alcoholic drinks without disgust and horror.

Many steady drinkers, even though they have never been drunk in their lives, are apt ultimately to acquire various diseased conditions of the body, into which we cannot enter in detail. The heart may be injured, or the arteries become diseased; the repeated irritation of the stomach may produce chronic gastritis; or the connective tissue of the liver and kidneys may increase, thus crowding upon the living cells and ultimately throwing a large part of them entirely out of use. While it must not be supposed that drinking alcohol is the sole cause of these troubles, for some or all of them may come from other causes, - the frequency of their occurrence in steady drinkers is suspiciously high, and this has led to the very strong conviction among medical men that alcohol plays a large rôle in producing them.

19. Summary of the Action of Alcohol as a Drug. - In small doses alcohol may be completely oxidized within the body without exerting any pharmacological action. In the forms and amounts usually employed in alcoholic beverages it exerts, in general, a hypnotic or anesthetic action; the result on the system as a whole depends on the amount taken, and varies from the paralysis of inhibitory processes to the depression of all nervous functions, ending in drunken stupor. Continued excess may produce exaggerated forms of temporary insanity, among which delirium tremens may be mentioned. There is, moreover, good reason for believing that steady drinking is very frequently an important agent in preparing the way for many other diseases, and is hence a serious menace to health.

20. The Seat of the Danger in Alcoholic Drink. - The regular use of alcoholic beverages is dangerous for the 
same reason that the regular use of any drug is dangerous. We are too apt to rely upon the drug to do for us what we ought to accomplish only by the hygienic conduct of life; the drug never satisfactorily does the work, and we go from bad to worse, and become its slave. But there is certainly greater danger in hypnotic drugs, like alcohol, than in true stimulants, like coffee, and cocoa, and tea. We need to have ourselves well under control when we use any drug; the highest faculties of the mind must keep tight rein or we may lose control of ourselves. With hypnotic drugs to which class belong not only alcohol but ether, chloroform, opium, chloral, etc. - there is special danger that these powers of control (inhibition) may be stealthily paralyzed before we know it. Of course thousands of people use alcohol in moderation and never become drunkards; but thousands also, with no intention of using it to excess, do unconsciously let the reins drop, and before they know it the drug gets the better of them. Experience shows that it is with the hypnotic drugs that this most frequently happens.

Again, if we make a habit of taking alcoholic drinks, we are specially exposed to temptation from our fellow-men to go too far. For the most part, people take coffee and tea or do not take them, as they please; no one urges them to use these drinks when they are disinclined to do so. To a less degree the same thing is true of tobacco, although here the force of fashion and example is stronger. But with alcoholic beverages the custom of "treating" makes the exercise of self-restraint more difficult than it would otherwise be; for here we are dealing with a drug which is capable of impairing self-control. Some one "treats" a friend to a drink; the friend wishes to return the compliment, and so they drink again; the person with deficient self-control - and what little he has now lessened insists upon a third, and so on, perhaps to intoxication. 
This, of course, does not always happen; thousands are strong and escape the danger, but thousands are weak or do not know better, and many a week's wages has gone in this way, leaving behind poverty and misery and impaired capacity before the close of Saturday night.

21. Concluding Remarks on the Use of Alcoholic Beverages. - In the foregoing pages we have stated the salient facts concerning the physiological action of alcohol and alcoholic drinks. It only remains to point out for the student the obvious conclusions to be drawn from them and from the long and on the whole very sad experience of the race with alcoholic drinks. The first is that, except in sickness and under the advice of a physician, alcoholic drinks are wholly unnecessary, and much more likely to prove harmful than beneficial. The last is that their frequent and especially their constant use is attended with the gravest danger to the user, no matter how strong or self-controlled he may be.

It is true that history and romance and poetry contain many attractive allusions to wine and other alcoholic drinks, and it may also be true that such drinks, by loosening tongues and breaking down social, political, or other barriers (removing inhibitions), may tend towards conviviality and good-fellowship; but it is no less true that the path of history is strewn with human wreckage directly due to alcohol; that many a promising career has been drowned in wine; and that indescribable misery follows in the trail of drunkenness. The only absolutely safe attitude toward alcoholic drinks is that of total abstinence from their use as beverages.

22. Opium, Morphine, and the Opium Habit. - The danger of the use of drugs as a regular habit of life is perhaps most painfully illustrated by what is known as the opium habit. Among the most valuable remedies at the physician's disposal is opium or its active principle, morphine, which 
possesses remarkable power to produce insensibility to pain. It sometimes happens, however, that by incautiously using this drug for this purpose men and women become addicted to the habit. They finally cannot do without the drug, and its constant use causes an appalling moral and physical degeneration; so far indeed does this often go that the victim will commit crime in order to obtain the drug. It should be clearly understood that it is unsafe for any one to use opiates to relieve pain; indeed, these should never be used except when prescribed by a careful physician.

23. Chloral, Cocaine, etc. - Men and women may become slaves to the use of other drugs, and in much the same way as they become slaves to alcohol and morphine. Among these drugs are chloral and cocaine. They belong in the same general group of hypnotics or anesthetics, and the habit acquired is perhaps no worse than the opium habit. It is certainly very little better. Let the student remember that the root of the evil here, as elsewhere, is the substitution of the use of the drug for normal habits of healthful living.

24. Tobacco. - The physiological effects of tobacco are quite complicated, so complicated that it is difficult to make general statements with regard to them. The effects of chewing are quite different from those of smoking, and those of smoking, no doubt, vary according as the smoke is or is not drawn into the lungs (inhaled).

The leaf of tobacco contains a poison, nicotine, which exerts a powerful action on the heart and on nerve cells. It is not, however, proved that the bad effects of the use of tobacco are due entirely or even chiefly to this substance; but it unquestionably contributes to the physiological effects.

The smoke from tobacco also contains ammonia vapor which locally irritates the mucous membrane of the mouth, throat, nose, etc., and this irritating action at times acts 
as a stimulant to the whole system in much the same manner as do "smelling salts."

It has been recently suggested that, owing to the incomplete character of the combustion, tobacco smoke contains a small amount of the poisonous gas, carbon monoxide (CO), and it is quite possible that some effects of smoking especially where the smoke is drawn into the lungs (inhaled) - may be attributed to this gas ; but the suggestion has not yet been submitted to the test of actual experiment.

Indeed, the physiological action of tobacco probably not only varies with the form in which the tobacco is used but is in any case the result of a combination of a number of factors partly physiological and partly psychical. We must, here, however, confine our attention to the purely hygienic aspects of the matter.

Human experience shows that the unwise use of tobacco may unfavorably affect digestion, cause serious disorders of the heart, and impair the work of the nervous system. Those training for athletic events are usually forbidden the use of tobacco because it "takes the wind," i.e. makes impossible the most efficient training of the heart. Many employers have found that youths who smoke cigarettes are less reliable in their work; and this is only one instance of the effect upon the nervous system already referred to, the same result being observed in a diminished steadiness of the hand, often amounting to actual tremor.

These effects do not, of course, manifest themselves in their extreme form whenever tobacco is used; but it is probable that they are always present in some degree. Whether they are noticeable or not depends largely upon the ability of the constitution to resist them. Tobacco is thus often used without demonstrable bad effects when one is leading a hygienic life; but very often the habit, formed under these conditions, persists after the increasing intensity 
of occupation, and the attendant cares and responsibilities of life result in neglect of muscular exercise and improperly directed nervous activity. And as this neglect begins to tell on general health it is found that the unfavorable effects of tobacco become more pronounced.

Especially to be condemned is its use by those who have not attained their full growth. During youth nothing should be allowed to interfere with the best development of the heart and nervous system, and the use of tobacco endangers the proper development of both of these most important parts of the human mechanism. It can hardly be doubted that many a young man has failed to make the most out of life because the habit contracted in youth has struck in this way at the foundations upon which he had subsequently to build. 


\section{CHAPTER XXI}

\section{THE PREVENTION AND CARE OF COLDS AND SOME OTHER INFLAMMATIONS}

1. Hygiene and Physical Efficiency. - A most important aim of personal hygiene is the maintenance of the highest working efficiency of the body. We should not be content with the avoidance of serious maladies like smallpox, diphtheria, and consumption, but should try also to avoid those minor ills which, though temporary and rarely fatal, may seriously interfere with our capacity for usefulness and enjoyment. The importance of avoiding constipation has already been pointed out (p. 128). The present chapter will be devoted to the practical consideration of such common complaints as colds, rheumatism, and diarrhea, all of which are accompanied by inflammatory conditions in some internal organ or organs, and are favored by exposure to cold, drafts, or dampness, which chill the skin and drive the blood into the internal organs.

2. Some Common Complaints and the Conditions which favor them. - We shall not give any extended account of the nature of the complaints mentioned in the preceding paragraph, for their exact causes are still obscure. Two points, however, should be emphasized for all of them.

1. The exposure to cold is not usually the cause of these diseases but only favors their development. It is the general experience of arctic travelers that they suffer very little or not at all from "colds." Nansen and his men were away in the Fram for more than three years, and during a large part of that time Nansen and Johannson journeyed on sleds 
or afoot, exposed to the worst rigors of an arctic climate. At times, after getting into their sleeping bags, they had to thaw out their frozen clothing by the heat of their own bodies before they could go to sleep; and yet not one of them had "a cold" until their return to Norway, when an epidemic of colds broke out among them. This and numerous similar experiences of others suggest strongly that colds are largely infectious diseases ; but we must not forget that dampness and drafts are favoring conditions for their development. The experience of the race on this point is abundant and conclusive.

2. Each of these diseases is characterized by a condition of inflammation. We shall not attempt to describe the exact nature of inflammation; it is sufficient to recall features of it familiar to every one. The sting of a bee or hornet or the bite of a mosquito results in local inflammation of the skin ; a severe case of sunburn presents a similar condition over larger areas; a wound of any kind often shows more or less of the same inflammatory process. The part becomes red, indicating the presence of an increased amount of blood; it is swollen, partly because of the greater quantity of blood and partly because of the greater quantity of lymph present in the tissue ; it is usually hot; and it is often painful. At times, as in the case of a wound or boil, pus, or " matter," may be formed.

One or more of these conditions is present in an inflamed organ during the diseases mentioned. When we have a cold in the head (rhinitis) the vascular membrane lining the nasal cavity is the seat of trouble ; in a sore throat it is the pharynx and larynx (pharyngitis and laryngitis); in a cold on the chest (bronchitis) it is the ciliated membrane of the trachea and bronchi; similarly in catarrhal attacks of the stomach and intestine it is the mucous membrane of these organs ; and we must think of these inflamed tissues of the respiratory and alimentary tracts as 
presenting somewhat the same condition as that seen in the skin during a bad case of sunburn. They all have an excessive amount of blood within them ; they are more or less swollen, _ as when one's " nose is stopped up"; there is an unusual amount of fluid in the tissue; and there is, besides, generally a transudation of this fluid to the surface, as in the "running of the nose."

3. Congestion during Inflammation. - The presence of an excessive quantity of blood in the capillaries of an organ is known as "congestion"; and this may be of two kinds, -active (or arterial), due to an excessive supply from the arterial reservoir; or passive (or venous), due to some interference with the outflow into the veins. ${ }^{1}$

In a cold, congestion of the inflamed area begins as an active congestion; the arteries are widened, the pressure in the capillaries is increased, and the blood flows much more rapidly. This is essentially the same thing - only in greater degree - that occurs when the arterioles of the stomach dilate during digestion, or those of the skin during exposure to warmth. This initial vascular stage is succeeded by one of passive congestion, caused by the adhesion of white blood corpuscles to the capillary walls, thereby diminishing the bore of the tube, and so making difficult the outflow into the veins; the velocity of the blood through the capillaries is lessened, pressure within them is increased (why?), and they become gorged with blood. Such is the vascular condition in an organ when an inflammatory process is at its height; the characteristic feature is the narrowing of the outlet of the capillaries and the consequent excess of pressure within them.

4. Dangers connected with Congestion. - A decidedly congested condition is undesirable because it is a predisposing

1 The artificial model described on page 142 may easily be used to show the difference between arterial and venous congestion. With the nozzle in the far end of the rubber tube, the tube may be congested (or swollen) with water by more rapid pumping (active congestion) or by narrowing the outlet (passive congestion). 
cause of these inflammatory diseases. It is not the only cause nor the exciting cause of the disease ; but a congested organ may succumb to an attack of disease and so become readily inflamed where it would have escaped had its vascular condition been normal. For example, the normal intestine may be the seat of some unusual bacterial action (see Chapter VIII, p. 125) and suffer no damage therefrom, while the same bacterial action may give rise to catarrhal inflammation, accompanied by diarrhea, if it occurs when the intestinal blood vessels are congested. Or again, whatever the cause of an ordinary cold may be, bacterial or otherwise, it is probable that its attack upon the perfectly normal organism may be and frequently is resisted; while at another time a congested condition of the nose, the throat, or the bronchial tubes may permit the disease to gain a foothold at that point. In other words, the congestion alone will not cause colds in the head or on the chest, or diarrheal troubles in the intestine; something else is needed. We may have the congestion without the cold, and we may also succumb to a cold without the preliminary congestion; but the presence of congestion often presents to an infecting agent the weak spot which is needed in order that the latter shall secure a foothold and do damage.

5. The Avoidance of Congestion during Colds, etc. The Care of Catarrhal Conditions. - Again, whenever an inflammatory process is established, there is, as we have seen, more or less of passive congestion; under these circumstances everything should be done to avoid arterial dilation in the inflamed area. Suppose there is catarrhal inflammation of some part of the small intestine, accompanied by diarrhea; the outlet into the veins is narrowed and there is consequently more or less "backing up" of the blood in the capillaries (passive congestion). This congestion is kept within moderate limits so long as the arterioles maintain a good tonic constriction and so limit the amount of blood 
which can flow in; if, however, they are made to dilate widely, - by eating a hearty meal for example, - this check is removed, blood flows in under high pressure, and the congestion is increased. Hence in all such catarrhal
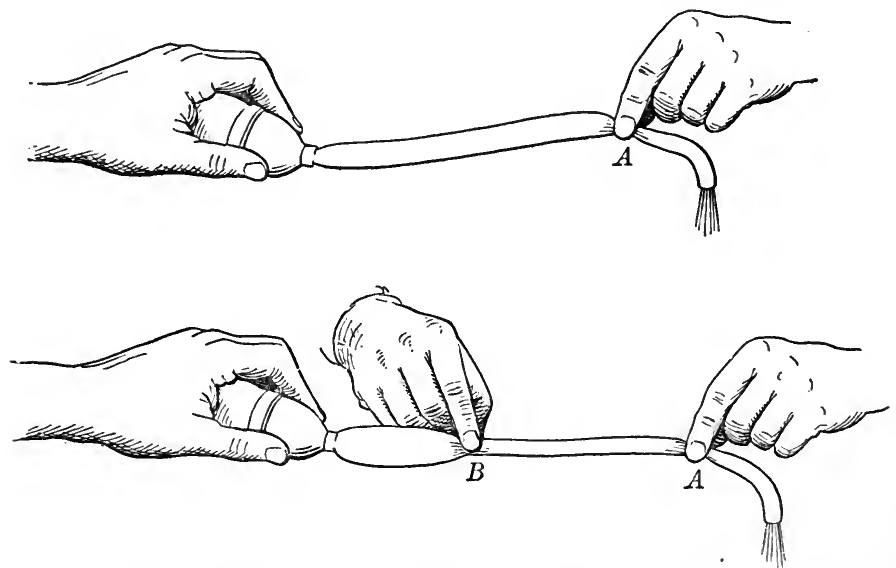

FIG. 111. Experiment to show the effect of arterial constriction in relieving capillary congestion

In the upper figure, constriction of the tube at $A$ results in distention (congestion) of the tube between the pump and the fingers; if, however, the tube be also constricted at $B$, as in the lower figure, pressure falls between $A$ and $B$, and the congestion is relieved.

attacks the diet should be very light and preferably confined to those things which are easily digested and absorbed.

6. The Care of Colds, etc. - Again, when suffering from any of these inflammatory diseases of internal organs the greatest care should be taken to avoid chilling the skin, because this means (Chapter XII) compensating dilation in the inflamed area, and therefore increase of congestion there. One should be warmly clad (not overclad); the living and sleeping rooms, though well ventilated, should not be cold; when a cold sleeping room cannot be avoided some covering for the head is often useful, as this part of the body is not protected by the bed covering; cold baths should be 
discontinued; and, above all, dampness should be avoided. - In severe cases it is often necessary for the patient to go away from a damp climate to a dry one. The key to the situation, so far as the management of the circulation is concerned, consists in keeping in the skin its full share of blood. A brief chilling of even a comparatively small area of the skin (e.g. cold feet) may produce a congestion in the inflamed organ capable of undoing the healing work of hours or days.

A word must be said in this connection about the "freshair" cure for colds, etc. There is no doubt that being in the fresh, dry air, even if it is cold air, and preferably out of doors, is better for a cold or any other catarrhal condition than remaining in a closed room. But this should never involve the chilling of any portion of the skin; one should be warmly clad, even the head and neck being well protected. It makes little or no difference that we breathe cold air; but it makes a very great difference whether or not the skin is exposed to cold air.

In taking care of colds and similar troubles it is well to remember that the inflammation is only one of the unfavorable conditions against which the system is struggling. Consequently we should not expect the disease to yield in all cases to our measures for keeping the skin warm. At times a hot bath, a drink of hot lemonade, or other measures for bringing the blood to the skin checks a threatened cold; but none of these measures is of great value after the disease has once obtained a foothold. It is then a struggle between the body and the disease; and we can do more by merely avoiding the chilling of the skin than by taking measures to produce marked cutaneous dilation. The true policy, in other words, is to give the living body every chance to cure itself, and this is best done by not calling on it to do too many other things at the same time. Thus muscular exercise, ordinarily one of our best means of keeping the blood in the skin, is not usually 
advisable when a cold is at its height, because an added strain would thereby be imposed on the already sorely taxed system. Later, when the worst is over, it is a valuable aid, though it should not be too vigorous until one is on the road to complete recovery.

"Stuff a cold and starve a fever" is one of those pithy sayings whose very pith may be poisonous. A full meal when we have a cold in the head often clears up the nasal congestion for a time (probably by drawing the blood to the stomach and intestine) and so deludes us into supposing that our "stuffing" has done good. It may also, and doubtless often does, support and reënforce the body in its battle with the disease. What it may do, however, is to overtax the body with the digestion of a heavy meal; the meal may not be properly digested; bacterial processes in the excessive mass of food may produce abnormal and poisonous substances (see Chapter VIII) which gain admission to the blood, and the "last state" of the patient may be "worse than the first."

7. Measures for stopping Colds. - When one "feels a cold coming on," that is, early in the struggle, active measures should first be taken to dilate the blood vessels of the skin. A hot bath before going to bed, and hot drinks such as hot lemonade, may be tried. If the cold does not promptly yield to these measures, rest in bed is usually the best treatment. The nervous system is frequently in no condition to sustain hard work of any kind, and hence, until the cold begins to clear up, it is well to confine the diet to easily digestible foods in moderate quantity, and to remain very quiet. Few people, unfortunately, act on this principle. "It's only a cold" is made the excuse for meeting every engagement that may have been made, or for attempting to do full work. Sometimes, perhaps generally, no serious results follow, but at other times the penalty is heavy. 
Very often a cold is a more serious matter than we suppose. Only physicians appreciate how often it is the sign of more serious disease. ${ }^{1}$ While we cannot say that one should always stop work until the cold is overcome, we do say that limiting work to the minimum and securing all the rest possible is always advisable and should be the rule rather than the exception. We may, unknown to ourselves, be nursing more than a cold; and, even if we are not, we always hasten the cure by taking care of ourselves:

8. The Use of Drugs for Catarrhal Conditions. - A remedy very frequently resorted to for colds and other inflammatory troubles is the taking of some drug. Large fortunes have been made by the sale of "cough medicines" and the like. Some of these "remedies" are worse than useless; others may do no harm, and some may be useful. But none of them are cures. The cure of the cold is effected not by the drug but by the system of the patient; the drug can do no more than remove some condition which stands in the way of the healing effort of the organism. The severe coughing of a bad case of bronchitis is often irritating to the inflamed surface of the air passages, and may stand in the way of clearing up the inflammation. Here a drug may do good, though it should be taken only on the advice of a physician, and never on the strength of newspaper testimonials to its wonderful virtues. But the use of these medicines does not render unnecessary the measures we have outlined as the proper treatment. It is worse than foolish to dose oneself with drugs when a cold is coming on, and then attempt to do full work; often the only result of such folly is a complete "knocking out of the stomach" by the drug. The average "cough medicine" is especially likely to do this.

1 In typhoid fever a "cold on the chest" is frequently the first outward indication of the disease. 
9. The Belief in Drugs. - A century ago the attitude of men and women toward practical hygiene consisted largely in living in ignorance of the workings of the body, taking little or no care of it, and then whenever bad feelings appeared "taking something simple" to cure them. This course of conduct was persisted in until something happened, - and something usually did happen, sooner rather than later, - when recourse was had at once to drugs. The doctor was the man who knew what drug to "give" for each disease. He was expected to "prescribe"; and if he did not prescribe something, he failed to satisfy his patient, who concluded that the physician did not understand the disease. The attitude of the public was largely that of neglecting personal, individual care of the health and meantime implicitly believing that, no matter what happened, some drug could be swallowed which would set matters right.

Medicine, and especially personal hygiene, have now advanced beyond this crude condition. To-day we realize as never before that the individual is responsible for the intelligent care of his health. The time is probably coming when he will be held as responsible for the care of his body as he is to-day for the care of his morals. At the same time, drugs are being much less used by the best physicians. It is not true that all drugs are useless; quite the contrary; but it is true that careful nursing often counts for more than does the use of drugs. Typhoid fever is to-day often treated with no drugs at all, and the tendency to use drugs in other diseases is distinctly lessening.

The wise physician is often hampered in his work by the survival of this old-fashioned belief in the all-sufficiency of drugs. Instances of it are sometimes encountered even among otherwise intelligent people. We should understand that in all cases of illness the one treatment which should be applied is good nursing, whether by a trained 
nurse, or by one's family, or by oneself. If medicine is given, it is usually subsidiary to the main procedure, although sometimes, as in the antitoxin treatment of diphtheria, it is the main thing. But in no case should we be so foolish, so unreasonable, as to distrust or lose confidence in a physician because he gives few drugs or none at all.

10. The Avoidance of Colds, etc. General Principles. - If the care of these slight ailments is of importance, their prevention is of much greater importance. And first of all among preventive measures must be placed not the avoidance of drafts and other chilling of the skin, not clothing, but the proper hygienic care of the body, regular and sufficient muscular exercise, the avoidance of improper feeding (for colds are often due to digestive disturbances resulting from overfeeding), and good habits of sleep and rest. When these things are properly attended to, one may usually suffer considerable chilling of the skin without ill effect. It is not true, as is often asserted, that by attention to these general matters the protection of the body from exposure to cold becomes unnecessary, but it is true that without this attention such protective measures are apt to be of little avail.

Among general measures none is more important than the avoidance of exposure to chilling influences when the nervous system is depressed by marked fatigue. We take cold more readily, much as we are more susceptible to any disease, when we are tired. It is a question of a struggle between the organism and unfavorable external or internal conditions, and, in general, the greater the fatigue of the organism the less is its chance of success in the struggle.

11. The Avoidance of Colds. Special Measures. - As to measures specially concerned with the avoidance of congestion in internal organs, let us first state clearly the principle involved and then pass to its practical applications. The condition to avoid is the undue constriction of 
the blood vessels of the skin, produced by chilling. The danger is not in the mere exposure to cold; people may be comparatively lightly clad on a cold dry day, when there is no wind, without chilling the skin, because the layer of air in contact with the skin becomes warmed, and in the absence of wind even light clothing, if dry, suffices to keep this warm air in contact with the body. But if the air is damp, so that it readily conducts heat, or if the wind is blowing, so that convection becomes important, or if the body is near cold objects to which it can radiate its heat, the skin may be easily chilled, especially if we are making no muscular exertion (pp. 193-198).

Again, during muscular exertion exposure to cold is usually harmless, even if the clothing be light, because the increased heat production within the body results in an adequate flow of warm blood through the skin. We seldom take cold during vigorous muscular work on a cold dáy. It is when we are sitting still or, even more, when we are lying down and the muscles are relaxed, that we should be on our guard.

Finally, and most important of all, is the fact that the dangerous region of atmospheric temperature is confined to the narrow limits of a few degrees just below the proper room temperature. This proper room temperature for light clothing is about $66^{\circ} \mathrm{F}$. with low or normal atmospheric humidity, and about $69^{\circ}$ or $70^{\circ} \mathrm{F}$. for high humidity. Above these points there is no chilling of the skin. Five or ten degrees below these points we feel so cold that we become uncomfortable and take steps to remedy matters, either by putting on heavier clothing or by heating the room. It is when the temperature is only slightly below what it should be that we are apt to be unaware of the insidious increase of arterial constriction and chilling in the skin, until, after an hour or more of it, we suddenly awake to the true condition of affairs. This is apt to 
happen when the fire in the stove or in an open grate goes down. It also happens at times when we come from a walk out of doors into a room of this "dangerous temperature," say $63^{\circ} \mathrm{F}$. on a day of high humidity; the skin is warmed by the exercise we have been taking, so that, as we enter the room, it does not seem cold (for the temperature we really notice is that of the skin, not that of the room at all); on sitting still in the room the cutaneous dilation of muscular exercise passes off so gradually that we do not notice the change, and, before we are aware that we are chilly, marked internal congestion may have been produced.

It is also well to remember that not all parts of the room have the same temperature. The floor is colder than the ceiling; it is colder nearer exposed walls and windows than away from them, and the common habit of sitting near a window on a cold day while reading or sewing is not wise.

12. Cooling off suddenly. - It is an old saying that it is not well to "cool off suddenly." While there is some truth in this, it is not true in general, nor in the form stated. It is perfectly safe for most healthy people to take a cold bath after exercise, or to pass directly from a hot bath into a cold one (see Chapter XXIV). The sudden cooling which experience has found to be harmful is where the clothing has been saturated with perspiration and one cools off by sitting still in a breeze or in a cool place. Here the clothing remains damp and so conducts heat readily from the skin ; and the danger lies not in the cooling off but in the prolonged chilling process which follows it. Consequently it is a general rule that clothing made damp by rain or perspiration should be changed as soon as possible, or else that drafts and cold rooms should be avoided until the clothing is dry.

It is unnecessary to multiply examples. In all the principle is the same, - the avoidance of conditions which 
produce marked constriction of cutaneous blood vessels, with the accompanying congestion of internal organs; and the student is again reminded that by this course we do not always secure immunity from internal inflammations; we merely remove one of the conditions which favor their development.

13. "Hardening" the System to Cold. - We must refer briefly to the importance of what is popularly known as hardening the system to cold. Cold unquestionably produces its effects in some people more readily than in others, and these differences are largely dependent upon habit or training. When the living rooms are kept above $70^{\circ}$, and heavy clothing is always worn out of doors, the skin is constantly subjected to a tropical climate and becomes more sensitive to external cold. Internal congestion will then be produced at $67^{\circ}$ or $68^{\circ} \mathrm{F}$. which would not take place above $60^{\circ}$ or $62^{\circ} \mathrm{F}$. in persons who have been accustomed to cold. In other words, it is possible to overdo the matter of protection from external cold. For this reason, overheated rooms and the use of heavy wraps while walking in moderately cold weather $\left(30^{\circ}-50^{\circ} \mathrm{F}\right.$.) are very objectionable.

We should thus harden ourselves to cold; but it should never be forgotten that this process of hardening may be carried too far. To harden oneself does not mean that the temperature of the living room should be kept below $65^{\circ} \mathrm{F}$., nor that sleeping rooms should be cold enough to freeze water at night. Severe colds and rheumatism have been contracted by this folly.

Many people fail to realize that because a little will do good, it does not necessarily follow that more will do better. One person is impressed by the undoubted fact that it is possible to eat too much meat, and thereupon abstains from meat altogether; another discovers that a sedentary life is a bad thing, and hastens intemperately 
to take "century rides" on a wheel. One finds that he has been overclad, and, discarding all warm clothing, shivers throughout the winter; another, on learning the possible value of cold bathing, enthusiastically but unwisely plunges into the coldest water he can get, and stays in it until his skin is blue. Very likely any one of these examples can be duplicated from the reader's own circle of acquaintances. It is important to remember that "nothing too much" is always a good rule, and nowhere is it more essential than in the hygienic conduct of life.

14. Reasons for avoiding Colds and Other Inflammatory Troubles. - We may conclude this chapter with some facts showing the hygienic importance of the prevention of colds and other inflammatory diseases, such as sciatica, lumbago, and rheumatism in its various forms. In all these diseases we find the same close connection between the chilling of the skin and the onset of the disease, so that what has been especially urged with regard to colds applies in large measure to the entire group. But some may say, "These are slight ailments; why not ignore and disregard them?"

1. The first and sufficient reason is that these ailments interfere seriously with our working power and with our capacity for usefulness and enjoyment. Every one knows from experience that the body is not as good a machine during the progress of a cold or a diarrheal attack, or while suffering from sciatica or slight attacks of rheumatism. We should strive not only to live, but to live well; not merely to do things, but to do them with our might; not merely to live and work, but to live happily and to work cheerfully.

2. The popular impression as to the frequency with which pulmonary consumption, pneumonia, etc., are preceded by common colds is much exaggerated. It is nevertheless probable that in some cases a cold is the means of lowering the power of resistance to the more serious 
disease, and we should take every reasonable precaution which will maintain the ability of the body to cope successfully with the inroads of diseases, especially of those for which there is no certain cure.

3. Colds and similar troubles have a well-known tendency to become chronic. Probably no sufferer from nasal catarrh, or chronic bronchitis, or chronic diarrhea, if he had his life to live over again, would neglect measures tending to avoid the occurrence of these conditions. Only those who do not know from experience the capacity of such troubles to produce annoyance and discomfort can regard their prevention as unworthy of serious attention. We cannot too strongly emphasize the fact that chronic troubles are very frequently the result of the repetitions of the neglected inflammations which accompany the acute attack; they are due not so much to inherent weakness of the tissue or organ as to the carelessness of the individual about avoiding them, or the failure to give them the attention they deserve when they occur. One of our leading physicians, a man of the widest experience and soundest judgment, writes, concerning chronic nasal catarrh, "It is sad to think of the misery which has been entailed upon thousands of people owing to the neglect of nasopharyngeal catarrh by parents and physicians." 


\section{CHAPTER XXII}

\section{THE CARE OF THE EYES AND EARS}

The visual apparatus (eye, optic nerve, nerve endings, etc.) furnishes one of the most important paths from the world without to the brain within, and it is of the utmost importance to the exercise of the highest functions of the human mechanism that this path be kept as smooth as possible. Unfortunately, however, the path is seldom either straight or smooth, and it frequently presents serious obstacles. The curvature of the cornea or of the lens may be irregular ; the muscle of accommodation may be weak; the retina may be too near or too far from the lens, or its sensitive cells may too readily become fatigued by the stimulation of light; finally, the path into the brain may be made of poorly constructed nervous tissue, or in the brain itself the coördinations upon which depend our visual judgments (p. 256) may be imperfect. The simplest act of vision is the end result of a most complicated series of events, difficulty with any one of which may make quick and accurate seeing impossible. Many a child has been considered stupid simply because an unrecognized condition of myopia or astigmatism renders it impossible to read clearly the printed page or the distant blackboard; and many people, adults as well as children, suffer from headaches and other troubles because of the strain thrown on the nervous system in the effort to work with defective vision.

When one is leading an outdoor life, occupied in the work of the farm or the lumber camp, and doing but little reading, the eyes usually give little trouble, because it is 
only when looking at near objects (three feet or less away) that the mechanism of accommodation is called into vigorous action. Eye strain is usually produced by prolonged near work with eyes incapable of enduring without undue fatigue what is demanded of them. Hence it is that defects of vision are more common to-day than they were a hundred years ago. Both the vocations and the avocations of modern life, with their large amount of reading, writing, and other forms of near work, impose upon the eye the most trying and difficult task it can be called upon to perform. The use of glasses is more common than formerly, and the care of the eyes is forced upon us as an important factor in the hygienic conduct of life.

1. The Necessity of Expert Advice. - In the care of the eyes expert advice is indispensable. 'The detection of defects of vision frequently demands the best skill of those who are thoroughly acquainted with the physiology of the entire visual apparatus, including its relation to other bodily functions, and who are also provided with every means for gaining an insight into the conditions which are giving trouble. The selection of the proper glass, for example, when lenses are needed is more than a mere matter of testing vision with test cards; and eyes may be seriously injured by using glasses prescribed on the basis of information gained by imperfect methods.

First of all, then, let us insist upon the necessity of competent medical advice whenever there is reason to suspect something wrong with the eyes. If vision is not distinct, if the eyes tire quickly when used for near work, and even when one suffers from headaches, "nervousness," and other forms of malaise without apparent cause, it is wise to find out whether some remediable defect of vision is not at the root of the trouble.

On first thought it may seem unreasonable to consult an oculist with regard to headaches or other troubles with 
organs having no obvious connection with the eye; but when we remember the fact that all parts of the central nervous system are connected with one another, it is easy to see how undue strain of one part in the effort to see with astigmatic or otherwise defective eyes may, by injuriously affecting other parts of the brain or spinal cord, unfavorably influence organs which themselves have nothing to do with vision. Over and over again it happens that headaches and other troubles are relieved, as by magic, when vision is made perfect by the use of proper glasses.

With these remarks as to the importance of skilled advice in the care of the eyes, we may pass to those practical measures which should be under the intelligent individual control of every man and woman. Suppose vision is perfect, or as nearly perfect as the best of medical skill can make it; what precautions in the use of the eyes favor the maintenance of their best working condition?

2. Resting the Eyes. - First of all we would suggest the importance of resting the eyes now and then while engaged in near work. This is accomplished by the simple expedient of looking for a few moments at some distant object (p. 250). The brief relaxation of the effort. of accommodation does for the neuromuscular mechanism involved exactly what a brief relaxation of the body in sleep accomplishes for the body as a whole.

3. Illumination of the Object. The Importance of Contrast. - The ease with which the details of an object are seen depends chiefly on the contrasts of shade and color which these details present to the eye, and nothing so influences this contrast as the amount of illumination. Thus as the light fades in the evening, the white paper of a printed page becomes darker and darker, until finally it reflects to the eye little more light than the black ink of the printed letters, which consequently no longer stand out clear and distinct. In order to admit all the light 
possible, the pupil enlarges, and in so doing lessens the distinctness of the retinal image (spherical aberration); more important than this, we hold the page closer to the eye, thereby enlarging the retinal image and increasing the intensity of stimulation, but throwing far more work upon the ciliary muscle to focus for the near object. All of these unfavorable conditions taken together place undue strain upon the mechanism of accommodation.

Hardly less objectionable is excessive illumination of an object. After a certain intensity of light is reached, the retina no longer responds to increase of stimulation with increase of visual reaction. If there were in addition to our sun a second sun which sent into the eye twice as much light, the second sun would seem no brighter than the first because the effect of the first upon the eye has already passed the point which calls forth the greatest possible reaction in the retina. To apply this principle to the case in point, we have only to remember that a printed letter is not absolutely "dead black," but reflects some light. When the illumination is moderate this reflected light hardly affects the retina at all, and the contrast between the black letter and the white paper is marked. As the intensity of illumination increases, however, the effect upon the retina of the light coming from the letters increases more rapidly than the effect of that coming from the paper. Contrast is lessened and sharper accommodation as well as closer attention is needed to see distinctly. Added to this, no doubt, is the fatigue and lack of sensitiveness in the retina, resulting from overstimulation.

4. The Size of Type. - The use of fine type should be reduced to a minimum, because it necessitates greater effort of accommodation and intensifies all the evils of improper illumination. Any printed matter which must be held less than eighteen inches from the eye in order to be seen clearly is undesiirable for long-continued reading. 
Especially is this true in youth, since then the eye is more plastic, and excessive strain of the muscle of accommodation, pulling as it does on the sclerotic and the choroid coats, may lead to permanent deformation of the curved surfaces. The marked increase of myopia within the past forty or fifty years is explained in this way.

5. Highly Calendered Paper Objectionable. - Closely connected with the size of the type is the character of the paper on which it is printed. This should be as dull as possible in order to avoid the confusing effect of a glossy surface. The use of highly calendered paper in many books and serial publications, because such paper lends itself more readily to the reproduction of pictures in half tone, is a sacrifice of hygienic considerations to cheapness.

6. Importance of a Steady Light. Reading on Railroad Trains. - The source of illumination for near work should be as free as possible from unsteadiness or flicker, since a flickering light necessitates the most accurate accommodation. A "student's lamp," "Rochester burner," or incandescent electric lamp is preferable in this respect to candles, gas jets, and arc lights for near work.

For the same reason caution is demanded in the matter of reading on railroad trains. American railway trains have recently become so heavy, and the roadbed, rails, etc., have been so much improved in various ways, that the danger of reading or writing while traveling by rail is much less than formerly. At the same time the danger still exists, and reading on many railway and trolley cars is still to be done with caution, or, better still, avoided altogether.

7. Microscopes, Telescopes, and other optical instruments require close and sometimes continuous use of one or both eyes, and are popularly supposed to be "hard on the eyes." But this is not necessarily the case, except for beginners and investigators; for beginners, because they 
try to see clearly by focusing with the eye rather than with the use of the focusing apparatus of the instrument; for investigators, because the eyes are used for too long periods at a time. Optical instruments are easily focused, and, if care be taken to provide good lighting, routine work with them need not be specially trying to the eyes.

8. The Removal of Cinders. - Particles of dust, cinders, etc., are often washed away from the surface of the eyeball by the copious secretion of tears which they call forth. Sometimes, however, they must be removed directly from the eyeball or the inner surface of the eyelid. In the case of the lower lid this operation presents little difficulty, for the eyelashes of this lid are easily seized, the lid drawn forward away from the eyeball, and the surfaces of the eyelid and eyeball readily inspected. If any foreign body is there located, it may be removed by the corner of a handkerchief. Successful manipulation of the upper lid is more difficult, because a piece of cartilage immediately above the eyelashes interferes with turning back the lid. The gaze of the patient should be directed downward, a small pencil or other cylindrical object pressed against the upper portion of the lid, above the cartilage, the eyelashes seized, and the lid turned upwards and backwards over the pencil.

9. Recapitulation. The Care of the Eyes. - To summarize, we may remind the student that the eyes, no less than other organs, should be kept sound and strong by attention to the general health and welfare of the body. Work, play, rest and sleep, muscular exercise, wise feeding, and regular removal of the wastes, - these and all other general hygienic habits help to keep the eyes sound and strong; but besides these, posture in work, lighting, paper (not forgetting wall paper), printing, dust, cinders, smoke, acid fumes, traveling, sight-seeing, and many other conditions have their effect. Finally, it must not be forgotten 
that the eyes are too precious to be trifled with, and that if one has sore or weak eyes, or pain in the eyes, or cannot see clearly to read or to write, or cannot plainly distinguish things near or at a distance, then it is always best to consult an oculist or the family physician for advice. Remedies or doctors puffed in high-sounding advertisements should be carefully avoided.

10. The Care of the Ears. - Besides good care of the general health, which common sense dictates and which we have repeatedly urged as the fundamental requirement in the hygiene of all organs, there is but little which the individual can do for the ears. Deafness, especially total deafness, is a defect or injury perhaps no less serious than blindness. Acute hearing is probably as valuable as acute vision, and a partial loss of hearing is a handicap often harder to overcome than are some defects of vision.

Keeping in mind the auditory apparatus and its connections (Chapter XIV), it is easy to see that the drum may be pierced or otherwise injured by slender objects thrust in from without; that catarrh of the throat may easily exten'd into the Eustachian tube, inflaming it or choking its lumen or outlet; and that any thickening of the drum must tend to make its vibrations slower and more difficult. In these possibilities we have some of the actual causes of deafness, and none of them is of a kind to be treated by the patient. Any recognition of incipient deafness in oneself should be regarded as cause for consulting a good physician. No attention should be paid to advertisements promising to relieve deafness, for these are usually traps calculated to catch the ignorant, unwary, or credulous. It is dangerous to explore the outer ears with hairpins or other pointed objects, as the drum may thus be broken or other harm done.

11. Noise, though delighted in by savages, who beat tom-toms, blow conch shells, or otherwise tickle the sense 
of hearing, and though in moderation often found stimulating and enjoyable by persons who have been living in solitude or isolation, is by adults among the most highly civilized peoples more and more regarded as a necessary evil, or even as a nuisance. Children, on the other hand, often delight in noise, and horn blowing, firecrackers, and pistol firing on holidays like the Fourth of July appear to give to them as much pleasure as to their elders pain. Adults also on occasions of rejoicing still ring bells, beat drums, blow horns, and fire cannon in order to express their emotions. Loud noise, like strong light, is unquestionably stimulating and exciting, and for these reasons, though justifiable at times of rejoicing, is something to be ordinarily avoided as far as possible in city life, itself already much too stimulating and exciting. One can, indeed, often learn to sleep even in the presence of distracting noises such as those of a busy city street; but such sleep cannot possibly be as wholesome as that enjoyed in quiet places. The constant whistling of locomotives, which was formerly a great nuisance in many American cities and towns, has been largely done away with, and the tendency of the times is to cultivate quiet, not only as a private luxury but also as a public necessity. 


\section{CHAPTER XXIII}

\section{THE HYGIENE OF THE FEET}

The hygienic care of the feet consists essentially in maintaining the ability of those organs to bear easily and without discomfort the weight of the body. "Weak feet" are to blame for many unhealthful conditions; the discomfort or pain which they cause as one goes about the ordinary occupations of life subjects their possessor to

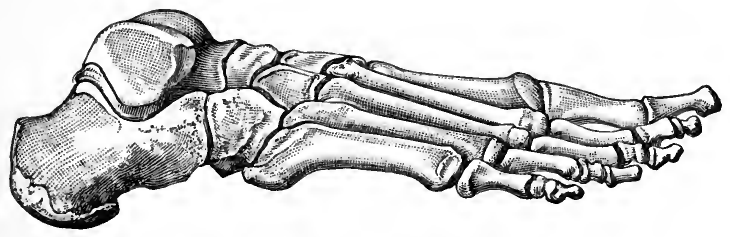

Fig. 112. Bones of the right foot

Seen from the outer side

nervous strain and often prevents the enjoyment of that muscular activity which the maintenance of health requires. Nor is it generally known that this state of affairs may be very largely avoided by intelligent care. In the majority of cases weakness of foot is the result of maltreatment of the foot, and not the result of inborn structural defects.

Each foot consists of no less than twenty-six small bones joined by ligaments and held in proper position relative to one another by the action of a number of muscles. The key to the understanding of the hygiene of the foot is the fact that it is upon the proper performance of the work of these muscles that the strength of the foot primarily 
depends, and that the weakening of the foot is due to interference with their action, chiefly by the use of wrongly shaped shoes.

1. The Arches of the Foot. - The bones of the foot should form two well-marked arches. One of these is the conspicuous arch of the instep and the other a less conspicuous but important transverse arch immediately back of the toes. Not only is the preservation of these arches important because they help to relieve the joints above them of jar, but also because under them lie nerves, blood vessels, lymphatics, and other tissues which are injured when the arch

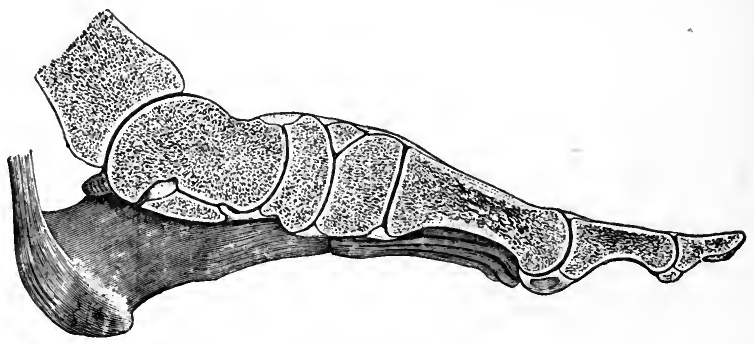

Fig. 113. Longitudinal section through the bones of the foot Showing the arch of the instep and the attachment of the tendon of the calf muscle to the heel bone

gives way and permits pressure upon them from above. Figure 144 shows the action of one of the groups of muscles which maintain the arch of the instep, and illustrates, in principle, how muscular action keeps the bones in proper relative positions. The muscles shown in the figure (the short flexors of the toes) act like the string of a bow, and by contracting resist the tendency of the weight of the body to break the arch down. Other groups of muscles are concerned, but it is unnecessary that we go into the details of their action. Enough has been said to show the importance of keeping these muscles strong, so that they may do the work imposed upon them. 
The groups of muscles specially concerned are those which move the toes, and these, like other muscles, can be kept strong only by use. Consequently interference with the freedom of action of the toes must lead to the disuse and partial degeneration (weakening) of the muscles in question. The fundamental principle in the care of the foot is none other than the maintenance of the freedom of motion of the toes, together with the use of the toes as well as the ankle in locomotion.

2. The Foot of the Infant and the Adult Foot. - Every human being begins life with a foot possessing wide range

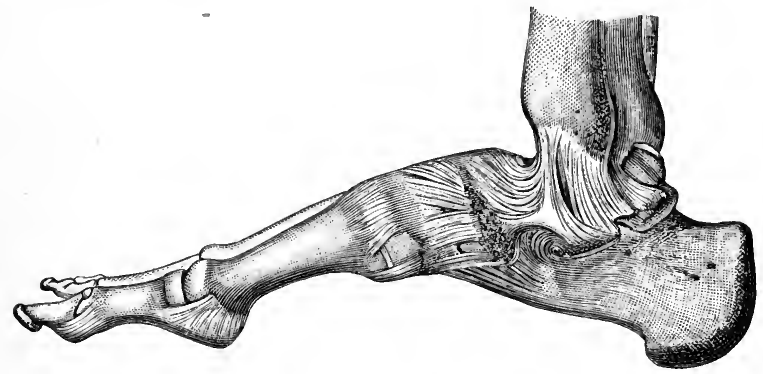

Fig. 114. Ligaments of the foot and ankle

of movement, amounting almost to a grasping power. It is most instructive to watch a baby use its toes; not only are they bent downward or upward (plantar and dorsal - flexion) and spread apart (abducted) with the greatest ease, but in walking the toes fairly grasp, or dig into, the ground. The adult foot of civilized man usually presents a painful contrast to this. Generally the toes are crammed together, their power of spreading apart is wholly lost, and their movements take no part whatever in walking. The foot, in other words, is reduced almost to the condition of a shoemaker's last.

Nor is this a natural change due to growth and development. It is produced by the use of shoes which permit no 
movement of the toes and therefore lead to disuse of the muscles in question. Walking thus comes to be performed almost entirely with muscles which act upon the ankle joint, the one articulation of the foot at which movement is still possible; whereas had the toes been allowed perfect freedom of action, the work of lifting the weight of the body from the ground with each step would have been shared by both groups of muscles, - those which raise the heel and those which flex the toes. That this is true is shown by the feet of people who have not worn constricting shoes, for in their case the toes are moved freely and perform an important share in locomotion. ${ }^{1}$

If it be asked why the flexors of the toes as well as the extensors of the ankle should take part in the act of walking, the answer is that it is precisely the disuse of the former which leads to their degeneration, so that they are no longer efficient in opposing the tendency of the weight of the body to break down the arches of the foot. It is true that "flat foot" is not the invariable result of this disuse, because some people are so fortunate as to possess ligaments of sufficient strength to hold the bones together despite the pressure of this weight, and also because tightly fitting shoes often assist in holding the bones in position. But it is also true that many others are not so fortunate; one or both arches give way, and some suffer agony as the result. Even if the arches do not break down, the foot is generally unable to stand the strain of prolonged walking

1 The action of each of these groups of muscles may be made clear as follows : with the bare feet take a step forward by first raising the heel and then pushing off by bending the toes downward as far as possible. It will be found that this second movement is capable of assisting to a very considerable extent in pushing the body forward. The student should thus make himself practically familiar with the difference between (1) walking when only the heel is raised and the toes passively bent upward as the step is completed, and (2) walking when the raising of the heel is followed by the active contraction of the plantar flexors of the toes. 
without marked discomfort, and it is not too much to say that this weakness of the foot is one of the chief reasons why most people regard a walk of ten or twelve miles as a great task.

The hygienic care of the foot in actual practice consists (1) in the use of properly fitting shoes, (2) in avoiding all

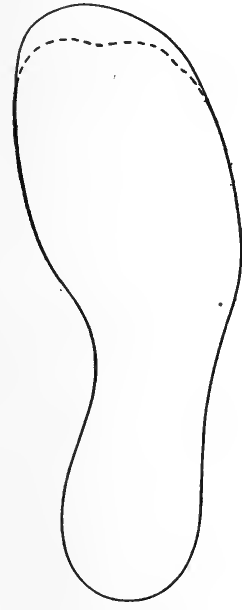

$A$

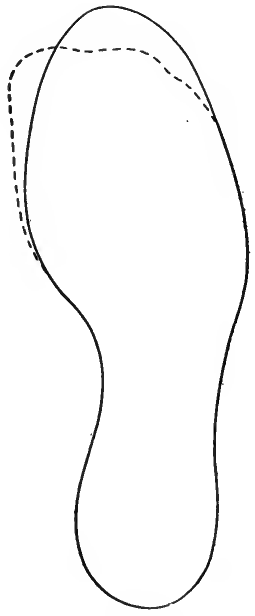

$B$

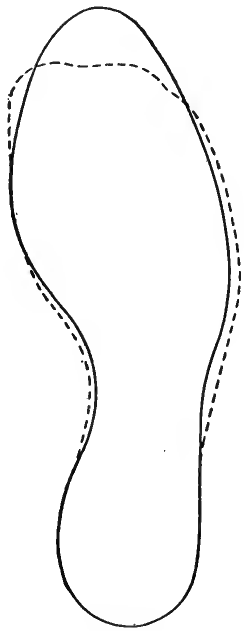

C

FIG. 115. Correct and incorrect shapes of the sole of the shoe

Outline of the sole in solid lines; of the natural shape of the foot in dotted lines. $A$, correct shape; in ' $B$ the shape is correct except that the median line of the sole is not straight in the region of the toes, thus pressing the great toe over toward the other toes; $C$ has not only this defect but is too narrow

interference with the circulation of blood in the foot, (3) in maintaining proper conditions of temperature and moisture within the shoe, and (4) in the training and use of the muscles of the foot, so as to keep them functionally strong and active.

3. Shoes. - Among the most important requirements of a good shoe are the following: (1) The sole of the shoe 
should everywhere be as wide as the sole of the foot when one is standing and the feet are warm. (2) The heels should be low and broad. (3) The sole and uppers should be sufficiently flexible to permit without great resistance the bending of the foot at the transverse line of articulation of the toes with the instep. Many shoes, otherwise correct, are faulty in that the sole or the upper from the heel forward is too stiff to permit the efficient action of the toe movements. (4) The inner (or median) side of the shoe should be "straight," i.e. the prolongation of the median line of the great toe should touch the heel. Figure $\mathbf{1 1 5}$ shows the proper and improper shape of the shoe in this respect. Unless the foot is already greatly deformed, no shoe should be tolerated which does not permit the great toe, and for that matter all the toes, to point straight forward, since otherwise it is not easy to flex them. Not only is the "toothpick" shoe a hygienic abomination, but any shoe in which the inner or median side slopes outward toward the toe is highly objectionable (see Fig. 115, B). (5) In the region of the toes the shoes should have sufficient room to permit perfect freedom of motion in the toes.

4. Shoes for Deformed Feet. - It must be frankly admitted that shoes which fill all the above requirements are uncomfortable to many feet. But this is only because such feet have already become deformed. In such cases the attempt should be made to bring the foot back toward its normal shape by gradually approaching the lines above indicated. With some hopelessly deformed feet this is, of course, impossible; but with many others great improvement is possible.

Upon one point there can be no yielding : children should wear only properly shaped shoes. It is a pitiable sight to see the foot of a child, broadening out as it does toward the mobile toes, forced into a shoe which seems to be constructed on the assumption that nature ought to have made 
the human foot wedge-shaped and that it is man's part to improve on nature.

Recent years have witnessed marked improvement in the shape of shoes. Fortunately it is now possible in many places to buy properly made shoes; but further improvement is still possible, both among those who make shoes and those who buy them. As a matter of common sense nothing could be more absurd than the custom of changing the shape of shoes each season merely to bring out a new style; nor would this be done if people were more generally informed as to the requirements of a good shoe and insisted on having only those which meet these requirements. In so far only as there is a general demand for such shoes in any community, will manufacturers supply them. The remedy lies with the public rather than with the manufacturers.

And this same public must learn that neither from the hygienic nor from the æsthetic standpoint is a small foot with a pointed toe and high heel the ideal foot. Such is not the foot of the Apollo Belvedere nor that of the Venus of Melos. It is simply a deformity, belonging in the same category with the constricted waist, and far more harmful to its possessor than the ear or nose ornaments of the Hottentot. No hygienic lesson is more important than that clothing should fit the body, and not the body the clothing.

5. Interference with the Circulation in the Foot. - By lacing the shoe too tightly, especially around the top, and by the use of tight garters the superficial veins which bring blood back from portions of the foot are often compressed. More or less of passive congestion results, and this not only produces discomfort but introduces in other ways conditions highly unfavorable for the free action of the foot; consequently it is part of the hygiene of that organ to avoid these congestions at all times. Garters 
should always be adjustable in length to the size of the leg, and shoes should not be laced tightly.

6. Proper Conditions of Temperature and Moisture within the Shoe. - Although the best of shoes are but poorly adapted to care for the perspiration and to maintain an equable temperature of the foot, some shoes are preferable to others in these respects. Thus any "patent" or "enamel" leather is objectionable for walking because it is almost impervious to moisture. In such shoes the foot becomes overheated while one is walking, because the perspiration does not evaporate from its surface; and if one afterwards sits still, the feet are apt to become cold because the wet stockings make a good conductor of heat. Because their surface radiates heat with such ease these shoes are cold in cold weather; and because they prevent the evaporation of perspiration they are hot in hot weather. While unobjectionable generally for dress occasions, they are wholly unsuitable for ordinary wear.

The "russet" shoe for summer wear is a great boon. The leather of which it is made is as porous as any on the market, and, because of its color, absorbs less heat in warm weather. The failure of the attempt a few years ago to retire these shoes from sale is good evidence that people can get a certain shoe if only they insist upon having it.

In brief, the feet should be dry and neither distinctly warm nor cold; and anything which interferes with these conditions should be attended to. Shoes and stockings should be changed as frequently as necessary and only such foot wear used as maintains as far as possible the ideal conditions given above.

7. The Proper Physical Training of the Foot. - It is quite possible to meet all the above hygienic conditions and yet have feet which are incapable of doing the work which we have a right to demand of them. As was shown at the outset, the action of the foot in bearing the weight 
of the body is not a passive but an active one. Muscles must assist in holding the bones in place when one is standing. still, and they must operate the foot during the act of locomotion. The physical training of the foot, therefore, consists (1) in securing adequate strength of these muscles, and (2) in establishing right habits in using them.

Since the muscles in which strength is especially needed are those which produce plantar (downward) flexion of the toes, we may strengthen these muscles by such exercises as pressing as hard as possible with the toes against the floor or the footboard of a bed, by attempting to "stand on tiptoe," and by the familiar gymnastic movement of "heels raise, knees bend," etc.

Among the habits which should be cultivated may be mentioned, first of all, walking and running with the foot straight forward instead of toeing outward. The bones which form any hinge joint, like that of the ankle, should move in a plane perpendicular to the axis of motion in the joint, and this is possible in the case in question only when the feet are pointed forward. It is absolutely wrong to teach children to toe outward in walking, and they would never do so were they left to themselves and their feet clothed in proper shoes.

In addition to this, the habit should be cultivated of completing each step by "digging into the ground" with all the toes. This cultivates the use of the foot muscles in locomotion along with the use of those which raise the heel, and the habit once acquired and regularly practiced keeps these muscles strong.

Finally, it must be remembered that the training of these muscles, like the training of all others, must be a gradual process. Where they have been weakened by improper use, one must proceed to strengthen them little by little from day to day, and in no case make the mistake of imposing upon them work which they are unable to bear. 
Most cases of "weak ankles" can be cured if taken in time and their muscles gradually trained. But these muscles can never be trained by imposing upon them sudden and severe work which, in their weakened condition, they are unable to perform. The fatigue thus induced too often prevents their working at all, thus leaving the weight of the body free to strain ligaments and do other damage which may leave the foot in a worse condition than before.

We have already insisted (p. 319) upon the importance of walking as a means of general muscular activity; and we may urge in concluding this chapter that the chief hygienic importance of the care and training of the feet lies, not so much in the fact that the danger of acquiring flat foot is thereby lessened, as in the fact that we thereby maintain in good working order this essential part of the mechanism of locomotion. American men, and especially American women, compare very unfavorably with their English cousins in the ability to enjoy walking and tramping; and while this is partly due to the general disuse of walking as a means of exercise, it is equally attributable to the deformation of the feet, which robs those organs of the power and even the possibility of performing with ease their proper function. 


\section{CHAPTER XXIV}

\section{BATHING}

1. The Hygiene of Bathing. - The principal hygienic purpose of bathing may be stated in one word, namely, cleanliness. A bath is often stimulating and refreshing, and special kinds of baths may be used upon occasions for good and useful ends; their value in the treatment of many diseases is coming to be widely recognized, and even in health they may be useful as aids to the best working power. But experience shows that it is not necessary, even if it be refreshing, for a healthy person leading a healthy life to use bathing for any other purpose than cleanliness.

The sweat glands and the sebaceous glands pour out upon the skin secretions which primarily serve the useful purposes of regulating the temperature of the body and keeping pliable the horny layer of the epidermis. Each of these secretions contains solid material, which, as the water of perspiration evaporates, is left on the surface of the skin or in the ducts of the sweat glands; some of the solids, too, are either themselves odorous or else are putrescible, giving rise to offensive decomposition products; consequently it is a duty which every one owes to his fellow-man to bathe so as to be clean and to render that bathing effective by wearing clean clothes. A clean person, clean clothing, a clean house, clean premises, clean streets, a clean town are so many forms of that habit of cleanliness which is one of the characteristics of high civilization, one of the fundamental elements of self-respect and proper living. 
Besides this, filth and dirt are effective carriers of disease; consequently bathing and the use of clean clothing diminish the chance of infection. Finally, personal cleanliness also keeps the skin in a healthy condition, and this alone is a sufficient reason for making it a rule in the hygienic conduct of life.

2. The Indifferent Bath. - A bath which is neither distinctly cold nor hot may be said, in general, to answer all purposes of cleanliness. The temperature of such a bath varies between $80^{\circ}$ and $90^{\circ} \mathrm{F}$. with different individuals. When soap is used, water of this temperature removes the waste products from the skin sufficiently for all practical purposes, especially when such a bath is taken daily. Indifferent baths are, however, without any stimulating (or depressing) physiological effect, provided they are not taken in a cold room; and for some people they are the most advisable form of bathing.

3. The Hot Bath used alone is not as a rule advisable. It has a well-recognized enervating effect, and experience shows that after it great caution is required as to exposure to cold. These effects, however, are generally obviated by following the hot bath with a cold needle bath, a cold shower, or a cold plunge, and possibly this procedure may be recommended as the most useful and beneficial form of bathing for the great majority of people. The hot bath serves the purposes of cleanliness more effectively than the indifferent bath, and the shock of the cold bath is not so trying to many people when taken immediately after the skin has thus been thoroughly warmed. Too frequent and especially too prolonged hot bathing, however, is apt to remove too much oil from the skin.

Hot baths, either of the body as a whole or at times a hot footbath, are often useful in bringing the blood to the skin and thus checking a threatened cold or other inflammatory process. Special care is needed, however, in this 
case to avoid subsequent exposure to cold. It should also be remembered that a very hot bath is a strong stimulus to the nervous system as a whole.

4. The Cold Bath is a powerful stimulus to the nervous system. When the irritability of the latter is low, as when we awake from slumber, it "wakes us up," and immediately after it we feel distinct exhilarating effects. In addition to this it probably serves as a training to the heat-regulating mechanism of the body, "hardening " the body to the effects of cold. Undoubtedly its influence with a large proportion of healthy people is beneficial, though, as we shall see, this is not the case with all. Before dealing with this side of the question we may give some rules which are always applicable in the use of such baths.

First, they should not be prolonged. To stay in a cold bath longer than one minute is undesirable save in a very few exceptional cases; thirty seconds is the usual time, while with some people ten seconds is the maximum.

Second, a cold bath should be taken when the skin is warm. Immediately on rising in the morning, immediately after muscular exercise, or immediately after a hot bath it is most beneficial and least likely to produce bad after effects.

Cold bathing should always be followed, except in warm weather, by a good rub-down with a rough towel. This promotes a good flow of blood through the skin and adds to the tonic effects.

A cold bath should not be taken in a cold room. Many profit by its use in summer, but experience undesirable effects in winter.

Third, cold bathing should not be used unless it is followed by what is called the "reaction"; that is, unless it produces a distinct glow in the skin. The persistence of pallor in the skin after the rub-down is proof that the 
system does not react properly, and is a warning that this form of bathing should be given up or at least modified. This does not mean that the bath necessarily agrees with us if it does produce the "glow," for this is only one of its after effects, and we must judge of its usefulness not by one but by the sum total of the effects produced.

Fourth, no bath, unless it be possibly the indifferent bath, should be taken within an hour or more after a meal. The evidence of experience on this point is so unmistakable that nothing more need be said about it.

It would be a mistake to discourage all bathing except that which is used for purposes of cleanliness; and when we insist that both hot and cold baths are an artificial element introduced into the environment, it is only to enforce the need of carefully observing the effects of their use. No one is justified in saying that these baths are necessarily good for all healthy people; no one is justified in recommending them as essential elements in the hygienic conduct of life. They must be judged by their effects, and when submitted to this standard it would appear that while they are beneficial to some people they are harmful to others.

We must furthermore distinguish between the immediate results, those noticed later in the day, and the remote results. The immediate effects may be exhilarating; we may "feel splendid" afterward, and yet this feeling may be succeeded by one of depression. At times cold bathing on rising in the morning results in constipation, although the bath itself may be enjoyable. This may be exceptional, but it shows that every one must determine for himself the value of the bath by the sum total of its after effects, and not alone by those which accompany or immediately follow it.

5. Swimming and Salt-Water Bathing. - When one is swimming, the heat produced within the body by muscular 
activity counteracts to some extent the effect of the cool or cold water applied to the skin. Hence it is possible to remain in the water a longer time with safety and even with profit than in the ordinary cold bath. It is quite impossible, however, to give definite rules as to the length of time one should remain in the water, since this depends on the amount of muscular activity, on the temperature of the water, and on the condition of the bather. But the hygienic value of swimming and sea bathing must be determined by the same tests as have been urged in the case of cold bathing in general.

It is also important to remember the danger of going into cold water when one is fatigued from muscular activity. The fatigued muscles seem especially liable to go into cramps under these conditions, and persons have been drowned in this way before help could reach them.

When one takes vigorous daily exercise the best time for the bath is immediately after the exercise. One is then in a perspiration and it is best to change the clothing. The skin is most readily cleaned in this condition, and most persons find a hot bath, with or without the use of soap, followed by a short, cold needle bath, shower, or plunge, preferable to other forms of bathing. The time for bathing, however, like the time for eating, must depend on one's work in life. We do not live to bathe, any more than we live to eat. 


\section{CHAPTER XXV}

\section{CLOTHING ${ }^{1}$}

1. The Hygienic Object of Clothing. - Even in the savage state some races clothe themselves thoroughly. The Eskimos, for example, go warmly clad in furs, and the wild Indians who once inhabited the northern United States wore, at least in winter, the skins of animals. In the tropics, on the other hand, as in northern Africa or the islands of the South Seas, very little clothing is worn, and that more for the sake of decency or ornament than for warmth. In these facts we find the hygienic reason for the use of clothing, namely, to aid the body in maintaining its constant temperature. In cold weather, clothing is a kind of portable house, a close and intimate shelter, an indispensable aid to the skin in preventing undue loss of heat; on the other hand, summer clothing should interfere no more than is unavoidably necessary with the dissipation of heat from the skin. If, in winter, warm days come, or if the body becomes heated by muscular activity, or if (as too often happens) houses or public places are overheated, then winter clothing may not only become a burden but may be actually unhygienic. Conversely, if in a changeable climate cold days or nights come in summer, or sea winds blow damp as well as cold, then ordinary summer clothing may prove to be insufficient. Here, as always, the individual must be the watchful guardian of his own welfare.

1 The student is advised to review Part I, Chapter XII, before studying this chapter. 
Clothing affects the temperature regulation of the body chiefly through its influence upon three processes by which heat is taken from the skin. These processes are (1) convection by wind or other currents of air, (2) conduction, and (3) the evaporation of perspiration. We shall deal with each of these in some detail.

1. Clothing and the Convection of Heat. - Any fabric whose texture permits the air warmed by contact with the skin to be replaced readily by colder air from without will obviously favor the cooling of the skin by convection; and conversely, any garment which lessens or altogether prevents these currents of air through it is to that extent a warm garment. The leather hunting jacket lined with wool or fur is especially warm, and a newspaper under one's coat or jacket similarly affords a large measure of protection against cold. On the other hand, a rubber coat may be very uncomfortable on a warm day, although the effect in this case is due to its interference with the evaporation of the perspiration as well as to the prevention of the passage of air through the garment.

2. Clothing and the Conduction of Heat.-Even when there is no passage of air through the clothing, heat may, of course, be transferred from the skin to the outer air by conduction, and some fabrics conduct heat more readily than others. Other things being equal, the rate at which clothing conducts heat depends on the amount of air within its meshes. 'Thus wool is warmer than cotton, not because of any difference in conductivity of the two kinds of fibers, but because when wool fibers are made into yarn their stiffness and elasticity keep them apart, so that garments woven from this yarn always contain spaces filled with air, which is a poor conductor of heat. Moreover, the same properties of the fibers prevent their being pressed and felted together in laundering, as ordinarily happens with cotton and linen fabrics. We shall see that cotton and linen may be so 
woven as to avoid this result, as in many "meshwork" fabrics, but they are not usually so woven.

A moment's thought will show that the warmth of a dry garment will depend on the size of its meshes. These may be so fine and close as to inclose an insufficient quantity of the non-conducting air, or they may be so large as to permit too free circulation. In the latter case heat is carried away from the skin by convection. It is also clear that the warmth of a garment is not determined by its weight or thickness alone.

3. Clothing and the Perspiration.- So long as the meshes of a fabric contain air, heat is conducted but slowly from the skin. When, however, this air is partially or entirely replaced by water, the fabric conducts heat from the skin much more rapidly; and if the surrounding atmosphere is distinctly colder than the body, the skin becomes chilled and internal organs congested; hence the danger of wet clothing.

More important still is the relation of clothing to the evaporation of perspiration. -We have learned that perspiration is useful to the body only as it evaporates. Consequently the clothing should be such as will permit the perspiration to evaporate almost as fast as it is secreted. The skin will thus be cooled at the time that the needs of the body require such cooling, and the clothing will not remain wet after the secretion of perspiration has ceased and the need for cooling the skin no longer exists. Or, if it is not possible to secure this rapid drying, the fabric should contain, even while moist, a considerable quantity of air within its meshes, thereby checking the loss of heat from the skin.

2. The Clothing worn next the Skin and the Outer Clothing. - Consideration of the above relations of clothing to convection, conduction, and the evaporation of perspiration shows at once that the clothing worn next the skin 
must fulfill requirements not demanded of the outer clothing. The sole hygienic purpose of the latter is warmth and the fabric should be chosen accordingly. In warm weather, in well-heated rooms, and during muscular activity, warm outer clothing is undesirable; on the other hand, when the body is exposed to cold and is not at the same time engaged in muscular exertion, the outer clothing should be chosen for warmth; and for this purpose woolen fabrics are superior to all others.

The clothing worn next the skin must, in addition, care for the perspiration. For those forced by age or other physical disability to lead sedentary lives, woolen underwear is very useful in cold weather. Since in the case of such persons the blood is not brought to the skin by muscular activity, it is necessary that the skin be kept warm and internal congestions prevented. For such persons woolen fabrics are probably superior to all others. Moreover, during exposure to extreme cold, when little or no perspiration is secreted even during vigorous muscular work, woolen underwear is superior for every one because of its greater warmth.

For healthy people, however, in the full vigor of life, taking daily muscular exercise but not exposed to extremes of cold, woolen underwear presents many serious drawbacks. In the first place its very warmth is objectionable during muscular activity, because it makes more difficult the discharge of the surplus heat. In the second place, wool absorbs the perspiration very slowly and so prevents its evaporation from the outer surface of the garment; the perspiration does not cool the body as it should, but remains between the skin and the garment, - an unhealthful condition for the skin. In the third place, when the garment has once become "wet through," i.e. the air within its meshes has been largely displaced by water, it dries more slowly than a linen or a cotton garment. 
It is better, in other words, for healthy people to depend upon the outer clothing, including overcoats, etc., for warmth, when protection against cold is needed, rather than upon even moderately heavy underwear. In this way it is possible readily to relieve the body of its heavier clothing when it becomes necessary to get rid of surplus heat, i.e. in warm rooms and during muscular activity in only moderate cold weather, and yet to protect oneself against cold when such protection is necessary.

Of late years the attempt has been made with considerable success to weave linen, and even cotton, so as to contain fairly large meshes between the threads. The perspiration is rapidly brought to the surface of the garment through the threads by capillary attraction and so evaporates quickly; for this reason the garment dries readily, and even while wet usually retains a considerable quantity of air within its meshes.

The thickness of underwear, as well as of the garments worn immediately over it, should be determined by the amount of exposure to cold when at rest. When our houses or offices are properly heated $\left(65^{\circ}-70^{\circ} \mathrm{F}\right.$.) in winter, heavy clothing is as much to be condemned as the too common overheating of our rooms, and for the same reason. When, on the other hand, our work is out of doors in cold weather but involves only a small amount of muscular activity, warmer clothing should be worn; in this case the use of heavy woolen underwear is advisable.

It is unnecessary to go further into details. The student can solve special problems for himself, always remembering that proper clothes are such as will prevent undue loss of heat and consequent chilling of the skin (with accompanying internal congestions) when the body is at rest.

3. The Outer Clothing. - Of this little need be said. By varying the thickness of the outer clothing we adapt it 
to the conditions of life. It must also be chosen with reference to its permeability to air. In hot summer weather it should be as thin and porous as possible; in winter it should protect from wind. When still further protection is needed, it may be obtained by the use of overcoats, gloves, muffs, lap robes, or other wraps.

Some people do not use sufficiently warm clothing in cold weather, but most adults make the opposite mistake. The custom of using very thick clothing in cold weather appears to have been inherited from the time when houses were poorly heated, when transportation from place to place was in cold cars or carriages, and when, in general, the human race was more exposed to cold than it is to-day. Where these conditions prevail, as they still do in many country districts, heavy clothing should no doubt be worn in winter. The same may be said of driving in open vehicles, such as sleighs, etc. But in cities, where houses are more likely to be overheated than underheated, where steam and electric cars are far from being chilly, where, in short, we need not generally be exposed to cold except when walking or taking other muscular exercise, the main dependence for protection against cold should be upon the outer wrappings rather than upon the underwear, the coat and trousers, or the dress. We do not change to heavy clothing in summer when the thermometer falls to $65^{\circ}$ or $70^{\circ} \mathrm{F}$, and there is no reason why we should use such clothing at these temperatures in winter. The precautions which many take against sudden changes of weather are often excessive.

4. Clothing not the only Protection against Cold. - It must be remembered that we have another means of protection against cold besides clothing, and that is muscular activity. Even if, as often happens, a balmy morning passes into a chilly afternoon, most people, especially those living in cities, should be able to keep warm by a brisk walk 
when going home; a little exposure to cold will not harm, but will rather harden, a healthy man or woman. If we are tired out and ought not to walk, we can usually ride in a heated car. To wear heavier clothing than the probable necessities of the case demand, merely because there is a chance that suitable weather for such clothing may overtake us, is in general unwise. Oppressed with its weight and warmth, the usual result is a disinclination to any vigorous muscular activity while out of doors, and this in the long run is more dangerous than a comparatively brief chilling of the skin.

5. Clothing should not be Heavy. - The reference in the last paragraph to the burden of heavy clothing deserves further consideration. The terms "warm," "thick," and "heavy," as applied to clothing, are often used as if they were synonymous, although a thick garment is not necessarily a heavy garment, and a thinner but more loosely woven coat may be warmer than one which is thicker but more closely woven. In the selection of clothing it is always advisable, not only as a matter of personal comfort but also as a matter of practical hygiene, to avoid heavy fabrics. While this holds especially for invalids and elderly people, to whom the burden is more oppressive, it also holds for the young and strong. The clothing should be such as will interfere in the least degree with the freedom of bodily movements. Not only should every one avoid such fashions as tight lacing and high-heeled boots, - so senseless as to be beneath the contempt of those who respect the human body and care for its physical wellbeing, - but care should be taken to have the clothing everywhere loose enough to be comfortable and, above all, light enough so that its weight is not a burden. For this reason a very close weave is objectionable except in windy weather, since it gives great weight of fabric with but small air contents. 


\section{DOMESTIC HYGIENE AND SANITATION}

\section{CHAPTER XXVI}

THE HOUSE: ITS SITE, CONSTRUCTION, FURNISHINGS, AND CARE

1. The Family a Private Community. - Every human being has not only individual or personal relations with his environment, but also various other, and public relations, since the life of an individual is always more or less closely connected with the lives of other human beings. Each individual or person is a member of some family, and also of some village, town, city, state, or nation. Connections of this kind constitute kinship, relationship, and fellowship, and are commonly described as social relations (socius, a fellow). They are nowhere more conspicuous than in matters of life and death, health and disease. The human infant is absolutely dependent upon parental care, and among civilized people the sick, the aged, the dying, and the dead must be tenderly cared for by those who are alive and well. But this is not all, for sickness is frequently "catching," and plagues, pestilences, and epidemics have often run like wildfire through families or communities, leaping from person to person, and from village to village, very much as a forest fire leaps from tree to tree.

A fundamental feature of all social relations is the fact that persons in families, villages, towns, cities, states, and nations have and use many things in common. This has caused such groups of human beings to be known as 
communities (communis, common). Of all communities the simplest, the most fundamental, and the most important is the family or household, in which the various individual members share a common shelter, a common fireside, a common table, and a common interest, based upon the allpowerful ties of blood or marriage. In these and many other respects the family is not only a community but a peculiar kind of community, namely, a private community. But precisely as the individual necessarily has relations to the world outside himself and is by nature not merely a man and an animal, but a social man and a social animal, so the civilized family or household, although essentially a private establishment, has certain public relations. It must draw its air supply from the aërial ocean common to all mankind; it must form a component unit in some village, township, state, or nation; it must buy sugar or salt, or tea, coffee, or spices, from overseas.

Midway between the more purely public relations which we shall presently discuss under public hygiene and sanitation, and those individual relations which we have considered in the foregoing chapters on personal hygiene, stand the hygiene and sanitation of the house and the family, subjects neither altogether public nor altogether personal. These we may describe as domestic hygiene and sanitation.

2. Housing and the House. - The chief function of clothing (p. 418) is to protect the body from cold by maintaining about the skin fairly constant temperature conditions, and accordingly clothing is of least importance in the tropics, where conditions of temperature are both constant and warm. The housing problem is very similar, for the principal function of the house is likewise to furnish for the body a favorable environment, and especially a fairly constant temperature; here also the problem is simplest in the tropics. The house, in fact, is a kind of outer clothing or protective shell, although usually designed 
not for a single individual but for an entire family, or, as in the case of tenement houses, apartment houses, or hotels, for many families, or for the public.

Savages and the lower races of men content themselves with caves, trees, and other ready-made shelters, or with huts, hovels, wigwams, and similar poor and primitive dwellings. The most highly civilized races, on the other hand, build carefully planned and sometimes elaborate houses, furnished with various devices, simple or complex, for heating, lighting, ventilation, water supply, drainage, cookery, and other necessaries or luxuries of a comfortable, as well as a uniform and favorable, environment.

Houses may be separated and detached, as on farms or in villages, or massed in groups or blocks, as in towns or cities; and owing to the fact that they are comparatively durable and costly, most people live in dwellings already built. But although very often a family cannot build its dwelling, but must take to some extent what it can get, it usually has, sooner or later, some choice; and even if it has not much choice, it may modify more or less from time to time the domicile which it must occupy.

3. The Site of the House is often determined more by necessity, taste, or convenience than by hygienic considerations; but in general it may be said that a human dwelling should be so situated as to afford good air, good light, good drainage, and good neighbors. If, in addition, beautiful, charming, or attractive surroundings can be had, these are of great importance, since beauty and charm often have a distinct hygienic value. A certain seclusion or privacy is also to be desired, for a quiet, retired, and restful home, removed from the distractions of publicity, is soothing to tired nerves as well as conducive to normal and wholesome family life. On the other hand, extreme isolation, such as is sometimes found in farmhouses, often produces a morbid feeling of loneliness. 
When possible the house should be placed upon open, porous, or gravelly soil, because such soil is less likely to be water-logged and is more easily drained. In the United States in general a southerly or southwesterly slope is usually preferable, as affording more sunshine in winter and more breeze in summer. It is also wise, of course, to have the principal living rooms on the side exposed to dry rather than to cold and damp winds.

Good air for a house is to be sought for in a clean neighborhood, a clean, dry cellar, and a free circulation, the latter impeded as little as possible by other buildings or, in the country, by too many trees. An elevation, therefore, rather than a depression is obviously desirable as an aid in securing these things, although the very top of a hill should usually be avoided because of its bleakness. A dry cellar and, if possible, a dry, open, and porous soil beneath the house are highly important, no matter where the house is placed. Cellar habitations are very objectionable and ought to be avoided by even the poorest family. Such dwellings were long since forbidden by law in England, and although sanitarians are not yet agreed as to all the reasons why cellar habitations are injurious, the principal reasons seem to be the well-known unwholesomeness of dampness, and want of sunlight. Undue dampness in air is believed to favor rheumatism and other disorders, and the absence of sunlight not only favors dampness and microbic life but also tends to mental depression. House cellars should be well drained.

Good light and, if possible, abundant sunshine are hygienic conditions of great importance, both as aids to cheerfulness and happiness and as powerful sanitary agents. Sunshine tends to remove dampness and to destroy the germs of infectious diseases. In winter, sunshine is valuable also for warmth.

Good drainage is no less (and perhaps no more) necessary for human habitations than good air and good light. With 
the abundant use of water in recent times for washing, bathing, cleaning, sewage disposal, and other purposes, it becomes necessary in modern houses to get rid somehow of a great deal of soiled and dirty water, and the possibility of easy and safe "drainage," or removal of such water, must be kept in mind in considering the sanitary aspects of the situation of any house, old or new. Here again the advantage is plain of some elevation of site.

4. The Construction of the House. - As the first object of any house is shelter from rain, snow, wind, dampness, and excessive heat or cold, its materials should be waterproof, windproof, and non-conducting for heat, as far as is consistent with a proper circulation of air. Wigwams or tents, at least in temperate latitudes, are clearly defective in some of these particulars. Houses built of glass or india rubber would answer most of these requirements but would still be most unhygienic, chiefly because glass houses would be too light and too hot, while both glass and india rubber would interfere seriously with that free circulation of air which takes place through relatively porous materials such as wood and brick. Buildings of wood, stone, brick, or steel and brick, rightly built, answer all requirements. A "double wall," i.e. a hollow wall, by providing a nonconducting air space is of great value for preventing rapid changes in the temperatures of houses under sudden changes of climate, as well as for protection against dampness and noise.

Much circulation of air usually takes place even through walls or partitions of plaster and wood, and a knowledge of this so-called "natural ventilation" helps us to understand how it is that many people live, and even thrive, in seemingly unventilated rooms and houses. It also helps us to understand how the damp air of a cellar finds its way upward into a house, and why a double floor (with air spaces between) is especially useful immediately above 
the cellar. Blinds or shutters, and shades or curtains, for darkening rooms, are of great hygienic value, since sleep is deeper in darkness than in light, and in summer these tend also to keep the house cooler.

5. The Furnishings of the House. - The walls of rooms may be of wood, - bare, painted, or varnished, — or of plaster, - either bare or covered by textiles such as burlap or tapestry, or "papered" by pasting upon them with thick paste, or "sizing," sheets of paper upon which designs, often in color, have been printed. Sometimes, instead of being papered, walls and partitions are painted, either with white paint or in colors, and sometimes simply a hard finish is given to plaster, which is afterwards "whitewashed " or " calcimined."

A good feature of painted walls is the fact that they may be washed, and of walls smoothly calcimined that they may be easily done over. In general, a smooth and washable surface is preferable to a rough one or one injured by washing, for these not only collect more dust but are harder to keep clean.

The most serious charge, from the hygienic point of view, thus far brought against wall papers, is that of the danger of poisoning for persons living in rooms papered with such papers as contain arsenic. The subject has been much in dispute, but the evidence of such occasional poisoning seems now convincing, especially since the work of Gosio, an Italian investigator, showed that molds or other microörganisms which grow in the paste used to stick the paper to the walls are capable of attacking the arsenic of some coloring matters, thereby producing volatile compounds of arsenic readily diffusible into the air of the room.

There is similar danger from arsenical poisoning in some tapestries or furniture coverings, and grave disorders have been attributed, apparently with reason, to this source. 
The iron bedstead, light, firm, cheap, and easy to keep clean, is a marked improvement upon the heavy wooden bedsteads formerly used. Curtains, canopies, valances, etc., either above or below beds, are objectionable, as they interfere with the free circulation of air. The modern open bedstead is an improvement upon the old-fashioned "fourposter" with its hangings, almost as great as is the modern "open," over the earlier concealed, plumbing.

Single beds possess many advantages over double beds. They are more easily cared for and kept clean; the amount of covering can be more accurately adapted to the individual needs of their occupants, who are also less exposed in cases of infectious disease; and the use of such beds is more conducive to undisturbed slumber.

Folding beds, mantle beds, sofa beds, and all similar devices for concealment of beds and bedding are subject to the objection that they are likely to be closed too soon after having been used, and before the bedding has been sufficiently aired or freshened. Certain forms of folding beds are further objectionable from the fact that by their closing automatically and unexpectedly the occupant has sometimes been imprisoned and even killed.

Floors are in America usually wooden and made of boards, "matched" or otherwise laid tight. Formerly the material used for inexpensive floors was of pine, spruce, hemlock, or other soft woods, oak being reserved for the more costly hard floors. Nowadays hard pine (Southern pine) is much used and many cheap yet good floors are made of this material. Softwood floors are apt to become dented and splintered unless covered and protected by carpets or mattings; but, if made of good stock and well cared for by frequent painting, such floors answer very well for a long time, especially in rooms, such as chambers, not subject to hard usage. Bare floors possess the immense sanitary advantage of being easy to clean and 
also of revealing dust and dirt. But they require, for the latter reason, more care, and are also open to the objection that they are comparatively noisy.

Fixed mattings are useful for the deadening of sounds, and fixed carpets not only for this but also for warmth; but both hold dirt and are hard to clean, while light, movable mattings, carpets, or rugs readily lend themselves to cleanliness, because they can be removed and, in their temporary absence, both they and the otherwise bare floors upon which they rest can be thoroughly cleaned.

6. The Care of the House. - The house, the outermost clothing of man and the family, like the dress of the body or the clothing proper, is subject to the wear and tear of time, season, and weather, and likewise requires care, consisting chiefly of cleaning and mending, or repair. Painting in the case of wooden houses (and for the steel parts of steel-and-brick structures), and pointing (or the renewal of mortar between bricks or stones) in the case of brick or stone houses, helps to make them waterproof and windproof and tends to keep out dampness.

The cellar, especially, requires watchful care, and should be kept not only dry, by windows or other ventilating devices, opened wide in favorable weather (and never wholly shut), but also clean and free from 'rubbish, decaying vegetables, or anything tending to dirt, dampness, or uncleanness.

The walls of the rooms, if papered, should from time to time be wiped with damp cloths, or if painted, they may be washed. Floors and the hard parts of furniture are thoroughly cleaned only by washing, but may also be well cared for by wiping with damp (not wet) cloths which are easily washed and wrung out. Brushes with long handles are also useful, though less effective, for ceilings and bare floors, and brooms for fixed mattings and carpets. Dust cloths and the little feather duster may be used upon books, 
fabrics, and other surfaces which would be injured by the damp cloth; but such "dusting," in so far as it lifts the dust into the air only to allow it to settle again somewhere in the same room, merely transfers it from one place to another, and, while perhaps better than nothing, does not produce true cleanness. The large feather duster often used to clean (?) things which should be wiped with damp cloths, easily washed or renewed, is simply a sanitary abomination. It makes a show of cleanliness but lacks the substance thereof. It is the favorite implement of the superficial, indolent, or shiftless, and especially of janitors of this class in public buildings.

Even when closed, houses quickly become dusty or dirty, because the air which finds its way into them through cracks or crevices is almost always more or less charged with dust, while the occupants of inhabited houses bring in upon clothes, shoes, and all kinds of articles more or less dust or dirt. Fires, such as those in stoves, fireplaces, or furnaces, also add greatly to the dust of houses. Dust and dirt are composed largely of inorganic or lifeless matters such as particles of sand, or coal, or ashes, or fibers of cotton or woolen, but also partly of microbes. Most of the latter are harmless, and some kinds of dust and dirt are of little sanitary importance, - a fact which helps us to understand why some people seem to have health even in dirty surroundings. But dust and dirt sometimes contain the germs of dangerous diseases, and the way of safety is the way of cleanliness. 


\section{CHAPTER XXVII}

\section{THE WARMING AND .LIGHTING OF THE HOUSE}

1. The Warming of the House. - The earliest method of warming human dwellings was the open fire, in hut, cave, or wigwam, and when chimneys were added to carry off smoke and improve combustion by creating drafts, the open fire still remained for a time the sole resource of mankind for heating purposes. It is still the most attractive and most cheerful method of heating, and has been well called "the eye of the room." It is a coveted luxury in all tasteful homes, not so much for the heat it furnishes as for its cheerful glow and the constant interest which it excites. The home and the fireside have become everywhere almost equivalent terms.

2. The Open Fire may be of either coal or wood. In England it is almost always of coal, and in that country is still the principal means of heating. In some other countries, and especially in the United States, it is made of either coal or wood, but is less depended upon for heating purposes. The open fire may be on a hearth in a fireplace, or in an open grate or an open stove. In all modern cases of true open fires, a chimney rises above the fire to carry off the smoke, and the draft of the chimney (caused by the rising of the column of lighter heated air) constantly sucks away the air of the room and produces considerable ventilation by removing vitiated air. The air thus removed is replaced by air from adjoining rooms or from outdoors, driven in by the atmospheric pressure through open doors or windows or through the walls themselves which, if of wood or 
plaster, or even of stone or brick, are to some extent porous. But while such ventilation has great advantages and is one of the best things about open fires, such fires are wasteful of heat and often do not effectively warm the entire room. This is because the warmed air is not returned to the room, but is drawn up the chimney, and because the movement of the cold air which is pressed in from the outside tends to make the room "drafty." Radiation from the fire itself, rather than convection by air currents, thus becomes the chief means of warmth; and the complaint against open fires that those gathered about them, whether indoors or out, are " roasted in front and frozen behind," is undoubtedly well founded. Open fires, nevertheless, serve admirably to "take the chill off" from a room in those days of late spring or early autumn when the temperature is only a few degrees below the proper point (see p. 201).

3. Stoves are superior to open fires as sources of warmth, but far inferior in attractiveness and as aids to ventilation. A stove is usually placed in a room at some distance from the wall, and connected with the chimney by a stovepipe to carry off the products of combustion. There is no such thing as an "air-tight" stove, a term often used because some stoves seem tightly closed, only enough air being allowed to enter to supply the actual need for combustion. A stove warms a room by the mixture of currents of heated air around the stove with the cooler air in other parts of the room, i.e. by convection, and also by direct radiation from the fire itself.

4. Hot-Air Furnaces are usually inclosed stoves placed in the basement or cellar. They are provided with smoke pipes and surmounted by a space, the hot-air chamber, to the lower portion of which a pipe, or "air box," conducts cold air, while a second pipe or system of pipes leading off from the upper portion of the chamber supplies the various rooms with the warmed air. This is a convenient, 
economical, and popular method of heating a house, and possesses the great advantage of bringing constantly into the various rooms supplies of fresh air. If this air has not been overheated while passing by the furnace, little objection can be brought against it on any ground. It is true that, having been 'warmed, its relative dryness has been increased; but this condition may be corrected to some extent by always keeping in the hot-air chamber of the furnace a vessel of water for evaporation.

If, however, the air supplied to the furnace is not fresh and drawn from the outer atmosphere, but is simply taken from the cellar in which the furnace stands; or if the furnace is not tight, but cracked or loose-jointed, so that the gases of combustion may escape and mingle with the air as the latter flows through the pipes and rises into the rooms of the house ; or if, as often happens, the air is overheated and greatly overdried, then furnaces of this kind may, and do, become objectionable. In very cold and windy climates, and for houses in bleak or exposed places, furnaces are less satisfactory than steam or hot-water heaters. As they usually deliver warm air under very small pressure, it is often impossible, especially in windy weather, "to put the heat where it is wanted," a difficulty not encountered in the use of steam or hot water. Another objection to hot-air furnaces is the fact that much dust finds its way in with the warm air; but fresh air without dust, at least in towns and cities, is rare.

A simple combination of stove and furnace is much used in some places, where a stove (usually in or against the fireplace) on the first floor heats not only the room in which it stands but also, by means of a pipe, or pipes, and registers, one or more rooms overhead. Unfortunately the air thus supplied to the upper rooms is not always pure air from out of doors; sometimes it is the already vitiated air of the room below. 
5. Warming by Steam and by Hot Water. - It is very common in the United States to find houses (and other buildings) heated by steam or hot water. Through the "radiators" or "coils" placed in the various rooms there is maintained a circulation of steam or of hot water from a "heater" below. Here the room is warmed partly by direct radiation and partly by convection currents, very much as in the case of the stove. The chief objection to this method of heating is that the heating and the ventilation of the room are not effected by the same process; the room must be ventilated by opening windows, and the air thus introduced is apt to be cold and to produce undesirable drafts. On the other hand, steam and hot water are both superior to hot air in convenience and efficiency. They can be carried anywhere and are free from disturbances by atmospheric conditions, wind-pressure, natural ventilation, etc., which greatly interfere with the proper distribution of hot air. I Sometimes a combination of direct and "indirect radiation ${ }^{2}$ is employed, the latter being used for all ordinary heating, and the former kept for aid in extremely cold or windy weather. On this plan fresh air drawn from outside is first passed over coils of pipes placed in a basement or cellar and containing steam or hot water, and then carried (as in the hot-air furnace) to the various rooms which it is desired to warm. In addition, radiators are placed in these rooms, often near doors or windows, and in extreme cold weather are charged with steam or hot water to furnish supplementary heating by direct radiation.

6. Oil Stoves, Gas Stoves, and Electric Heaters. - These do not greatly differ from other stoves except in the sources

1 The hot-water system is rapidly coming into favor to replace steam heat, because a given volume of water will carry a larger amount of heat than the same volume of steam; consequently the water can be sent from the heater at a lower temperature than the steam, the supply pipe is not so hot, and the heat is more evenly distributed through the house.

2 "Indirect radiation" is, of course, really convection. 
of the heat which they provide, and in the important fact that in the first two the products of combustion are not usually carried off by chimneys but, as in oil or gas lamps (which are also powerful room warmers and really only luminous stoves), escape directly into the room and thus tend to vitiate its atmosphere without causing any compensating ventilation. In the case of gas stoves special care must be taken to see that the unburnt gas does not escape into the room from leaks in the connections or elsewhere. Here the same considerations apply as in the case of gas used for lighting. The flexible rubber tubes often used for supplying gas stoves deteriorate with age and then frequently permit the escape of gas directly into the room. Whenever possible, permanent (metallic) connection of the stove with the iron gas pipe is advisable. Where rubber connections are used, the gas should always be turned off at the cock on the main pipe, never at that on the stove.

Electric stoves, like electric lights, are heated by electricity, and even electric lights, though inferior in this respect to oil or gas lights, are often noteworthy factors in the warming of houses. Stoves used for cooking add materially to the warmth of houses, and hence gas or oil stoves may be used with advantage when, as in summer, heat is undesirable.

7. Solar Heating. Glass Verandas. - Less use is made of the direct heat of the sun than is often advisable or advantageous. Rooms flooded with sunshine are always more economically warmed than those without it, and a solarium, or glass-covered room or veranda, on the south side of a house is often useful as well as agreeable in winter. If provision is made for heating it at night, and in cold and cloudy weather, it may be made to answer also as a plant' conservatory, or greenhouse, and thus become a source of added interest and pleasure. 
8. Overheating. - If the temperature of the house is too high, we suffer from many of the objectionable conditions of hot weather; mental work is more difficult and we are disinclined to muscular exercise. It is probably unwise to keep the temperature of the house above $68^{\circ}$ or $70^{\circ} \mathrm{F}$. A good rule is to keep it between $65^{\circ}$ and $70^{\circ} \mathrm{F}$. (see pp. 389-391). Those who, by reason of infirmities of age, cannot enjoy regular muscular activity often find rooms of this temperature too cold; but the aged should be encouraged and even urged to keep up at all hazards the habit of doing some muscular work every day. With careful attention to muscular exercise and outdoor life they can not only endure but also enjoy lower room temperatures than is generally supposed, and thus permit the younger members of the household to live under more wholesome temperature conditions. Appetite is also improved by this practice and old age made in general more comfortable and more cheerful. Youth should remember, however, that the aged, largely because they cannot "get warm from the inside," not only desire but actually require warm clothing and often very warm rooms.

9. The Lighting of the House. - The fire light and the light of the pine knot, with which the hut, the hovel, or the wigwam were lighted, were objectionable chiefly because of their inconvenience, smoke, and flare or flicker. The invention of the lamp without chimney, and of the candle, marked a step forward, though their light was weak and flickering. A much greater advance was the invention of the lamp chimney, as it provided what nothing else had done, steadiness of flame, and avoided flare and flicker. Once the latter was overcome, it became easy to improve the fuel, until now the oil lamp with chimney not only illuminates and decorates the home of wealth but also brightens and cheers the hut of the fisherman and the cabin of the sailor; it aids and comforts the seamstress in her 
toil, in the humblest lodging; it warns the mariner by night from dangerous coasts by lighthouses, and throws about the student a warm and cheerful radiance as he "burns the midnight oil." Candles are still much used both in churches and in houses, but chiefly because of sentiment or for decoration. They still furnish the softest and most beautiful light, especially for quiet places;' but they are unfit for reading purposes because of their flickering and their feebleness.

The introduction of gas lighting was a great advance over lighting by lamps, owing to its convenience and cleanliness and the intensity of the light afforded. But gas lights, unless provided with chimneys, are generally unsteady and therefore objectionable for use in reading. Gas lights (and oil lamps) also produce much heat, and by this as well as by their products of combustion may greatly vitiate the air of rooms in which they are used.

While some kinds of illuminating gas are more poisonous than others, all manufactured - as distinguished from "natural" - gas contains a considerable percentage of poisonous constituents. When the gas is burned, these are oxidized and form harmless substances, and hence there is little or no danger from the products of its combustion. But the greatest care should be taken to avoid the entrance in any way of unburned gas into the air of a room. This may happen by the gas escaping through leaky fixtures, or after "blowing it out" instead of turning it off. It may also occur when the light has been turned down very low in the sleeping room and is afterwards blown out by a draft, or goes out because of lessened pressure in the main, and the unburned gas escapes freely when the pressure is restored. Still another source of danger exists when the gas cock used to turn off the gas works too easily in its socket, and so is capable of being turned on by slight jars, touches, - etc. The student is referred to Chapter XXXII for a full 
description of the dangers of inhaling unburned illuminating gas. Illuminating gas is also explosive when mixed with air in certain proportions.

Electric lighting is in many respects an ideal method, giving a convenient, steady, and powerful light; but, as is stated in the next paragraph, care must be exercised that such light is not too bright.

10. The Best Light. - Probably there is no one kind of light which is best for all purposes. For general illumination of public squares and public buildings the electric light seems to be generally preferred. The same thing is probably true of private houses. For reading and for microscopic work, on the other hand, the electric light may easily be too bright; but this objection can be overcome by using lamps of proper candle power, by having the lamp at a suitable distance, or by using bulbs with ground glass. The same thing may be true of the light yielded by any incandescent solid, such as the "lime" (oxyhydrogen) light and the various "mantles" made from incombustible earths, such as that in the Welsbach light. In general, for reading a "soft" light is best, and it is desirable to have the larger part of the light come to the book by reflection from the walls of the room rather than solely and directly from any source of light near by. For this reason, dark-colored walls are objectionable for rooms in which a number of people do much reading, sewing, or other near work. 


\section{CHAPTER XXVIII}

\section{THE AIR SUPPLY OF THE HOUSE. VENTILATION}

Besides the relatively permanent furnishings and fixtures of the house there are other necessaries of civilized domestic life, such as air, water, oil, gas, coal, and provisions, which come into the house only to be consumed, their waste materials being cast out again. These are commonly called the domestic supplies, - air supply, water supply, gas supply, etc., - and they are usually derived from much larger public supplies which are used in common by many families. All such public supplies, although convenient, may, under certain circumstances, become dangerous to human life and health.

1. The Air Supply of the house is probably more neglected than any other. Water, gas, coal, and provisions are costly and often difficult to get, but air is always abundant and cheap. The familiar saying, "as free as air," applies best, however, to outdoor air; for, as we shall see, good air in houses is not always very abundant, nor always cheap and easy to provide.

Inasmuch as the adult human body requires for its regular uses about five hundred cubic inches of air per minute, the air in the immediate vicinity of the nose is quickly used up; and as an equal amount of exhausted or vitiated air is discharged per minute at the same place, the need is obvious of a constant streaming of air about the body which shall remove vitiated air and supply fresh air. This circulation or flow of air is just as necessary as is the circulation of the blood; but, as the movement always 
goes on unseen, through the diffusion of gases and by other natural and invisible agencies, it is harder to realize the need. Out of doors the air supply is ordinarily sufficient and of good quality, especially while the body is in motion, in walking, driving, riding, wheeling, skating, sailing, rowing, or in doing much of the manual work on farms, forests, ships, in fishing, etc.

2. Stagnant Air. - Indoors, conditions are very different. Life is more sedentary, the body is more quiet, and natural wind currents or drafts are intentionally prevented; the air of houses tends to become stationary and even stagnant, and with it the air supply about the bodies of the house dwellers. Since it is this stagnant air which is steadily exhausted or vitiated by the air discharged from the nose and mouth, a blanket of increasingly stagnant and impure air tends to accumulate about the body of a sitting or sleeping person. To prevent this stagnation, and the consequent impurity of the air supply, movement of the body or, better, movement of the air is a prime necessity.

3. Ventilation (Latin, ventus, wind) is the name usually given to any circulation or movement of the air of rooms or buildings by which fresh, pure air, preferably from outdoors, is introduced and vitiated air is removed, the movement of the air being rapid enough to meet all the needs of the body, but not so rapid as to cause dangerous currents or drafts. This movement or circulation may be either intermittent and occasional, as when a window is opened for a little while and then shut, or more or less regular and constant, as in all efficient "systems" of ventilation or even in such primitive methods as that of the chimney above an open fire.

4. Natural Ventilation. - The walls of most houses are more or less porous and permeable for gases. Cracks and crevices around doors and windows also allow gases to 
leave and enter. In an experiment made by one of the authors, four ordinary gas jets in a small room were left open (but not lighted) all night, and after the gas had poured in for eight hours it was found that the room contained only three per cent of gas, the remainder having escaped by natural ventilation. It is largely because of the cracks, crevices, and pores in the walls that human beings get on as well as they do in rooms and houses seemingly wholly unventilated. Wood, brick, stone, and plaster are more porous than glass, iron, or glazed brick, and dry walls more porous than those wet or damp. Painted and papered walls are less porous than those left bare, and accordingly the walls of summer houses are often loosely made, preferably of "natural" woods.

5. What do we mean by Air Good or Bad, Pure or Impure? - Air is not a chemical compound of fixed composition, but a mixture of gases containing, even when pure, varying amounts of nitrogen, oxygen, carbonic acid, ammonia, and water, - this last in the form of invisible (aqueous) vapor. Moreover, the density of the air varies not only at different places, but also at the same place at different times. Impure air contains all these gases, and may contain in addition any other gas capable of mixing with them, such as hydrogen sulphide, carbonic oxide, marsh gas, etc. The terms "good" and "bad" air, " moist," " fine," " dry," " bracing," " muggy," " humid," " heavy," "foul," "fetid," "stagnant," “ dead," “thick," or "lifeless" air, and all similar terms, are popular descriptions of atmospheric conditions real or imaginary, testifying to the wonderful variety of this part of the environment of mankind.

We may define "pure" air as any portion of the atmosphere free from noxious gases or vapors and from infectious microörganisms. Such air may, however, be unfit for breathing, as is the case with those higher portions 
of our atmospheric ocean into which aëronauts have sometimes ventured. At the height of three miles above the sea the air no doubt is very "pure," but yet too thin to support human life readily.

Air may be considered as polluted or "impure" when it contains noxious gases, or floating particles in large numbers (as in smoke), or disease-producing germs or microörganisms (perhaps derived from dust).

6. The Sources of Discomfort and Danger in Air. - We must be careful to discriminate between discomfort and danger in atmospheric conditions. Positive danger comes chiefly from deficiency of oxygen, excess of carbonic acid, admixture of poisonous gases, or from infectious microorganisms. Aëronauts, explorers on high mountains, and persons living at great altitudes are apt to suffer from oxygen deficiency. Miners, charcoal burners, and well cleaners sometimes suffer from carbonic acid excess. Laborers in gas works and consumers of illuminating gas may be poisoned by carbon monoxide; and workers in sewers, by various gases, especially by illuminating gas which may have leaked in. Air may also contain, and thus convey, germs of infectious diseases such as smallpox, scarlet fever, measles, etc.

On the other hand, air that is not dangerous, and even perfectly "pure" air, may be a source of great discomfort, simply because of its temperature or moisture, or its temperature and moisture taken together. The air in the "dog days" of August is no less pure than that of June or October; yet it is often oppressive because it is both too warm and too moist. It has been shown in Chapter XII how greatly the regulation of the temperature of the body depends upon the capacity of the atmosphere to take up moisture, and it is plain that any atmosphere saturated or nearly saturated with aqueous vapor must seriously interfere with the cooling of the body. A careful review of that 
chapter will greatly help the student to an understanding of the sources of discomfort in the atmosphere of houses or rooms.

A shut and uninhabited room often becomes "musty" or "damp," because of a want of circulation to remove air containing traces of odoriferous gases and excess of moisture; the former perhaps derived from carpets or furniture, the latter from the basement or cellar.

The air of an inhabited room may prove a source of discomfort to its inmates, and therefore deserve to be called bad for any or all of the following reasons: (1) the air may be overheated or underheated; (2) it may contain an excess of moisture due either to its dampness of location or to the breath of its inmates; (3) it may be both overheated and overmoist; (4) it may contain odoriferous gases which cause displeasure or discomfort.

What such rooms do not often suffer from is oxygen deficiency or carbonic acid excess; for experiments have proved that unless the oxygen falls below twelve per cent, or the carbonic acid rises above three per cent (conditions which are very rarely met with in ordinary human habitations), no marked discomfort ensues.

7. Ventilation replaces Bad Air with Good Air and causes Aërial Movement or Circulation. - It is now easy to see precisely how ventilation aids us in securing comfortable and agreeable atmospheric conditions. It removes bad air and supplies good (that is fresh) air and, by causing movement, favors evaporation from the skin and consequent cooling on muggy days. It is also easy to see why ventilation is at times ineffective. No system of ventilation can wholly overcome the "mugginess" of a "close" room in August, because the pure outer air is itself unpleasant and uncomfortable; but active ventilation, by producing a breeze, can do more than anything else to make the conditions tolerable (see Part I, p. 203). 
8. Fans and Fanning. - It is an old point of dispute whether or not a person who "fans" himself grows cooler or warmer. However this may be, there can be no question that persons who use fans feel cooler, and there is no doubt that any one fanned by another or by a breeze not only feels, but actually is, cooled thereby. The great and growing use of electric fans in hotels, houses, etc., testifies to the same fact.

9. A Room may be well Ventilated but Oppressive from Overheating. - This fact, though perfectly obvious, and familiar to all who have been in well-ventilated boiler rooms, or who have lived in the tropics, is too little attended to. Many public halls, Pullman and other railway cars, steamboats, and private houses, especially in the northern United States, are rendered almost intolerable and very unhygienic by simple overheating. Elderly people and infants require higher room temperatures than do active persons in youth and middle life, but in general any temperature above $70^{\circ} \mathrm{F}$. must be regarded as excessive, and $65^{\circ} \mathrm{F}$. to $68^{\circ} \mathrm{F}$. is a better temperature (pp. 201, 390). Housekeepers, at least in the northern United States, would do well to try to keep the mercury in their houses between these lower limits. When the outdoor air is moist, as in rainy weather, a somewhat higher temperature is often required than when it is dry.

10. A Room may be Comfortable in Temperature but Defective in Ventilation. - This fact is less obvious than that just considered, but nevertheless true. It may be because of excessive moisture, or because of odors, or for other reasons; but those entering such rooms from out of doors are often able to remark and deplore the fact. It is perhaps oftenest exemplified in warm countries or in warm seasons when people close and darken rooms to "keep out the heat." Such closing and darkening interferes with a good circulation of air and thus hinders ventilation. It also favors dampness by excluding sunshine. 
11. Some Practical Hints about the Ventilation of Rooms. - We have already referred to the great value of chimneys and open fireplaces as ventilators, and to the "natural" ventilation by porous walls, and by cracks above and below doors and windows. The simplest and most usual artificial method of ventilation is the opening more or less widely of windows or doors, and this is a means which should never be disregarded. The great drawback associated with it is the fact that uncomfortable and frequently unwholesome drafts are likely to ensue. It should be remembered, however, that the existence of a decided draft is usually an indication that the amount of ventilation is greater than is necessary. When the wind is blowing against a window it is enough to open this an inch or less; if the wind is blowing very hard, the natural ventilation may be sufficient. Moreover, the amount of natural ventilation secured depends quite as much on the ease of egress as of ingress of air. It often happens that if the window be closed or very slightly opened on the windward side of the house, enough natural ventilation will be secured by opening other windows on the side away from the wind.

It is often possible, especially in warm or temperate weather, to secure satisfactory ventilation by opening windows both at the top and the bottom, the warm air passing out above, while cooler, fresh air comes in below. This, however, is not advisable when the temperature of the incoming air is too low, since the air then sinks at once to the floor and chills the feet. Another good plan is to raise the lower sash three or four inches and place under it a board made to fit the space. Air now enters between the sashes and, the air being directed upward, the occupants of the room are protected from drafts. Where electricity is available, an electric fan placed in one of the upper sashes is frequently very effective in hastening the 
removal of vitiated air. Fans for this purpose are now readily obtainable, and have often proved to be serviceable.

In winter, fresh air and good ventilation cost something, as the air must be heated; but it is poor economy to use stagnant air for the sake of saving fuel. The keen edge of capacity for good work is dulled by bad air, the vital resistance is lowered, and the susceptibility to disease increased. On the other hand, there is such a thing as too much ventilation; for if it causes dangerous drafts or leads to actual chilling of the body, it may do almost as much harm as too little ventilation. Here again each individual must study and determine his own needs.

The hot-air furnace is capable of supplying fresh air in abundance and, if the air be not overheated or overdried, gives an admirable method of heating and ventilation, combined in a single device, - the jacketed stove, - provided always that the air supplied to its heating chamber is unobjectionable.

\section{Mechanical Systems of Ventilation. - Buildings} larger than dwelling houses, such as large schoolhouses or public halls, are, or should be, ventilated by some mechanical system. These are of two principal types, known as the "vacuum" and the "plenum" systems. In the former an' attempt is made to effect good ventilation by sucking out the air from the building by an exhaust fan or blower attached to one main pipe or duct, to which are led tributary ducts connected with the various rooms. To supply the air thus removed, fresh air is supposed to make its way in, either by " natural " ventilation (p.443) or through inlet ducts specially provided, in either case being pressed in by the outer atmospheric pressure. In the plenum system this arrangement is reversed, and air, previously warmed if need be, is driven by a fan into a main duct or space, from which smaller ducts carry it to the various rooms, circulation being favored by outlet ducts through 
which the air flows away. Sometimes an effective combination of the two systems is used, in which case the air is not only pumped in but also at the same time sucked out.

In favor of the plenum system it may be said that, instead of currents of (often) cold air pressing in by the paths of natural ventilation, about doors, windows, etc., the direction of the aërial current at these places is reversed, owing to the pressure to which the air is subjected, so that it is more often possible to sit very near such windows and doors.

The combination of the two systems offers many practical advantages, but is obviously relatively costly. It is sometimes forgotten that air, quite as truly as water, possesses inertia and moves along paths of least resistance; but experience has shown that in order to govern the direction of flow of the aërial stream it is often necessary, as well as advisable, to control the outgo as well as the income of air. 


\section{CHAPTER XXIX}

THE WATER SUPPLY, PLUMBING, AND DRAINAGE OF THE HOUSE. GARBAGE AND RUBBISH

1. Water Supply. - The water supply of the house should be first pure, and second, abundant. No exact figures can be given as to the amount required, but for kitchen and laundry use, bathing, and good drainage, it is safe to say that thirty gallons per day per capita are ample. An ordinary barrel holds this amount. Most families get on with very much less; but for the greatest convenience and cleanliness some such quantity, if not absolutely needed, can be used to advantage, and no domestic supply is a greater luxury than abundant water.

The purity of the domestic water supply should be above suspicion. In a following chapter we shall emphasize (Chapter XXXII) the requirements as to purity of a proper public water supply from which the domestic supply may be drawn; but we may here consider briefly those private supplies, such as wells, springs, and brooks, from which many houses, especially in the country, must obtain their water. It is worth remembering that all water in or upon the earth was originally rain water (i.e. distilled water from the atmosphere). This, when it flows over the surface of the earth, or percolates through the ground, is known later as surface water, or ground water. Streams, such as brooks, creeks, and rivers, are composed largely, but by no means wholly, of surface water; deep wells, dug or driven, and many springs, contain a mixture of surface and ground waters. Surface waters are particularly 
exposed to pollution by dirt and filth from roads, manured fields, and the surface of the ground generally. Ground waters, on the other hand, although subject to pollution by percolating through buried filth, and by surface waters mingling with them through cracks or fissures in the earth, are, in general, subject to great purification by filtration during their percolation through earth, which often acts as a porous filter (Fig. 116).

2. Domestic Wells. - The well of water has an ancient reputation and has long been celebrated in song and story. As a supply more or less public it has often served as a meeting place and a social center, and has frequently been ornamented with decorated curbs or covers testifying to popular esteem. Until 1854 the common well was, with rare exceptions, regarded as a perfectly safe and satisfactory method of securing water for public as well as private water supplies; but in that year all wells began to be regarded with suspicion, because of a disastrous outbreak of Asiatic cholera in London, which was conclusively traced to a polluted public well on Broad Street in that city. It was found on investigation that a privy vault, probably infected by the discharges of a cholera patient, had leaked directly into the well; and immediately all wells, especially those near any source of pollution, fell under suspicion.

The truth, in brief, appears to be that many wells are absolutely innocent of all contamination and yield excellent water. Some wells undoubtedly contain water originally impure, but coming from a long distance through the soil before reaching the well and thoroughly purified by filtration. Others, however, are in more direct connection with cesspools, privy vaults, barnyards, stables, or similar objectionable and perhaps dangerous sources, and are utterly unfit to serve as water supplies for domestic uses. Still others, though receiving good water from 
the earth about them, yield bad water because objectionable matters find their way in at the top. Poultry should be prevented from walking over loose planking which only partially covers the well, and farmers whose boots have

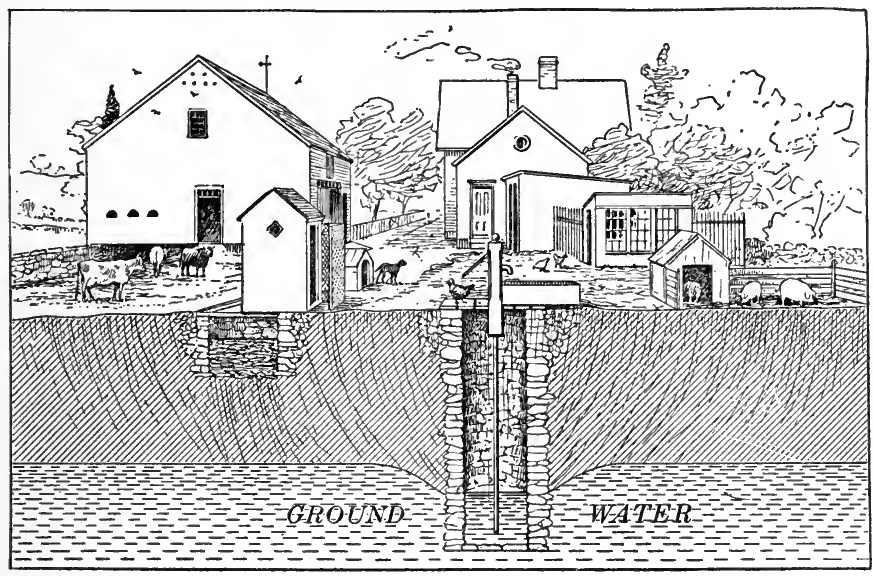

Fig. 116. A domestic well badly situated in a farmyard

Observe that the water which it yields is partly drainage from the barnyard,

privy, pigpen, etc. It is also exposed to pollution at the top

become fouled by walking in barnyards, or on fields heavily manured with stable manure, should be careful to avoid doing likewise. The danger of even worse contamination of wells from manure or other surface dirt washed in at the top during heavy rains is also very great (Figs. 116 and 117).

3. Springs are usually sources of pure water, but a spring in a barnyard or a cemetery would be plainly objectionable, and care should always be taken to ask whence comes the water which the spring yields. Springs often occur on hillsides; in such cases they should be protected from the possibility of surface pollution, while sources of pollution of any sort higher up the hill should not be tolerated, since from these the spring may become contaminated. 
4. Cisterns of Rain Water are often used for domestic supply in country houses and in some places, such as New Orleans and the Bermuda Islands, where wells are not available. There is no objection to this practice, which should secure a pure and very soft water, provided the roofs, cisterns, reservoirs, and other receptacles employed in collection or storage of the water are clean and suitable. Painted roofs, and pipes, roofs, or reservoirs containing any exposed metallic copper or lead should be avoided, since rain water may attack these metals, forming with them soluble and poisonous salts.

After a long dry period in summer, roofs are often dusty and dirty, and the first washings of dirty roofs should be allowed to go to waste. Rain water collected in winter or spring, and subjected to long storage, is probably the purest and most desirable cistern water.

5. Streams, such as brooks and creeks, are sometimes used as sources of private domestic water supply, and if the places which they drain are wooded and entirely uninhabited, i.e. not manured in tillage or pasturage, the surface water which they yield may answer fairly well for house use. But, even at its best, such water is exposed to pollution by wild animals and by passing tramps, fishermen, or gunners, and a carefully protected well or spring is, as a rule, a safer supply. Well water is often more palatable, especially in summer, but is sometimes very hard. For washing, surface or rain water is generally softer and of course unobjectionable.

6. Hard Waters and Soft. - Rain water contains few or no salts in solution, and is therefore called "soft" water. Many surface waters and some well and spring waters are also soft. All such waters readily dissolve soap, and because soapy waters are sticky and easily form air bubbles, a "lather" or "soapsuds" is easily made in soft waters with a very little soap. 
Other waters, and especially ground waters, contain salts in solution, some of which, notably those of calcium and magnesium, form compounds and even precipitates with soap, thus removing it from the water in which it is placed. Such waters, therefore, require more soap to make them soapy, lathery, or sudsy, and are known as " hard" waters, because they feel less bland, or soft, to the skin.

In some parts of the United States the well waters (and sometimes even the surface waters) are so hard as to be almost or quite useless for washing, and even for drinking. It has never been shown that moderately hard waters are necessarily any more harmful for drinking than soft waters. Persons used to either kind are apt to suffer temporary disturbances, such as diarrhea, when they change suddenly from one to the other; but otherwise no great or permanent harm ordinarily happens. If, however, a drinking water is very hard and heavily charged with mineral salts so that it becomes essentially a mineral water, it may be unfit for regular use.

7. House Filters for water are not needed if the water supply is pure and colorless, but in many places this is not the case. If the water supply is impure, it should either be carefully filtered by a germ-proof filter (several kinds of which are on the market, but all of which are costly), or else boiled for a few minutes and cooled before it is used for drinking. If the water is pure but colored or turbid, it may be made bright and attractive by filtering through a charcoal filter; but this also, if durable and effective, is sometimes costly.

8. The Ice Supply of the house is one of the greatest of modern conveniences. Ice in summer was formerly a luxury, but in northern latitudes ice is now generally harvested in winter and stored for the following summer. In warmer climates the so-called artificial or manufactured ice 
brings the same luxury within reach of persons of moderate means. Provided the water from which it is made is pure, manufactured ice is as wholesome as the best natural ice. The economical value of ice in preserving foods is very great, as is also its sanitary importance in hindering harmful decomposition and decay, for example, in milk.

Ice water, so generally used as a beverage in America, is probably harmless enough when not drunk in too large quantities or too rapidly; although, as a matter of fact, thirst is normally slaked by cool water more effectively than by very cold water. The ice added to drinking water should be pure; that is, ice obtained from ponds, streams, or other waters unfit to serve as sources of domestic water supply, should never be used in water intended for drinking purposes, and all ice should be carefully washed before being so used.

9. The Plumbing of the House. - Almost all houses have a sink of some sort; from this there runs a drainpipe, which should be tight, and large enough to carry off readily the drainage from the sink. Many houses have in addition more or less complex systems of water supply and drainage, requiring piping and plumbing.

The plumbing for water calls for brief comment only. Lead service pipes should, as a rule, be avoided, for experience has shown that if the water passing through lead pipes happens to contain an excess of free carbonic acid $\left(\mathrm{CO}_{2}\right)$, this may attack the lead and form with it a very soluble bicarbonate which is a dangerous poison. In Massachusetts there have been several epidemics of lead poisoning due to this cause.

Service pipes, i.e. pipes leading from street mains into houses, may often be made of lead without any bad consequences, and are usually preferred by plumbers because they are more easily worked; but it is much safer to use 
iron for all water pipes, although iron pipes are less convenient to install and sometimes get badly clogged with rust.

The plumbing for drainage should aim to provide against escape or leakage of both liquids and gases. As drainpipes are not usually filled with liquids or gases under pressure, leaky joints and even small holes may, and often do, occur without detection. If under such circumstances any stoppage happens, pressure may arise and the liquid or gaseous contents escape. It was formerly believed that great danger existed in defective plumbing, owing to the escape of sewer gas or gases by leakage, and particularly from the pressing backward, or " rising," of sewer gas into bath rooms, or sleeping rooms provided with set bowls, etc. The present view is that while such gases may, and probably sometimes do, escape into houses, they are usually greatly diluted before they are breathed and, at the worst, are much less harmful than was formerly supposed. They are, nevertheless, highly objectionable, and it is likely that they occasionally produce serious poisoning. If breathed for a long time, even in small amounts, they probably lower vital resistance and increase susceptibility to infectious disease, and are thus not merely objectionable but also dangerous.

Pains should be taken to ventilate thoroughly all places, such as sleeping rooms, bath rooms, and water-closets, into which sewer gases may find their way, and it is advisable and customary to seal up the various drainpipes by water seals, or traps. If, in addition, the main drainpipes are provided with vents to allow the escape of any gases accumulated in the pipes, the essentials of sanitary plumbing have been secured. Good workmanship is, however, indispensable in all water and drain pipes, as well as in all gas pipes, in the house, to prevent serious damage from breaks or leaks. 
The main drainpipe in the house is called the soil pipe. This usually empties into an underground drain or sewer outside the house, which discharges its contents, - now known as sewage, - into a cesspool, or a stream, or upon a sand bed, a sewage filter, a cultivated field, or some other place of sewage disposal.

10. Drainage and the Disposal of Household Wastes. The consumption of the solid and liquid supplies of the house - water, ice, coal, food, etc. - is accompanied by the formation of various wastes which for sanitary as well as æsthetic reasons must be promptly got rid of. Waste water and melted ice necessitate drainage; the dust and ashes of fuel remain to be disposed of, and from food, putrescible remnants, known as garbage. Dirt, bottles, papers, boxes, tin cans, old clothes, worn-out mattresses, broken furniture, crockery, and glass must also be removed. Among all the wastes of the house, however, the discharges of human bodies are of the first importance, not only because of their putrescible and disagreeable character, but also because they frequently contain the germs of dangerous diseases.

Drainage is often necessary for a house merely to carry off rain water from the roofs, and to keep the cellar dry. It is very important to remove all surplus water from the house and its vicinity in order to prevent dampness, - this being one of the most unfavorable conditions in the environment of mankind.

If the drains of houses or lands carry water only, they keep the name of drains, and the water in them is called drainage; but if such drains carry household wastes, and especially human or animal excreta, they are more often called sewers, their contents being known as sewage. The process or act of removing sewage from a house or a city, and the systems of sewers, are both known as sewerage, although this same term is sometimes popularly applied to the sewage itself. 
11. The Disposal of Drainage and Sewage. - Cellar drains, and drains for the removal of roof water, usually discharge, especially in the country, upon the surface of the ground at some distance from the house, and give little trouble; but sink drains, since they contain dish washings, soapsuds, and the liquid wastes of the kitchen, are apt to become choked with grease. Grease is dissolved by alkalies, and common lye (potash), allowed to dissolve and flow down the sink waste pipe, will often remove greasy obstructions and give at least temporary relief. The final disposal of sink water, however, is more difficult, and a greasy, slimy, malodorous, and unsightly channel or area behind a country house too often tells of trouble. The only complete remedy is a large waste pipe, as straight as possible, going to an equally large or larger (underground) drain, which ends in a covered pit or tank placed in porous or gravelly soil. This pit must be cleaned out from time to time, and if no open porous soil is available, a tight tank or pit must be used and frequently emptied.

Sewage disposal is a more difficult matter, for sewage contains not only the sink wastes just mentioned but also washings from the human body, human excreta, and other putrescible matters, all in comparatively large volume. We shall discuss beyond the problem of the disposal of the mixed sewage of numerous houses combined into communities (p. 519), and therefore at this point need consider only the disposal of the sewage of separate houses, such as country homes or farmhouses. If these are so placed as to be readily drained or sewered into the sea, or into some large lake or stream nowhere used for drinking purposes, the solution is simple. If not, the cesspool or disposal upon land, as described in the next following paragraphs, are among the best expedients.

12. The Cesspool is a receptacle or tank in the earth, at some distance from the house, - not less than one hundred 
feet away, and the farther off the better, - into which sewage is conveyed by a drain or sewer directly connected with the soil pipe of the house. The cesspool may be either water-tight to prevent leakage, or loosely built to favor it. A common construction is one in which the cesspool is water-tight and has an outlet pipe just below the surface. This outlet pipe may run into a drain, loosely laid to facilitate leakage from its joints and thereby the escape of liquid sewage into the earth.

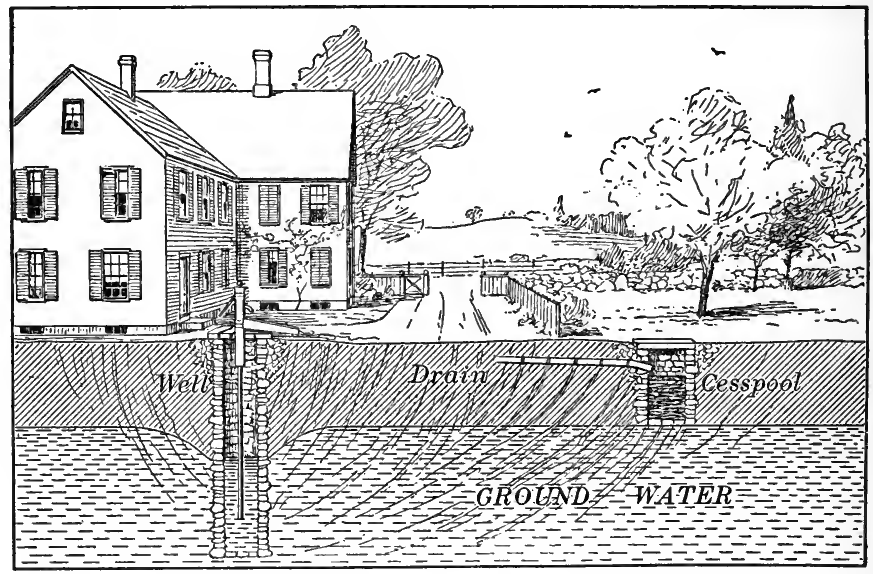

FIg. 117. Disposal of household sewage by meáns of a cesspool

Observe that the loosely laid drainpipe, or sewer, allows some sewage to leach away before reaching the cesspool. If no well were near, this would be a distinct advantage

It was formerly held that the cesspool was a sanitary abomination; that it favored putrefaction and the development of sewer gas; and that it ought always to be avoided if possible. Experience has shown, however, that thousands of houses have been drained into cesspools, not only conveniently but without the slightest discoverable sanitary injury; and sanitary science now recognizes in the septic tank (a special form of cesspool) a useful and popular means of sewage disposal (Fig. 117). 


\section{Irrigation and Subsurface Disposal of Sewage.} Another method often successful, especially in soil which is open and porous, i.e. in sandy soil, is that of simply discharging the sewage upon the surface of land set apart for the purpose at a distance from the house. This method may be recommended in many cases, but is often less satisfactory than the use of a well-regulated cesspool. A modification of it, in which the sewage is distributed under, instead of upon, the surface by means of a system of branching pipes loosely laid, is frequently preferable, even in the same porous or sandy soil; but neither of these methods is to be recommended as compared with the cesspool, if the soil is impervious or clayey.

14. The Care and Disposal of Garbage is a matter of much importance both to the housekeeper and to the community. Garbage consists chiefly of the more solid refuse from the kitchen and, since it is composed of the remnants of food, - bits of meat and fish, either cooked or raw, bones, vegetables, fruit, and the like,-it is highly putrescible. On the farm, garbage may be fed to swine, and in many towns and cities it is collected by farmers and used to maintain large (and often offensive) piggeries, in or near a city or town. More rarely, garbage is fed to milch cows, the milk from such cows being known as swill milk; but this use of garbage is rightly forbidden by boards of health.

In the house, garbage is simply a nuisance, to be got rid of as quickly and as completely as possible. It should be either burned (in which case disagreeable odors are often produced), or kept as short a time as possible in a clean receptacle in or near the kitchen. The garbage receptacle is usually the dirtiest and foulest smelling household utensil. Nothing about the house requires more careful attention. The receptacle itself should be of metal rather than wood; it should be no larger than is necessary, because a small can is easier than a large one to keep clean; it 
should have a tight-fitting cover; and it should be kept where dogs and cats cannot overturn or open it. Above all, it should be frequently emptied and cleaned.

15. The Disposal of Ashes, Dirt, and Refuse is perhaps as much a question of good taste as of sanitation. Nothing is more unsightly than a dump, especially if its papers, boxes, and other combustible materials are set on fire, as often happens, and left to smolder. In towns and cities ashes and rubbish (as well as garbage) are usually removed periodically by public carts, but isolated householders must ordinarily look after their refuse disposal themselves; and of all methods, burial in pits, when possible, is the least objectionable. For garbage, especially, when a piggery is undesirable and cremation not possible, burial in sandy land at a distance from the house has much to recommend it. 


\section{PUBLIC HYGIENE AND SANITATION}

\section{CHAPTER XXX}

\section{PUBLIC HEALTH. INFECTIOUS AND CONTAGIOUS}

\section{DISEASES. MICROBES}

1. The Public Health. - The environment of man consists of two principal, and very different, parts : one near, and chiefly personal, domestic, or private, including his clothing, house, family, and estate; the other more remote, impersonal, and public, including his neighborhood, village, town or city, state, and country (p. 299).

In sparsely settled districts little attention is paid to any health beyond that of the person or the family; and the family, as we have shown (p. 425), is really a small and private community; but in thickly settled regions, such as cities and towns, conditions and problems arise involving numbers of families, and public hygiene and sanitation become necessities. In the country each family generally has its own private water supply, milk supply, food supply, and drains; but in cities and towns mutual convenience, economy, and safety require public supplies and public drains. Instead of private roads we find public streets; instead of private estates, public parks. Public gardens and public markets furnish flowers and vegetables; public conveyances, such as cars, steamboats, and carriages, serve public needs; public institutions arise, such as hospitals, schools, almshouses, and jails; and public buildings, such as halls, hotels, churches, schoolhouses, shops, factories, stores. 
In all such cases numbers, groups, or masses of individual families - called collectively the people, or the public are at times and as a whole exposed to unfavorable conditions, such as a general want of muscular exercise, lack of sleep, a too sedentary life, and overwork; or to germs of infectious and contagious diseases in public supplies of water, milk, etc.; or to foul air, overheating, defective lighting, gas poisoning, noise, dust, smoke, or impure food; some of which conditions are chiefly personal, affecting more or less directly the bodies of the people, while others are more remote, or environmental.

By the public health we mean the health of the public, i.e. of the people as a whole; and the health of the public depends - just as the health of the individuals who compose that public depends - on a great variety of conditions, some of which, as just stated, are chiefly internal, or in intimate relation with the persons of the people, and may conveniently be called hygienic; while others are chiefly external, or at least not in intimate relation with the persons of the people but rather in their environment, and may be described as sanitary (pp. 301, 302).

The applications of the various branches of science, such as physiology, chemistry, bacteriology, vital statistics, climatology, medicine, engineering, etc., to the control of these various hygienic and sanitary conditions, and thereby to the protection and promotion of the public health, constitute the science of public health; and of this, as indicated in the last paragraph, there are two grand divisions, namely, hygiene and sanitation.

2. Public Health Rules and Regulations. - For the regulation and control of those conditions which are personal and domestic we must look, even in large communities, chiefly to individuals and families; but even if individuals and families always obeyed the laws of personal hygiene and domestic sanitation, the protection of the public health would 
still require special supervision and control of public supplies, public drains, public vehicles, public institutions, and the like, because these things are outside and beyond the control of private individuals or families and stand in a class by themselves. In point of fact, however, private persons and families are often negligent in matters of this kind, inflicting damage upon their neighbors by maintaining nuisances of one kind or another, or else by their carelessness in respect to filth or in respect to the spread of infectious or contagious diseases. Hence it has come to pass that sanitary and hygienic rules and regulations have been adopted by the citizens of most civilized communities for mutual benefit.

3. Public Health Authorities. - For the enforcement of these rules and regulations (sanitary laws) special public officials are usually elected or appointed, such as boards of health, health officers, city physicians, sanitary inspectors, medical inspectors, quarantine officers, school nurses, sanitary police, vaccinating physicians, etc. By common consent of the majority of the citizens these officers are authorized and required under the laws to prepare, publish, and enforce needful sanitary rules and regulations for the protection and promotion of the public health.

4. Public Health Problems. - In this and the following chapters we can touch upon only a few of those more elementary and important problems of the public health of which every educated citizen should have some knowledge. Such problems are almost all fundamentally concerned with the control of infectious diseases, to the nature of which we shall therefore at once turn our attention.

Much of what follows was formerly the exclusive possession of the medical profession and has only recently become a part of the common knowledge of mankind. Much of it also is comparatively new, and among the best 
fruits of the splendid advances of the last half century in the sciences of pathology, hygiene, and sanitation.

5. Plagues, Pestilences, and Epidemics are the most striking examples of influences affecting both personal and public health. Only wars, riots, and great conflagrations are capable of throwing communities into such terror as has often been caused by plagues or pestilences of some swiftly fatal disease. Such was the plague in London described by Defoe in his Journal of the Plague Year, a story which has been well called "that truest of all fictions." History is full of similar instances. Even as late as 1892 the rich and powerful city of Hamburg, Germany, was terrorized by a severe epidemic of Asiatic cholera due to a polluted public water supply, while still more recently the cities of Ithaca in New York and Butler in Pennsylvania have been ravaged by the plague of typhoid fever.

Plagues and pestilences are simply older names for great epidemics of much dreaded diseases, such as smallpox, yellow fever, Asiatic cholera, or the bubonic plague, and the pesthouse which formerly existed in many towns and cities was a remote and isolated shelter, or primitive hospital, often of the crudest and poorest kind, to which the victims of pestilence were taken (or driven) by a frightened public. The true sources of epidemics, plagues, and pestilences have only recently become known. Savages often attribute these to supernatural causes, such as evil spirits or demons, and even for civilized people pestilences were until recently mysterious in origin and incomprehensible in behavior. It is now known, however, that such outbreaks are simply extensive epidemics of contagious or infectious diseases, which may often be controlled and even prevented; but in order that control or prevention shall be effective, the intelligent coöperation of all good citizens is essential. It is one of our first duties to acquaint ourselves with the nature and the methods of prevention of 
contagious and infectious diseases, and thus at the same time of plagues, pestilences, and epidemics.

6. What are Infectious and Contagious Diseases? - The discoveries of Pasteur, Koch, and their successors in the last half of the nineteenth century have brought to light the remarkable fact that those "fevers" - typhoid fever, malarial fever (malaria), diphtheria, smallpox, cholera, tuberculosis, etc., and probably also measles, chicken pox, scarlet fever, and many " colds" - which attack apparently healthy persons and cause a severe but brief sickness that seems to run its course and then cease, are due to invasions of the body by microparasites ${ }^{1}$ called microbes. Each of these contagious or infectious diseases has its own special microbe to which it owes its origin ; and it is customary to speak of the microbes of diphtheria, of typhoid fever, of the bubonic plague, of Asiatic cholera, etc., as the cause of these diseases. Although in some contagious and infectious diseases the microbe has not yet been discovered, all these diseases are nevertheless so much alike, and causative microbes have been found in so many cases, that all are believed to have a similar microbic origin.

The view or theory just outlined is known as the germ theory of infectious and contagious diseases, and the causative microbes are known as disease germs. It is easy, on this theory, to see why these diseases are "catching." It is,

1 Microparasites. - A parasite is a plant or animal which feeds upon another plant or animal (called its host), and renders it no services in return. Some parasites, like fleas, lice, the pork worm (Trichina) and " ringworm," are visible or nearly visible to the naked eye ; but many others are invisible and may be called microparasites. Of these the most important belong among the microbes (see p. 468); but, as the microbes form an enormous group of plants and animals, most of which are in no way parasitic or harmful to mankind, but on the contrary are highly useful, we must be careful not to regard as parasites more than a very few of the microbes. Those that do not lead a parasitic life are usually scavengers and lead a saprophytic life; that is, they feed upon dead organic matters, often helping greatly to clean and to keep clean the surface of the earth. 
of course, not the disease but the parasitic microbes which can be "caught" or "taken," as fleas can be "taken " from a dog, or bedbugs carried from place to place in bedding or clothing, or lice "caught" by children from lousy playmates. It is easy, also, to understand how destructive epidemics, plagues, and pestilences can occur, if public food supplies, water supplies, milk supplies, carriages, steamers, caravans, cars, or other conveyances have become infected with infectious microbes or disease germs.

An infectious disease is one in which the disease germs infect (that is, invade) the body from without. Such are diphtheria, typhoid fever, tuberculosis, trichinosis, scarlet fever, smallpox, measles, chicken pox, and all the more common "fevers." Among these some are ordinarily conveyed quite directly and quickly from person to person, and to such infectious diseases the term "contagious" is often applied. Formerly a sharp line was drawn between infection and contagion, but to-day it is recognized that no such line exists. Typhoid fever, for example, is still sometimes said to be "infectious but not contagious." If by this is meant that it is not as often spread through the air or by mere "contact" as are smallpox and some other diseases, then it is true. But if the saying means that it cannot be transmitted by mere contact with the patient or his excreta, then it is false (see p. 485). It would be better to drop altogether the term "contagious," for its use is antiquated and misleading.

7. Microbes. - Brief references have frequently been made on previous pages to microbes and their work, but we must now give them special consideration.

As the word implies, microbes (micros, small; bios, life) are little living things, and they have been described as "all forms of life, whether animal or vegetable, invisible or barely visible to the naked eye." It is customary to regard them as the smallest of all living things, and sometimes 
as identical with microörganisms, germs, or bacteria. All bacteria, however, are plants, so that a broader term, such as "germs," "microörganisms," or " microbes," is required if the lowest forms of animal life are also to be covered. In these pages we shall use the term microbes for those forms of life, either plant or animal, which are invisible or barely visible

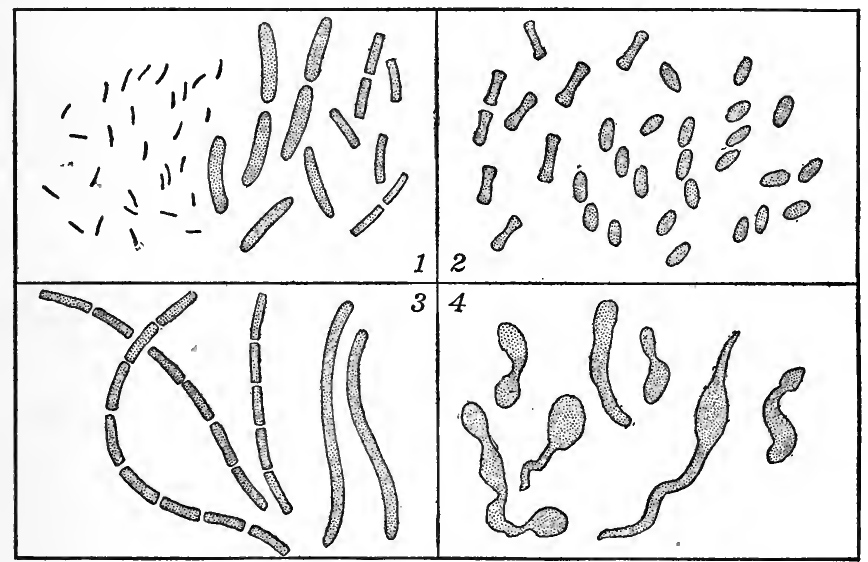

Fig. 118. Microbes (rod-shaped bacteria, or bacilli). (All very highly magnified)

1, bacilli, some very large and some very small; 2 , other forms of bacilli; 3 , bacilli forming threads or filaments; 4 , dead or dying bacilli (involution forms)

to the naked eye, and of interest or importance in physiology, hygiene, and sanitation.

For our purposes microbes may be divided into bacteria, or vegetable microbes, and protozoa, or animal microbes. The bacteria are unicellular plants of the simplest structure and of three principal forms, viz. rods, berries or balls, and spirals. The rods form the group bacilli (Fig. 118), the balls the cocci (pronounced cock's eye) (Fig. 119), and the spirals the spirilla (Fig. 120). Bacteria often grow and multiply (by simple cell division) very rapidly, and some are 
capable of producing within themselves smaller cells, called spores, which have thick walls and possess great powers of resistance (see Fig. 121).

The protozoa are unicellular animals, also of the simplest structure, and among them one group, the sporozoa, is of

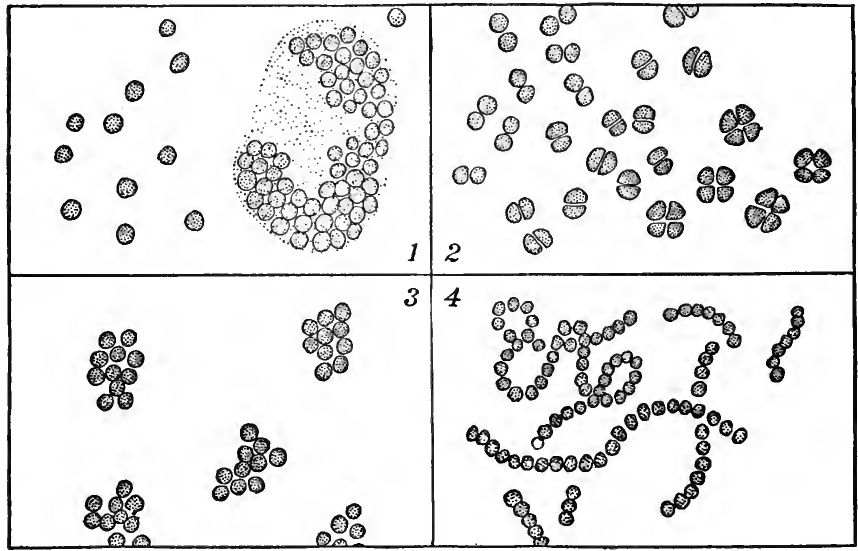

Fig. 119. Microbes (ball-shaped bacteria, or cocci). (All very highly magnified)

1 , cocci single, and cocci united in a jelly mass or zoöglœe $; 2$, in twos and fours (diplococci and tetrads); 3 , in clusters (staphylococci); 4, in chains or necklaces (streptococci)

especial interest because it certainly includes the microbes of malaria, and probably also those of smallpox and of scarlet fever (see Fig. 126).

Microbes are of interest and importance to the physiologist, hygienist, and sanitarian, first, because they are nature's scavengers, i.e. removers of organic waste matters; second, because they are the ordinary agents of the decomposition, putrefaction, and decay of foods and other valuable organic matter; and third, and especially, because among them are found many microparasites, and especially those germs which cause infectious and contagious diseases such as consumption, typhoid fever, diphtheria, and malaria. 
1. Microbes as Scavengers. - Whenever the dead body of a plant or animal, or any part of it, is left upon the ground, or in water, or is buried in the earth, it soon crumbles or decays and disappears, turning, as we say, to dust and ashes. It was formerly universally believed that this

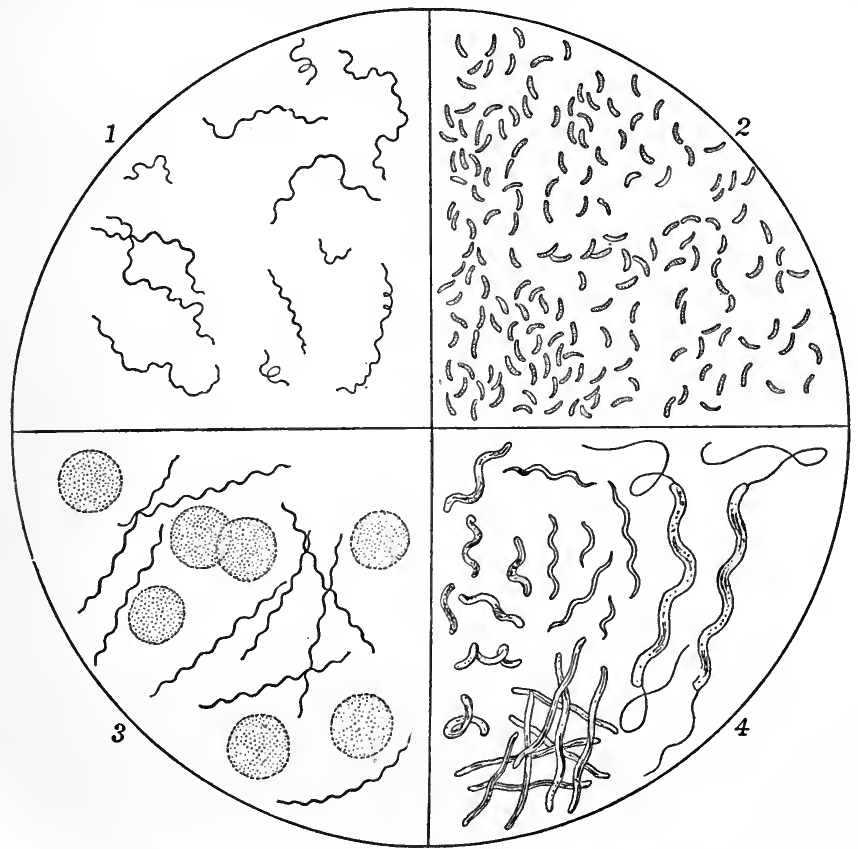

Fig. 120. Microbes (spiral or screw-shaped bacteria, or spirilla). (All very highly magnified)

1, spirilla from the human mouth; 2 , microbes of Asiatic cholera; 3 , spirilla of relapsing fever; 4 , large spirilla from ditch water

change was a slow combustion or oxidation caused by the direct action of the oxygen of the atmosphere. It is now known, however, that this oxidation is due to the influence of microbes which abound in the upper layers of the earth, and to a less extent in air and water. These oxidize the waste organic matters to carbon dioxide, water, etc., much 
as the muscle fiber oxidizes the food brought to it by the blood (see Chapter IV).

It is now established that scavenging is one of the principal functions of microbes, for they abound in sewage, which they readily decompose and, under favorable circumstances, completely purify; in excrement, which they work over and change to harmless, inoffensive, and even useful mineral matters; and in many organic wastes, which they reduce to simple and harmless chemical compounds (Figs. 120, 4, and 122, 4).

2. Microbes as Agents of Decomposition and Decay.The peculiar property which makes microbes destroyers of waste organic matters, and therefore useful as scavengers, makes them also troublesome, if not dangerous, agents of the decomposition, decay, and consequent destruction of useful organic substances, such as foods. Milk, for example, may be spoiled by lactic acid microbes which feed upon its sugar and, by producing lactic acid in the course of their feeding, cause the milk to turn sour; but, on the other hand, this very change wrought by the microbes, though dreaded by the milkman, may be desired by the cheese-maker, in whose work the souring of the milk is necessary. The spoiling of meat, fish, fruit, and many other forms of food is due almost wholly to the vital activity of microbes, and we have to use cold and heat for protection against their inroads. Cold, by chilling and benumbing them, checks their growth and multiplication; while heat, if sufficiently intense, destroys them altogether. Upon the former fact are based the important arts of refrigeration and cold storage; upon the latter the great modern industry of canning.

Microbes have a wide and useful employment in the arts and industries, - such as the souring of milk in cheesemaking, the flavoring of cheese and butter, the preparation of hides for tanning, the ripening of manures, the fixation 
of free nitrogen in agriculture, and many other processes depending upon their vital activity. But, on the other hand, spoiled foods - especially meat, eggs, and fish may be not only disagreeable but also dangerous, owing to the formation by microbes of poisonous by-products known as ptomaines, to whose agency have been attributed severe outbreaks of acute disease. Ptomaines are bodies of uncertain chemical composition, which cause intense general prostration and sometimes death. It is a good rule to avoid carefully

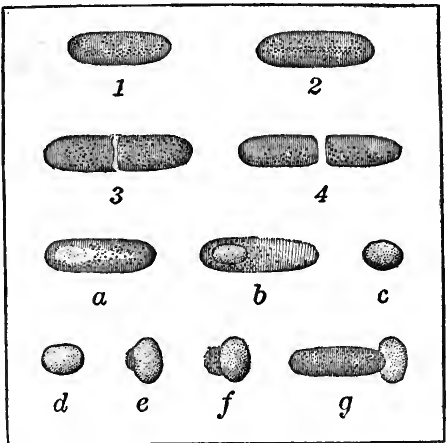

Fig. 121. Diagram of the growth, multiplication, and spore formation of a bacterial microbe

all meat or fish which is 1-4, growth and multiplication by cell "tainted," or suspected of putrefactive decomposition. division (fission) ; $a-g$, formation, liberation, and germination of a spore At the same time there is reason to believe that some outbreaks of obscure poisoning have been charged to this rather uncertain source largely because no other explanation could be as easily given.

3. Microbes as Disease Germs. - But it is as disease germs that microbes are of the greatest hygienic importance. Long before the nineteenth century it had been suspected that infectious and contagious diseases were caused by invisible "germs" of some kind, but it was not until the last half of that century that the responsibility for some of the worst diseases that afflict the human race was clearly and specifically fastened upon certain microbes. For this great discovery we are indebted chiefly to Louis Pasteur, a French mineralogist and biologist, and Robert Koch, a German physician and bacteriologist. Thanks 
mainly to their genius and their patient labors, we now know that many of the infectious and contagious diseases of animals and plants are due to the entrance into the living body of specific microbes which, growing in it or upon it, poison it, and cause it to sicken or even to die.

But if infectious and contagious diseases are thus due to the attacks of microbes coming from the environment,

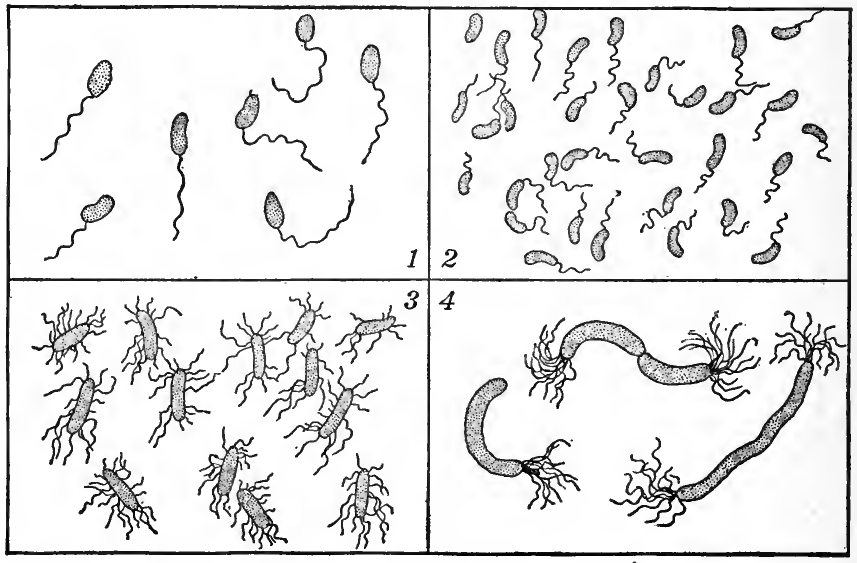

Fig. 122. Some motile bacterial microbes, showing flagella or cilia (all very highly magnified)

1, 4, large water bacteria; 2, microbes of Asiatic cholera; 3, microbes of typhoid fever

we may hope to prevent these diseases, either by warding off the microbes or by making the body competent to resist or overcome them, or both; and it is one of the chief lessons of sanitation and hygiene to show how the warding off, the resistance, and the overcoming, of infectious and contagious microbes may be most effectively accomplished.

8. The Biology and Control of Microbes. - It must never be forgotten that microbes are living cells, and as such subject to the laws which govern all living things. As a rule, they work best at about the blood heat. They feed 
and grow and multiply by cell division. Like muscle fibers and gland cells, they decompose their food materials and produce secretions and by-products, some of which may be harmless and some harmful. Microbes, or germs, are killed by strong acids, strong alkalies, and various other substances, which, for this reason, are called germicides or disinfectants. Sterilization is complete removal of microbic life. It may be effected by germicides, or by intense heat, such as boiling or burning, or by various other means. Pasteurization is incomplete sterilization by heat, at a temperature sufficient to destroy most, but not all, germs. Antiseptics inhibit, but do not necessarily destroy, microbic activity.

9. The Prevention of Microbic Diseases. - For the occurrence of any infectious or contagious disease two conditions must be fulfilled, viz. (a) a specific disease germ, microparasite, or microbe, and (b) a person susceptible to the disease in question. Without the microbe the disease cannot arise, no matter how favorable for it the condition of the person may be; and, on the other hand, the microbe appears to be powerless to produce the disease, unless the condition of the person is favorable for its reception, life, and activity. This being the case, we obviously have at our command two principal lines of defense against the attacks of infectious and contagious disease; on the one hand we must seek to obtain (a) control of disease-producing microbes, and on the other to secure $(b)$ insusceptibility or invulnerability of the human mechanism to their attacks.

Boards of health are constantly seeking to destroy or control dangerous microbes, by requiring the reporting of all cases of infectious diseases, by isolation of such cases, by the placarding of houses, by disinfection, by the inspection of food and other materials, and by quarantine. Cities are purifying their water supplies, their sewage, and their milk supplies with a view to warding off the attacks 
of disease germs. Individuals also, if wise, will take all reasonable care to avoid exposure to infection by such germs, and will endeavor, as far as is practicable, to secure food and drink free from microparasites capable of causing disease.

To measures of this kind, devoted to the destruction or avoidance of the active agents of infectious disease, should be added efforts calculated to lessen personal susceptibility to their influence if, as sometimes happens in spite of all precautions, they find entrance into the body. The wise individual will study himself and learn from experience how to avoid colds and other slight ailments; he will regulate his diet and his exercise, his sleep and his bathing, his work and his play, in order to build up and maintain abounding health with which to meet any microbic invasions which may chance to occur. The wise and enlightened community will also provide parks and playgrounds, gymnasia and baths, and other means which will facilitate the cultivation of this abounding health among its citizens, young and old. 


\section{CHAPTER XXXI}

\section{SOME MICROBIC DISEASES AND THEIR PREVENTION. VACCINATION AND ANTITOXIC SERUMS}

1. Tuberculosis. - One of the first diseases of which microbes were conclusively proved to be the cause was tuberculosis, a disease to which more deaths are annually attributed than to any other. For the latter reason it has

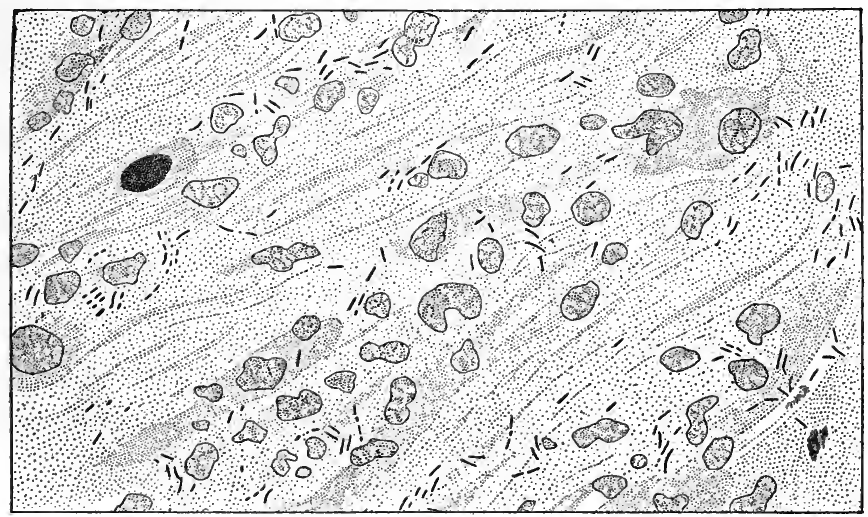

Fig. 123. The microbe of tuberculosis (Bacillus tuberculosis). (Very highly magnified)

A minute amount of the spit (sputum) of a consumptive has been spread out thin and photographed. The tubercle bacilli are the little sticks or small rods scattered all about among larger irregular epithelial cells, mucus, etc.

been called the "great white plague," and under the common name of "consumption" one form of it is only too familiar. The name "tuberculosis" was given to the disease because of certain characteristic cheesy nodules or tubercles (little tubers) found in the lungs and other tissues of 
persons affected with it, and until 1882 it was generally regarded as a constitutional disease, readily inherited and often "running in families." In that year, however, Koch announced the great discovery that in the tubercles could be found peculiar and apparently characteristic microbes belonging among the bacteria; also, that by special methods he had cultivated these microbes pure, or free from all others; and, finally, that with them he had inoculated healthy guinea pigs and actually produced tuberculosis in the infected animals.

Intense interest was everywhere felt in Koch's splendid discovery, which was quickly confirmed, and soon became an established and universally accepted fact. It is now known that tuberculosis is not ordinarily an inherited or constitutional, but an acquired, disease, infectious, contagious, and environmental in origin, and due to the ravages of a special microparasite, a bacterial microbe named Bacillus tuberculosis. It is true that the disease often runs in families, but infectious diseases frequently do this; and the reason why it affects some families so much more than others is believed to be simply that some families are more susceptible to it, more adapted to its growth, than others; precisely as some soils are better suited than others for growing wheat or grass or corn.

2. How Tuberculosis is spread. - As this disease is caused by the invasion of a specific microbe, it can only be caused by the entrance of that microbe into susceptible bodies. Sanitarians generally believe that the principal ways in which tuberculosis is conveyed from one patient to another are the following : $(a)$ by contagion, that is, by personal contact of tuberculous with non-tuberculous persons, and especially by kissing. A consumptive mother or sister or friend, fondling a baby and "smothering it with kisses," may thus transmit the germs of the disease to the child; (b) by objects handled or mouthed (such as food, 
forks, drinking cups, pencils, or towels) first by consumptives and then by susceptible non-consumptives; $(c)$ by $d u s t$ containing sputum expectorated upon streets or floors, and then dried and carried in the air; (d) by milk or meat of tuberculous animals; and (e) by the moisture of the breath thrown off not as vapor but as fine droplets or spray, in coughing or even while talking. It is not certain just what part any or all of these play in the dissemination of the disease. At one time it was thought that the principal vehicle of tuberculosis was the spit, or sputum, of tuberculous patients expectorated upon floors, sidewalks, or streets and afterwards dried, pulverized, and driven about as dust particles, which might readily find access to healthy throats and lungs. There is no doubt that the spit of consumptives may, and often does, contain the germs of the disease, and for this reason "spit cups" and destructible handkerchiefs (e.g. of paper) should always be used by them; but further investigations have shown that drying and exposure to sunlight both tend to weaken and to destroy microbes, so that to-day, while dust is still regarded by all as a vehicle of very great importance in the spread of tuberculosis, more significance than formerly is attributed to other factors.

It is an open question how far milk is a carrier of tuberculosis. Cows undoubtedly often suffer from a form of the disease not readily distinguishable from that which occurs in man, and the frequency of tuberculosis in children certainly suggests that infected milk may have been the cause of it. But, on the other hand, it is far from certain that bovine tuberculosis is readily transferable to man; and at present, while it may be that milk is an important vehicle of the disease, the whole question is much in doubt. The same thing is true of meat from tuberculous animals, although in this case thorough cooking must destroy most, if not all, of the germs. The safest plan is to use for food 
no milk or meat which is in the least doubtful in quality, at least not without first subjecting it to thorough cooking. At present the dissemination of the bacilli through the air by means of finely divided droplets of mucous or moisture, as suggested above, is regarded as of special importance.

3. The Prevention of Tuberculosis. - No successful steps have yet been taken toward the prevention of tuberculosis by vaccination, or in any other ways than by warding off the microbe and by helping the patient in his struggle with it by giving him good air, good food, rest, and all other favorable conditions which shall aid the body in resisting infection and the ravages of the disease.

It has been proposed to isolate cases of tuberculosis and in general to deal with them very much as the more contagious diseases are ordinarily dealt with. There is something to be said in favor of this plan, but the general opinion is that such isolation is a hardship to the patient and not often necessary for the safety of the community. Consumptives should, however, be expected, and even required, to be especially cleanly in their habits, and to collect and destroy their sputum in cheap paper cups or in paper handkerchiefs, which can readily be burned. They should never spit upon floors or streets, or cough into the faces of friends or attendants, and they should wash their hands and mouths frequently and thoroughly.

Milk or meat derived from tuberculous animals should not be used, and dust, which may at any time contain the germs of tuberculosis, should be kept down as far as possible, both in houses and in streets. Above all, every means of direct conveyance of the fresh virulent microbes from persons having the disease to new victims should be carefully avoided. Some of these means are kissing and coughing, by which latter minute infectious particles may be thrown to a distance and caught upon the face or hands of friends, or upon food, tableware, or linen; and 
any lack of absolute cleanliness in washing dishes, cups, spoons, napkins, etc., recently used by consumptives, is to be scrupulously avoided. Those, also, who do the washing need to be on their guard against infection, by exercising extreme care and cleanliness.

There are many other forms of tuberculosis besides consumption, but this is the form of principal interest to students beginning the study of hygiene and sanitation.

4. Hygiene in the Treatment of Tuberculosis. - While consumption is the cause of so many deaths, it is not necessarily a fatal disease. The Bacillus tuberculosis is usually of very slow growth and low virulence; it does not, like some microbes, produce large quantities of poisonous toxins which, upon entering the blood, cause rapid and extensive injuries to most organs. On the contrary, its action is at first largely confined to the spot in the lungs where it has gained a lodgment, and at the outset the constitutional disturbances are slight. So insidious is the attack and growth of the germ that the patient does not at first even suspect its presence, and merely feels "out of sorts" or "run down." Only later, when the pathological processes have spread over a considerable area of tissue, are the symptoms serious; and frequently the disease is not recognized until irreparable damage is done.

It is chiefly for this reason that consumption claims so many victims; for the inroads of the disease are by no means unresisted by the living cells of the body. From the outset a struggle between these cells and the invading microbes takes place; and it should be better known than it is that in the majority of cases the human mechanism is the victor in the struggle. This is shown by the fact that autopsies on persons who have died of other diseases disclose in a surprisingly large percentage of cases healed tuberculous lesions, where the presence of the disease had not been suspected. In other words, the disease moves on 
to a fatal issue only when the vital resistance proves unequal to the defense, and the mortality from consumption would undoubtedly be exceedingly low were sufficient attention paid to the hygienic care of the body and the sanitation of its surroundings, by both of which the vital resistance is powerfully reënforced.

This is in itself a powerful argument for attention to general hygiene, and it points out unmistakably the hygienic treatment of the disease when once recognized. No reliance whatever should be placed in drugs. On the contrary, the patient, his family, and friends should recognize that the one hope lies in the hygienic conduct of life. The patient should live and sleep out of doors, if possible; he should fearlessly breathe cold air, but should protect the skin from chilling by warm clothing; if he cannot live out of doors, the windows of the living and sleeping room should be kept wide open, even in winter weather; the sleeping room should have light walls, and all curtains and draperies which limit the amount of sunlight should be dispensed with; the furniture of the room should be reduced to a minimum and should be such as can be easily cleaned; rest from anything but very moderate muscular activity, and from nervous strain, is absolutely essential; and all these measures should be reënforced by abundant feeding with appetizing and easily digested food; the feeding, indeed, should be pushed to the extreme, should even be forced feeding, but only with easily digestible foods. In brief, rest, fresh and cool air, sunshine, and abundant food are the cures for tuberculosis, and, unless the disease has gone too far before it is recognized, they are almost certain cures.

While some climates are more favorable for the treatment of tuberculosis than others, - a cold, dry climate being preferable, - it should be understood that the treatment we have outlined has been used with excellent results 
even in the patient's own home, wherever that may be; and this hygienic treatment should be employed whether or not it is possible to go away from home.

Continuous high temperature and high humidity, or dampness, are the main conditions which make change of climate desirable; and where these are not present it is generally better to treat the patient at his own home, where family and friends may not only minister to his needs but may also maintain those cheerful surroundings which count for so much both in sickness and in health.

5. Typhoid Fever. - This disease is now believed to be due to the bacterial microbe known to bacteriologists as

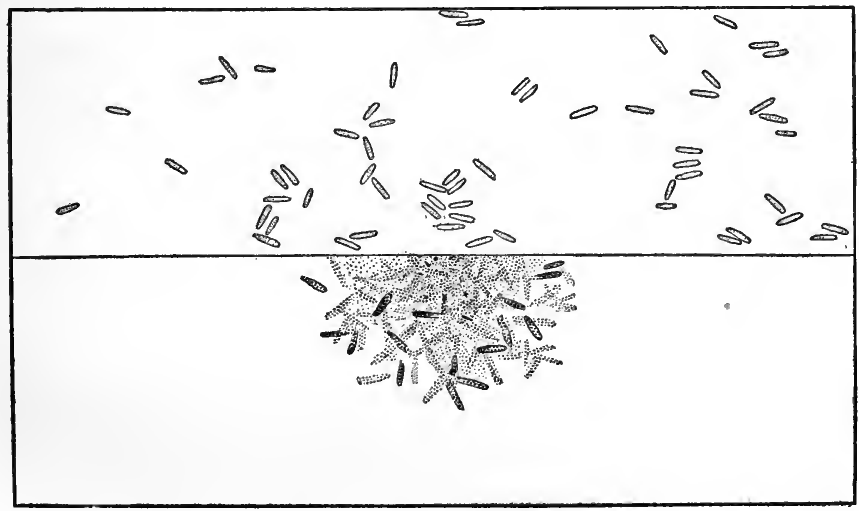

FIG. 124. Typhoid fever germs (Bacillus typhi). (Highly magnified) Above, swimming free in normal blood serum ; below, " clumped" or "agglutinated" in the serum of a typhoid fever patient

Bacillus typhi. The latter was observed by Koch, Eberth, and others about 1879, but first thoroughly worked out by a pupil of Koch, Dr. Gaffky, in 1884 .

Typhoid fever has been well known since about 1840 , previous to which time it was confused with typhus (jail, spotted, or ship) fever, - a disease now not often met with. It is one of the worst maladies that afflicts mankind, for 
although not generally fatal in more than about ten per cent of the cases, it is a slow fever, disabling the patient even when it does not kill, and requiring weeks and often months for its course and for convalescence. It is widely distributed, probably all over the world; and, although less widespread than tuberculosis, it is still one of the chief causes of death. It is a disease which seriously damages the intestine and is one form of what is sometimes called " inflammation of the bowels." The microbe finds its way into the alimentary canal with food or drink, and is believed to multiply in the intestine and to invade the body proper, producing a poisonous substance (typhotoxin) which causes the fever and otherwise injures the whole organism. The germs are probably cast off in abundance with the various excreta, and as diarrhea is a frequent (though not invariable) accompaniment of the disease, typhoid fever is called a diarrheal disease. At its beginning and, in mild attacks, throughout the whole disease, the victim may not be confined to his bed, but may "keep about." Such cases are known as "walking" typhoid.

6. How Typhoid Fever is spread. - Since the germs of this disease are present in the excreta, - both urine and fæces, and even in the saliva, - it has been well called a " filth" disease. The old idea of a filth disease, however, was that filth bred disease, and that almost any heap of dirt or rotting material might generate disease, especially typhoid fever, and inflict it on persons in the vicinity. This idea is now abandoned, for it is held that the germs of any one kind or species of disease can come only from other germs of the same kind; that is to say, typhoid fever can come only from a person or persons now having, or having recently had, that disease. The excreta of such persons may, nevertheless, readily convey it; and if food or drink are polluted in any way by such excreta, then the germs readily find access to fresh victims. 
Unfortunately, food and drink are oftener polluted than most persons realize. Water may be contaminated by sewage; milk, by the dirty hands of careless or unclean milkers ; oysters growing in harbors or estuaries, by city sewers discharging therein; vegetables, by manure; and fruits or berries, by filthy hands. When we stop to think that filth. may readily find access to food and drink in these and many other ways, it is clear that typhoid fever may still be called a filth disease, even if we understand by the term that it is a disease conveyed by infected filth, but not bred or generated by filth alone.

7. The Prevention of Typhoid Fever. - This disease can be vastly reduced in amount and destructiveness in any community in which it abounds, by careful attention to the avoidance and destruction of its microbes, and by maintaining high vital resistance through hygienic and wholesome living. No means are yet known of vaccinating against its attacks, as is done for smallpox, nor has any antitoxic serum yet been discovered effective for its cure, as is antitoxin for diphtheria.

The microbes of typhoid fever may generally be avoided by the use of pure drinking water, pure milk, clean vegetables and fruits, raw oysters derived only from harbors and estuaries free from sewage, and, in general, by the use of pure foods and drinks. Microbes are readily destroyed by cooking at a high temperature and, in the case of the excreta of patients suffering from typhoid, by disinfection, which, under the direction of an attending physician, should always be thoroughly carried out.

It should never be forgotten that, contrary to the general impression, typhoid fever is really contagious, i.e. may be "taken" by contact, not necessarily with the patient, but very readily with his excreta, or with his linen, or with any of his belongings soiled with his excreta. Even trained nurses sometimes seem to forget this fact, for not 
infrequently a trained nurse contracts the disease from her patient. Similar secondary cases of typhoid fever, especially in families in which the mother or sister attends the patient and at the same time prepares food for the rest of the family, are painfully common.

It has been proved again and again that persons overworked or otherwise in poor condition are those most susceptible to this disease, and we could hardly have a better instance than typhoid fever affords of the double duty which a sound hygiene imposes upon us, namely, $(a)$ the duty of avoiding infection and $(b)$ the duty of keeping the mechanism in such good condition that if by accident infection occurs, the microbes shall not be able to overcome its vital resistance.

8. Diphtheria. - This disease has long been known under the name of "membranous croup" and "malignant sore throat," but it was not until 1884 that Loeffler, one of the pupils of Koch, detected the microbe now generally agreed to be its sole and only cause (Fig. 125).

It is believed that this microbe, Bacillus diphtherioe, finding lodgment in the throat of a susceptible person, grows and multiplies there, secreting meanwhile a poisonous substance, or toxin, which injures the tissues of the throat and causes the formation of the white spots and false membrane so characteristic of the disease, and also damages the rest of the body after its absorption into the circulation, by producing fever, paralysis of particular parts, and sometimes death.

9. How Diphtheria is spread. - Inasmuch as diphtheria is a disease of the throat especially, it is easy to see that it must be chiefly conveyed from one person to another by sputum and by objects which come in contact with the mouth or lips. Kissing and fondling among children, or parents and children; fingers, which, especially in children, are too often in the mouth; books, handkerchiefs, pencils, 
playthings, and food bitten and passed from hand to hand, - all these may be vehicles of contagion and infection in this microbic disease. It may be spread by dried sputum

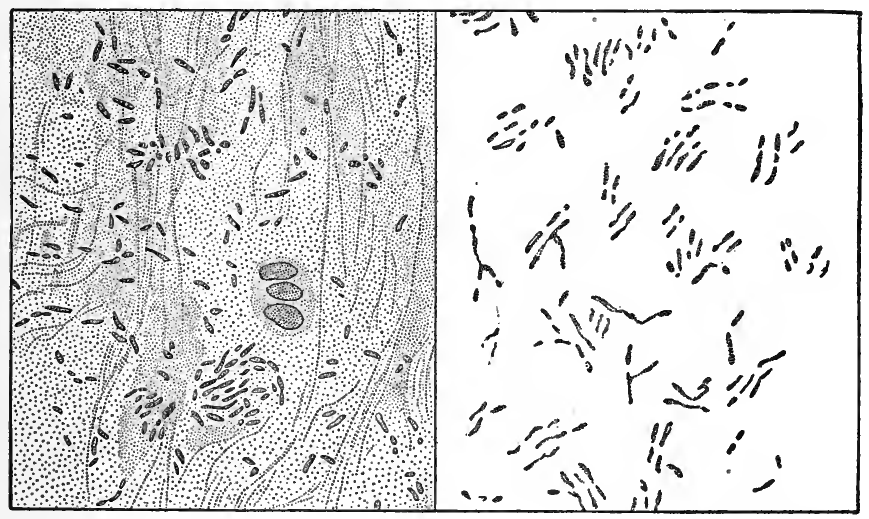

Fig. 125. Microbes of diphtheria and croup (Bacillus diphtheria)). (Highly magnified)

On the left, as they appear under the microscope in discharges from the throat of a diphtheritic patient. On the right, after cultivation in the bacteriological laboratory

in dust, by milk infected by milkers suffering with the disease, by infected food, and possibly by pets, such as cats and dogs and even birds, suffering from the disease.

10. The Prevention of Diphtheria. - Diphtheria being primarily a disease of the throat, and therefore distributed both by personal contact and by spittle, it would seem to suffice for its prevention to isolate patients having the disease as long as they are capable of communicating it to others, and thus cut off the escape and distribution of the germs. Unfortunately, however, in this, as in many other infectious diseases, persons often have the disease for some time before they or their friends discover the fact; and some mild cases of this malady (as also of typhoid fever and other infectious diseases) probably occur and run their 
course without ever having revealed their true character. Hence arises the difficulty of accounting for the origin of some apparently inexplicable cases, and also the difficulty of stamping out a disease of this kind. Diphtheria is not an eruptive disease, and ought, therefore, to be more readily controlled than smallpox, scarlet fever, or measles, which are doubtless most often disseminated by means of scales shed off from the skin during the "peeling" which follows the eruption.

It should be clearly understood that all kissing by persons having sore throats, or the mouthing of pencils or other objects by children, is a dangerous practice ; and that fingers, which so readily find their way into mouths, may as easily as not carry infection to books, playthings, food, letters, or other objects which are " handled." Letters sealed or handled by diphtheritic patients, or by persons attending them, have probably at times conveyed the germs of disease to persons at a distance in whom the appearance of the illness seemed quite unaccountable. Here also, as in tuberculosis, care in the disposal of sputum is of great importance.

Within a decade the discovery of an antitoxic serum, antitoxin (see p. 499), has given us a novel and invaluable means of defense against the microbes of diphtheria by increasing the resistance of the human body so that it shall be no longer susceptible to the disease. But as this discovery means much more to hygiene than the control of this one disease, we shall devote to its careful consideration an entire paragraph beyond.

11. The Spitting Nuisance, as the habit of public spitting is often called, is not only a disgusting nuisance but a real menace to the public health, because, as will now readily be seen, it may be the means of spreading abroad diseases, such as tuberculosis and diphtheria, with which many persons - incipient cases, or "walking" cases_may be 
moving about. Fortunately, the habit is chiefly confined to one sex, and this fact shows how easily it might be controlled if custom demanded.

12. Malarial Fever, or "Malaria," is a world-famous disease, especially common in warm climates but also frequently occurring in the more temperate zones. It is probably by far the most important of all tropical diseases, for while it does not kill as do yellow fever and Asiatic chol- 1 era, it is much more common and disables a far greater number of victims.

Malaria has long been associated in the popular mind with low grounds and swamps. Experience has shown, however, that it cannot be caused by swamps alone, for many swamps and marshes are entirely free from malaria. Sometimes it has seemed to go with the digging up of earth; yet the earth has very often been opened and thrown about without causing any malaria whatever.

The true source of this disease remained an absolute mystery until, in 1880, Laveran, a French investigator resident in Algiers, discovered in the blood of malarial patients a microbe of a very peculiar kind, - not a bacterium, nor even a plant, like the microbes of tuberculosis, typhoid fever, and diphtheria, but an animal known as a hoematozoön, or sporozoön, belonging among the simplest animals, or protozoa (see p. 469). Figure 126 gives illustrations of some of the various forms of the microbe, and its life history is outlined in the description of the figure.

But even after the microbe of malaria had been detected no one knew, for ten years longer, whence it came, or whether it lived outside of man at all, or, if not, how it was conveyed from victim to victim. When, at last, in 1899, as if for a final benediction upon mankind by a century wonderfully rich in human and especially in medical progress, the whole subject was cleared up, the solution of the riddle was found to be as simple as it was unexpected, for the 


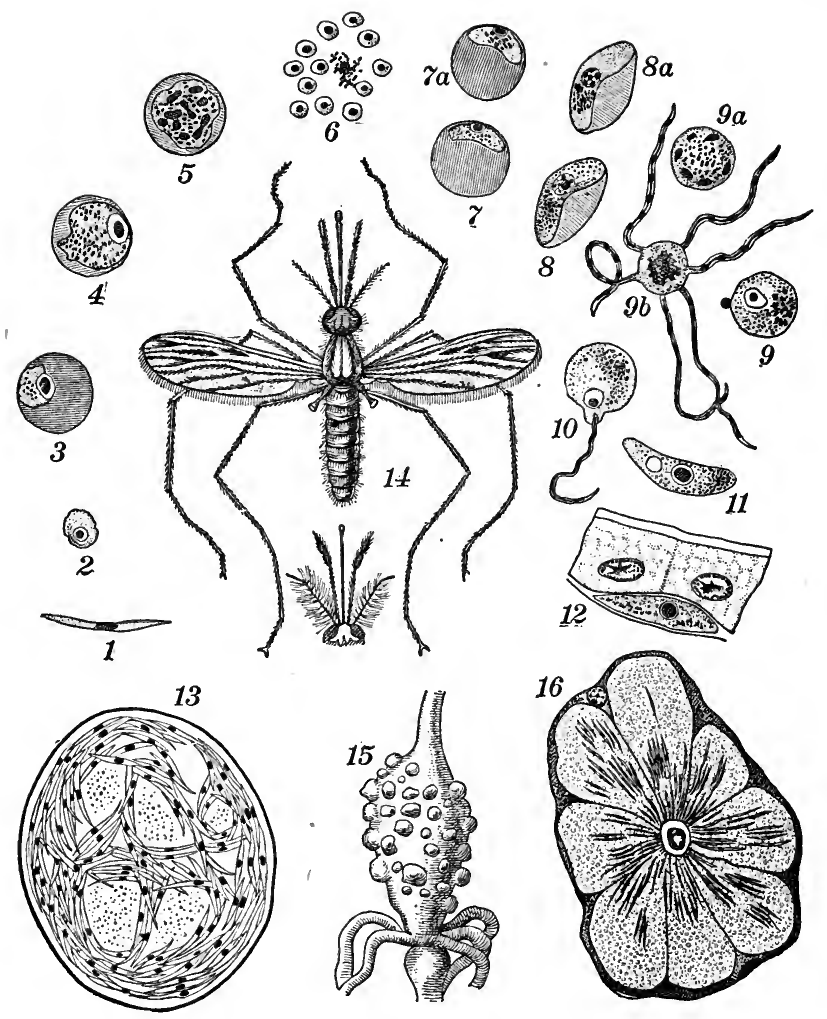

FIG. 126. The malaria microbe (Plasmodium malarice) and a malarial mosquito. After Leuckart-Chun's wall diagram

1 , the microbe; $2-5$, its growth in a red blood corpuscle; 6 , its multiplication and escape from the corpuscle (it is now ready to infect fresh blood corpuscles) ; 7, 8, crescentic forms of the microbe. For further development the microbe must be transferred from man to mosquito (by a biting, i.e. bloodsucking, mosquito).

In the stomach cavity of Anopheles: 9, female stage of the microbe; $9 a, 9 b$, male stage; 10 , union of 9 with one of the vibratile arms of $9 b ; 11$, the microbe resulting from 10.

In the body proper of Anopheles: 12, The microbe (11) has penetrated the stomach wall of the mosquito and embedded itself on the outer (body) side of the stomach. Here it undergoes a process of growth, cell division, and multiplication (13), eventually forming " tumors" on the outside (body side) of the mosquito's stomach, as shown in 15. From these tumors the microbes escape into the body cavity of the mosquito and find their way into the salivary glands (shown in section in 16). From these they are readily transferred (with the saliva) into a human body bitten by Anopheles.

14, female malarial mosquito (head of male below). 
vehicle proved to be a common insect, a special mosquito, long known as a pest, but never hitherto suspected or dreaded as a bearer of disease. At once it became clear why malaria is a disease of some warm climates and some seasons, and why it "hangs about" some swamps and not about others.

13. How Malaria is spread. - The malarial microbe is a microparasite (p. 467), spending a part of its life in man and a part in certain mosquitoes, which are thus its "hosts." A mosquito of the right kind bites and sucks the blood of a man having malaria, and having thus become infected bites other persons, injecting into them germs of malaria, along with that poison which causes the familiar swelling often following a mosquito bite.

It is important to note that only one genus of mosquito (Anopheles), and that not the commonest in most places, seems capable of conveying the disease. Moreover, it is

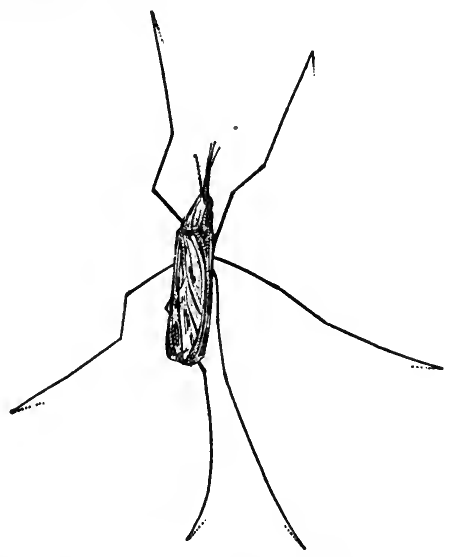

Fig. 127. Anopheles punctipennis (female). After a photograph from life by W. Lyman Underwood

Common in the northern United States only the female Anopheles - and even that only after it has become infected by biting a person having the disease - which can transmit malaria. Hence many mosquitoes, even if Anopheles, are harmless, as are all mosquitoes in regions in, which no Anopheles or no malarial microbes exist. For the causation of malaria three things are required, namely, (a) malarial microbes, $(b)$ female Anopheles, and $(c)$ susceptible victims. Fortunately the first two do not always coexist, and in case either is missing malaria 
is absent. Many places abounding in mosquitoes have no malaria, either because there are no Anopheles, or be-
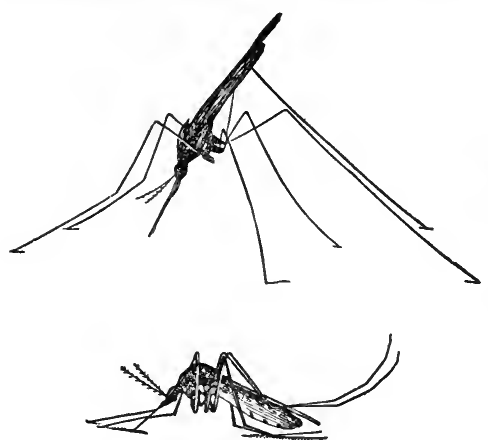

FIg. 128. The malaria mosquito (Anopheles) above, and the common mosquito (Culex) below. After photographs from life by W. Lyman Underwood

Showing a characteristic difference in the resting attitudes cause the Anopheles present have never become infected.

14. The Prevention of Malaria. - Beyond a general reënforcement of the body by wholesome living, the only means yet known of avoiding this disease is the avoidance of mosquitoes in those regions in which they abound, and in which malaria also occurs. If a region contains no malaria, the mosquitoes in it cannot produce the disease. If the region is malarious but has no malarial mosquitoes, no fresh cases, so far as known, can occur. But if malarial fever and malarial mosquitoes coexist, then the only hope is to remove one or the other, and if possible both. For relief from malaria already fastened upon a patient, application should be made to a physician. For the extermination of mosquitoes from a neighborhood, all swamps and marshes must be drained, and pools of stagnant water either treated with crude petroleum or stocked with fishes that will feed upon and destroy the mosquito larvæ. ${ }^{1}$

15. Yellow Fever is an infection greatly dreaded in the tropics. It is now attributed to a microbe somewhat similar

1 For further information on this subject see Dr. L. O. Howard, Mosquitoes, How they live, How they carry Disease, etc., New York (McClure), 1901; W. L. Underwood, Mosquitoes and their Extermination, Boston (W. B. Clarke), 1903; Celli, Malaria according to the New Researches, New York (Longmans), 1900. 
to that of malaria, and which is believed to be conveyed, like the malarial microbe, by a mosquito, though of another kind, Stegomyia by name. If this view is correct, yellow fever is to be prevented only by avoiding these mosquitoes. Havana, which was formerly cursed by yellow fever, has been virtually freed from it by preventive action based wholly on the mosquito theory of its causation; and the suppression of yellow fever in Cuban ports, from which formerly the disease was frequently exported to the United States, means much to the southern states of the Union.

In 1905 a disastrous outbreak of yellow fever occurred in New Orleans and vicinity. Its germs were probably brought in from

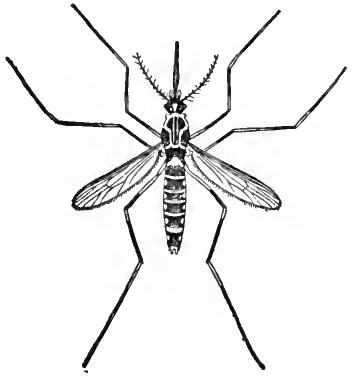

Fig. 129. The yellow fever mosquito (Stegomyia). After a drawing by $\mathrm{L}$. $\mathrm{O}$. Howard some infected district nearer the tropics by mosquitoes (Stegomyia) on ships and on fruit (bunches of bananas). The epidemic was fought wholly upon the mosquito theory, and with results entirely satisfactory to the sanitarians in charge.

16. Some Other Infectious or Contagious Diseases, and how they are supposed to be spread. - In the case of some of the commonest infectious or contagious diseases we are still in the dark both as to their precise causation and the ways in which they are scattered abroad. Concerning measles, chicken pox, and whooping cough, for example, we are still awaiting such discoveries as have already been made for tuberculosis and the other diseases described above.

We have also referred above (p. 380) to the fact that some colds or influenzas appear to be infectious. Attempts have been made to detect the microbes of the "grippe" 
and other influenzas, and figures are often given of germs found associated with disorders of this class. Figure 130 is an example of this kind, although it is not safe to say positively that the microbes chiefly concerned have really been as yet identified.

But while we patiently wait for more light, we have good reason, because of their general character, to believe that these also are microbic diseases, caused likewise by microparasites transmitted either directly or indirectly from

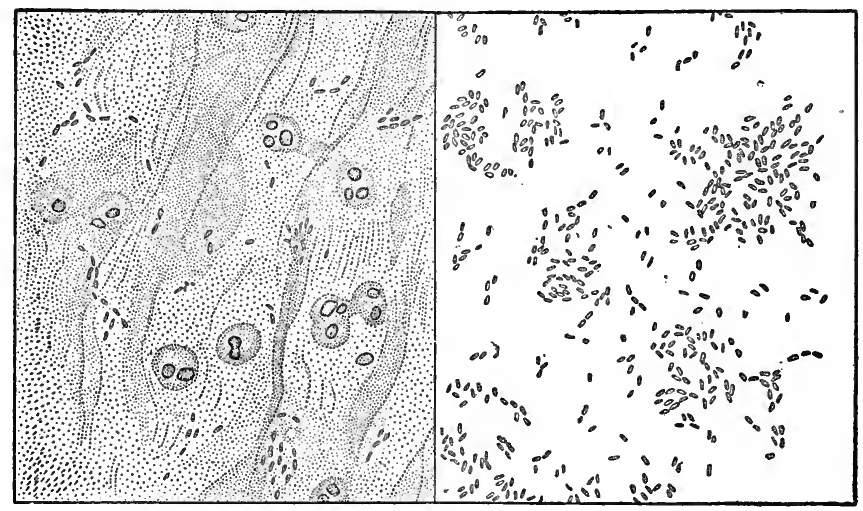

FIG. 130. A so-called "influenza bacillus"

On the left, as found in the sputum in some "colds"; on the right, after cultivation in the bacteriological laboratory

victim to victim. Experience has shown that the same kind of effort which tends to prevent diseases undoubtedly microbic tends to prevent these also ; so that at present, and for all practical purposes, we may consider that we know enough about their causation and spreading to enable us to deal with them intelligently, fearlessly, and hopefully.

17. Scarlet Fever, Measles, and Chicken Pox belong to the group of eruptive diseases, a term derived from the fact that persons having any of them are usually, sooner or 
later, "broken out," or more or less covered with an eruption, or rash. When this eruption heals, scales of the skin are shed off, and the wide dissemination of these scales during the process of "peeling" is believed to be one reason why eruptive diseases are more contagious than some microbic diseases (like tuberculosis and diphtheria) which are not eruptive. Persons suffering from an eruptive disease should not be allowed to go about among other people until they have ceased peeling.

As for the prevention of measles, chicken pox, scarlet fever, and whooping cough, the only means at hand at present seem to be isolation and non-intercourse. To maintain or increase the resistance of the body no better means are known than good feeding, temperate living, and, in general, a wise and wholesome conduct of life; yet even so, immunity is often purchased only at the cost of one or more attacks, and prevention by isolation is frequently difficult under the conditions of modern life, especially in tenements and crowded districts.

18. Summer Complaint in Children is a severe and often dangerous summer diarrhea, believed to be caused by microbes and apparently due in part to wrong feeding. In cities it appears to be closely connected with the use of stale milk, and is often prevented or overcome by using very fresh milk, or even by pasteurizing ordinary milk (p. 475). This is readily done by immersing bottles of milk in water and then heating the water to a temperature of about $160^{\circ} \mathrm{F}$. for five minutes. If no thermometer is available, it will suffice to bring the milk nearly (but not quite) to the boiling point and keep it at that temperature for a few minutes.

19. Smallpox, a disease once so common that "scarce one in a thousand" escaped it, is now happily rare in most highly civilized countries. It is an eruptive fever, small pustules or pocks giving it its name, and while not 
as fatal as some of the diseases shortly to be considered, it is peculiarly loathsome and very apt to leave after it lifelong disfigurement, or pitting. It is hard to realize to-day the dread and fear with which our ancestors rightly regarded smallpox, and for this reason some people make much more of the slight discomfort and insignificant danger of vaccination than of the loathsome smallpox itself; but if communities ever cease to take the simple but indispensable steps required to prevent smallpox, outbreaks of the disease will undoubtedly remind them in alarming fashion of what they had previously escaped.

It is not yet absolutely proved, although it is now generally believed, that smallpox is caused by microbic activity; but it is certain that it is extremely contagious, probably through the scales cast off from the skin of those suffering from it, with which scales the specific microbes are blown or handed about.

Experience has shown that great gain results from "isolating" or "quarantining" smallpox patients in a detention hospital (to which the name of "pesthouse" was formerly given). Smallpox patients are thus removed and isolated, while those suffering from typhoid fever or consumption are not, partly because of the much greater contagiousness of smallpox, and partly because of its graver and more loathsome character.

In smallpox, as in other diseases, wholesome living diminishes the danger of infection; but it is a matter of history that in the days when the disease was prevalent and the question was put to the severest test, general good health proved an unreliable defense. Fortunately, however, the human race, which was once so frightfully scourged by this disease, has discovered a more certain means of protection, which consists not in the warding off or destruction of microbes but in an enhancement of the powers of resistance of the organism, so remarkable as 
to constitute for extended periods virtual exemption, or immunity, from smallpox. The methods by which this extraordinary result is reached are known as inoculation and vaccination.

20. Immunity. - The best of all defenses against any disease would be complete insusceptibility, or immunity, to it; for no matter how ingenious, elaborate, or complete the devices may be for preventing disease germs from finding access to the body, accidents may always happen which will allow them to enter. Immunity, or insusceptibility, to disease is therefore one of the principal aims of hygiene, one of the goals of sanitary science. Unfortunately, natural immunity is not common, and artificial immunity is not easily conferred or acquired, except in the case of one or two diseases; but there is good reason to hope that the future may have in store for the human race great gains in this direction.

Natural immunity means a natural insusceptibility to disease. It is usually constitutional and inherited. The lower animals, for example, are not susceptible to typhoid fever, and birds are immune to anthrax, although mammals take it readily. Diseases common to many species of animals appear to be the exception. In general, each species is immune to many, if not most, of the diseases of other species.

By artificial immunity is meant a similar exemption from disease, not constitutional or inherited, but acquired in one way or another. The most familiar method of becoming immune to any disease is to have the disease in question. For example, long before inoculation and vaccination were known, it was well recognized that persons who had once had smallpox were not likely to have it a second time, and such persons were, and still are, in demand as nurses for cases of that disease. Again, children who have had scarlet fever or measles or whooping cough are believed 
(and rightly) for that reason to be less likely to have the same disease a second time. There can be no question that protection is generally secured in this way, although cases are not rare in which such protection, even if once secured, is ultimately lost; for people sometimes have measles, typhoid fever, and diphtheria twice, or even oftener.

21. Inoculation for Smallpox. - The first great step towards the prevention of infectious diseases by producing artificial immunity from them was that of inoculation for smallpox.

For a century after the first English settlements in this country smallpox ravaged Europe and America. But in 1721 a novel and ingenious method of producing immunity from the disease was introduced into England from Constantinople, and quickly reached the United States. This method, known as inoculation, consisted in inoculating persons while in good health with the virus of true smallpox (not vaccine), for the purpose of causing them to undergo a mild attack of the disease while well and in good condition, so that they might avoid having a severe attack when unwell and in poor condition. Inoculation for smallpox was an effective preventive and met with wide acceptance and approval both in England and in the United States. It was extensively practiced for nearly a century, but was finally supplanted by the much safer process of vaccination, in which the inoculation was with vaccine (the mild and comparatively harmless virus of cowpox), instead of with the always dangerous smallpox virus.

22. Vaccination, one of the greatest blessings ever conferred upon mankind, was first invented in England in 1796 by Edward Jenner, a young physician of Gloucestershire. It consists in the inoculation of the human being with virus derived from a cow having cowpox. A spot, usually upon the upper arm, is scraped by a lancet, so that the outer layers of the epidermis are removed; the spot 
is then rubbed with an ivory point, quill, or tube, carrying the virus. A slight and usually unimportant illness or indisposition follows, and the arm is sore for a time, a characteristic scar remaining. In some cases the illness is more serious; but deaths plainly due to mere vaccination very rarely, if ever, occur.

The immunity from smallpox produced by vaccination is remarkable, and has been proved over and over again, not only by the experience of armies and nations, but also by actual experiment. It was formerly thought that "once vaccinated" was "always protected"; but to-day it is recognized that occasional revaccination is essential to complete immunity, the length of the period of protection usually fixed nowadays being not more than ten years. Indeed, so variable is the duration of the immunity in different individuals, and in the same individual at different times, that the only safe course is to revaccinate whenever there is an appearance of smallpox in the community. It should also be remembered that the vaccination may fail to "take" merely because the virus has been rubbed off by the clothing, or because it was not effective to begin with. Consequently when any vaccination fails to "take," it is safest to try again a second or even a third time, especially if we are unusually exposed to the contagion of the disease.

\section{Diphtheria Antitoxin, and Other Antitoxic Serums. -} As has been said above, inoculation against smallpox was begun in western Europe and America about 1721, and vaccination for the same disease at about the beginning of the nineteenth century (1796). No further progress was made in the art of immunizing until, in 1879, Pasteur succeeded in extending vaccination to some species of the lower animals, upon which he conferred immunity from certain diseases by using a modified, or as he called-it, attenuated, virus of the disease itself. 
A much more important discovery than this of Pasteur was made in 1892 when von Behring, a German bacteriologist, found that the serum of the blood of an animal immune to diphtheria differs from that of one not immune, in that it is capable of neutralizing, both in a test tube and in the body of another animal, the poison (toxin) produced by diphtheria germs. This great discovery naturally led to the use of such neutralizing, antidotal, or antitoxic serum (antitoxin) in cases of diphtheria in man, and such use of it has now become general.

In order to obtain the antitoxin, horses are inoculated hypodermically with virus, or toxin, ${ }^{1}$ of diphtheria (from which all germs have been removed), at first in small doses but gradually with larger amounts, until they have become immune to heavy doses. The serum of the horse's blood under this treatment gradually becomes changed, so that it possesses antitoxic or antidotal properties. This serum (or antitoxin) is then carefully collected, bottled, and afterwards used to cure, and sometimes to prevent, cases of diphtheria in human beings.

Von Behring's discovery is probably one of the most beneficent ever made, because it has pointed out the way for the prevention or cure of other infectious diseases, by showing that when the disease is due to a toxin it may be possible in any case to produce an antidote (antitoxin) which shall neutralize and overcome it. Good progress

1 If the animal were inoculated with the germ of diphtheria, instead of its toxin, we should have no control of its growth and the severity of the disease produced. By inoculating with carefully measured doses of the toxin, however, - which does not increase in amount, - we may produce the symptoms of diphtheria in very mild form, and always have the course of the disease under control. Immunity is gradually acquired with but trifling discomfort to the animal, and the antitoxic serum is absolutely free from the germs of the disease. The statement sometimes made, that the use of antitoxin is liable to produce diphtheria because the animal was inoculated with the germs of that disease, is untrue, because such germs are never present in antitoxin properly made. 
has, indeed, been made already in this direction for a few other diseases than diphtheria, especially for tetanus (lockjaw).

24. Tetanus, or Lockjaw, is a comparatively rare disease, although in America about the Fourth of July it is quite common among boys as a consequence of accidents attending the celebration of that anniversary. The disease is a

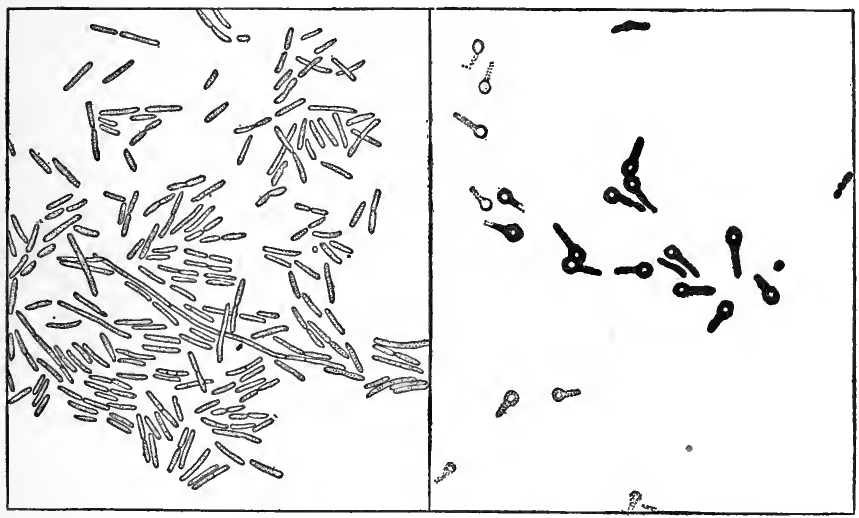

Fig. 131. Microbes of lockjaw (tetanus). After Kolle and Wassermann On the left, in the ordinary rodlike stage of active growth; on the right, after having formed resting "spores" in the resting stage

peculiar one, and prolonged muscular contractions or spasms are a characteristic symptom of its advanced stages. These spasms may cause the lower jaw to become more or less set or fixed ; hence the popular name, "lockjaw."

The microbe of tetanus is well known, and is common in the intestine of herbivorous animals and in dirt in many places. It grows best in the absence of oxygen, and deep or lacerated wounds, such as are made by toy pistols, etc., appear specially to favor its development.

25. Asiatic Cholera is a microbic fever formerly greatly dreaded all over the civilized world, and still very destructive of human life in the East, - for example, in the 
Philippine Islands. The germ of the disease was discovered by Koch in 1883, in the bowel discharges of cholera patients in Egypt. Cholera appeared in Hamburg, Germany, in epidemic form as late as 1892 , causing great alarm and about eight thousand deaths. It is easily prevented by the same means used to prevent typhoid fever

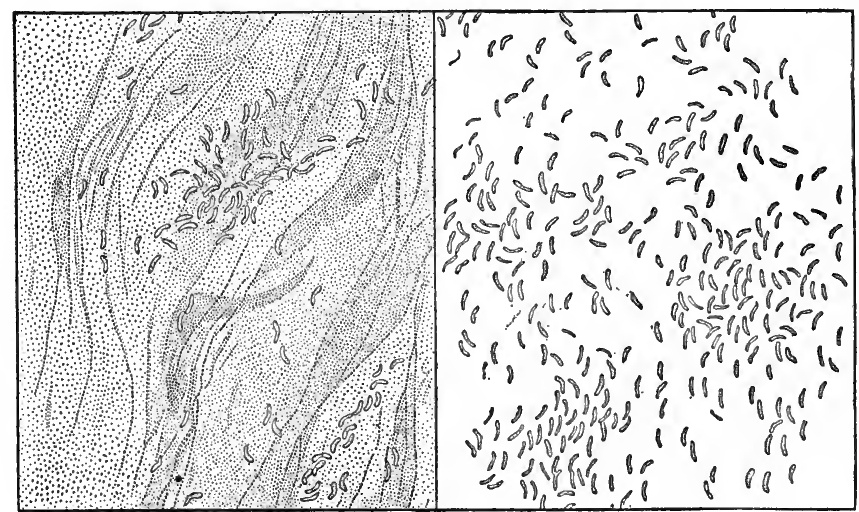

Fig. 132. Microbes of Asiatic cholera

On the left, as they occur in the feces of victims of the disease; on the right, after cultivation in the bacteriological laboratory

(p. 485), and need no longer be greatly feared in any clean and well-ordered community supplied with pure food and pure water.

26. The Plague (Bubonic Plague) is the most famous of all the great epidemic diseases of history. It has repeatedly ravaged Europe, and is still very common in some parts of Asia, such as India and China. The Black Death, which was probably a severe form of the bubonic plague, was a severe epidemic disease of the fourteenth century, when it is said to have killed off twenty-five millions of people, or from one fourth to one half of the entire population of Europe. 
The bubonic plague is believed to be transmitted through the agency of rats and fleas, - rats, like human beings, being susceptible to the disease. Rats having the plague are supposed to be bitten by fleas, which thus become infected and, later, feeding upon human beings, inoculate them with the germs of the disease. In order to prevent

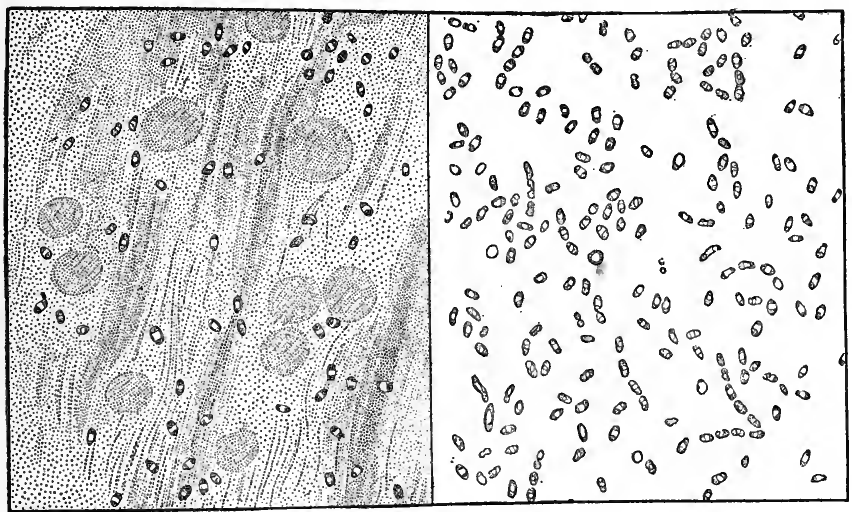

Fig. 133. Microbes of plague

On the left, as they occur in the swollen lymph glands; on the right, after cultivation in the bacteriological laboratory

the plague it is important, therefore, to do away as far as possible with fleas and rats ; and in plague-stricken districts preventive measures are directed chiefly to the destruction of rats. In the Philippines, for example, rat catching is an important branch of sanitary work in combating the plague.

27. The Care of Wounds. - Proper care is usually taken of a severe wound by the physician or surgeon who is summoned to attend the case. Slight cuts, on the other hand, are frequently neglected as trivial affairs; and without question these cuts usually heal with no bad after-effects, either because no pathogenic organisms are introduced, or because, if introduced, they are killed by one or another 
of the means of defense possessed by the body against microbic invasion. Infections from such wounds are, however, by no means of rare occurrence, and it is safer to care for them whenever possible. The wound should be washed thoroughly with some antiseptic, - such as a solution of corrosive sublimate (1 to 1000) or carbolic acid ( 1 to 40$)$, - and then protected by absorbent cotton until healed. Since the bacillus of tetanus grows only in the absence of oxygen, it is generally safer not to close the wound with anything like collodion, which entirely prevents access of air. The bleeding from an ordinary cut presents no danger of undue loss of blood from the body, and, by washing out the wound before clotting takes place, is an important safeguard against infection. 


\section{CHAPTER XXXI!}

\section{PUBLIC SUPPLIES OF FOOD, WATER, AND GAS. PUBLIC SEWERAGE}

1. Communities and Public Supplies. - Communities (Latin, communis, common) may be of neighborhoods, villages, towns, or cities, and are so called because they possess many things, such as roads, bridges, post offices, churches, and general situation, in common. Some supplies, such as air, sunlight, and rainfall, are naturally and necessarily public and shared in common. Others, such as water, ice, gas, fuel, milk and other foods, may or may not be so shared. In villages some families keep their own cows, while some buy their milk from their neighbors, or from a milkman supplying several families; but in cities few people keep cows, while most people buy milk. In villages private wells abound, but in large communities most people give up private wells or springs and use a public water supply.

Public supplies and public services are designed to meet those wants or demands which many families have in common, and the chief supplies are those of water, ice, gas, and milk. Fuel, transportation facilities, libraries, parks, playgrounds, baths, laundries, bakeries, and the like are examples of other public supplies or services. Formerly in rural life each family was comparatively independent, but the modern town or city family, and to a great extent the rural family, depend upon some public supply for nearly everything that it has or uses; for books, upon public libraries or bookshops; for clothing, 
furniture, household utensils, etc., upon shops or stores; for food, upon markets; and sometimes even for housing, upon hotels or other public houses.

2. Public Supplies as Public Conveniences and Safeguards. - When such public supplies or services are well regulated, cheap, and abundant they may often be superior in safety, comfort, and convenience to private arrangements for the same purpose. In a city it is easier and cheaper to buy milk than it is to keep a cow. It is also better to do so, because cows in cities must be under unnatural, if not unwholesome, conditions, and the milk may suffer in quality. It is more convenient to draw water from a tap than from a well, and city wells are generally objectionable because usually subject to contamination. It is more convenient, more cleanly, and safer in a city to connect a house with a good sewer than to supply it with a privy and a sink drain. Public supplies may thus serve not only as conveniences but also as sanitary safeguards.

3. Public Supplies as Public Dangers. - On the other hand, public supplies must be well arranged and well regulated, or they may become sources of public danger. If the water supply, for example, is allowed to become polluted, a whole community may be stricken with typhoid fever or some other infectious disease. Hundreds of cases of typhoid fever have been known to occur among the customers of a single milkman whose milk supply had become infected. Sewage-polluted raw oysters have been known to cause the illness of dozens of persons at a single public banquet.

It is easy. to see that the very convenience and widespread use of public supplies which are not pure, makes them doubly dangerous. If a private supply becomes polluted, ordinarily only a single family suffers; but if a public supply is impure, hundreds or even thousands of persons may perish. The moral is plain: the purity of 
public supplies should be thoroughly established at the outset and carefully maintained. If, as is often the case, public supplies are owned or controlled by the municipality, then no persons should be put in charge of them who are mere politicians, or in any other way unfit to act as guardians of the public welfare. Expert scientific supervision of public supplies is indispensable for efficiency, for economy, and for public safety.

4. Food Supplies, Public and Private. - The supply of foods to families or individuals may be largely from private sources, as in the case of a farm upon which many foods may be produced. But with the growth of cities and large towns food supplies are more and more shared in common by many persons or families; while certain necessaries or luxuries of life, such as fish, sugar, salt, tea, coffee, spices, are, with rare exceptions, always obtained from public supplies.

Furthermore, even on the farm, specialization often leads to the raising of only one thing or a few things, such as cotton, corn, or wheat, and so to dependence on public supplies for other things which might be raised if it were worth while. For example, most farmers in New England might cultivate sugar maples and make from the sap of their own trees a year's supply of maple sugar, the purity of which they could control; but most of them prefer to raise other things which they can sell or exchange for ordinary cane sugar, of the purity of which they have no knowledge. Flour nowadays is generally bought in barrels or bags taken at random from the output of distant mills over which the buyer has no control. Meat of various kinds is often purchased from a public cart, shop, or market; fish and shellfish, yeast, butter, eggs, cream, spices, canned and dried foods, are likewise obtained from special dealers, whose stores are drawn upon by many families and are therefore public supplies. Obviously 
impurity or adulteration in any of these public supplies may injure, or at least defraud, an entire community.

5. The Impurity of Foods may be of many kinds and many degrees, some of them of little or no hygienic significance. An excellent spring water, for instance, may not be "chemically pure" (i.e. containing nothing but water), and yet may be hygienically wholesome; and milk might conceivably be somewhat adulterated with distilled water without perceptible damage to the health of the community.

We may, for convenience, distinguish three principal kinds of impurity in foods: the first kind, caused by the addition of some cheaper substance, either already present in the food (as of water to milk) or altogether foreign to it (as of sawdust to ground spices). Such impurity is produced artificially, intentionally, and fraudulently, and is known as adulteration. It may or may not be prejudicial to health, but it is always a cheat.

The second kind of impurity of foods, known as their infection, consists in the occurrence in them of parasites or microparasites, such impurity being as a usual thing entirely accidental and unintentional, though often due to ignorance, negligence, carelessness, or uncleanliness. It is always prejudicial to the public health, but is not often due to a desire to cheat.

A third kind of impurity is that due to the use of unfit or unclean or diseased raw materials, disgusting to the taste and destructive to the appetite. This again arises either from negligence or the desire to cheat.

6. The Adulteration of Foods. - The commonest and most familiar adulteration of food is that of milk by water. Water is so abundant and cheap, and mixes so readily with milk, that it offers a constant temptation to dishonest milkmen who profit by the sale of such milk. It is often difficult for the consumer to detect this adulteration, 
even if he suspects the cheat; but it is easy for the chemist, and large cities should keep milk inspectors and analysts constantly on the watch, in the interests of the public welfare. In some cities the fines imposed upon dishonest milkmen more than repay the cost of the service.

But while milk is the food whose adulteration is most familiar, it is by no means the only adulterated food. Coffee, spices, beverages, sirups, honey, vinegar, and many other foods are subject to serious adulteration; and most states and countries are obliged to maintain laboratories devoted to the protection of the public against the adulteration of foods and drugs. Massachusetts has such a laboratory in the Statehouse in Boston, conducted by the State Board of Health. Some of its revelations are surprising and instructive. Milk, for example, is treated not infrequently with artificial coloring materials, and preservatives such as formic aldehyde, sodium carbonate, and boracic acid. Of about one thousand samples of suspected milk examined during July and August, 1898, nearly three per cent contained preservatives. Chocolate and cocoa likewise have been found to be frequently adulterated with wheat or sugar ; coffee with roasted peas, wheat, pea hulls, chicory, and sometimes bark, wood, and charcoal; honey with cane sugar or glucose; lard with cotton-seed oil; maple sugar with other sugars; maple sirup and molasses with glucose; pepper with rice and buckwheat; cloves with bran, sawdust, and charcoal; mustard, one of the most commonly adulterated of all spices, with rice, cornstarch, etc.; cider with salicylic acid. Worse yet, some so-called "patent medicines" which profess to effect cures contain the very substances - such as alcohol and morphine the effects of which they are supposed to overcome.

7. The Infection of Foods by Parasites and Microparasites. - Another, and from our standpoint much more important kind of impurity sometimes occurring in foods 
consists in their infection by disease-producing organisms, such as parasitic worms or microbes, - e.g. the germs of typhoid fever, scarlet fever, diphtheria, etc. Here again milk has the unenviable distinction of serving as a familiar example, for some of the worst epidemics of typhoid fever that have ever occurred have been traced conclusively to the infection of some milk supply by persons suffering with that disease.

But milk is by no means the only food that may be rendered impure and dangerous by infection. Raw hams, bacon, and sausages may contain a kind of almost microscopic worm called Trichina, which sometimes occurs in the muscles of hogs, and which, if not killed by cooking, is capable of developing in the alimentary canal of man, boring into the tissues and encysting in the muscles (especially the diaphragm), thereby causing severe disease and even death. Extensive epidemics of this disease (trichinosis) have occurred in Germany, in America, and elsewhere, due to ham and other pork products (sausages) either raw or imperfectly cooked. Such foods are fortunately seldom eaten underdone or "rare," in America, but even here one should be careful to eat them only when thoroughly cooked, or well done. It is required by law that hogs exported to foreign countries shall be carefully examined for Trichina, and numbers of experts are constantly employed by the United States Bureau of Animal Industry in making the necessary microscopic tests.

Other foods subject to infection and capable of conveying disease are those which are either occasionally or regularly eaten uncooked; for example, shellfish, such as oysters and clams; vegetables, such as celery, parsley, water cress, lettuce, tomatoes, cabbage; and fruits, berries, and the like. The danger lies in the fact that they may have been handled by persons themselves dirty and suffering from infectious diseases ; or they may have been grown on 
fields manured with sewage or other fecal matters containing germs of disease. For all these dangers there is but one sure remedy, namely, sterilization by cooking at a high temperature. But this in the nature of the case is impossible for many of the foods cited above.

Some fruits (oranges, bananas, melons, etc.) are naturally protected by their skins, and are consequently especially wholesome. Others (such as cherries, plums, apples, pears) should be washed thoroughly or rubbed with a damp clean cloth before being eaten. Still others (grapes, raspberries, strawberries) may be immersed in water and imperfectly washed, though they are seldom really cleaned by this process. Moreover, such "washing" is apt to injure the texture or flavor of delicate fruits, and is sometimes avoided on that account.

After all has been said and done, preventive measures may fail and some risks must be taken. The final defense must often come from that vital resistance, that good general health which it is the special object of hygiene to secure and promote. Life is valuable and health is precious, but either or both may be safeguarded at too great cost. Undue anxiety about foods, or even about life and death, is unworthy of those who have at most but a few short years to live, and who in those few years have many better things to do than merely to keep alive. "'T is not the whole of life to live."

8. Food Preserving and Preservatives. - Processes such as canning, and cold storage in wells, cellars, refrigerators, etc., are of immense value to the human race as conveniences and for the saving of surplus foods. The packing of pork in brine, the salting, smoking, and drying of fish, the "corning" of beef, and the pickling of vegetables are familiar examples of other kinds of food preserving. In these latter cases the foods are saved from spoiling by substances (brine or vinegar) which inhibit the growth of putrefactive microbes and are therefore called antiseptics. There 
are many other antiseptics besides brine and vinegar, and chemistry is constantly adding to the number. Some of the more important are boracic acid, formaldehyde (formalin or formol), and salicylic acid.

A difficult and delicate question arises when we come to ask, Does the introduction of chemical antiseptics into foods make them impure or dangerous? It is obvious that the use of salt to preserve fish, of brine for packing pork or corning beef, of smoke for preserving fish, hams, and dried beef, and of vinegar for pickling have been approved and sanctioned by generations. On the other hand, the use of boracic acid or formalin in milk is an undesirable practice, and at present the employment of any chemical antiseptic in food preserving must be regarded as of doubtful justification.

Some food substances contain acids which may attack the tins in which they are put up for the market. Blueberries, for example, readily corrode tin cans, forming salts of tin which in large amounts are harmful. The use of glass is therefore preferable for preserved foods whenever practicable; but the long-continued and very extensive use of tin cans for tomatoes, peas, beans, pears, etc., without known harm, indicates that for many foods tin cans may be used without much, if any, danger.

Sometimes food products, such as peas, clams, etc., are chemically treated in order to make them more attractive. French peas (canned) have frequently been found to contain copper, and canned clams are sometimes bleached to make them whiter. It is needless to say that such treatment is almost always objectionable, even if not positively dangerous.

The best preservative, hygienically speaking, is heat, and this, carefully applied, may be made wonderfully effective. The processes of canning and preserving are too familiar to need description, but it is not always understood that if the temperature employed is high enough and 
sufficiently long-continued it is of great hygienic value, because it tends to destroy any disease germs which may be present. It is, in brief, a kind of cookery, and cooking tends not only to preserve but also to purify foods.

9. The Purity of Public Water Supplies. - Public water supplies should be derived from the purest possible sources. Villages and small cities are often supplied from driven wells or open basins located near a lake or a river, and thus receive ground water (see p. 451). Large cities and many small ones often secure their supplies from lakes, ponds, or rivers, or from smaller streams, the water of which is stored in reservoirs. Supplies of this sort are called surface-water rather than ground-water supplies, and the water from them is naturally softer (see p. 451).

Ground-water supplies are apt to be of good quality but limited in quantity. Surface-water supplies are generally ample in quantity but more easily subject to pollution. For this reason they should not, as a rule, be drawn from thickly inhabited districts or from rivers, lakes, or smaller streams into which sewage or other polluting matters may find their way; and they should never be drawn from such sources unless they have first been purified in some manner.

Some cities, like Brooklyn (New York) and Lowell (Massachusetts), rely for their public water supply in part or wholly upon driven wells; some, like Boston, New York, and Liverpool, upon water collected in large reservoirs from streams upon comparatively uninhabited watersheds; and some, like Philadelphia, Paris, St. Louis, London, Hamburg, Lawrence, Albany, upon impure river water which is purified by filtration, or otherwise treated, before it is distributed to the citizens. ${ }^{1}$

1 The student, unless already informed, should familiarize himself with the sources and the possibility of pollution of the public water supply, if any, of his own village, town, or city, and should satisfy himself, if possible, as to its purity. 
It was formerly thought that running water sufficiently purified itself, although as early as 1874 a Royal Commission of experts on water supply reported in England that " there is no river in the United Kingdom long enough to purify itself from any sewage introduced into it even at its source," and the river Thames is more than two hundred miles long. It is true that sewage or other filth in streams often disappears, and that great improvement in polluted streams frequently takes place; but such "self-purification" is too often partial, incomplete, and untrustworthy.

In many cases the disappearance of obvious pollution is due to a mere dilution of the filth with purer water, and such dilution may greatly improve or even "purify" it. A drop of ink in a quart of water makes a mixture far less inky than the original drop. On the other hand, dilution does not necessarily mean destruction. A flock of birds may be lost sight of, but not destroyed, by scattering, and the purification of sewage filth should mean its destruction as such and its conversion into harmless substances. Much true purification does take place in a flowing stream, but this is not usually adequate, and towns and cities nowadays are generally turning toward filtration, or other artificial treatment on a large scale, of waters which for any reason are suspected of possible contamination. Some of these municipal purification works are elaborate and costly, as, for example, those in Albany, Philadelphia, St. Louis, Ithaca (New York), Lawrence (Massachusetts), Washington.

10. Public Gas Supplies and their Dangers. - There is no more danger from the products of combustion of illuminating gas than from those of oil or other illuminating materials. The air of rooms naturally becomes heated and more or less vitiated by these products, just as it does by human breath or any other waste product of oxidation; but illuminating gas properly burned is no more dangerous to life than is kerosene oil or any similar illuminant. 
Unburned gas, on the other hand, escaping from pipes or fixtures is often extremely dangerous, both because it is poisonous and because in certain proportions it forms with air an explosive mixture.

Illuminating gas is generally either "natural " gas drawn ready-made from the earth, or gas made from gasoline, oil, wood, coal, or coal and water, and hence known as "oil gas," "wood gas," "coal gas," or "water gas," as the case may be.

11. Natural Gas consists chiefly of marsh gas, or methane $\left(\mathrm{CH}_{4}\right)$, this making from ninety to ninety-seven per cent of the whole. It never contains more than one half of one per cent of carbonic oxide (CO), - a quantity too small to do serious damage. Though irrespirable, - that is, not fitted to support life, - natural gas is not poisonous. It may even leak into an apartment in considerable quantities without endangering life or seriously damaging health.

12. Coal Gas is made by distilling "soft" or bituminous coal, and consists chiefly of hydrogen and marsh gas, with smaller amounts of carbonic oxide and other compounds of carbon. It contains from six to ten per cent of carbonic oxide, a highly poisonous gas, and cannot be admitted into living or sleeping rooms in any great quantity without extreme danger to life. It also readily forms explosive mixtures with air.

13. Water Gas is made by passing steam over red-hot coal or coke (carbon), which decomposes the water vapor, producing, among other gases, an abundance of carbon monoxide. As it leaves the generator, water gas burns with a pale blue flame only. For lighting purposes it is therefore enriched by the addition of naphtha or other vapors which give it good illuminating qualities. But even after this treatment water gas generally contains from twenty-five to thirty per cent of carbonic oxide and is therefore extremely poisonous. 
In cities supplied with water gas, cases of asphyxiation and death from gas poisoning are common. These come chiefly from ignorance (in blowing out the gas instead of shutting it off) or carelessness (in turning the gas on again after extinguishing the light), or from suicidal intent, or drunkenness, or from leaky fixtures, or from change of pressure, - a light turned low being extinguished by a decrease of pressure in the pipes and the gas escaping into the room afterwards when the pressure is renewed.

The most remarkable (and often the most extensive) cases of poisoning by illuminating gas are those in which the inhabitants of houses or apartments not piped for gas have been poisoned by gas which has escaped from a broken or leaking main in an adjoining street. In these cases the gas makes its way underground to the basement of the house in question, and then, perhaps partly robbed of its warning odors by passage through the earth, rises through the house to sicken or kill those within. Whole families, and even groups of families, have occasionally been poisoned in this way, even in houses or tenements not piped for gas at all. The fact is that heated houses act like chimneys in producing a strong up-draft; and in winter, when windows and doors are shut tight, this draft sucks in air from the surrounding ground. If the ground air happens to be charged with gas from a leaky main, both air and gas may enter the house and sicken or even kill the inmates, although the house itself is not supposed to receive any gas. It has been estimated that "fourteen per cent of the total product of gas plants leaks into the streets and houses of the cities supplied."

Headaches and malaise (a convenient term for "feeling poorly") may be caused by small and imperceptible leaks of illuminating gas, and great care should be taken to have all gas fitting well done, and all leaky joints or fixtures made perfectly tight, especially if the gas used is 
water gas, now very generally supplied to the public in American cities.

One of the great advantages of lighting houses by electricity is that it does away with all possibility of gas poisoning except that from leaky mains in public streets, already referred to.

The use of gas for heating and cooking requires especial caution, owing to the large quantities used and the temporary connections often employed (pp. 438 and 440).

14. The Purity of Public Milk Supplies. - Milk is one of the most universal and most important of foods. It is also one of the most peculiar, in that it is a secretion drawn directly from the bodies of living animals. This remarkable animal secretion, when fresh, is very sweet, smooth, and bland to the taste, but on exposure to the air generally spoils quickly and sours. It is obviously not the air alone which causes it to sour, for milk is easily kept sweet a long time if kept in a cold place, or if scalded when it threatens to turn sour.

The spoiling and souring of milk are caused by certain bacterial microbes which, having got into the milk as it was drawn, or later from dust, air, dirt, or unclean pails or strainers, live and multiply enormously at the expense of the sugar and other food stuffs which milk contains. The so-called lactic-acid bacteria, in particular, thrive in milk, especially if it is kept warm, and spoil it by converting the milk sugar (lactose) into milk acid (lactic acid).

Milk that is pure should be free from dirt, and sweet rather than sour, but such milk is unfortunately not always easy to obtain, especially in cities. A black sediment in milk indicates dirt (usually cow dung), and so does a "cowy" taste. Milk may also be adulterated with water, with antiseptics, or with other substances, as has been shown above (p. 509); but the most serious impurity in public milk supplies is the occurrence of germs of contagious 
or infectious diseases. Many epidemics of typhoid fever and diphtheria have been conclusively traced to a public milk supply which served as the unsuspected vehicle of the disease. In all of these cases uncleanness of some sort - on the farm, in the dairy, among the milkmen, or elsewhere - is believed to have been always at the bottom of the trouble.

Persons supplying milk to the public should take pains to keep their cows healthy and their cow stables clean; to milk only after careful washing of the hands, pails, cans, strainers, etc., and also only after washing the udders of the cow; and it should always be remembered that milk is a rich animal secretion which readily supports bacterial life and therefore should be scrupulously guarded against any invasion of dirt or disease. To secure rich, pure, clean, and fresh milk in cities, a higher price must probably be paid than has been the custom hitherto. The demand is for better, purer, cleaner milk; and for this it is reasonable to expect that more must be charged.

It should also be remembered that the number of bacteria in milk, unlike that in water, does not depend simply on the number that get in, since germs multiply very rapidly in this rich food supply. Hence milk as soon as drawn should be chilled as far as possible before delivery. The mere souring of milk lessens its digestibility, especially in the case of infants, so that it is a matter of hygienic importance, particularly in warm weather, to lessen the growth of bacteria in it by immediate cooling as soon as drawn from the cow, and keeping as cold as possible afterwards.

It must also be borne in mind that the milk-producing industry, while one of the oldest known to man, is still largely in a primitive condition. What is needed is a more scientific knowledge of the subject, more intelligence, skill, and cleanliness among those engaged in it, and, finally, 
expert supervision both on the part of the producer and of the sanitary authorities of cities.

15. Public Sewerage and the Disposal of Sewage. - One of the most beneficent procedures in any community is the establishment of a system of public drains which shall quickly and effectually remove all liquid and many solid wastes, especially the excreta of human beings and other animals. Well-built sewers not only do this but also carry off much "ground" water, making the soils of cities drier and therefore more wholesome. The term "sewerage" is applied both to the act of draining and to the system of sewers; the word "sewage," to the contents of sewers.

The disposal of the sewage of cities and towns is often a very serious, difficult, and costly problem. Sometimes the sewage can be safely emptied into a river, a lake, or the sea, but more often it is necessary to purify it, either upon land (where it may be made useful, though rarely profitable, for agricultural purposes), or by chemical treatment, or by microbic (bacterial) action during cesspool or filtration processes. The problem of the final disposal of sewage is not yet fully solved, and at the present time is engaging the anxious attention of the world's ablest sanitary engineers. 


\section{CHAPTER XXXIII}

\section{THE HYGIENE AND SANITATION OF TRAVELING, PUBLIC CONVEYANCES, PUBLIC HOUSES, ETC.}

1. Migration, Past and Present. - One of the most striking characteristics of the present as compared with the past is the increased and increasing movement of masses of people not only permanently out of one country (emigration) and into another (immigration), but also temporarily from place to place, and back and forth (traveling). Such migration inevitably removes the traveler, temporarily at least, from one environment, and subjects him to another and often very different one; so that from the hygienic point of view a change of this sort is of great importance and interest. It also often affects the environments of others besides the migrant himself, by introducing into those environments new elements of disease.

2. Traveling and Change of Scene.- Even before starting upon a journey conditions for the prospective traveler have often begun to change. The bustle and the thought of the necessary preparations constitute a kind of excitement, sometimes pleasurable, sometimes wearisome, accompanied, it may be, by temporary loss of appetite or even sleeplessness (especially in children), or by other abnormal conditions sometimes described by the phrase "journey proud." With the start come leave takings, farewells, and partings more or less unusual and exciting, and then begins a series of tolerably rapid changes of environment or scene. The body is moving and possibly shaken about or jarred; unusual and shifting scenes fall upon the retina and come 
before the mind, calling for attention and arousing new sensations; strange sounds are heard, strange odors detected; the air (if in an open vehicle) beats against the face and the ordinary atmospheric " blanket" is diminished or otherwise interfered with.

Arrived at a stopping place or the journey's end, streets, houses, and hotels are new or strange ; strange faces meet the traveler; there are strange rooms and walls, strange furnishings, strange sounds and odors, — in short, a strange or unusual environment.

All this may or may not be wholesome, according to circumstances on the one hand and the individual on the other; but it is certainly stimulating and physiologically exciting, as may be readily proved by observing its effects upon children and the aged. The change of scene is only one element in the hygiene of travel, and its value must be determined by weighing it together with other equally influential factors, namely, the change of occupation, the change of air, and the change of food, and finally by applying all of these considerations to particular cases or individuals.

3. The Change of Occupation. - It is an old saying that "all work and no play makes Jack a dull boy," and experience teaches clearly enough that a change of occupation is wholesome. One of the best features of travel is that it necessitates a change of occupation. A common expression contains the idea of "going away from home to get a change." One of the most valuable characteristics of the home is the repose and restfulness which result from its uniformity of conditions, and one of the best things about travel is the mild stir and excitement involved. Routine and regularity of occupation are on the whole the more natural and normal, and "a steady job," whether it be in shop, mine, or factory ; on farm, plantation, or shipboard; in bank, school, or professional life, is naturally sought and prized by everybody. 
And yet most persons profit from time to time by "a day off," or a vacation, or a journey which affords change of occupation with freedom from responsibility. Once on the way, the traveler is not responsible for the train or the steamer, for the cookery or the beds, for the house or hotel, or its furnishings or management; and this freedom from responsibility is a complete and often refreshing change.

4. The Change of Air. - It is difficult to say in what way and to what extent a change of air is beneficial in traveling. Much of the benefit, even when attributed to the "change of air," is no doubt really due to other things, such as the change of work and the change of scene; but after making all allowances, it would still seem to be true that a change of air has a perceptible effect, and often does great good or great harm. Air that is drier or damper, or warmer or cooler than usual, or air in the forest or by the sea, often seems to have decided effects for good or for evil, all other conditions remaining apparently much the same. At times, obscure atmospheric influences at home, unknown to ourselves, may be the source of lessened vital resistance, and so of a lowered tone of general health, and the change of air may be the means of restoring normal conditions by removing the obscure cause of trouble. Moreover, when the change is from the close, "stuffy" air of an office to the open air of country or of sea, with their agreeable odors, there is a "bracing" or stimulating effect which reacts favorably upon the entire constitution, but especially upon the nervous system. The tendency to "fill our lungs" with it is only a sign of the general beneficial influence upon the system as a whole. Many a case of " the blues" has been successfully overcome by this simple expedient.

On the other hand, air as a vehicle of infection may affect the traveler unfavorably; for he must almost inevitably be exposed to air (as well as to other things) which has 
recently been in contact with persons having incipient tuberculosis, diphtheria, measles, typhoid fever, or other infectious diseases. To this subject we shall return in the next section but one.

5. The Change of Food. - It is uncertain how much or how little influence a change of food has upon the organism. It is commonly believed that a change of food is often beneficial, or the reverse, and that much of the good or bad effects of travel is due to the inevitable change of diet and of cookery which goes with it. How far this is true is unknown, but it is easy to see that a simpler diet for some and a more abundant diet for others may in itself alone be helpful. It is doubtful if any special virtue resides in "sea food," or in "country living," or in " camp cookery," apart from that which consists in its palatability or its novelty, - qualities which affect appetites and therefore nutrition; but in so far as a change makes food appetizing or acceptable, such food is, of course, more valuable to the body. However this may be, there can be no question about the increased danger of infection from food and drink taken at random from unknown sources.

6. The Dangers of Infection away from Home. - At home the traveler, in theory at least, has an environment well under his control; but when he starts upnn a journey, whether afoot, or riding, or driving; by bicycle, railway, steamship, or other means of conveyance, - he enters into new environments, of whose precise nature he is ignorant, and which are usually beyond his control. Of the sanitary or unsanitary condition of the water supply, ice supply, milk supply, etc., he is entirely ignorant; and he may at any time be thrown in contact with persons suffering from infectious diseases, especially in a mild or incipient form. The public vehicle (carriage, wagon, car, or omnibus) in which he travels, the hotels, rooms, chairs, and even the beds which he uses may have been recently 
occupied by diseased persons. His laundry work may be done or delivered by workers suffering from contagious diseases ; uncleanness may attend the preparation and serving of his food. In short, in leaving his own familiar and controllable environment and passing into others unfamiliar and beyond his control, the traveler clearly takes large risks.

7. Safeguards of the Traveler. - If it be asked what one can do to protect himself or his family from the dangers of travel, it may be pointed out, in the first place, that it is often better not to travel at all. When one is in poor condition, although a change to some new scene whose hygienic conditions are known to be good is likely to be beneficial, a railroad journey with frequent stops is apt to increase the danger of infection at a time when vital resistance is low. When a journey is necessary the traveler should try to avoid marked fatigue, which always diminishes vital resistance and thus predisposes to disease; he should seek to avoid unclean hotels, unclean conveyances, badly aired rooms, and unclean fellow-travelers; he should avoid the use of public drinking cups, public towels, public razors, and the like; he should, if possible, drink only waters of established reputation; he can, if need be, forego the use of rav milk, raw oysters, and other uncooked foods the antecedents of which he knows nothing about, and he can tate other obvious and useful precautions that will suggest themselves as he goes along.

But, after ail, it must be admitted that precautions, even if rigorously observed, will often prove insufficient, and also thiat too much thought about them, or about the dangers of travel, would rob it of most of its advantages. People always have traveled and probably always will travel without much consideration of the dangers involved in traveling. Some risks must always be taken, even at home, and most travelers cheerfully accept the necessary 
risks for the sake of the gains to be derived. With the increase in the amount of traveling, many of the risks are gradually decreasing, and in highly civilized countries adults in robust health who know how to take care of themselves may now go upon a journey without very much more risk of infection than they would undergo if they stayed at home. This is probably less true of children, for children and old people are not only more easily excited and more easily fatigued, but they also suffer more severely from exposure, and children are especially apt to contract infectious diseases when away from home.

8. Public Drinking Cups should be avoided by travelers, theater goers, and all persons in parks or other public places. Few sights are more distressing to a sanitarian than to see (on a hot day in a crowded railway car) men, women, and children, of all ages, sorts, and conditions, clean and unclean, sick and well, one after another in rapid succession applying their mouths to the one public drinking cup. If the student will once carefully observe for himself the use to which this cup is put during even a short journey under such conditions, he will realize that every traveler had better carry his own drinking cup, or, in default of this, go thirsty. In some theaters, between the acts, trays containing glasses of water are passed to patrons in their seats. Here also the lips of many persons touch successively the same glasses, and one who is wise will avoid the obvious danger involved in using one of these glasses, which may have become infected. Sanitary drinking fountains in which, by a simple device, the obnoxious common drinking cup is made unnecessary, are now being gradually introduced in parks, schools, and other public places.

9. The Influence of Travelers upon the Environment. We have thus far considered chiefly the effects of strange environments upon the traveler, but before leaving the 
subject we must not fail to point out some of the reactions of travelers upon the environments in which they journey or linger. Many epidemics of infectious diseases have sprung from germs left by travelers, and most of the great plagues and pestilences of history have followed the routes of pilgrims, caravans, crusaders, conquerors, traders, or travelers. "Walking cases" of typhoid fever, diphtheria, etc., are perhaps most dangerous to the public health, and tramps, peddlers, and other roving characters do much to spread disease. Persons coming down with an infectious disease, such as typhoid fever, are very apt to leave off work and go a-fishing, sometimes upon or along the shores of a public water supply, which they may unwittingly contaminate. Life away from home has its dangers for the traveler; it is no less true that life at home has its dangers, these often arising from travelers themselves.

10. Public Conveyances, because they are used promiscuously by the well and the ailing alike, are subject to infection, and for this reason carriages, cars, and steamboats should be kept clean and occasionally should be thoroughly disinfected. Steamboats and steamships are essentially floating hotels, and should be treated as such. Sleeping cars bear less resemblance to public houses, and may be cleaned partly by washing, partly by blasts of compressed air, and partly by disinfectants, and when properly cared for are less likely to endanger health than are many hotels. Their lavatories should be kept scrupulously clean and should be frequently disinfected. In modern times vast improvements have been made in all kinds of public conveyances in the direction of greater steadiness, less noise, better heating, better air, and better lighting. The public drinking cup, however, remains as objectionable as ever, and its use ought to be abolished.

11. Public Houses. - Hotels and other public houses may be either clean, wholesome, and restful, or unclean, 
noisy, and unsanitary. Owing to the fact that their population is constantly changing they are far more exposed to infection than are private houses, and great pains should be taken to keep them always in good sanitary condition. The simplest (iron) bedsteads are the best, and in hotels all carpets, draperies, etc., should either be avoided or subjected to frequent and thorough cleaning. The kitchen, especially, requires careful supervision to insure cleanliness, and in the laundry the linen should be so treated as to be sterilized during the process of washing. Employees should be instructed in the art of cleanliness, and any suffering from contagious or infectious diseases should be excluded or quarantined. All lavatories should be kept scrupulously clean and should be frequently disinfected.

12. Public Places, such as streets, parks, playgrounds, and cemeteries, are dangerous only when infected. Dirty streets are unsightly and disagreeable, but it is very hard to trace the source of much disease directly to them. Nevertheless, few things sooner or more agreeably impress a visitor than clean streets, and in the lower portions of the town or city clean streets are particularly important because the streets are the home and the playground of the children of the poor. Pavements in cities should be hard and nonabsorbent rather than porous, and should be kept clean and free from rubbish.

13. Public Parks are desirable for fresh air, recreation, rest, and change of scene, and in these respects are important hygienic factors in city life. They are of special benefit to those living in tenement houses or under crowded conditions. Public playgrounds minister to the needs of the same class of people. Their importance can hardly be overestimated, since they furnish to city children almost the sole opportunity for normal physical development and some contact with nature. It has been well said that "the boy without a playground is the father of the man without 
a job." But here also wise supervision and cleanliness are the conditions of hygienic success.

Public cemeteries in America are usually well conducted and unobjectionable from the hygienic standpoint. The objections sometimes urged against them as centers of infection and sources of disease are seldom well founded. Cremation, or the burning of the dead, is slowly but steadily growing in favor and has much to recommend it from the sanitary standpoint, since it prevents slow decay and destroys completely all germs of disease. Near most of the larger American cities there are now one or more crematories. 


\section{CHAPTER XXXIV}

\section{PUBLIC PROTECTION OF THE PUBLIC HEALTH}

1. The Public Health. - By this term is meant the health of the community; and of some community every family and every individual is a member. The public health is obviously of vital importance to the individual, and, conversely, the health of the individual is of vital importance to the community. Personal hygiene, or the hygiene of the individual, and public hygiene, or the hygiene of the community, are thus closely bound together. Not only because it is his duty, but also because from the selfish point of view it is to his advantage, the individual should, therefore, interest himself in and seek to promote the public health. If, for example, smallpox appears in his community, he cannot afford, even from a selfish point of view, to fail to do his best to aid in suppressing it. Conversely, if he himself falls ill of smallpox, his neighbors and the whole public naturally feel a similar interest in isolating him and preventing the spread of the disease.

For these and similar reasons, people living in communities, and especially in villages, towns, and cities, by common consent usually elect or appoint a few of their own number as sanitary authorities or officials to attend to matters affecting the public health. The citizens thus chosen are endowed with special powers and privileges, and, taken together, are generally designated as the Board of Health, or Health Commissioners. Sometimes, especially in small communities, there is no formally organized board of health, the duties of such a board being performed by 
some governing body of the community, such as the selectmen, etc.

2. Boards of Health, their Powers and Duties. - Very much as boards of police are chosen by the people to preserve public order and to prevent disturbance and crime, so boards of health are chosen to preserve the public health and prevent disease and death. And as the police officer could not possibly do the work assigned to him without unusual powers and privileges, these sometimes involving a considerable interference with personal liberty, so the health officer cannot do the work expected of him without unusual powers and privileges. But it should never be forgotten that in each case both the officers themselves and the powers which they possess exist by the common consent of the community, which desires, and thus provides for, protection at the cost of surrendering some personal rights and privileges. Boards of this sort derive their powers solely from the consent of the majority of the community which they serve, and those members of the community who disapprove of their existence, powers, and acts must either persuade the majority to do otherwise, or must submit, or must go elsewhere.

Among the important powers of boards of health are the right of quarantine, isolation, entrance and search, and vaccination. A board of health may, in many cities of the United States, detain a vessel, perhaps full of passengers impatient of delay and eager to land, at quarantine, even for many days, subjecting the owners, passengers, and others to great inconvenience, expense, and damage. A board of health, finding smallpox in a hotel or boarding house, may quarantine or isolate the building, surround it by police, and forbid all persons to enter or leave it, thus causing great alarm and annoyance to the inmates, great damage to the proprietor, and a heavy expense to the community. A board of health may declare general vaccination 
necessary for the protection of the public health, and may even enforce vaccination upon the careless, reluctant, or resisting. It may forbid a dairyman to sell milk thought to contain typhoid fever or other disease germs, thus causing the dairyman great inconvenience and even financial ruin. In all these cases the board is, as a rule, simply obeying the wishes of a majority of the community, and those who are delayed, constrained, or financially injured have to submit as best they may, unless the general sentiment of the community undergoes a change in their favor.

The duties of boards of health are manifold. Some of the most obvious and general are usually prescribed by public statute or ordinance. Such, for example, are the following in the state of Massachusetts :

The State Board of Health shall take cognizance of the interests of health and life among the citizens of the Commonwealth. It shall make sanitary investigations and inquiries in respect to the causes of disease, and especially of epidemics and the sources of mortality, and the effects of localities, employments, conditions, and circumstances on the public health; and shall gather such information in respect to those matters as it may deem proper, for diffusion among the people. It shall advise the government in regard to the location and other sanitary conditions of any public institutions.

Others are less general and more specific, like the following :

The State Board of Health shall have the general supervision of all streams and ponds used by a city or town as sources of water supply, with reference to their purity, together with all springs, streams, and water courses tributary thereto ; and shall have authority to examine the same from time to time and inquire what pollutions exist and what are their causes.

When the Board of Health of any city or town has had notice of the occurrence of a case of smallpox or of any other disease dangerous to the public health in such city or town, such Board of Health shall, within twenty-four hours after the receipt of such notice, notify the State Board of Health of the same. 
A nation may and should hare a national sanitary authority charged with the protection and promotion of the public health and provided with large powers; it should also be supplied with trained experts and money enough to enable these to deal with emergencies, to study large practical problems, and to carry on researches into the causes of disease and the improvement of methods for their prevention. Germany has such an organization in its Imperial Board of Health, and the United States, for a short time, had a National Board of Health. At present the United States Public Health and Marine Hospital Service does this same work to some extent.

The states also, in the United States, hare for the most part their own boards of health; but such boards do not, as a rule, have very large powers, these being reserved for the so-called " local boards" of the rarious cities and towns. It is believed by many experts that a larger grant of powers and resources to state and national boards would be of immense benefit to the public, and would secure for all a much more constant and efficient sanitary protection.

3. What the Individual may do to protect the Public Health. - The first duty of the individual to the public health is to remember that he himself, his family, his house, and all his belongings constitute one important and fundamental element in the health of the community of which he is a unit. He should therefore seek, first of all, to maintain and promote good health in himself, in his family, and in all his household; for the prevention of disease and premature death in one household is a distinct and genuine contribution to the public health of all other households.

In the next place, he should cheerfully conform to all reasonable regulations of the board of health or other sanitary authority of his community, duly prescribed by them under powers conferred by the community as a whole. 
Finally, he should inform himself as fully and as accurately as possible upon hygienic and sanitary subjects, in order not only to protect and promote his own health and that of his household, but also to enable him to become an intelligent, critical, and yet coöperative member of the community, thus doubly aiding in preserving and promoting the public health.

Having done, or tried to do, these three things, the good citizen has still one further duty of the utmost importance to perform for the maintenance and betterment of the public health, - which, as we have shown above is also of great consequence both to himself and his family, - and that is to aid and assist in all their good works boards of health and all others in sanitary authority. This, one may do by reporting the existence of cases of infectious disease, nuisances, etc. ; by trying to secure the election or appointment of intelligent, upright, and expert officials ; by loyally upholding such officials in the performance of their duty; by refusing to countenance opposition to necessary public procedures such as vaccination, gas inspection, plumbing inspection, the placarding of houses containing cases of infectious disease, the isolation of patients, etc., and in many other ways which are certain to arise.

At times this individual responsibility for public health involves personal inconvenience and hardship, severely testing the good citizenship even of those most desirous of coöperating with the public health authorities. This is well illustrated in the case of diphtheria. When antitoxin is given in this disease, the toxin produced by the bacteria is neutralized in the blood and tissues and is thus prevented from injuring the organism; but not all the bacteria are immediately killed by this treatment. Consequently it sometimes happens that, long after the clinical symptoms have disappeared and when the patient is apparently perfectly normal, examination of the throat reveals the presence 
of the bacillus; and it has been proved beyond question that germs from this source are often capable of transmitting the disease to healthy persons. It is a real hardship to such a patient to be kept in quarantine for days and weeks, until the disappearance of the germ in the throat is established by bacteriological examination, and boards of health are frequently criticised severely for enforcing quarantine under such circumstances; but it is obvious that these measures are demanded in the interests of the community and that resistance to them can arise only from ignorance or selfishness, or both.

4. What the Public may do to protect and promote the Health of the Individual. - On the other hand, the community, through its paid or unpaid officials, may do much to protect and promote the health of its individual members. It may see to it that the public water supply, if any, is pure; it may maintain an efficient system of milk inspection; it may provide investigations of food adulteration, and prosecutions and penalties for the same ; it may require prompt and efficient scavenging, and the collection and removal of wastes such as sewage, garbage, and other refuse ; it may establish a wholesome system of school hygiene ; it may prevent the concealment of the existence of cases of infectious or contagious disease ; it may provide for vaccination against smallpox, and for the use of antitoxic serum in diphtheria; and in many other ways it may protect the individual and his family even better than he, unaided, could protect himself. 


\section{CHAPTER XXXV}

\section{THE HEALTH OF NATIONS}

1. The Modern World One Vast Community. - Ever since the invention of the mariner's compass, followed as this was by the voyages of discovery of Columbus, Vasco da Gama, and Magellan, the world has become, century by century, more and more one great community or neighborhood. With the introduction of steam transportation on land and sea our globe has practically shrunk so that intercourse between the various nations of the earth has become both frequent and easy, and if no other means than those formerly known existed for the prevention of disease, plagues and pestilences would, without question, ravage mankind worse than ever before. The isolation of the ancient world was its sanitary salvation, but to-day there is no isolation. Steamers ply regularly and frequently between Orient and Occident, commingling the people and the products of the whole world. Books, newspapers, letters, food materials, fabrics, and many other sorts of merchandise pass freely back and forth, and yet plague and pestilence to-day seldom follow in their train. It is possible, and even probable, that some milder diseases such as influenza, or the grippe, may still owe their sudden and wide distribution to the modern ease and extent of communication; but mails and steamers come and go, and bubonic plague, and Asiatic cholera, and smallpox, though occasionally brought by them to Europe or America, do not make great headway there after their arrival.

2. Ancient Paths of Pestilence and Plague. - Although it is true that modern civilization is indebted to the Orient 
for its first knowledge of the art of inoculation for the prevention of smallpox, it is no less true that many of its worst epidemic diseases have often come from the same source.

The plague, a world-famous disease (p. 502), has afflicted mankind for centuries, and has repeatedly appeared in Europe, traveling westward from the Orient and from Africa. The Black Death, which is held to have destroyed one fourth of the population of Europe in the fourteenth century, was probably a virulent form of the oriental plague which entered Europe from the south and east. The Great Plague of London (in 1665) probably' came from Holland, in bales of merchandise brought from the Levant.

The Asiatic cholera, as its name suggests, has repeatedly come to Europe and America from the East, and is believed to exist almost constantly in India, from which place its germs are readily conveyed to western countries. The germs of the great Hamburg (Germany) epidemic of 1892 were probably brought there by immigrants from Russia.

3. The Modern Impotence of Pestilence and Plague. The modern increase of the means of communication has no doubt tended to spread far and wide all sorts of contagious and infectious diseases, but with that increase there has come, especially within the last few years, such a scientific knowledge of these diseases and of the ways of holding them in check that, in spite of vastly greater facilities for their distribution, they are actually less dangerous to mankind, and far less dreaded, than formerly. The appearance of the bubonic plague in China or in India, or of Asiatic cholera in Japan or in the Philippines, still causes international anxiety, and vigorous local precautionary or corrective measures are taken to overcome them; yet little widespread alarm is felt. The closer intimacy between 
Cuba and the United States since the Spanish War of 1898, while in itself favoring the spread of yellow fever, has had the marvelous and happy consequence - thanks to the brilliant researches and able administration of the medical and sanitary officers of the American army - not of bringing more yellow fever to the United States, as would formerly have been the case, but of virtually extirpating that disease, for the present at least, in Cuba.

4. The Use and Abuse of Quarantine. - The word "quarantine" comes from the French word quarante, meaning forty, because a detention of forty days was formerly enforced upon travelers crossing frontiers. Quarantine is of great value in some cases, as, for example, in ports like Boston or New York, and when thoroughly enforced may be an important means of protecting the country against infectious disease. When a vessel which has been long enough at sea to give contagious disease (if present) time to appear, comes into port with cases of such disease on board, its detention is a wise precaution. On the other hand, indiscriminate quarantine between states or cities, or of vessels that have come from near ports, so that little or no time has been given for disease, if present, to show itself, is necessarily severe and often useless and unwarrantable.

Quarantine is also liable to abuse on other grounds, for it is claimed that it has sometimes been unjustifiably employed to keep out of a country foods or other products which came into competition with domestic products, the plea of sanitary danger being raised for commercial reasons.

5. International Sanitary Congresses. - From time to time there are held nowadays international sanitary congresses which undertake to deal with the larger questions affecting the health of nations. There is also held annually an International Congress of Hygiene and Demography; and the annual meetings of the American Public Health 
Association, in which the United States, Canada, Mexico, and Cuba are represented, are really international congresses for a large part of the western hemisphere.

6. Health and Longevity in Various Countries. - It is interesting to inquire how different nations compare, one with another, in respect to health and longevity. It might be supposed that somewhere on the earth's surface the climate should be so salubrious, the food so wholesome, the conditions so favorable, and life so normal, that sickness would be unknown and death indefinitely postponed. Invalids in large numbers do, in fact, turn to Colorado or California, to Madeira or to the Riviera, seeking in these places more favorable conditions for sustaining or prolonging life; but no place. has ever been found altogether free from disease, and no climate, however salubrious, seems capable of causing any great increase in longevity. It was many centuries ago in the Orient, and of a race singularly strong and persistent, that the Jewish poet wrote those majestic lines which for every land and every people are no less true to-day: "The days of our years are threescore years and ten; and if by reason of strength they be fourscore years, yet is their strength labor and sorrow; for it is soon cut off and we fly away."

The general death rate - that is, the number of deaths per year per thousand of the population - is not a complete measure either of health or of longevity, but is sometimes the only test we have, and the following table for 1900 shows how great is the difference in the death rates of some of the larger cities of the world.

London

18.7

New York . . . . . 20.6

Paris . . . . . . 20.5

Berlin . . . . . . 18.9

Vienna . . . . . . 20.6

Moscow

Rome . . . . . . 16.5

Madrid . . . . . . 33.3

St. Petersburg $\quad$. $\quad . \quad$. 27.0

Boston . . . . . 20.8 
The following table (from the United States Census of 1900) gives the death rates for the periods specified of some of the principal countries of the civilized world.

Comparative Death Rates per 1000 Population for Certain Countries

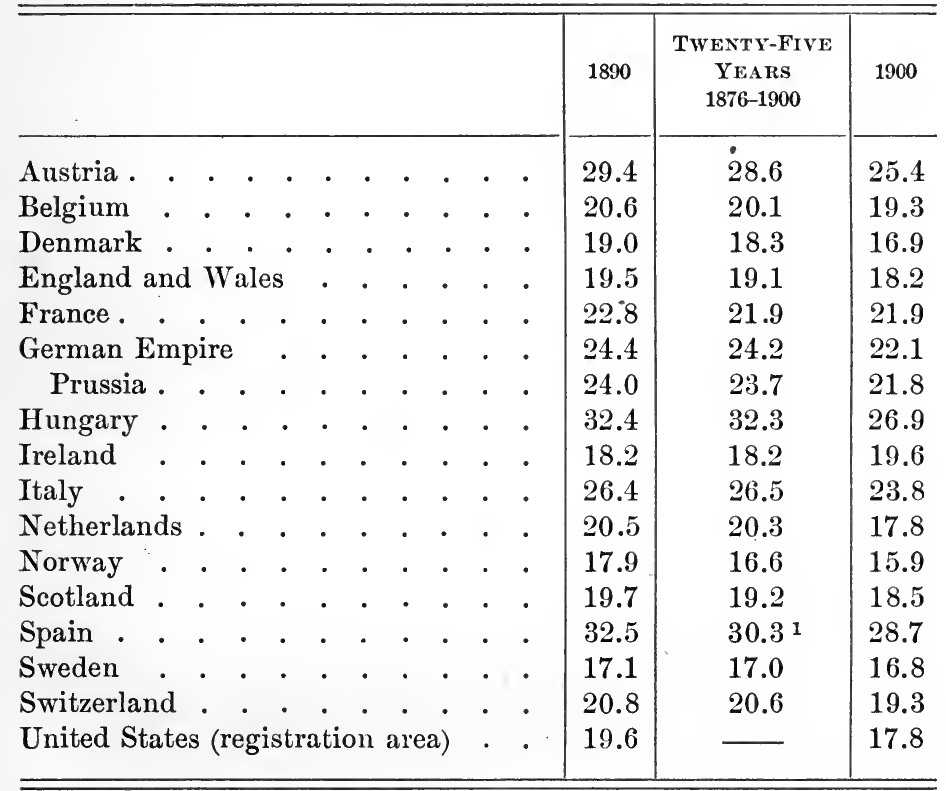

7. The Sanitation of the World. - Enough has been said in the foregoing paragraphs to show that while the hopes of dreamers seeking after an elixir of life have no foundation, and while a wholly salubrious environment cannot greatly prolong human life beyond the usual period, much is being done and much still remains to be done for a more complete and perfect sanitation. Infectious diseases

1 Average for twenty years, 1878-1884, 1888-1900. 
still sweep over communities, carrying sickness and death among the people, increasing the death rate, and diminishing longevity. Here and there nations and individuals are devoting themselves with energy, public spirit, and wisdom to investigation of the causes of disease, and to improvement of the environment by careful organization of boards of health, by municipal sanitation, by sanitary engineering, by purer water and milk supplies, by proper sewerage and sewage disposal, by food inspection, and the like. All this is wise and encouraging, but it is only a beginning. Far more might and ought to be done both by nations and by individuals. Many of the nations, especially those known as half-civilized or barbarous, have as yet hardly made a beginning in hygiene or sanitation, and as long as this is the case they are, and will continue to be, a menace not only to themselves but to the whole world, which, as one vast community, is in these respects closely bound together.

The student should never forget that the foundation of municipal, national, and international hygiene and sanitation, and therefore of the health of nations, ultimately rests upon the hygiene and sanitation of individuals, - that is, upon personal hygiene and sanitation. If all human beings were healthy and clean, the nations of the world would of necessity be in the same condition. Personal hygiene and personal sanitation thus form the basis of all hygiene and sanitation, whether of home or village, of town or city, or of the world; and the essentials of personal hygiene and sanitation are simply the proper management and care of the human mechanism and its surroundings. 


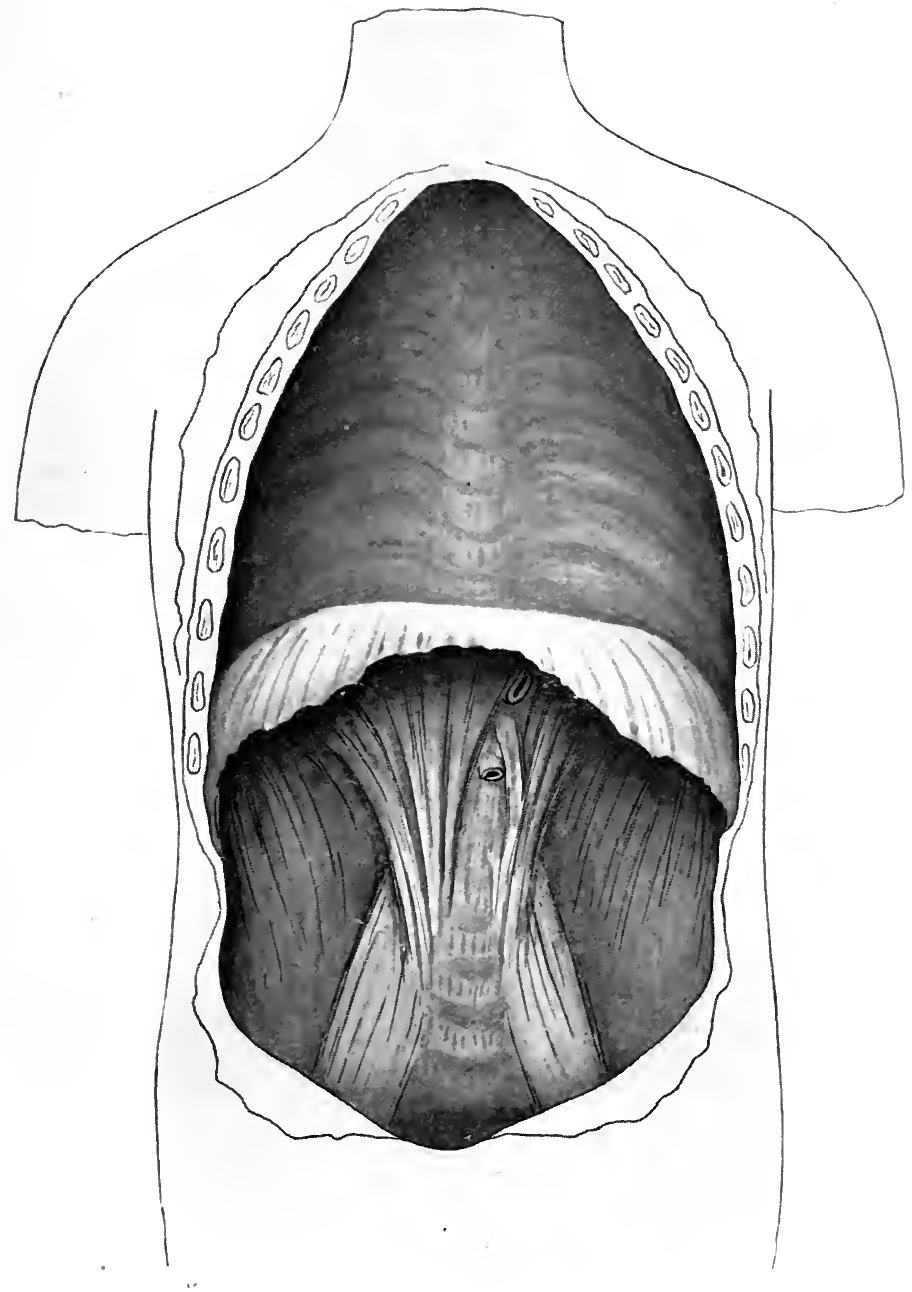

Fig. 134. The thoracic and abdominal cavities, after the removal of the organs shown in Fig. 2

The diaphragm has been drawn somewhat forward 


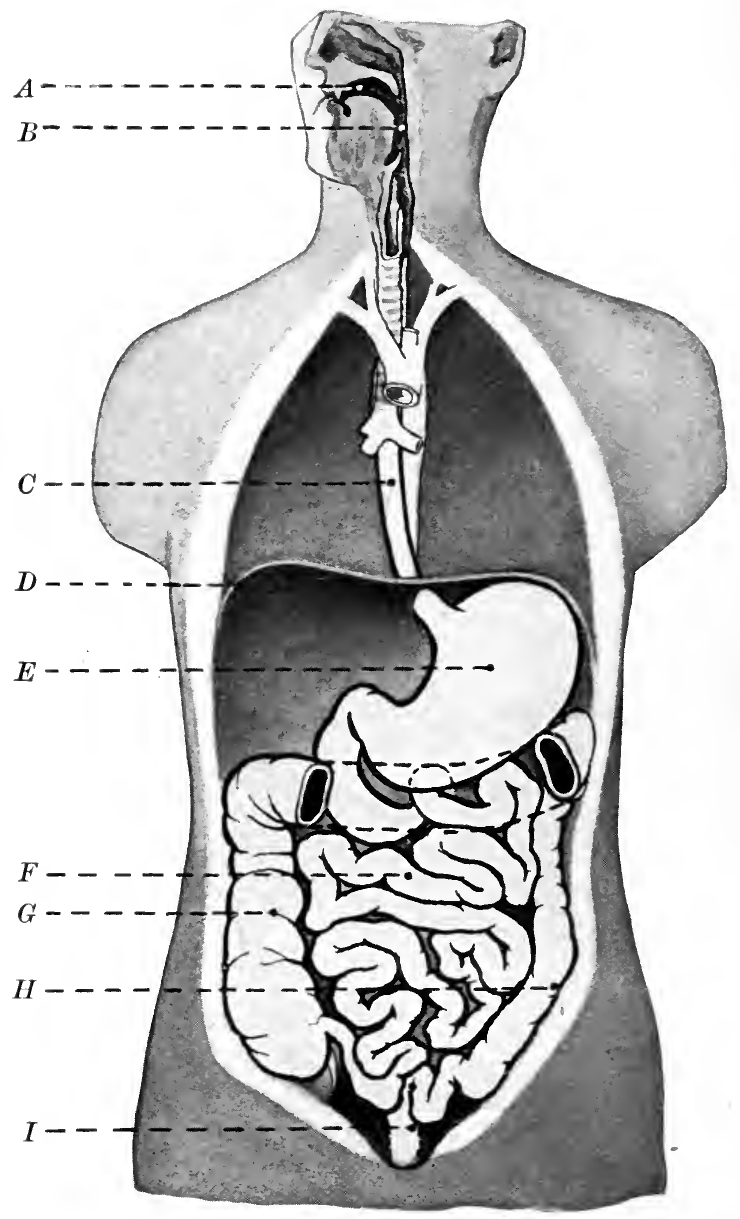

FIG. 135. General view of the digestive tract. After Spalteholz

$A$, mouth cavity ; $B$, pharynx; $C$, œsophagns ; $D$, diaphragm ; $E$, stomach ; $F$, small intestine; $G$, ascending colon; $H$, descending colon; $I$, rectum. The transverse colon has been cut away, its position being indicated by dotted lines 


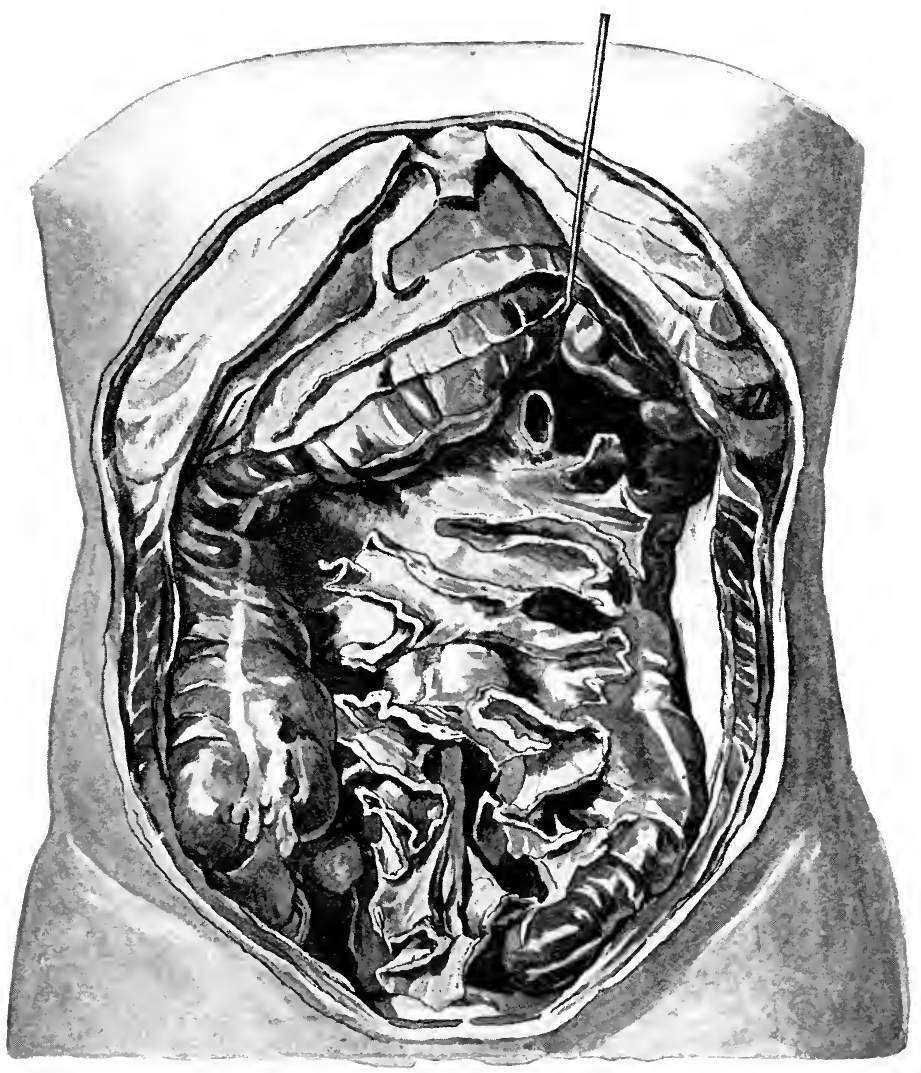

Fig. 136. The flouncelike folding of the mesentery, as seen after removing the small intestine. After Spalteholz 


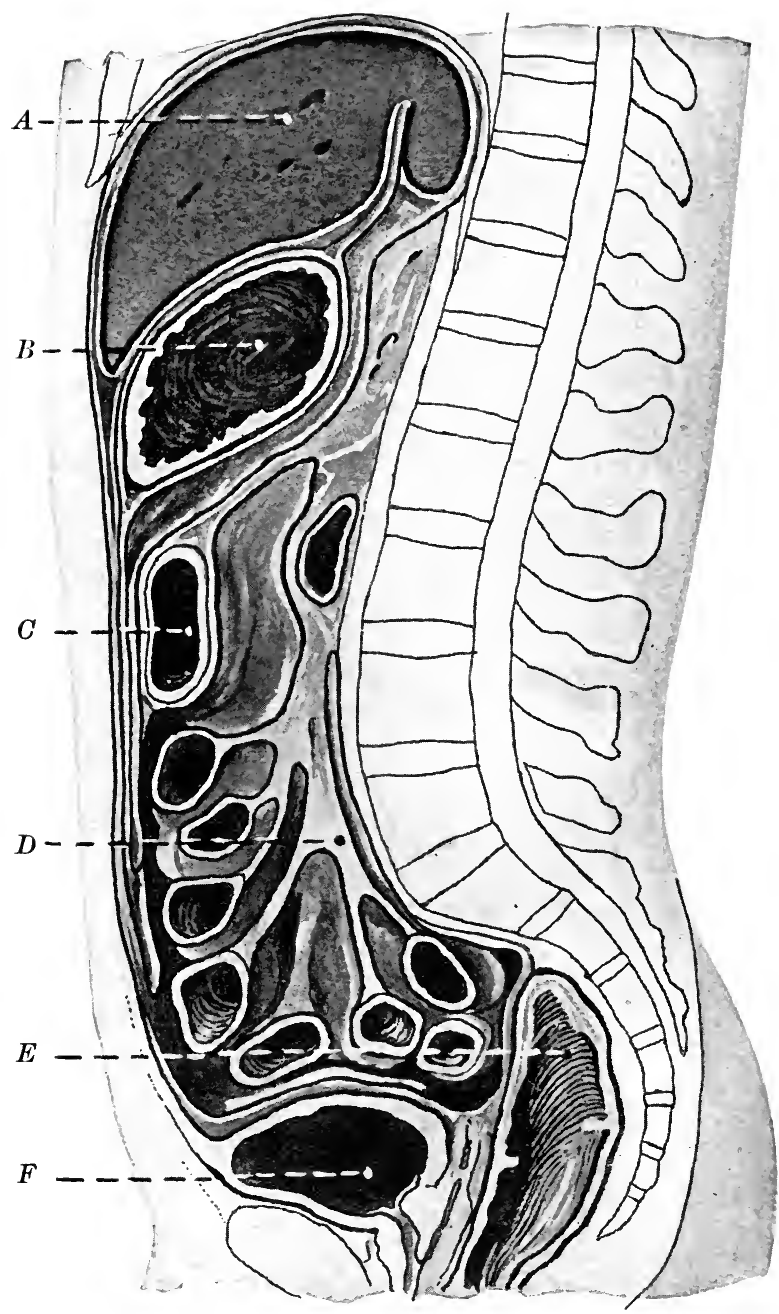

FIg. 137. Median dorso-ventral section of the trunk in the abdominal region, showing the suspension of the stomach and intestine by the mesentery. After Spalteholz

$A$, liver; $B$, stomach ; $C$, transverse colon; $D$, mesentery ; $E$, rectum; $F$, urinary bladder 


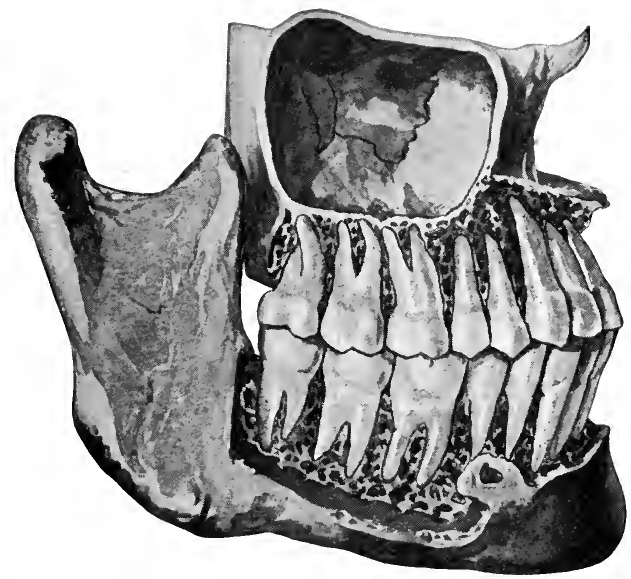

Fig. 138. 'The permanent teeth in the jaw-bones, viewed from the right. After Spalteholz

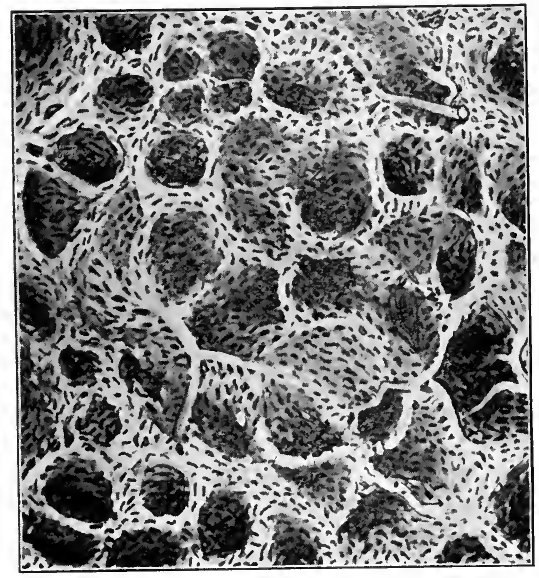

Fig. 139. The network of capillaries on the lining of the air cells of the lungs. After Kölliker 


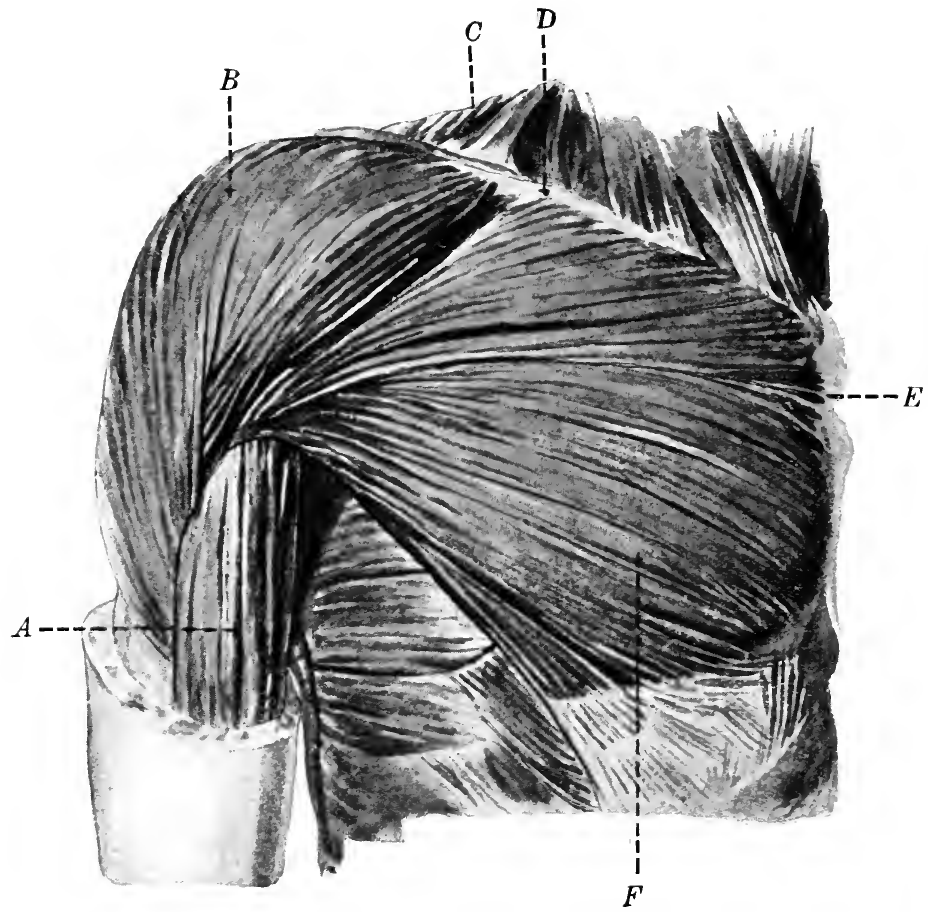

Fig. 140. First layer of muscles of the breast and shoulder region. After Spalteholz

$A$, biceps of the arm (p. 34); $B$, deltoid; $C$, portion of the trapezius (see Figs. 106 and 107$) ; D$, elavicle; $E$, sternum or breastbone; $F$, pectoralis major (see p. 324 and Fig. 107) 


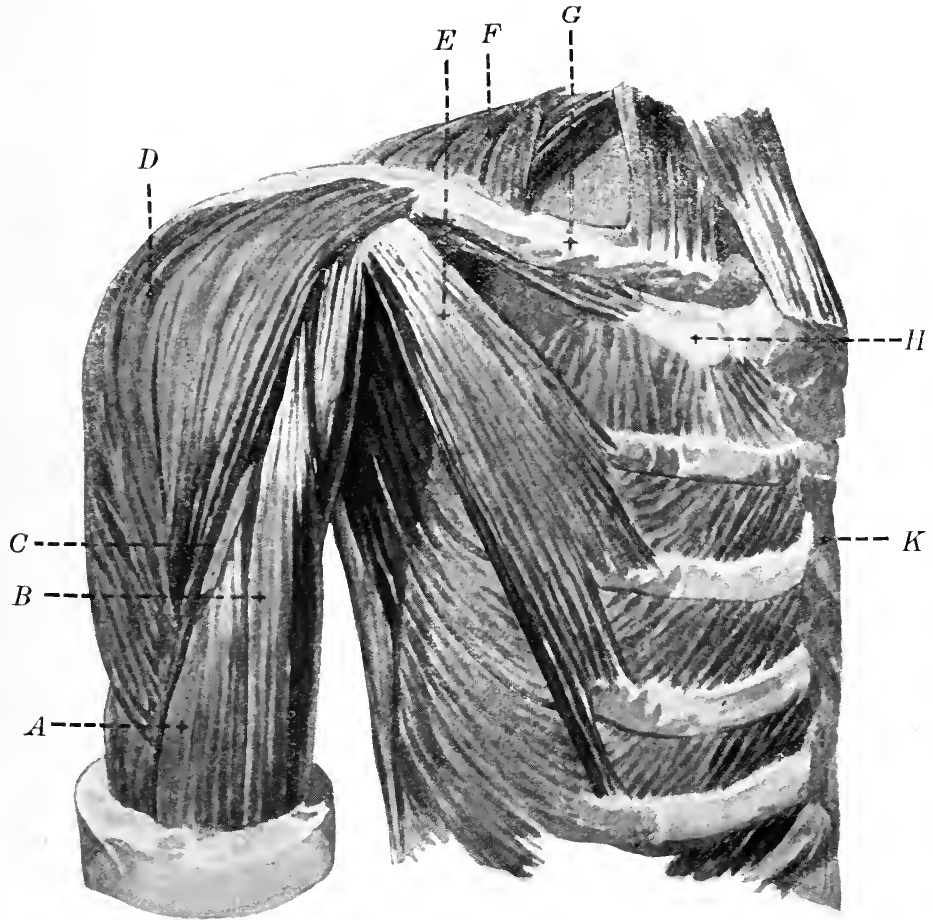

Fig. 141. Second layer of muscles of the breast, exposed by dissecting away the pectoralis major in Fig. 140. After Spalteholz

$A, B$, the two "heads" of the biceps ; $C$, cut end of the pectoralis major; $D$, deltoid ; $E$, pectoralis minor ; $F$, trapezins ; $G$, clavicle; $H$, first rib; $K$, sternum. Note the direct attachment of the intercostal muscles to the ribs (p. 8). Compare Fig. 140 


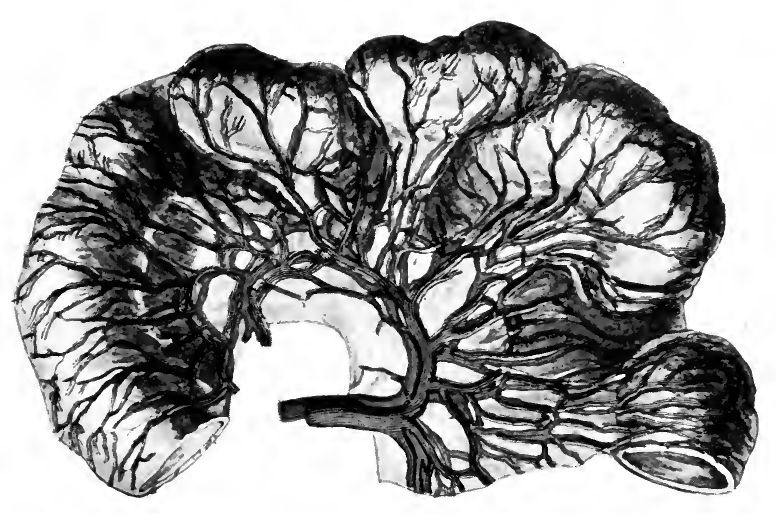

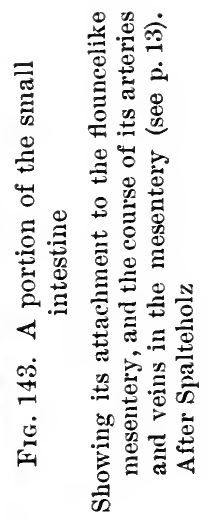
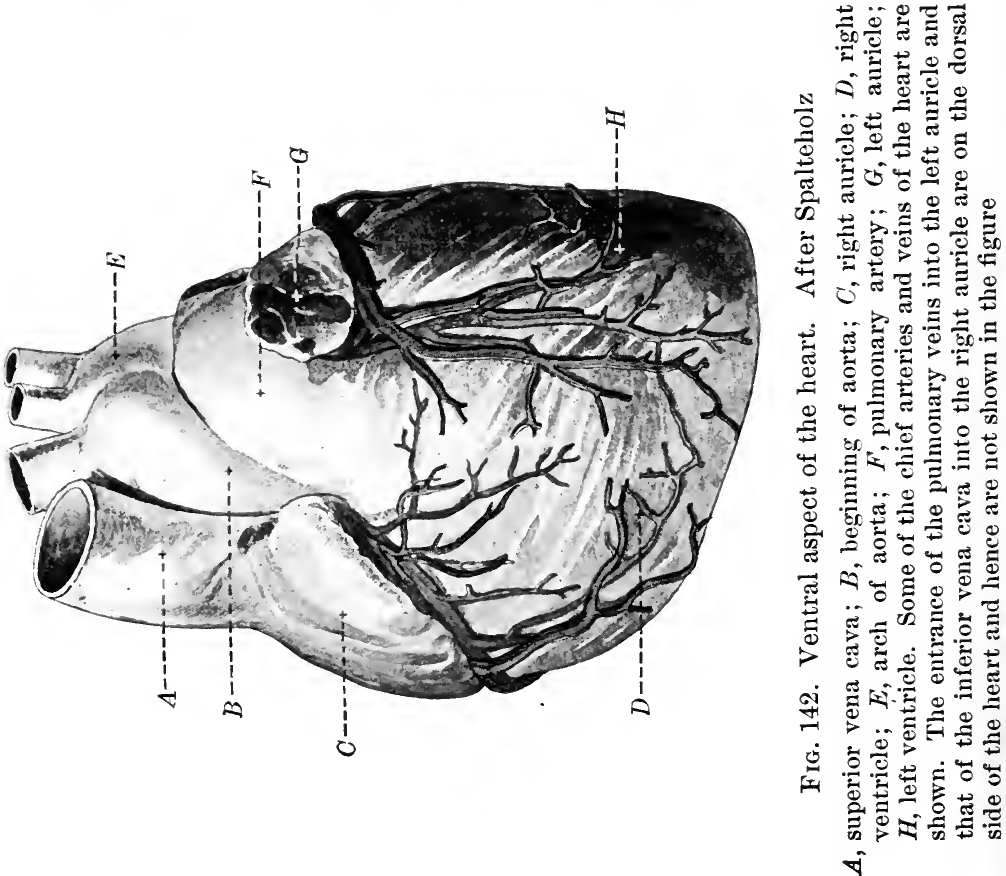


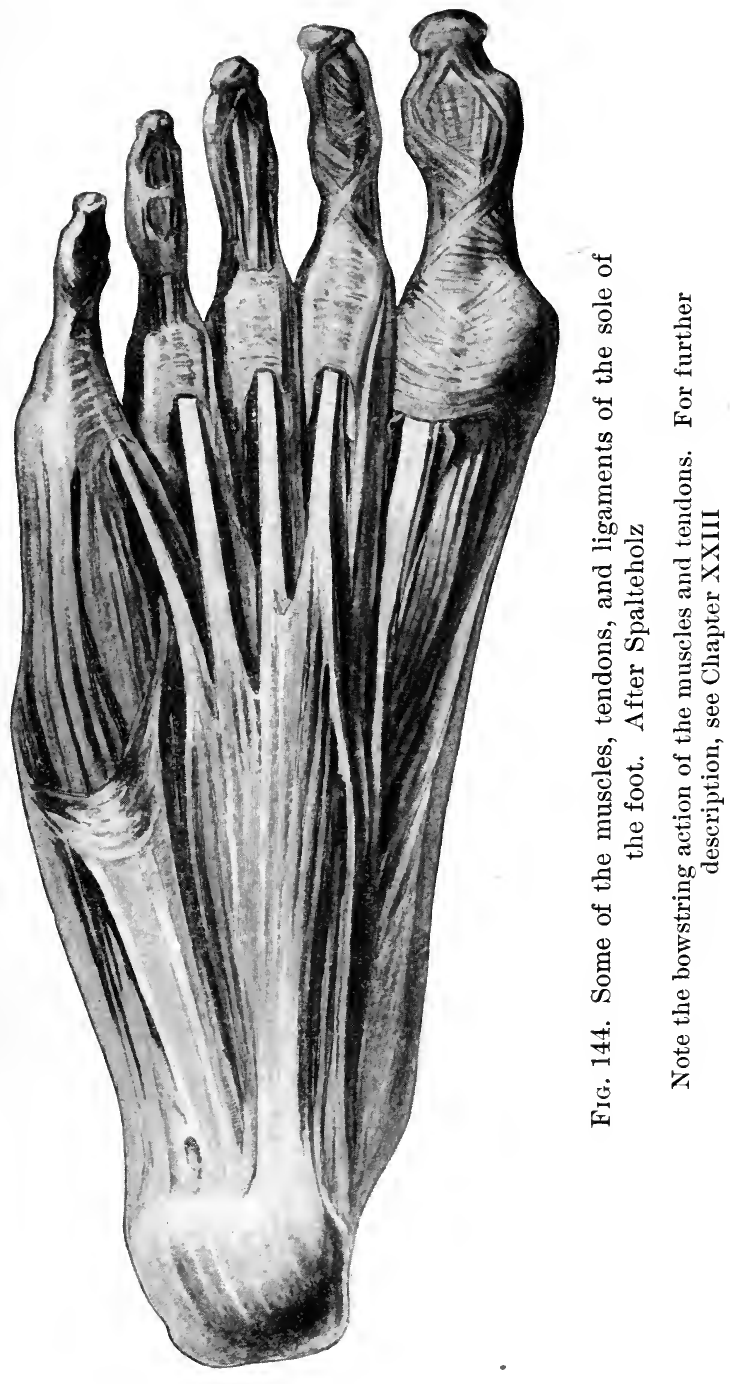




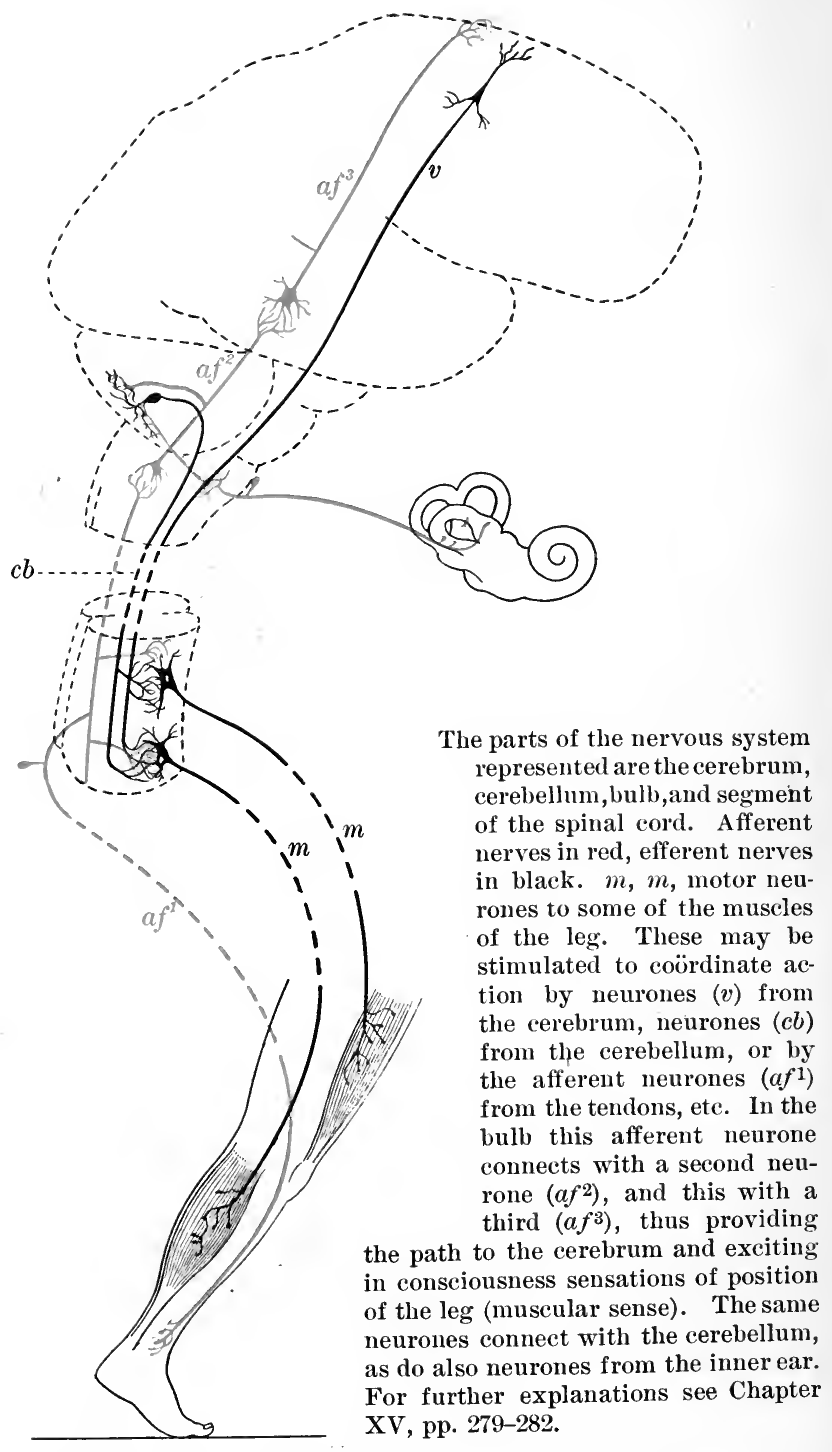

Fig. 145. Diagram of the nervous mechanism of walking 

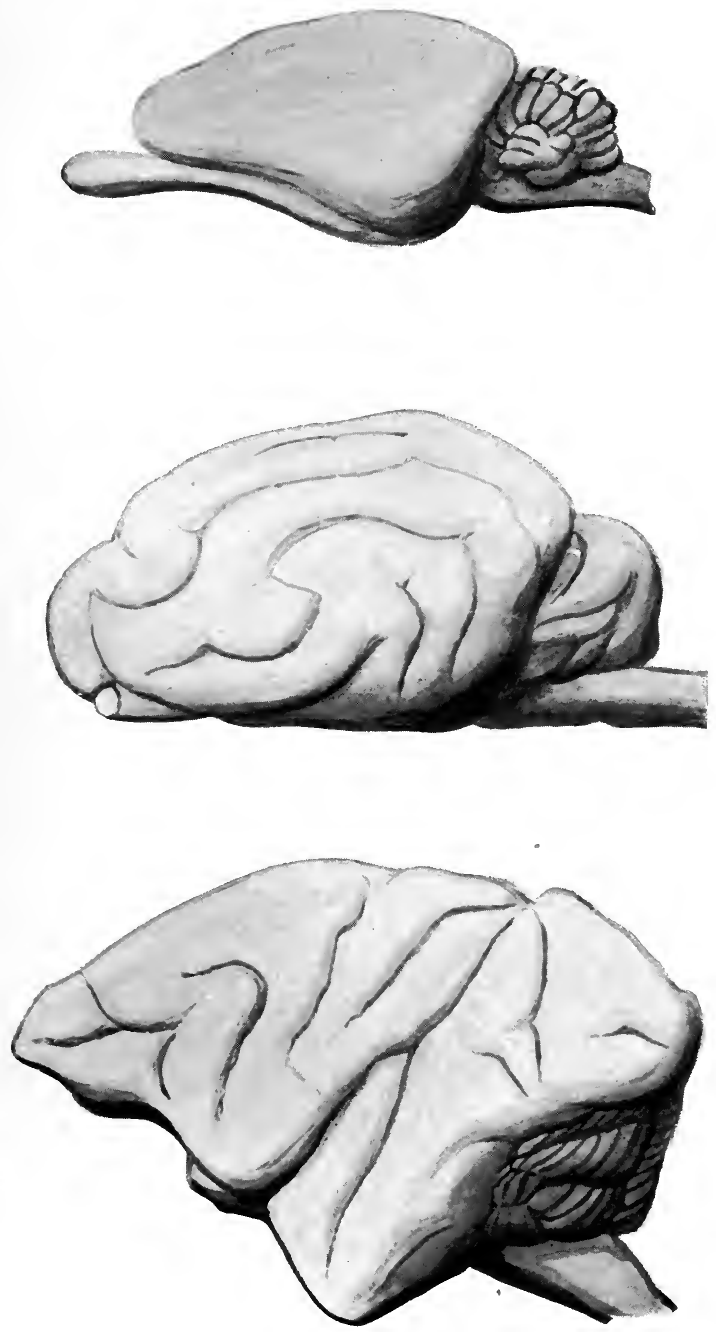

Fig. 146. Side view of the brains of rabbit, cat, and monkey See page 271 


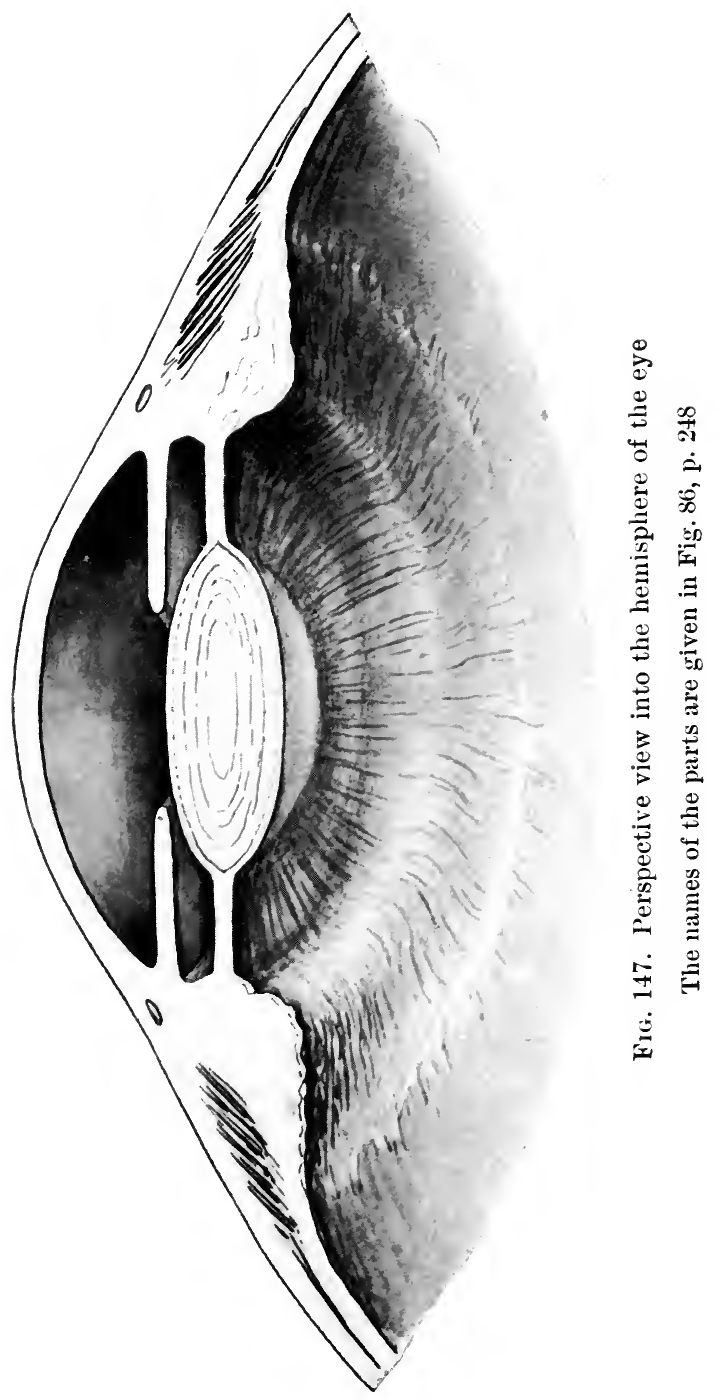




\section{N D E X}

Abdominal breathing, 170 .

Abdominal cavity. See Peritoneal cavity.

Abdominal muscles, action in breathing, 172.

Absorption from the intestine, 123.

Accommodation, for near objects, 248 ; muscle of, 247.

Adipose tissue, 182, 230.

Adrenal, 68.

Adulteration of foods, 508.

Afferent impulses, 77 ff.; reflex and conscious effects of, 281 .

Afferent neurones, 77, 78.

Air cell, 168.

Air, good and bad, pure and impure, 444 ; change of, 522. See also Fresh air.

Albuminoids, 91, 218.

Alcohol, physiological action of, 366 ; as a stimulant, 367,369 ; as a food, 372 ; as a defense against cold, 372 ; pathological conditions due to, 373; in muscular work, 370 ; influence on self-control, 375 .

Alcoholic beverages, composition of, 363.

Alimentary canal, structure of, 20 ; as a digestive laboratory, 97 . Alimentation, 88, 96.

Alveolus of gland, 31, 32 (fig.), 33 (fig.); of lungs, 167, 168 (fig.). Amœba, amœboid movement, 135. Amylopsin, 119. Anesthetics, 284.

Animal foods, 94, 111, 240, 241.
Ankle, bones of, 19, 403 (fig.), 404 (fig.).

Anopheles, 490, 491 (fig.), 492 (fig.). Anterior, 9.

Antiseptics, 475, 504 ; in food preservation, 512.

Antitoxin, 488, 499.

Aorta, 13, 22, 24 (fig.), 25, 141. Apical lobes of lungs. See Lungs. Appendicular skeleton, 14, 19.

Appetite, 265 ; as a guide in feeding, 348.

Aqueduct of Sylvius, 268, 270 .

Aqueous humor, 247.

Arborization. See Synapse.

Arterial reservoir, 139.

Arterial tone, 158.

Arteries, 22, 38, 144 (fig.).

Asphyxia, 168.

Astigmatism, 253.

Auditory nerve, 28, 259.

Augmentor nerves of heart, 157.

Auricle, 22, 24 (fig.), 137.

Auriculo-ventricular valves, 138.

Automatic nervous actions, 82.

Axial skeleton, 14.

Axon or axis cylinder, 73, 77.

Bacilli, 469.

Bacteria, 469. See also Microbes.

Balance exercises, 331.

Balance movements, 276.

Bathing and baths, 413 ff., 476.

Beds and bedsteads, 431.

Bile, 119.

Bile duct, 107 (fig.).

Bladder, urinary, 179, 180 (fig.). 
Blood, arterial and venous, 23,163 ; microscopic structure of, 133 ; gases of, 163 ; as a common carrier, 132; distribution among organs, 144.

Blood corpuscles, red, $133-135$; as carriers of oxygen, 164 .

Blood corpuscles, white, 133, 134; behavior during inflammation, 382.

Blood plasma, 134, 135; gases of, 163.

Blood vessels, 38. See also Arteries, Capillaries, and Veins.

Boards of Health. See Health.

Body cavity, 10.

Bone, 37. See also Skeleton.

Brain, 27 ; of frog, 267 ; of mammal, 270; histological structure of, 271 ; the seat of sensations, 245 ; functions of, $274 \mathrm{ff}$.

Breastbone, 17.

Breathing movements, 168, 169; effect on circulation, 145,173 ; effect on flow of lymph, 148, 173; hygiene of, 172 ; in muscular activity, 308, 315 .

Breathlessness, 174.

Bronchiole, 168.

Bronchitis, 381.

Bronchus, 12, 21, 167.

Bulb, 267 (fig.), 268, 270 (fig.); functions of, 275 .

Caffeine, 361.

Calendered paper, 399.

Calorie, 212.

Canal, spinal or vertebral, 18.

Canning of foods, 512.

Capillaries, 25 (fig.), 27, 32 (fig.), 39.

Capsule of gland, 31 .

Carbohydrates, 91,218 ; digestion of, $103,110,119$; waste products of, 177 ; as source of power for work, 213; fuel value of, 213 ; as food in cold climates, 216; as source of fat, 231.

Carbon dioxide (carbonic acid) formed during muscular work, $49,60,175,306$; in lymph, 162; in blood plasma, 163 .

Cardiac region of stomach, 107.

Carpets, 432.

Catalytic actions, 106.

Catarrh, chronic, 394.

Catarrhal conditions, care of, 383.

Cellar, sanitation of, 428.

Cell walls in plants, 94 .

Cells, 32, 33, 36, 38, 40, 41 (fig.), 43 ; as chemical factories, 52 ; as living mechanisms, 63 ; waste and repair of, 220.

Cellulose, 94.

Cement of tooth, 99 .

Cemeteries, 528.

Central canal of spinal cord, 268.

Cerebellum, 267 (fig.), 268, 273 (fig.).

Cerebrum, 83, 266 (fig.), 267 (fig.), 269 (fig.), 270 (fig.); connections with other parts of the nervous system, 279 ; functions of, 277 , 283.

Cervical vertebræ, 14 .

Cesspools, 459.

Chemical change, relation to work, 52 ; as source of heat, 193 ; as influenced by temperature, 187 .

Chicken pox, 493, 494.

Chloral, 377.

Chloroform, 284.

Chocolate, 362.

Cholera, Asiatic, 501.

Choroid, 247, 248, 251 (fig.).

Chyme, 112.

Cilia, 474.

Ciliary muscle, 248.

Ciliary region of eye, 248 (fig.).

Cinders, removal from eyes, 400 .

Circulation, organs of, 22, 132, 140 (fig.); time of, 136 ; mechanics of, 136; in warm and cold 
weather, 150, 200 ; during muscular activity, 151; during digestion, 155,156 ; during mental work and sleep, 155 ; nervous factors of, $156 \mathrm{ff}$.; essential to respiration, 174; in foot, 409.

Cisterns, 454.

Clavicle, 20.

Cleanliness, sanitary value of, 433 . Climate, and mental work, 204 ; and feeding, 236 ; in treatment of tuberculosis, 482 .

Clothing, hygiene of, $418 \mathrm{ff}$.

Coagulation of proteids, 91 .

Coarse foods, importance of, 355 .

Cocaine, 377.

Cocci, 469, 470 (fig.).

Coccygeal vertebræ, 14.

Cochlea, 259.

Cocoa, 362.

Coffee, 361 .

Cold, effect on circulation of the blood, 150; effect on body as a whole, $200 \mathrm{ff}$.

Cold, sensations of, 261.

Cold-blooded animals, 189.

Colds, nature, prevention, and care of, $380 \mathrm{ff}$.

Collagen, 91, 218.

Collar bone. See Clavicle.

Collaterals, 79.

Colon, 21.

Color, sensations of, 256.

Compensatory adjustments of the circulation, 150.

Conduction of heat, 194-196.

Congestion of blood in internal organs, 307 ; during inflammation, 382.

Connective tissues, structure of, 7 , $8,37,228,230$; of glands, 31,32 ; of muscles, 34,35 ; of nerves, 72 ; of lungs, 167 ; of skin, 182 ; relation to blood vessels, 38 ; to lymphatics, 39 ; digestion of, 109 , 115 (footnote).
Consciousness, 244, 275, 277, 283, 285.

Consciousness of correct posture, education of, 326 .

Constant temperature of the body, 188.

Constipation, 130.

Consumption. See Tuberculosis.

Contagious diseases. See Diseases.

Contraction of muscle, $34,48,56$.

Convection of heat, 194, 195.

Convolutions of cerebrum, 270 (fig), 271.

Cooking, 243 ; as an aid in nutrition, 349 ; sterilization of food by, 511.

Cooling off suddenly, 391.

Coördination, $70,80,275$; training of, $86,280,330$.

Corium, 182.

Cornea, 247, 248 (fig.), 251 (fig.).

Corpuscles. See Blood corpuscles.

Corrective muscular exercises, 321 ; hygienic value of, 332 .

Cortex of cerebrum and cerebellum, 272.

Costal breathing, 170.

Cotton underwear, 421.

Cough medicines, 387.

Course dinners, 242.

Cranial nerves, 271.

Cranium, 18.

Creatinin, 178.

Culex, 492 (fig.).

Curd of milk, 90, 108.

Curvatures of vertebral column, 16.

Curve of fatigue, 58.

Cutaneous sensations, 261.

Cutis, 182.

Cycling, 317.

Cytoplasm, 32, 36, 43, 76.

Dampness, 380.

"Dangerous region": of atmospheric temperature, 201, 390.

Deafness, 401. 
Dendrites, 76, 77, 272, 273, 274.

Dentine, 99.

Dermis, 182.

Dextrines, 92, 105.

Dextrose, 98 .

Diaphragm, 10 ; action in respiration, 170-172.

Diarrhea, 130, 380, 455.

Diastase, 364 .

Diastole, 137, 139 (fig.).

Diet, 226.

Digestibility of food, 226 .

Digestion, organs of, 20 ; nature of, 88, 98; external and internal, 97 ; in the mouth, 98 ; in the stomach, 105 ; in the intestine, 115 ; coöperation of processes of, 129 ; and the circulation, 155 ; and temperature regulation, 205; as affected by muscular activity, 309 ; during fatigue, 342.

Diphtheria, 486, 533.

Diseases, 293 ; mental cures of, 345; infectious and contagious, 467 ; prevention of microbic, 475 .

Disinfectants, 475 .

Distilled liquors, 365 .

Divisive movements of intestine, 121.

Dorsal, 9.

I)rainage in house sanitation, 428 , 458.

Drinking cups, public, 525.

Driving force for circulation through capillaries, 144.

Drug habit, 358.

Drugs, $357 \mathrm{ff}$. ; in the operation of the nervous system, 344 ; in the catarrhal conditions, 387 ; as a substitute for hygienic living, 388 ; in treatment of tuberculosis, 482.

Ducts of glands, 20, 29, 32 (fig.), 33 (fig.).

Dust as vehicle of tuberculosis, 479 .

Dyspepsia, 113.
Ear, structure of, 258 ; care of, 401. Efferent nerve fibers and impulses, 76,80 (fig.).

Elasticity of arteries, 142.

Elimination of intestinal waste, 128.

Emmetropia, 250.

Enamel, 99.

End organs of nerves, 28, 76-84, 245.

Engine, analogy of, 62.

Enjoyment of food, hygienic value of, 114.

Enjoyment of muscular activity, 311.

Environment, 299.

Enzyme，45，48，52; action of, $101 \mathrm{ff}$. ; of saliva, 103 ; of gastric juice, 108 ; of small intestine, $118,120$.

Epidemics, 466. See also Plagues. Epidermis, 182.

Epiglottis, 20 (fig.).

Equilibrium, nervous factors in, 276 ; in physical training, 330.

Esophagus. See Esophagus.

Ether, 284.

Eustachian tube, 259, 401.

Excitation, 285, 367.

Excretion, 177 ; in relation to feeding, 225 ; essential and incidental organs of, 178.

Extractive, 135, 218.

Eye, structure of, 246; care of, 395.

Face, bones of, 18.

Farsightedness, 253.

Fasciculus of muscle, 34, 35.

Fatigue, $55 \mathrm{ff}$; hygienic value of, 66 ; influence on digestion, 353 ; and taking cold, 389 ; practical considerations concerning, 316 .

Fatigue level, 58, 61.

Fats, 92,218 ; of meat, 115 ; digestion of, 119 ; waste products of, 177 ; fuel value of, 213 ; as food 
in cold climates, 217 ; storage of, $224,227,229,231$; a hindrance to output of heat, 205.

Fatty acids, 92, 119.

Feces, 128.

Feeding, muscular activity after, 156 ; effect on chemical changes in the body, 221; for muscular activity, heat, cold, mental work, and sedentary occupations, 235237 ; hygiene of, 347 ; during colds, 386.

Femur, 19.

Fermentation, 364 .

Fever, 210.

Fibula, 19.

Filters, house, 455.

Filth and filth diseases, 484.

Filtration of public water supplies, $513 \mathrm{f}$.

Fires, open, 196, 434.

Flagella, 474 (fig.).

Flat foot, 406.

Flesh of body, 227; increase of, 228.

Food accessories, $357 \mathrm{ff}$.

Food stuff. See Nutrient.

Foods, chemical composition of, 89 , 95; animal and vegetable, 94, 240 ; as source of energy or power, 88,211 ; as material for growth and repair, 88,220-222; fuel value of, 211 ; heating, 216 ; choice of, 233 ; adulteration and infection of, $508 \mathrm{ff}$; preservatives and antiseptics in, 511; canning of, 512.

Foot, hygiene of, $403 \mathrm{ff}$; arches of, 404; deformation of, 405 ; physical training of, 410 .

Force-pump action of heart, 137, 139 (fig.).

Forebrain, 267 (fig.), 283.

Fresh air, as a substitute for exercise, 320 ; as a cure for colds, 385 .

Fried foods, 352 .
Fuel substances, storage of, in muscle, 49.

Fuel value of food, 211.

Gall bladder, 107 (fig.).

Games, 318.

Ganglion, 73 ; of the dorsal root, 77.

Garbage, disposal of, 461.

Gas, illuminating, 440; natural, coal, and water, 515 ; poisoning by, 516 .

Gaseous exchange in capillaries, 164,165 ; during muscular activity, 175 .

Gastric digestion, 350 .

Gastric juice, 108; secretion of, 113. Gelatin, 91.

General muscular exercise, $314,332$. Germ theory of disease, 467 .

Germicides, 475.

Glands, 20, 29, 31, 32 (fig.), 33 (fig.); working and resting, 44, 47 ; blood supply during activity, 45 ; ductless, $29,68$.

Glottis, 20 (fig.).

Gluten, 90.

Glycerin, 92.

Glycogen, 227, 232.

Granules, storage of, in gland cells, 47.

Gray matter of spinal cord and brain, 75 .

Grippe, 493.

Ground water, 451, 513.

Growth, favorable period for the acquisition of deformities, 325 .

Gums or dextrines, 92.

Gymnasium, 332, 476.

Habits, physical basis of, 287.

Hair and hair follicle, 183, 185 (fig.).

"Hardening" to cold, 392.

Harvey, William, 136.

Headaches, 128, 396. 
Health, 293 ; public, 529 ; national, state, and local boards of, 530 ; in different countries, 538. See also Public health.

Hearing, 258.

Heart, 10, 12, 22; valves of, 138 ; nerves of, 157; effect of muscular activity on, 307 .

Heart beat, 136 ; force-pump action of, 137, 139 (fig.); regulation of, 157.

Heat, effect of, on the circulation of the blood, 150; unit of, 212 ; transfer of, from internal organs to the skin, 195; production and transfer of, 192, 198; supply of energy for production of, 216; as a food preservative, 512 .

Heating foods, 216.

Hemoglobin, 134, 135, 165.

Hepatic artery, 24 (fig.).

Hepatic vein, 24 (fig.), 27.

Hind brain, 267.

Hip bones, 20.

Hoarseness, 21.

Horny layer of skin, 182, 183.

Hotels, sanitation of, 526 .

House, sanitation, $425 \mathrm{ff}$; ; construction, 429; furnishings, 430 ; floors, 431 ; care of, 432.

Humerus, 19, 20.

Humidity, influence on temperature regulation, 202; influence on mental work, 204.

Hunger, 264.

Hygiene, scope and subdivisions of, 301 ; personal, 304 ; domestic, 425 ; public, 463.

Hypermetropia, 253, 255.

Hypnotics, 344.

Ice supply of the house, 455 .

Illumination for near work, 255.

Illusions, optical, 257.

Immunity, natural and artificial, 497.
Indigestible material in food, 93.

Indigestion, 113, 125.

Infection of foods, 509.

Infectious diseases. See Diseases.

Inflammation, 381 .

Influenza, 493.

Inhibition, 158, 367; in the nervous system, 285,367 ; an active process in muscular relaxation, 343.

Inhibitory nerves of heart, 157.

Inorganic salts. See Salts.

Instep, bones of, 19.

Interdependence of organs, 65 .

Internal secretion, 67.

Intestinal juice, 117, 120.

Intestinal waste, elimination of, 128.

Intestine, small, 10, 21, 24 (fig.), 26,116 ; large, 11, 21, 127; action of muscular coat of, 120 .

Iris, 247, 248 (fig.), 251 (fig.).

Irritability, 46, 50 .

Jugular vein, 24 (fig.).

Junket tablets, 108.

Kidneys, 11, 13, 24 (fig.), 26, 68 ; structure of, 179, 181.

Kilogrammeter, 212.

Koch, Robert, 473, 478.

Labyrinth of ear, 260.

Large intestine. See Intestine.

Laryngitis, 381.

Larynx, 21.

Lateral costal breathing, 171.

Lateral curvature of spine, 329 .

Lens of eye, 247, 248 (fig.), 251 (fig.); formation of image by, 249,250 (fig.).

Levulose, 98.

Ligaments, 8, 14, 16, 17.

Lighting of the house, 439.

Linen underwear, 421.

Lipase, 119. 
Liver, 11, 21, 24 (fig.), 26, 27, 29, 68, 107 (fig.), 117.

Lobes and lobules of glands, 30, 31 (fig.); of the lung, 13, 172.

Lockjaw, 501.

Locomotion, nervous factors in, $276,277,280$.

Longevity, 538.

Lumbar vertebræ, 14.

Lungs, 10, 12, 21, 24 (fig.) ; structure of, 167 ; apical lobes of, $172,309$.

Lymph, 39 ; origin of, 40 ; environment of cells, 40,135 ; gases of, 162.

Lymph flow, function of, 42 ; cause of, 137 ; influenced by respiratory movements, 173 ; influenced by muscular activity, 308 .

Lymph spaces, 39 .

Lymphatics, 41, 147.

Malaise, $128,396$.

Malaria, $489 \mathrm{ff}$.

Malt liquors, 364.

Massage, 147.

Master neurones, 84.

Mastication, hygienic aspects of, 349.

Mattings, 432.

Measles, 493, 494.

Meat, a proteid food, 90 ; composition, 220 ; in diet, 242 ; eating of, 350 ; as a vehicle of infection, 479.

Mediastinum, 11, 21.

Medulla oblongata. See Bulb.

Mental cures of disease, 345 .

Mental states, influence on the health of the nervous system, 344.

Mental work and the circulation, 155 ; after meals, 156,353 ; as influenced by climatic conditions, 204; feeding for, 237; effect on nutrition, 237.
Mesentery, 12, 26.

Meshwork underwear, 421.

Microbes, 467,468 ; as scavengers, 471 ; in decomposition and decay, 472 ; as disease germs, 473 ; growth, multiplication, and spore formation, 473 (fig.).

Microbic action on food, 225.

Microbic life in the intestine, 125, 128.

Micromillimeter, or micron, 133.

Microparasites, 467.

Microscopic work and the eyes, 399.

Midbrain, 267 (fig.).

Milk, 108; as a vehicle of infection, $479,517,531$.

Milk supplies, purity of, 517. .

Moisture, influence on temperature regulation, 202.

Monosaccharides, 92.

Moral conduct as a part of nervous hygiene, 344 .

Morphine, 376.

Mosquito, as transmitter of malaria, 491 ; as transmitter of yellow fever, 493.

Motor nerves, 28.

Mouth, 183.

Movements, active or passive, effect on circulation, 146 ; respiratory. See Breathing movements.

Mucin, 45, 127.

Mucous coat of stomach, 108; of intestine, 116, 127.

Muscle fibers, 35, 36 ; of stomach and intestine, 108, 116, 127 ; of arteries and veins, 144 .

Muscles, 8, 16 ; structure of, 34 ; isolated, 49, 56; physiology of, 48,56 ; and temperature regulation, 207 ; antagonistic action of, 17, 208 ; in faulty carriage, $322 \mathrm{ff}$.

Muscular activity, effect on circulation, $146,151,156$; on respiration, 175 ; and the regulation of 
the temperature of the body, 203, 390,423 ; hygiene of, 304 ; ministry to body as a whole, 304 ; after meals, 156,353 ; and fatigue, 316 ; for women, 313 ; physiology of, 306.

Muscular exercises, general character of the most useful, 312 ; for special purposes, 321 .

Muscular sense, relations to locomotion and maintenance of equilibrium, 280.

Muscular work, power for, 213 ; feeding for, 235 ; of stomach, 110 ; of intestine, 120.

Myopia, 252.

Narcotics, 344. See also Anesthetics.

Nasal cavity, 20.

Nearsightedness, 252.

Near vision, 249, 254, 396.

Neck, carriage of, 328 .

Nerve cells, 73 ff., 271.

Nerve fibers, structure of, 73 ; afferent and efferent, 76, 77; of spinal cord and brain, 75, 271.

Nerve roots, 75 .

Nerves, 28; distribution to organs, 42 ; structure of, 72 ; cranial, 271.

Nervous impulse, nature of, 87 ; in reflexes and sensation, 246, 266.

Nervous strain, $335 \mathrm{ff}$.

Nervous system, general anatomy of, $26,27,266$; structural organization of, $72 \mathrm{ff}$. ; training by practice, 86,286 ; physiology of, 70 , 273 ; and the circulation, $157-$ 161 ; and respiration, 173 ; and secretion of perspiration, 185 ; and regulation of body temperature, 209 ; and carriage of body, 324 ; hygiene of, 334.

Nervousness, 396.

Neurones, 77 ; "master," 84 ; of brain, 272.
Nitrogenous equilibrium, 223.

Noise, 401.

Non-proteids, 218

Nucleus, $32,36$.

Nutrients, 89 ; classification of, 218 ; special effects of each on oxidations of the body, 221 ; on flesh weight, 222 ; on storage of fat, 224 ; choice of, $233,239$.

Nutrition, $211 \mathrm{ff}$.

(Esophagus, 10, 12, 20 (fig.), 183.

Opium, 376.

Optic lobes, 267.

Optic nerve, 28, 247, 251 (fig.).

Organs, typical structure of, 41 (fig.), 42.

Overfeeding, 351.

Overheating of houses, 439.

Oxidation, 49, 50, 52, 92, 162 ; products of, 177 .

Oxygen, rôle of, in cell life, 48, 162 ; in lymph, 162 ; in blood plasma, 163; absorbed during muscular activity, 175.

Pain, sensations of, 263 ff. ; signs of, 284.

Palate, 20.

Pancreas, anatomical relations, 11, $21,26,29,107$ (fig.), 117 ; the source of an internal secretion, 68.

Pancreatic duct, 107.

Pancreatic juice, 109, 118.

Paper, in printing, 399.

Papillæ of skin, 182.

Parasites, 467.

Parks, 476, 527.

Parotid gland, 30.

Pasteur, Louis, 473.

Pasteurization, 475.

Pelvis, of skeleton, 19 (fig.), 20 (footnote); of ureter, 180, 181 (fig.).

Pepsin, 108, 118. 
Peptones, 98.

Peristalsis, 122.

Peritoneal cavity, 10, 11, 12.

Peritoneum, 10, 11, 13.

Peritonitis, 11.

Perspective, idea of, 256.

Perspiration, composition of, 179 ; secretion of, 184 ; sensible and insensible, 185 ; and the output of heat, 197 ; in relation to feeding, 352.

Pharyngitis, 381.

Pharynx, 20, 21, 183, 259.

Plague, bubonic, 502 .

Plagues, 466, 535.

Plasma. See Blood plasma.

Play, 287, 318.

Playgrounds, 476.

Pleura, 10, 11.

Pleural cavity, 10,11 ; pressure in, 169.

Pleurisy, 11.

Plumbing of the house, 456 .

Pons Varolii, 270 (fig.).

Portal vein, 24 (fig.), 27, 125.

Position, sense of, 262, 280, 327.

Posterior, 9.

Posture, nervous factors in, 276 ; faulty, as a cause of deformity, 322.

Presbyopia, 253.

Preservatives of food, 511.

Pressure in arteries and veins, 142, 143, 152 (fig.); in pleural space, 169.

Processes of nerve cells, 76 .

Proteids, nature of, 90,115, 218; in blood plasma, 135 ; in living cells, 220 ; digestion of, in stomach and intestine, 109, 118; fuel value of, 213 ; quantity in body, 222 ; waste products of, 177 ; influence on secretion of urine, 181 ; disintegration of, 221 ; native, 126.

Proteoses, 109.

Protozoa, 469, 470.
Pseudopodium, 134.

Psychic secretion of gastric juice, $113,350$.

Ptyalin, 103.

Public health, $463 \mathrm{ff}$; rules and regulations, 464 ; authorities, 465 ; problems, 465.

Pulmonary arteries, 22, 24 (fig.), 25, 167.

Pulmonary circulation, 23,140 (fig.).

Pulmonary veins, 22, 23, 24 (fig.), 25, 167.

Pulp cavity, of tooth, 99 .

Pupil of eye, 247.

Purposeful character of reflex and volitional actions, 71, 80, 275.

Pylorus, 107 (fig.).

Quarantine, 530, 537; in tuberculosis, 480 ; in smallpox, 496 ; in diphtheria, 533.

Radiation of heat, 195, 196.

Radius, 19.

Railroad trains, reading on, 399 .

Rectum, 130, 183.

Reflexes, 81 ; of locomotion, etc., 280 ; disappearance of, during anesthesia, 284.

Relaxation, muscular, in sleep, 342.

Renal arteries, 26.

Rennin, 108, 118.

Repair of cells, 220.

Reservoirs, arterial and venous, 139.

Resistance to the flow of blood, 143, 152 (fig.).

Respiration, organs of, 21 ; of the cells, 162 ; nervous factors in, 173 ; and muscular activity, 175,308 , 315.

Respiratory movements. See Breathing movements.

Rest, in relaxation and sleep, 334, 338 ; in change of work, 340.

Retina, 247. 
Rheumatism, 380.

Rhinitis, 381 .

Ribs, 17 ; action in respiration, 170 , 172.

\section{Rugs, 432.}

Sacrum, 14, 20.

Saliva, chemical composition of, 45 ; secretion of, 44-48; action in digestion, 101, 105.

Salivary glands, 21,30 .

Salts, inorganic, 45, 93, 218, 219.

Sanitation, scope and subdivisions of, 301 ; domestic, 425 ; public, $303,463$.

Saprophyte, 467 .

Sarcolactic acid, 49, 60.

Sarcolemma, 35.

Sarcostyles, 36, 53 .

Scapula, 20.

Scarlet fever, 494.

Sclerotic coat, 247, 248.

Sebaceous glands, 184 .

Secondary aids to the circulation, 145.

Secretion, general physiology of, 44-48; internal, 67 ; of gastric juice, 113; of pancreatic juice, bile, and intestinal juice, 116-120; of urine, 180 ; of perspiration, 184.

Sedentary occupations, 237.

Segmenting movements of intestine, 121.

Semicircular canals, 259, 260 (fig.), $263,280$.

Sensations, 244, 245, 246 ; reference of, 244.

Sense organs, 78, 244, 245.

Sense of position, importance of, in physical training, 327.

Septum, of gland, 31 ; of muscle, 34.

"Setting-up" drill, 326.

Sewage, disposal of, 459, 519.

Shivering, 208.
Shoes, 405, 407; for deformed feet, 409 ; temperature and moisture within, 410.

Shoulder blade, 20.

Shoulder girdle, 20.

Sinuses of temporal bone, 260 .

Skeleton, 14, 15, 19.

Skin, 7 ; structure and functions, 181 ; regulator of the output of heat, 199 ; as an organ of absorption, 186; care of, 185, 413.

Skull, 19.

Sleep, circulation during, 153 ; hygiene of, $334 \mathrm{ff}$.

Smallpox, 495 ; inoculation and vaccination for, 498.

Smell, sensations of, 261.

Soaps, 92, 119.

Soda water, 363 .

Soil, an element in house sanitation, 428.

Solidity, ideas of, 256 .

Somnambulism, 278.

Speech, 282.

Spinal column, 14, 17; faults of carriage of, 328 .

Spinal cord, gross anatomy of, 18 , 27 ; structure of, 74,267 ; functions of, 274.

Spirilla, 469, 471 (fig.).

Spitting nuisance, 488.

Spleen, 11, 26.

Sporozoa, 470.

Springs, 453.

Sputum, as vehicle of tuberculosis, 479.

Starch, 91, 94 (fig.); digestion of, $102,110,119$. See also Carbohydrates.

Steapsin. See Lipase.

Stegomyia, 493.

Sterilization, 475 ; of food by cooking, 511.

Sternum. See Breastbone.

Stimulants, 344, $357 \mathrm{ff}$.

Stimulation, 46, 52, 80, 81 . 
Stomach, anatomical relations of, $10,21,26$; structure of, $107 \mathrm{ff}$. ; digestive work of, 108-115.

Storage of material in the cell, 52 .

Stoves, oil and gas, 437 .

Streets, sanitation of, 527.

Submaxillary gland, 30 .

Submucous coat of intestine, 116 (fig.), 127 (fig.).

Suction action of breathing movements, 148.

Sugars, 92, 104, 105, 119. See also Carbohydrates.

Summer complaint in children, 495 .

Sunshine, 428.

Supplies, public, of food, water, and gas, $505 \mathrm{ff}$.

Supporting organs and tissues, 37, 220.

Suprarenal, 68.

Surface water, 451, 513.

Suspensory ligament of eye, 248.

Sweat glands, structure of, 184 .

Synapse, 79, 273 (fig.).

Systemic circulation, 23.

Systole, 137.

Tannic acid, 361.

Tartar, 100.

Taste sensations, 261.

Tea, 361 .

Teeth, 98.

Temperature, external, influence on chemical change and vital activities, 187, 188; reactions of body to changes of, 200 ; "dangerous region" of, 201, 390 ; of living rooms, $390,439,447$; influence on secretion of urine, 181.

Temperature of the body, 188-205. Temperature sensations, 192, 195, 262.

Tendon, 8, 34, 35.

Tetanus, 501.

Theine, 361.
Thermal phenomena of the body, 187.

Thirst, 264.

Thoracic cavity. See Pleural cavity. Thyroid glands, 66 .

Tibia, 19.

Tobacco, 377.

Toes, bones of, 19 ; flexion of, in hygiene of foot, 405 .

Tone, arterial, 158; of skeletal muscle, 209.

Touch, 261 .

Trachea, 10, 11, 21.

Traveling, hygiene and sanitation of, $520 \mathrm{ff}$.

Trichina, 510.

Trunk movements, 315.

Trypsin, 118.

Tuberculosis, $477 \mathrm{ff}$.

'Tweenbrain, 267 (fig.).

Tympanic membrane, 258, 259 (fig.).

Tympanum, 259.

Type, size of, 398.

Typhoid fever, $483 \mathrm{ff}$.

Ulna, 19 .

Underclothing, 420.

Urea, 177.

Ureter, 179, 181 (fig.).

Uric acid, 178.

Urine, secretion of, 180 .

Use and disuse in the training of the nervous system, 286.

Vaccination, 498, 530.

Valves, of heart, 22, 138, 141; in . veins, 146.

Vasoconstrictor nerves, 159.

Vasodilator nerves, 160.

Vasomotor. See Vasoconstrictor and Vasodilator.

Vegetable foods, 94, 111, 240, 350.

Vegetarianism, 240.

Veins, 7. 23, 26, 38, 39, 144 (fig.) ; intermittent compression of, in muscular activity, 146. 
Venæ cavæ, 13, 22, 24 (fig.), 26.

Venous reservoir, 139.

Ventilation, 442 ff.; natural, 443; mechanical systems of, 449 .

Ventral, 9.

Ventricles, of heart, $22,24,137$; of brain, 268, 269.

Vertebra, 14, 16.

Vertebral column. See Spinal column.

Villus of intestine, 116 (fig.), 117, 118 (fig.), 123 (fig.).

Visual judgments, 256.

Visual sensations, 255.

Vital resistance, $297,482$.

Vitreous humor, 247, 251 (fig.).

Volitional actions or movements, 82, 279, 282.

Walking as a means of exercise, 319. See Locomotion.

Wandering cells, 134 .

Warm weather, and feeding, 156 ; circulation in, 150.

Warm-blooded animals, 189.

Warming of the house, $434 \mathrm{ff}$.; by open fire, 434 ; by stoves, 435 ; by hot-air furnaces, 435 ; by steam and hot water, 437.

Warmth, sensations of, 261.

Waste products, 49, 52, 59, 65, 177 ; excretion of, 225.
Water, as a food, 93, 218, 219; and the secretion of urine, 181 ; use as a drink, 354.

Water supply, of the house, 451; purity of public, 513,526 .

Waters, hard and soft, 454.

Wells, 452.

White matter of spinal cord and b: ain, 75 .

Wh' oping cough, 493.

Will, 82.

Wind and temperature regulation, 203.

Wines, 365 .

Winking, muscular and nervous mechanism of, 70 .

Woolen underwear, 421.

Work, unit of, 212.

Wort, 365.

Wounds, care of, 503.

Wrist, bones of, 19 .

Writing, 283.

Xanthin bases, 178.

Yeast, 364.

Yellow fever, 492.

Zymogen, 48. 


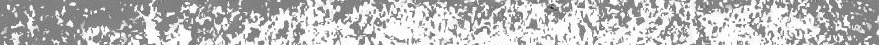

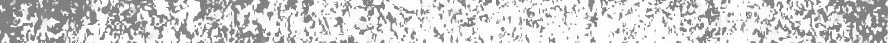

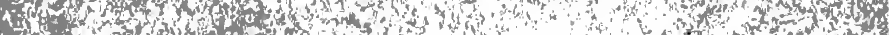

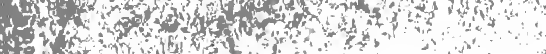

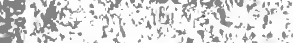

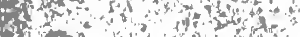

$+1$

H The

ant

Q

* a

"द्धार?"

$3 \lim ^{2}$

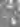

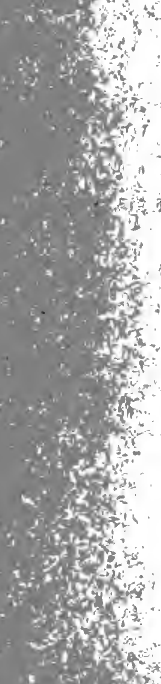

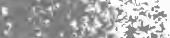
$4 x^{2}=30$

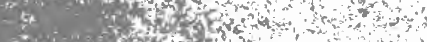

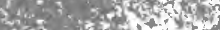

is: Ex

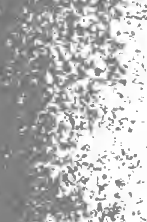
angers? $+3$ $-10$

(3)

$+4$

Pij

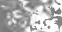


RETURN TO the circulation desk of any

University of California Library

or to the

NORTHERN REGIONAL LIBRARY FACILITY

Bldg. 400, Richmond Field Station

University of California

Richmond, CA 94804-4698

\section{ALL BOOKS MAY BE RECALLED AFTER 7 DAYS}

- 2-month loans may be renewed by calling (510) 642-6753

- 1-year loans may be recharged by bringing books to NRLF

- Renewals and recharges may be made 4 days prior to due date

\section{DUE AS STAMPED BELOW}

AUJG 052005

DD20 6M 9-03 
$38 \% 3 \%$

Hough, T.

H6

The human mechanism.

$2 p 34$

Hough, T.

$1+6$

38237

LIBRARY, COLLEGE OF AGRICULTURE, DAVIS

UNIVERSITY OF CALIFORNIA 
